\title{
DIGITALCOMMONS
}

$5-1-2013$

\section{Vol. 12, No. 1 (Full Issue)}

JMASM Editors

Follow this and additional works at: http:// digitalcommons.wayne.edu/jmasm

\section{Recommended Citation}

Editors, JMASM (2013) "Vol. 12, No. 1 (Full Issue)," Journal of Modern Applied Statistical Methods: Vol. 12 : Iss. 1 , Article 31. DOI: $10.22237 /$ jmasm/1367382600

Available at: http://digitalcommons.wayne.edu/jmasm/vol12/iss1/31 


\section{In Memory}

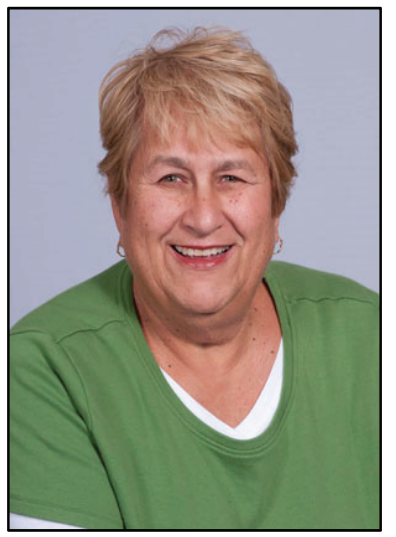

Dr. Gail Frances Fahoome

Wayne State University, Detroit, MI

We at JMASM were saddened by the unexpected passing of our colleague and friend Dr. Gail Fahoome on May 27, 2013. She completed her Bachelor of Science at Wayne State University (WSU) in 1992. She obtained a teaching certificate in secondary mathematics and in computer science, and taught high school mathematics in the Detroit Public Schools. She returned to WSU in 1994 to pursue a Masters' degree in Educational Evaluation and Research (EER), conducting her thesis under the supervision of Prof. Shlomo S. Sawilowsky. Subsequently, she earned her Ph. D. in EER in 1999, with Prof. Sawilowsky serving as her major professor.

Dr. Fahoome became an adjunct instructor at WSU in 1999. Her dissertation results appeared in $J M A S M$ (2002, Twenty nonparametric statistics and their large-sample approximations, p. 248268), a massive undertaking that became the definitive source on this topic. In 2003, she coauthored (with Prof. Sawilowsky) Statistics through Monte Carlo Simulation with Fortran (ISBN: 0-9740236-0-4). She also published in the Annals of Family Medicine, the Journal of Sport and Exercise Physiology, the Academic Emergency Medicine journal, and the Encyclopedia of Behavioral Statistics. She was a reviewer for the Journal of the American Board of Family Medicine, Family Practice, and Sage Publications.

She arose through the ranks at WSU, as Lecturer (2000), Senior Lecturer (2006), and Assistant Professor - Clinical (2008). She also served as an adjunct faculty member from $1998-2005$ at Davenport University. A member of the American Evaluation Association, her areas of expertise included quantitative program evaluation, Monte Carlo methods, and structural equation modeling. In 2010, Dr. Fahoome became the Chair of the EER program. She was the advisor for EER Masters' students, served on over forty doctoral dissertation committees, and taught twelve different graduate level research methods, evaluation, and applied statistics courses.

Gail Fahoome will be missed and fondly remembered by all who knew her. 


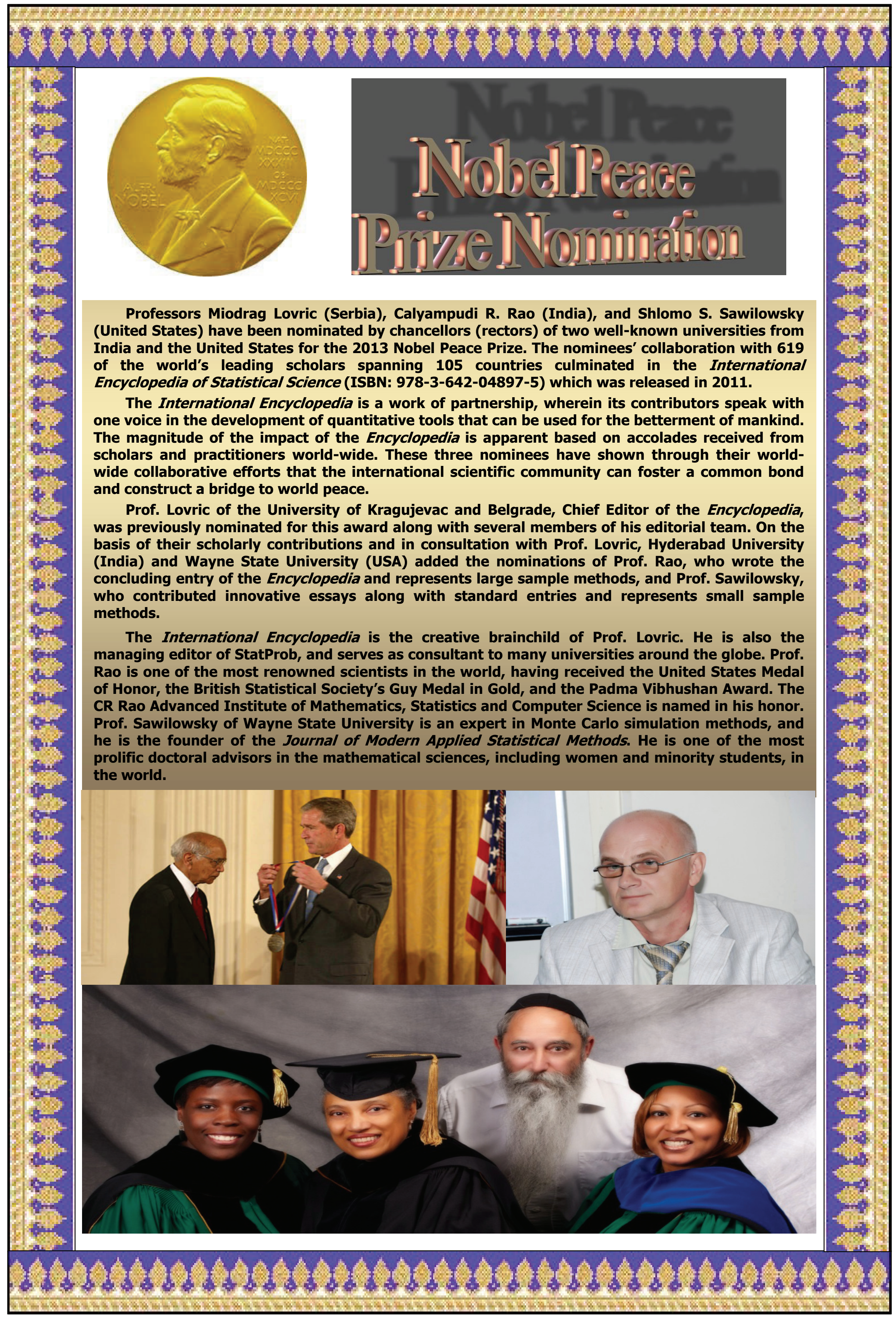




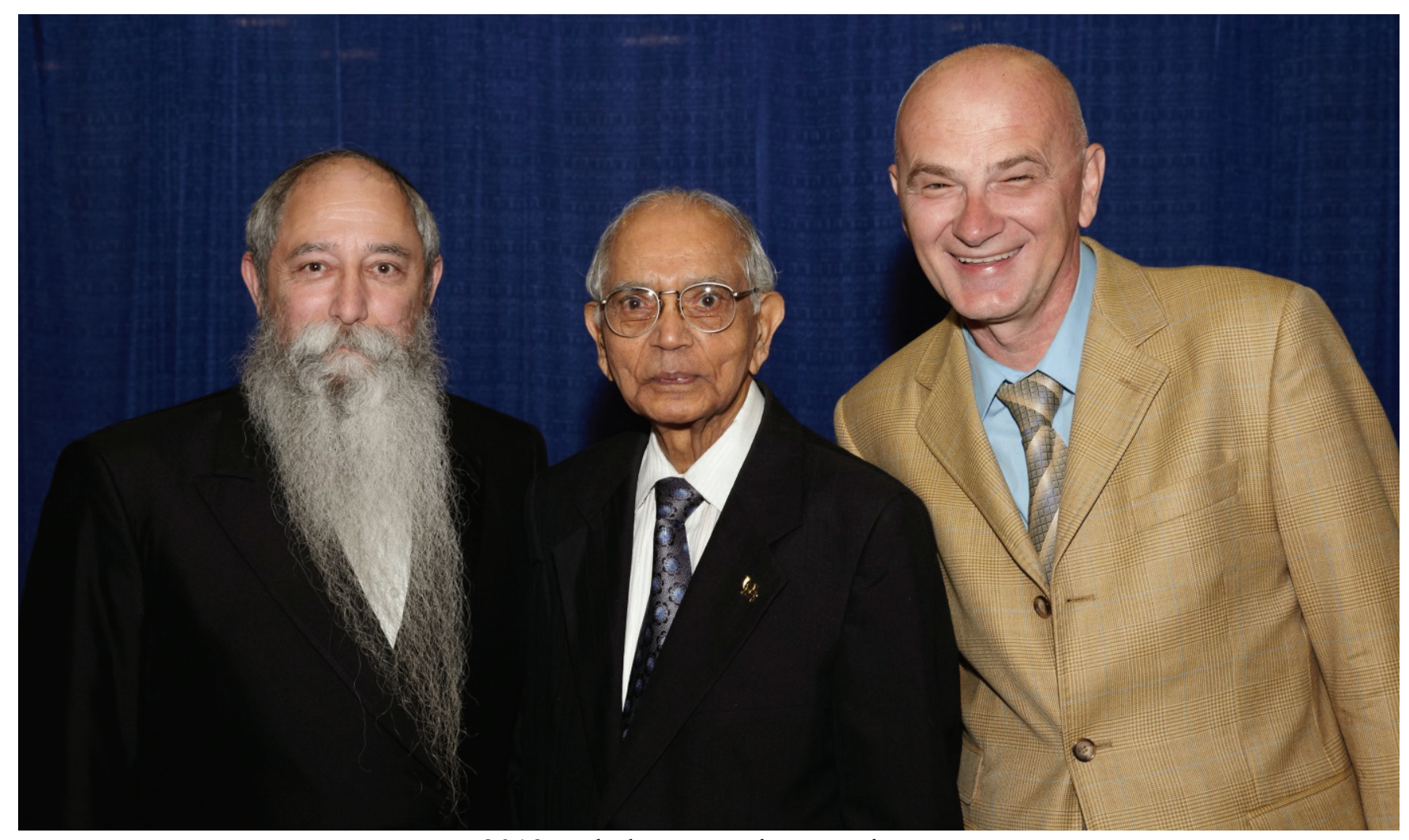

2013 Nobel Peace Prize Nominees

L to R: Prof. Shlomo S. Sawilowsky (USA), Prof. Calyampudi R. Rao (India), Prof. Miodrag Lovric (Serbia) 


\section{Journal Of Modern Applied Statistical Methods}

Shlomo S. Sawilowsky

Editor

College of Education

Wayne State University

Harvey Keselman

Associate Editor

Department of Psychology

University of Manitoba

Bruno D. Zumbo

Associate Editor

Measurement, Evaluation, \& Research Methodology

University of British Columbia

Vance W. Berger

Assistant Editor

Biometry Research Group

National Cancer Institute

John L. Cuzzocrea

Assistant Editor

Educational Research

University of Akron

Todd C. Headrick Assistant Editor

Educational Psychology and Special Education

Southern Illinois University-Carbondale

Alan Klockars
Assistant Editor

Educational Psychology

University of Washington 


\section{Journal Of Modern Applied Statistical Methods}

Invited Debate

$2-8$

$9-10$

$11-17$

18

$19-20$

Regular Articles

$21-33$

$34-44$

$45-53$

$54-57$

$58-64$

$65-77$
Richard B. Anderson

Philip Good

Fortunato Pesarin, Stefano Bonnini

Bryan Manly

Richard B. Anderson

William T. Mickelson

Spyros Konstantopoulos

Maher Qumsiyeh

Makarand Ratnaparkhi, Uttara Naik-Nimbalkar

J. Subramani,

G. Kumarapandiyan

Stan Lipovetsky
Conceptual Distinction between the Critical $p$ Value and the Type I Error Rate in Permutation Testing

$p$-Values versus Significance Levels

A Response to Anderson's (2013)

Conceptual Distinction between the Critical $p$ value and Type I Error Rate in

Permutation Testing

Randomization Test $p$-Values versus Significance Levels

Conceptual Distinction between the Critical $p$ Value and the Type I Error Rate in Permutation Testing: Author Response to Peer Comments

A Monte Carlo Simulation of the Robust Rank-Order Test Under Various Population Symmetry Conditions

Constructing a More Powerful Test in Two-Level Block Randomized Designs

Using the Bootstrap for Estimating the Sample Size in Statistical Experiments

The Length-Biased Versus Random Sampling for the Binomial and Poisson Events

Estimation of Variance Using Known Coefficient of Variation and Median of an Auxiliary Variable

Priorities in Thurstone Scaling and SteadyState Probabilities in Markov Stochastic Modeling 


$\begin{array}{ll}78-89 & \begin{array}{l}\text { Miguel A. Padilla, } \\ \text { Jasmin Divers }\end{array} \\ 90-102 & \begin{array}{l}\text { Thomas Laloë, } \\ \text { Rémi Servien }\end{array} \\ 103-119 & \begin{array}{l}\text { Ayman Alzaatreh, } \\ \text { Kristen Knight }\end{array} \\ 120-127 & \begin{array}{l}\text { Sunil Kumar } \\ 128-147\end{array} \\ & \begin{array}{l}\text { Grace Lee Ching Yap, } \\ \text { Wan Rosmanira Ismail, } \\ \text { Zaidi Isa }\end{array}\end{array}$

$148-163$

$164-169$

$170-180$

$181-197$

$198-206$

$207-219$

$220-226$

\author{
Saad Saeed Alkahtani
}

Mehdi Razzaghi

M. A. A. Cox

E. B. Nkemnole,

O. Abass,

R. A. Kasumu

R. R. L. Kantam,

V. Ramakrishna,

M. S. Ravikumar

Emily A. Blood, Leslie A. Kalish, Lydia A. Shrier

Zakaria M. Sawan
Bootstrap Interval Estimation of Reliability via Coefficient Omega

The X-Alter Algorithm: A Parameter-Free Method of Unsupervised Clustering

On the Gamma-Half Normal Distribution and Its Applications

Improved Estimators in Finite Population Surveys: Theory and Applications

An Alternative Approach to Reduce Dimensionality in Data Envelopment Analysis

Robustness of DEWMA versus EWMA Control Charts to Non-Normal Processes

The Probit Link Function in Generalized Linear Models for Data Mining Applications

An Approximate Approach to the Economic Design of Charts By Considering the Cost of Quality

Parameter Estimation of a Class of Hidden Markov Model with Diagnostics

Estimation and Testing in Type I

Generalized Half Logistic Distribution

Estimating Heterogeneous Intra-class Correlation Coefficients in Dyadic Ecological Momentary Assessment

An Approach for Dealing with Statuses of Non-Statistically Significant Interactions between Treatments 
$227-234$

$235-248$

Xing Liu,

Hari Koirala

Brief Report

$249-254$

Makarand Ratnaparkhi, Uttara Naik-Nimbalkar
Bayesian Inference of Pair-Copula

Constriction for Multivariate Dependency

Modeling of Iran's Macroeconomic

Variables

Fitting Proportional Odds Models to Educational Data with Complex Sampling

Designs in Ordinal Logistic Regression
A Note on $\alpha$-Curvature of the Manifolds of the Length-biased Lognormal and Gamma Distributions in View of Related Applications in Data Analysis

Emerging Scholars $255-260$

\section{Nur Syabiha Zafakali, Wan Muhamad Amir W Ahmad}

JMASM Algorithms and Code $261-275$

\author{
Modeling and Handling Overdispersion \\ Health Science Data with Zero-Inflated \\ Poisson Model
}

$J M A S M$ is an independent print and electronic journal (http://www.jmasm.com/), publishing (1) new statistical tests or procedures, or the comparison of existing statistical tests or procedures, using computer-intensive Monte Carlo, bootstrap, jackknife, or resampling methods, (2) the study of nonparametric, robust, permutation, exact, and approximate randomization methods, and (3) applications of computer programming, preferably in Fortran (all other programming environments are welcome), related to statistical algorithms, pseudorandom number generators, simulation techniques, and self-contained executable code to carry out new or interesting statistical methods.

Editorial Assistant: Julie M. Smith, Ph. D. 


\title{
Invited Debate \\ Conceptual Distinction between the Critical $p$ Value and the Type I Error Rate in Permutation Testing
}

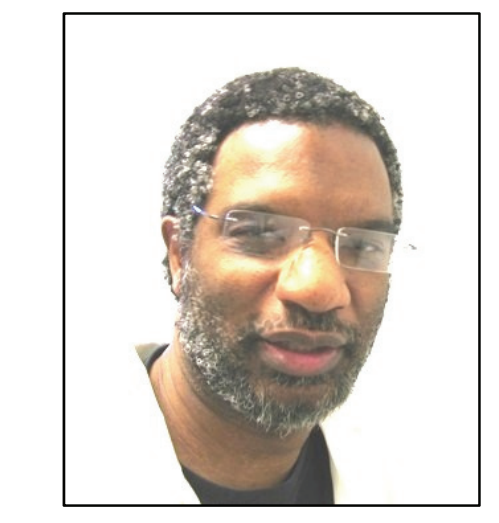

\author{
Richard B. Anderson \\ Bowling Green State University \\ Bowling Green, $\mathrm{OH}$
}

To counter past assertions that permutation testing is not distribution-free, this article clarifies that the critical $p$ value (alpha) in permutation testing is not a Type I error rate and that a test's validity is independent of the concept of Type I error.

Key words: Statistics, null hypothesis, non-parametric, permutation test, exchangeability; Type I error; validity.

Introduction

Traditional parametric tests, such as $t$ and $F$ tests, are said to be robust against violation of the normality assumption (e.g., Keppel \& Wickens, 2004), but researchers often hesitate to apply such tests when the extent of the violation is obvious or severe. For example, it is known

Richard Anderson is an Associate Professor of Psychology in the Department of Psychology. His research interests include topics in judgment, decision-making, reasoning, memory, statistical cognition and statistical methods. He teaches courses in cognition, statistics and research methods and holds memberships in the Psychonomic Society and the Cognitive Science Society. Email him at: randers@bgsu.edu. that most parametric tests are not robust against violations of equal variance and that the situation is exacerbated when sample sizes are unequal (Howell, 2012). Thus, non-parametric tests, including permutation tests (e.g., Edgington \& Onghena, 2007; Fisher, 1935; Good, 2011; Ludbrook \& Dudley, 1998), have become increasingly popular. Some critiques of permutation tests have questioned whether such tests are genuinely distribution-free in the sense of being valid irrespective of the shapes of the population distributions (Hayes, 1997, 2000; Mewhort, Kelley \& Johns, 2009). This article clarifies and demonstrates that the critical $p$ values for permutation tests are not estimates of Type I error probability and that the divergence of the two values does not impugn the validity of the permutation test's $p$ value. 


\section{RICHARD B. ANDERSON}

The Logic of the Permutation Test

As described by Edgington \& Onghena (2007), Fisher (1935), Good (2011) and others, permutation testing entails the following steps. First, the investigator must formulate the null hypothesis as one that meets the exchangeability requirement. That is, when the null hypothesis is true, the coupling of particular values of the dependent variable with particular values of the independent variable is random. This idea can be further explicated by imagining a failed experiment in which a researcher randomly assigns each of several participants to complete a state-anxiety questionnaire while experiencing either silence (the no noise group) or loud automobile traffic noise (the loud noise group). When the experiment ends, it is discovered that someone forgot to plug-in the machine that plays the recorded noise. Each score in the data set has a label, no noise or loud noise, but the labels are meaningless because of the failed manipulation. Thus, each score's attachment to the no noise versus the loud noise label might as well be random; in other words, when the null hypothesis is true, the scores are exchangeable across the labels. This concept shall be referred to as exchangeability under the null, to emphasize that the exchangeability defines the null hypothesis only. (The scores would not, and could not, be exchangeable when the null hypothesis is false.)

In permutation testing, the null hypothesis is not more specific than the proposition that the coupling of particular values of the dependent variable with particular values of the independent variable is random. For example, whereas a parametric test may assess the null hypothesis that the means are equal across groups, a permutation test is restricted to testing the null hypothesis of random coupling of values to condition labels. (Note: Howell (2013) states that this is also true for the Wilcoxon Rank-Sum test, which is a form of permutation test wherein the data are transformed to ranks prior to permutation.) Having conceptualized the null hypothesis as entailing the exchangeability of scores, the next step is to characterize the sample at hand in terms of one or more test statistics, such as the difference between the means, between the medians or between the variances.
Finally, the principle of exchangeabilityunder-the-null permits computation of a form of $p$ value that is the probability that an effect as large as (or larger than) one computed prior to permutation would occur by chance. This chance process is simulated by repeatedly reassigning the scores to the labels that represent the levels of the independent variable. Following each permutation - that is, each set of reassignments - the test statistic is recomputed. The recomputed statistic is counted as having met the threshold of the original statistic if the former is equal to or more extreme than the latter. (In the case of a two-tailed test, the recomputed and the original statistic are transformed to their absolute values prior to comparison to one another.) An exact $p$ value is obtained by calculating the proportion of all possible unique permutations that produce an outcome at least as extreme as the statistic computed for the original, unpermuted data (Fisher's Exact test is an example). Unless the data set is small, it is often more practical to obtain an approximate, Monte Carlo $p$ value by calculating the aforementioned proportion for a large number of random permutations (which may occasionally, and by chance, include repetitions of particular permutation patterns) rather than for all possible permutations. In the remainder of this article, permutation test will refer to the Monte Carlo variety unless otherwise specified.

Note that permutation tests can be conceptualized as drawing samples (permutations) from a population, where the socalled population is what a parametric test would regard as a sample (Rodgers, 1999). However, the relationship between the sample and the population in parametric testing is not parallel to the relationship between the samples and the population in permutation testing. In parametric testing the goal is to use a sample statistic to infer a population parameter; in permutation testing the parameters of the so-called population are known and need not be inferred. Permutation-test logic does not use samples to make inferences about population parameters. Rather, a permutation test makes inferences about process. Specifically, it assesses the probability that a random process in which data values are coupled to condition labels would 


\section{THE CRITICAL $P$ VALUE AND TYPE I ERROR RATE IN PERMUTATION TESTING}

produce a data set characterized by a given test statistic.

The permutation test is widely used in the form of Fisher's Exact test (and the Monte Carlo variant of Fisher's test) for analyzing relative frequencies in dichotomous data. Other forms of the permutation test have been used infrequently to date but can be implemented with the help of statistical software (e.g., Anderson, 2012; R, version 2.15.1; Stata, version 12.0).

A Permutation Test's Critical $p$ Value $(\alpha)$ is not an Estimate of the Type I Error Rate

Some have argued that permutation tests entail highly restrictive assumptions. Hayes (1996), for example, wrote that the "permutation test is not distribution free" (p.1). As an example, he found that in simulated correlational data, under conditions in which the $x, y$ correlation was zero and in which the variance among $y$ observations differed as a function of $x$, both permutation tests and parametric tests rejected the null hypothesis more often than the rate suggested by the tests' $p$ values.

Mewhort, et al. (2009) explored a phenomenon in which, when the null hypothesis is true, unequal variance interacts with unequal sample size. In a set of simulations, the researchers created pairs of populations that could have equal or unequal means and that could have equal or unequal variances. They then sampled from the populations to produce a large number of two-group data sets; the two groups could be equal or unequal in size. Thus, some of the data sets consisted of groups that differed both in size and in variance. The researchers conducted a permutation test on each set to assess the rate at which the tests produced Type I errors. With the critical $p$ value $(\alpha)$ set at 0.05 , the researchers found that when the population variances and the sample sizes were unequal and when the smaller sample had been drawn from the population with the higher variance, the actual Type I error rate was somewhat higher than 0.05 . Conversely, when the smaller sample had been drawn from the population with the lower variance, the actual Type I error rate was somewhat lower than 0.05 . The authors went on to propose an algorithm to correct the permutation test's apparent bias (for situations which the smaller group is characterized by higher variance).

I argue that there is a conceptual difficulty in using a permutation test's $p$ value as a standard to evaluate a test's liberalness or conservativeness. In a traditional, parametric test, $p$ refers to a portion of a hypothetical distribution of the values of a test statistic (e.g., a distribution of $t$ scores) that would be obtained if one were to generate multiple data sets, via random sampling from a population or set of populations, and then compute the test statistic for each data set. This meaning of $p$ allows a researcher to evaluate empirically whether $p$ is liberal, conservative or unbiased. This is done by drawing multiple data sets from a population (or set of populations) in which the null hypothesis is known to be true. The researcher then establishes a critical $p$ value $(\alpha)$ and computes the obtained Type I error rate as the proportion of simulated data sets in which the $p$ value is less than or equal to $\alpha$. The statistical test is liberal or conservative to the degree that the obtained Type I error rate tends to be higher or lower (respectively) than $\alpha$.

The $p$ value of a permutation test has a meaning very different from the one previously described, thus the term $p_{t}$ will be used to refer to the $p$ value produced by a traditional, parametric test, and $p_{\text {perm }}$ will be used to refer to the kind of $p$ value produced by a permutation test.

The value $p_{\text {perm }}$, in contrast to $p_{t}$, is about only the data at hand. Therefore, $p_{\text {perm }}$ does not pertain to populations, or to multiple samples that could have been draw from a population or to multiple values of a statistic that could have been computed from multiple episodes of random sampling. Instead, $p_{\text {perm }}$ is the rate at which the various possible re-assignments of scores to condition labels lead to an effect that matches or exceeds the magnitude of the effect in the un-permuted data. Likewise, $p_{\text {perm }}$ 's critical value is conceptually distinct from $p_{t}$,'s critical value. This can be observed clearly when considering that a permutation test is valid and useful even when conducted on the entirety of a finite population. Consider the following example: For the uppercase letters in the Modern English alphabet is the central tendency of their ordinal positions significantly different for 


\section{RICHARD B. ANDERSON}

symmetrical letters (e.g., "A") than for asymmetrical letters (e.g., "B")?

The question is nonsensical within the framework of parametric testing because the letters in this data set are not sampled (randomly or otherwise) from populations. But the question is eminently sensible from the standpoint of permutation testing. The null hypothesis is that the coupling of ordinal positions with the condition labels symmetrical and asymmetrical has occurred by chance. An alternative hypothesis, that the two conditions differ in the medians of their ordinal positions, is assessed by repeatedly permuting the values of the serial positions across the condition labels and recalculating the medians. For this example, the median ranks for the symmetrical and asymmetrical letters (uppercase) are 20 and 11, respectively (Test Statistic $[50,000$ permutations $]=$ Median $_{\mathrm{A}}-\operatorname{Median}_{\mathrm{B}}, p_{\text {perm }} \approx$ .047). Thus, there is a significant tendency for symmetrical uppercase letters to occur later rather than earlier in the alphabet. Such a finding sets the stage for further scientific inquiry into the genesis of the alphabet, and more importantly, could not have arisen from classical, parametric statistical analysis.

There are two reasons why it would not make sense to ask whether the $p_{\text {perm }}$ value, above, is liberal or conservative. First, there is no imaginable population - simulated or otherwise - from which additional Modern English alphabets could be sampled. Consequently, there is no basis for computing a Type I error rate across samples from such a population. Second, and just as importantly, even if one could imagine that the Modern English alphabet is just one random sample among many possible random samples, $p_{\text {perm }}$ would still pertain only to permutations of the data at hand and not to a sampling distribution. Therefore, simulated (or otherwise obtained) Type I error rates such as those generated by Hayes (1996, 1997) and Mewhort, et al. (2009) cannot serve as standard to assess bias in $p_{\text {perm }}$ because $p_{\text {perm }}$ is unrelated to Type I error and $p_{\text {perm }}$ 's critical value does not estimate a Type I error rate.
Is Possible to Estimate a Legitimate Type I Error Rate for a Permutation Test?

When a permutation test is conducted on an entire population of values, the concept of a Type I error rate is meaningless because there is no family of alternate samples over which a Type I error rate can be computed, thus in some circumstances, it is impossible to estimate a Type I error rate. But what about situations in which one computes $p_{\text {perm }}$ for data that happen to have been sampled randomly from a population? In such a situation, can a Type I error rate be defined? The difficulty in answering yes in this case is that any such error rate must be defined with respect to the permutation test's null hypothesis and not with respect to a parametric null hypothesis. For example, imagine a simulation in which two populations have identical means and in which random samples are repeatedly drawn from the two populations. Computing the Type I error for a parametric test involves simply counting the proportion of samples that lead to the rejection of the null hypothesis of equal population means are equal. However, for a permutation test, such a parametric null hypothesis is not the relevant null hypothesis. Instead, the relevant null is that the arrangement of the data within a fixed set (not multiple, randomly sampled sets) reflects the random coupling of data values to condition labels. Thus, the random sampling procedure described above does not provide a basis on which to assess the rate of incorrect rejection of the particular null hypothesis tested by a permutation test.

Tests on Simulated Data

I now present what I believe to be a conceptually coherent assessment of Type I errors in permutation testing. Within a given simulation, the following procedure is employed:

(1) Decide on a set of numbers (i.e., a seed set) that will be constant throughout the simulation.

(2) Prior to conducting any permutation tests, perform many permutations of the data set. Each result of this initial set of permutations 


\section{THE CRITICAL $P$ VALUE AND TYPE I ERROR RATE IN PERMUTATION TESTING}

is not part of a permutation test, but instead constitutes a data set to be analyzed via a permutation test. Such permutation-prior-totesting is necessary because it produces data sets in which the null hypothesis (which is the true hypothesis in the present simulations) is of the permutation-test variety rather than the parametric-test variety.

It should be noted that while it would be possible to arrange things so that the two groups would differ systematically on some dimension (in their variances, for example), this would make the null hypothesis false rather than true. Such a situation would allow a permutation-test assessment of Type II errors (i.e., erroneous acceptance of the null hypothesis of random coupling of data values to condition labels), but it would miss the point of the present simulations, which is to assess Type I rather than Type II errors.

(3) Conduct the permutation tests in the usual manner. However, unlike typical simulations (e.g., Hayes, 1996, 1997; Mewhort, et al., 2009), each test is conducted on a data set generated by permuting a set that has not been sampled from a population and whose membership is the same for all tests.

(4) Compute the Type I error rate as the proportion of tests that reject the null hypothesis. But do not subsequently compare the Type I error rate to the conceptually distinct, critical $p_{\text {perm }}$.

Three simulations were conducted. In Simulation 1, the seed data set was the set of consecutive, non-repeating integers 1 through 30. Thus, the seed data were uniformly distributed. In Simulation 2, the seed data consisted of 30 values that were the squares of the 30 values in Simulation 1. Thus in Simulation 2, the seed data were exponentially distributed. In Simulation 3 the seed data consisted of the cubes of values in Simulation 1.

In all simulations, the procedure was as follows. The 30 seed values were randomly permuted 1,000 times across two equal-sized groups, yielding 1,000 data sets, with each set composed of 30 unique values divided among two groups ( $n=15$ per group). Each of the 1,000 two-group data sets was then submitted to a permutation test of the difference between means $\left(\right.$ Test Statistic $=$ mean $_{1}-$ mean $_{2} ; 1,000$ random permutations) and a permutation test of the difference between variances (Test Statistic $=$ variance $_{1}-$ variance $_{2} ; 1,000$ random permutations). Overall, each simulation included 1,000 times 1,000 permutations of data. For each permutation test, the critical $p_{\text {perm }}$ was set at .05 . The results are shown on Table 1.

Perhaps the most important aspect of Table 1 is that is does not permit assessment of the validity of $p_{\text {perm. }}$. Because neither $p_{\text {perm }}$ nor its critical value pertain to the Type 1 error rate, the table does not permit comparison of the Type I error rates to a standard (Note: The critical $p_{\text {perm }}$ is not such a standard.) Another result is that the computed Type I error rates happen to correspond (roughly) to the value of $p_{\text {perm }}$, irrespective of whether the test assesses differences in means or differences in variances and irrespective of which seed data set served as the basis for generating the tested data. Finally, the simulations show that Type I error rates in permutation testing can meaningful (though such error rates are not essential), despite the absence of random sampling from populations.

\section{Implications}

The present arguments show that the critical $p_{\text {perm }}$ for a permutation test is not a Type I error rate, and that consequently, a permutation test's validity does not depend on whether the numerical value of $p_{\text {perm }}$ matches the Type I error rate that would be relevant to a parametric test. It is also clear that the true Type I error rate for a family of permutation tests will likely depend on how one chooses to define the family tests. In my view, the absence of a well-defined Type I error rate in no way impugns the validity or usefulness of permutation testing. The test computes the probability that a random process could have produced the observed assignment of data to condition labels, and this is a sufficient basis for deciding whether to reject the hypothesis of a random process. Thus, unlike other statistical approaches, permutation testing entails no parametric assumptions, and does not require the to-be-analyzed data to be randomly sampled from a population. 
Table 1: The Mean of Group Means, Mean of Group Variances, Central Tendencies of $p_{p e r m}$ and Type I Error Rates for Three Different Seed Data Sets

\begin{tabular}{|c|c|c|c|c|c|c|c|c|}
\hline \multirow{2}{*}{ Seed } & \multicolumn{2}{|c|}{ Descriptives } & \multicolumn{3}{c|}{ Test of Means } & \multicolumn{3}{c|}{ Test of Variances } \\
\cline { 2 - 9 } & $\begin{array}{c}M \text { of } M_{1} \\
\left(M \text { of } S_{1}{ }^{2}\right.\end{array}$ & $\begin{array}{c}M \text { of } M_{2} \\
\left(M \text { of } S_{2}{ }^{2}\right.\end{array}$ & $\begin{array}{c}M \text { of } \\
p_{\text {perm }}\end{array}$ & $\begin{array}{c}M d n \text { of } \\
p_{\text {perm }}\end{array}$ & $\begin{array}{c}\text { Type } 1 \\
\text { Error Rate }\end{array}$ & $\begin{array}{c}M \text { of } \\
p_{\text {perm }}\end{array}$ & $\begin{array}{c}M d n \text { of } \\
p_{\text {perm }}\end{array}$ & $\begin{array}{c}\text { Type 1 } \\
\text { Error Rate }\end{array}$ \\
\hline Uniform & $\begin{array}{c}15 \\
(72)\end{array}$ & $\begin{array}{c}16 \\
(73)\end{array}$ & .509 & .501 & .047 & .504 & .512 & .049 \\
\hline Squared & $\begin{array}{c}316 \\
(73623)\end{array}$ & $\begin{array}{c}315 \\
(73797)\end{array}$ & .493 & .487 & .058 & .486 & .474 & .056 \\
\hline Cubed & $\begin{array}{c}7251 \\
(62408539)\end{array}$ & $\begin{array}{c}7164 \\
(62667777)\end{array}$ & .500 & .500 & .046 & .500 & .488 & .045 \\
\hline
\end{tabular}

Notes: For each permutation test, the critical value of $p_{\text {perm }}$ was 0.05 . This critical value does not refer to and is conceptually distinct from the Type I error rate. For each tested data set, the null hypothesis is true in that the coupling of data values to group labels is random.

\section{Conclusion}

Although this article demonstrates that permutation testing's validity is independent of the idea of Type I error, it does not resolve all of the outstanding questions concerning permutation tests. For example, there is some uncertainty as to the formal relationship between the null hypothesis and the test statistic used to reject that null hypothesis. Consider the following data set: Group A $(10,8,77,2,40,92$, 88), Group B (7, 4, 2, 5, 5, 3, 3). With a critical $p_{\text {perm }}$ of 0.05 and with 50,000 permutations, the null hypothesis of random coupling of values to group labels is rejected, whether the test statistic is the difference between means $\left(p_{\text {perm }} \approx 0.01\right)$, between medians $\left(p_{\text {perm }} \approx 0.03\right)$, or between variances $\left(p_{\text {perm }} \approx 0.02\right)$. Yet, in all three cases, the rejected null hypothesis - that is, that the coupling of values to condition labels is random - is precisely the same hypothesis. Thus, there is unresolved ambiguity concerning the degree to which each of the three tests, above, provides unique information about the null hypothesis.

\section{Limitations}

There is also a question about the possible definitions of a type one error rate, within permutation testing. The simulations in this study utilized just a few of an infinite number of possible seed data sets. In principle each seed set could yield a different Type I error rate, therefore, it is not known whether this type of simulation typically yields Type I error rates that approximate the critical $p_{\text {perm. }}$. Moreover, there are likely other methods (besides the permutation of seed data) for generating a family of data sets to serve as the basis for computing Type I error. This article does not resolve these questions. Nevertheless, if future research on permutation testing is to provide such answers, the Type I error rates must be established either empirically or by some means that does not interpret $p_{\text {perm }}$ to refer to a Type I error rate. It should be reiterated, however, that the logic of permutation testing does not require establishing a Type I error rate.

\section{Acknowledgements}

The author wishes to thank Dr. Michael Doherty, Dr. John Tisak, Dr. Mark Appelbaum, Dr. Joseph Rodgers and Don Zhang for their helpful comments on earlier drafts of the manuscript.

\section{References}

Anderson, R. B. (2012). Anderson's permutation tester. Available at: https://sites.google.com/site/complexcognitionb $\mathrm{gsu} /$ calculators. 


\section{THE CRITICAL $P$ VALUE AND TYPE I ERROR RATE IN PERMUTATION TESTING}

Edgington, E. S., \& Onghena, P. (2007). Randomization tests ( $4^{\text {th }} E d$.). Boca Raton, FL: Taylor \& Francis Group.

Fisher, R. A. (1935). The design of experiments. Edinburgh: Oliver \& Boyd.

Good, P. I. (2011). A practitioner's guide to resampling for data analysis, data mining, and modeling. Boca Raton, FL: Taylor \& Francis Group.

Hayes, A. F. (1996). Permutation test is not distribution-free: Testing $\mathrm{H}_{0}: \quad \rho=0$. Psychological Methods, 1, 184-198.

Hayes, A. F. (1997). Cautions in testing variance equality with randomization tests.

Journal of Statistical Computation and Simulation, 59, 25-31.

Hayes, A. F. (2000). Randomization tests and the equality of variance assumption when comparing group means. Animal Behaviour, 59, 653-656.
Howell, D. C. (2012). Statistical methods for psychology ( $8^{\text {th }} E d$.). Belmont, CA: Wadsworth Publishing.

Keppel, G., \& Wickens, T. D. (2004). Design and analysis: A researcher's handbook $\left(4^{\text {th }} E d\right.$.). Upper Saddle River, NJ: Pearson Education.

Ludbrook, J., \& Dudley, H. (1998). Why permutation tests are superior to $t$ and $F$ tests in biomedical research. American Statistician, 52, 127-132.

Mewhort, D. J. K., Kelly, M., \& Johns, B. T. (2009). Randomization tests and the unequal-N/unequal-variance problem. Behavior Research Methods, 41, 664-667.

Rodgers, J. L. (1999). The bootstrap, the jackknife, and the randomization test: A sampling taxonomy. Multivariate Behavioral Research, 34, 441-456. 


\section{$p$-Values versus Significance Levels}

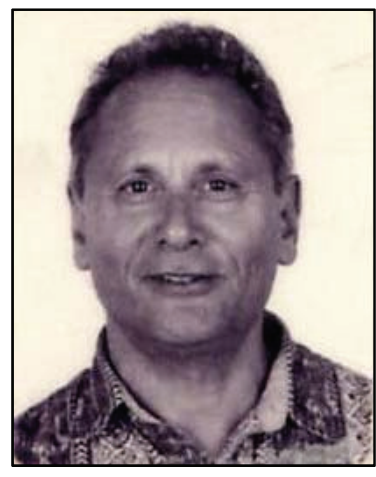

Phillip Good

Information Research, Huntington Beach, CA

In this article Phillip Good responds to Richard Anderson's article Conceptual Distinction between the Critical $p$ Value and the Type I Error Rate in Permutation Testing.

Key words: Statistics, null hypothesis, non-parametric, permutation test, exchangeability; Type I error; p-value, discrete set, significance level, validity.

\section{Introduction}

As a consequence of Richard Anderson eschewing formal definitions, I wish to clarify our understanding of permutation procedures.

A statistical procedure is said to have significance level $\alpha$ if one can expect the hypothesis $\mathrm{H}$ to be rejected in error $\alpha \%$ of the time under the following conditions:

- $\mathrm{H}$ is true,

- The assumptions underlying the procedure are valid,

Philip Good is a statistical consultant. He authored numerous books that include Introduction to Statistics via Resampling Methods and R/S-PLUS and Common Errors in Statistics (and How to Avoid Them). Email: drgood@statcourse.com.
- Repeated samples are taken (not necessarily from the same population) and the procedure is applied to each of the samples independently.

Thus, a significance level is a property of a statistical procedure and takes a fixed value. In contrast, a p-value is a random variable whose value depends upon the composition of the individual sample.

These definitions apply regardless of the nature of the statistical procedure, whether it is permutation or parametric, optimal or suboptimal, providing the assumptions underlying the procedure are valid each time it is applied.

One can apply a permutation procedure and achieve or closely approximate the anticipated frequency of type I errors only if the observations that compose the sample are exchangeable (See Good (2002) for a formal definition of exchangeability.) 


\section{P-VALUES VERSUS SIGNIFICANCE LEVELS}

The failure to satisfy the requirement of exchangeability explains and renders invalid the so-called counter-examples offered by Hayes and by Mewhort and his colleagues.

Hayes (1996) draws samples from a population that is a mixture of bivariate normal distributions, each with the same mean and variance but differing rho. Although the couples $(\mathrm{x}, \mathrm{y})[\mathrm{i}]$ are exchangeable in this false counterexample as Hayes asserts, the variables y[i] are not when they are exchanged independently of the $\{x[j]\}$. Thus, a permutation distribution is not applicable for testing the hypothesis rho $=0$, and the simulations performed by Hayes were not necessary to confirm this.

As there are only a finite number of rearrangements of observations, permutation procedures yield only a finite number of possible p-values. They may or may not be able to achieve a predetermined significance level exactly. This is the case in Mewhort, et al. (2009). In addition, his observations are not exchangeable as the observations in the groups he examines have different variances.
References

Anderson, R. B. (2013). Conceptual Distinction between the Critical $\mathrm{p}$ Value and the Type I Error Rate in Permutation Testing. Journal of Modern Applied Statistical Methods, 12(1), 2-8.

Good, P. I. (2002). Extensions of the concept of exchangeability and their applications. Journal of Modern Applied Statistical Methods, 1, 243-247.

Hayes, A. F. (1996). Permutation test is not distribution-free: Testing $\mathrm{H}_{0}: \quad \rho=0$. Psychological Methods, 1, 184-198.

Mewhort, D. J. K., Kelly, M., \& Johns, B. T. (2009). Randomization tests and the unequal-N/unequal-variance problem. Behavior Research Methods, 41, 664-667. 


\section{A Response to Anderson's (2013) Conceptual Distinction between the Critical $p$ value and Type I Error Rate in Permutation Testing}

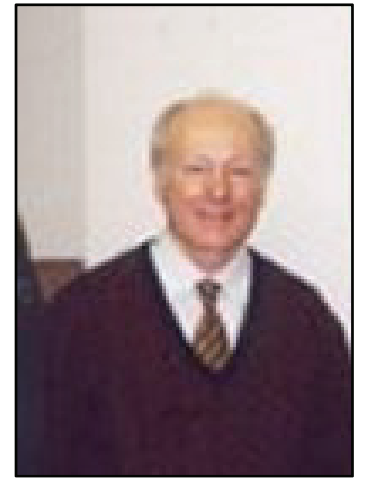

Fortunato Pesarin University of Padova, Padova, Italy

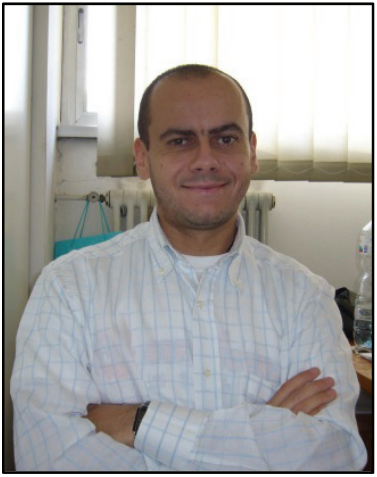

Stefano Bonnini

University of Ferrara, Ferrara, Italy

Pesarin and Bonnini respond to Anderson's (2013) Conceptual Distinction between the Critical p Value and the Type I Error Rate in Permutation Testing.

Key words: Statistics, null hypothesis, non-parametric, permutation test, exchangeability; Type I error; p-value, discrete set, significance level, validity.

Introduction

Anderson (2013) referred to various aspects of permutation $\mathrm{p}$-values. We agree with most of the notions and ideas heuristically discussed there; however, we present a more formal discussion to confirm and be more precise. The approach adopted in Pesarin (2001), Pesarin (2013) and Pesarin \& Salmaso (2010a, 2010b) considered permutation tests in light of the conditionality and sufficiency principles of inference, and we borrow freely from those citations.

Fortunato Pesarin is a Professor Emeritus of Statistics in the Department of Statistical Sciences. Email him at: pesarin@stat.unipd.it. Stefano Bonnini is Assistant Professor of Statistics in the Department of Economics and Management. Email him at: bnnsfn@unife.it.
Without loss of generality, consider a two-sample one-dimensional design for onesided alternatives where: $\boldsymbol{X}_{1}=\left(X_{1 i} ; i=1, \ldots, n_{1}\right)$ are data of first sample, $\boldsymbol{X}_{2}=\left(X_{2 i}, i=1, \ldots, n_{2}\right)$ are those of the second, and $\boldsymbol{X}=\left(\boldsymbol{X}_{1}, \boldsymbol{X}_{2}\right)=\left(X_{i}, i\right.$ $\left.=1, \ldots, n ; n_{1}, n_{2}\right)$ is the pooled set from which it is intended that the first $n_{1}$ are from the first sample and the remainder from the second. Denoting by $\Pi(\boldsymbol{u})$ the set of permutations of unit labels $\boldsymbol{u}=(1, \ldots, n)$ and by $\boldsymbol{u}^{*}=$ $\left(u_{1}^{*}, \ldots, u_{n}^{*}\right) \in \Pi(\boldsymbol{u})$ one of these, the related permutation of $\boldsymbol{X}$ is $\boldsymbol{X}^{*}=\left\{X_{i}^{*}=X\left(u_{i}^{*}\right), i=\right.$ $\left.1, \ldots, n ; n_{1}, n_{2}\right\}$. Thus, $\boldsymbol{X}_{\mathbf{1}}^{*}=\left\{X_{1 i}^{*}=X\left(u_{i}^{*}\right), i=\right.$ $\left.1, \ldots, n_{1}\right\} \quad$ and $\quad \boldsymbol{X}_{2}^{*}=\left\{X_{2 i}^{*}=X\left(u_{i}^{*}\right), i=n_{1}+\right.$ $1, \ldots, n\}$ denote the two permuted samples; in multidimensional problems vectors associated with units are permuted (Basso, et al., 2009; Pesarin, 2001; Pesarin \& Salmaso, 2010a; Pesarin \& Salmaso, 2011). The hypotheses are $H_{0}: X_{1} \stackrel{d}{=} X_{2}$ and $H_{1}: X_{1} \stackrel{d}{\geq} X_{2}$, respectively stating the equality and the dominance in 
distribution of two population variables. Note that $H_{0}$ implies data are exchangeable without being necessarily independent; in practice the exchangeability condition is usually attained when subjects are randomized to treatments or when related to observations on independent units.

Permutation tests are ordinary tests conditional on the observed data set $\boldsymbol{X}$, which in $H_{0}$ is always a sufficient $n$-dimensional statistic for the underlying (known or unknown) distribution $f$ in the sense that it contains all the information on $f$ that are contained in $\boldsymbol{X}$. Outside the regular exponential family of distributions $\boldsymbol{X}$ is generally minimal sufficient. In particular this implies that no suitable univariate statistic exists for summarizing the whole information on the aspect of interest for the analysis; therefore, no parametric or nonparametric method can aspire to be uniformly better than others.

One way to attenuate this drawback within the multi-aspect methodology (Pesarin, 2001; Pesarin \& Salmaso, 2010a; Pesarin \& Salmaso, 2011; Salmaso \& Solari, 2005) is to use several partial test statistics, each specialized to put into evidence one aspect of interest and to nonparametrically combine their dependent results by means of a suitable function.

The act of conditioning on $\boldsymbol{X}$ implies referring to the related conditional reference space, denoted by $\chi_{/ X}$. Essentially $\chi_{/ X}$ contains points of sample space $\chi^{n}$ that are equivalent to $\boldsymbol{X}$ in terms of information carried by the underlying - and usually unknown likelihood function $f^{(n)}(\boldsymbol{X})$, which in turn is assumed to be positive on all observed data $\boldsymbol{X}$. Thus, it contains all points $\boldsymbol{X}^{*}$ such that the likelihood ratio $f^{(n)}(\boldsymbol{X}) / f^{(n)}\left(\boldsymbol{X}^{*}\right)$ is $\left(\boldsymbol{X}, \boldsymbol{X}^{*}\right)$ invariant and $f$-independent; so it corresponds to the orbit of equivalent points associated with $\boldsymbol{X}$. Data exchangeability in $H_{0}$ implies that the density $f^{(n)}(\boldsymbol{X})$ is invariant on permutations of its arguments, thus $f^{(n)}(\boldsymbol{X})=f^{(n)}\left(\boldsymbol{X}^{*}\right)$ for every permutation $\boldsymbol{X}^{*}$ of $\boldsymbol{X} ; \chi_{/ \boldsymbol{X}}$ then contains all distinct permutations of $\boldsymbol{X}$. That is $\chi_{/ \boldsymbol{X}}=$ $\left\{\bigcup_{\boldsymbol{u}^{*} \in \Pi(\boldsymbol{u})}\left[X\left(u_{i}^{*}\right), i=1, \ldots, n\right]\right\} . \quad$ Therefore, because every $\boldsymbol{X}^{*} \in \chi_{/ \boldsymbol{X}}$ is sufficient for $f$ in $H_{0}$, $\chi_{/ \boldsymbol{X}}$ can be considered as playing the role of a sufficient space.
In $H_{l}$ the set of sufficient statistics is the pair $\left(\boldsymbol{X}_{1} ; \boldsymbol{X}_{2}\right)$ because data are only exchangeable within - but not between - groups. As a consequence of sufficiency of $\chi_{/ X}$ for $f$ under $H_{0}$ the null conditional probability of any event $A$, given $\chi_{/ X}$, is independent of $f-$ whatever it is - because $\operatorname{Pr}\left\{\boldsymbol{X}^{*} \in A ; f \mid \chi_{/ \boldsymbol{X}}\right\}=$ $\operatorname{Pr}\left\{\boldsymbol{X}^{*} \in A \mid \chi_{/ \boldsymbol{X}}\right\}$. Therefore, the null permutation (conditional) probability is distribution-free and nonparametric as well as the distribution of any statistic $T: \chi^{n} \rightarrow \mathcal{R}^{1} \quad$ conditional on $\chi_{/ \boldsymbol{X}}$. Moreover, because for finite sample size $n$ the number $M^{(n)}=\sum_{\chi_{/ X}} 1\left(X^{*} \in \chi_{/ X}\right)$ of points in $\chi_{/ X}$ is finite, in $H_{0}$ the conditional probability of any $A$ is calculated as

$$
\begin{aligned}
\operatorname{Pr}\left\{\boldsymbol{X}^{*} \in A \mid \chi_{/ X}\right\} & =\frac{\sum_{\boldsymbol{X}^{*} \in A} f\left(\boldsymbol{X}^{*}\right) d \boldsymbol{X}^{*}}{\sum_{\boldsymbol{X}^{*} \in \chi_{/ X}} f\left(\boldsymbol{X}^{*}\right) d \boldsymbol{X}^{*}} \\
& =\sum_{\chi_{/ X}} \frac{1\left(\boldsymbol{X}^{*} \in A\right)}{M^{(n)}}
\end{aligned}
$$

because $f\left(\boldsymbol{X}^{*}\right) d \boldsymbol{X}^{*}=f(\boldsymbol{X}) d \boldsymbol{X}$ for every $\boldsymbol{X}^{*} \in \chi_{/ \boldsymbol{X}}$. Note that in carrying out the calculations for this conditional probability it is not necessary to make reference to the so-called hypothetical repeated sampling principle; that is, it is not necessary to examine the whole population sample space $\chi^{n}$, which in turn has generally a virtual existence, and to consider all sample points that could have been realized but only the point $\boldsymbol{X}$ of which has been actually observed. In fact, as the observed data $\boldsymbol{X}$ are exclusively taken into consideration, $\operatorname{Pr}\left\{A \mid \chi_{/ X}\right\}$ simply requires the complete enumeration of $\chi_{/ X}$ which, as it consists of all permutations $\boldsymbol{X}^{*}$ of $\boldsymbol{X}$, has an objective existence.

With different arguments, a similar notion is also expressed in Anderson's article. In practice, when sample sizes $\left(n_{1}, n_{2}\right)$ are not small, to overcome the too large cardinality of $\chi_{/ X}$, the probability $\operatorname{Pr}\left\{A \mid \chi_{/ X}\right\}$ can be estimated at any degree of accuracy by a conditional Monte Carlo simulation on $\chi_{/ X}$ as described by Anderson.

A first property of the permutation distribution is that in $H_{0}$ the data set $\boldsymbol{X}$ is 


\section{FORTUNATO PESARIN \& STEFANO BONNINI}

uniformly distributed over $\chi_{/ X}$ conditionally. In fact, $\quad \operatorname{Pr}\left\{\boldsymbol{X}=\boldsymbol{x} \mid \chi_{/ \boldsymbol{X}}\right\}=\operatorname{Pr}\left\{\boldsymbol{X}^{*}=\boldsymbol{x} \mid \chi_{/ \boldsymbol{X}}\right\}=$ $1 / M^{(n)}$ if $\boldsymbol{x} \in \chi_{/ X}$ and 0 elsewhere. Essentially, this property states that, under $H_{0}$, the permutations in $\chi_{/ X}$ are equally likely; whereas under $H_{1}$, because the set of sufficient statistics is the pair $\left(\boldsymbol{X}_{\mathbf{1}} ; \boldsymbol{X}_{\mathbf{2}}\right)$, no data can be exchanged between two samples and permutations in $\chi_{/ X}$ are not equally likely. This property may suggest ways for finding test statistics provided with good inferential properties. Further, assuming that the exchangeability condition on data $\boldsymbol{X}$ is satisfied in $H_{0}$, for any randomized test based on a statistic $T$ for which large values are significant,

$$
\phi_{R}(\boldsymbol{X})=\left\{\begin{array}{l}
1 \text { if } T^{0}>T_{\alpha} \\
\gamma^{n} T^{0}=T_{\alpha} \\
0 " T^{0}<T_{\alpha}
\end{array}\right.
$$

where $T^{0}=T(\boldsymbol{X})$ is the observed value of $T$ on data $\boldsymbol{X}$, and

$$
\gamma=\left[\alpha-\operatorname{Pr}\left\{T^{0}>T_{\alpha} \mid \chi_{/ X}\right\}\right] / \operatorname{Pr}\left\{T^{0}=T_{\alpha} \mid \chi_{/ X}\right\},
$$

the $\alpha$-size conditional critical value $T_{\alpha}=T_{\alpha}(\boldsymbol{X})$ which, if $H_{0}$ were true, could be determined by complete enumeration of $\chi_{/ X}$. The conditional rejection probability of $\phi_{R}(\boldsymbol{X})$ for any given $\alpha \in(0,1)$ is then $\mathbb{E}\left\{\phi_{R}(\boldsymbol{X}) \mid \chi_{/ X}\right\}=\alpha$. Thus, under $H_{0}$, due to sufficiency of $\boldsymbol{X}$, test $\phi_{R}(\boldsymbol{X})$ is invariant with respect to all underlying distributions $f$ and all sample points $\boldsymbol{X}$ and so independently on how they are selected from $\chi^{n}$. This property defines the so-called uniform similarity property of randomized permutation tests, corresponding to the stronger version of the Neymann $\alpha$-structure.

One consequence, but not the most important, of conditioning on $\boldsymbol{X}$ is that the permutation critical value $T_{\alpha}=T_{\alpha}(\boldsymbol{X})$ does not need to be determined in practice. Unless it is known that $H_{0}$ is true - in which case knowing $T_{\alpha}$ would be essentially useless - its determination would require the ordering of $\left\{T\left(\boldsymbol{X}^{*}\right), \boldsymbol{X}^{*} \in \chi_{/ X}\right\}$, that is, that of all permutation values of $T$, thus it necessarily depends on actual data $\boldsymbol{X}$ and on some unknown treatment effect $\delta$ (if any). One efficient way to overcome this apparent drawback is by making reference to the significance level transformation of $T$, that is, by considering the so-called observed $p$-value-like score. The observed $p$-value-like score of test $\phi_{R}$, which is defined as $\lambda_{R}^{0}=\lambda_{R}(\boldsymbol{X}, T)=\operatorname{Pr}\left\{T^{*}>T^{0} \mid \chi_{/ X}\right\}$, is a non-increasing function of $T^{0}$ and is one-toone related with the $\alpha$-value of $T$. Because $T^{0}<T_{\alpha}$ implies $\lambda_{R}^{0}>\alpha$ and $T^{0}>T_{\alpha}$ implies $\lambda_{R}^{0}<\alpha$ :

$$
\phi_{R}(\boldsymbol{X})=\left\{\begin{array}{l}
1 \text { if } \lambda_{R}^{0}<\alpha \\
\gamma " T^{0}=T_{\alpha} \\
0 " \lambda_{R}^{0}>\alpha
\end{array}\right.
$$

is the general form of all permutation tests. Of course, simple or non-randomized or ordinary test $\phi(X)=1$ if $T \geq T_{\alpha}$ and 0 elsewhere, the $p$ value score $\lambda^{0}=\operatorname{Pr}\left\{T^{*} \geq T^{0} \mid \chi_{/ X}\right\}$ is defined in the discrete set of attainable values, $\Lambda(\boldsymbol{X}, T) \in$ $(0,1)$.

The statistic $\lambda^{0}$ coincides with the $p$ value of test $T$ if $H_{0}$ were true, then it works as a $p$-value score. Thus, we agree with Anderson's notion that $\lambda^{0}$ is not a true $p$-value, unless $H_{0}$ were true. We partially disagree, however, because $\alpha$ is simply the critical value (i.e., the type I error rate $\alpha$ ) of any randomized permutation test (for non-randomized tests, the resulting type I error rate is $\alpha^{\prime} \leq \alpha$ so giving rise to conservative inferences); exactly as for parametric tests.

In order to establish whether the $p$-value score is a test statistic furnished with good inferential properties it is useful to observe that:

i. in $H_{0}$ the distribution of $\lambda^{0}$ is uniform on its attainable set $\Lambda(X, T)$;

ii. in $H_{1}:\left(\begin{array}{c}d \\ X_{1} \geq X_{2}\end{array}\right)=\left(\begin{array}{c}d \\ X+\delta \geq X_{2}=X\end{array}\right) \equiv$ $(\delta>0)$, where the unknown treatment effect $\delta$ can be either fixed or random and, in the latter case, it could depend on latent unobservable values $X$. The permutation distribution of the $p$-value score is such that $d \quad d$ $\lambda^{0}(\boldsymbol{X}, \delta) \leq \lambda^{0}(\boldsymbol{X}, 0)=\mathcal{U}[\Lambda(\boldsymbol{X}, T)]$, and thus it is uniformly dominated by the uniform 
$\begin{array}{llrr}\begin{array}{l}\text { distribution } \\ \delta^{\prime}>\delta>0\end{array} \quad \begin{array}{c}\Lambda(\boldsymbol{X}, T) ; \\ \text { implies }\end{array} & \begin{array}{r}\text { moreover, } \\ \text { that }\end{array} \\ \lambda^{0}\left(\boldsymbol{X}, \delta^{\prime}\right) \leq \lambda^{0}(\boldsymbol{X}, \delta) \leq \lambda^{0}(\boldsymbol{X}, 0), & \text { so } \quad \text { the }\end{array}$

dominance in distribution is monotone with respect to $\delta$ for every $\boldsymbol{X}$.

Provided that the exchangeability property is satisfied in $H_{0}$, these properties imply that the test statistic based on $\lambda^{0}$ is uniformly unbiased for all data sets $\boldsymbol{X}$, all distributions $f$, all sample sizes $\left(n_{1}, n_{2}\right)$ and independently on how subjects are selected from a population (i.e., possibly under selection-bias sampling if they are randomized to treatments). These properties guarantee that tests based on the permutation $p$ value score are valid for making inference whenever the permutation testing principle applies (Pesarin, 2001; Pesarin \& Salmaso, 2010a; Pesarin \& Salmaso, 2010b). In this we agree with Anderson's view-point that the permutation $p$-value does not impugn the validity of related tests.

With respect to the consistency property, if in $H_{0}$ the population mean value of $X$ is finite, that is, $\mathbb{E}(|X|)<\infty$, then the permutation test based on difference of two sample means, $T=\bar{X}_{1}-\bar{X}_{2}$ for example, is consistent (Pesarin \& Salmaso, 2013), that is, its rejection probability converges to 1 as $\min \left(n_{1}, n_{2}\right)$ diverges. Thus, not all test statistics are "valid irrespective of the shapes of the population distributions," in the sense that there exist population $f$ for which there are permutation tests $T$ that are not consistent. A way to obtain valid consistent test statistics is described in (Pesarin \& Salmaso, 2010a; Pesarin \& Salmaso, 2010b; Salmaso \& Solari, 2005) within the socalled multi-aspect testing.

We would like to also underline some few other points:

1. The permutation test is unbiased and consistent when the effect $\delta$ is such that the distribution of $X_{1}(\delta)$ in the alternative is not only shifted, but also when the dispersion increases or decreases provided $d$ that $X_{1}(\delta) \geq X_{2}$. For example, the effect on high blood pressure of a suitable drug is to reduce it towards smaller values by diminishing both mean and dispersion. That is, it is valid under homoscedasticity in $H_{0}$ (when the exchangeability is satisfied) and for possible non-homoscedasticity in $H_{l}$. When $\mu_{1}=\mu_{2}$ and homoscedasticity is not satisfied in $H_{0}$, so the exchangeability is also not satisfied, it falls within the so-called Behrens-Fisher problem for which no exact parametric and non-parametric nonrandomized solutions exist. In Pesarin (2001, Chapter 10) an almost exact (but not exact) univariate and multivariate permutation solution is provided for symmetric distributions. Thus, in our opinion, some of the related arguments in Anderson's paper should be accordingly modified.

2. Traditional parametric tests are said to be generally valid under their conditions. This, however, is not always true. For example, in a simulation under multivariate normality we found that as the number $q$ of processed variables increases, while keeping sample sizes fixed, the power of Hotelling's $T^{2}$ increased up to a maximum at approximately $q \cong(n-2) / 2$, then it decreased and was not calculable when $q \geq(n-1)$, whereas that of a permutation test based on $T_{p}=\sum_{h \leq q}\left(\bar{X}_{1 h}-\bar{X}_{2 h}\right)^{2}$ always increased up to 1 for divergent $q$. Moreover, depending on the correlation matrix, there is a value $q$ ' such that the permutation test $T_{p}$ is more powerful than $T^{2}$. To this end we would like to report a brief simulation study from Pesarin and Salmaso (2010b). The results in Table 1, where $\alpha=0.01, \mathbf{0 . 0 5}$ (bold face), $\mathrm{B}=1000$ random permutations and $M C=1000$ Monte Carlo simulations from $q$-dimensional $d$

normal distributions $X_{1}=\mathcal{N}_{q}(\boldsymbol{\delta}, \mathbf{1})$ and $d$

$X_{2}=\mathcal{N}_{q}(\mathbf{0}, \mathbf{1})$ were considered, show that:

(i) as $q$ increases, the power of Hotelling's $T^{2}$ increases up to a maximum and then decreases to a minimum for $q=n-2$, after 


\section{FORTUNATO PESARIN \& STEFANO BONNINI}

that it cannot be calculated due to singularity of estimated covariance matrix; (ii) power of $T_{p}$ increases monotonically with $q$; (iii) power of $T_{p}$ is not invariant with respect to alternatives lying at Mahalanobis distance from $H_{0}$ and so in some circumstances and even for small $q$ it can be more powerful than $T^{2}$, which in stringent conditions is the uniformly most powerful unbiased similar invariant (whereas $T_{p}$ is simply unbiased). A similar result, however less general, is also in Blair, Higgins, Karniski and Kromrey (1994).

Table 1: Simulations under

\begin{tabular}{|c|c|c|}
\multicolumn{3}{|c}{$H_{1}: n_{1}=n_{2}=10, \mu=0, \delta=0.40$} \\
\hline $\mathrm{q}$ & $\mathrm{T}^{2}$ & $\mathrm{~T}_{\mathrm{p}}$ \\
\hline 4 & $.079 / .219$ & $.081 / .237$ \\
\hline 8 & $.063 / .234$ & $.126 / .347$ \\
\hline 12 & $.037 / .186$ & $.176 / .436$ \\
\hline 18 & $.013 / .067$ & $.253 / .543$ \\
\hline 19 & & $.244 / .544$ \\
\hline 22 & & $.340 / .618$ \\
\hline 25 & & $.365 / .656$ \\
\hline
\end{tabular}

3. Under selection-bias sampling, any parametric inference cannot be extended to the target population, even asymptotically, unless a suitable treatment independent selection model is well-defined, identifiable and estimable. Conversely, under mild conditions and based on the uniform similarity and the uniform unbiasedness, permutation inference can be extended (Pesarin, 2001; Pesarin, 2002; Pesarin \& Salmaso, 2010a, Pesarin \& Salmaso, 2010b) to the possibly unknown population from which selected subjects belong. Therefore, the result of the inferential conclusion is not only valid to the actual subjects (e.g., treatment is effective on actual subjects), as suggested by Edgington and Onghena (2007) and by Lehmann (2009), it is also valid to the latent population (e.g., treatment is effective), at least in a weak sense. This in part contradicts one of Anderson viewpoints.

4. When in a multivariate testing problem the alternatives are restricted to be one-sided and the underlying distribution is not multivariate normal with known covariance matrix, no parametric solution is known (under multivariate normality some asymptotic solution are known (Silvapulle \& Sen, 2005)); whereas within the permutation testing principle these solutions are straightforward through the so-called nonparametric combination of dependent partial tests, one for each component variable (Basso, Pesarin, Salmaso \& Solari, 2009; Basso \& Salmaso, 2011; Finos, Salmaso \& Solari, 2007; Pesarin \& Salmaso, 2010a). This, together with the fact that no single test can recover all of the information on $f$ contained in $\boldsymbol{X}$, may solve the question regarding the "unresolved ambiguity concerning the degree to which each of the ... tests ... provides unique information about the null hypothesis" expressed in Andersons manuscript.

5. Reported results from Hayes (1996) regarding the non "distribution-free" property of permutation tests are, in our opinion, misleading because in order for the permutation test based on $\sum X_{i} Y_{i}$ for testing $H_{0}: \rho=0$ to be valid, the null hypothesis must be $H_{0}: X \perp Y$, that is, independence must be assumed between $X$ and $Y$, not only zero correlation. In other words it is not possible to test $\rho=0$ via permutation without assuming independence due to the lack of exchangeability (remember that $\rho=0$ characterizes independence on bivariate normal distributions). In a sense, this implies some limitations on the inferential possibilities of permutation tests.

6. One important - and very useful property of permutation tests is when there are missing or censoring or zero-inflated data informative on the treatment effect (see Pesarin, 2001; Pesarin \& Salmaso, 2010a; Pesarin \& Salmaso, 2010b) where, unless a 
suitable, identifiable and separately estimable model is provided, no parametric test exists (Sen, 2007).

7. Permutation testing can be used in an intriguing problem as that in which some treated subjects have negative and others positive effect, as for example with some drugs sensitive to genetic interaction, so that both $\delta<0$ and $\delta>0$ can be jointly true in the alternative. This problem is studied in Bertoluzzo, Pesarin \& Salmaso (2013).

8. In Pesarin and Salmaso (2010a) and Pesarin and Salmaso (2010c) an extended notion of consistency is provided for permutation tests where it is assumed that sample sizes remain fixed and the number of informative variables, those which contribute with nonnull effect, diverge to the infinity. A notion which can have application, for example, in many problems of modern biological analyses where the number of observed variables per subject can be practically unlimited or even in problems where data are curves or images which can be reduced to a large number of coefficients (e.g., functional principal components, Fourier and/or wavelets coefficients, area under the curve and/or number of picks over a list of thresholds, etc.).

9. It is possible to download without cost the software NPC-Test R.10 from the Wiley website http://www.wiley.com/go/npc for Pesarin and Salmaso (2010a), which may solve the large majority of unidimensional and multidimensional permutation testing problems. $\mathrm{R}$ coded programs are also available for download from the same website.

\section{References}

Anderson, R. B. (2013). Conceptual Distinction between the Critical $\mathrm{p}$ Value and the Type I Error Rate in Permutation Testing. Journal of Modern Applied Statistical Methods, 12(1), 2-8.
Basso, D., Pesarin, F., Salmaso, L., \& Solari, A. (2009). Permutation tests for stochastic ordering and ANOVA: Theory and applications. New York, NY: Springer.

Basso, D., \& Salmaso, L. (2011). A permutation test for umbrella alternatives. Statistics and Computing, 21, 45-54.

Bertoluzzo, F., Pesarin, F., \& Salmaso, L. (2013). On multi-sided permutation tests. Communications in Statistics -Simulation and Computation, 42, 1380-1390.

Blair, R. C., Higgins, J. J., Karniski, W., \& Kromrey, J. D. (1994). A study of multivariate permutation tests which may replace Hotelling's t2 test in prescribed circumstances. Multivariate Behavioral Research, 29, 141-163.

Finos, L., Salmaso, L., \& Solari, A. (2007). Conditional inference under simultaneous stochastic ordering constraints. Journal of Statistical Planning and Inference, 137, 2633-2641.

Lehmann, E. L. (2009). Parametric versus nonparametrics: two alternative methodologies. Journal of Nonparametric Statistics, 21, 397-405.

Pesarin, F. (2001). Multivariate permutation tests: with application in biostatistics. Chichester, UK: John Wiley \& Sons.

Pesarin, F. (2002). Extending permutation conditional inference to unconditional one. Statistical Methods and Applications, 11, 161-173.

Pesarin, F. (2013). Some elementary theory of permutation tests. Communications in Statistics - Theory and Methods. Submitted, R1 version.

Pesarin F., \& Salmaso, L. (2010a). Permutation tests for complex data. Theory, applications and software: Wiley series in probability and statistics. Chichester, UK: John Wiley \& Sons.

Pesarin F., \& Salmaso, L. (2010b). The permutation testing approach: a review. Statistica, LXX(4), 1-29.

Pesarin, F., \&. Salmaso, L. (2010c). Finite-sample consistency of combination-based permutation tests with application to repeated measures designs. Journal of Nonparametric Statistics, 22, 669-684. 


\section{FORTUNATO PESARIN \& STEFANO BONNINI}

Pesarin, F. \& Salmaso, L. (2011). A review and some new results on permutation testing for multivariate problems. Statistics and Computing, 22(2), 639-646.

Pesarin, F., \& Salmaso, L. (2013). On the weak consistency of permutation tests. Communications in Statistics - Simulation and Computation, 42, 1368-1379.

Salmaso, L., \& Solari, A. (2005). Multiple aspect testing for case-control designs. Metrika, 12, 1-10.
Sen, P. K. (2007). Union-intersection principle and constrained statistical inference. Statistical Planning and Inference, 137, 37413752.

Silvapulle, J. S., \& Sen, P. K. (2005). Constrained statistical inference: Inequality, order and shape restrictions. Hoboken, NJ: Wiley. 


\section{Randomization Test $p$-Values versus Significance Levels}

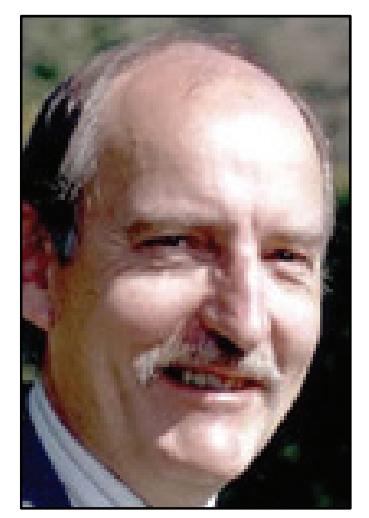

Bryan Manly

Western EcoSystem Technology, Inc., Cheyenne, WY

Bryan Manly responds to Richard Anderson's article Conceptual Distinction between the Critical p Value and the Type I Error Rate in Permutation Testing.

Key words: P-value, randomization test.

\section{Response}

The $p$-value from a randomization test is no more and no less than the probability of getting a test statistic as extreme or more extreme than that observed if the data are effectively in a random order. The examples by Hayes (1996) and Mewhort, et al. (2009) do not meet that criterion and, therefore, are not relevant.

Dr. Bryan Manly is a consultant with Western EcoSystem Technology, Inc. Before that, he was Chair of Statistics and Director of the Center for Applications of Statistics and Mathematics at the University of Otago, New Zealand. $\mathrm{He}$ specializes in applications of statistics in ecology and environmental science. He has particular expertise in the design and analysis of biological sampling programs, multivariate analysis, population modeling, computerintensive methods and the design and analysis of studies on resource selection by animals. Email him at: bmanly@west-inc.com.

\section{References}

Anderson, R. B. (2013). Conceptual Distinction between the Critical $\mathrm{p}$ Value and the Type I Error Rate in Permutation Testing. Journal of Modern Applied Statistical Methods, 12(1), 2-8.

Hayes, A. F. (1996). Permutation test is not distribution-free: Testing $\mathrm{H}_{0}: \quad \rho=0$. Psychological Methods, 1, 184-198.

Mewhort, D. J. K., Kelly, M., \& Johns, B. T. (2009). Randomization tests and the unequal-N/unequal-variance problem. Behavior Research Methods, 41, 664-667. 


\section{Conceptual Distinction between the Critical $p$ Value and the Type I Error Rate in Permutation Testing: Author Response to Peer Comments}

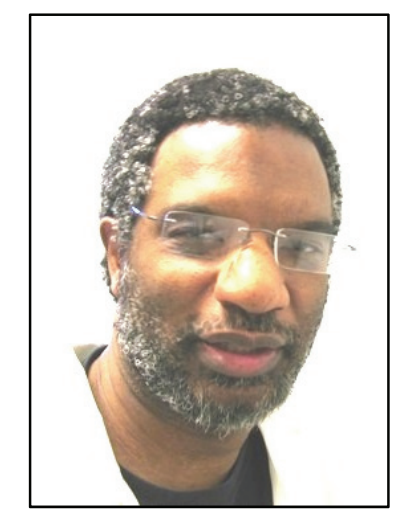

Richard B. Anderson

Bowling Green State University

Bowling Green, $\mathrm{OH}$

Richard Anderson responds to comments regarding his target article Conceptual Distinction between the Critical $p$ Value and the Type I Error Rate in Permutation Testing.

Key words: Statistics, null hypothesis, non-parametric, permutation test, exchangeability; Type I error; validity.

\section{Response}

Some of the replies to my article, Conceptual Distinction between the Critical $p$ Value and the Type I Error Rate in Permutation Testing, have focused on what is called an exchangeability requirement, and on the question of whether previous evaluations of permutation test validity

Richard Anderson is an Associate Professor of Psychology in the Department of Psychology. His research interests include topics in judgment, decision-making, reasoning, memory, statistical cognition, and statistical methods. He teaches courses in cognition, statistics, and research methods and holds memberships in the Psychonomic Society and the Cognitive Science Society. Email him at: randers@bgsu.edu. have violated exchangeability. It should be clarified that the aim was not to critique previous analyses on grounds of exchangeability violation: The purpose was to address exchangeability as a characteristic of how the permutation test's null hypothesis is formulated. No claims were advanced concerning the idea that exchangeability is an assumption to be met or violated by a given dataset. Instead, the central thesis was that when a significance criterion is adopted, for example 0.05, for rejecting the null hypothesis of random coupling of data points to condition labels, that 0.05 is not a Type I error probability. 


\section{RICHARD ANDERSON RESPONSE TO PEER COMMENTS}

\section{References}

Anderson, R. B. (2013). Conceptual Distinction between the Critical $p$ Value and the Type I Error Rate in Permutation Testing. Journal of Modern Applied Statistical Methods, 12(1), 2-8.

Good, P. (2013). p-Values versus Significance Levels. Journal of Modern Applied Statistical Methods, 12(1), 9-10.
Manly, B. (2013). Randomization Test $p$-Values versus Significance Levels. Journal of Modern Applied Statistical Methods, 12(1), 18.

Pesarin, F., \& Bonnini, S. (2013). A Response to Richard Anderson's Conceptual Distinction between the Critical $p$ value and Type I Error Rate in Permutation Testing. Journal of Modern Applied Statistical Methods, 12(1), 11-17. 


\title{
Regular Articles \\ A Monte Carlo Simulation of the Robust Rank-Order Test Under Various Population Symmetry Conditions
}

\author{
William T. Mickelson \\ University of Wisconsin - Whitewater \\ Whitewater, WI
}

The Type I Error Rate of the Robust Rank Order test under various population symmetry conditions is explored through Monte Carlo simulation. Findings indicate the test has difficulty controlling Type I error under generalized Behrens-Fisher conditions for moderately sized samples.

Key words: Robustness, hypothesis testing, Monte Carlo.

Introduction

Statistical significance tests are widely used in empirically based quantitative research and have been applied in virtually every field of study to test research hypotheses. Although many applied researchers use statistical methods, the pitfalls and limitations of statistical hypothesis tests due to violations of underlying assumptions are often overlooked (Kesselman, et al., 1998, Snyder \& Thompson, 1998). It is known that the most commonly used statistical tests, the ANOVA F and Student's T-test, have underlying assumptions of independence of observations that data are obtained from normally distributed populations having equal variances. Furthermore, these commonly used tests suffer potentially severe performance degradation when underlying assumptions of normality and equality of variance are not met (Glass, Peckham \& Sanders, 1972). The violation of normality and equality of variance assumptions are often referred to as the generalized BehrensFisher problem.

William T. Mickelson is an applied statistician and consultant. Current interests include measurement, statistical robustness, modern resampling and nonparametric methods and the teaching and learning of statistics. Email him at: mickelsw@uww.edu.
When researchers are interested in testing the equality of two means - or medians it is generally recommended that a nonparametric inference procedure, such as the Wilcoxon-Mann-Whitney test, be used (Harwell \& Serlin, 1989). The Wilcoxon-Mann-Whitney test, however, is inappropriate for cases with unequal variances (Harwell, et.al. 1992; Zimmerman \& Zumbo, 1993a, 1993b): It behaves much like the traditional student's t-test where the Type I error rate is depressed when the larger sample size is associated with the larger variance and, when the smaller sample size is associated with the larger variance, there is an inflation of the Type I error rate. Siegel and Castellan (1988) recommend that, in the case of non-normality and unequal variances, an alternative nonparametric inference procedure, the Robust Rank Order (RRO) test be used.

The Robust Rank Order (RRO) test (Fligner \& Policello, 1981) is a modified version of the Mann-Whitney-Wilcoxon test designed to maintain both the nominal Type-I error rate and statistical power under generalized BehrensFisher conditions (Behrens,1929; Fisher, 1939; Scheffé, 1970; Zumbo \& Coulombe, 1997). According to Siegel and Castellan (1988), the RRO test statistic approximates a normal distribution quickly as sample size increases; however, there is an underlying assumption of population distribution symmetry that is essential for the RRO test to be truly robust. Zumbo and Coulombe (1997) indicate this 


\section{ROBUST RANK-ORDER TEST UNDER POPULATION SYMMETRY CONDITIONS}

quality is lacking under conditions of heterogeneity of variance.

Zumbo and Coulombe (1997) examined the performance of the RRO test for non-normal populations with unequal variances but confined their study to small sample size cases (n between 3 and 12) where the exact level of the RRO test was known. They found that the RRO test was conservative for symmetric distributions and that it performed inconsistently when the population distribution was skewed. Vargha and Delaney (2000) also conducted a simulation study to examine the performance of the RRO test, the results of which conflicted with Zumbo and Coulombe.

In this study, the Type I Error Rate of the RRO test is examined for moderately sized samples. Sample sizes examined are larger than those for which an exact test is possible, yet potentially before the asymptotic convergence to the standard normal distribution has occurred. This work extends Zumbo and Coulombe's (1997), and Vargha and Delaney's (200) simulation research on the RRO test to a larger range of variance inequality and non-normality situations, and sample sizes that are typically found in researcher practice.

The Robust Rank Order Test

Let $\mathrm{X} 1, \mathrm{X} 2, \ldots, \mathrm{Xm}$ and $\mathrm{Y} 1, \mathrm{Y} 2, \ldots, \mathrm{Yn}$ denote two independent random samples from parent populations with continuous distribution functions $F(X)$ and $G(Y)$, respectively. If it is assumed that a treatment effect will manifest itself as a difference in the location of the experimental group's location, then the null hypothesis for the RRO test is:

$$
\begin{gathered}
\mathrm{H}_{0}: \operatorname{Median}(\mathrm{X})=\operatorname{Median}(\mathrm{Y}) \\
\text { versus } \\
\mathrm{H}_{\mathrm{a}}: \operatorname{Median}(\mathrm{X}) \neq \operatorname{Median}(\mathrm{Y})
\end{gathered}
$$

The RRO test is a distribution-free test of $\left(\mathrm{H}_{\mathrm{a}}\right)$. The following steps are used to compute the RRO test:
1. For each observation in group 1 , let $\mathrm{Pi}=$ [Number of observations in group $2<\mathrm{Xi}$ ], for $\mathrm{i}=1,2, \ldots, \mathrm{m}$.

2. For each observation in group 2 , let $\mathrm{Qj}=$ [Number of observations in group $1<\mathrm{Yj}$ ], for $\mathrm{j}=1,2, \ldots, \mathrm{n}$. The Pi and $\mathrm{Qj}$ are called the placements.

3. Compute the average of the placements for group 1 and group 2, termed $\bar{P}$ and $\bar{Q}$, respectively.

4. Compute the sum of squares of placement deviations for each group. The formulas for these computations are:

$$
V_{I}=\sum_{i=1}^{n}\left(P_{i}-\bar{P}\right)^{2}
$$

and

$$
V_{2}=\sum_{j=1}^{m}\left(Q_{i}-\bar{Q}\right)^{2}
$$

5. The Robust Rank Order test statistic is:

$$
T S=\frac{\left(n^{*} \bar{P}\right)-\left(m^{*} \bar{Q}\right)}{2 \sqrt{V_{1}+V_{2}+\bar{P} * \bar{Q}}}
$$

Fligner \& Policello (1981) give critical values for small sample sizes. For larger sample sizes, the test statistics TS is distributed as a standard normal distribution.

\section{Methodology}

Monte Carlo simulation was used to estimate Type I error rates for the Robust Rank Order (RRO) Test under various population symmetry conditions. All individual estimates are based on 20,000 iterations. Three replications per condition were obtained in order to model and graph the Type I error rates as response surfaces. The conditions modified in the simulation consisted of:

- $\quad$ Total Sample Size $(\mathrm{N}=30,50,100,150)$

- Unequal sample sizes per group in terms of sample size ratios of: 


\section{WILLIAM T. MICKELSON}

$$
\begin{array}{ll}
\circ & 1: 1 \\
\circ & 1.5: 1 \\
\circ & 2.3: 1 \\
\circ & 4: 1 \\
\circ & 9: 1
\end{array}
$$

- Variance ratios ranging from 1:1 to $20: 1$

- Inverse and direct pairing of sample size with variances

- Population distributions consisting of:

○ Symmetric Normal

- Symmetric Uniform

- Symmetric T-distribution with $\mathrm{df}=3$ (heavy tailed)

- Moderately skewed (Weibull with parameters $a=2$ and $b=2$ )

- Heavily skewed (Weibull with parameters $a=1.5$ and $b=1$ )

Type I error estimation was defined and calculated as the number of times the test statistics for both tests rejected the null hypothesis divided by the maximum number of iterations $(20,000)$ when the null hypothesis is true. GAUSS programming language was used to run the simulation.

Consistent with recommendations of Bradley (1978) for evaluating Type I error rate estimates, multiple benchmarks for the criteria of robustness were used. Specifically, Type I error rate estimates between $\alpha \pm \alpha / 10$, or $[0.045$, 0.055] for a nominal 0.05 level test, were considered robust at a stringent level. Other benchmarks used include: a) intermediate level, $\alpha \pm \alpha / 4$; b) liberal, $\alpha \pm \alpha / 2$; and c) very liberal, $\alpha$ $\pm 3 \alpha / 4$. Graphical representations of the data are presented for selected conditions to illustrate the primary findings of this simulation study. A database of the Type I Error rate estimates under all of the simulated conditions is available from the author (mickelsw@uww.edu). Selected graphical representations of the data are presented herein.

\section{Results}

Type I error rate results are organized by the combinations of sample size paired with variance ratios.
Test Performance: Equal Sample Sizes per Group

Figures 1 - 5 present the results of the Robust Rank Order (RRO) test when sample sizes are equal under 5 different population symmetry situations. The $\mathrm{X}$-axis in these plots corresponds to increasing levels of population variance heterogeneity, where the variance ratio ranges from 1:1 to 20:1. The Y-axis corresponds to the Type I Error rate. The results delineated by total sample size. The lines in the graph relative to each total sample size level represent interpolation between means.

As shown in Figures 1,2 and 3 the RRO Test is relatively robust for the symmetric population distribution conditions and sample sizes under consideration. All Type I error rates are within liberal robustness standards, and within intermediate robustness standards for total sample sizes of 50 and larger. There is some Type I Error rate inflation for the smaller sample size. Not surprisingly, the error rates improve with increasing total sample size.

Figure 4 illustrates that the RRO is also relatively robust under the condition of the moderately skewed population distribution. Total sample sizes of 50 or larger result in error rate estimates within the intermediate robustness standard, however the smallest sample size under consideration does experience Type I Error Rate inflation approaching the very liberal standard, furthermore the error rate appears to increase as the variance ratio increases. Figure 5 demonstrates that the RRO is not robust to moderately extreme asymmetry in the population distribution. Further analysis indicated that this phenomenon was prevalent under all conditions evaluated when the population distribution was heavily skewed, as such, this distribution is not presented.

In sum, the RRO test can be considered robust when sample sizes are equal and the population distribution can be considered symmetric or at worst, moderately skewed. For small total sample size $(\mathrm{N}=30)$, the $\mathrm{RRO}$ exhibits some Type I Error rate inflation, however sample sizes of 50 or larger have Type I Error rate controlled at the intermediate level of robustness. 
Figure 1: RRO Test Type I Error Rate - Normal Distribution

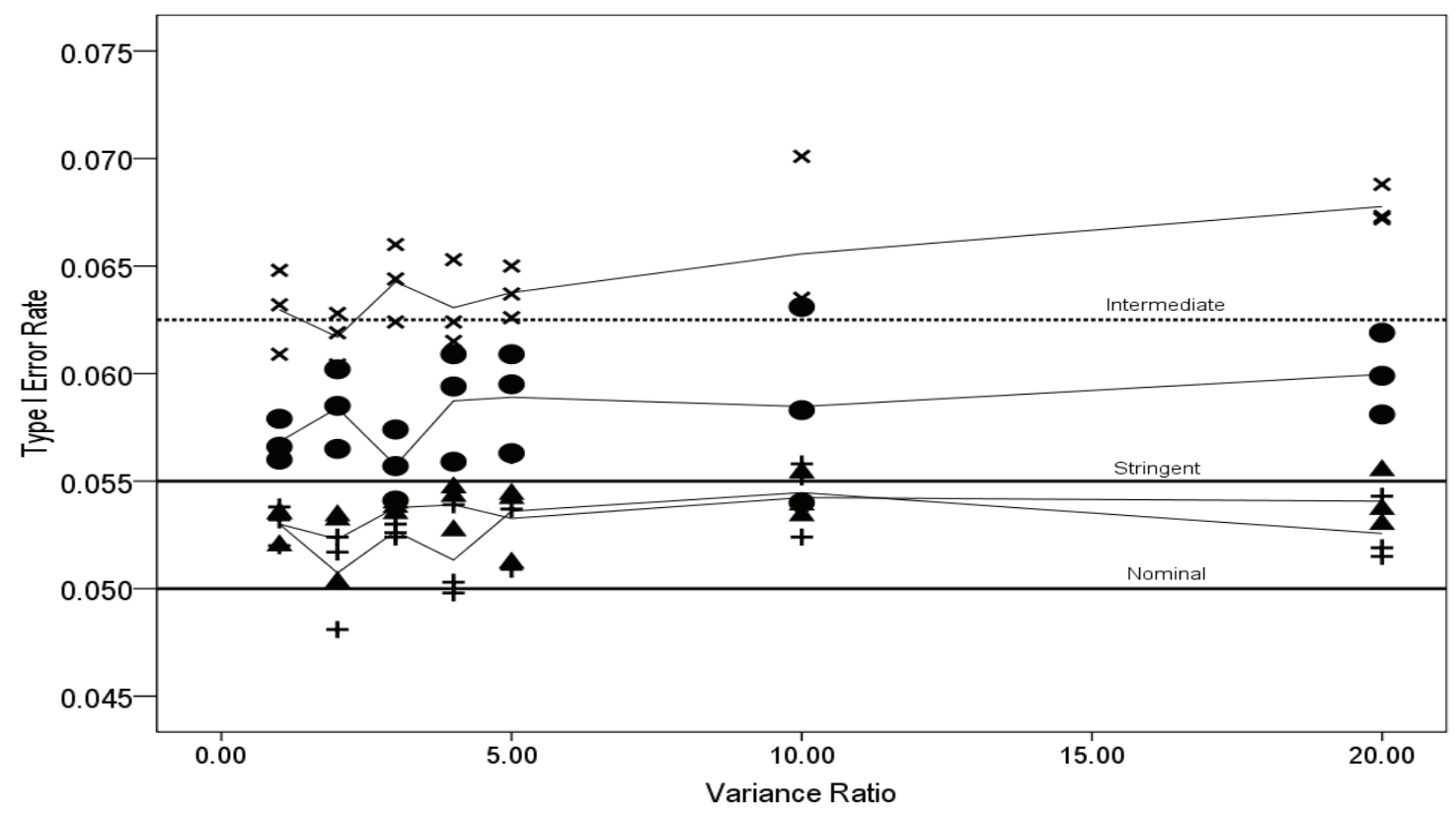

Sample Size

X N=30

? $\mathrm{N}=50$

? $\quad \mathrm{N}=100$

$+\mathrm{N}=150$

Figure 2: RRO Test Type I Error Rate - Uniform Distribution

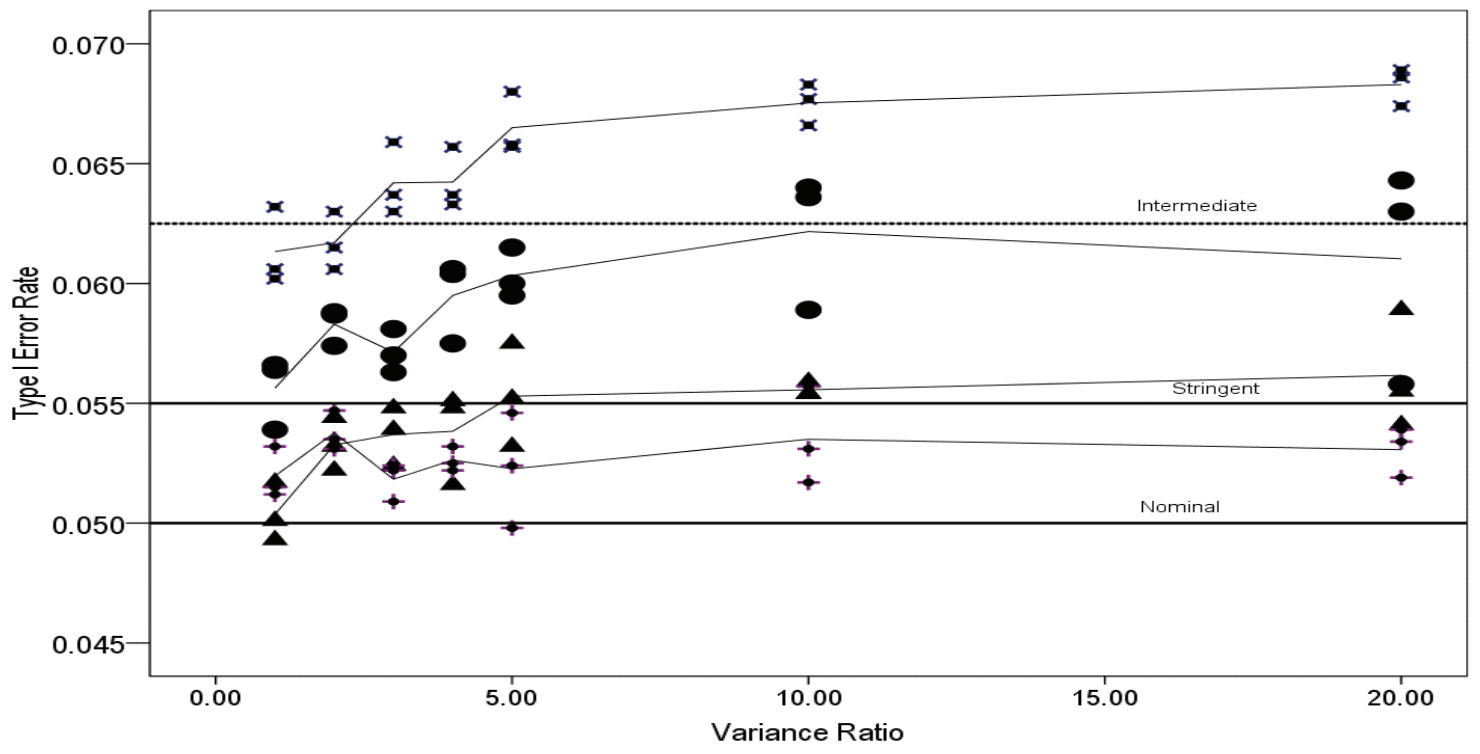




\section{WILLIAM T. MICKELSON}

Figure 3: RRO Test Type I Error Rate - Heavy-Tailed Distribution

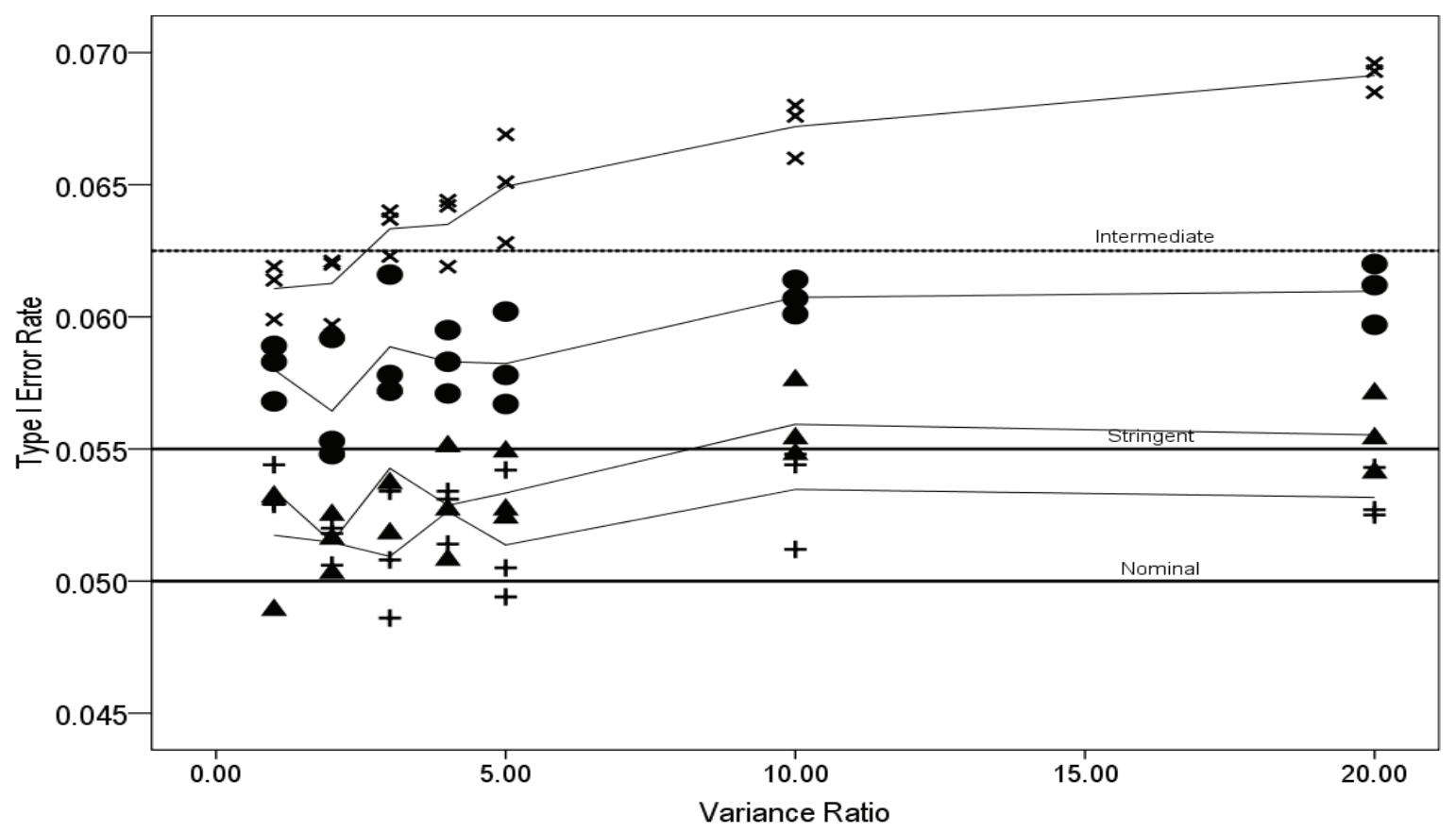

$\underline{\text { Sample Size }}$

X N=30

? $\mathrm{N}=50$

? $\mathrm{N}=100$

$+\mathrm{N}=150$

Figure 4: RRO Test Type I Error Rate - Moderately Skewed Distribution

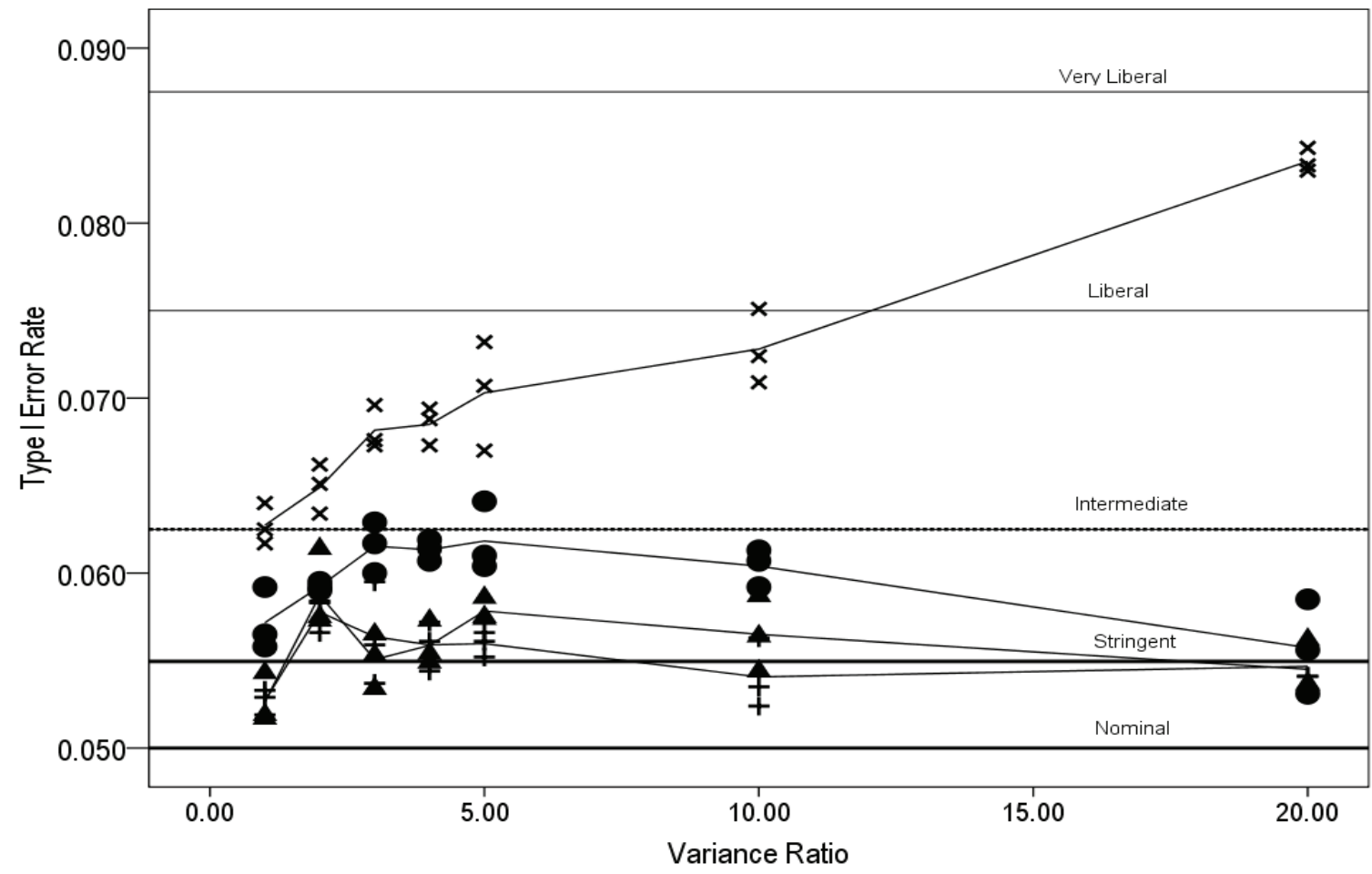

$\underline{\text { Sample Size }}$

X N=30

? $\mathrm{N}=50$

? $\mathrm{N}=100$

$+\mathrm{N}=150$ 


\section{ROBUST RANK-ORDER TEST UNDER POPULATION SYMMETRY CONDITIONS}

Figure 5: RRO Test Type I Error Rate - Heavily Skewed Distribution

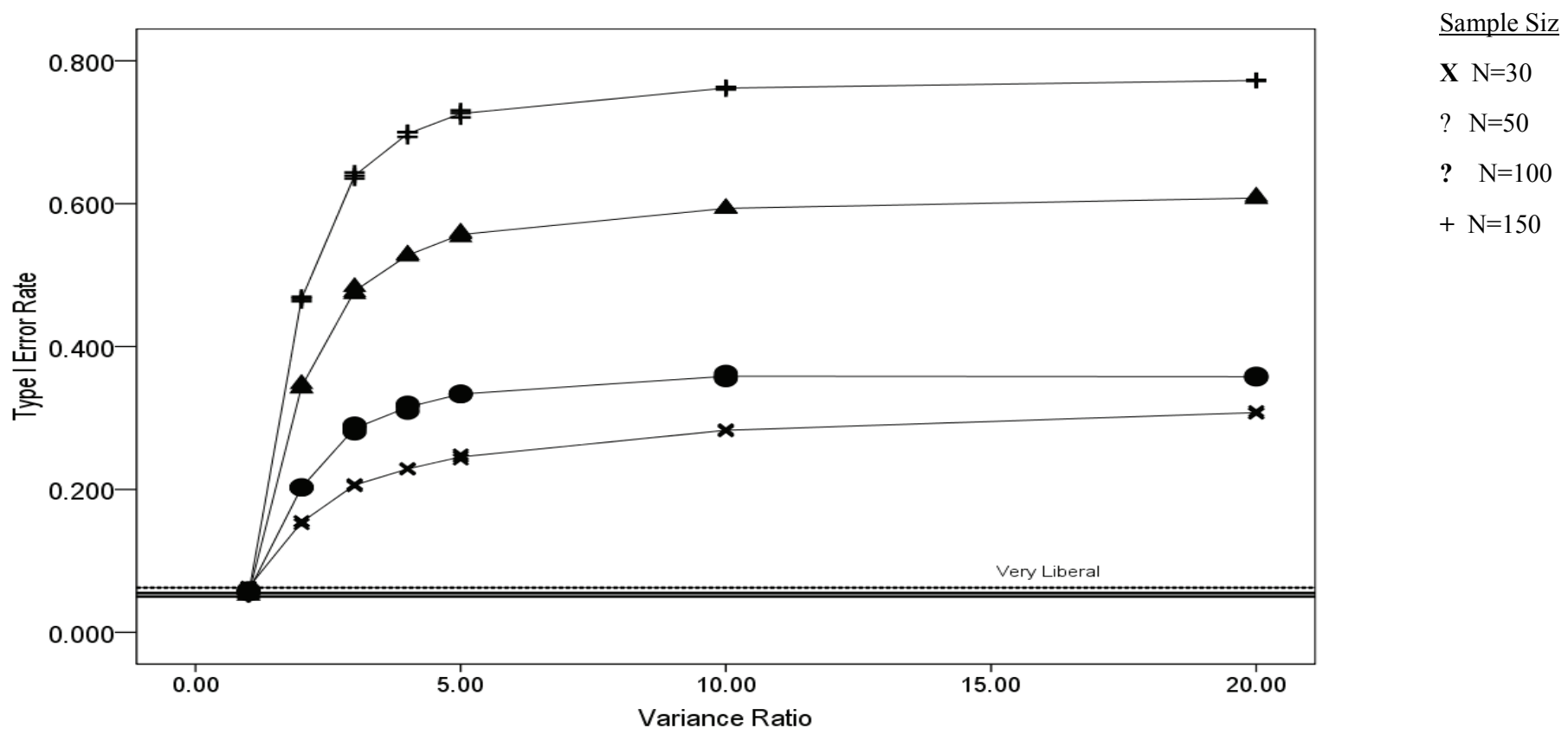

Test Performance: Inverse Pairing

Selected Type I Error results for the Robust Rank Order (RRO) test under the situation of inverse pairing (sample sizes in each group are unequal and the group with the largest sample is paired with the smallest population variance) are presented in Figures 6 - 11. The Xaxis in these plots corresponds to increasing levels of population variance heterogeneity, where the variance ratio ranges from 1:1 to 20:1. The Y-axis corresponds to the Type I Error rate. The degree of sample size inequality is given in the legend of the graph; lines in the graph represent interpolation between means within levels of the sample size ratio.

Figures 6 and 7 present the Type I error rate results for the RRO test under the situation of inverse pairing when the population is normally distributed for total overall sample size of 30 and 150, respectively. Figure 6 shows that when the total sample size is small, $\mathrm{N}=30$, the $\mathrm{RRO}$ is not robust under inverse pairing when the sample size ratio is 4:1 or larger. In general, Type I Error Rate inflates as the sample size ratio and variance ratios increase, either independently or together. Across Figures 6 and 7 , the situation generally improves as the total sample size increases. Figure 7 shows that when $\mathrm{N}=150$, RRO test appears to be robust at the moderate robustness standard or better provided the sample size ratio is $4: 1$ or less. The RRO cannot be considered robust when there is great discrepancy between the sample sizes and the sample size ratio is $9: 1$ or larger. When the overall sample size increases to $\mathrm{N}=150$, the RRO test is robust at the most liberal robustness standard for the largest sample size ratio and can be considered robust at the intermediate robustness standard for sample size ratios of $4: 1$ or smaller.

In examining the Type I Error Rate performance when the population has a uniform distribution, the pattern to the results is highly similar to the patterns observed when the population is normally distributed. As such, those findings are not summarized here and the reader is referred to the Type I Error Rate database available by request from the author.

Figures 8 - 11, present the Type I error rate results for the RRO test under the situation of inverse pairing when the population distribution is heavy tailed, and moderately skewed, respectively. Results are presented for 
Figure 6: RRO Test Type I Error Rate - Normal Distribution N=30

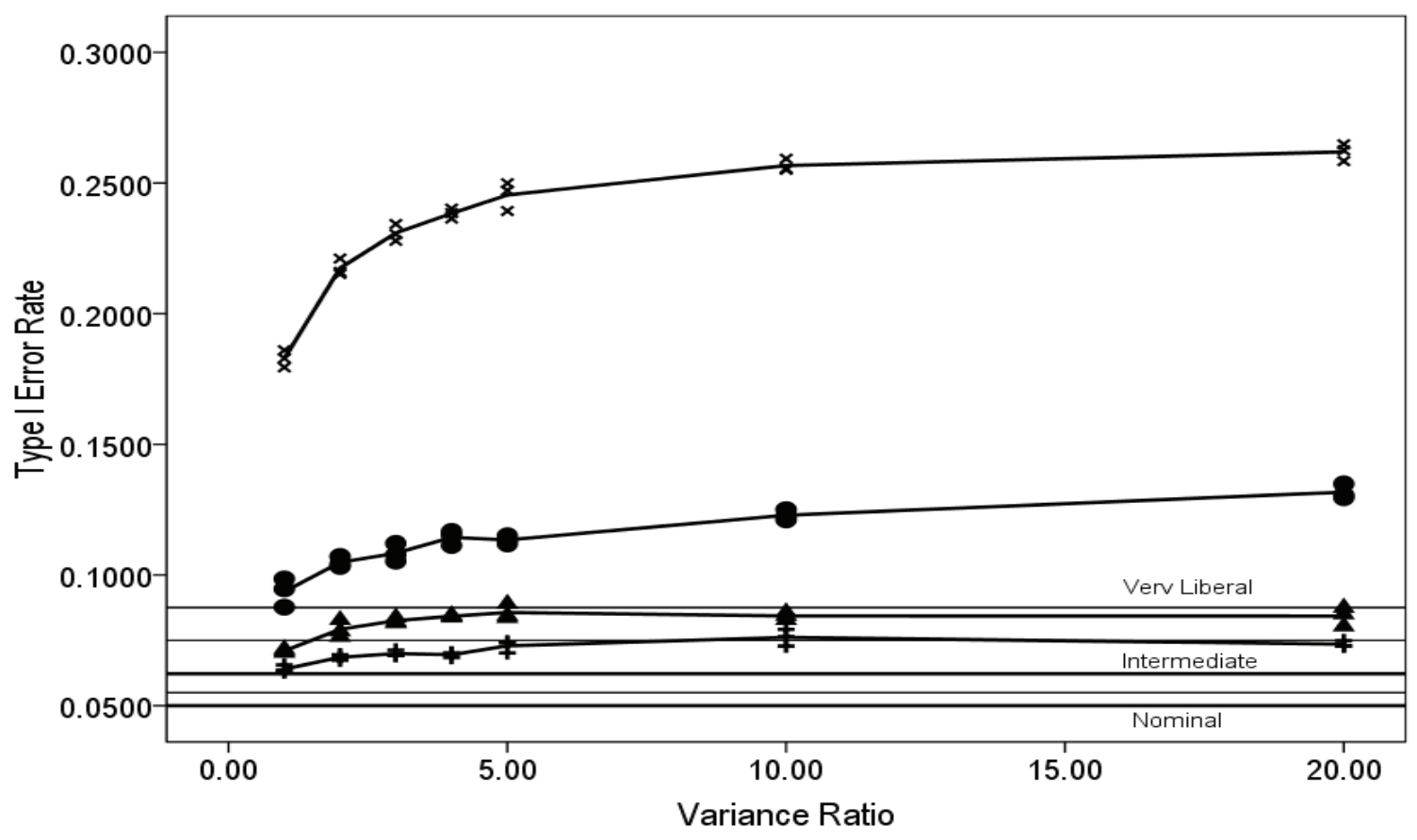

$\underline{\text { Sample Size Ratio }}$

X $9.0: 1$

? $4.0: 1$

? $2.3: 1$

$+1.5: 1$

Figure 7: RRO Test Type I Error Rate - Normal Distribution N=150

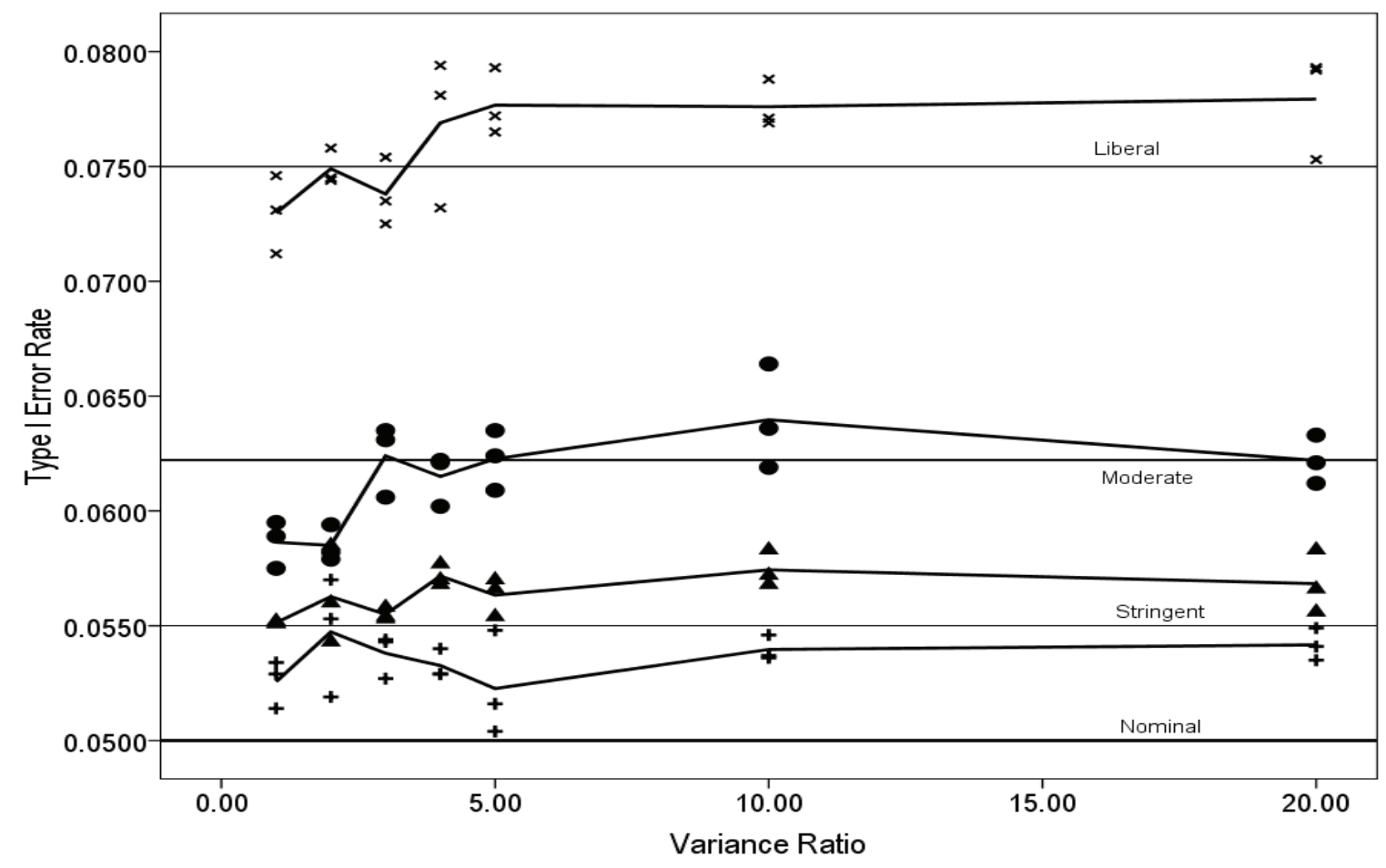

$\underline{\text { Sample Size Ratio }}$

X $9.0: 1$

? $4.0: 1$

? $2.3: 1$

$+1.5: 1$ 


\section{ROBUST RANK-ORDER TEST UNDER POPULATION SYMMETRY CONDITIONS}

total overall sample sizes of 30 and 150, respectively. Type I Error Rate estimates for intermediate sample sizes and additional population distributions are available by request from the author.

The Type I Error Rate estimates for the heavy tailed (Figures 8 and 9) and moderately skewed, (Figures 10 and 11) population distributions demonstrate patterns that are very similar to those previously observed when the population distribution was normally distributed. The RRO test does not perform well under inverse pairing for small sample sizes and performance degrades as the discrepancy in sample size increases, that is, increasing sample size ratio. Performance does improve with increasing sample size, however, even with sample sizes as large as $\mathrm{N}=150$ the higher the discrepancy in sample size situations still have not become sufficiently robust to claim Type I Error rate is controlled. The RRO performs less well when the population distribution is moderately skewed. The same general patterns as those of the symmetric distributions emerge, however, with Type I Error rates becoming more controlled and robust as the sample size ratio becomes closer to $1: 1$.

The claim by Fligner and Policello (1981) that the RRO test statistic converges to a standard normal distribution with increasing sample size for symmetric population distributions appears to be confirmed from this evidence. Even at the sample size of $\mathrm{N}=150$, however, the convergence has not fully materialized to warrant calling the RRO test truly robust at the most stringent level of robustness for heavily-tailed symmetric distributions. There is some evidence that the RRO test could be used in situations with data from moderately skewed population distributions, but only in cases where the sample size ratios are $4: 1$ or less.

\section{Test Performance: Direct Pairing}

Type I Error results for the Robust Rank Order (RRO) test under the situation of direct pairing (unequal sample sizes between groups and the group with the largest sample is paired with the largest population variance) when the populations are normally distributed. Direct pairing occurs when population variances are unequal and the group with the smallest variance is paired with the smallest sample size. The Xaxis in these graphs corresponds to increasing levels of population variance heterogeneity, where the variance ratio ranges from 1:1 to 20:1. The Y-axis corresponds to the Type I Error rate. The degree of sample size inequality is measured by the ratio of the sample sizes and is given in the legend of the graph; lines in the graph represent interpolation between means within levels of the sample size ratio.

Figures 12 and 13 present the Type I error rate results for the RRO test under the situation of direct pairing when the population is normally distributed for total overall sample sizes of 30 and 150, respectively. Figure 12 shows that when the total sample size is small, $\mathrm{N}=30$, the RRO test is predominantly robust and controls the Type I Error Rate rather well. There is one exception, however, in that the RRO test is not robust under direct pairing when the sample size ratio is 9:1 and there is some indicate that there are some issues with error rate control when the sample size ratio is $4: 1$. In general, as sample size ratio increases, the RRO test becomes less robust and this pattern is more pronounced as the sample size ratio exceeds $4: 1$. As the overall sample size increases, however, the RRO is robust for the direct pairing situation (see Figure 13).

Interestingly, the Type I Error Rate patterns exhibited by the RRO when the population is normally distributed are the same for the heavy-tail and moderately skewed distributions. Lack of robustness occurs with the low overall sample size, $\mathrm{N}=30$, and dramatically improves with increasing $\mathrm{N}$. When overall sample sizes is large, $\mathrm{N}=150$, the RRO test can be considered robust, even for the moderately skewed distribution (these findings are not illustrated here and the reader is referred to the Type I Error Rate database available by request from the author. Only for the heavily skewed population distribution does the RRO fail to control Type I error rate well for the direct pairing situation when sample size is large. 
Figure 8: RRO Test Type I Error Rate - Heavy Tail Distribution N=30

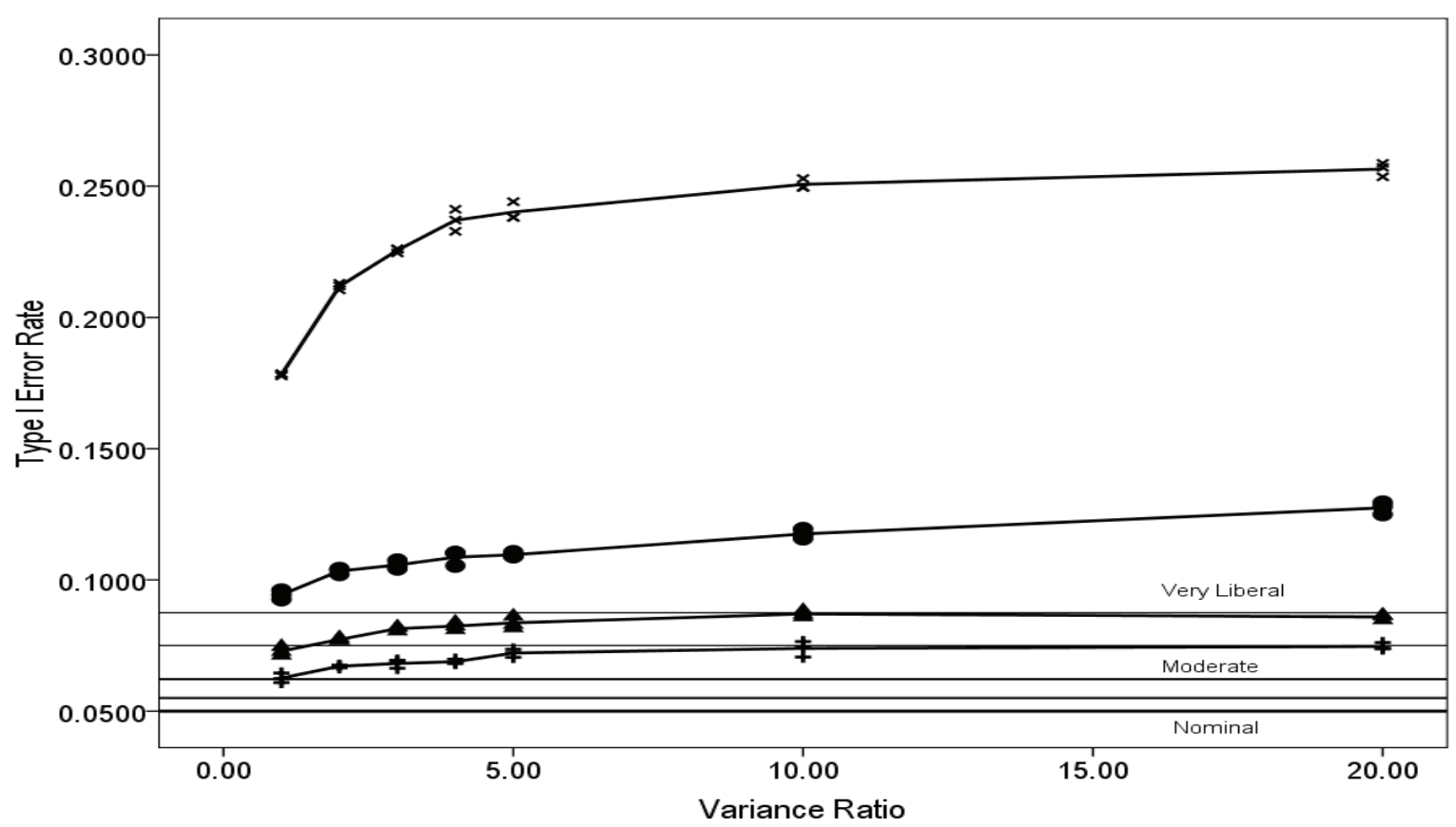

Sample Size Ratio

X $9.0: 1$

? $4.0: 1$

? $2.3: 1$

$+1.5: 1$

Figure 9: RRO Test Type I Error Rate - Heavy Tail Distribution N=150

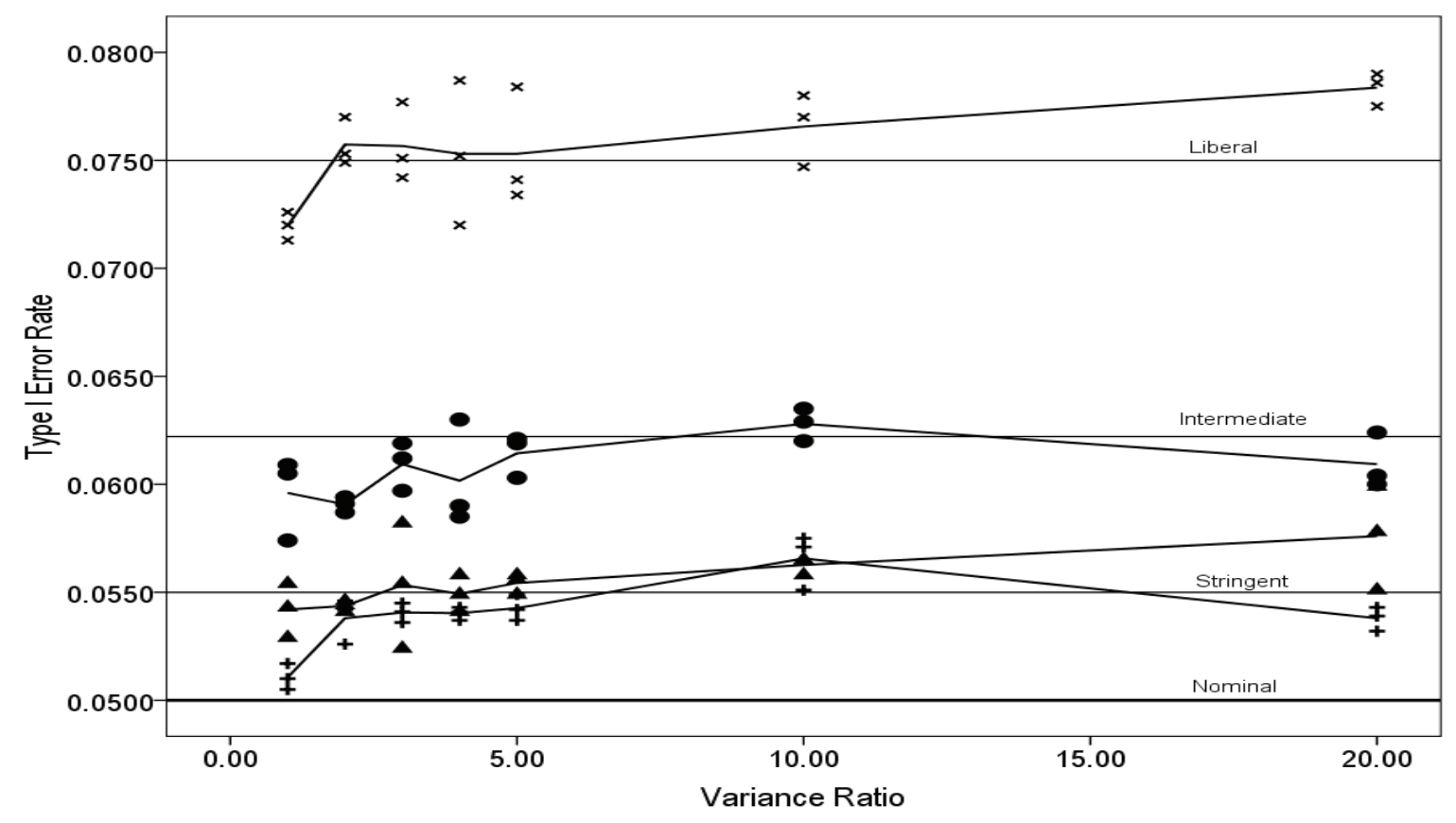

$\underline{\text { Sample Size Ratio }}$

X $9.0: 1$

? $4.0: 1$

? $2.3: 1$

$+1.5: 1$ 
ROBUST RANK-ORDER TEST UNDER POPULATION SYMMETRY CONDITIONS

Figure 10: RRO Test Type I Error Rate - Moderately Skewed Distribution N=30

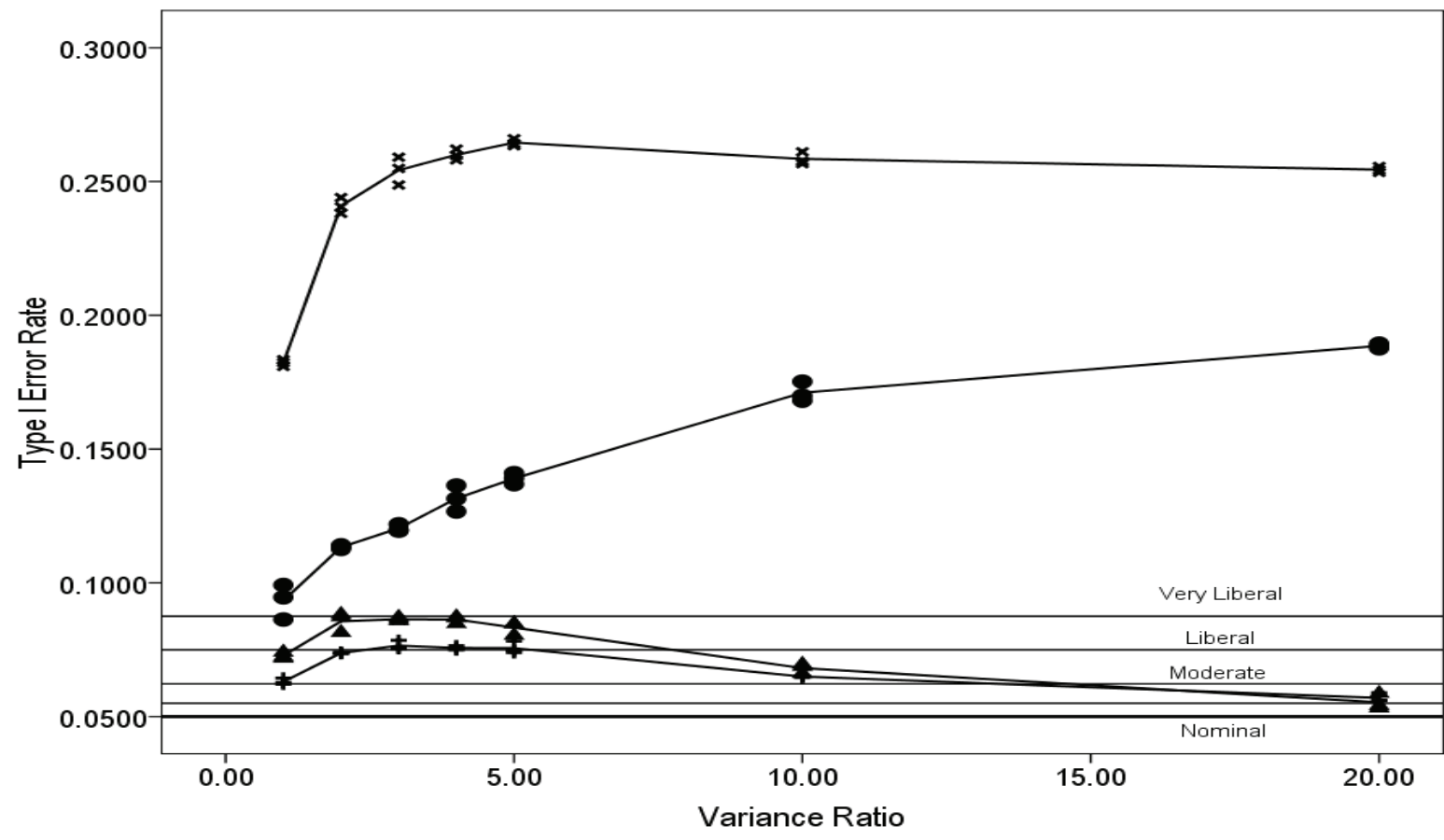

$\underline{\text { Sample Size Ratic }}$

X $9.0: 1$

? $4.0: 1$

? $2.3: 1$

$+1.5: 1$

Figure 11: RRO Test Type I Error Rate - Moderately Skewed Distribution N=150

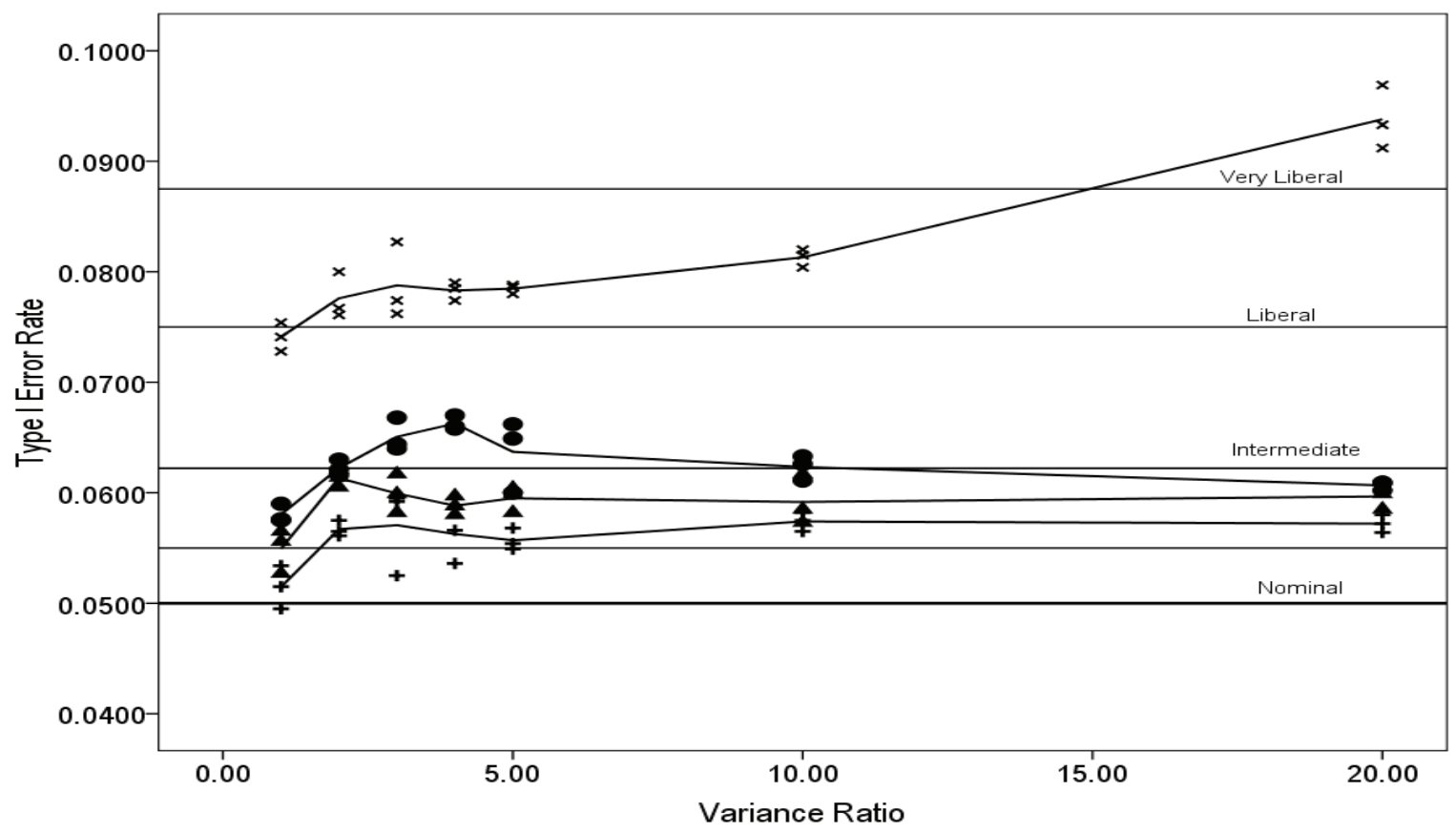

$\underline{\text { Sample Size Ratio }}$

X $9.0: 1$

? $4.0: 1$

? $2.3: 1$

$+1.5: 1$ 
Figure 12: RRO Test Type I Error Rate - Normal Distribution N=30

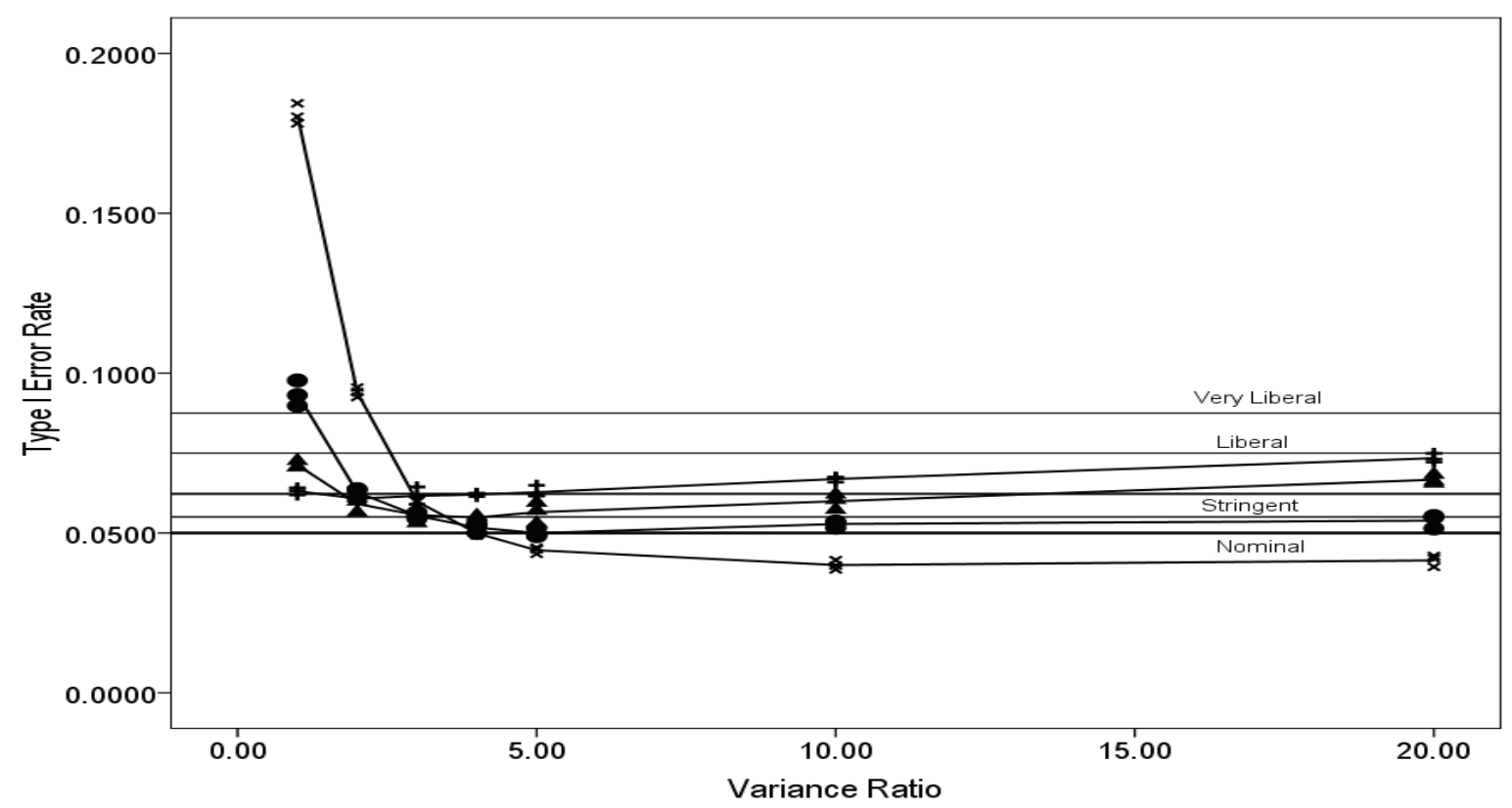

$\underline{\text { Sample Size Ratio }}$

X $9.0: 1$

? $4.0: 1$

? $2.3: 1$

$+1.5: 1$

Figure 13: RRO Test Type I Error Rate - Normal Distribution N=150

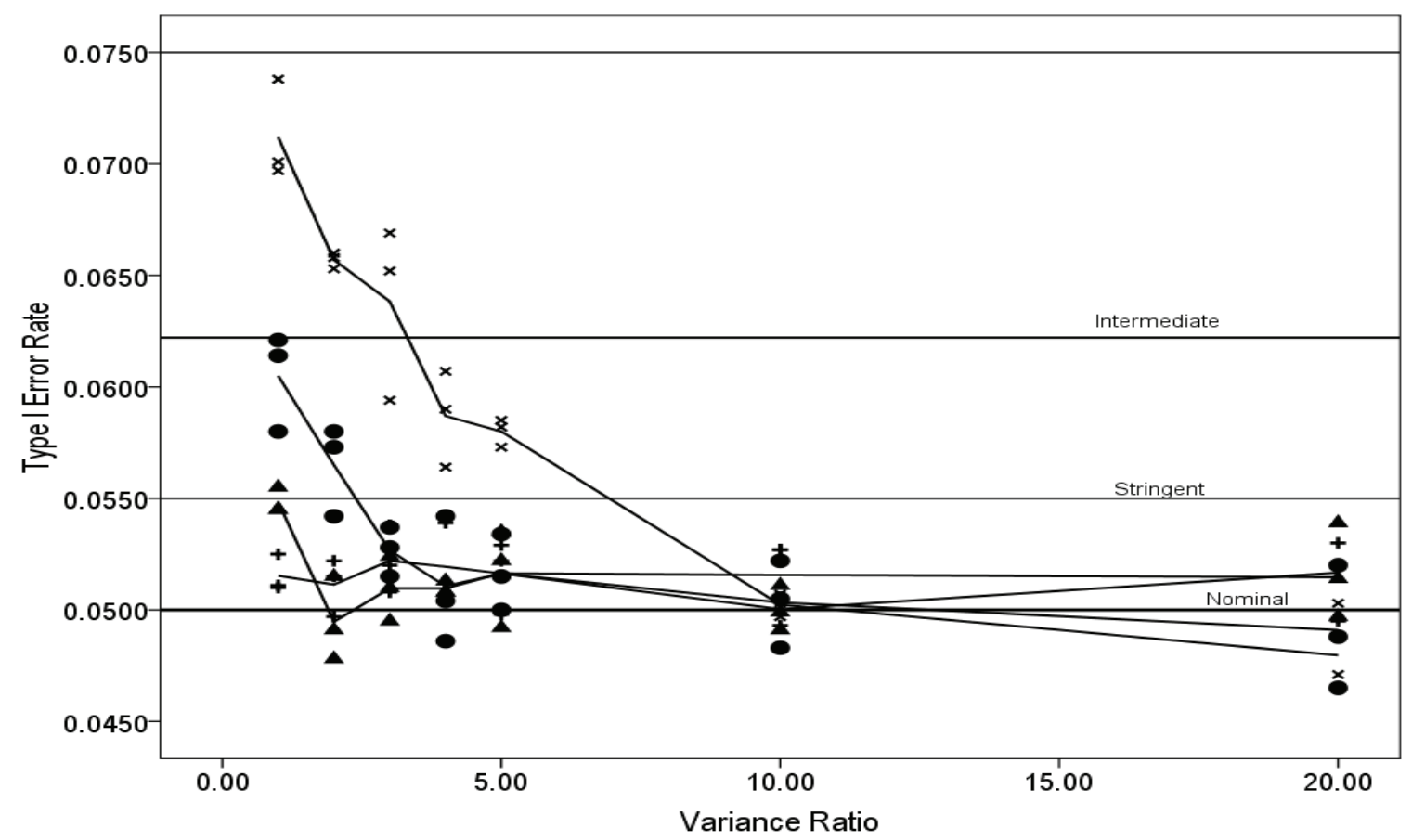

$\underline{\text { Sample Size Ratio }}$

X $9.0: 1$

? $4.0: 1$

? $2.3: 1$

$+1.5: 1$ 


\section{ROBUST RANK-ORDER TEST UNDER POPULATION SYMMETRY CONDITIONS}

\section{Conclusion}

This study investigated the performance of the Robust Rank Order (RRO) test (Fligner \& Policello, 1981) under various population symmetry conditions in the intermediate sample size range prior to the asymptotic distribution holding. First, the claim by Fligner and Policello (1981) that it is necessary to assume the underlying population distributions are symmetric is confirmed. However, to a modest degree, the RRO test does control the Type I Error for moderately skewed population distributions. In general, it appears that the RRO test has the tendency to be liberal, with Type I error rate estimates becoming increasingly inflated:

- as the population distribution becomes more skewed;

- when variance ratios and/or sample size ratios become larger; and

- when overall sample size is smaller.

Particularly interesting is the finding that the RRO appears to perform better under the situation of direct pairing than it does when inverse pairing is present.

When sample sizes are equal, the RRO test controls Type I error rate at essentially the nominal level. The RRO has a slightly inflated Type I error rate, but for the symmetric population distributions, this rate inflation is moderate, at worst, and performance improves with increasing sample size. Under inverse pairing, the RRO test does not perform particularly well in controlling Type I error rate. There is considerable rate inflation that increases as the sample size ratio and/or variance ratio's increase. Performance does improve with increased sample sizes, but even when total sample size reaches $\mathrm{N}=150$ the Type I Error rate is not fully controlled; this is particularly true the more sample sizes become disparate. For smaller sample sizes the RRO test cannot be recommended under conditions of inverse pairing. However, for the direct pairing situation the RRO performs moderately well in controlling Type I error rate. There is some rate inflation for the large sample size and variance ratios when overall sample size is small, but performance does improve dramatically with increased sample size.

In summary, the RRO test, billed as a statistical test designed to maintain the nominal Type-I error rate under generalized BehrensFisher conditions, did not perform uniformly well. The RRO improves with increasing sample size, but has a difficult time with inverse pairing of sample size and variance inequality. Overall, results of this study indicate that the asymptotic result of the RRO test has not sufficiently come into play when overall sample size is between $\mathrm{N}=30$ and $\mathrm{N}=150$ to make the test uniformly robust. The RRO test can be cautiously used in these overall sample size ranges, provided the sample size ratios are less than 4:1 and it can be reasonably assumed that the population distribution is symmetric or - at worst moderately skewed.

\section{References}

Behrens, W. V. (1929). Ein beitrag zur fehlerberechnung bei wenigen beobachtungen. Landwirtsch, Jahrbucher, 68, 807-837.

Bradley, J. V. (1978). Robustness? British Journal of Mathematical and Statistical Psychology, 31, 144-152.

Fisher, R. A. (1939). The comparison of samples with possibly unequal variances. Annals of Eugenics, 9, 174-180.

Fligner, M. A., \& Policello, G. E. II (1981). Robust rank procedures for the BehrensFisher problem. Journal of the American Statistical Association, 76, 162-168.

Glass, G. V., Peckham, P. D., \& Sanders, J. R. (1972). Consequences of failure to meet assumptions underlying the fixed effects analysis of variance and covariance. Review of Educational Research, 42(3), 237-288.

Harwell, M. R., Rubinstein, E. N., Hayes, W. S., \& Olds, C. C. (1992). Summarizing Monte Carlo results in methodological research: The one- and twofactor fixed effects ANOVA cases. Journal of Educational Statistics, 17, 315-339.

Harwell, M. R., \& Serlin, R. C. (1989) A nonparametric test statistic for the general linear model. Journal of Educational Statistics, 14, 351-371. 


\section{WILLIAM T. MICKELSON}

Keselman, H.J., et al. (1998). Statistical practices of educational researchers: An analysis of their ANOVA, MANOVA, and ANCOVA analyses. Review of Educational Research, 68(3), 350-386.

Scheffé, H. (1970). Practical solutions of the Behrens-Fisher problem. Journal of the American Statistical Association, 65, 15011508.

Siegel, S., \& Castellan, N. J. (1988). Nonparametric statistics for the behavioral sciences. New York, NY: McGraw-Hill.

Snyder, P. A., \& Thompson, B., (1998). Use of tests of statistical significance and other analytic choices in a school psychology journal: Review of practices and suggested alternatives. School Psychology Quarterly, 13(4), 335-348.

Vargha, A., \& Delaney, H. D. (2000). A critique and improvement of the CL common language effect size statistics of McGraw and Wong. Journal of Educational and Behavioral Statistics, 25, 101-132.
Zimmerman, D. W., \& Zumbo, B. D. (1993a). Rank transformations and the power of the Student $t$ test and Welch $t$ test non-normal populations with unequal variances. Canadian Journal of Experimental Psychology, 47, 523539.

Zimmerman, D. W., \& Zumbo, B. D. (1993b). The relative power of parametric and nonparametric statistical methods. In $A$ handbook for data analysis in the behavioral sciences: Methodological issues, G. Keren \& C. Lewis (Eds.), 481-517. Hillsdale, NJ: Erlbaum.

Zumbo, B. D., \& Coulombe, D. (1997). Investigation of the robust rank order test for non-normal populations with unequal variances: The case of reaction time. Canadian Journal of Experimental Psychology, 51, 139-149. 


\title{
Constructing a More Powerful Test in Two-Level Block Randomized Designs
}

\author{
Spyros Konstantopoulos \\ Michigan State University \\ East Lansing, MI
}

A more powerful test is proposed for the treatment effect in two-level block randomized designs where random assignment takes place at the first level. When clustering at the second level is assumed to be known, the proposed test produces higher estimates of power than the typical test.

Key words: Block randomized designs, statistical power, clustering, experiments.

\section{Introduction}

One important consideration when designing large-scale experiments is to ensure that the design is sensitive enough to detect the expected intervention effect. This task involves making decisions about sample sizes to ensure sufficient statistical power of the test of the treatment effect. Statistical power is defined as the probability of rejecting the null hypothesis when it is false. Extensive literature exists on the computation of statistical power (e.g., Cohen, 1988; Kraemer \& Thiemann, 1987; Lipsey, 1990; Murphy \& Myors, 2004). Much of the literature, however, involves the computation of power in studies that use simple random samples; hence nesting effects are typically not included in power computations.

In education and the social sciences, populations of interest have often multilevel structures, for example, students are nested within classrooms or schools. Because individuals within aggregate units are often more alike than individuals in different units,

Spyros Konstantopoulos is Associate Professor of measurement and quantitative methods at the department of counseling, educational psychology, and special education at the College of Education at Michigan State University. Website: http://spyros.wiki.educ.msu.edu/. Email him at: spyros@msu.edu. this nesting produces an intraclass correlation structure which is often called clustering in the sampling literature (Kish, 1965). Clustering should be taken into account both in experimental design and statistical analysis. Treatment conditions in experiments may be assigned either to individuals (e.g., students), subgroups (e.g., classrooms) or entire groups (e.g., schools). When treatments are assigned to individuals or subgroups within entire groups the designs are called block randomized designs; these designs are also known as multisite experiments or multisite trials because each site runs a self-sufficient experiment.

In designs that involve clustering, the computation of statistical power is more complicated than in simple random samples designs. First, nested factors are usually assumed to have random effects, and hence, power computations should involve the variance components structures which are typically expressed via intraclass correlations of these random effects. In education for example, schools are clusters that are typically treated as random effects. Second, there is more than one sample size involved because there are units at different levels in the hierarchy. For example, in education where students are nested within schools the power of the test of the treatment effect depends not only on the number of students within a school, but also on the number of schools (Hedges \& Hedberg, 2007; Raudenbush, 1997). The sample sizes at different levels may affect power estimates differently. Statistical theory for computing 


\section{SPYROS KONSTANTOPOULOS}

power in two-level balanced designs has been documented (Barcikowski, 1981; Hedges \& Hedberg, 2007; Raudenbush, 1997; Raudenbush \& Liu, 2000). Hedges and Hedberg provided methods for computing statistical power in twolevel balanced cluster randomized designs where the second level units (e.g., schools) are randomly assigned to a treatment and a control group. Raudenbush and Liu (2000) provided methods for power analysis in two-level balanced block randomized designs where the first level units (e.g., students within schools) are randomly assigned to a treatment or a control group within second level units. These methods are helpful for a priori power analysis during the designing face of the experiment. Methods for power computations of tests of treatment effects in multi-level designs have also been discussed in the health sciences (Donner \& Klar, 2000; Murray, 1998).

Previous methods for power analysis in two-level balanced designs (e.g., students nested within schools) involved the computation of the non-centrality parameter of the non-central $F$ - or $t$-distribution (Hedges \& Hedberg, 2007; Raudenbush \& Liu, 2000). Power is a function of the non-centrality parameter and of the degrees of freedom of the test, and higher values of these two factors correspond to higher values of statistical power. The non-centrality parameter is a function of clustering at the second level, which is typically expressed as an intraclass correlation, the number of level-1 and level-2 units, and the magnitude of the treatment effect. The degrees of freedom are a function of the number of the level-2 units.

Previous work has demonstrated that statistical power is an increasing function of the number of level-1 (e. g. students) and level-2 units (e.g., schools), and the magnitude of the treatment effect, but a decreasing function of the intraclass correlation (Hedges \& Hedberg, 2007; Raudenbush, \& Liu, 2000). Also, the number of level-2 units (e.g., schools) has a larger impact on power than the number of level-1 units (e.g., students). An implicit assumption in these methods, that are useful for a priori power analysis, is that the researcher has an idea about the value of the population intraclass correlation and the treatment effect in order to conduct the necessary power computations.
Hedges and Hedberg (2007) showed that in two-level balanced cluster randomized designs, where, for example, entire groups such as schools are randomly assigned to a treatment and a control condition, the power of the $t$-test has $2(\tilde{m}-1)$ degrees of freedom (assuming no covariates), where $\widetilde{m}$ is the number of level-2 units (e.g., schools) assigned to each condition. When $w$ covariates are included at the second level the degrees of freedom of the $t$-test are 2( $\widetilde{m}-1)-w$. In such designs however, a more powerful test can be constructed when the intraclass correlation structure is assumed to be known (Blair \& Higgins, 1986). Blair and Higgins showed that in two-level cluster randomized designs using an exact $F$-test with larger degrees of freedom is more powerful than that used by Hedges and Hedberg. Specifically, the test provided by Blair and Higgins had 2( $\tilde{m} \tilde{n}-1$ ) degrees of freedom (assuming no covariates), where $\tilde{n}$ is the number of level-1 units (e.g., students) within each level-2 unit (e.g., school).

As in two-level cluster randomized designs a test with larger degrees of freedom can also be constructed for two-level block randomized designs when the intraclass correlation (clustering) structure is assumed to be known. This test is more powerful than the typical test based on level-2 unit means because it preserves the degrees of freedom that are associated with level-1 units (e.g., students). Also, the test is exact, examines the same hypothesis about the treatment effect and has the same non-centrality parameter as the test presented by Raudenbush and Liu (2000) in the balanced case using the ANOVA framework.

The only difference between the two tests is in the degrees of freedom. The exact $t$ test for the treatment effect carried on level-2 unit (e.g., school) means assuming one treatment and one control group has $m-1$ degrees of freedom when no covariates are included at any level, and $m-1-w$ degrees of freedom when $w$ covariates are included at the second level, where $m$ is the total number of level-2 units. Note that in this test the number of first level units is not taken into account in the degrees of the freedom of the test. However, following 


\section{A MORE POWERFUL TEST IN TWO-LEVEL BLOCK RANDOMIZED DESIGNS}

Blair and Higgins (1986) a more powerful test for two-level block randomized designs can be constructed that includes the number of level-1 units in the degrees of freedom of the test. This article provides methods for constructing a more powerful test for treatment effects in two-level block randomized designs; these methods are useful for a priori power computations during the design phase of an experiment.

$$
\text { Methodology }
$$

A Two-Level Block Randomized Design

Following Graybill (1976) and Blair and Higgins (1986) consider a simple case of the general linear model in matrix notation as

$$
\mathbf{y}=\mathbf{X} \boldsymbol{\beta}+\boldsymbol{\varepsilon}
$$

where $\mathbf{y}$ is a $N \times 1$ vector ( $N$ is the total number of observations), $\mathbf{X}$ is a $N \times 2$ (assuming one treatment and one control group) design matrix for the regression coefficients, $\boldsymbol{\beta}$ is a $2 \times 1$ vector of the regression coefficients to be estimated (i.e., treatment and control means), and $\boldsymbol{\varepsilon}$ is a $N \times 1$ vector of residuals that follows a multivariate normal distribution with a mean of zero and a variance matrix $\sigma^{2} \mathbf{V}$, that is $\boldsymbol{\varepsilon} \sim \mathbf{N}\left(\mathbf{0}, \sigma^{2} \mathbf{V}\right)$, where $\sigma^{2}$ is the total variance in the outcome and is factored out of the variance covariance matrix $\mathbf{V}$. If the level-1 units are nested within level-2 units (the clusters), then matrix $\mathbf{V}$ has elements that represent variances or intraclass correlations and ones within each cluster and zeroes between clusters. If these variances or intraclass correlations are assumed to be known, then matrix $\mathbf{V}$ is positive definite and known (Graybill, 1976).

Consider a two-level block randomized design where level-1 units such as students are randomly assigned to one treatment and one control condition within level-2 units such as schools (the blocks). Suppose that there are $m$ level-2 units overall and that the total variance is $\sigma^{2}=\sigma_{e}^{2}+\tau^{2}$, where $\sigma_{e}^{2}$ is the level-1 variance and $\tau^{2}$ is the level-2 variance; matrix $\mathbf{V}$ then has the same structure as the matrix $\mathbf{V}^{*}$ which is block diagonal $\mathbf{V}^{*}=\mathbf{I}_{m} \otimes\left\{\mathbf{V}_{j}^{*}\right\}$ with $m$ blocks (the total number of level-2 units in the sample), I is the identity matrix, and $\otimes$ is the kronecker product. The diagonal elements of matrix $\mathbf{V}_{j}^{*}$ for cluster $j$ are $v_{i i t j}^{*}=\sigma_{e}^{2}+(1+\vartheta) \tau^{2}$ for level-1 units that receive the treatment within the level-2 unit, and $v_{i i c j}^{*}=\sigma_{e}^{2}+\tau^{2}$ for level-1 units that do not receive the treatment within a level-2 unit, where $\vartheta=\tau_{t}^{2} / \tau^{2}$ is the proportion of level-2 unit by treatment variance to the total level-2 variance $(0 \leq \vartheta \leq 1)$ and subscripts $i, j, t, c$ represent level-1, level-2 units, and treatment and control groups respectively. The off diagonal elements of matrix $\mathbf{V}_{j}^{*}$ are $v_{i k j}^{*}=\tau^{2}$. If the intraclass correlation are defined as the proportion of the between level-2 unit variance to the total variance, namely $\rho=\tau^{2} / \sigma^{2}$ and the total variance $\sigma^{2}$ is factored out from matrix $\mathbf{V}^{*}$ matrix $\mathbf{V}$ is constructed, which has ones in the main diagonal for level-1 units in the control group, $1+\vartheta \rho$ in the main diagonal for level-1 units in the treatment group, $\rho$ in the off diagonal between level-1 units within each level-2 unit and zeroes between level-2 units (see Appendix). If the intraclass correlation $\rho$ and $\vartheta$ are known, which essentially means that the proportion of the level-2 unit by treatment variance to the total variance is known, then matrix $\mathbf{V}$ is known.

To illustrate the structure of matrix $\mathbf{V}$ consider a simple case where there are two schools and within each school two students are randomly assigned to a treatment and two students to a control group. Assuming the first two students receive treatment, $\mathbf{V}_{j}=\mathbf{V}_{j}^{*} / \sigma^{2}$ for school $j$ is

$$
\mathbf{V}_{j}=\left[\begin{array}{llll}
1+\vartheta \rho & \rho & \rho & \rho \\
\rho & 1+\vartheta \rho & \rho & \rho \\
\rho & \rho & 1 & \rho \\
\rho & \rho & \rho & 1
\end{array}\right],
$$

and $\mathbf{V}$ is 


$$
\mathbf{V}=\left[\begin{array}{ll}
\mathbf{V}_{j} & \mathbf{0} \\
\mathbf{0} & \mathbf{V}_{j}
\end{array}\right]
$$

where $\mathbf{0}$ is a $2 \times 2$ matrix of zeros, namely $\mathbf{0}=[0,0,0,0]$, expressed as a row vector. In this simple case when no covariates are included the matrix $\mathbf{X}$ is

$$
\mathbf{X}^{T}=\left[\begin{array}{llllllll}
1 & 1 & 0 & 0 & 1 & 1 & 0 & 0 \\
0 & 0 & 1 & 1 & 0 & 0 & 1 & 1
\end{array}\right]
$$

and

$$
\boldsymbol{\beta}=\left[\begin{array}{l}
\beta_{1} \\
\beta_{2}
\end{array}\right]
$$

Finally, the vectors $\mathbf{y}$ (the outcome) and $\mathbf{e}$ (the residuals of $\mathbf{y}$ ) are expressed as row vectors

$\mathbf{y}^{T}=\left[\mathrm{y}_{1}, \ldots, \mathrm{y}_{8}\right], \quad \mathbf{e}^{T}=\left[\mathrm{e}_{1}, \ldots, \mathrm{e}_{8}\right]$.

According to Graybill (1976) when matrix $\mathbf{V}$ is known the regression estimates of the general linear model are computed as

$$
\widehat{\boldsymbol{\beta}}=\left(\mathbf{X}^{T} \mathbf{V}^{-1} \mathbf{X}\right)^{-1} \mathbf{X}^{T} \mathbf{V}^{-1} \mathbf{y},
$$

the total variance is estimated as

$$
\hat{\sigma}^{2}=\frac{1}{N-2} \mathbf{y}^{T}\left(\mathbf{V}^{-1}-\mathbf{V}^{-1} \mathbf{X}\left(\mathbf{X}^{T} \mathbf{V}^{-1} \mathbf{X}\right)^{-1} \mathbf{X}^{T} \mathbf{V}^{-1}\right) \mathbf{y}
$$

and the variances of the regression estimates are computed as

$$
\operatorname{var}(\widehat{\boldsymbol{\beta}})=\sigma^{2}\left(\mathbf{X}^{T} \mathbf{V}^{-1} \mathbf{X}\right)^{-1}
$$

Following Graybill (1976) and Blair and Higgins (1986) the test constructed for the hypothesis

$$
\mathbf{H} \boldsymbol{\beta}=0
$$

is a general $F$-test

$$
F=\frac{(\mathbf{H} \widehat{\boldsymbol{\beta}}-0)^{T}\left(\mathbf{H}^{T}\left(\mathbf{X}^{T} \mathbf{V}^{-1} \mathbf{X}\right)^{-1} \mathbf{H}\right)^{-1}(\mathbf{H} \widehat{\boldsymbol{\beta}}-0)^{T}}{\hat{\sigma}^{2}} / 1
$$

with 1 and $\mathrm{N}-2$ degrees of freedom (assuming one treatment and one control group). Matrix $\mathbf{H}$ is a $1 \times 2$ design matrix that facilitates the contrast among the two treatment conditions and $\wedge^{2}$

$\hat{\sigma}$ is defined in equation (3). Specifically, when there is one treatment and one control group and a researcher is interested in testing the equality between the two means, the vector of contrasts $\mathbf{H}=[1,-1]$, and the vector of coefficients is $\boldsymbol{\beta}^{T}=\left[\beta_{1}, \beta_{2}\right]$. Note that the proposed test can be used to test hypotheses for many general linear models including one-way, factorial ANOVA, and ANCOVA (Blair \& Higgins, 1986; Graybill, 1976). When the null hypothesis is false the test follows a non-central $F$ distribution with a noncentrality parameter

$$
\lambda^{2}=\frac{(\mathbf{H} \boldsymbol{\beta}-0)^{T}\left(\mathbf{H}^{T}\left(\mathbf{X}^{T} \mathbf{V}^{-1} \mathbf{X}\right)^{-1} \mathbf{H}\right)^{-1}(\mathbf{H} \boldsymbol{\beta}-0)^{T}}{2 \sigma^{2}}
$$

and $1, \mathrm{~N}-2$ degrees of freedom. This test can be used for unbalanced or balanced data.

\section{Clustering}

The test proposed herein assumes that matrix $\mathbf{V}$ is known. This implies that the variance of the treatment effect across level-2 units is known. Eventually, this translates to knowing the intraclass correlation $\rho$ and $\vartheta$, which means knowing the proportion of level-2 unit by treatment variance to the total level-2 variance. Typically values of population parameters are not likely to be known exactly. A more realistic assumption for a priori power analysis is that there is a range of intraclass correlations which will most likely capture the real value of the population parameter. Hedges \& Hedberg (2007) provided a comprehensive collection of intraclass correlations for achievement data based on national representative samples of students. Specifically, they gave an array of plausible values of intraclass correlations for achievement outcomes using recent large-scale studies that surveyed national probability samples of elementary and secondary students in America. This compilation of intraclass correlations is useful for planning 


\section{A MORE POWERFUL TEST IN TWO-LEVEL BLOCK RANDOMIZED DESIGNS}

two-level designs. The values of intraclass correlations ranged typically between 0.10 and 0.20 for typical samples and were smaller than 0.10 for more homogeneous samples (e.g., lowachieving schools). Evidence from two-level models of the National Assessment of Educational Progress (NAEP) trend data and Project STAR data (Konstantopoulos \& Hedges, 2008; Nye, Hedges \& Konstantopoulos, 2000) also points to intraclass correlations between 0.10 and 0.20. Finally, evidence from Project STAR has suggested that the between school variance of the small class effect is typically less than 50 percent of the between school variance.

\section{The ANOVA Model}

The proposed test presented in equation (5) is a general test that can be used in both unbalanced and balanced designs. To simplify computations, how the proposed test can be used in simple two-level balanced block randomized designs is now discussed. Using the ANOVA framework the noncentrality parameter of a test can be computed, which facilitates power computations. In this model, level-1 units are randomly assigned to treatment and control groups within level-2 units. The number of level-2 units are represented by $m$, and the number of level-1 units within each condition by $n$. The assumption is that there is one treatment and one control group and hence the total sample size is $N=2 m n$. At this point the model does not include any covariates. A structural model for a student outcome $Y_{i j k}$, the $k^{\text {th }}$ level-1 unit in the $j^{\text {th }}$ treatment in the $i^{\text {th }}$ level2 unit can then be described as

$$
Y_{i j k}=\alpha_{j}+\beta_{i}+\alpha \beta_{i j}+\varepsilon_{i j k},
$$

where $\alpha_{j}$ is the (fixed) effect of the $j^{\text {th }}$ treatment $(j=1,2)$ within level-2 unit $i, \beta_{i}$ is the random effect of level-2 unit $i(i=1, \ldots, \mathrm{m}), \alpha \beta_{i j}$ is the treatment by level-2 unit interaction random effect, and $\varepsilon_{i j k}$ is the error term of student $k(k=$ $1, \ldots, \mathrm{n})$ within treatment $j$, within level-2 unit $i$. The level-1, level-2 and treatment by level-2 unit random effects have variances $\sigma_{e}^{2}, \tau^{2}, \tau_{t}^{2}$ respectively. The random effects at different levels are orthogonal to each other.

The objective is to examine the statistical significance of the treatment effect, meaning to test the hypothesis:

$$
\mathrm{H}_{0}: \alpha_{1}=\alpha_{2} \text { or } \alpha_{1}-\alpha_{2}=0 \text {. }
$$

Suppose that a researcher wants to test the hypothesis and carries out the usual $t$ - or $F$-test. When the null hypothesis is false, the test statistic $F$ has the non-central $F$-distribution with a non-centrality parameter $\lambda^{2}$. In the balanced case the non-centrality parameter is defined as the expected value of the estimate of the treatment effect divided by the square root of the variance of the estimate of the treatment effect, namely

$$
\lambda^{2}=\delta^{2} \frac{m n}{2} \frac{1}{1+(n \vartheta-1) \rho}
$$

(Hedges \& Hedberg, 2007; Raudenbush \& Liu, $2000)$. This $F$-test is based on the level-2 unit means and hence the degrees of freedom of the denominator of the test are $m-1$ assuming no covariates at the second level. The power of the $F$-test at level $\alpha$ is

$$
p=1-\mathrm{H}\left[c(\alpha, 1, m-1), 1, m-1, \lambda^{2}\right],
$$

where $c(\alpha, 1, v)$ is the level $\alpha$ critical value of the $F$-distribution with $1, v$ degrees of freedom (e.g., $c(0.05,1,20)=4.35)$ and $\mathrm{H}(x, 1, v, \lambda)$ is the cumulative distribution function of the noncentral $F$-distribution with $1, v$ degrees of freedom and non-centrality parameter $\lambda^{2}$. Equivalently, the test of the treatment effect and statistical power can also be computed using the $t$-statistic that has a non-central $t$-distribution with $m-1$ degrees of freedom and a noncentrality parameter $\lambda$ (the square root of equation (8)).

When the intraclass correlation structure is assumed known, however, a more powerful $F$ or $t$-test can be constructed (see equation (5)). In the balanced case the non-centrality parameter of the test is the same as that reported in 


\section{SPYROS KONSTANTOPOULOS}

equation (8). However, this test has larger degrees of freedom, because $\sigma$ in equation (5) is estimated by $\hat{\sigma}$ in equation (3). Because the degrees of freedom associated with $\hat{\sigma}$ are $N-2$, the degrees of freedom of the denominator of the proposed test are $\mathrm{N}-2=2(m n-1)$ assuming one treatment and one control and no covariates. The power of the $F$-test at level $\alpha$ is

$$
p=1-\mathrm{H}\left[c(\alpha, 1,2(m n-1)), 2(m n-1), \lambda^{2}\right] .
$$

Equivalently, the $t$-statistic has a non-central $t$ distribution with $2(m n-1)$ degrees of freedom and a non-centrality parameter $\lambda$.

\section{The ANCOVA Model}

When covariates are included at the first and second level the linear model is

$$
Y_{i j k}=\alpha_{A j}+\beta_{A i}+\boldsymbol{\theta}_{1}^{T} \mathbf{X}_{i j k}+\boldsymbol{\theta}_{2}^{T} \mathbf{Z}_{i}+\alpha \beta_{A i j}+\varepsilon_{A(i j) k}
$$

where $\boldsymbol{\theta}_{1}^{T}=\left(\theta_{11}, \ldots, \theta_{1 r}\right)$ is a row vector of $r$ first-level covariate effects, $\boldsymbol{\theta}_{2}^{T}=\left(\theta_{21}, \ldots, \theta_{2 w}\right)$ is a row vector of $w$ second-level covariate effects, $\mathbf{X}_{i j k}$ is a column vector of $r$ first-level covariates (e.g., student characteristics) in the $j^{\text {th }}$ treatment in the $i^{\text {th }}$ second level unit, $\mathbf{Z}_{i}$ is a column vector of $w$ second-level covariates (e.g., school characteristics); all other terms have been previously defined. The subscript A indicates that both the treatment and the random effects are adjusted by the covariates in the model. In principle however, assuming randomization is successful, the treatment effect is orthogonal to the covariates and the error term and the expected value of the adjustment is zero. The first and second level random effects are adjusted by first and second level covariates respectively. The first level covariates are centered around their second level unit means and therefore they do not explain variance of the random effects at the second level (i.e., groupmean centering). Centering also ensures orthogonality among predictors at the first and second level. All first level covariates are treated as fixed at the second level. When covariates are included in the model the level-1 and level-2 residual variances are defined as $\sigma_{R e}^{2}, \tau_{R}^{2}$ respectively, and the residual total variance is $\sigma_{R T}^{2}=\sigma_{R e}^{2}+\tau_{R}^{2} \quad$ (and $\mathrm{R}$ indicates residual variances because of the adjustment for the effect of covariates). The adjusted level-2 intraclass correlation is defined then as

$$
\rho_{A 2}=\frac{\tau_{R}^{2}}{\sigma_{R T}^{2}} .
$$

Covariates are useful when conducting power analysis because they typically increase the power of the test for the treatment effect. Specifically, covariates that are significantly associated with the outcome typically explain some proportion of the variance in the outcome, which in turn results in a reduction of the unconditional intraclass correlations and the standard errors of the treatment effects. In experimental studies this indicates that the $F$ - or the $t$-tests for the treatment effects will have higher values when covariates are included in the model because the treatment effect remains virtually unchanged due to the fundamental principle of randomization, which assumes independence between treatment effects and covariates. That is, a researcher can achieve optimal power estimates (e.g., 0.80) without having to increase sample size. In fact, as Cook (2005) argues covariates with considerable predictive power are important for reducing the number of larger units such as schools needed, and for making the study less expensive or affordable given a fixed budget. Powerful covariates at the first level, when modeling achievement data, include previous achievement and socioeconomic status (Hedges \& Hedberg, 2007). Powerful covariates at the second level include school aggregate measures of achievement or socioeconomic status.

In a balanced design within the ANCOVA framework the objective is to examine the statistical significance of the treatment effect net of the possible effects of covariates, namely to test the hypothesis

$$
\mathrm{H}_{0}: \alpha_{A 1}=\alpha_{A 2} \text { or } \alpha_{A 1}-\alpha_{A 2}=0
$$




\section{A MORE POWERFUL TEST IN TWO-LEVEL BLOCK RANDOMIZED DESIGNS}

which involves the computation of the typical $t$ or $F$-test statistic. When the null hypothesis is false, the $F$-test statistic has the non-central $F$ distribution with $a$ non-centrality parameter

$$
\lambda_{A}^{2}=\delta^{2} \frac{m n}{2} \frac{1}{\eta_{1}+\left(n \eta_{2} \vartheta-\eta_{1}\right) \rho},
$$

where

$$
\eta_{2}=\tau_{R}^{2} / \tau^{2}, \eta_{1}=\sigma_{R e}^{2} / \sigma_{e}^{2}
$$

(Hedges \& Hedberg 2007; Murray, 1998). The $\eta$ 's indicate the proportion of the variances at each level of the hierarchy that is still unexplained (percentage of residual variation). For example, when $\eta_{1}=0.25$, this indicates that the variance at the first level decreased by $75 \%$ due to the inclusion of covariates such as pretreatment measures. The degrees of freedom of the $F$-test are $1,2(m n-1)-w-r$. The power of the $F$-test at level $\alpha$ is

$$
p=1-\mathrm{H}\left[\begin{array}{l}
c(\alpha, 1,2(m n-1)-w-r), \\
1,2(m n-1)-w-r, \lambda_{a}^{2}
\end{array}\right]
$$

Equivalently, the $t$-statistic has a non-central $t$ distribution with $2(m n-1)-w-r$ degrees of freedom and a non-centrality parameter $\lambda_{A}$ (square root of equation (13)).

\section{Computational Example}

Power comparisons between two $t$-tests are now discussed: the typical $t$-test carried out on level-2 unit means with $m-1-w$ degrees of freedom and the proposed $t$-test with $2(m n-1)-$ $w-r$ degrees of freedom. The power computations are presented in Tables 1 and 2 and apply to balanced designs. For this exercise power is computed assuming one treatment and one control group for two-tailed $t$-tests, or equivalently an $F$-test, at the 0.05 significance level assuming no covariates in the model. In Table 1 the effect size parameter is $\delta=0.25$, and in Table 2 the effect size parameter is $\delta=0.40$. Three values of intraclass correlations were used: $0.05,0.10$, and 0.20 . These values have been reported in previous work as typical values for homogeneous and more heterogeneous samples (Hedges \& Hedberg, 2007; Raudenbush $\&$ Liu, 2000). Results from Project STAR have also indicated that $\vartheta \leq 0.50$. The first step in the power analysis is to compute the noncentrality parameter. Suppose that there are a total of $m=6$ schools, $n=15$ students in each condition (30 students total per school) within each school and that $\delta=0.40, \rho=0.10$, and $\vartheta$ $=0.50$. The noncentrality parameter using equation (8) is

$$
\lambda=0.4^{2} \frac{6 * 15}{2} \frac{1}{1+(15 * 0.5-1) * 0.10}=4.36
$$

and the degrees of freedom are $6-1=5$ for the test using the level- 2 means and $2 *(6 * 15-1)=$ 178 for the proposed test. Using equation (10) the power is 0.39 and using equation (11) the power is 0.55 (see seventh row in Table 2). The functions for the noncentral $F$ - or $t$-test are available in mainstream packages such as SPSS (the functions are Ncdf.F or Ncdf.T), SAS (using the cumulative distribution functions, CDF, of the $F$ - or $t$-distribution), S-Plus (the functions are pf or ptnoncent) or $\mathrm{R}$ (the functions are $\mathrm{pf}$ or $\mathrm{pt}$ ).

The first column of Table 1 shows the number of level-2 units in the sample. The second column shows the number of level-1 units within each condition within each level-2 unit. The third and fourth columns show values of $\rho$ and $\vartheta$, and columns five and six show the degrees of freedom for each test. Finally, columns seven and eight show power values for each test. The number of level-2 units ranges from 6 to 12, and the number of level-1 units per condition per level-2 unit ranges from 15 to 30 . Results from Table 1 suggest that the power of the proposed test is always higher than the power of the typical test based on level-2 unit means. The difference in power is more pronounced when the number of level-2 units is smaller, the number of level-1 units is larger, and $\rho, \vartheta$ are small. For example, when the total number of level-2 units $m=6$, the number of level-1 units $n=30$ in each condition per level-2 unit, $\delta=0.25, \rho=0.05$, and $\vartheta=0.25$ 


\section{SPYROS KONSTANTOPOULOS}

the power is 0.54 for the proposed test and 0.39 for the typical test that uses level-2 unit means. The difference in power becomes smaller however, as the number of level-2 units increases.

The structure of Table 2 is identical to that in Table 1. As expected, because the effect size is larger, power estimates in Table 2 are larger. Again, the power of the proposed test is always higher than the power of the typical test based on level-2 unit means. As in Table 1, the difference in power is more pronounced when the number of level-2 units is smaller, the number of level-1 units is larger, and $\rho, \vartheta$ are smaller. For example, when the total number of level-2 units $m=6$, the number of level-1 units $n$ $=30$ in each condition per level- 2 unit, $\delta=0.40$, $\rho=0.10$, and $\vartheta=0.25$ the power is 0.84 for the proposed test and 0.66 for the typical test based on level-2 unit means. The difference in power becomes smaller as the number of level-2 units becomes larger. Overall, power is positively affected by the effect size and the number of level-1 and level-2 units, and negatively affected by $\rho, \vartheta$, which suggests that the larger the between level-2 unit variance of the treatment effect the smaller the power, other things being equal.

These findings replicate the results presented by Blair and Higgins for two-level cluster randomized designs. The power estimates of the proposed test will always be larger than those obtained by the test based on the level-2 unit means, and the difference in power is larger when the number of the level-2 units is smaller, the number of level-1 units is larger and the between level-2 unit variance of the treatment effect is smaller. However, as the number of level-2 units increases, the difference in power between the two tests decreases, and when the number of level-2 units becomes infinitely large the two tests provide almost identical estimates of power.

\section{Conclusion}

This study proposed a more powerful test for treatment effects in two-level block randomized designs where, for example, students within schools are randomly assigned to a treatment and a control group. The proposed test statistic is more powerful than the typical test based on level-2 unit means because it preserves the degrees of freedom that are associated both with level-2 and level-1 units. The test can be used to compute power both in unbalanced and balanced designs. However, this study focused on the balanced case. The assumption of the proposed test is that the between level-2 unit variance of the treatment effect is known, that is, $\rho, \vartheta$ are known.

In education, when the outcome is achievement, there is evidence that the level-2 intraclass correlation ranges typically from 0.10 to 0.20 , and it is less than 0.10 for more homogeneous samples. There is also some evidence that the between level-2 unit variance of the treatment effect is typically less than 50 percent of the between level-2 unit variance. As with some statistical procedures a limitation of the current test is that the information used to compute power is not always known exactly. Nonetheless, for a priori power analysis some knowledge of clustering and effect sizes is always necessary for computing power of the typical test based on level-2 unit means (Raudenbush \& Liu, 2000).

It is important to stress that the methods for a priori power computations provided are intended to serve simply as useful guides for experimental designs; the sample sizes proposed, although informative, should be treated as approximate and not exact (Kraemer \& Thieman, 1987). The results of the methods presented are accurate as long as the assumptions about the model and the tests, as well as the estimates of effect sizes and intraclass correlations, are accurate. Regardless, assuming educated or accurate guesses for the information used to compute the power in the proposed test produce higher estimates of power than in the typical test, especially when the number of level-2 units and the intraclass correlations are small. The findings of this study are useful because in education and the social sciences many times researchers focus on homogeneous groups (e.g., minorities, disadvantaged students). In addition, sampling fewer level-2 units (e.g., schools) is costeffective because it reduces the cost of the study overall without compromising statistical power. 
A MORE POWERFUL TEST IN TWO-LEVEL BLOCK RANDOMIZED DESIGNS

Table 1: Power Comparisons between a F-test Based on Level-2 Unit Means and the Proposed F-test: Effect Size is 0.25

\begin{tabular}{|c|c|c|c|c|c|c|c|}
\hline $\begin{array}{l}\text { Number of } \\
\text { Level-2 } \\
\text { Units }\end{array}$ & $\begin{array}{l}\text { Number } \\
\text { of Level-1 } \\
\text { Units }\end{array}$ & $\begin{array}{l}\text { Intraclass } \\
\text { Correlation }\end{array}$ & Theta & $\begin{array}{c}\mathrm{df} / \text { Level-2 } \\
\text { Unit Means }\end{array}$ & $\begin{array}{c}\text { df/ } \\
\text { All } \\
\text { Observations }\end{array}$ & $\begin{array}{c}\text { Power/Level-2 } \\
\text { Unit Means }\end{array}$ & $\begin{array}{c}\text { Power/ } \\
\text { All } \\
\text { Observations }\end{array}$ \\
\hline 6 & 15 & 0.05 & 0.25 & 5 & 178 & 0.25 & 0.35 \\
\hline 6 & 30 & 0.05 & 0.25 & 5 & 358 & 0.39 & 0.54 \\
\hline 6 & 15 & 0.05 & 0.50 & 5 & 178 & 0.22 & 0.31 \\
\hline 6 & 30 & 0.05 & 0.50 & 5 & 358 & 0.32 & 0.44 \\
\hline 6 & 15 & 0.10 & 0.25 & 5 & 178 & 0.23 & 0.31 \\
\hline 6 & 30 & 0.10 & 0.25 & 5 & 358 & 0.32 & 0.45 \\
\hline 6 & 15 & 0.10 & 0.50 & 5 & 178 & 0.19 & 0.25 \\
\hline 6 & 30 & 0.10 & 0.50 & 5 & 358 & 0.24 & 0.33 \\
\hline 6 & 15 & 0.20 & 0.25 & 5 & 178 & 0.20 & 0.27 \\
\hline 6 & 30 & 0.20 & 0.25 & 5 & 358 & 0.25 & 0.34 \\
\hline 6 & 15 & 0.20 & 0.50 & 5 & 178 & 0.15 & 0.20 \\
\hline 6 & 30 & 0.20 & 0.50 & 5 & 358 & 0.17 & 0.23 \\
\hline 12 & 15 & 0.05 & 0.25 & 11 & 358 & 0.53 & 0.60 \\
\hline 12 & 30 & 0.05 & 0.25 & 11 & 718 & 0.76 & 0.83 \\
\hline 12 & 15 & 0.05 & 0.50 & 11 & 358 & 0.47 & 0.54 \\
\hline 12 & 30 & 0.05 & 0.50 & 11 & 718 & 0.65 & 0.73 \\
\hline 12 & 15 & 0.10 & 0.25 & 11 & 358 & 0.48 & 0.55 \\
\hline 12 & 30 & 0.10 & 0.25 & 11 & 718 & 0.66 & 0.74 \\
\hline 12 & 15 & 0.10 & 0.50 & 11 & 358 & 0.39 & 0.45 \\
\hline 12 & 30 & 0.10 & 0.50 & 11 & 718 & 0.50 & 0.58 \\
\hline 12 & 15 & 0.20 & 0.25 & 11 & 358 & 0.41 & 0.48 \\
\hline 12 & 30 & 0.20 & 0.25 & 11 & 718 & 0.52 & 0.60 \\
\hline 12 & 15 & 0.20 & 0.50 & 11 & 358 & 0.30 & 0.34 \\
\hline 12 & 30 & 0.20 & 0.50 & 11 & 718 & 0.35 & 0.40 \\
\hline
\end{tabular}




\section{SPYROS KONSTANTOPOULOS}

Table 2: Power Comparisons between a F-test Based on Level-2 Unit Means and the Proposed F-test: Effect Size is 0.4

\begin{tabular}{|c|c|c|c|c|c|c|c|}
\hline $\begin{array}{l}\text { Number of } \\
\text { Level-2 } \\
\text { Units }\end{array}$ & $\begin{array}{l}\text { Number } \\
\text { of Level-1 } \\
\text { Units }\end{array}$ & $\begin{array}{l}\text { Intraclass } \\
\text { Correlation }\end{array}$ & Theta & $\begin{array}{l}\mathrm{df} / \text { Level-2 } \\
\text { Unit Means }\end{array}$ & $\begin{array}{c}\text { df/ } \\
\text { All } \\
\text { Observations }\end{array}$ & $\begin{array}{c}\text { Power/Level-2 } \\
\text { Unit Means }\end{array}$ & $\begin{array}{c}\text { Power/ } \\
\text { All } \\
\text { Observations }\end{array}$ \\
\hline 6 & 15 & 0.05 & 0.25 & 5 & 178 & 0.53 & 0.71 \\
\hline 6 & 30 & 0.05 & 0.25 & 5 & 358 & 0.75 & 0.91 \\
\hline 6 & 15 & 0.05 & 0.50 & 5 & 178 & 0.47 & 0.64 \\
\hline 6 & 30 & 0.05 & 0.50 & 5 & 358 & 0.65 & 0.83 \\
\hline 6 & 15 & 0.10 & 0.25 & 5 & 178 & 0.48 & 0.66 \\
\hline 6 & 30 & 0.10 & 0.25 & 5 & 358 & 0.66 & 0.84 \\
\hline 6 & 15 & 0.10 & 0.50 & 5 & 178 & 0.39 & 0.55 \\
\hline 6 & 30 & 0.10 & 0.50 & 5 & 358 & 0.51 & 0.69 \\
\hline 6 & 15 & 0.20 & 0.25 & 5 & 178 & 0.42 & 0.57 \\
\hline 6 & 30 & 0.20 & 0.25 & 5 & 358 & 0.52 & 0.70 \\
\hline 6 & 15 & 0.20 & 0.50 & 5 & 178 & 0.30 & 0.42 \\
\hline 6 & 30 & 0.20 & 0.50 & 5 & 358 & 0.35 & 0.49 \\
\hline 12 & 15 & 0.05 & 0.25 & 11 & 358 & 0.90 & 0.94 \\
\hline 12 & 30 & 0.05 & 0.25 & 11 & 718 & 0.99 & 1.00 \\
\hline 12 & 15 & 0.05 & 0.50 & 11 & 358 & 0.85 & 0.91 \\
\hline 12 & 30 & 0.05 & 0.50 & 11 & 718 & 0.96 & 0.98 \\
\hline 12 & 15 & 0.10 & 0.25 & 11 & 358 & 0.86 & 0.92 \\
\hline 12 & 30 & 0.10 & 0.25 & 11 & 718 & 0.97 & 0.99 \\
\hline 12 & 15 & 0.10 & 0.50 & 11 & 358 & 0.77 & 0.84 \\
\hline 12 & 30 & 0.10 & 0.50 & 11 & 718 & 0.88 & 0.93 \\
\hline 12 & 15 & 0.20 & 0.25 & 11 & 358 & 0.79 & 0.86 \\
\hline 12 & 30 & 0.20 & 0.25 & 11 & 718 & 0.90 & 0.94 \\
\hline 12 & 15 & 0.20 & 0.50 & 11 & 358 & 0.65 & 0.70 \\
\hline 12 & 30 & 0.20 & 0.50 & 11 & 718 & 0.71 & 0.79 \\
\hline
\end{tabular}




\section{A MORE POWERFUL TEST IN TWO-LEVEL BLOCK RANDOMIZED DESIGNS}

\section{References}

Barcikowski, R. S. (1981). Statistical power with a group mean as the unit of analysis. Journal of Educational Statistics, 6, 267-285.

Blair, R. C., \& Higgins, J. J. (1986). Comment of statistical power with group mean as the unit of analysis. Journal of Educational Statistics, 11, 161-169.

Cohen, J. (1988). Statistical power analysis for the behavioral sciences ( $2^{\text {nd }} E d$.). New York, NY: Academic Press.

Cook, T. D. (2005). Emergent principles for the design, implementation, and analysis of cluster-based experiments in social science. The Annals of the American Academy of Political and Social Science, 599, 176-198.

Donner, A., \& Klar, N. (2000). Design and analysis of cluster randomization trials in health research. London: Arnold.

Graybill, F. A. (1976). Theory and application of the linear model. Boston, MA: Duxbury Press.

Hedges, L. V., \& Hedberg, E. (2007). Intraclass correlation values for planning group randomized trials in Education. Educational Evaluation and Policy Analysis, 29, 60-87.

Kish, L. (1965). Survey sampling. New York, NY: Wiley.

Konstantopoulos, S., \& Hedges, L. V. (2008). How Large an effect can we expect from school reforms? Teachers College Record, 110, 1613-1640.

Kraemer, H. C., \& Thieman, S. (1987). How many subjects? Statistical power analysis in research. Newbury Park, CA: Sage.

Lipsey, M. W. (1990). Design sensitivity: Statistical power analysis for experimental research. Newbury Park, CA: Sage Publications.

Murphy, K. R., \& Myors, B. (2004). Statistical power analysis: A simple and general model for traditional and modern hypothesis tests $\left(2^{\text {nd }} E d.\right)$. Mahwah, N.J.: Lawrence Erlbaum.

Murray, D. M. (1998). Design and analysis of group-randomized trials. New York, NY: Oxford University Press.
Nye, B., Hedges, L. V., \& Konstantopoulos, S. (2000). Effects of small classes on academic achievement: The results of the Tennessee class size experiment. American Educational Research Journal, 37, 123-151.

Raudenbush, S. W. (1997). Statistical analysis and optimal design for cluster randomized trials. Psychological Methods, 2, 173-185.

Raudenbush, S. W., \& Liu, X. (2000). Statistical power and optimal design for multisite randomized trials. Psychological Methods, 5, 199-213.

\section{Appendix}

The diagonal elements of matrix $\mathbf{V}_{j}^{*}$ for cluster $\mathrm{j}$ are $v_{i t j}^{*}=\sigma_{e}^{2}+(1+\vartheta) \tau^{2}$ for level-1 units that receive the treatment within the level-2 unit, and $v_{i i c j}^{*}=\sigma_{e}^{2}+\tau^{2}$ for level-1 units that do not receive the treatment within a level-2 unit. The off diagonal elements of matrix $\mathbf{V}_{j}^{*}$ are $v_{i k j}^{*}=\tau^{2}$ . The structure of the block diagonal matrix $\mathbf{V}^{*}$ is

$$
\mathbf{V}^{*}=\left[\begin{array}{llll}
\mathbf{A}_{1} & \mathbf{0} & \cdots & \mathbf{0} \\
\mathbf{0} & \mathbf{A}_{2} & \cdots & \mathbf{0} \\
\vdots & & & \\
\mathbf{0} & \mathbf{0} & \cdots & \mathbf{A}_{m}
\end{array}\right]
$$

assuming $m$ level- 2 units, where $\mathbf{A}_{\mathbf{j}}$ is a $2 n \times 2 n$ matrix

$$
\mathbf{A}_{j}=\left[\begin{array}{llll}
\sigma^{2}(1+\vartheta \rho) & \sigma^{2} \rho & \ldots & \sigma^{2} \rho \\
\sigma^{2} \rho & \sigma^{2}(1+\vartheta \rho) & \ldots & \sigma^{2} \rho \\
\vdots & & & \\
\sigma^{2} \rho & \sigma^{2} \rho & \ldots & \sigma^{2}
\end{array}\right]
$$

assuming $n$ level-1 units per condition. 


\title{
Using the Bootstrap for Estimating the Sample Size in Statistical Experiments
}

\author{
Maher Qumsiyeh \\ University of Dayton, \\ Dayton $\mathrm{OH}$
}

Efron's (1979) Bootstrap has been shown to be an effective method for statistical estimation and testing. It provides better estimates than normal approximations for studentized means, least square estimates and many other statistics of interest. It can be used to select the active factors - factors that have an effect on the response - in experimental designs. This article shows that the bootstrap can be used to determine sample size or the number of runs required to achieve a certain confidence level in statistical experiments.

Key words: Efron's bootstrap, experimental factors, statistical estimation, confidence level.

\section{Introduction}

Traditional methods of finding sample sizes depend on knowing the underlying distribution. For example, to determine a sample size that will result in $(1-\alpha) \times 100 \%$ confidence that the sample mean is within $\mathrm{E}$ units from the population mean the following is used:

$$
n=\frac{z_{\alpha / 2}^{2} \sigma^{2}}{E^{2}}
$$

assuming normality, or using the central limit theorem, and determining an approximate value for $\sigma$. For a multiple regression model

$$
y=\beta_{0}+\beta_{1} x_{1}+\beta_{2} x_{2}+\ldots+\beta_{k} x_{k}+\varepsilon
$$

a $(1-\alpha) \times 100 \%$ confidence interval for $\beta_{\mathrm{j}}$ is given by

$$
\widehat{\beta}_{j} \pm t_{\propto / 2} \sqrt{\frac{1-R^{2}}{\left(1-R_{j}^{2}\right)(N-p)}} .
$$

Maher Qumsiyeh is an Assistant Professor in the Department of Mathematics. Prior to joining the University of Dayton he was a Professor at Bethlehem University for over 25 years, with 10 years as Department Chair. Email him at: qumsiyeh@udayton.edu. where, $\mathrm{R}^{2}$ is the multiple coefficient of determination and $R_{j}{ }^{2}$ is the same when $x_{j}$ is predicted from the remaining $k-1$ regressors.

Using equation (3), the sample size to predict the standardized coefficient within $\mathrm{E}$ units of the true value (replacing $t$ with normal) is given by

$$
n=\frac{z_{\propto / 2}^{2}}{E^{2}}\left(\frac{\left(1-R^{2}\right)}{\left(1-R_{j}^{2}\right)}\right)+p .
$$

However with this $n$ there is approximately a $50 \%$ chance that the interval will be longer than 2E (Kelly \& Maxwell, 2003).

Hahn and Meker (1991) provide a value for $N$ for which the confidence is $(1-\delta) \times 100 \%$ that the interval obtained is of a length less than or equal $2 \mathrm{E}$. The value of such $N$ is

$$
N=\frac{z_{\propto / 2}^{2}}{E^{2}}\left(\frac{\left(1-R^{2}\right)}{\left(1-R_{j}^{2}\right)}\right)\left(\frac{\chi_{\delta}^{2}(n-1)}{n-p}\right)+p .
$$

where $n$ is the value found in equation (4).

An alternative is to use the bootstrap method to determine if the sample size calculated using equations (1) and (5) is necessary or if it is larger than what is needed to achieve a certain confidence. The bootstrap has been shown to provide better than normal estimates of distribution functions of studentized 


\section{BOOTSTRAP USE FOR ESTIMATING SAMPLE SIZE IN STATISTICAL EXPERIMENTS}

statistics (Singh, 1981; Bickle \& Freedman, 1980; Babu and Singh, 1983, 1984). Qumsiyeh (1994) showed that bootstrap approximation for the distribution of the studentized least square estimate is asymptotically better, not only than the normal approximation, but also than the twoterm Edgeworth expansion. Lahiri (1992) showed the superiority of the bootstrap for approximating the distribution of M-estimators. Bhattacharya and Qumsiyeh (1989) preseneted an $\mathrm{L}^{\mathrm{p}}$-comparison between the bootstrap and Edgeworth expansions. Finally, Qumsiyeh and Shaughnessy $(2008,2010)$ showed that the bootstrap can be used to determine the active factors in two level designs and how to estimate missing responses in those designs. In this study the bootstrap was applied to three data sets; SAS and the SQL procedure in SAS were used to perform calculations and resampling.

\section{Data Set 1}

Data set 1 is comprised of 1,000 randomly selected samples of size 61 each from a normal distribution with mean 20 and standard deviation 2 (61 is the number $\mathrm{n}$ obtained using Equation (1) with $\mathrm{E}=0.5$ and $\alpha=0.05$ ). An example of one such sample of size 61 is:

\begin{tabular}{|l|l|l|l|}
\hline 21.39 & 19.92 & 19.08 & 19.86 \\
\hline 19.98 & 20.47 & 22.84 & 16.87 \\
\hline 20.86 & 21.29 & 22.25 & 20.14 \\
\hline 23.20 & 19.86 & 21.95 & 19.11 \\
\hline 19.74 & 23.06 & 17.06 & 19.06 \\
\hline 19.92 & 21.22 & 25.37 & 21.60 \\
\hline 19.06 & 20.87 & 22.99 & 21.77 \\
\hline 22.14 & 21.83 & 19.61 & 17.87 \\
\hline 19.59 & 18.42 & 17.43 & 18.98 \\
\hline 19.16 & 20.49 & 19.19 & 19.07 \\
\hline 18.10 & 19.12 & 21.01 & 19.69 \\
\hline 19.13 & 19.20 & 19.55 & 18.51 \\
\hline 17.66 & 20.90 & 21.88 & 21.09 \\
\hline 17.53 & 20.97 & 20.41 & 21.68 \\
\hline 18.93 & 19.56 & 19.56 & 19.17 \\
\hline 15.91 & \multicolumn{3}{|l}{} \\
\cline { 1 - 2 } & &
\end{tabular}

The mean for this sample was 20.068 and the standard deviation was 1.75 .

\section{Data Set 2}

Data set 2 is real data that correlates the GPA y (out of 4) of 194 students from Bethlehem University, with their high school Math $\left(\mathrm{x}_{1}\right)$ and English $\left(\mathrm{x}_{2}\right)$ scores (out of 100 points). The first few observations are shown in Table 1. The model for data set two is: $y=\beta_{0}+\beta_{1} x_{1}+\beta_{2} x_{2}+\varepsilon$.

Table 1: Data Set 2 Example

\begin{tabular}{|c|c|c|}
\hline $\mathrm{y}$ & $\mathrm{x}_{1}$ & $\mathrm{x}_{2}$ \\
\hline 2.55 & 75 & 77 \\
\hline 3.69 & 87 & 99 \\
\hline 2.48 & 80 & 70 \\
\hline 1.90 & 70 & 65 \\
\hline 2.07 & 70 & 89 \\
\hline 2.73 & 72 & 64 \\
\hline 1.81 & 80 & 66 \\
\hline 2.30 & 71 & 67 \\
\hline 1.76 & 83 & 66 \\
\hline 2.17 & 78 & 89 \\
\hline 1.77 & 65 & 60 \\
\hline
\end{tabular}

Data Set 3

Data set 3 is an example provided by Bisgaard and Fuller (1995). It is a $2^{4}$ full factorial experiment to determine if blade size (A), centering (B), leveling (C) and speed (D) had an effect on the occurrence of undesirable marks on a steel sample. The design matrix is shown in Table 2, where $\mathrm{Y}$ represents the number of defective (undesirable marks) among 20 samples at each setting and $\widehat{P}$ is the proportion of defects at each setting.

The Bootstrap

The bootstrap was used to analyze the three data sets. SAS programming and the SQL procedure in SAS were used to perform the analyses. Resampling with replacement was conducted 1,000 times based on Efron and Tibshirani (1993) finding that 1,000-2,000 works best. The SAS program used for data set 1 


\section{MAHER QUMSIYEH}

Table 2: Data Set 3 Design Matrix

\begin{tabular}{|c|c|c|c|c|c|c|}
\hline Run & A & B & C & D & Y & $\widehat{P}$ \\
\hline 1 & -1 & -1 & -1 & -1 & 0 & 0 \\
\hline 2 & 1 & -1 & -1 & -1 & 16 & 0.8 \\
\hline 3 & -1 & 1 & -1 & -1 & 0 & 0 \\
\hline 4 & 1 & 1 & -1 & -1 & 20 & 1 \\
\hline 5 & -1 & -1 & 1 & -1 & 0 & 0 \\
\hline 6 & 1 & -1 & 1 & -1 & 10 & 0.5 \\
\hline 7 & -1 & 1 & 1 & -1 & 0 & 0 \\
\hline 8 & 1 & 1 & 1 & -1 & 14 & 0.7 \\
\hline 9 & -1 & -1 & -1 & 1 & 0 & 0 \\
\hline 10 & 1 & -1 & -1 & 1 & 10 & 0.5 \\
\hline 11 & -1 & 1 & -1 & 1 & 0 & 0 \\
\hline 12 & 1 & 1 & -1 & 1 & 20 & 1 \\
\hline 13 & -1 & -1 & 1 & 1 & 1 & 0.05 \\
\hline 14 & 1 & -1 & 1 & 1 & 12 & 0.6 \\
\hline 15 & -1 & 1 & 1 & 1 & 0 & 0 \\
\hline 16 & 1 & 1 & 1 & 1 & 20 & 1 \\
\hline
\end{tabular}

is provided in Appendix A; due to the length of the programs for data sets two and three they are not provided. A different procedure was used for each data set.

\section{Data Set 1}

For the first example the sample size was 61 , this is the sample size necessary for $95 \%$ confidence that the sample mean is within 0.5 units from the population mean using the equation: $n=\frac{z_{\alpha / 2}^{2} \sigma^{2}}{E^{2}}$. Using resampling and taking a random sample of size 61 from a $\mathrm{N}(20$, $2^{2}$ ) distribution, it is resampled 1,000 times with replacement, the mean of each of the 1,000 samples is calculated and half the difference between the 2.5 and 97.5 percentiles of the 1,000 means is found. This should be the value of $E$. One sample of size 61 from a $\mathrm{N}\left(20,2^{2}\right)$ distribution to another the value of such $\mathrm{E}$ will vary to a great degree, thus, this procedure is repeated several times (500 in this case) and an interval for the values of such E's is listed. The sample size continued to decrease and the values of $\mathrm{E}$ continued to be recorded. Results are shown in Table 3.

Table 3: Data Set 1 Results

\begin{tabular}{|c|c|}
\hline$n$ & $E$ \\
\hline 61 & $0.403-0.458$ \\
\hline 53 & $0.451-0.478$ \\
\hline 48 & $0.463-0.509$ \\
\hline 40 & $0.538-0.567$ \\
\hline
\end{tabular}

Table 3 shows that a sample of size 61 was not necessary; 48 would have been sufficient. The bootstrap was repeated 500 


\section{BOOTSTRAP USE FOR ESTIMATING SAMPLE SIZE IN STATISTICAL EXPERIMENTS}

times, each resampling 1,000 times using a different 61 randomly selected data points with replacement from a $\mathrm{N}\left(20,2^{2}\right)$ distribution and the values of all 500 replications were in the interval given which is $0.403-0.458$ for $\mathrm{n}=61$. (See Appendix A for the SAS program used for data set 1 with $\mathrm{n}=48$.)

\section{Data Set 2}

The second data set, which correlates the university GPA to high school English and math scores, the model is: $y=\beta_{0}+\beta_{1} x_{1}+\beta_{2} x_{2}+\varepsilon$. The initial results using SAS for the whole data set are shown in Table 4.

First using equation (4),

$$
n=\frac{z_{\propto / 2}^{2}}{E^{2}}\left(\frac{\left(1-R^{2}\right)}{\left(1-R_{j}^{2}\right)}\right)+p,
$$

to find an initial $\mathrm{n}$ using $\alpha$ as 0.05 and $\mathrm{E}$ as 0.2 for the standardized betas (the value of $E$ depends on the type of data at hand). Using all 194 data points and the values of $R^{2}$ and $R_{j}{ }^{2}$ from the data set $\left(\mathrm{R}^{2} \approx 0.4192\right.$ and $\left.\mathrm{R}_{\mathrm{j}}^{2} \approx 0.0163\right)$ and equation (4), the value of $n$ is 60 (approximating to the next integer), however, with this $n$ there is approximately a $50 \%$ chance that the interval will be longer than 2E. (Kelly \& Maxwell, 2003). By contrast, using equation (5),

$$
N=\frac{z_{\propto / 2}^{2}}{E^{2}}\left(\frac{\left(1-R^{2}\right)}{\left(1-R_{j}^{2}\right)}\right)\left(\frac{\chi_{\delta}^{2}(n-1)}{n-p}\right)+p,
$$

$\delta=0.05$ and $\mathrm{j}=2$ the value of $\mathrm{N}$ is determined to be 78 ; this results in $95 \%$ confidence that the interval obtained is of a length less than or equal 2E (Hahn \& Meker, 1991). Note that the value of $E$ used is for the standardized betas; thevalue of $E$ for the non-standardized betas (EN) will be approximately

$$
\mathrm{EN} \approx \frac{S_{y}}{S_{x_{2}}} \mathrm{E}=\frac{0.51651973}{10.5807703}=0.0097
$$

Next, the half-length of the confidence interval for $\beta_{2}$ is determined using the bootstrap method and a random sample of size 78 from the
194 original observations. The procedure is as follows:

1. Select 78 points using random sampling without replacement from the 194; name this as subset and perform a regular regression procedure obtaining $\left(\mathrm{X}_{1,1}, \mathrm{X}_{2,1}, \mathrm{Y}_{1}, \mathrm{E}_{1}\right)$, $\left(\mathrm{X}_{1,2}, \mathrm{X}_{2,2}, \mathrm{Y}_{2}, \mathrm{E}_{2}\right), \ldots,\left(\mathrm{X}_{1,78}, \mathrm{X}_{2,78}, \mathrm{Y}_{78}\right.$ , $\left.\mathrm{E}_{78}\right)$. Here, $E_{i}=Y_{i}-\widehat{Y}_{i}$, is the residual for the $i^{\text {th }}$ observation.

2. Select 1,000 samples with replacement from the subset, this is the bootstrap sample. Each sample has 78 points and samples are designated as $\left\{\right.$ sample $\left._{1}\right\}, \quad\left\{\right.$ sample $\left._{2}\right\}, \ldots$, $\left\{\right.$ sample $\left._{1000}\right\}$.

3. Examine $\left\{\right.$ sample $\left._{1}\right\} ; \mathrm{n}=78$ points taken with replacement from the subset. We have sets of points $\left(\mathrm{X}_{1,1}{ }^{*}, \mathrm{X}_{2,1}{ }^{*}, \mathrm{Y}_{1}{ }^{*}, \mathrm{E}_{1}{ }^{*}\right),\left(\mathrm{X}_{1,2}{ }^{*}\right.$, $\left.\mathrm{X}_{2,2^{*}}, \mathrm{Y}_{2}^{*}, \mathrm{E}_{2}^{*}\right), \ldots, \quad\left(\mathrm{X}_{1,78}{ }^{*}, \mathrm{X}_{2,78^{*}}\right.$, $\left.\mathrm{Y}_{78}{ }^{*}, \mathrm{E}_{78}{ }^{*}\right)$. Each $\left(\mathrm{X}_{1, \mathrm{j}}{ }^{*}, \mathrm{X}_{2, \mathrm{j}}{ }^{*}, \mathrm{Y}_{\mathrm{j}}^{*}, \mathrm{E}_{\mathrm{j}}^{*}\right)$ can be any of the $\left(X_{1,1}, X_{2,1}, Y_{1}, E_{1}\right),\left(X_{1,2}, X_{2,2}\right.$, $\left.\mathrm{Y}_{2}, \mathrm{E}_{2}\right), \ldots,\left(\mathrm{X}_{1,78}, \mathrm{X}_{2,78}, \mathrm{Y}_{78}, \mathrm{E}_{78}\right)$ with probability $1 / 78$.

4. Find the average of the $E_{i}^{*}$ 's, and name this $\mathrm{ME}_{1}$. Due to the fact that the mean of the errors is assumed to be 0 , standardize the errors by subtracting $\mathrm{ME}_{1}$ from each of them.

5. Still using $\left\{\right.$ sample $\left._{1}\right\}$, the new Y's are obtained by the adding the respective standardized error term to the predicted values and these are termed as new $\mathrm{Y}_{\mathrm{j}}$ 's.

6. Continue to examine $\left\{\right.$ sample $\left._{1}\right\}$; using the least-square method, find the slope and the intercept, ( slope $_{1}$, slope $_{2}$, intercept $t_{1}$ ), based on $\left(\mathrm{X}_{1,1}{ }^{*}, \mathrm{X}_{2,1}{ }^{*},\right.$, new $\left.\mathrm{Y}_{1}^{*}\right),\left(\mathrm{X}_{1,2}{ }^{*}, \mathrm{X}_{2,2}{ }^{*}\right.$, new $\left.Y_{2}^{*}\right), \ldots,\left(X_{1,78}{ }^{*}, X_{2,78}{ }^{*}\right.$, new $\left.Y_{78}{ }^{*}\right)$.

7. Repeat steps 3-6 for the other 999 samples to obtain 1,000 estimates for the intercept $\beta_{0}$ and the slopes $\beta_{1}$ and $\beta_{2}$. Interest is in $\beta_{2}$.

8. Estimate the value of $\beta_{2}$ by averaging the 1,000 estimates of $\beta_{2}$ and calculate a $95 \% \mathrm{CI}$ for $\beta_{2}$ by finding the 2.5 and 97.5 percentile 


\section{MAHER QUMSIYEH}

of those 1,000 values. Half the length of this interval, $\mathrm{E}^{*}$, will be compared with the $\mathrm{EN}=$ 0.0097 previously obtained.

Based on this procedure, how is it known that there is $95 \%$ confidence that half the length of the interval will not exceed EN? The answer is that by repeating steps 1-8, 1,000 times to obtain 1,000 EN's and then finding the top 95 percentile, it should not exceed EN.

The estimate for $\beta_{2}$ from one random subset of 78 points was $\widehat{\beta_{2}}=0.01655$ and a $95 \%$ CI for $\beta_{2}$ was $(0.0089,0.0242)$. This assumes that all conditions, such as normal residuals and constant variances, hold; in addition, half the length of the interval is 0.0077 , which is smaller than expected $(0.0097)$. The $\mathrm{n}=78$ guarantees that $95 \%$ of the cases will result in smaller half lengths.

Using the bootstrap method discussed results in a mean half-length of 1,000 runs of the bootstrap method of 0.0095 and a 95\% confidence interval of $(0.00915,0.0102)$ : this is without any assumptions on the model. An estimate for $\beta_{2}$ was calculated as an average of the 1,000 bootstrap sample estimate for $\beta_{2}$ it was $\widehat{\beta_{2}}=0.01643$ for one run with a $95 \%$ CI of $(0.0 .0162,0.0167)$. The bootstrap was repeated 1,000 times and the average value for the estimated values of $\beta_{2}$ was 0.01633 with a $95 \%$ CI of (0.01607. 0.01662). Without any assumption on the model, the bootstrap produced an estimate for $\beta_{2}$ that was close to that produced assuming the regular model assumptions hold, in addition, the length of the 95\% CI was a little shorter than expected using a sample of size $78(0.0095$ vs. 0.0097). It is important to note that the calculations were carried out without assuming the error terms to be normal, however, it is valuable to understand what will happen if the error terms in the example are exactly normal.

Table 4: SAS Results for Data Set 2

\begin{tabular}{|c|c|c|c|c|c|c|c|}
\hline \multicolumn{8}{|l|}{ Model: Model1 } \\
\hline \multicolumn{8}{|c|}{ Dependent Variable: y } \\
\hline \multicolumn{8}{|c|}{ Number of Observations Read: 194} \\
\hline \multicolumn{8}{|c|}{ Number of Observations Used: 194} \\
\hline \multicolumn{8}{|c|}{ Analysis of Variance } \\
\hline Source & $\mathrm{DF}$ & $\begin{array}{c}\text { Sum of } \\
\text { Squares }\end{array}$ & $\begin{array}{c}\text { Mean } \\
\text { Square } \\
\end{array}$ & F Value & $\operatorname{Pr}>F$ & & \\
\hline Model & 2 & 21.58370 & 10.79185 & 68.92 & $<0.0001$ & & \\
\hline Error & 191 & 29.90728 & 0.15658 & & & & \\
\hline Corrected Total & 193 & 51.49098 & & & & & \\
\hline Root MSE & & 0.39571 & & R-Square & 0.4192 & & \\
\hline Dependent Mean & & 2.65381 & & Adj R-Sq & 0.4131 & & \\
\hline Coeff Var & & 14.91081 & & & & & \\
\hline \multicolumn{8}{|c|}{ Parameter Estimates } \\
\hline Variable & $\mathrm{DF}$ & $\begin{array}{c}\text { Parameter } \\
\text { Estimate }\end{array}$ & $\begin{array}{l}\text { Standard } \\
\text { Error }\end{array}$ & t value & $\operatorname{Pr}>|t|$ & \multicolumn{2}{|c|}{ 95\% Confidence Limits } \\
\hline Intercept & 1 & -0.73516 & 0.29076 & -2.53 & 0.0123 & -1.30868 & -0.16163 \\
\hline $\mathrm{x} 1$ & 1 & 0.02696 & 0.00294 & 9.16 & $<.0001$ & 0.02116 & 0.03277 \\
\hline $\mathrm{x} 2$ & 1 & 0.01659 & 0.00271 & 6.11 & $<.0001$ & 0.01124 & 0.02194 \\
\hline
\end{tabular}




\section{BOOTSTRAP USE FOR ESTIMATING SAMPLE SIZE IN STATISTICAL EXPERIMENTS}

$2^{\text {nd }}$ Data Set (Normal Errors)

For a random sample of size 78, the predicted values of the $y_{i}{ }^{\prime} s, \widehat{y_{i}}$ were calculated and a new variable $\mathrm{w}_{\mathrm{i}}$ was defined as:

$$
\mathrm{w}_{\mathrm{i}}=\widehat{\mathrm{y}}_{\mathrm{i}}+\mathrm{c} \varepsilon_{\mathrm{i}}
$$

where $\varepsilon_{\mathrm{i}}$ is randomly chosen from a $\mathrm{N}(0,1)$ distribution. The new $\mathrm{w}_{\mathrm{i}}$ 's with the original $\mathrm{x}$ 's will have normal errors with constant variance. Note that the variance of the $\mathrm{w}_{\mathrm{i}}$ 's must be the same as the original $y_{i}$ 's to be able to compare the length of the confidence interval for the new $\beta_{2}$ with the previous one. The solver function in Microsoft Excel was used to provide a value for $\mathrm{c}$ to achieve this; this value of $\mathrm{c}$ was calculated to be 0.4123 . This improved the previous results and half the length of the $95 \% \mathrm{CI}$ for $\beta_{2}$ using the bootstrap method was much smaller $(0.0089$ vs. 0.0097). This shows that using the bootstrap requires a smaller sample size than the previous estimate of $n=78$.

\section{Data Set 3}

Bisgaard and Fuller (1995) provided a table that gives estimated sample sizes (n) for the number of runs at each setting for two level full factorial experiments using proportions as a response. Their estimate for $\mathrm{n}$ which represents the number of runs needed to detect an error of size $\Delta$ in the untransformed scale is given by

$$
n=\frac{\left(z_{\propto / 2}-z_{\beta}\right)^{2}}{N \delta^{2}}
$$

where $\mathrm{N}$ is the total number of basic runs in a $2^{\mathrm{k}}$ factorial experiment $(4,8,16, \ldots), \alpha$ and $\beta$ are the probabilities of type I and type II errors, 0.05 and 0.1 respectively, and $\delta$ is the expected value of the effect (Bisgaard \& Fuller, 1995). Bisgaard and Fuller's table presents values of $\Delta$ that vary from $10 \%$ to $90 \%$ of the proportion of defective $\left(\mathrm{p}_{0}\right)$ and shows that sample size depends on the average defective level. If the average defective level is low, for example $5 \%$, a larger sample size is needed to indicate that a change has truly occurred.

For the $3^{\text {rd }}$ data set the current level of defective $\left(\mathrm{p}_{0}\right)$ was not known, it was approximated with the average proportion of defective in the sample at each setting which is $\hat{\bar{p}} \approx 0.384$. Because $\mathrm{n}$ is given in this experiment as 20, the method described in Bisgaard and Fuller (1995) or the table they provide can be used to determine the minimum size of detectable error. For $\alpha$ to be at most 0.05 , the minimum size is $|\Delta|>0.185$; calculating the effect size of each factor, it was found that factors $\mathrm{A}, \mathrm{B}$ and the $\mathrm{AB}$ interaction have effect sizes larger than this $(0.984,0.208,0.247$ respectively). This agrees with the half normal plot (Daniel, 1959) which states that factors B and the $\mathrm{AB}$ interaction appear to be slightly active (not very clear) and that factor $\mathrm{A}$ is a definitely active factor (see Figure 1).

In the calculations described it is not certain that a 95\% confidence interval for the effect size will have its lower bound less than 0.185 for those factors (A, B and $A B)$.

Qumsiyeh and Shaughnessy (2008, 2010) showed that the bootstrap can be used (under no assumptions) to determine active factors in factorial experiments, to estimate the size of the effect and to determine a confidence interval for the effect size. The method can be described with the following steps using factor A for illustration purposes:

1. Sample N/2 responses with replacement from data at the +1 level of the given factor A.

2. Sample N/2 responses with replacement from data at the -1 level of the given factor A.

3. Estimate the effect of that factor using the difference between +1 level and -1 level.

4. Repeat the sampling procedure a large number of times (1,000 in this example).

5. Find the average of the 1,000 values; this is an estimate of the effect size of factor A.

Determine the upper $(1-\alpha / 2)$ and lower $\alpha / 2$ percentile points of the resampled effect values found in step 4. Use these values to construct the 


\section{MAHER QUMSIYEH}

Figure 1: Half-Normal Plot for the Effects in Data Set 3

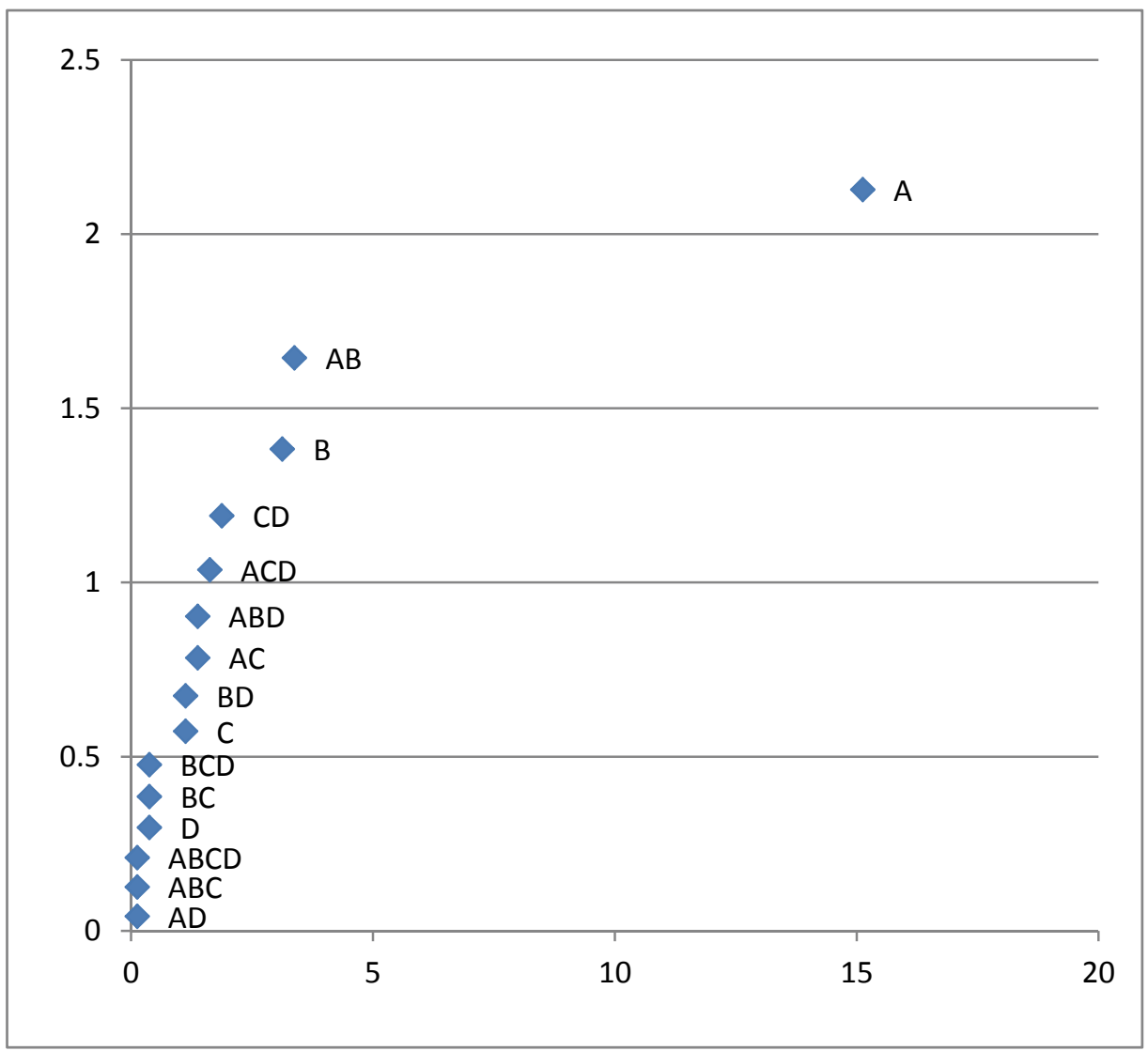

effect size. If the confidence interval doesn't contain 0 then this factor is an active factor $-\mathrm{a}$ factor that has an effect on the response.

Using the procedure described previously for data set 3, the following were determined: All confidence intervals for the effect size for all factors except factor A contained 0 , therefore they must be assumed as inactive factors. For factors $\mathrm{A}, \mathrm{B}$ and the $\mathrm{AB}$ which appear to be effective using the normal plot and are reported as active factors by Bisgaard and Fuller (1995), using the proportion of defectives, the results were as follows:

- Factor A: The mean effect size for the 1,000 runs was 0.7566 and a $95 \%$ confidence interval for the effect size was $(0.6188$, 0.8875).
- Factor B: The mean effect size for the 1,000 runs was 0.1500 and a $95 \%$ confidence interval for the effect size was $(-0.2313$, 0.5406).

- Factor $\mathrm{AB}$ (The $\mathrm{AB}$ interaction): The mean effect size for the 1,000 runs was 0.1776 and a $95 \%$ confidence interval for the effect size was $(-0.2406,0.5894)$.

Results show that only factor A can be considered active. If this is the case, the confidence interval for effect $\mathrm{A}$ has a lower bound of 0.618 which leads to a much higher value than the least expected of 0.185 . This indicates that a sample size smaller than 20 would have been sufficient. 


\section{BOOTSTRAP USE FOR ESTIMATING SAMPLE SIZE IN STATISTICAL EXPERIMENTS}

\section{Conclusion}

The bootstrap method can be used to determine sample sizes in statistical experiments and to check whether a certain sample size used is more than is needed by examining the length of the confidence interval resulting from using the bootstrap method. The bootstrap is also good for selecting active factors and in constructing confidence intervals for effect size. The availability of computers and statistical software make using re-sampling (bootstrap) easy and fast and provides good predictions.

Acknowledgment

This research is supported by University of Dayton Seed Grant.

\section{References}

Babu, G., \& Singh, K. (1983). Inference on means using the bootstrap. The Annals of Statistics, 11, 999-1003

Babu, G. J., \& Singh, K. (1984). On one term Edgeworth correction by Efrons bootstrap. Sankhya, 46, Series A, 219-232.

Bhattacharya, R. N., \& Qumsiyeh, M. (1989). Second order and $\mathrm{L}^{\mathrm{p}}$ - comparison between the bootstrap and empirical Edgeworth expansion methodologies. Annals of Statistics, 17, 160-169.

Bickel, P. J., \& Freedman, D. A. (1980). On Edgeworth expansions for the bootstrap. Unpublished.

Bisgaard, S., \& Fuller, H. (1995). Sample size estimate for $2^{\mathrm{k}-\mathrm{p}}$ designs with binary responses. Quality Engineering, 27(4), 344-354.

Daniel, C. (1959). Using of half normal plots in interpreting factorial two-level experiments. Technometrics, 1, 311-341.

Efron, B. (1979). Bootstrap methods: Another look at jacknife. The Annals of Statistics, 7, 1-26.

Efron, B., \& Tibshirani, R. (1993). An introduction to the bootstrap. New York, NY: Chapman and Hall.

Hahn, G. J., \& Meeker, W. Q. (1991). Statistical intervals: A guide for practitioners. New York, NY: Wiley.
Kelley, K., \& Maxwell, E. (2003). Sample size for multiple regression: Obtaining regression coefficients that are accurate, not simply significant. Psychological Methods, 8(3), 305-321.

Lahiri, S. (1992). Bootstrapping Mestimators of a multiple linear regression parameter. The Annals of Statistics, 20(3), 15481570 .

Qumsiyeh, M. (1994). Bootstrapping and empirical Edgeworth expansions in multiple linear regression models. Communications in Statistical Theory and Methods, 23(11), 32273239.

Qumsiyeh, M., \& Shaughnessy, G. (2008). Using the bootstrap to select active factors in unreplicated factorial experiment. In JSM Proceedings, Statistical Computing Section. Alexandria, VA: American Statistical Association.

Qumsiyeh, M., \& Shaughnessy, G. (2010). Bootstrapping Un-replicated two-level designs with missing responses. Journal of Statistics: Advances in Theory and Applications, 4, 91-106.

Singh, K. (1981). On the asymptotic accuracy of Efron's bootstrap. The Annals of Statistics, 9, 1187-1195.

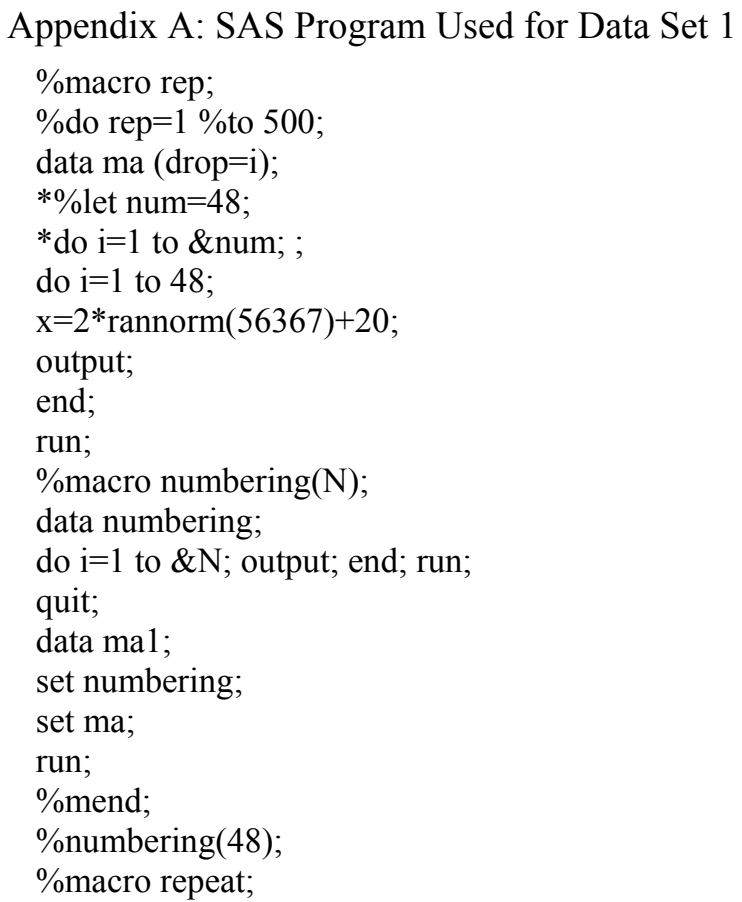




\section{MAHER QUMSIYEH}

Appendix A (continued): SAS Program Used for Data Set 1

$\%$ do repeat $=1 \%$ to 1000 ;

$\%$ macro distinct(nThrow, N);

data table2;

do $\mathrm{j}=1$ to \&nThrow;

$\mathrm{pt}=\operatorname{int}(\operatorname{int}(\operatorname{ranuni}(0) * \& \mathrm{~N})+1.5)$;

set mal point $=p t$;

output;

end;

stop; $\quad *$ required for point $=$;

run;

$\%$ mend;

$\%$ distinct $(48,48)$;

run;

proc means data $=$ Table $2 \mathrm{n}$ mean noprint;

var x;

output out=Table $3 \mathrm{n}=\mathrm{n}$ mean $=\mathrm{mx}$;

run;

quit;

proc sql;

create table tableF as

select a1.mx as mx1

from table 3 as a1;

run;

proc append data $=$ TableF base $=$ summary force;

run;

$\%$ end;

$\%$ mend;

$\%$ repeat;

proc univariate data $=$ summary noprint; var $\mathrm{mx} 1$;

output out $=\mathrm{z} 1$ mean $=$ tm pctlpts $=2.5,97.5$ pctlname $=\mathrm{p} 25 \mathrm{p} 975$ pctlpre $=\mathrm{mx} 1$;

run;

data $\mathrm{E}$;

set $\mathrm{z} 1$;

$\mathrm{E}=(\mathrm{mx} 1 \mathrm{p} 975-\mathrm{mx} 1 \mathrm{p} 25) / 2$;

run;

proc append data $=\mathrm{E}$ base $=\mathrm{E} 1$ force;

run;

proc sql;

drop table $\mathrm{E}$;

drop table $\mathrm{Ma}$;

drop table Ma1 ;

drop table Numbering;

drop table Summary ;

drop table Table2;

drop table Table3;

drop table Tablef;

drop table $\mathrm{zl}$;

run;

quit;

$\%$ end;

$\%$ mend;

$\%$ rep;

proc univariate data $=\mathrm{E} 1$ noprint; var $\mathrm{E}$;

output out $=$ Length mean $=$ tm pctlpts $=2.5,97.5$ pctlname $=$ p25 p975 pctlpre $=E$; run; quit; 


\section{The Length-Biased Versus Random Sampling for the Binomial and Poisson Events}

\author{
Makarand V. Ratnaparkhi \\ Wright State University, \\ Dayton, $\mathrm{OH}$
}

\author{
Uttara V. Naik-Nimbalkar \\ Pune University, \\ Pune, India
}

The equivalence between the length-biased and the random sampling on a non-negative, discrete random variable is established. The length-biased versions of the binomial and Poisson distributions are discussed.

Key words: Length-biased data, weighted distributions, binomial, Poisson, convolutions.

Introduction

The occurrence of so-called length-biased data has been documented by many researchers. No formal mathematical definition of lengthbiasedness exists; however, if for collected data the probability of inclusion of an observation in a sample is proportional to the magnitude of an observation then the data is referred to as the length-biased data. Further, if the probability of inclusion depends on a certain known function (weight function) of an observation then it is referred to as the size-biased data.

The realization of such data as a sample, as opposed to the realization of a well-defined random sampling procedure, appeared for the first time in a paper by Fisher (1934). In particular, the data collected for estimating the proportion of individuals having certain rare genetic traits such as albinism are length-biased. However, in statistical literature, the term length-biased data was introduced during the 1960s. Fisher (1934) referred to the data collection procedure for counting the number of albino children in a family as the method of ascertainment, which clearly is not a random sampling procedure. For the analysis of the collected data he considered the modifications of

Makarand V. Ratnaparkhi is a Professor of Statistics. Email him at: makarand.ratnaparkhi@wright.edu. Uttara V. Naik-Nimbalkar is a Professor of Statistics. Email her at: uvnaik@stats.unipune.ac.in. binomial distribution (probabilities), which was appropriate at that time. The methodology for such discrete data analysis was formalized by Rao (1965) who introduced the concept of weighted distributions (or in informal nomenclature, the distorted distributions). Rao also considered the weighted distributions for the analysis of various discrete data sets. Recently, Gao, et al. (2011) found that the length-biased Poisson (events) data arise in bioinformatics. There are a number of fields such as ecology, geology, medical science and engineering where the data is length-biased and the researchers have used the weighted distribution for the analysis of such data.

This study follows Ratnaparkhi and Naik-Nimbalkar (2012) regarding the lengthbiased data arising in oil filed exploration and seeks to associate length-biased data with a random sampling procedure. It is hoped that these efforts will lead to new methods for building appropriate models for length-biased data and for the estimation of the related parameters using such data. Since, the general discussion of length-biasedness (regardless of whether the data is on continuous or discrete random variables is more involved, here, the discussion is limited to the discrete random variables. Further, the binomial and Poisson distributions are commonly used for modeling discrete data. Thus, the derivation of results and the discussion are restricted to these distributions; however, the extensions and modifications to other discrete distributions are possible. 


\section{RATNAPARKHI \& NAIK-NIMBALKAR}

Length-Biased Data versus Random Sample Data: Examples

Example 1: Estimation of Proportion of Families with Albino Children (Fisher, 1934)

An albino child is located and his/her family is included in a sample. Therefore, larger the number of albino children in a family higher is the probability of inclusion of the family in the sample. Let $X=$ the number of albino children in a family. Clearly, the data on $X$ is length-biased. The binomial probabilities are considered for the analysis if the observed frequencies of the values of $X$.

Example 2: Estimation of the Population of Moose Using Aerial Transect Sampling

Moose, in general, stay and move as groups. The group is located (aerial survey) and the numbers of animals in a group are recorded by a team on the ground. For locating the group, at least one animal from the group must be visible while traveling on a randomly selected transect in the aerial survey of the population. The Poisson distribution is considered for the analysis of these data. Note that larger the number of animals in group, the higher is the probability of sighting at least one animal and hence the length-biasedness in the collected data.

Example 3: Length-Biased Data on RNA Sequences (Gao, et al., 2011)

The quantification of transcripts (RNAsequences) and related properties, such as the differential expression of the transcripts, arise in microarray data analysis. The differential expression of longer transcripts is more likely to be identified than that of a shorter transcript with the same effect size. This situation is described as length-biasedness in the related data. For the analysis of such data, the Poisson distribution is considered as a model.

\section{Methodology}

The derivations of the results for this study are related to the distributions arising in the analysis of length-biased data on discrete random variables. These are based on certain definitions and the probability generating function of the random variables. The derivations are either simple or are the known properties of the distributions of random variables. Therefore, the detailed proofs are omitted for brevity. For future reference, the notations and definitions used herein are listed below.

- $X:$ A random variable representing the populations of interest (the original random variable) $X$ is assumed to be a non-negative, non-degenerate discrete random variable.

- $Y, Z_{1}, Z_{2}$ : Other random variables that will be introduced as needed.

- $f_{X}(x ; \theta)$ : The pdf of the original distribution of a random variable $X$ where $\theta$ is a scalar or a vector of parameters.

- $g_{X}(x ; \theta)$ : The pdf of the length-biased version of $f_{X}(x ; \theta)$.

- LBS: Length-biased sampling

The pdf of the length-biased version of $f_{X}(x ; \theta)$ is given by

$$
g_{X}(x ; \theta)=x f_{X}(x ; \theta) / E[X], E[X]<\infty .
$$

For a more general case of (1), $x$ is replaced by $w(x)>0$, and $E[X]$ by $E[w(X)]<\infty$.

- $G_{X}(t)=E\left[t^{X}\right]$ :The probability generating function (pgf) of $X$. The subscript of $G(t)$ denotes the pgf of the corresponding variable.

- $\quad B(n, p)$ : The binomial distribution with parameters $n$ and $p$.

- $\operatorname{LBB}(m, p)$ : The length-biased version of $B(n, p)$.

- $P(\lambda)$ : The Poisson distribution with parameter $\lambda$.

- $\operatorname{LBP}(\lambda)$ : The length-biased version of the Poisson distribution.

\section{Results}

Convolution of the Poisson and Degenerate Random Variables

Suppose that the original distribution of a random variable $X \sim P(\lambda)$ with pdf 


\section{LENGTH-BIASED VS. RANDOM SAMPLING FOR BINOMIAL AND POISSON EVENTS}

$$
\begin{aligned}
& f_{X}(x ; \lambda)=e^{-\lambda} \lambda^{x} / x !, \\
& x=0,1,2, \ldots, \infty .
\end{aligned}
$$

then using (1), the pdf of the length biased Poisson distribution is given by

$$
\begin{aligned}
& g_{X}(x ; \lambda)=e^{-\lambda} \lambda^{(x-1)} /(x-1) !, \\
& x=1,2, \ldots, \infty .
\end{aligned}
$$

Further, if $\mathrm{Z}_{1} \sim P(\lambda), z_{1}=0,1,2, \ldots, \infty$, then $G_{Z_{1}}(t)=\exp (-\lambda(1-t))$. And, if $\mathrm{Y}$ has a degenerate distribution at $Y=1$ with $G_{Y}(t)=t$, then, $G_{\left(Z_{1}+Y\right)}(t)=t \exp (-\lambda(1-t))$, which is the pgf of (3). Thus, the length-biased version of the Poisson distribution is a convolution of the Poisson random variable and the degenerate distribution of $Y$. Further, this result shows that the length-biased version of $X$ has the same distribution as that of $\left(Z_{1}+Y\right)$.

This observation, interpreted in terms of sampling, implies that the random sampling on the original Poisson variable denoted by, $Z_{1}$, and the degenerate random variable $Y$ is equivalent to the so-called $L B S$ on random variable $X$.

It is known that the chance mechanism, namely, the well-defined Poisson process, leads to the pdf given by (2) and a well-defined $P(\lambda)$ exists as a corresponding probability model. However, no known stochastic process can be associated that will lead to the pdf (3), the socalled the $\operatorname{LBP}(\lambda)$ distribution; therefore, in a strict sense the pdf in (3) is an artificial mathematical construct if the above convolution result is not associated for deriving (3). The related implication is: because $Z_{1}$ and $Y$ are random variables, the random sampling on $Z_{1}$ and $Y$ is well defined and, as a result, the random sampling on a length-biased random variable $X$ is in order. Thus, the data on $X$ is a realization of a random sample on $X=\left(Z_{1}+Y\right)$ and there seems to be no need to refer to the data as a length-biased sample. Regarding the theory of statistics, this provides a theoretical basis for length-biased data as a random sample. Note that, in practice, there is a need for providing a practical justification for introducing variable $Y$ and its interpretation; however, such interpretation must come from the description of the research problem form where such data is arising in practice.

For practical purposes, if it is known that while sampling for the original random variable $X$ the probability of inclusion of an observation is proportional to the magnitude of the observation, a researcher should define the random variables $Z_{1}$ and $Y$ appropriately and then collect sample data on these variables independently, for example, take a sample first on $Y$ followed by a sample on $Z_{1}$ and then define $X=Z_{1}+Y$ for practical purposes. It is likely that such a sampling plan is more involved in terms of its execution and planning as compared to the practice of collecting the data on $X$ and calling it a length-biased variable without any justification with reference to the theory of probability. But, it provides a theoretical base for the collected data and a general theoretical justification to the statistical inference based of these data.

Amari (1985) introduced the role of differential geometrical properties of statistical manifolds, which are well defined objects, in estimation theory. Clearly, the manifolds of the family of distributions given by (2) and (3) are not the same. Therefore, due care is needed when using (3) for estimating parameter $\lambda$ when it is known that it is associated with a different manifold corresponding to the family of distributions given by (2) and not the one associated with distribution (3).

In view of the discussion related to the Poisson distribution, it seems reasonable to consider other discrete distributions for which the above conclusions are applicable. In general, it can be shown that these conclusions are applicable to the commonly used original discrete distributions with support $\Omega=\{0,1,2$, $\ldots, \infty\}$. For example, it was observed that the method based on the convolution of random variables was useful for generating the lengthbiased version of the original geometric random variable defined on $\Omega$.

Convolution of the Binomial and Degenerate Random Variables

As noted, the length-biased binomial distribution also arises as a model for the lengthbiased data on the original discrete random variable $X$; therefore, it seems reasonable to 


\section{RATNAPARKHI \& NAIK-NIMBALKAR}

obtain the results, similar to those obtained for the Poisson distribution, for the binomial case and to further interpret these results in view of applications.

Suppose that the original distribution of a random variable $X \sim B(n, p)$ with pdf:

$$
\begin{aligned}
& f_{X}(x ; n, p)=\left(\begin{array}{l}
n \\
x
\end{array}\right) p^{x}(1-p)^{n-x}, \\
& x=0,1,2, \ldots n .
\end{aligned}
$$

Using (1) the pdf of the length-biased binomial distribution is given by:

$$
\begin{aligned}
& g_{X}(x ; n, p)=\left(\begin{array}{c}
n-1 \\
x-1
\end{array}\right) p^{x-1}(1-p)^{n-x}, \\
& x=1,2, \ldots n .
\end{aligned}
$$

If $\quad \mathrm{Z}_{2} \sim B(n, p), z_{2}=0,1,2, \ldots, n, \quad$ then $G_{Z_{2}}(t)=(q+p t)^{n}$. If $Y$ has a degenerate distribution at $\mathrm{Y}=1$ with $G_{Y}(t)=t$, then $G_{\left(Z_{2}+Y\right)}(t)=t(q+p t)^{n}$, which is not the pgf of (5). Thus, the length-biased version of the binomial distribution of random variable is not a convolution of a binomial distribution and the degenerate distribution of $\mathrm{Y}$. Thus, the situation is different than for $P(\lambda)$ and $\operatorname{LBP}(\lambda)$; further, the length-biased sampling on the original random variable $X$ is not equivalent to the random sampling on $\left(Z_{2}+Y\right)$.

It is not possible to associate a natural chance mechanism with the length-biased binomial distribution - either as repetitions of Bernoulli trials or as a convolution of random variables. Therefore, the $\operatorname{LBB}(m, p)$ is a mathematical construct lacking statistical significance. Under such a situation, as opposed to using $L B B$ distribution it might be necessary to investigate other distributions as a model for the length-biased data on (original) binomial random variable $X$. The lack of convolution property and, hence, the non-availability of corresponding chance mechanism for the $L B B$ distribution appears to be due to the finite support for binomial random variable $X$.
Therefore, a similar situation will be present in other distributions with finite support. This raises the following question: Can the so-called length-biased binomial distribution be considered as a model for the so-called lengthbiased binomial data when it is known that the support of the random variable of interest is finite? At this stage, the exact answer is unknown; therefore, it is left to the reader.

\section{Conclusion}

It has been shown that, at least for some situations where data are so-called lengthbiased, the convolution property could be used for providing a more rigorous treatment for modeling such data within the framework of random sampling and thereby justifies the probability models such as a Poisson distribution. Length-biased data on binomial random variables needs further investigation in data analysis rather than using the mathematical constructs such as the weighted binomial distributions. The extension of results obtained in this study is not straightforward; in general, for length-biased data on continuous random variables, for example, survival data arising in medical science. However, it is worth investigating such cases in view of the number of practical situations where the collected data are on continuous random variables.

\section{References}

Amari, S. I. (1985). Differentialgeometrical methods in statistics. New York, NY: Springer-Verlag.

Fisher, R. A. (1934). The effects of the methods of ascertainment upon the estimation of frequencies. Annals of Eugenics, 6, 13-25.

Gao, L., et al. (2011). Length bias correction for RNA-seq gene set analyses. Bioinformtics, 27,662-669.

Rao, C. R. (1965). On discrete distributions arising out of methods of ascertainment. Sankhya A, 27, 311-324.

Ratnaparkhi, M. V., \& Naik-Nimbalkar, U. V. (2012). Length-biased lognormal distribution and its application in the analysis of data from the oil field exploration studies. Journal of Modern Applied Statistical Methods, 11(1), 255-260. 


\title{
Estimation of Variance Using Known Coefficient of Variation and Median of an Auxiliary Variable
}

\author{
J. Subramani G. Kumarapandiyan \\ Pondicherry University \\ R V Nagar, Kalapet, Puducherry
}

A modified ratio type variance estimator for estimating population variance of a study variable when the population median and coefficient of variation of an auxiliary variable are known is proposed. The bias and mean squared error of the proposed estimator are derived and conditions under which the proposed estimator performs better than the traditional ratio type variance estimators and modified ratio type variance estimators are obtained. Using a numerical study results show that the proposed estimator performs better than the traditional ratio type variance estimator and existing modified ratio type variance estimators.

Key words: Bias, mean squared error, natural populations, ratio type estimators, simple random sampling.

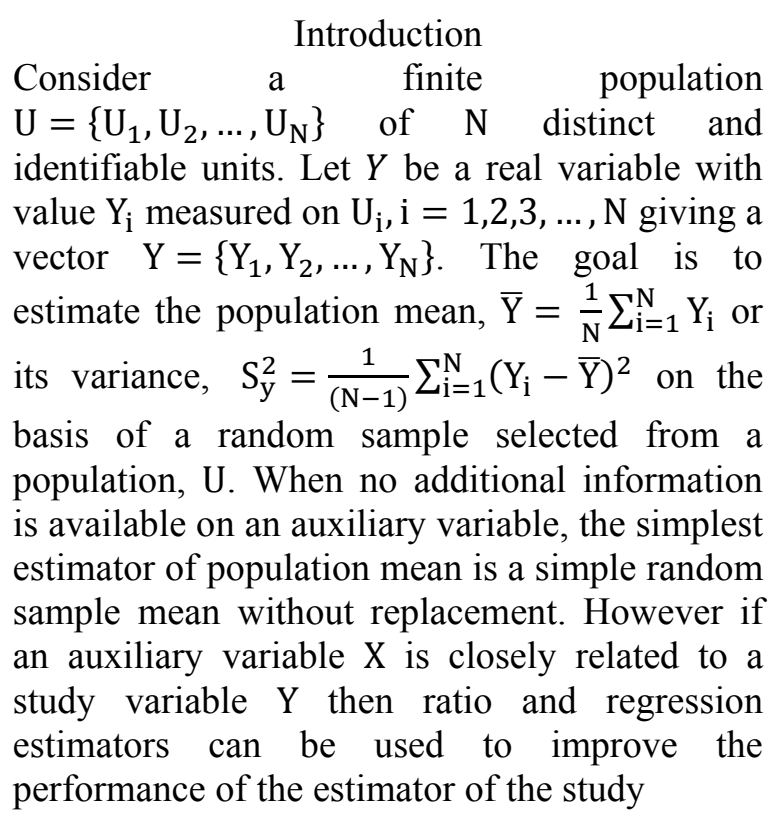

J. Subramani is an Associate Professor and Head in the Department of Statistics in the Ramanujan School of Mathematical Sciences. Email him at: drjsubramani@yahoo.co.in.

G. Kumarapandiyan is a Research Scholar in the Department of Statistics in the Ramanujan School of Mathematical Sciences. Email him at: kumarstat88@gmail.com. variable. This article considers the problem of estimating a population variance and uses auxiliary information to improve the efficiency of the population variance estimator

$$
\mathrm{S}_{\mathrm{y}}^{2}=\frac{1}{(\mathrm{~N}-1)} \sum_{\mathrm{i}=1}^{\mathrm{N}}\left(\mathrm{Y}_{\mathrm{i}}-\overline{\mathrm{Y}}\right)^{2} .
$$

Population variance estimation is considered by Isaki (1983) who proposed ratio and regression estimators. Prasad \& Singh (1990) considered a ratio type estimator for estimating population variance by improving Isaki's estimator (1983) with respect to bias and precision. Arcos, et al. (2005) introduced another ratio type estimator that also improved the Isaki's estimator (1983) and is less biased and more precise than other estimators. Notations used throughout this article are:
$\mathrm{N}$ : population size
n: sample size
$\gamma: 1 / n$
Y: study variable
$\mathrm{X}$ : auxiliary variable
$\overline{\mathrm{X}}, \overline{\mathrm{Y}}:$ population means 


\section{SUBRAMANI \& KUMARAPANDIYAN}

$\overline{\mathrm{x}}, \overline{\mathrm{y}}:$ sample means

$\mathrm{S}_{\mathrm{y}}^{2}, \mathrm{~S}_{\mathrm{x}}^{2}:$ population variances

$\mathrm{s}_{\mathrm{y}}^{2}, \mathrm{~s}_{\mathrm{x}}^{2}:$ sample variances

$\mathrm{C}_{\mathrm{x}}, \mathrm{C}_{\mathrm{y}}$ : coefficients of variation

$\rho:$ correlation coefficient

$\mathrm{B}($.$) : bias of the estimator$

MSE(.): $\begin{aligned} & \text { mean squared error of the } \\ & \text { estimator }\end{aligned}$

$\hat{\mathrm{S}}_{\mathrm{R}}^{2}$ : traditional ratio type variance

$\mathrm{S}_{\mathrm{R}}^{2}$ : estimator of $\mathrm{S}_{\mathrm{y}}^{2}$

$\hat{\mathrm{S}}_{\mathrm{KCi}}^{2}: \begin{aligned} & \text { existing modified ratio type } \\ & \text { variance estimator of } \mathrm{S}_{\mathrm{y}}^{2}\end{aligned}$

$\hat{\mathrm{S}}_{\mathrm{JG}}^{2}: \begin{aligned} & \text { proposed modified ratio type } \\ & \text { variance estimator of } \mathrm{S}_{\mathrm{y}}^{2}\end{aligned}$

Isaki (1983) suggested a ratio type variance estimator for population variance $S_{y}^{2}$ when the population variance $S_{x}^{2}$ of an auxiliary variable $X$ is known together with its bias and mean squared error as:

$$
\begin{gathered}
\hat{\mathrm{S}}_{\mathrm{R}}^{2}=\mathrm{s}_{\mathrm{y}}^{2} \frac{\mathrm{S}_{\mathrm{x}}^{2}}{\mathrm{~s}_{\mathrm{x}}^{2}} \\
\mathrm{~B}\left(\hat{\mathrm{S}}_{\mathrm{R}}^{2}\right)=\gamma \mathrm{S}_{\mathrm{y}}^{2}\left[\left(\beta_{2(\mathrm{x})}-1\right)-\left(\lambda_{22}-1\right)\right] \\
\operatorname{MSE}\left(\hat{\mathrm{S}}_{\mathrm{R}}^{2}\right)=\gamma \mathrm{S}_{\mathrm{y}}^{4}\left[\begin{array}{l}
\left(\beta_{2(\mathrm{y})}-1\right)+\left(\beta_{2(\mathrm{x})}-1\right) \\
-2\left(\lambda_{22}-1\right)
\end{array}\right]
\end{gathered}
$$

where

$$
\beta_{2(\mathrm{y})}=\frac{\mu_{40}}{\mu_{20}^{2}}, \beta_{2(\mathrm{x})}=\frac{\mu_{04}}{\mu_{02}^{2}}, \lambda_{22}=\frac{\mu_{22}}{\mu_{20} \mu_{02}}
$$

and

$$
\mu_{\mathrm{rs}}=\frac{1}{\mathrm{~N}} \sum_{\mathrm{i}=1}^{\mathrm{N}}\left(\mathrm{y}_{\mathrm{i}}-\overline{\mathrm{Y}}\right)^{\mathrm{r}}\left(\mathrm{x}_{\mathrm{i}}-\overline{\mathrm{X}}\right)^{\mathrm{s}}
$$

The ratio type variance estimator in (1) is used to improve the precision of a population variance estimate compared to simple random sampling when a positive correlation exists between $\mathrm{X}$ and $\mathrm{Y}$. Further improvements are achieved on the classical ratio type variance estimator by introducing a number of modified ratio type variance estimators using known parameters such as the coefficients of variation and kurtosis. The problem of constructing efficient estimators for a population variance has been discussed by many authors including: Agarwal \& Sithapit (1995), Ahmed, et al. (2000), Al-Jararha \& Al-Haj Ebrahem (2012), Arcos, et al.(2005), Bhushan (2012), Das \& Tripathi (1978), Garcia \&Cebrain (1997), Gupta \& Shabbir (2008), Isaki (1983), Kadilar \& Cingi (2006a, 2006b), Prasad \& Singh (1990), Reddy (1974), Singh \& Chaudhary (1986), Singh, et al. (1988, 2003), Subramani \& Kumarapandiyan (2012b, 2012c), Upadhyaya \& Singh (2006) and Wolter (1985)

Based on works by Sisodia \& Dwivedi (1981), Upadhyaya \& Singh (1999) and Singh, et al. (2004), Kadilar \& Cingi (2006a) suggested four ratio type variance estimators using known values of coefficient of variation $C_{X}$ and coefficient of kurtosis $\beta_{2(\mathrm{x})}$ of an auxiliary variable $X$ together with their biases and mean squared errors (see Table 1).

The modified ratio type variance estimators discussed are biased but have minimal mean squared errors compared to traditional ratio type variance estimators. The list of estimators in Table 1 uses known values of the parameters such as $\mathrm{S}_{\mathrm{x}}^{2}, \mathrm{C}_{\mathrm{x}}, \beta_{2}$ and their linear combinations. Subramani \& Kumarapandiyan (2012a) used the linear combination of a known value of the population median $\mathrm{M}_{\mathrm{d}}$ and Coefficient of variation $\mathrm{C}_{\mathrm{x}}$ of an auxiliary variable to improve the ratio estimators in estimating a population mean. Based on Subramani \& Kumarapandiyan (2012a), a modified ratio type variance estimator using a linear combination of known value of a population median and coefficient of variation of an auxiliary variable is proposed. Results show that the proposed estimator performs better than the traditional ratio type and existing modified ratio type variance estimators. 
Table 1: Existing Modified Ratio Type Variance Estimators with their Biases and Mean Squared Errors (Source: Kadilar \& Cingi, 2006a)

\begin{tabular}{|c|c|c|}
\hline Estimator & Bias B(.) & Mean Squared Error MSE (.) \\
\hline $\begin{array}{l}\hat{\mathrm{S}}_{\mathrm{KCl} l}^{2}= \\
\quad \mathrm{s}_{\mathrm{y}}^{2}\left[\frac{\mathrm{S}_{\mathrm{x}}^{2}+\mathrm{C}_{\mathrm{x}}}{\mathrm{s}_{\mathrm{x}}^{2}+\mathrm{C}_{\mathrm{x}}}\right]\end{array}$ & $\gamma \mathrm{S}_{\mathrm{y}}^{2} \mathrm{~A}_{1}\left[\mathrm{~A}_{1}\left(\beta_{2(\mathrm{x})}-1\right)-\left(\lambda_{22}-1\right)\right]$ & $\gamma \mathrm{S}_{\mathrm{y}}^{4}\left[\left(\beta_{2(\mathrm{y})}-1\right)+\mathrm{A}_{l}^{2}\left(\beta_{2(\mathrm{x})}-1\right)-2 \mathrm{~A}_{l}\left(\lambda_{22}-1\right)\right]$ \\
\hline $\begin{array}{l}\hat{\mathrm{S}}_{\mathrm{KC} 2}^{2}= \\
\quad \mathrm{s}_{\mathrm{y}}^{2}\left[\frac{\mathrm{S}_{\mathrm{x}}^{2}+\beta_{2(\mathrm{x})}}{\mathrm{S}_{\mathrm{x}}^{2}+\beta_{2(\mathrm{x})}}\right]\end{array}$ & $\gamma \mathrm{S}_{\mathrm{y}}^{2} \mathrm{~A}_{2}\left[\mathrm{~A}_{2}\left(\beta_{2(\mathrm{x})}-1\right)-\left(\lambda_{22}-1\right)\right]$ & $\gamma \mathrm{S}_{\mathrm{y}}^{4}\left[\left(\beta_{2(\mathrm{y})}-1\right)+\mathrm{A}_{2}^{2}\left(\beta_{2(\mathrm{x})}-1\right)-2 \mathrm{~A}_{2}\left(\lambda_{22}-1\right)\right]$ \\
\hline $\begin{array}{l}\hat{\mathrm{S}}_{\mathrm{KC} 3}^{2}= \\
\quad \mathrm{S}_{\mathrm{y}}^{2}\left[\frac{\mathrm{S}_{\mathrm{x}}^{2} \beta_{2(\mathrm{x})}+\mathrm{C}_{\mathrm{x}}}{\mathrm{S}_{\mathrm{x}}^{2} \beta_{2(\mathrm{x})}+\mathrm{C}_{\mathrm{x}}}\right.\end{array}$ & $\gamma \mathrm{S}_{\mathrm{y}}^{2} \mathrm{~A}_{3}\left[\mathrm{~A}_{3}\left(\beta_{2(\mathrm{x})}-1\right)-\left(\lambda_{22}-1\right)\right]$ & $\gamma \mathrm{S}_{\mathrm{y}}^{4}\left[\left(\beta_{2(\mathrm{y})}-1\right)+\mathrm{A}_{3}^{2}\left(\beta_{2(\mathrm{x})}-1\right)-2 \mathrm{~A}_{3}\left(\lambda_{22}-1\right)\right]$ \\
\hline $\begin{array}{l}\hat{\mathrm{S}}_{\mathrm{KC} 4}^{2}= \\
\quad \mathrm{S}_{\mathrm{y}}^{2}\left[\frac{\mathrm{S}_{\mathrm{x}}^{2} \mathrm{C}_{\mathrm{x}}+\beta_{2(\mathrm{x})}}{\mathrm{S}_{\mathrm{x}}^{2} \mathrm{C}_{\mathrm{x}}+\beta_{2(\mathrm{x})}}\right.\end{array}$ & $\gamma \mathrm{S}_{\mathrm{y}}^{2} \mathrm{~A}_{4}\left[\mathrm{~A}_{4}\left(\beta_{2(\mathrm{x})}-1\right)-\left(\lambda_{22}-1\right)\right]$ & $\gamma \mathrm{S}_{\mathrm{y}}^{4}\left[\left(\beta_{2(\mathrm{y})}-1\right)+\mathrm{A}_{4}^{2}\left(\beta_{2(\mathrm{x})}-1\right)-2 \mathrm{~A}_{4}\left(\lambda_{22}-1\right)\right.$ \\
\hline
\end{tabular}

Notes: $A_{I}=\frac{S_{x}^{2}}{S_{x}^{2}+C_{x}}, A_{2}=\frac{S_{x}^{2}}{S_{x}^{2}+\beta_{2(x)}}, A_{3}=\frac{S_{x}^{2} \beta_{2(x)}}{S_{x}^{2} \beta_{2(x)}+C_{x}}, A_{4}=\frac{S_{x}^{2} C_{x}}{S_{x}^{2} C_{x}+\beta_{2(x)}}$

\section{Proposed Estimator}

The performance of the estimator of the study variable can be improved by using known population parameters of an auxiliary variable, which are positively correlated with a study variable. A modified ratio type variance estimator using the linear combination of known value of the population median $\mathrm{M}_{\mathrm{d}}$ and coefficient of variation $\mathrm{C}_{\mathrm{x}}$ of an auxiliary variable is proposed; this modified ratio type variance estimator for population variance $S_{y}^{2}$ is defined as

$$
\hat{S}_{\mathrm{JG}}^{2}=\mathrm{s}_{\mathrm{y}}^{2}\left[\frac{\mathrm{C}_{\mathrm{x}} \mathrm{S}_{\mathrm{x}}^{2}+\mathrm{M}_{\mathrm{d}}}{\mathrm{C}_{\mathrm{x}} \mathrm{s}_{\mathrm{x}}^{2}+\mathrm{M}_{\mathrm{d}}}\right] .
$$

The bias and mean squared error of $\widehat{\mathrm{S}}_{\mathrm{JG}}^{2}$ to the first degree of approximation are:

$$
\begin{aligned}
& \mathrm{B}\left(\hat{\mathrm{S}}_{\mathrm{IG}}^{2}\right)=\gamma \mathrm{S}_{\mathrm{y}}^{2} \mathrm{~A}_{\mathrm{JG}}\left[\begin{array}{l}
\left.\mathrm{A}_{\mathrm{JG}}\left(\beta_{2(\mathrm{x})}-1\right)-\left(\lambda_{22}-1\right)\right] \\
\operatorname{MSE}\left(\hat{\mathrm{S}}_{\mathrm{JG}}^{2}\right)=\gamma \mathrm{S}_{\mathrm{y}}^{4}\left[\begin{array}{l}
\left(\beta_{2(\mathrm{y})}-1\right)+\mathrm{A}_{\mathrm{JG}}^{2}\left(\beta_{2(\mathrm{x})}-1\right) \\
-2 \mathrm{~A}_{\mathrm{JG}}\left(\lambda_{22}-1\right)
\end{array}\right]
\end{array}\right.
\end{aligned}
$$

where $A_{J G}=\frac{S_{x}^{2}}{S_{x}^{2}+M_{d}}$ 


\section{SUBRAMANI \& KUMARAPANDIYAN}

Efficiency Comparison of the Proposed Estimator

The bias and mean squared error of the traditional ratio type variance estimator are:

$$
\begin{gathered}
\mathrm{B}\left(\hat{\mathrm{S}}_{\mathrm{R}}^{2}\right)=\gamma \mathrm{S}_{\mathrm{y}}^{2}\left[\left(\beta_{2(\mathrm{x})}-1\right)-\left(\lambda_{22}-1\right)\right] \\
\operatorname{MSE}\left(\hat{\mathrm{S}}_{\mathrm{R}}^{2}\right)=\gamma \mathrm{S}_{\mathrm{y}}^{4}\left[\begin{array}{l}
\left(\beta_{2(\mathrm{y})}-1\right)+\left(\beta_{2(\mathrm{x})}-1\right) \\
-2\left(\lambda_{22}-1\right)
\end{array}\right]
\end{gathered}
$$

For convenience and for the ease of comparisons, the biases mean squared errors and constants of the modified ratio type variance estimators shown in Table 1 are represented in a single class as:

$$
\begin{aligned}
& \mathrm{B}\left(\hat{\mathrm{S}}_{\mathrm{KCi}}^{2}\right)=\gamma \mathrm{S}_{\mathrm{y}}^{2} \mathrm{~A}_{\mathrm{i}}\left[\mathrm{A}_{\mathrm{i}}\left(\beta_{2(\mathrm{x})}-1\right)-\left(\lambda_{22}-1\right)\right], \\
& \mathrm{i}=1,2,3,4
\end{aligned}
$$

$\operatorname{MSE}\left(\hat{\mathrm{S}}_{\mathrm{KCi}}^{2}\right)=\gamma \mathrm{S}_{\mathrm{y}}^{4}\left[\begin{array}{l}\left(\beta_{2(\mathrm{y})}-1\right)+\mathrm{A}_{\mathrm{i}}^{2}\left(\beta_{2(\mathrm{x})}-1\right) \\ -2 \mathrm{~A}_{\mathrm{i}}\left(\lambda_{22}-1\right)\end{array}\right]$, $\mathrm{i}=1,2,3,4$

where

$$
\begin{aligned}
& \mathrm{A}_{1}=\frac{\mathrm{S}_{\mathrm{x}}^{2}}{\mathrm{~S}_{\mathrm{x}}^{2}+\mathrm{C}_{\mathrm{x}}}, \\
& \mathrm{A}_{2}=\frac{\mathrm{S}_{\mathrm{x}}^{2}}{\mathrm{~S}_{\mathrm{x}}^{2}+\beta_{2(\mathrm{x})}}, \\
& \mathrm{A}_{3}=\frac{\mathrm{S}_{\mathrm{x}}^{2} \beta_{2(\mathrm{x})}}{\mathrm{S}_{\mathrm{x}}^{2} \beta_{2(\mathrm{x})}+\mathrm{C}_{\mathrm{x}}}, \\
& \mathrm{A}_{4}=\frac{\mathrm{S}_{\mathrm{x}}^{2} \mathrm{C}_{\mathrm{x}}}{\mathrm{S}_{\mathrm{x}}^{2} \mathrm{C}_{\mathrm{x}}+\beta_{2(\mathrm{x})}}
\end{aligned}
$$

The bias and mean squared error of the proposed modified ratio type variance estimator are:

$$
\mathrm{B}\left(\hat{\mathrm{S}}_{\mathrm{JG}}^{2}\right)=\gamma \mathrm{S}_{\mathrm{y}}^{2} \mathrm{~A}_{\mathrm{p}}\left[\mathrm{A}_{\mathrm{JG}}\left(\beta_{2(\mathrm{x})}-1\right)-\left(\lambda_{22}-1\right)\right]
$$

$$
\operatorname{MSE}\left(\hat{\mathrm{S}}_{\mathrm{JG}}^{2}\right)=\gamma \mathrm{S}_{\mathrm{y}}^{4}\left[\begin{array}{l}
\left(\beta_{2(\mathrm{y})}-1\right)+\mathrm{A}_{\mathrm{JG}}^{2}\left(\beta_{2(\mathrm{x})}-1\right) \\
-2 \mathrm{~A}_{\mathrm{JG}}\left(\lambda_{22}-1\right)
\end{array}\right]
$$

$$
\text { where } A_{J G}=\frac{S_{x}^{2} C_{x}}{S_{x}^{2} C_{x}+M_{d}}
$$

The condition for which the proposed estimator $\widehat{\mathrm{S}}_{\mathrm{JG}}^{2}$ is more efficient than the traditional ratio type variance estimator derived from (6) and (10) is:

$$
\begin{aligned}
& \operatorname{MSE}\left(\hat{\mathrm{S}}_{\mathrm{JG}}^{2}\right)<\operatorname{MSE}\left(\hat{\mathrm{S}}_{\mathrm{R}}^{2}\right) \\
& \text { if } \lambda>1+\frac{\left(\mathrm{A}_{\mathrm{JG}}+1\right)\left(\beta_{2(\mathrm{x})}-1\right)}{2}
\end{aligned}
$$

The conditions for which the proposed estimator $\widehat{\mathrm{S}}_{\mathrm{JG}}^{2}$ is more efficient than the existing modified ratio type variance estimators in Table 1, $\widehat{\mathrm{S}}_{\mathrm{KCi}}^{2} ; \mathrm{i}=1,2,3$ and 4 derived from (8) and (10) are:

$$
\begin{aligned}
& \operatorname{MSE}\left(\hat{\mathrm{S}}_{\mathrm{JG}}^{2}\right)<\operatorname{MSE}\left(\hat{\mathrm{S}}_{\mathrm{KCi}}^{2}\right) \\
& \text { if } \lambda>1+\frac{\left(\mathrm{A}_{\mathrm{JG}}+\mathrm{A}_{\mathrm{i}}\right)\left(\beta_{2(\mathrm{x})}-1\right)}{2} ; \\
& \mathrm{i}=1,2,3 \text { and } 4
\end{aligned}
$$

\section{Numerical Study}

The performance of the proposed modified ratio type variance estimator was assessed and compared with a traditional ratio type and existing modified ratio type variance estimators (see Table 1) for natural populations. Populations 1 and 2 are a real data set taken from the Italian Bureau for Environment Protection (APAT) 2004 Report on Waste 2004. (Data and reports are available at: http://www.osservatorionazionalerifiuti.it.) For 


\section{VARIANCE ESTIMATION USING COEFFICIENT OF VARIATION AND MEDIAN}

Table 2: Parameters and Constants of the Populations

\begin{tabular}{|c|c|c|c|c|}
\hline Parameters & Population1 & Population2 & Population3 & Population4 \\
\hline $\mathrm{N}$ & 103 & 103 & 80 & 49 \\
\hline$n$ & 40 & 40 & 20 & 20 \\
\hline$\overline{\mathrm{Y}}$ & 626.2123 & 62.6212 & 51.8264 & 116.1633 \\
\hline$\overline{\mathrm{X}}$ & 557.1909 & 556.5541 & 11.2646 & 98.6765 \\
\hline$\rho$ & 0.9936 & 0.7298 & 0.9413 & 0.6904 \\
\hline $\mathrm{S}_{\mathrm{y}}$ & 913.5498 & 91.3549 & 18.3569 & 98.8286 \\
\hline $\mathrm{C}_{\mathrm{y}}$ & 1.4588 & 1.4588 & 0.3542 & 0.8508 \\
\hline $\mathrm{S}_{\mathrm{x}}$ & 818.1117 & 610.1643 & 8.4563 & 102.9709 \\
\hline $\mathrm{C}_{\mathrm{x}}$ & 1.4683 & 1.0963 & 0.7507 & 1.0435 \\
\hline$\beta_{2(\mathrm{x})}$ & 37.3216 & 17.8738 & 2.8664 & 5.9878 \\
\hline$\beta_{2(\mathrm{y})}$ & 37.1279 & 37.1279 & 2.2667 & 4.9245 \\
\hline$\lambda_{22}$ & 37.2055 & 17.2220 & 2.2209 & 4.6977 \\
\hline $\mathrm{M}_{\mathrm{d}}$ & 308.0500 & 373.820 & 7.5750 & 64.0000 \\
\hline $\mathrm{A}_{1}$ & 0.9999 & 0.9999 & 0.9896 & 0.9999 \\
\hline $\mathrm{A}_{2}$ & 0.9999 & 0.9999 & 0.9615 & 0.9994 \\
\hline $\mathrm{A}_{3}$ & 0.9999 & 0.9999 & 0.9964 & 1.0000 \\
\hline $\mathrm{A}_{4}$ & 0.9999 & 0.9999 & 0.9493 & 0.9995 \\
\hline $\mathrm{A}_{\mathrm{JG}}$ & 0.9996 & 0.9990 & 0.8763 & 0.9942 \\
\hline & & & & \\
\hline
\end{tabular}

each Italian province, three variables are considered: the total amount (tons) of recyclable-waste collection in Italy in 2003 (Y), the total amount of recyclable-waste collection in Italy in $2002\left(X_{1}\right)$ and the number of inhabitants in $2003\left(\mathrm{X}_{2}\right)$. Population 3 is from Murthy (1967, p. 228) and population 4 is from Cochran (1977, p. 152). The population parameters and constants computed from these populations are shown in Table 2. The biases and mean squared errors for the existing and proposed modified ratio type variance estimator for the populations are shown in Tables 3 and 4 .

Table 3 shows that the proposed modified ratio type variance estimator has less bias compared to the biases of the traditional and existing modified ratio type variance estimators. It is observed that the mean squared error of the proposed modified ratio type variance estimator is lower than the mean squared errors of the traditional and existing modified ratio type variance estimators.

\section{Conclusion}

This article proposed a modified ratio type variance estimator using a known value of median of an auxiliary variable. The bias and mean squared error of the proposed estimator were obtained and compared with the traditional ratio type and existing modified ratio type variance estimators. Further the conditions for which the proposed estimator is more efficient than the traditional and existing estimators were derived. The performance of the proposed estimator was assessed using four known populations. Results show that the bias and mean squared error of the proposed estimator are less than the biases and mean squared errors of the traditional and existing estimators for the known populations considered. Based on results, the proposed modified ratio type variance estimator may be preferred over traditional ratio type and existing modified ratio type variance estimators for the use in practical applications. 


\section{SUBRAMANI \& KUMARAPANDIYAN}

Table 3: Biases of the Existing and Proposed Modified Ratio Type Variance Estimators

\begin{tabular}{|c|c|c|c|c|c|}
\hline \multirow{2}{*}{ Source } & \multirow{2}{*}{ Estimator } & \multicolumn{4}{|c|}{ Bias $\mathrm{B}()}$. \\
\cline { 3 - 6 } & & Population 1 & Population 2 & Population 3 & Population 4 \\
\hline $\begin{array}{c}\text { Isaki } \\
(1983)\end{array}$ & $\hat{\mathrm{S}}_{\mathrm{R}}^{2}$ & 2422.3488 & 135.9935 & 10.8762 & 630.0302 \\
\hline \multirow{2}{*}{$\begin{array}{c}\text { Kadilar \& Cingi } \\
(2006 \mathrm{a})\end{array}$} & $\hat{\mathrm{S}}_{\mathrm{KC} 1}^{2}$ & 2420.6810 & 135.9827 & 10.4399 & 629.7285 \\
\cline { 2 - 6 } & $\hat{\mathrm{S}}_{\mathrm{KC} 2}^{2}$ & 2379.9609 & 135.8179 & 9.2918 & 628.3006 \\
\cline { 2 - 6 } & $\hat{\mathrm{S}}_{\mathrm{KC} 3}^{2}$ & 2422.3041 & 135.9929 & 10.7222 & 629.9798 \\
\cline { 2 - 6 } & $\hat{\mathrm{S}}_{\mathrm{KC} 4}^{2}$ & 2393.4791 & 135.8334 & 8.8117 & 628.3727 \\
\hline $\begin{array}{c}\text { Proposed } \\
\text { Estimator }\end{array}$ & $\hat{\mathrm{S}}_{\mathrm{JG}}^{2}$ & 2184.1910 & 132.6505 & 6.1235 & 612.4793 \\
\hline
\end{tabular}

Table 4: Mean Squared Error of Existing and Proposed Modified Ratio Type Variance Estimators

\begin{tabular}{|c|c|c|c|c|c|}
\hline \multirow{2}{*}{ Source } & \multirow{2}{*}{ Estimator } & \multicolumn{4}{|c|}{ Bias $\mathrm{B}()}$. \\
\cline { 3 - 6 } & & Population 1 & Population 2 & Population 3 & Population 4 \\
\hline $\begin{array}{c}\text { Isaki } \\
(1983)\end{array}$ & $\hat{\mathrm{S}}_{\mathrm{R}}^{2}$ & 670393270 & 35796612 & 3925.1627 & 7235508 \\
\hline \multirow{3}{*}{$\begin{array}{c}\text { Kadilar \& Cingi } \\
(2006 \mathrm{a})\end{array}$} & $\hat{\mathrm{S}}_{\mathrm{KC} 1}^{2}$ & 670384403 & 35796605 & 3850.1552 & 7234298 \\
\cline { 2 - 6 } & $\hat{\mathrm{S}}_{\mathrm{KC} 2}^{2}$ & 670169790 & 35796503 & 3658.4051 & 7228570 \\
\cline { 2 - 6 } & $\hat{\mathrm{S}}_{\mathrm{KC} 3}^{2}$ & 670393032 & 35796611 & 3898.5560 & 7235306 \\
\cline { 2 - 6 } & $\hat{\mathrm{S}}_{\mathrm{KC} 4}^{2}$ & 670240637 & 35796512 & 3580.8342 & 7228859 \\
\hline $\begin{array}{c}\text { Proposed } \\
\text { Estimator }\end{array}$ & $\hat{\mathrm{S}}_{\mathrm{J} \mathrm{H}}^{2}$ & 669188376 & 35794559 & 3180.7740 & 7165517 \\
\hline
\end{tabular}

Acknowledgements

The authors wish to express their gratitude and thanks for financial assistance received from UGC-MRP and the Department of Science and Technology (DST), New Delhi, India.

\section{References}

Agarwal, M. C., \& Sithapit, A. B. (1995).Unbiased ratio type estimation. Statistics and Probability Letters, 25, 361-364.
Ahmed, M. S., Raman, M. S., \& Hossain, M. I. (2000).Some competitive estimators of finite population variance Multivariate Auxiliary Information. Information and Management Sciences, 11(1), 49-54.

Al-Jararha, J., \& Al-Haj Ebrahem, M. (2012).A ratio estimator under general sampling design. Austrian Journal of Statistics, 41(2), 105-115. 


\section{VARIANCE ESTIMATION USING COEFFICIENT OF VARIATION AND MEDIAN}

Arcos, A., et al. (2005). Incorporating the auxiliary information available in variance estimation. Applied Mathematics and Computation, 160, 387-399.

Bhushan, S. (2012). Some efficient sampling strategies based on ratio type estimator. Electronic Journal of Applied Statistical Analysis, 5(1), 74-88.

Cochran, W. G. (1977). Sampling techniques, $3^{\text {rd }}$ Edition. New York, NY: John Wiley \& Sons.

Das, A. K., \& Tripathi, T. P. (1978).Use of auxiliary information in estimating the finite population variance. Sankhya, 40, 139-148.

Garcia, M. K., \& Cebrain, A. A. (1997). Variance estimation using auxiliary information: An almost unbiased multivariate ratio estimator. Metrika, 45, 171-178.

Gupta, S., \& Shabbir, J. (2008).Variance estimation in simple random sampling using auxiliary information. Hacettepe Journal of Mathematics and Statistics, 37, 57-67.

Isaki, C. T. (1983). Variance estimation using auxiliary information. Journal of the American Statistical Association, 78, 117-123.

Kadilar, C., \& Cingi, H. (2006a). Improvement in variance estimation using auxiliary information. Hacettepe Journal of Mathematics and Statistics, 35(1), 111-115.

Kadilar, C., \& Cingi, H. (2006 b).Ratio estimators for population variance in simple and stratified sampling. Applied Mathematics and Computation, 173, 1047-1058.

Murthy, M. N. (1967). Sampling theory and methods. Calcutta, India: Statistical Publishing Society.

Prasad, B., \& Singh, H. P. (1990). Some improved ratio type estimators of finite population variance in sample surveys. Communication in Statistics: Theory and Methods, 19, 1127-1139.

Reddy, V. N. (1974).On a transformed ratio method of estimation. Sankhya, C, 36, 5970.

Singh, D., \& Chaudhary, F. S. (1986). Theory and analysis of sample survey designs. New Delhi: New Age International Limited.
Singh, H. P., Chandra, P., \& Singh, S. (2003). Variance estimation using multiauxiliary information for random non-response in survey sampling. STATISTICA, LXIII, 1, 2340.

Singh, H. P., Tailor, R., Tailor, R., \& Kakran, M. S. (2004). An improved estimator of population mean using power transformation. Journal of the Indian Society of Agricultural Statistics, 58(2), 223-230.

Singh, H. P., Upadhyaya, U. D., \&Namjoshi, U. D. (1988). Estimation of finite population variance. Current Science, 57, 13311334.

Sisodia, B. V. S., \& Dwivedi, V. K. (1981).A modified ratio estimator using coefficient of variation of auxiliary variable, Journal of the Indian Society of Agricultural Statistics, 33(1), 13-18.

Subramani, J., \& Kumarapandiyan, G. (2012a). Estimation of population mean using coefficient of variation and median of an auxiliary variable. International Journal of Probability and Statistics, 1(4), 111-118.

Subramani, J., \& Kumarapandiyan, G. (2012b). Variance estimation using median of the auxiliary variable.International Journal of Probability and Statistics, 1(3), 36-40.

Subramani, J., \& Kumarapandiyan, G. (2012c). Variance estimation using quartiles and their functions of the auxiliary variable. International Journal of Statistics and Applications, 2(5), 67-72.

Upadhyaya, L. N., \& Singh, H. P. (1999). Use of transformed auxiliary variable in estimating the finite population mean. Biometrical Journal, 41(5), 627-636.

Upadhyaya, L. N., \& Singh, H. P. (2006).Almost unbiased ratio and product-type estimators of finite population variance in sample surveys. Statistics in Transition, 7(5), 1087-1096.

Wolter, K. M. (1985). Introduction to variance estimation. New York, NY: SpringerVerlag. 


\title{
Priorities in Thurstone Scaling and Steady-State Probabilities in Markov Stochastic Modeling
}

\author{
Stan Lipovetsky \\ GfK Custom Research North America, \\ Minneapolis, MN
}

Thurstone scaling is widely used in marketing and advertising research where various methods of applied psychology are utilized. This article considers several analytical tools useful for positioning a set of items on a Thurstone scale via regression modeling and Markov stochastic processing in the form of ChapmanKolmogorov equations. These approaches produce interval and ratio scales of preferences and enrich the possibilities of paired comparison estimation applied for solving practical problems of prioritization and probability of choice modeling.

Key words: Thurstone scale, regression estimation, Bradley-Terry model, Markov model, ChapmanKolmogorov equations, steady-states probability.

\section{Introduction}

Thurstone scaling is a method of priority evaluation among items by the frequency of their empirical pairwise preferences (Thurstone 1927, 1959; Thurstone \& Jones, 1957). This technique is widely used in fields of applied psychology, particularly, in marketing and advertising research (Edwards, 1957; Torgerson, 1958; Bock \& Jones, 1968; Green \& Tull, 1978; Conklin \& Lipovetsky, 1999, 2004a, 2004b; Lipovetsky, 2007a, 2007b). Thurstone scaling transforms ranked or paired comparison data into a scale that is used for displaying the results of a ranking procedure. Statistical properties of Thurstone multiple comparisons were considered by Mosteller (1951) and Daniels

Stan Lipovetsky, Ph.D., is Senior Research Director at the GfK Research Center for Excellence, Marketing Sciences. $\mathrm{He}$ has numerous publications in multivariate statistics, multiple criteria decision making, econometrics, microeconomics and marketing research. He is a member of the editorial boards of the International Journal of Operations and Quantitative Management, the Journal of Electronic Modeling and the Journal of Model Assisted Statistics and Applications. Email him at: stan.lipovetsky@gfk.com.
(1950) and this technique is also known as Thurstone-Mosteller-Daniels (TMD) model (David, 1988; Stern, 1990; Ennis \& Johnson, 1993). Connections of TMD with other methods of multiple paired comparisons, particularly, with the Analytic Hierarchy Process (AHP), are considered in several studies (Zinnes \& MacKay, 1989; MakKay, Bowen \& Zinnes, 1996; Lipovetsky \& Conklin, 2001, 2002).

Positioning the items on a Thurstone scale consists in taking the proportions of respondents who prefer one item over each of the others, finding the corresponded percentiles (z-scores) of the cumulative normal distribution and averaging them. In practice it is convenient to rescale the obtained scores so that the best and the worst performing items will have scores 1 and 0 , respectively. A Thurstone scale is typically constructed from ranked data when it is determined how often one item ranked ahead of another one, thus, the data could be reduced to or collected as paired comparisons and their frequencies. The paired approach to analysis also means that it is not required for every respondent to have ranked or compared every item; however, because the result is a relative scale, it is important that the pairwise comparisons be balanced. Thurstone scales can also be created from rating data, although this approach can produce a large number of ties that make the TMD unstable. 
This article considers several possibilities of priority estimation by pairwise data. One is evaluation of the TMD scale and the statistical significance of the obtained levels. For this purpose TMD is as presented a regression model by a special design of dummy variables. In constructing such a regression the standard errors and t-statistics for the levels of the compared items are obtained simultaneously in order to estimate precision and statistical significance of the differences among the items. The Thurstone model defines a scale of differences; standardizing to zero-one range corresponds to the interval scale. Together with the TMD model, the Bradley-Terry-Luce (BTL) model is also considered for pair comparison (Bradley \& Terry, 1952; Luce (1959); Luce \& Suppes, 1965; Lipovetsky, 2008) that corresponds to applying the logistic as opposed to the normal probability function.

Another possibility for pair comparisons evaluation may be found in stochastic Markov chain modeling via Chapman-Kolmogorov equations for discrete states and continuous time of transitions probabilities (Bellman, 1960; Hillier \& Lieberman, 1974; Bar-Niv \& Lipovetsky, 1995; Lipovetsky, 2005, 2006). This approach uses pair comparison data for intensity of transitions among the states (items) for constructing Chapman-Kolmogorov system of differential equations and solving for the dynamic as well as for the eventually reached steady-state probabilities. Although in the Thurstone model only differences are meaningful, the Markov states approach elaborates a ratio scale of probabilities to choose each of the items in comparison. Thus, the Thurstone and Markov models correspond to relative and absolute preference estimates.

\section{Thurstone Scale as a Regression Model}

The TMD general model is defined due by Thurstone's law of comparative judgment. According to Thurstone, a psychological characteristic $x_{i}$ (where $i=1,2, \ldots, m$ denotes different characteristics) can be presented as a random normal variable $x_{i}=N\left(v_{i}, \sigma_{i}\right)$ with a mean value $v_{i}$ and standard deviation $\sigma_{i}$. The problem consists in estimating $v_{i}$ values as the positions of the stimulus on the total psychological scale. The random variable of the difference $y=x_{i}-x_{j}$ between two psychological values (stimulus) has probability density function

$$
p(y)=\frac{1}{\sqrt{2 \pi} \bar{\sigma}} \exp \left(-\frac{\left(y-\left(v_{i}-v_{j}\right)\right)^{2}}{2 \bar{\sigma}^{2}}\right),
$$

where the standard deviation for the difference of two stimulus is

$$
\bar{\sigma}=\left(\sigma_{i}^{2}+\sigma_{j}^{2}-2 \sigma_{i} \sigma_{j} r_{i j}\right)^{1 / 2}
$$

with $r_{i j}$ denoting the correlation between $i^{\text {th }}$ and $j^{\text {th }}$ variables. The cumulative probability is then:

$$
p_{i j}=\Phi\left(v_{i}-v_{j}\right)=\frac{1}{\sqrt{2 \pi}} \int_{-\left(v_{i}-v_{j}\right) / \bar{\sigma}}^{\infty} e^{-y^{2} / 2} d y .
$$

The Case- $\mathrm{V}$ of TMD, which is the most widely used of these models, corresponds to equal standard deviations $\bar{\sigma}$ (2) for all paired differences of stimulus (it is fulfilled by the assumption of equal variances of the independent/uncorrelated variables).

If the values $p_{i j}$ at the left-hand side of (3) are given, then the corresponded quantiles, or $\mathrm{z}$-values can be defined as

$$
z_{i j}=\Phi^{-1}\left(p_{i j}\right)=v_{i}-v_{j} .
$$

In (4) there are more equations for the pairs $(i>$ $j, j=1, \ldots, m-1)$ than $m$ values $v_{i}$ themselves. For estimation of $v_{i}$ values Mosteller (1951) suggested to use the Least Squares (LS) objective:

$$
L S=\sum_{i \neq j}^{m}\left(z_{i j}-\left(v_{i}-v_{j}\right)\right)^{2} \rightarrow \min .
$$

The objective (5) is homogeneous by the parameters $v_{i}$ of estimation, therefore, it needs a normalizing condition:

$$
\sum_{i=1}^{m} v_{i}=0
$$




\section{STAN LIPOVETSKY}

The first order condition $d L S / d v_{i}=0$ for minimizing (5) yields the estimate

$$
v_{i}=\frac{1}{m} \sum_{j=1}^{m} z_{i j}+\frac{1}{m} \sum_{j=1}^{m} v_{j}=\frac{1}{m} \sum_{j=1}^{m} z_{i j},
$$

where the relation (6) is accounted. So the position on the psychological scale for each $i^{\text {th }}$ item equals the mean value of $z$-scores of comparison of this item with the others.

In practical TMD modeling (for example, in comparison of a product's flavors or brands) as opposed to probabilities $p_{i j}$ (3), sample estimates of frequencies corresponding to the observed proportions of cases with item $j$ preferred to item $i$ are available. These frequencies are usually presented in a matrix

$$
F=\left(\begin{array}{cccc}
f_{11} & f_{12} & \cdots & f_{1 m} \\
f_{21} & f_{22} & \cdots & f_{2 m} \\
\cdots \cdots & \cdots \cdots & \cdots \cdots & \cdots \cdots \\
f_{m 1} & f_{m 2} & \cdots & f_{m m}
\end{array}\right)
$$

where each element $f_{i j}$ corresponds to the preference of the item $j$ over the item $i$. If in the pair comparison $n_{i}$ respondents preferred the $i^{\text {th }}$ item and $n_{j}$ respondents preferred the $j^{\text {th }}$ one, then $i j^{\text {th }}$ and $j i^{\text {th }}$ elements of frequency matrix (8) are

$$
f_{i j}=\frac{n_{j}}{n_{i}+n_{j}}, \quad f_{j i}=\frac{n_{i}}{n_{i}+n_{j}} .
$$

In a general case the frequencies can be obtained by a different number of respondents in each pair comparison. The elements in (8) are positive and satisfy the relation of symmetry

$$
f_{i j}+f_{j i}=1,
$$

thus, the diagonal elements are $f_{i i}=0.5$. The quantiles $z_{i j}$ of normal distribution (4) obtained for the elements of matrix (8) are

$$
z_{i j}=\Phi^{-1}\left(f_{i j}\right)
$$

The values $z_{i j}=-z_{j i}$ and $z_{i i}=0$, thus they define the elements of a skew-symmetric matrix $Z$. Due to the definition of the matrix (8) with elements (9), where each element $f_{i j}$ corresponds to the prevalence of the item $j$ over the item $i$, the means in the columns of matrix $Z$ correspond to the estimates (7) obtained by the empirical frequencies (8), that is,

$$
v_{j}=\frac{1}{m} \sum_{i=1}^{m} z_{i j}=\frac{1}{m} \sum_{i=1}^{m} \Phi^{-1}\left(f_{i j}\right) .
$$

The total of these means equals zero, so condition (6) is satisfied. Thus, the averaged zvalues (12) are used as positions of items on the Thurston scale of preferences. These values are usually reduced to the standard zero-one scale of preferences by the transformation

$$
\tilde{v}_{j}=\frac{v_{j}-\min (v)}{\max (v)-\min (v)} \text {. }
$$

Using (13) a Thurstone scale is reduced to an interval scale.

Consider the Bradley-Terry-Luce (BTL) model. The BTL model for pair comparisons defines probability that an item $a$ is preferred to an item $b$ as a share $p(a, b)=v(a) /(v(a)+v(b))$ where $v$ denotes a utility function. Using a logarithmic scale $A=\ln (v(a))$ and $B=\ln (v(b))$ results in $p(a, b)=\exp (A) /(\exp (A)+\exp (B))$ $=1 /(1+\exp (-(A-B)))$ that is a logistic probability function. The standardized logistic cumulative probability

$$
p=\frac{1}{1+\exp (-\gamma z)}
$$

practically coincides with the standardized cumulative normal probability (3) when the parameter $\gamma=\pi / \sqrt{3} \approx 1.81$; this choice defines the logistic probability density function with a unit variance (see Long, 1997, chapter 3). Thus, for a simpler estimation the logistic as opposed to normal probability can be used when the $z$-value defined from (14) equals 


$$
z=\frac{1}{\gamma} \ln \frac{p}{1-p} .
$$

Using in (12) $\mathrm{z}(15)$ defined by the empirical frequencies in (8)-(9) results in the values:

$$
\begin{aligned}
v_{j} & =\frac{1}{m} \sum_{i=1}^{m} z_{i j} \\
& =\frac{1}{\gamma m} \sum_{i=1}^{m} \ln \frac{f_{i j}}{1-f_{i j}} \\
& =\frac{\sqrt{3}}{\pi} \ln \left(\prod_{i=1}^{m} \frac{f_{i j}}{1-f_{i j}}\right)^{1 / m} .
\end{aligned}
$$

Therefore, the Thurstonian logistic scale for a $j^{\text {th }}$ item in comparison is proportional to the logarithm of the geometric mean of odds of the frequency in the $j^{\text {th }}$ column of matrix (8). This solution is convenient for analytical consideration.

Returning to LS objective (5) that yields solution (12) for a matrix of paired comparison (8) notice that it corresponds to minimizing deviations for the linear regression:

$$
\begin{aligned}
z_{i j} & =v_{j}-v_{i}+\varepsilon_{i j} \\
& =a_{1} u_{i j, l}+a_{2} u_{i j, 2}+\ldots+a_{m} u_{i j, m}+\varepsilon_{i j},
\end{aligned}
$$

where each $i j^{\text {th }}$ value of the dependent variable $z_{i j}$ is represented by its theoretical model $v_{j}-v_{i}$ and random noise $\varepsilon_{i j}$. The theoretical model consists of a set of $m$ dummy variables $u_{i j, l}, \ldots, u_{i j, m}$ combined with the regression coefficients $a_{1}, \ldots$, $a_{m}$. If all $z_{i j}$ values are stacked into one vector of $\mathrm{m}^{2}$ observations by the dependent variable and all vectors of the variables $u_{i j, 1}, \ldots, u_{i j, m}$ are arranged as a design matrix $U$ of $m^{2}$ by $m$ order, then in the row of matrix $U$ defined by any $i j^{\text {th }}$ pair of indices the only non-zero elements are in the $j^{\text {th }}$ and $i^{\text {th }}$ columns, and they equal 1 and -1 , respectively. Therefore the dummy variables can be defined as:

$$
u_{i j, k}=\left\{\begin{array}{lll}
+1, & \text { if } & k=j \\
-1, & \text { if } & k=i
\end{array}\right.
$$

where $k=1, \ldots, m$ corresponds to different dummies.

For linear regression model (17) with the predictors (18) the least squares objective is

$$
\begin{aligned}
L S & =\sum_{i, j=1}^{m}\left(z_{i j}-a_{1} u_{i j, 1}-a_{2} u_{i j, 2}-\ldots-a_{m} u_{i j, m}\right)^{2} . \\
& =\rightarrow \min
\end{aligned}
$$

The coefficients $a_{k}$ are the estimates of the Thurstone scale levels $v_{k}$. The totals in each row of the design matrix (18) equal zero, thus, this matrix has the rank $m-1$ and in regression modeling it is only necessary to use $m-1$ dummy variables. One of the coefficients, for example, $a_{m}=0$, can be fixed to construct the regression by other $m-1$ variables, and then renormalize all $m$ coefficients by condition (6).

Numerically the coefficients of regression (17) or (19) coincide with the explicit solution (12), the regression approach, however, yields much richer results. To name some of them, besides the coefficients themselves, their standard errors and t-statistics, the coefficient of multiple determination as a characteristic of the quality of the approximation, deviations in each point of observation, etc., are obtained. The statistical difference between the Thurston scale levels can be checked, or the minimum distance found, between the significantly different levels.

In applied research with a large number of items, the pair comparison is usually arranged by experimental design when each respondent compares several items (not all) from a total set. In this case the frequencies (9) can correspond to different numbers of respondents in each paired comparison. Suppose, there are $n_{i j}=n_{i}+n_{j}$ counts in the $i j^{\text {th }}$ pair of the items, so the variance of the proportion in this comparison equals

$$
\sigma_{i j}^{2}=\frac{f_{i j}\left(1-f_{i j}\right)}{n_{i j}-1} .
$$

The standardized normal probability density function can then be written as 


\section{STAN LIPOVETSKY}

$$
d p=\frac{1}{\sqrt{2 \pi}} \exp \left(-\frac{z^{2}}{2}\right) d z
$$

thus, due to the rule of error propagation the relation for the variances is

$$
\sigma^{2}(z)=2 \pi \exp \left(z^{2}\right) \sigma^{2}(p) .
$$

Taking (20) for the variance of empirical frequency as the estimate for the variance $\sigma^{2}(p)$ in (22) results in the variance for zvalues in each $i j^{\text {th }}$ pair comparison. In place of (19) the Gauss-Markov weighted least squares objective can then be used

$$
\begin{aligned}
L S & =\sum_{i, j=1}^{m} w_{i j}\left(z_{i j}-a_{1} u_{i j, 1}-a_{2} u_{i j, 2}-\ldots-a_{m} u_{i j, m}\right)^{2} \\
& =\rightarrow \min
\end{aligned}
$$

with the weights of the observations defined as follows:

$$
\begin{aligned}
w_{i j} & =\frac{1}{\sigma^{2}\left(z_{i j}\right)} \\
& =\frac{1}{2 \pi} \exp \left(-z_{i j}^{2}\right) \frac{n_{i j}-1}{f_{i j}\left(1-f_{i j}\right)} .
\end{aligned}
$$

The weighted regression (23)-(24) can be constructed without difficulty and used as a weighted estimation for the TMD model.

Stochastic Modeling by Chapman-Kolmogorov Equations.

Return to frequency matrix (8)-(9) and consider other possibilities to estimate preferences among compared items. In the approach developed in (Lipovetsky \& Conklin, 2002) the analytic hierarchy process (AHP) matrix of pairwise ratios was transformed to a share matrix with the elements of the kind (9), and a specific eigenproblem was designed for evaluating the priorities among the items. The results of that work applied to a Thurstone matrix (8) (that corresponds to the transposition of a transformed AHP matrix) can be presented in the eigenproblem:

$$
\left[F^{\prime}+\operatorname{diag}\left(F^{\prime} e\right)\right] \alpha=\lambda \alpha,
$$

where prime denotes transposition of the matrix $F(8), \operatorname{diag}\left(F^{\prime} e\right)$ is a diagonal matrix of the totals in the columns of matrix $F$, and $e$ denotes a uniform vector of the $m^{\text {th }}$ order. Solving (25) for the maximum eigenvalue $\lambda$ yields the estimate $\alpha$ for the priority vector.

The matrix at the left-hand side (25) is proportional to a transposed stochastic matrix. It means that totals in the columns of this matrix equal the following vector:

$$
\left[F^{\prime}+\operatorname{diag}\left(F^{\prime} e\right)\right]^{\prime} e=F e+F^{\prime} e=m e,
$$

where the property (10) is used, so each element of the vector (26) equals $m$. Dividing (25) by this term the eigenproblem is represented as

$$
\left(\frac{1}{m} F^{\prime}+\frac{1}{m} \operatorname{diag}\left(F^{\prime} e\right)\right) \alpha=\mu \alpha \text {. }
$$

Totals in every column of matrix (27) equal one. A positive matrix with such property is a transposed stochastic matrix. Such matrices have a maximum eigenvalue equal to one. Due to the Perron-Frobenius theory for positive matrices the principal eigenvector always exists, is unique and has all positive elements; the desired properties of the priority vector are thus ensured.

Consider the eigenproblem (27) from the point of view of Markov chain modeling one of the most widely used tools in theoretical and applied statistics. A discrete state and continuous time model are presented via a system of Chapman-Kolmogorov differential equations used for a stochastic process of transitions among the states. These well-known (especially in queuing theory) equations express change in probability to be found in any of $m$ states as a linear combination of these probabilities with the coefficients of the transition intensities. 
Any pair of elements $f_{i j}$ and $f_{j i}(9)$ of Thurstone matrix (8) can be interpreted in terms of frequency to prefer one of the items over the other. Thus, each element $f_{i j}$ can be used to describe the preference of the $j^{\text {th }}$ item over the $i^{\text {th }}$ item that corresponds to transition to the preferred state $j$ from the state $i$ with the intensity of transition $f_{i j}$. The frequency matrix $F$ (8) can be presented as a connected oriented graph with $m$ nodes of states (items) and two edges between each pair of nodes - the one going to state $j$ from state $i$ corresponds to transition intensity $f_{i j}$, and the other going from state $j$ to state $i$ corresponds to transition intensity $f_{j i}$. An example of such graph for three states is presented in Figure 1.

The system of Chapman-Kolmogorov equations can thus be written as:

$$
\frac{d p_{k}}{d t}=\sum_{i \neq k}^{m} f_{i k} p_{i}-\sum_{j \neq k}^{m} f_{k j} p_{j}, \quad k=1, \ldots, m,
$$

where $p_{i}$ denotes probability of belonging to each of the states. Items with positive signs at the right-hand side (28) define influx to each state from all the others and those with negative signs define departure from a state to all the other states. If $0.5 p_{k}$ is added into each sum in each $k^{\text {th }}$ equation (28) this system can be represented in a matrix form:

$$
\dot{p}=\left(F^{\prime}-\operatorname{diag}(F e)\right) p,
$$

where $p$ is a vector consisting of the probabilities $p_{i}$ for all the states, $\dot{p}$ denotes the vector of their derivatives (as in the left-hand side (28)). Using property (26) that sum of totals in any $k^{\text {th }}$ column and row of the matrix $F$ equals $m$, (29) can be rewritten as

$$
\dot{p}=\left(F^{\prime}+\operatorname{diag}\left(F^{\prime} e\right)-m I\right) p,
$$

where $I$ denotes the identity matrix of the $m^{\text {th }}$ order.

Figure 1: Transition Intensities for Markov Modeling

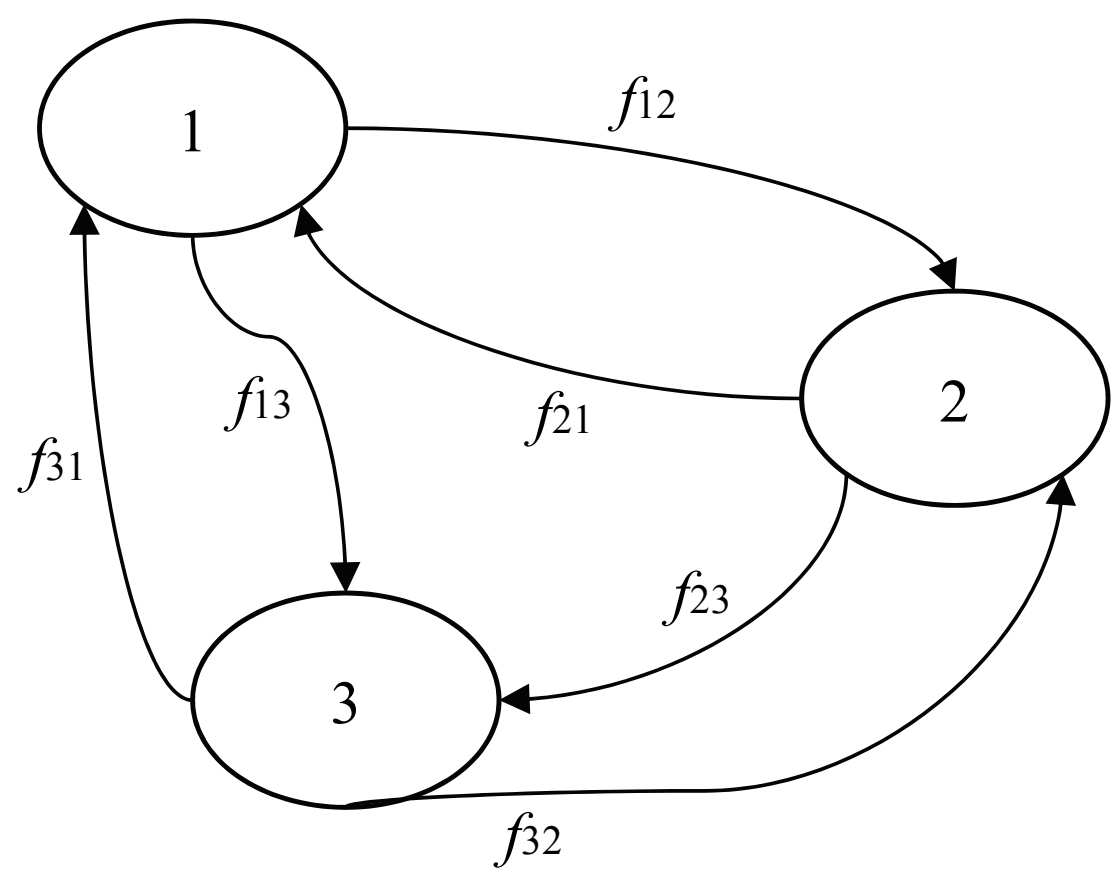




\section{STAN LIPOVETSKY}

Consider the solution of the ChapmanKolmogorov equations for the steady-state probability when the process is stabilized. If the derivatives in the right-hand side (30) equal zero, then (30) is reduced to

$$
\left(F^{\prime}+\operatorname{diag}\left(F^{\prime} e\right)\right) p=m p,
$$

which is the same as eigenproblem (25) with the maximum eigenvalue equal $m$ and a unique positive main eigenvector, as discussed in relation to expression (27). Coincidence of these results suggests a useful interpretation: the priority vector (27) makes sense of the eventual probabilities to belong to the discrete states corresponded to the compared items. These probabilities define the preferences among the compared items.

The general dynamic solution for system (30) can be useful for problems in priority modeling. For example, item preference depending on different initial conditions is of interest in maximum differences among the preferences and in their specific behavior (monotonic increase or decrease, oscillating) before the process is stabilized. Priorities behavior after adding new items to the original set may also be considered, taking initial probabilities of the new items equal zero and assuming the pair relations kept due to the given data.

As Bellman (1960) showed, the solution of a homogeneous linear system of differential equations with constant coefficients can be presented as

$$
p(t)=P \operatorname{diag}\left(\exp \left(\lambda_{j} t\right)\right) c
$$

where $c$ is a vector of constants, $\lambda_{j}$ are the eigenvalues and $P$ is a corresponded matrix of columns $p_{j}$ of eigenvectors obtained in solving the problem

$$
\left(F^{\prime}+\operatorname{diag}\left(F^{\prime} e\right)-m I\right) p=\lambda p
$$

Expression (33) defines the eigenproblem with the matrix at the right-hand side of ChapmanKolmogorov system (30), and its solution coincides with the solution for problem (25) up to reducing the latter eigenvalues by $m$.
For the moment $t=0$, solution (32) reduces to $p(0)=P c$, and solving this linear system with a known vector of initial conditions $p(0)$ results in the vector of constants $c=P^{-1} p(0)$. Thus, the general solution of the differential system is

$$
p(t)=P \operatorname{diag}\left(\exp \left(\lambda_{j} t\right)\right) P^{-1} p(0)
$$

The expression $P \operatorname{diag}\left(\exp \left(\lambda_{j} t\right)\right) P^{-1}$ in (34) is known as matrix exponent. Each component of the vector $p(t)$ is a linear combination of the exponents in (34), and functions $\exp \left(\lambda_{j} t\right)$ behave in accordance with the specific values of $\lambda_{j}$ obtained in eigenproblem (33).

As noted, the main eigenvalue in (33) is less by $m$ than the main eigenvalue in (25), so it equals zero, $\lambda_{1}=0$, which corresponds to the constant part of (34) behavior. The other eigenvalues (33) are real numbers or conjugated pairs of complex numbers. As per the PerronFrobenius theory, all other eigenvalues have less real value than the main eigenvalue, meaning that all real eigenvalues, or real parts in complex eigenvalues, are negative. Thus, the general behavior of solution (34) is defined by a constant part $\left(\lambda_{1}=0\right)$, by diminishing exponents (real negative eigenvalues), and by oscillating diminishing exponents (complex eigenvalues giving sine and cosine parts of functions). There can also be polynomial items corresponding to equal eigenvalues, although in practical numerical evaluations such cases are rare. The eigenvectors $p$ corresponding to the complex eigenvalues are also complex, however, the total expression (34) yields real values.

The total of the eigenvalues equals the trace of the matrix, which for the matrix in (33) is

$$
\begin{aligned}
-\sum_{j=1}^{m} \lambda_{j} & =-\operatorname{Tr}\left(F^{\prime}+\operatorname{diag}\left(F^{\prime} e\right)-m I\right) \\
& =-\frac{m}{2}-\frac{m^{2}}{2}+m^{2} \\
& =\frac{m(m-1)}{2}
\end{aligned}
$$


The inverted values of the reciprocal intensities in the exponents (34) make sense of the mean time of the transitions among the states, $-1 / \lambda_{j}=\bar{t}_{j}$, similarly to the interpretation of the parameters in the exponential and Erlang distributions from queueing theory. Thus, (35) corresponds to an ergodic relation saying that the total of the intensities equals the number of connections $m(m-1) / 2$ among $m$ states. The mean intensity can be defined from (35) as

$$
\bar{\lambda}=-\frac{1}{m} \sum_{j=1}^{m} \lambda_{j}=\frac{m-1}{2}
$$

that corresponds to a mean number of links from initial to final states (Levene \& Loizou, 2002). A value $\lambda_{j}$ defines the decay of the exponent, therefore, several first eigenvalues from the main $\lambda_{1}=0$ to about the mean (36) are important in the solution (34). It is interesting to note that the relation (36) can be also interpreted as a harmonic mean for the mean times:

$$
\frac{1}{H}=\frac{1}{m} \sum_{j=1}^{m} \frac{1}{\bar{t}_{j}}=\frac{m-1}{2} .
$$

The mean time for the exponential decay is about $2 /(m-1)$, and after this time the process is stabilizing.

\section{Numerical Example}

Consider a numerical example using data from a real research project. Twenty flavors (see Table 1) of a snack were ranked by 151 respondents. The first 6 flavors (A to $F$ ) were in the current line and the others 14 flavors $(G$ to $\mathrm{T})$ were considered for possible addition to the production line.

The results of the modeling are presented in Table 2. The table shows Thurstone Scaling - coefficients of regression (19), their tstatistics, raw Thurstone scale (12), standard scale in 0-1 range (13), and ranks of the flavors. In the regression approach (12) the upper triangle (190 values) of the z-matrix with elements (11) is used, $m-1$ dummies (18) without the first flavor A, and no intercept. Centering the coefficients of regression by
Table 1: Flavors Tested

\begin{tabular}{|c|c|}
\hline Code & Flavor \\
\hline A & Hot Fudge Swirl \\
\hline B & Caramel Swirl \\
\hline C & Cheesecake Swirl \\
\hline D & Walnut \\
\hline E & Chocolate Chunk \\
\hline F & Double Chocolate \\
\hline G & Peanut Butter Chunk \\
\hline H & Peanut Butter Frosted \\
\hline I & Peanut Butter Swirl \\
\hline J & White Chocolate Chunk \\
\hline K & German Chocolate \\
\hline L & Raspberry Swirl \\
\hline M & Snickers \\
\hline N & Chocolate Frosted \\
\hline O & Mocha Swirl \\
\hline P & Chocolate Chip Blonde \\
\hline Q & S'mores \\
\hline R & Bailey’s Irish Crème Swirl \\
\hline S & Icing \\
\hline T & Frosted Mint \\
\hline
\end{tabular}

subtracting their mean from each, the coefficients are rescaled until their total equals zero (6). The rescaled coefficients of regression coincide with the raw Thurstone scale (12). In regression, t-statistics are also obtained for the coefficients that correspond to checking the significance of the difference between the level of each flavor in comparison with the first flavor A (thus, except for flavor E, all the others are different from flavor A). Flavor A was best one on the Thurstone scale, this explains the negative signs of the regression coefficients and $\mathrm{t}$-values. If any other flavor is excluded from the regression, its coefficient would be of positive and negative signs for the flavors preferred and non-preferred to this one, respectively, and the tvalues would estimate the significance of the difference of all the levels from the fixed one. Coefficient of multiple determination in the 


\section{STAN LIPOVETSKY}

model equals $R^{2}=0.966$, so approximation of the data by the Thurstone scale is very high. If the total $z$-matrix is used to construct a regression (19) with an intercept, again by $m-1$ dummies (18), then the coefficients of regression will be the same as described above. Also in the rescaled set of coefficients the value of the intercept equaled the coefficient for the excluded flavor. The results of the weighted regression (23)-(24) practically coincide with the regular regression by this data.

Table 2 also presents a Thurstonian estimation by the logistic BTL model (16) and its standard scaling by (13) to 0-1 range. Note that the raw logistic scale slightly differs from the Thurstone raw scale, however, the standardized scales in $0-1$ range are practically undistinguishable and both the normal and logistic estimations also yield the same ranks.

Finally, Table 2 shows the results of Markov modeling for the eventually reached steady-state probabilities. These probabilities correspond to the elements of the main eigenvector in problems (25), (31) or (33) and define shares of choosing each of the flavors under consideration. Ranks of flavors by the Markov probabilities coincide with the ranks by the Thurstone scale for this data.

Reaching the steady-states in Markov processing can be considered by solution (34) of the Chapman-Kolmogorov differential equations (28). At first the behavior of the current six flavors in the line is constructed, using initial conditions with equal probability for all 6 states (see Figure 2). The reached steady-state probabilities in this set are $0.219,0.123,0.135$, $0.123,0.205$, and 0.195 , for the flavors from $\mathrm{A}$ to $\mathrm{F}$, respectively. Using these probabilities as initial values for the current flavors and zero initial values for other possibly introduced flavors, another Markov model by all 20 flavors is constructed (see Figure 3). Note that 14 possibly introduced flavors would push down the current flavors' shares. The flavors A, E and $\mathrm{F}$ remain best, however, two new flavors $-\mathrm{M}$ and $\mathrm{N}$ - could become more attractive than the other current flavors. Thus, the mutual behavior of all current flavors are considered with these two best candidates for the line extension (see Figure 4). Figure 4 shows that newly introduced flavors $\mathrm{M}$ and $\mathrm{N}$ can overcome three of the current flavors, thus, it makes sense to substitute the current $\mathrm{B}$ and $\mathrm{D}$ flavors for these new ones if the size of the line will continue to consist of only six flavors. It is interesting to note that the eigenvalues (34) in all these eigenproblems are real numbers so the flavor curves behavior consists in just exponential change, without oscillations corresponding to the complex numbers. This indicates a consistent relation among the pair comparison data and the robust results of both Thurstone and Markov evaluations.

\section{Conclusion}

This article considered preference evaluation by pair comparison data. Thurstonian scaling via multiple regression and Markov chain modeling by Chapman-Kolmogorov equations was explored. A Thurstone scale as a regression model a special design of dummy variables was used for estimation. Coefficients of regression represent the levels of the items by the Thurstone scale. Simultaneously the standard errors and t-statistics for the coefficients of regression were obtained along with the coefficient of multiple determination so that precision and statistical significance of the differences among the items could be estimated.

The Thurstone model defines a scale of differences, and its standardized zero-one range corresponds to the interval Thurstone scale. With regression, non-linear scaling can be considered, a hierarchy Bayesian model using other variables (for example, demographics) or any other technique known in regression modeling. Also considered was the BradleyTerry-Luce logistic model of pair comparison that produces a scaling of the Thurstonian type with the results very close to the ThurstoneMosteller-Daniels model.

Another possibility for multiple pair comparison evaluation was suggested based on stochastic Markov chain modeling for discrete states and continuous time of transitions probabilities. This approach uses pair comparisons data for intensity of transitions among the states (items) for constructing the Chapman-Kolmogorov system of differential equations and solving for the dynamic as well as for the eventually reached steady-state probabilities. While in the Thurstone model only 
differences are meaningful, the Markov states approach elaborates a ratio scale of probabilities to choose each of the items in comparison. Thus, the Thurstone and Markov models correspond to the relative and absolute preference estimates. The considered methods of priority evaluation are convenient and simple and could enrich both theoretical modeling and practical applications for various multiple criteria decision making problems.

\section{References}

Bar-Niv, M., \& Lipovetsky, S. (1995). Deciding circular priorities in insolvency situations, International Journal of Operations and Quantitative Management, 1, 183-195.

Bellman, R. (1960). Introduction to matrix analysis. New York, NY: McGraw-Hill.

Table 2: Thurstone Scale, Logistic Scale and Markov States

\begin{tabular}{|c|c|c|c|c|c|c|c|c|}
\hline \multirow{2}{*}{ Flavor } & \multicolumn{5}{|c|}{ Thurstone Scale } & \multicolumn{2}{|c|}{ Logistic Scale } & \multirow{2}{*}{\begin{tabular}{|c|} 
Markov \\
State \\
$\begin{array}{c}\text { Probability } \\
\%\end{array}$ \\
\end{tabular}} \\
\hline & $\begin{array}{l}\text { Regression } \\
\text { Coefficients }\end{array}$ & t-statistics & $\begin{array}{l}\text { Raw } \\
\text { Scale }\end{array}$ & $\begin{array}{c}\text { Range } 0- \\
1\end{array}$ & Rank & $\begin{array}{l}\text { Raw } \\
\text { Scale }\end{array}$ & $\begin{array}{c}\text { Range } \\
0-1\end{array}$ & \\
\hline A. Hot Fudge Swirl & 0 & 0 & 0.49 & 1.00 & 1 & 0.44 & 1.00 & 9.93 \\
\hline B. Caramel Swirl & -0.36 & -14.1 & 0.12 & 0.64 & 7 & 0.11 & 0.63 & 5.51 \\
\hline C. Cheesecake Swirl & -0.35 & -13.4 & 0.14 & 0.65 & 6 & 0.13 & 0.65 & 5.75 \\
\hline D. Walnut & -0.38 & -14.8 & 0.11 & 0.62 & 8 & 0.09 & 0.61 & 5.42 \\
\hline E. Chocolate Chunk & -0.01 & -0.3 & 0.48 & 0.99 & 2 & 0.43 & 0.99 & 9.75 \\
\hline F. Double Chocolate & -0.05 & -2.1 & 0.44 & 0.95 & 3 & 0.39 & 0.95 & 9.13 \\
\hline G. Peanut Butter Chunk & -0.47 & -18.2 & 0.02 & 0.53 & 10 & 0.02 & 0.53 & 4.59 \\
\hline H. Peanut Butter Frosted & -0.80 & -30.3 & -0.31 & 0.20 & 18 & -0.28 & 0.20 & 2.72 \\
\hline I. Peanut Butter Swirl & -0.42 & -16.3 & 0.07 & 0.58 & 9 & 0.06 & 0.58 & 4.99 \\
\hline J. White Chocolate Chunk & -0.55 & -21.4 & -0.06 & 0.45 & 11 & -0.06 & 0.45 & 4.04 \\
\hline K. German Chocolate & -0.60 & -23.2 & -0.11 & 0.40 & 13 & -0.10 & 0.40 & 3.86 \\
\hline L. Raspberry Swirl & -1.00 & -38.6 & -0.51 & 0.00 & 20 & -0.46 & 0.00 & 1.97 \\
\hline M.Snickers & -0.33 & -12.6 & 0.16 & 0.67 & 5 & 0.15 & 0.67 & 5.90 \\
\hline N. Chocolate Frosted & -0.17 & -6.5 & 0.32 & 0.83 & 4 & 0.29 & 0.83 & 7.39 \\
\hline O. Mocha Swirl & -0.71 & -27.5 & -0.22 & 0.29 & 17 & -0.20 & 0.29 & 3.12 \\
\hline P. Chocolate Chip Blonde & -0.66 & -25.3 & -0.17 & 0.34 & 14 & -0.15 & 0.35 & 3.43 \\
\hline Q. S'mores & -0.57 & -21.9 & -0.08 & 0.43 & 12 & -0.07 & 0.43 & 3.96 \\
\hline R. Bailey's Irish Crème Swirl & -0.68 & -26.2 & -0.19 & 0.32 & 15 & -0.17 & 0.33 & 3.37 \\
\hline S. Icing & -0.71 & -27.4 & -0.22 & 0.29 & 16 & -0.20 & 0.29 & 3.11 \\
\hline T. Frosted Mint & -0.96 & -37.2 & -0.47 & 0.04 & 19 & -0.43 & 0.04 & 2.07 \\
\hline
\end{tabular}


Figure 2: State Probability of Current Six Flavors in Markov Model

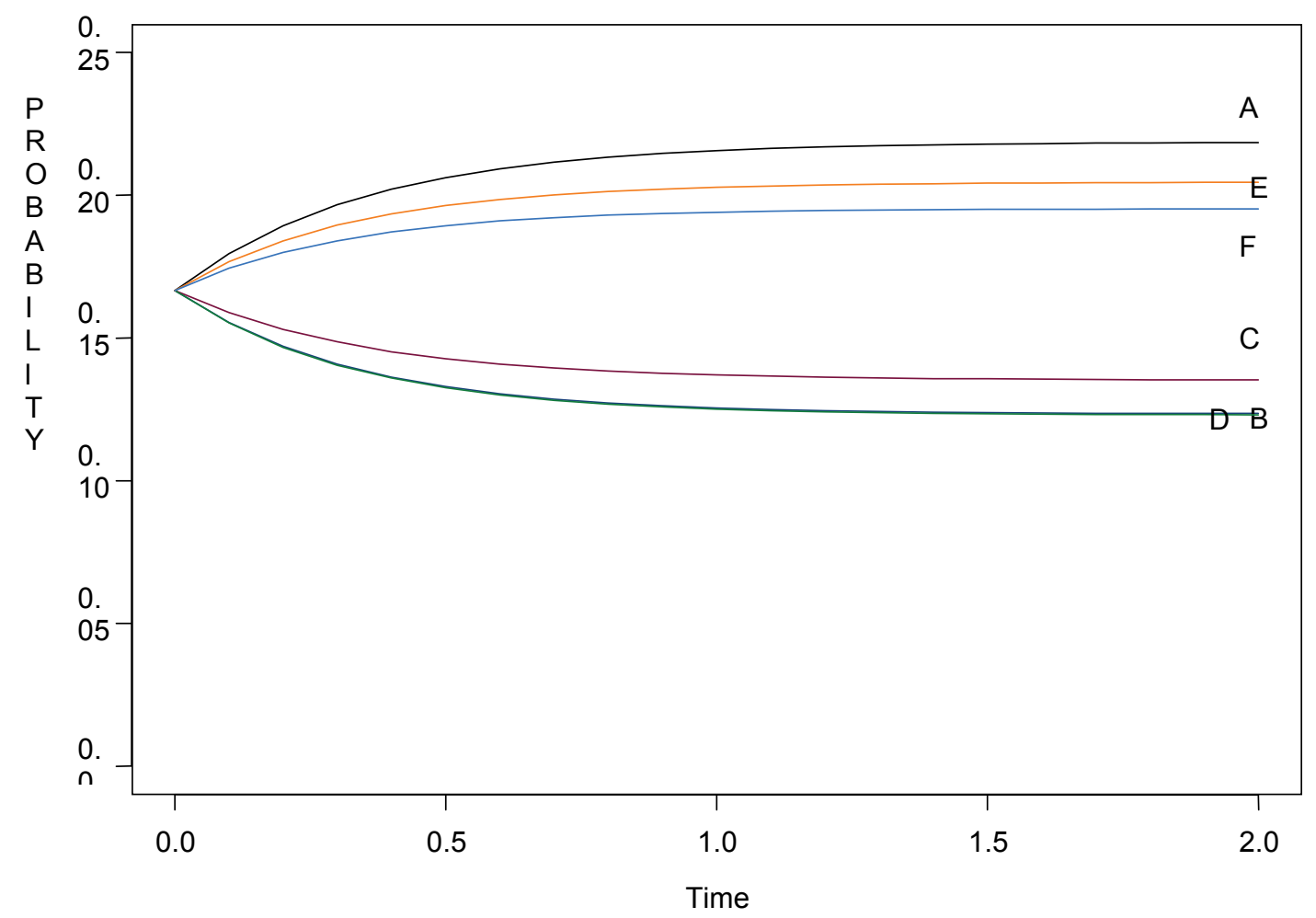

Figure 3: State Probability for 20 Flavors in Markov Model

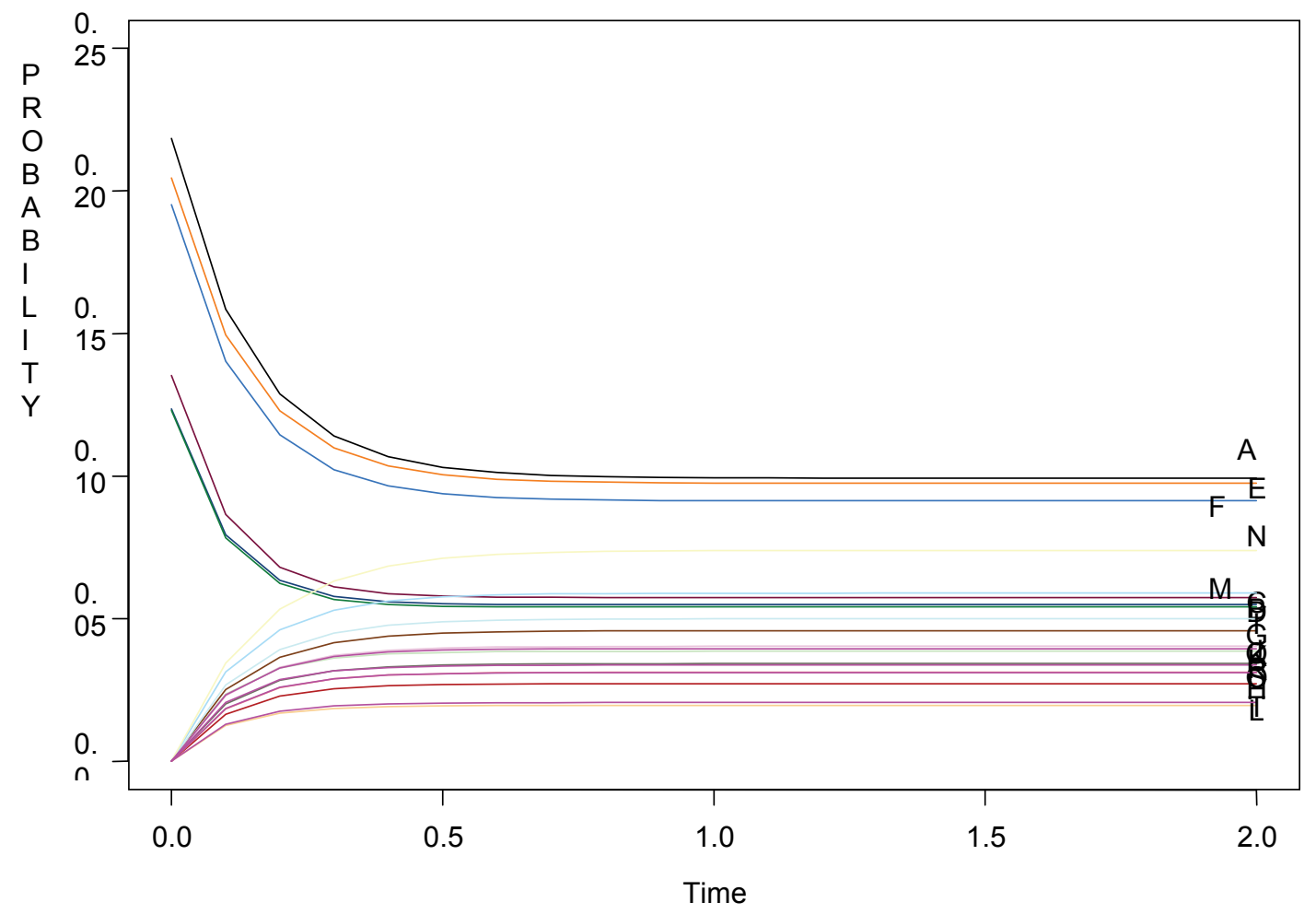


Figure 4: State Probability for Chosen Eight Flavors in Markov Model

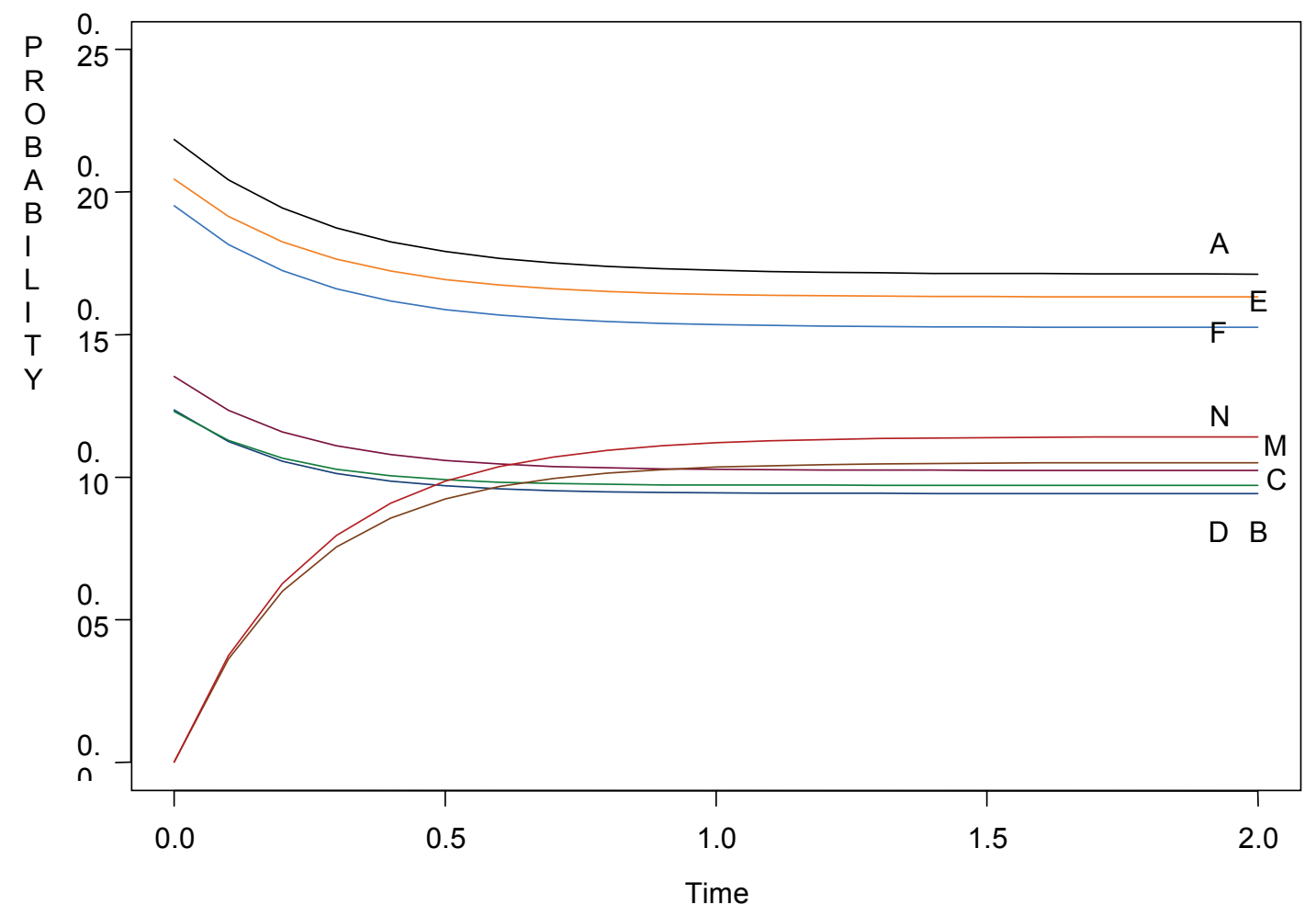

Bock, R. D., \& Jones, L. V. (1968). The measurement and prediction of judgment and choice. San Francisco, CA: Holden-Day.

Bradley, R. A., \& Terry, M. E. (1952). Rank analysis of incomplete block designs: The method of paired comparisons. Biometrika, 39, 324-345.

Conklin, M., \& Lipovetsky, S. (1999). Efficient assessment of self-explicated importance using latent class Thurstone scaling. The $10^{\text {th }}$ Annual Advanced Research Techniques Forum, Santa Fe, NM: American Marketing Association.

Daniels, H. E. (1950). Rank correlation and population models, Journal of the Royal Statistical Society, Series B(12), 171-181.

David, H. A. (1988). The method of pair comparisons, $2^{\text {nd }} E d$. London: Griffin.

Edwards, A. L. (1957). Techniques of attitude scale construction. New York, NY: Appleton-Century-Crofts.
Ennis, D. M., \& Johnson, N. L. (1993). Thurstone-Shepard similarity models as special cases of moment generating functions. Journal of Mathematical Psychology, 37,104-110.

Green, P. E., \& Tull, D. S. (1978). Research for marketing decisions. New Jersey: Prentice-Hall.

Hillier, F. S., \& Lieberman G. J. (1974). Introduction to operations research. San Francisco, CA: Holden-Day.

Levene, M., \& Loizou, G. (2002). Kemeny's constant and the random surfer. The American Mathematical Monthly, 109, 741-745.

Lipovetsky, S., \& Conklin, M. (2001). Dual priority-antipriority Thurstone scales as AHP eigenvectors. Engineering Simulation, 18, 631648.

Lipovetsky, S., \& Conklin, M. (2002). Robust estimation of priorities in the AHP. European Journal of Operational Research, 137, 110-122. 


\section{STAN LIPOVETSKY}

Lipovetsky, S., \& Conklin, M. (2004a). Thurstone scaling via binary response regression. Statistical Methodology, 1, 93-104.

Lipovetsky, S., \& Conklin, M. (2004b). Nonlinear Thurstone scaling via SVD and Gower plots. International Journal of Operations and Quantitative Management, 10, 259-273.

Lipovetsky, S. (2005). Analytic hierarchy processing in Chapman-Kolmogorov equations. International Journal of Operations and Quantitative Management, 11, 219-228.

Lipovetsky, S. (2006). Van Westendrop price sensitivity in statistical modeling, International Journal of Operations and Quantitative Management, 12, 141-156.

Lipovetsky, S. (2007a). Thurstone Scaling in Order Statistics, Mathematical and Computer Modelling, 45, 917-926.

Lipovetsky, S. (2007b). Priority and choice probability estimation by ranking, rating, and combined data. Journal of Statistical Theory and Practice, 1, 265-278.

Lipovetsky, S. (2008). Bradley-Terry choice probability in maximum likelihood and eigenproblem Solutions. International Journal of Information Technology \& Decision Making, 7, 395-405.

Long, J. S. (1997). Regression models for categorical and limited dependent variables. London: SAGE Publications.

Luce, R. D. (1959). Individual choice behavior: A theoretical analysis. New York, NY: Wiley.
Luce, R. D., \& Suppes, P. (1965). Utility, preference and subjective probability. In Handbook of Mathematical Psychology, R. D. Luce, R. R. Bush \& E. Galanter (Eds.), 249-410. New York, NY: Wiley.

MakKay, D. B., Bowen, W. M., \& Zinnes, J. L. (1996). A Thurstonian view of the analytic hierarchy process. European Journal of Operational Research, 89, 427-444.

Mosteller, F. (1951). Remarks on the method of paired comparisons. Psychometrika, 16, 203-218.

Stern, H. (1990). Models for distributions on permutations. Journal of the American Statistical Association, 85, 558-564.

Thurstone, L. L. (1927). A law of comparative judgment. Psychological Reviews, 34, 273-286.

Thurstone, L. L. (1959). The measurement of values. Chicago, IL: University of Chicago Press.

Thurstone, L. L., \& Jones L. V. (1957). The rational origin for measuring subjective values. Journal of the American Statistical Association, 52, 458-471.

Torgerson, W. S. (1958). Theory and Methods of Scaling. New York, NY: Wiley.

Zinnes, J. L., \& MacKay, D. B. (1989). Probabilistic multidimensional analysis of preference ratio judgments. In New developments in psychological choice modeling, G. De Soete, H. Feger \& K. C. Klauer (eds.), 177-205. Amsterdam: North-Holland. 


\section{Bootstrap Interval Estimation of Reliability via Coefficient Omega}

\author{
Miguel A. Padilla \\ Old Dominion University \\ Norfolk, VA
}

\author{
Jasmin Divers \\ Wake Forest School of Medicine \\ Winston-Salem, NC
}

Three different bootstrap confidence intervals (CIs) for coefficient omega were investigated. The CIs were assessed through a simulation study with conditions not previously investigated. All methods performed well; however, the normal theory bootstrap (NTB) CI had the best performance because it had more consistent acceptable coverage under the simulation conditions investigated.

Key words: Coefficient omega, reliability, composite reliability, bootstrap, confidence interval, interval estimate.

\section{Introduction}

Coefficient omega was proposed in the literature over 40 years ago (McDonald, 1970) as a reliability measure of homogenous items from a measurement instrument. It indexes the consistency with which the items measure the underlying latent variable (or construct). Based on a factor analytic model, coefficient omega uses the item factor loading and uniqueness to estimate reliability. Therefore, coefficient omega can be viewed as a more intuitive measure of reliability compared to coefficient alpha. However, it is rarely used in practice for two reasons: (1) it is largely overshadowed by coefficient alpha (Cronbach, 1951; Guttman, 1945), and (2) its theoretical framework is narrow with a limited body of knowledge about its properties with respect to statistical inference.

One issue faced by behavioral/social science researchers is the presence of

Miguel A. Padilla is an Assistant Professor of Quantitative Psychology in the Department of Psychology. His research interests are in applied statistics and psychometrics. Email him at: mapadill@odu.edu. Jasmin Divers is an Associate Professor of Biostatistics in the Department of Biostatistical Sciences. His research interests are in statistical genetics and biostatistics. Email him at: jdivers@wfubmc.edu. measurement error in data collected through multiple-item questionnaires, inventories and other measurement instruments. The most common estimator of reliability used in the behavioral/social sciences is coefficient alpha (Hogan, Benjamin \& Brezinski, 2000), at times referred to as Cronbach's coefficient alpha or Cronbach's alpha (Peterson, 1994). With reliability coefficients such as alpha, behavioral/social science researchers are able to evaluate the reliability of their items to aid in the creation of reliable measurement instruments.

Coefficient alpha's dominance is a result of five features. First and most notably, it is relatively simple to calculate and is a common option in popular statistical packages such as SAS and SPSS. Second, coefficient alpha can be calculated after a single test administration as opposed to requiring at least two test administrations. Third, it can be computed for continuous, ordinal or binary items; this advantage is notable when working with binary items such as right/wrong, true/false, etc. Fourth, different types of interval estimates for coefficient alpha have been developed (MaydeuOlivares, Coffman \& Hartmann, 2007; Padilla, Divers \& Newton, 2012; Romano, Kromrey \& Hibbard, 2010; van Zyl, Neudecker \& Nel, 2000; Yuan, Guarnaccia \& Hayslip, 2003). Fifth, a lack of other options, as well as more than a half century of cited research gives the impression that coefficient alpha is the only viable estimate of reliability. 


\section{PADILLA \& DIVERS}

Although coefficient alpha is an excellent estimator of internal consistency when used correctly, it is biased when items are not at least Tau-equivalent or essentially Tauequivalent (Graham, 2006; Lord, Novick \& Birnbaum, 1968; McDonald, 1999; Zinbarg, Revelle, Yovel \& Li, 2005). Tau-equivalence of items can best be described within the framework of the classical true score model (CTSM) from classical test theory. The CTSM for items can be ordered from most to least restrictive as follows: (1) parallel, (2) Tau - or essentially Tau-equivalent, and (3) congeneric. For items under conditions 1 and 2, coefficient alpha is equal to the reliability of the set of items. Under condition 3, coefficient alpha underestimates the reliability for a set of items (Zinbarg, et al., 2005). However, coefficient omega is equal to the reliability of a set of items for all 3 conditions (McDonald, 1999; Zinbarg, et al., 2005).

As stated, a reason for the limited use of coefficient omega is the limited knowledge about its statistical properties. One noteworthy drawback is a lack of development and investigation of a confidence interval (CI) for coefficient omega.

Raykov (1998) proposed a bootstrap percentile CI for the composite reliability of congeneric items measuring a common dimension (Raykov, 1997). The method is specified as a structural equation model (SEM) and shows promise. An illustration of the method was applied to a small simulation that included a sample size of 400,6 multivariate normal congeneric items, and assumed unidimensionality. The bootstrap estimates were based on 1,000 bootstrap samples.

In another study Raykov (2002) derived the standard error for composite reliability CIs via the delta method. As previously, the model was specified through an SEM framework and showed promise when illustrated with a small simulation $(\mathrm{n}=500,5$ multivariate normal congeneric items) assuming unidimensionality. The delta method CI was also compared to the bootstrap percentile CI with 2,000 bootstrap samples; both methods had similar results.

In a parallel study, Raykov and Shrout (2002) presented a more general form of composite reliability in a SEM framework with bootstrapped percentile CIs. The method extends the previous method by Raykov (1997, 1998). The authors applied the method to a small simulation with a sample size of 300,6 multivariate normal congeneric items, and assumed two dimensions. The study results show that the composite reliability estimate is unbiased and the CIs contain the population parameter. The bootstrap percentile CIs were based on 1,000 bootstrap samples.

More recently (Raykov, 2012; Raykov \& Marcoulides, 2011), the non-bootstrap method has been illustrated using large example data sets (i.e., $n \geq 350$ ). Results indicate that the method is applicable to approximately continuous items having a multi-normal distribution. In addition, the method is applicable to non-normal items with at least 5 to 7 response categories with the use of the robust maximum likelihood estimator (MLR). Study results also indicate that the MLR estimator can be used with items with less than 5 response categories by using parcels. For further details about using the MLR estimator with parcels see Raykov and Marcoulides (2011).

Hence, studies of CIs for composite reliability have been conducted (Raykov, 1998, 2002), but there are several limitations. First, it is difficult to generalize findings based on small simulations or example data. Second, the simulation studies designed to test the proposed methods were based on continuous items; such items are rare in the behavioral/social sciences (Raykov, 2002), most are Likert/ordinal or, in some cases, binary. Third, the methods require large sample sizes based on the asymptotic theory that underlies SEMs - recall that maximum likelihood (ML) is the standard method of estimating SEMs (Bollen, 1989; Raykov, 1998, 2002; Raykov \& Shrout, 2002). Lastly, the methods require specialized SEM software (e.g., EQS, Mplus, etc.).

This study assesses the performance of bootstrap CIs for composite reliability as specified through coefficient omega in terms of a one-factor model under simulation conditions that investigate the limitations above. Of particular interest is the impact of binary and Likert/ordinal (e.g., categorical) items, and a sample size less than 300 on the coefficient omega bootstrap CIs. 


\section{BOOTSTRAP INTERVAL ESTIMATION OF RELIABILITY}

Coefficient Omega and Reliability

Consider a set of $k$ items $x_{1}, x_{2}, \ldots, x_{k}$ designed to measure a single construct or attribute. A common procedure in behavioral/social science research is to estimate the reliability of the composite or sum score $x=\sum_{j=1}^{k} x_{i}$. This represents the reliability of the measurement instrument or test reliability. Reliability of the composite or sum score is defined as

$$
\rho=\frac{\operatorname{var}(\tau)}{\operatorname{var}(x)}=\frac{\sigma_{\tau}^{2}}{\sigma_{\tau}^{2}+\sigma_{u}^{2}}
$$

where $\operatorname{var}($.$) denotes the variance operator, \sigma_{\tau}^{2}$ the true score variance, and $\sigma_{u}^{2}$ the error variance. This definition of reliability assumes that all items are parallel (Allen \& Yen, 1979; Crocker \& Algina, 1986).

The analogous composite reliability for congeneric items via coefficient omega is defined as

$$
\omega=\frac{\left(\sum_{j=1}^{k} \lambda_{j}\right)^{2}}{\left(\sum_{j=1}^{k} \lambda_{j}\right)^{2}+\sum_{j=1}^{k} \psi_{j}}
$$

where $\lambda_{j}$ and $\psi_{j}$ are the $j^{\text {th }}$ factor loading and uniqueness, respectively (McDonald, 1970, 1999). Note that this model assumes the items are measuring a single construct or factor (i.e., a one-factor model). Coefficient omega is estimated by replacing $\lambda_{j}$ and $\psi_{j}$ with the sample estimates $\hat{\lambda}_{j}$ and $\hat{\psi}_{j}$ in equation 2 . Although several methods are available for estimating the factor loadings, ML will be used herein.

\section{Bootstrapped Coefficient Omega CIs}

The bootstrapping algorithm for coefficient omega can be summarized in three steps. Suppose $\mathbf{X}=\left(\mathbf{x}_{1}, \mathbf{x}_{2}, \ldots, \mathbf{x}_{n}\right)^{t}$ are the observed data where each $\mathbf{x}_{\mathrm{i}}$ is a $1 \times k$ vector. First, obtain a bootstrap sample $\mathbf{X}^{(b)}=\left(\mathbf{x}_{1}^{(b)}, \mathbf{x}_{2}^{(b)}, \ldots, \mathbf{x}_{n}^{(b)}\right)^{t}$, which is the $b^{\text {th }}$ random resample from $\mathbf{X}$ selected with replacement. Note that $\mathbf{X}$ and $\mathbf{X}^{(b)}$ have the same sample size. Second, compute the $b^{\text {th }}$ bootstrap estimate of coefficient omega $\left(\hat{\omega}^{(b)}\right)$ from $\mathbf{X}^{(b)}$. Lastly, $\hat{\omega}^{(1)}, \hat{\omega}^{(2)}, \ldots, \hat{\omega}^{(B)}$ represents the empirical sampling distribution (ESD) for $\hat{\omega}$ for $b=1,2, \ldots, B$ bootstrap samples. The ESD can then be summarized for statistical inference about $\omega$. Typical parameter estimates are the bootstrap mean, percentiles, quantiles and standard error (SE). The bootstrap estimate of $\mathrm{SE}$ is

$$
S E(\hat{\omega})=\left[\frac{1}{B-1} \sum_{b=1}^{B}\left(\hat{\omega}^{(b)}-\bar{\omega}\right)^{2}\right]^{1 / 2}
$$

where

$$
\bar{\omega}=\frac{1}{B} \sum_{b=1}^{B} \hat{\omega}^{(b)}
$$

The three most common bootstrap CIs were examined. First, the normal theory bootstrap (NTB) CI is computed as $\hat{\omega} \pm Z_{\alpha / 2} S E(\hat{\omega})$. Second, the percentile based (PB) CI is obtained by computing the $\alpha / 2$ and $1-\alpha / 2$ percentiles from the $\hat{\omega}$ ESD where $\alpha$ is the type I error rate. Third, the bias-corrected and accelerated (BCa) $\mathrm{CI}$ is an improved version of the PB CI in that it adjusts the PB CI $\alpha / 2$ and $1-\alpha / 2$ percentiles in two ways: (1) it makes a correction for bias, and (2) a correction for skewness (or acceleration). Note that the NTB CI assumes that the ESD is normally distributed, whereas the $\mathrm{PB}$ and $\mathrm{BCa}$ make no assumption about the shape of the ESD. For technical and theoretical details concerning the three bootstrap CIs investigated see Efron and Tibshirani (1998). 


\section{PADILLA \& DIVERS}

\section{Simulation Design}

Methodology

Four different conditions were investigated in a 4 (number of items) $\times 3$ (correlation type) $\times 5$ (number of item response categories) $\times 4$ (sample size) Monte Carlo simulation design. A total of 240 conditions were investigated. All simulated items were binary or Likert-type (ordinal) in order to mimic items commonly found in behavioral/social science research; none of the items were continuous. For each simulation condition, 1,000 replications were obtained.

Binary and Likert-type items were generated using the Maydeu-Olivares et al. (2007) method. In brief, the method is:

1. Select a $k \times k$ population correlation matrix $\mathbf{P}$, where $k$ is the number of items and a set of thresholds $\boldsymbol{\tau}$ for categorization so that resultant items have a predetermined skewness and kurtosis.

2. Generate an $n \times k$ multivariate data $\mathbf{X}^{*} \sim N(\mathbf{0}, \mathbf{P})$, where $n$ is the sample size.

3. Categorize the generated data $\mathbf{X}^{*}$ with $\boldsymbol{\tau}$ into data $\mathbf{X}$. Each variable $x$ in $\mathbf{X}$ is categorized by the thresholds as follows: $x=m$ if $\tau_{m}<x^{*}<\tau_{m+1}$ for $m=0,1, \ldots, M-1$ where $\tau_{0}=-\infty$ and $\tau_{M}=\infty$, and $M$ is the number of categories.

4. Compute the true population coefficient omega $(\omega)$ according to $\mathbf{P}$ and the thresholds in $\boldsymbol{\tau}$. See Maydeu-Olivares et al. (2007) for details.

5. Estimate coefficient omega $(\hat{\omega})$ bootstrapped CIs from the categorized data $\mathbf{X}$.

6. Determine if the bootstrapped CIs includes the population coefficient omega $(\omega)$.

The specific simulation conditions investigated are as follows.
Number of Items $(k)$.

Past research on coefficient alpha has examined various numbers of items ranging from two to twenty (Duhachek \& Iacobucci, 2004; Enders, 2003; Maydeu-Olivares et al. (2007). To make results consistent, the following number of items were selected for this study: $k=5,10,15,20$.

Correlation Type $(\rho)$.

Three different item correlation structures for $\mathbf{P}$ were investigated. The first two correlation structures were from a parallel-item one-factor model with common loadings $\lambda=.55$ or .705 . These two models generated compound symmetric item correlation structures with $\rho=.30$ or .50 , respectively. The third correlation structure was generated from a congeneric item one-factor model with loadings of $\lambda=.3, .4, .5, .6, .7$. The third item correlation was unstructured, and the same as the one generated by Maydeu-Olivares et al. (2007), but modified for cases with multiples of 5 items (the original called for multiples of 7 items).

Item Response Categories (IRCs).

Five item response categories were investigated: 2, 2, 3, 5 and 7. None of the items were continuous. For each response category, the first category was set to 0 . For example, for an item with seven categories, the first category was set to 0 and the last to 6 . The data generated from the specified item correlation matrix above (P) were categorized with $\boldsymbol{\tau}$ using the same methodology as Maydeu-Olivares et al. (2007).

For the binary items, $\tau$ was chosen so that the resultant categorized items had skewness $=0$ and kurtosis $=-2$, and skewness $=$ 0.41 and kurtosis $=-1.83$, respectively. The second condition was investigated by MaydeuOlivares et al. (2007). For the Likert-type items, $\tau$ was chosen so that the resultant categorized items had skewness $=$ kurtosis $=0$.

Sample Size $(n)$. The following sample sizes were investigated: $n=50,100,150,200$. These are common sample sizes in behavioral/social science research. In addition, Duhachek and Iacobucci (2004) noted that going 


\section{BOOTSTRAP INTERVAL ESTIMATION OF RELIABILITY}

beyond a sample size of 200 reaches a point of diminishing returns for reliability estimates.

In each simulation replication, coefficient omega was estimated along with its corresponding bootstrap CIs. In this study, the $100(1-\alpha) \%$ CIs for coefficient omega were estimated from a total of 2,000 bootstrap samples, where $\alpha=.05$. Relative bias for coefficient omega was calculated as

$$
\hat{\omega}_{\text {bias }}=\frac{\hat{\omega}-\omega}{\omega} .
$$

CI coverage was assessed using Bradley's (1978) liberal criteria, which is defined as $1-1.5 \alpha \leq 1-\alpha^{*} \leq 1-0.5 \alpha$ where $\alpha^{*}$ is the true Type I error probability. Coverage is defined as the proportion of estimated CIs that contain the true population coefficient omega. Therefore, acceptable coverage for $\alpha=.05$ is given by $[.925, .975]$.

\section{Point Estimate Bias}

\section{Results}

The estimate of bias was investigated because it can have a major impact on bootstrap CIs. However, tables with all combinations of the simulation conditions were inspected and no bias was observed. In fact, the largest bias observed was $\hat{\omega}_{\text {bias }}=.04$.

\section{Confidence Interval Coverage}

The NTB method had the best performance in terms of coverage. However, the major impact on the CIs was the number of items. Thus, results are presented in the context of number of items.

\section{Items}

Only the BCa method was impacted by 5 items (see Table 1). The PB method had acceptable coverage under all simulation conditions, however, the $\mathrm{BCa}$ method tended to be impacted when the sample size was 100 or less. In this case the $\mathrm{BCa}$ coverage probability was below the acceptable range. There were three instances where the $\mathrm{BCa}$ coverage probability was below the acceptable range when the sample size was 150 or more.

\section{Items}

The NTB and PB methods had unacceptable coverage in one instance each (see Table 2). In each case the unacceptable coverage occurred with a sample size of 50 and with a compound item correlation matrix with $\rho=0.56$.

\section{Items}

In this situation, all methods had at least one instance of unacceptable coverage (see Table 3). The NTB method had unacceptable coverage for the unstructured item covariance matrix with a sample size of 50 and 2 and 3 item response categories. For the PB method, unacceptable coverage occurred in two instances with a sample size of 50 and 3 item response categories. For the $\mathrm{BCa}$ method, the unacceptable coverage occurred with a sample size of 150 and 5 item response categories.

\section{Items}

In this condition, only the NTB and PB methods were impacted (see Table 4). The NTB method had unacceptable coverage in two instances in the unstructured item covariance matrix with a sample size of 50 and 2 item response categories. Conversely, unacceptable coverage for the PB method occurred with 3 or more item response categories and with a sample size of 100 or less.

\section{Coverage Bands}

In Figure 1 the $95 \% \mathrm{CI}$ coverage band is displayed for each method by number of items across all simulation conditions and shows the impact of five items on all methods. In particular, the $\mathrm{BCa}$ is most impacted by five items because it tended to be the furthest from acceptable coverage and has the most variance. Another noticeable feature is that the NTB method tended to have coverage bands that were slightly above $95 \%$, whereas the $\mathrm{PB}$ and $\mathrm{BCa}$ methods tended to have coverage bands below $95 \%$. The PB method appears to be the most conservative. 
PADILLA \& DIVERS

Table 1: 95\% Coverage Probabilities for 5 Items

\begin{tabular}{|c|c|c|c|c|c|c|c|c|c|c|c|c|c|}
\hline \multirow[b]{2}{*}{ IRC } & \multirow[b]{2}{*}{$\mathrm{n}$} & \multicolumn{4}{|c|}{$\rho=0.30$} & \multicolumn{4}{|c|}{$\rho=0.56$} & \multicolumn{4}{|c|}{$\rho=$ Unstructured } \\
\hline & & 50 & 100 & 150 & 200 & 50 & 100 & 150 & 200 & 50 & 100 & 150 & 200 \\
\hline \multirow{3}{*}{$2^{\mathrm{a}}$} & NTB & .945 & .932 & .944 & .951 & .940 & .948 & .944 & .941 & .925 & .935 & .942 & .934 \\
\hline & PB & .949 & .941 & .945 & .954 & .949 & .956 & .948 & .944 & .944 & .939 & .940 & .947 \\
\hline & $\mathrm{BCa}$ & .928 & .919 & .934 & .948 & .948 & .952 & .951 & .945 & .953 & .926 & .917 & .920 \\
\hline \multirow{3}{*}{$2^{\mathrm{b}}$} & NTB & .936 & .940 & .937 & .949 & .940 & .950 & .952 & .940 & .940 & .936 & .948 & .949 \\
\hline & PB & .954 & .944 & .943 & .954 & .942 & .948 & .946 & .945 & .954 & .946 & .942 & .955 \\
\hline & $\mathrm{BCa}$ & .940 & .929 & .939 & .949 & .942 & .950 & .950 & .945 & .941 & .932 & .928 & .934 \\
\hline \multirow{3}{*}{3} & NTB & .936 & .950 & .953 & .937 & .953 & .940 & .940 & .949 & .943 & .950 & .945 & .938 \\
\hline & PB & .935 & .941 & .963 & .937 & .939 & .939 & .939 & .944 & .958 & .948 & .939 & .940 \\
\hline & $\mathrm{BCa}$ & .912 & .929 & .957 & .929 & .936 & .938 & .938 & .947 & .934 & .926 & .923 & .928 \\
\hline \multirow{3}{*}{5} & NTB & .938 & .940 & .944 & .932 & .948 & .943 & .943 & .945 & .933 & .940 & .947 & .950 \\
\hline & PB & .936 & .947 & .940 & .942 & .933 & .940 & .940 & .943 & .937 & .937 & .951 & .956 \\
\hline & $\mathrm{BCa}$ & .920 & .939 & .938 & .937 & .932 & .936 & .936 & .943 & .920 & .914 & .932 & .953 \\
\hline \multirow{3}{*}{7} & NTB & .940 & .930 & .946 & .943 & .941 & 949 & .949 & .935 & .937 & .940 & .956 & .944 \\
\hline & PB & .942 & .934 & .935 & .944 & .935 & .943 & .943 & .936 & .949 & .953 & .954 & .948 \\
\hline & $\mathrm{BCa}$ & .925 & .926 & .935 & .938 & .932 & .946 & .946 & .934 & .927 & .938 & .945 & .944 \\
\hline
\end{tabular}

Notes: For IRC $=2^{\mathrm{a}}$, skewness $=0$ and kurtosis $=-2$; for IRC $=2^{\mathrm{b}}$ skewness $=0.41$ and kurtosis $=-1.83$. Bold numbers indicate unacceptable coverage outside [0.925, 0.975]. NTB = normal theory bootstrap; PB $=$ percentile bootstrap; $\mathrm{BCa}=$ biased-corrected and accelerated bootstrap. All methods based on 2,000 bootstrap samples. 


\section{BOOTSTRAP INTERVAL ESTIMATION OF RELIABILITY}

Table 2: 95\% Coverage Probabilities for 10 Items

\begin{tabular}{|c|c|c|c|c|c|c|c|c|c|c|c|c|c|}
\hline \multirow[b]{2}{*}{ IRC } & \multirow[b]{2}{*}{$\mathrm{n}$} & \multicolumn{4}{|c|}{$\rho=0.30$} & \multicolumn{4}{|c|}{$\rho=0.56$} & \multicolumn{4}{|c|}{$\rho=$ Unstructured } \\
\hline & & 50 & 100 & 150 & 200 & 50 & 100 & 150 & 200 & 50 & 100 & 150 & 200 \\
\hline \multirow{3}{*}{$2^{a}$} & NTB & .970 & .961 & .957 & .957 & .958 & .957 & .940 & .957 & .974 & .968 & .967 & .949 \\
\hline & PB & .954 & .955 & .946 & .950 & .945 & .949 & .936 & .961 & .954 & .964 & .958 & .945 \\
\hline & $\mathrm{BCa}$ & .964 & .956 & .951 & .955 & .950 & .957 & .939 & .960 & .963 & .971 & .964 & .944 \\
\hline \multirow{3}{*}{$2^{b}$} & NTB & .970 & .963 & .951 & .970 & .979 & .946 & .945 & .941 & .968 & .961 & .952 & .956 \\
\hline & PB & .953 & .952 & .944 & .963 & .970 & .947 & .942 & .941 & .957 & .948 & .955 & .944 \\
\hline & $\mathrm{BCa}$ & .955 & .954 & .944 & .964 & .975 & .952 & .944 & .944 & .957 & .964 & .962 & .947 \\
\hline \multirow{3}{*}{3} & NTB & .965 & .955 & .944 & .950 & .951 & .957 & .951 & .954 & .950 & .964 & .950 & .957 \\
\hline & PB & .926 & .937 & .951 & .938 & .927 & .944 & .944 & .948 & .926 & .943 & .943 & .951 \\
\hline & $\mathrm{BCa}$ & .942 & .942 & .949 & .944 & .938 & .955 & .946 & .954 & .930 & .952 & .947 & .950 \\
\hline \multirow{3}{*}{5} & NTB & .961 & .957 & .946 & .947 & .960 & .950 & .949 & .960 & .966 & .955 & .950 & .946 \\
\hline & PB & .941 & .945 & .944 & .941 & .944 & .943 & .942 & .950 & .936 & .949 & .947 & .947 \\
\hline & $\mathrm{BCa}$ & .952 & .953 & .941 & .944 & .948 & .941 & .946 & .953 & .941 & .945 & .948 & .945 \\
\hline \multirow{3}{*}{7} & NTB & .961 & .956 & .947 & .943 & .946 & .942 & .943 & .952 & .964 & .951 & .938 & .945 \\
\hline & PB & .931 & .951 & .944 & .938 & .923 & .928 & .943 & .945 & .9630 & .936 & .933 & .942 \\
\hline & $\mathrm{BCa}$ & .935 & .950 & .946 & .937 & .928 & .933 & .939 & .947 & .939 & .941 & .934 & .942 \\
\hline
\end{tabular}

Notes: For IRC $=2^{\mathrm{a}}$, skewness $=0$ and kurtosis $=-2$; for IRC $=2^{\mathrm{b}}$ skewness $=0.41$ and kurtosis $=-1.83$. Bold numbers indicate unacceptable coverage outside [0.925, 0.975]. NTB = normal theory bootstrap; PB $=$ percentile bootstrap; $\mathrm{BCa}=$ biased-corrected and accelerated bootstrap. All methods based on 2,000 bootstrap samples. 
PADILLA \& DIVERS

Table 3: 95\% Coverage Probabilities for 15 Items

\begin{tabular}{|c|c|c|c|c|c|c|c|c|c|c|c|c|c|}
\hline \multirow[b]{2}{*}{ IRC } & \multirow[b]{2}{*}{$\mathrm{n}$} & \multicolumn{4}{|c|}{$\rho=0.30$} & \multicolumn{4}{|c|}{$\rho=0.56$} & \multicolumn{4}{|c|}{$\rho=$ Unstructured } \\
\hline & & 50 & 100 & 150 & 200 & 50 & 100 & 150 & 200 & 50 & 100 & 150 & 200 \\
\hline \multirow{3}{*}{$2^{\mathrm{a}}$} & NTB & .968 & .973 & .954 & .949 & .964 & .955 & .937 & .943 & .979 & .966 & .952 & .952 \\
\hline & PB & .956 & .963 & .956 & .952 & .950 & .950 & .938 & .944 & .968 & .950 & .945 & .951 \\
\hline & $\mathrm{BCa}$ & .966 & .966 & .962 & .956 & .959 & .949 & .942 & .948 . & .980 & .955 & .950 & .953 \\
\hline \multirow{3}{*}{$2^{\mathrm{b}}$} & NTB & .971 & .965 & .943 & .955 & .954 & .949 & .959 & .944 & .972 & .961 & .953 & .943 \\
\hline & PB & .949 & .944 & .940 & .956 & .945 & .944 & .958 & .941 & .946 & .951 & .941 & .944 \\
\hline & $\mathrm{BCa}$ & .965 & .957 & .947 & .958 & .947 & .948 & .959 & .945 & .961 & .962 & .943 & .951 \\
\hline \multirow{3}{*}{3} & NTB & .970 & .953 & .949 & .953 & .951 & .955 & .952 & .954 & .976 & .957 & .949 & .953 \\
\hline & PB & .934 & .938 & .945 & .945 & .921 & .940 & .944 & .950 & .922 & .937 & .947 & .946 \\
\hline & $\mathrm{BCa}$ & .952 & .946 & .949 & .949 & .933 & .945 & .954 & .952 & .954 & .939 & .945 & .951 \\
\hline \multirow{3}{*}{5} & NTB & .956 & .947 & .930 & .950 & .956 & .950 & .949 & .941 & .965 & .948 & .951 & .946 \\
\hline & PB & .928 & .941 & .926 & .952 & .934 & .943 & .947 & .947 & .939 & .950 & .950 & .945 \\
\hline & $\mathrm{BCa}$ & .934 & .945 & .919 & .952 & .941 & .950 & .952 & .937 & .949 & .944 & .950 & .947 \\
\hline \multirow{3}{*}{7} & NTB & .974 & .945 & .955 & .947 & .939 & .950 & .946 & .940 & .960 & .950 & .958 & .944 \\
\hline & PB & .937 & .930 & .939 & .941 & .929 & .947 & .947 & .931 & .928 & .943 & .950 & .945 \\
\hline & $\mathrm{BCa}$ & .943 & .931 & .942 & .938 & .929 & .951 & .947 & .933 & .932 & .943 & .952 & .945 \\
\hline
\end{tabular}

Notes: For IRC $=2^{\mathrm{a}}$, skewness $=0$ and kurtosis $=-2$; for IRC $=2^{\mathrm{b}}$ skewness $=0.41$ and kurtosis $=-1.83$. Bold numbers indicate unacceptable coverage outside [0.925, 0.975]. NTB = normal theory bootstrap; PB $=$ percentile bootstrap; $\mathrm{BCa}=$ biased-corrected and accelerated bootstrap. All methods based on 2,000 bootstrap samples. 
BOOTSTRAP INTERVAL ESTIMATION OF RELIABILITY

Table 4: 95\% Coverage Probabilities for 20 Items

\begin{tabular}{|c|c|c|c|c|c|c|c|c|c|c|c|c|c|}
\hline \multirow[b]{2}{*}{ IRC } & \multirow[b]{2}{*}{$\mathrm{n}$} & \multicolumn{4}{|c|}{$\rho=0.30$} & \multicolumn{4}{|c|}{$\rho=0.56$} & \multicolumn{4}{|c|}{$\rho=$ Unstructured } \\
\hline & & 50 & 100 & 150 & 200 & 50 & 100 & 150 & 200 & 50 & 100 & 150 & 200 \\
\hline \multirow{3}{*}{$2^{\mathrm{a}}$} & NTB & .971 & .961 & .943 & .955 & .965 & .944 & .935 & .956 & .976 & .964 & .948 & .947 \\
\hline & PB & .953 & .955 & .940 & .957 & .955 & .942 & .941 & .963 & .940 & .956 & .945 & .950 \\
\hline & $\mathrm{BCa}$ & .971 & .960 & .943 & .960 & .960 & .949 & .942 & .961 & .966 & .966 & .952 & .953 \\
\hline \multirow{3}{*}{$2^{b}$} & NTB & .972 & .969 & .963 & .965 & .945 & .947 & .947 & .951 & .979 & .958 & .958 & .958 \\
\hline & PB & .947 & .964 & .961 & .965 & .941 & .949 & .945 & .944 & .956 & .943 & .947 & .955 \\
\hline & $\mathrm{BCa}$ & .968 & .972 & .966 & .960 & .943 & .953 & .949 & .944 & .965 & .948 & .951 & .958 \\
\hline \multirow{3}{*}{3} & NTB & .971 & .947 & .956 & .958 & .948 & .949 & .958 & .951 & .973 & .947 & .947 & .945 \\
\hline & PB & .913 & .941 & .951 & .958 & .927 & .936 & .953 & .960 & .947 & .936 & .943 & .938 \\
\hline & $\mathrm{BCa}$ & .940 & .942 & .955 & .957 & .935 & .939 & .958 & .956 & .963 & .935 & .946 & .942 \\
\hline \multirow{3}{*}{5} & NTB & .965 & .936 & .957 & .949 & .956 & .948 & .943 & .949 & .968 & .959 & .953 & .945 \\
\hline & PB & .939 & .922 & .948 & .949 & .924 & .934 & .936 & .946 & .923 & .952 & .948 & .946 \\
\hline & $\mathrm{BCa}$ & .940 & .934 & .947 & .946 & .933 & .939 & .941 & .948 & .932 & .950 & .950 & .942 \\
\hline \multirow{3}{*}{7} & NTB & .961 & .939 & .944 & .944 & .953 & .943 & .956 & .938 & .966 & .940 & .939 & .957 \\
\hline & PB & .929 & .934 & .940 & .947 & .928 & .932 & .949 & .936 & .921 & .929 & .939 & .952 \\
\hline & $\mathrm{BCa}$ & .934 & .934 & .938 & .947 & .937 & .939 & .953 & .944 & .929 & .931 & .935 & .952 \\
\hline
\end{tabular}

Notes: For IRC $=2^{\mathrm{a}}$, skewness $=0$ and kurtosis $=-2$; for $\mathrm{IRC}=2^{\mathrm{b}}$ skewness $=0.41$ and kurtosis $=-1.83$. Bold numbers indicate unacceptable coverage outside [0.925, 0.975]. NTB = normal theory bootstrap; PB $=$ percentile bootstrap; $\mathrm{BCa}=$ biased-corrected and accelerated bootstrap. All methods based on 2,000 bootstrap samples. 


\section{PADILLA \& DIVERS}

Figure 1: Distribution of 95\% Bootstrap CI coverage for Estimation Method by Number of Items

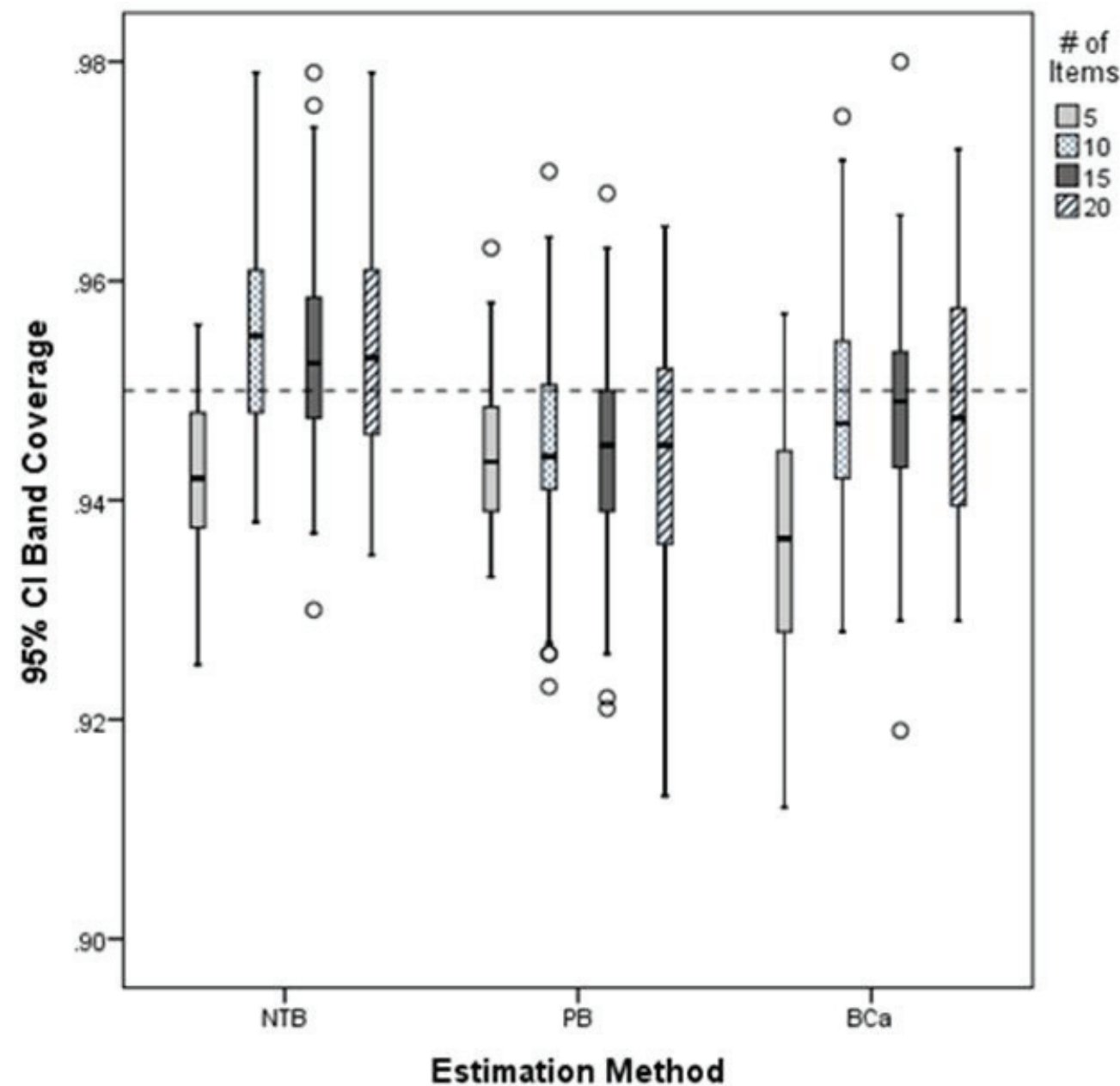

Notes: $\mathrm{CI}=$ confidence interval; $\mathrm{NTB}=$ normal theory bootstrap; $\mathrm{PB}=$ percentile bootstrap; $\mathrm{BCa}=$ biased-corrected and accelerated bootstrap; dashed line is at 0.95 .

\section{Conclusion}

Coefficient omega bootstrap CIs were proposed and their performance was investigated under several simulation conditions. Coefficient omega is a reliability index for a composite of congeneric items measuring a common dimension (i.e., factor or latent variable). To date, no study has investigated the performance of composite reliability CIs for congeneric items measuring a common dimension in such a simulation design. Results indicate that the NTB CI had the best coverage across all of the simulation conditions investigated. Even so, the major impact on the coefficient omega bootstrap CIs were the number of items.

Although the number of items impacted all three bootstrap CIs, it was most noticeable for the $\mathrm{BCa}$ method. In general, all methods were impacted when the number of items was set to five in that they tended to have coverage probability below $95 \%$ on average. However, only the BCa method had unacceptable coverage with five items. In fact, it had unacceptable coverage in eight instances, five of which had the unstructured covariance matrix. This suggests that unstructured covariance matrices 


\section{BOOTSTRAP INTERVAL ESTIMATION OF RELIABILITY}

for five items do not provide enough information for the $\mathrm{BCa}$ method to make the proper adjustments.

When there are ten or more items, with the exception of the $\mathrm{BCa}$ method, the results were somewhat sporadic. In this case, the $\mathrm{BCa}$ method had acceptable coverage under all but two simulation conditions. The NTB method tended to have unacceptable coverage with fifteen or more items, a sample size of 50, and an unstructured item covariance matrix. Conversely, the PB method tended to have unacceptable coverage with a sample size of 50 . For the NTB and PB method, most of the unacceptable coverage was so close to the $[.925, .975]$ boundaries that failure to fall within the interval was likely due to sampling variability.

Within the contexts of the simulation conditions investigated there is a clear order of preference of the bootstrap CIs investigated. The NTB method had the best performance in that it had consistent acceptable coverage under all but five simulation conditions $(235 / 240=0.979)$. This was followed by the PB and BCa methods, whose performances were comparable $(232 / 240$ $=0.967$ for $\mathrm{PB}$ vs. $230 / 240=0.958$ for $\mathrm{BCa}$ ). Another noticeable feature was that the NTB method tended to be the most liberal and the PB method most conservative. Nevertheless, a recommendation can be made. When there are 10 or less items the NTB method performed well, however, when there are 15 or more items, the BCa method was superior. In light of these findings, it is important to emphasize that all three methods had an acceptable range of coverage within the context of the investigated simulation conditions.

Despite these promising results, more research is needed. These results were obtained assuming that the Likert/ordinal items were normally distributed or that the underlying distribution did not depart greatly from normality. However, it is unlikely that data will follow a normal distribution in applied settings. Therefore, future research should focus on the CI estimation of coefficient omega using data that deviate from normality.
Through the simulation results provided and because coefficient omega is a general index of reliability, five advantages can be pointed out about its corresponding bootstrap CIs. First, the investigated items were not continuous and this had no significant impact on the CIs (recall that all items investigated were binary or Likert/ordinal with response categories that ranged from 2 to 7). Second, a sample size of 50 to 200 did not have a major impact. This is a significant finding because the factor loadings that are used by coefficient omega are estimated through ML which is based on the law of large numbers. Therefore, the literature has noted that this is a condition in need of investigation (Raykov, 1998, 2002; Raykov \& Shrout, 2002). Only the PB method appeared to be somewhat affected by a small sample size. Third, the type of correlation structure did not have a major impact, thus, coefficient omega appears to be appropriate for items that range from parallel to congeneric. Fourth, though not investigated in this study, coefficient omega can be used with measures that have multiple factors or latent variables (McDonald, 1970, 1999). Lastly, the methods investigated do not require specialized SEM software; they only require the freely available and general $\mathrm{R}$ statistical package (http://www.r-project.org/). As such, interested researchers can obtain an easy-to-use R function for the coefficient omega bootstrap CIs with example data free of charge by visiting the corresponding author's website (http://www.omegalab-padilla.org/).

\section{References}

Allen, M. J., \& Yen, W. M. (1979). Introduction to measurement theory. Monterey, CA: Brooks/Cole Pub. Co.

Bollen, K. A. (1989). Structural equations with latent variables. New York, NY: Wiley.

Bradley, J. V. (1978). Robustness? British Journal of Mathematical and Statistical Psychology, 31(2), 144-152.

Crocker, L. M., \& Algina, J. (1986). Introduction to classical and modern test theory. New York, NY: Holt, Rinehart, and Winston.

Cronbach, L. (1951). Coefficient alpha and the internal structure of tests. Psychometrika, 16(3), 297-334. 


\section{PADILLA \& DIVERS}

Duhachek, A., \& Iacobucci, D. (2004). Alpha's standard error (ASE): An accurate and precise confidence interval estimate. Journal of Applied Psychology, 89(5), 792-808.

Efron, B., \& Tibshirani, R. (1998). An introduction to the bootstrap. Boca Raton, FL: CRC Press.

Enders, C. K. (2003). Using the expectation maximization algorithm to estimate coefficient alpha for scales with item-level missing data. Psychological Methods, 8(3), 322337.

Graham, J. M. (2006). Congeneric and (essentially) tau-equivalent estimates of score reliability: What they are and how to use them. Educational and Psychological Measurement, 66(6), 930-944.

Guttman, L. (1945). A basis for analyzing test-retest reliability. Psychometrika, $10(4), 255-282$.

Hogan, T. P., Benjamin, A., \& Brezinski, K. L. (2000). Reliability methods: A note on the frequency of use of various types. Educational and Psychological Measurement, 60(4), 523-531.

Lord, F., Novick, M., \& Birnbaum, A. (1968). Statistical theories of mental test scores. Reading, MA: Addison-Wesley.

Maydeu-Olivares, A., Coffman, D. L., \& Hartmann, W. M. (2007). Asymptotically distribution-free (ADF) interval estimation of coefficient alpha. Psychological Methods, 12(2), 157-176.

McDonald, R. P. (1970). The theoretical foundations of principal factor analysis, canonical factor analysis, and alpha factor analysis. British Journal of Mathematical and Statistical Psychology, 23(1), 1-21.

McDonald, R. P. (1999). Test theory: A unified treatment. Mahwah, NJ: L. Erlbaum Associates.

Padilla, M. A., Divers, J., \& Newton, M. (2012). Coefficient Alpha bootstrap confidence interval under nonnormality. Applied Psychological Measurement, 36(5), 331-348.

Peterson, R. A. (1994). A Meta-Analysis of Cronbach's Coefficient Alpha. Journal of Consumer Research, 21(2), 381-391.
Raykov, T. (1997). Estimation of composite reliability for congeneric measures. Applied Psychological Measurement, 21(2), 173-184.

Raykov, T. (1998). A method for obtaining standard errors and confidence intervals of composite reliability for congeneric items. Applied Psychological Measurement, 22(4), 369-374.

Raykov, T. (2002). Analytic estimation of standard error and confidence interval for scale reliability. Multivariate Behavioral Research, 37(1), 89-103.

Raykov, T. (2012). Scale construction and development using structural equation modeling. In Handbook of structural equation modeling, R. H. Hoyle (Ed.), 472-492. New York, NY: Guilford Press.

Raykov, T., \& Marcoulides, G. A. (2011). Introduction to psychometric theory. New York, NY: Routledge.

Raykov, T., \& Shrout, P. E. (2002). Reliability of scales with general structure: Point and interval estimation using a structural equation modeling approach. Structural Equation Modeling, 9(2), 195-212.

Romano, J. L., Kromrey, J. D., \& Hibbard, S. T. (2010). A Monte Carlo study of eight confidence interval methods for coefficient alpha. Educational and Psychological Measurement, 70(3), 376-393.

van Zyl, J., Neudecker, H., \& Nel, D. (2000). On the distribution of the maximum likelihood estimator of Cronbach's alpha. Psychometrika, 65(3), 271-280.

Yuan, K-H., Guarnaccia, C. A., \& Hayslip, B., Jr. (2003). A study of the distribution of sample coefficient alpha with the Hopkins Symptom Checklist: Bootstrap versus asymptotics. Educational and Psychological Measurement, 63(1), 5-23.

Zinbarg, R. E., Revelle, W., Yovel, I., \& Li, W. (2005). Cronbach's $\alpha$, Revelle's $\beta$ and Mcdonald's $\omega_{H}$ : Their relations with each other and two alternative conceptualizations of reliability. Psychometrika, 70(1), 123-133. 


\title{
The $X$-Alter Algorithm: A Parameter-Free Method of Unsupervised Clustering
}

\author{
Thomas Laloë \\ Rémi Servien \\ Université of Nice Sophia-Antipolis, \\ Nice, France \\ UMR Toxalim, INRA, \\ Toulouse, France
}

Using quantization techniques, Laloë (2010) defined a new clustering algorithm called Alter. This $L^{1}$ based algorithm is shown to be convergent but suffers two major flaws. The number of clusters, $K$, must be supplied by the user and the computational cost is high. This article adapts the $X$-means algorithm (Pelleg \& Moore, 2000) to solve both problems.

Key words: Clustering, quantization, K-means, free-parameter algorithm.

Introduction

Clustering consists in partitioning a data set into subsets (or clusters) so that the data in each subset share some common trait; proximity is determined according to a distance measure (for a thorough introduction to this subject please see Kaufman \& Rousseeuw, 1990). The origin of clustering goes back over 45 years when some biologists and sociologists began to search for automatic methods to build different data groups. Today, clustering is used in many fields, for example, in medical imaging it can be used to differentiate between types of tissue and blood in a three dimensional image. Market researchers use clustering to partition the general population of consumers into market segments and to better understand the relationships between different groups of consumers/potential customers. There are also many applications in artificial intelligence, sociology, medical research and political science.

Thomas Laloë is Assistant Professor at the University of Nice Sophia-Antipolis. Email him at: laloe@unice.fr. Rémi Servien is Permanent Researcher for the National Institute for Agronomic Research (INRA). Email him at: remi.servien@toulouse.inra.fr.
$K$-means clustering is the most popular method (Hartigan \& Wong, 1979; MacQueen, 1967); its attractiveness lies in its symplicity and its fast execution. It has however two main drawbacks. First, the number of clusters $K$ must be supplied by the user; for this reason, different ways to determine $K$ have been studied in the literature ( $\mathrm{Li}$, et al., 2008; Pham, et al., 2005). Second, the algorithm strongly depends on initialization and can easily converge to a local minimum. Pelleg and Moore (2000) offered a solution for the first problem with a buildingblock algorithm called $X$-means that quickly estimates $K$. After each run of 2-means, local decisions are made regarding whether subsets of the current centroid should be split; the splitting decision is accomplished by computing the Bayesian Information Criterion (BIC). In a different approach, Laloë (2010) proposed a consistent algorithm, called Alter, which also requires specification of $K$.

This article combines the $X$-means and the Alter algorithm to overcome the drawbacks of both algorithms. The complexity of the Alter algorithm decreases and an automatic selection of the number of clusters is simultaneously performed. In addition, the convergence properties of the Alter algorithm overcomes the local optimality problem of the $X$-means algorithm inherited from the $K$-means algorithm. 


\section{THOMAS LALOË \& REMI SERVIEN}

The Alter Algorithm

\section{Methodology}

The Alter algorithm method is based on quantization. It is a commonly used technique in signal compression (Graf \& Luschgy, 2000; Linder, 2002). All theoretical results presented herein are from Laloë (2010). Consider (HH, $||$.$) a$ normed space. Let $X$ be a $H /$-valued random variable with distribution $\mu$ such as $E|X|<\infty$. Given a set $C$ of points in $H^{k}$, any Borel function $q: H \leftrightarrow C$ is called a quantizer. The set $C$ is called a codebook, and the error made by replacing $X$ by $q(X)$ is measured by the distortion:

$$
D(\mu, q)=E d(X, q(X))=\int_{H}|x-q(x)| \mu(d x) .
$$

Note that $D(\mu, q)<\infty$ because $E|X|<\infty$. For a given $k$, the aim is to minimize $D(\mu,$.$) among the set$ $Q_{k}$ of all possible $k$-quantizers. The optimal distortion is then defined by

$$
D_{k}^{*}(\mu)=\inf _{q \in Q_{k}} D(\mu, q) \text {. }
$$

When it exists, a quantizer $q^{*}$ satisfying $D\left(\mu, q^{*}\right)=D_{k}^{*}(\mu)$ is said to be an optimal quantizer. Laloë (2010) showed that only nearest neighbor quantizers can be considered, that is, a quantizer $q$ will be characterized by its codebook $C=\left\{y_{i}\right\}_{i=1}^{k}$ and the rule:

$$
q(x)=y_{i} \Leftrightarrow \forall 1 \leq j \leq k, j \neq i,\left\|x-y_{i}\right\| \leq\left\|x-y_{j}\right\| .
$$

Thus, a quantizer can be defined by its codebook only. Moreover the aim is to minimize the distortion among all possible nearest neighbor quantizers. However, in practice, the distribution $\mu$ of the observations is unknown, and only $n$ independent observations $X_{1}, \ldots, X_{n}$ with the same distribution than $X$ are available. The goal is then to minimize the empirical distortion:

$$
\frac{1}{n} \sum_{i=1}^{n} d\left(X_{i}, q\left(X_{i}\right)\right)
$$

The $L^{1}$-based distortion is chosen to obtain more robust estimators (Kemperman, 1987). The clustering is accomplished by regrouping the observations that have the same image by $q$; more precisely, a cluster $C$ is defined by $C=\left\{X_{i}: q\left(X_{i}\right)=\hat{x}_{C}\right\}, \hat{x}_{C}$ being representative of cluster $C$.

Laloë (2010) presented theoretical results of consistency and rate of convergence. In particular, he stated that the rate of convergence is closely related to the metric entropy, however, the minimization of the empirical distortion is not possible in practice and Laloë (2010) proposed an alternative to perform the Alter algorithm. The idea is to select an optimal codebook among the data set. The outline of the algorithm is:

1. List all possible codebooks, i.e., all possible $K$-tuples of data;

2. Compute the empirical distortion associated to the first codebook. Each observation $X_{i}$ is associated with its closed center;

3. For each successive codebook, compute the associated empirical distortion. Each time a codebook has an associated empirical distortion smaller than the previous smallest one, store the codebook;

4. Return the codebook that has the smallest distortion.

Theoretical results of consistency and rate of convergence have been shown for the Alter algorithm. In particular it has been stated that the convergence rate is of the same order as the theoretical method described previously. Moreover, this algorithm does not depend on initial conditions (unlike $K$-means) and it converges to optimal distortion; unfortunately its complexity is $O\left(n^{K+1}\right)$ and it is not possible to use it for high values of $n$ or $K$.

The X-Means Algorithm

Pelleg and Moore (2000) define the $X$ means algorithm adapted from a $K$-means algorithm. The $X$-means algorithm goes into action after each run of $K$-means, making local decisions about which subset of the current centers should split themselves in order to better 


\section{X-ALTER ALGORITHM: A PARAMETER-FREE METHOD OF CLUSTERING}

fit the data. The splitting decision is done by computing the BIC criterion. This new approach proposes an efficient solution to one of the major drawbacks of $K$-means: the search for the number of clusters $K$. In addition, $X$-means has a low computational cost. However, results suffer from the non-convergence property of the $K$ means algorithm. The outline of this algorithm is:

1. Perform 2-means to obtain clustering $C$;

2. Evaluate the relevance of the classification $C$ with a BIC Criterion; and

3. Iterate step one and two in each cell of $C$. Continue until there is no more relevant discrimination.

The $X$-Alter Algorithm

Following $X$-means, a recursive use of Alter with $K=2$ can simultaneously allow both advantages of these two methods to be combined: estimation of $K /$ low computational cost for $X$-means and convergence/parameterfree character for Alter. An aggregation step is added at the end of the algorithm to prevent the creation of too many clusters. Note that no parameter is needed by the algorithm, although a user can specify a range in which the true $K$ reasonably lies if desired (this would be $[2,+\infty[$ if no information was available). The outline of the algorithm is:

1. Perform Alter with $K=2$ to obtain clustering $C$;

2. Evaluate the relevance of the classification $C$ (see Figure 1) with a BIC criterion;

3. Iterate steps one and two in each cell of $C$ (see Figure 2); continue until there is no more relevant discrimination (see Figure 3);

4. Final aggregation; aggregation can be considered if $B I C(K=1)>B I C(K=2)$; aggregations are successively made according to decreasing values of $B I C(K=1)-B I C(K=2)$ (see Figure 4).

The algorithm starts by performing Alter with $K=2$ centers. A model selection criterion
(BIC) is performed on all data in the set. Using this criterion, the suitability of the discrimination is checked by comparing $B I C(K=1)$ and $B I C(K=2)$. The criterion asks if the two cluster model is better than the one cluster model. If the answer is yes, the iterative procedure occurs in the two subsets.

The structure improvement operation begins by splitting each cluster into two subsets. The procedure is local in that the children are fighting each other for the points in the parent's region, no others; when the discrimination is not validated by BIC criterion the algorithm ends in this region. Up to that point, the only difference with $X$-means is the utilization of Alter as opposed to 2-means because the consistent property of Alter must improve results. When all regions are asleep and no more clusters are needed, the aggregative step prevents the creation of too many clusters or the presence of split clusters (see Figure 2).

The complexity of this algorithm in the worst case scenario, that is when it creates $n$ clusters with one data set, is $O\left(n^{4}\right)$ which is less than the initial Alter algorithm. However, the computational cost is still higher than for $X$ means. For several thousand points, this complexity is not a critical practical concern but, if the database exceeds several tens of thousands of points, it could still be too high.

The BIC Criterion

Pelleg and Moore (2000) used the formula from Kass and Wasserman (1995) that evaluates the relevance of the classification $C$ with

$$
B I C(C)=l-\frac{p}{2} \log n
$$

where $l$ is the log-likelihood of the data according to clustering $C$ and taken at the maximum likelihood point and $p$ is the number of parameters in $C$. The number of free parameters $p$ is the sum of $K-1$ class probabilities, $d^{*} K$ centroids coordinates and one variance estimate. Data in each cluster are supposed to be normally distributed around the center. The empirical study shows that it performs well on real data. 
THOMAS LALOË \& REMI SERVIEN

Figure 1: First Iteration of $X$-Alter

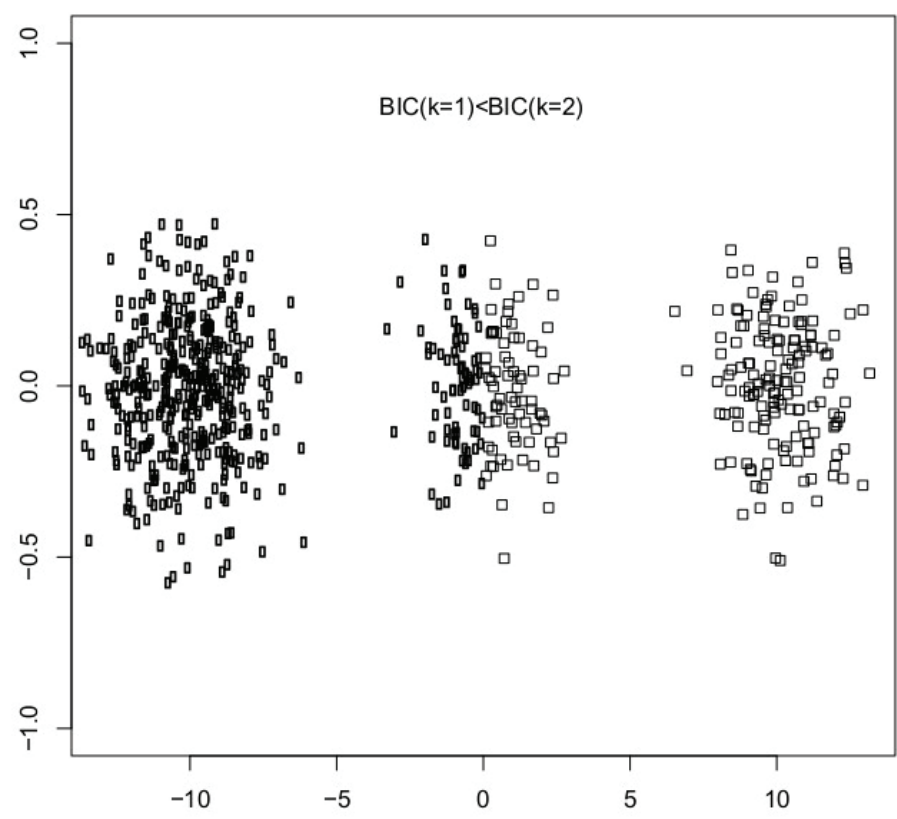

Notes: The discrimination in 2 clusters (Step 1) was validated by the BIC criterion (Step 2); in each cluster, observations are represented by a different symbol.

Figure 2: Second Iteration of $X$-Alter

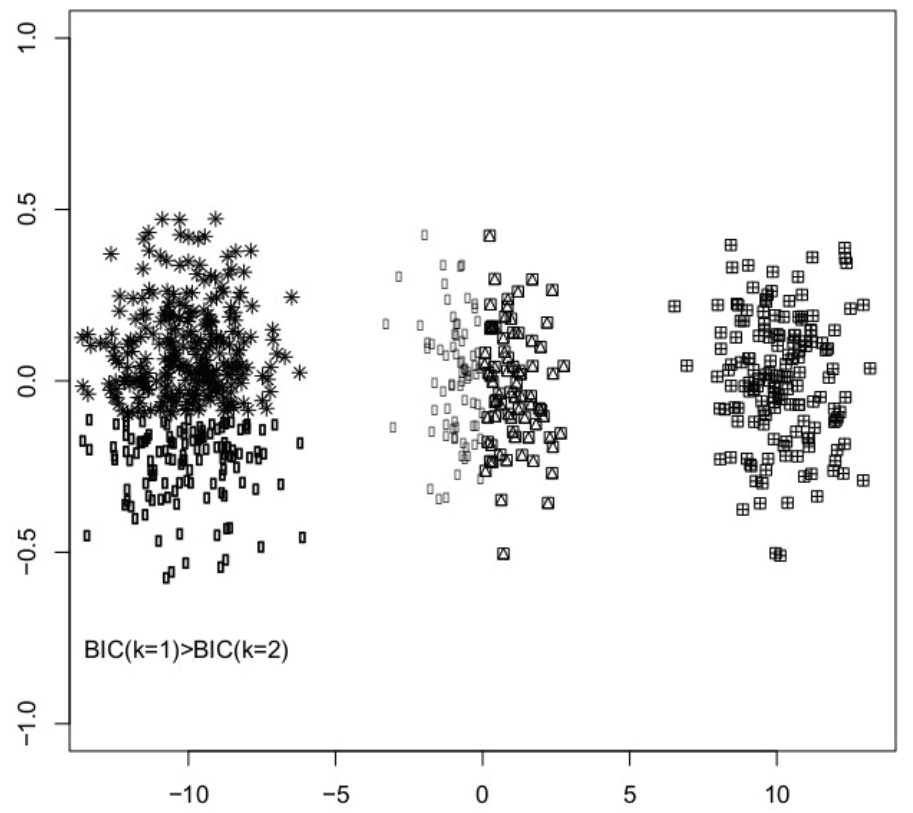

Notes: The sub-classification is done in the two relevant clusters (Step 1). Sub-classifications are validated by BIC (Step 2) and four clusters are obtained. 
Figure 3: No Relevant Sub-Classification in the Left Cluster According to BIC

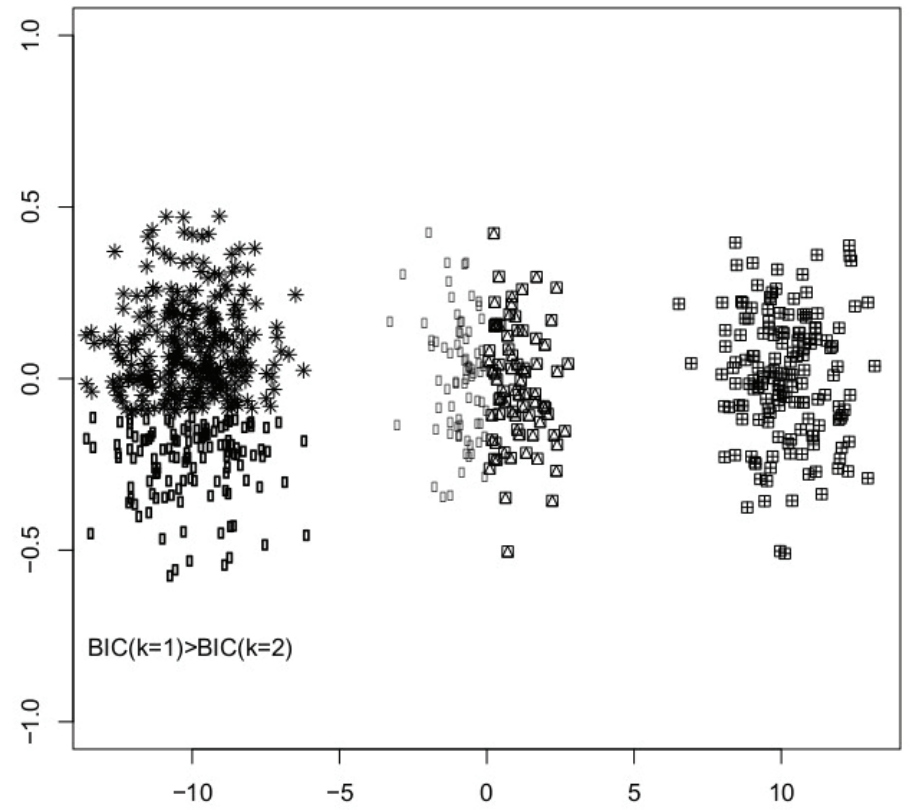

Note: In the three other clusters, the same rejection of sub-classification was obtained (Step 3).

Figure 4: Final Discrimination

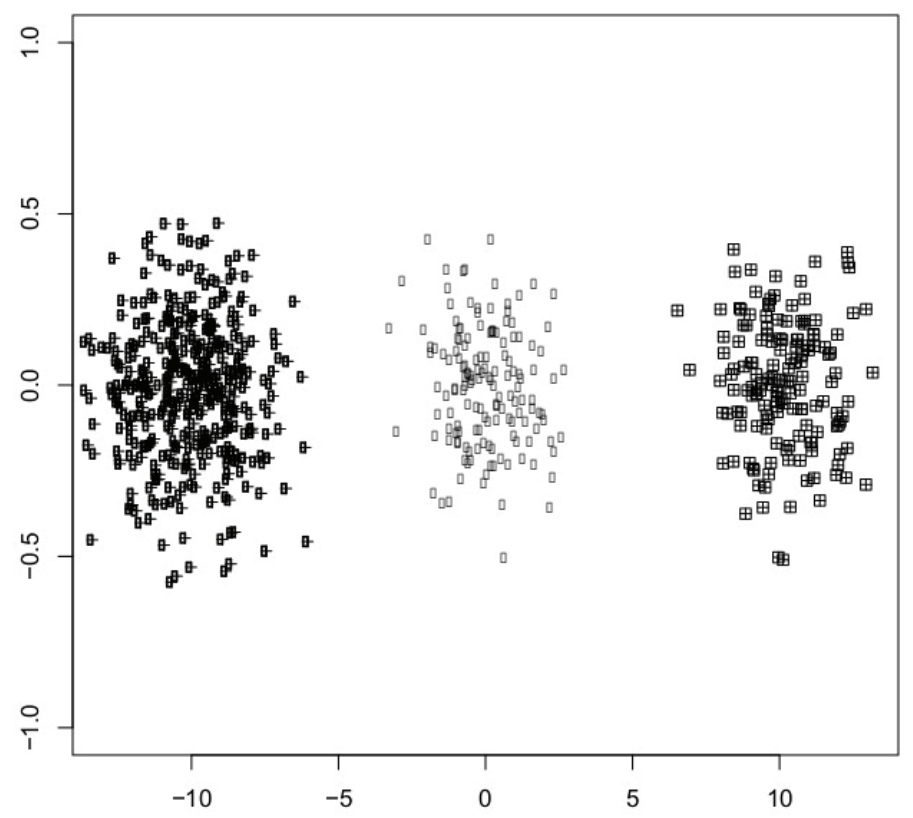

Note: The two middle clusters were aggregated in Step 4 


\section{THOMAS LALOË \& REMI SERVIEN}

\section{Results}

An empirical study was performed to show the relevance of the proposed method. Three criterion were considered: the number of detected clusters, the Adjusted Rand Index (A.R.I.) (Rand, 1971; Hubert \& Arabie, 1985) and the Dunn index (Dunn, 1974; Handl, et al., 2005). The Rand Index is a measure of the similarity between two clusters. A problem with the Rand index is that the expected value of the Rand index of two random partitions does not take a constant value (for example, zero). Thus, Hubert and Arabie (1985) defined the A.R.I. which is a corrected-for-chance version of the Rand index. Studies have shown the need and usefulness of the adjusted measures (Nguyen, et al., 2009); more clusters are similar (respectively dissimilar) closer to 1 (respectively 0 ).

Alternatively, the Dunn Index measures the compactness of the clusters and is a worst case indicator. The goal is to identify sets of clusters that are compact, with a small variance between individuals in the same cluster, and well separated, where the centers of different clusters are sufficiently far apart, as compared to the within cluster variance. The higher the Dunn Index, the better the clustering. For more details on this classical cluster validation indexes the interested reader is referred to Dunn (1974) or Handl, et al. (2005).

Pelleg and Moore showed that $X$-means performs better and faster than repeatedly using accelerated $K$-means for different values of $K$. Thus, the $X$-Alter algorithm is compared to $X$ means and to $X$-means with the aggregation step, called $X$-means- $\mathrm{R}$, that is, a clustering is obtained using $X$-means and then the aggregation procedure is computed (Step 4 in the $X$-Alter algorithm ) on this clustering. This allows the usefulness and the computational time of the aggregation step to be assessed.

Simulated data

\section{A Simple Case}

Clusters of Gaussian vectors were simulated in $\mathbb{R}^{d}$. First, two clusters well identified in $\mathbb{R}^{20}$ were considered (see Table 1 ). More precisely, two clusters of 25 vectors (in $\mathbb{R}^{20}$ ) with $\mu_{1}=-\mu_{2}=15$ and $\sigma_{1}^{2}=\sigma_{2}^{2}=100$ were simulated. The covariance matrices are given by
$\Sigma^{2}=100 I_{20}$ where $I_{20}$ is the identity $20 * 20$

matrix and the mean vectors are:

$$
M_{1}=-M_{2}=15\left(\begin{array}{l}
1 \\
\vdots \\
1
\end{array}\right) \text {. }
$$

This results in $X_{1}, \ldots, X_{25} \sim N\left(M_{1}, \Sigma\right)$ and $X_{25}, \ldots, X_{50} \sim N\left(M_{2}, \Sigma\right)$. The results are averaged on 300 simulations.

Table 1: Results of the Three Algorithms for the Two Well-Defined Clusters

\begin{tabular}{|c|c|c|c|}
\hline Algorithm & $\begin{array}{c}\text { \% of Correct } \\
\text { Number of } \\
\text { Clusters }\end{array}$ & A.R.I. & $\begin{array}{c}\text { Dunn } \\
\text { Index }\end{array}$ \\
\hline$X$-means & 99 & 1 & 1.62 \\
\hline$X$-means-R & 100 & 1 & 1.64 \\
\hline$X$-Alter & 100 & 1 & 1.64 \\
\hline
\end{tabular}

As expected, the three methods perform well on this very simple case. Next three simulated clusters well identified in $\mathbb{R}^{5}$ were considered. This allows the relevance of the aggregation step to be observed because $X$ means will often cut the middle cluster in its first iteration. Two clusters of 20 vectors (in $\mathbb{R}^{5}$ ) with $\mu_{1}=-\mu_{2}=20$ and $\sigma_{1}^{2}=\sigma_{2}^{2}=100$ were simulated and one cluster of 20 vectors with $\mu_{3}=0$ and $\sigma_{3}^{2}=100$. The results were averaged on 300 simulations (see Table 2).

The influence of the aggregation step can be remarked upon; $X$-means-R found the good number of clusters almost forty percent more often than $X$-means. Moreover, the proposed $X$-means algorithm obtained better results than the other two: the inherited convergence property of Alter clearly improves results. 


\section{X-ALTER ALGORITHM: A PARAMETER-FREE METHOD OF CLUSTERING}

Table 2: Results for the Three Algorithms on the Three Clusters

\begin{tabular}{|c|c|c|c|}
\hline Algorithm & $\begin{array}{c}\text { \% of Correct } \\
\text { Number of } \\
\text { Clusters }\end{array}$ & A.R.I. & $\begin{array}{c}\text { Dunn } \\
\text { Index }\end{array}$ \\
\hline$X$-means & 55 & 0.82 & 0.22 \\
\hline$X$-means-R & 76 & 0.82 & 0.22 \\
\hline$X$-Alter & 86 & 0.84 & 0.22 \\
\hline
\end{tabular}

Finally, tests with random values for the numbers of clusters were performed, the mean, standard deviation and number of data in cluster. The $\mu_{i}$ are randomly selected between -50 and 50 , the $\sigma_{i}$ between 5 and 15 , the number of clusters between 2 and 10 and the number of vectors in each cluster between 8 and 25 . The dimension of the data is fixed to 10 . Table 3 summarizes the results averaged over 300 simulations. The proposed algorithm obtains better results than the other two for the estimated number of clusters, also the A.R.I. and Dunn Index are approximately the same.

Table 3: Results for the Three Algorithms on the Random Clusters

\begin{tabular}{|c|c|c|c|}
\hline Algorithm & $\begin{array}{c}\text { \% of Correct } \\
\text { Number of } \\
\text { Clusters }\end{array}$ & A.R.I. & $\begin{array}{c}\text { Dunn } \\
\text { Index }\end{array}$ \\
\hline$X$-means & 63 & 0.96 & 0.60 \\
\hline$X$-means-R & 71 & 0.97 & 0.60 \\
\hline$X$-Alter & 91 & 0.96 & 0.59 \\
\hline
\end{tabular}

Functional Case

Functional data are now considered along with computing times. When a dimension is small (as in the previous examples), the CPU times were approximately the same. Two configurations were considered: First, functions $\sqrt{x}+\cos (10 x+\pi / 2-10) / 5$, $x+\cos (10 x+\pi / 2-10) / 5$

and

$x^{2}+\cos (10 x+\pi / 2-10) / 5$ were taken in $[0,1]$ discretized 20 times. The term $\cos (10 x+\pi / 2-10) / 5$ was added to disturb functions $\sqrt{x}, x$ and $x^{2}$. Each data in $\mathbb{R}^{20}$ was noised with a vector composed by twenty Gaussian law $N(0, \sigma)$ where the value of $\sigma$ is selected for each data using $\sigma \sim N(0.1,0.02)$. Figure 5 shows examples of some of the functions generated. Three clusters of size randomly chosen between 15 and 25 were simulated 300 times. Results are presented in Table 4 (time is given in seconds).

The proposed method gives better results, mainly regarding the search of the number of clusters. A slightly more difficult case was also considered. This configuration was constructed on the same model as the first, but based on functions $\sqrt{x}, x^{3 / 4}$ and $x$ which are closer than previous ones (see Figure 6 and Table 5).

The proposed method retrieves the correct number of clusters more often. Note that if the complexity of the algorithm is larger than that of the $X$-means, it is still much smaller than the Alter. Moreover Alter does not estimate the number of clusters.

Robustness Study

The robustness properties of the $L_{1}$ distance are now illustrated. As a starting point, the first functional configuration shown in Figure 5 was considered. To obtain noisy data the following protocol was used: a value $x \in[-0.30 ;-0.15] \cup[0.15 ; 0.30]$ was added to $a \in[10 ; 25]$ percent of points (randomly chosen) of $b \in[10 ; 25]$ percent of data (randomly chosen) (see Figure 7 for an example). This procedure was repeated 300 times and averaged results are provided in Table 6 .

The relevance of the $L^{1}$-based distance error, which is much more robust to extreme values, is shown. If results are compared to those shown in Table 4 the correct number of clusters is found $95 \%$ of the time, while $X$ means and $X$-means-R do not perform as well and $X$-means-R do not perform as well (a loss of respectively $4 \%$ and $6 \%$ ). 
THOMAS LALOË \& REMI SERVIEN

Figure 5: Example of Functions

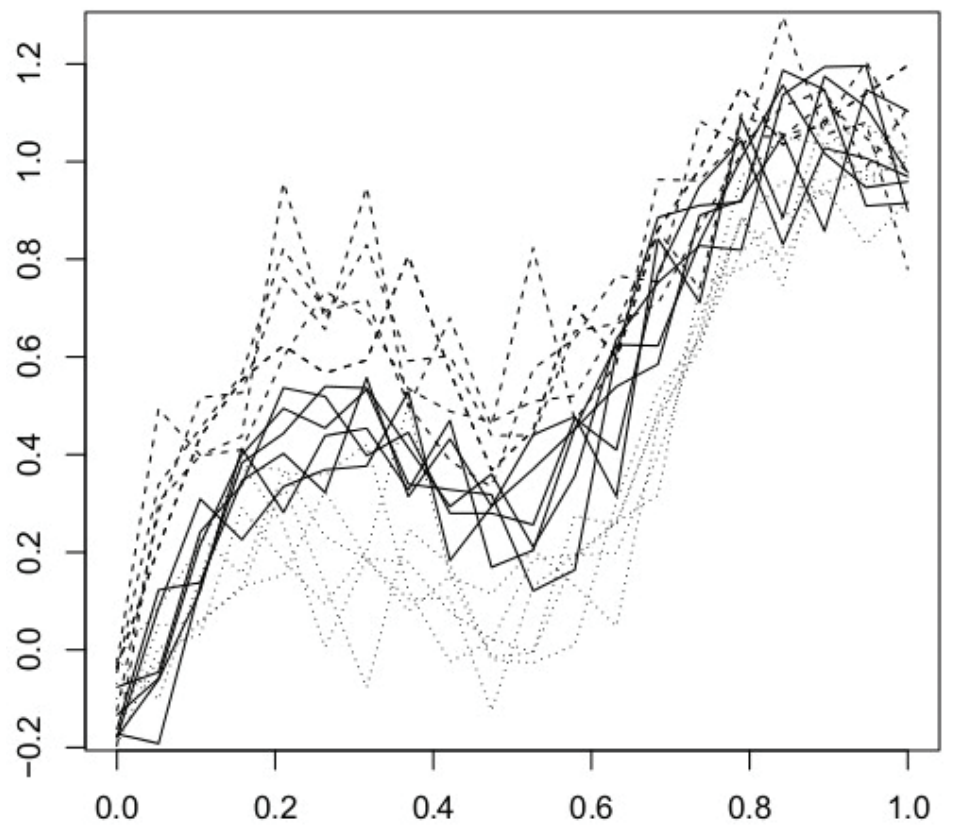

Notes: Functions based on $\sqrt{x}$ are on dashed lines, ones based on $x$ are on solid lines and ones based on $x^{2}$ are on dotted lines.

Table 4: Results for the Three Algorithms on the Functional Data

\begin{tabular}{|c|c|c|c|c|}
\hline Algorithm & $\begin{array}{c}\text { \% of Correct } \\
\text { Number of } \\
\text { Clusters }\end{array}$ & A.R.I. & $\begin{array}{c}\text { Dunn } \\
\text { Index }\end{array}$ & Time \\
\hline$X$-means & 81 & 0.88 & 0.63 & 2.0 \\
\hline$X$-means-R & 85 & 0.88 & 0.63 & 3.5 \\
\hline$X$-Alter & 95 & 0.89 & 0.63 & 27.6 \\
\hline
\end{tabular}


Figure 6: Example of Functions

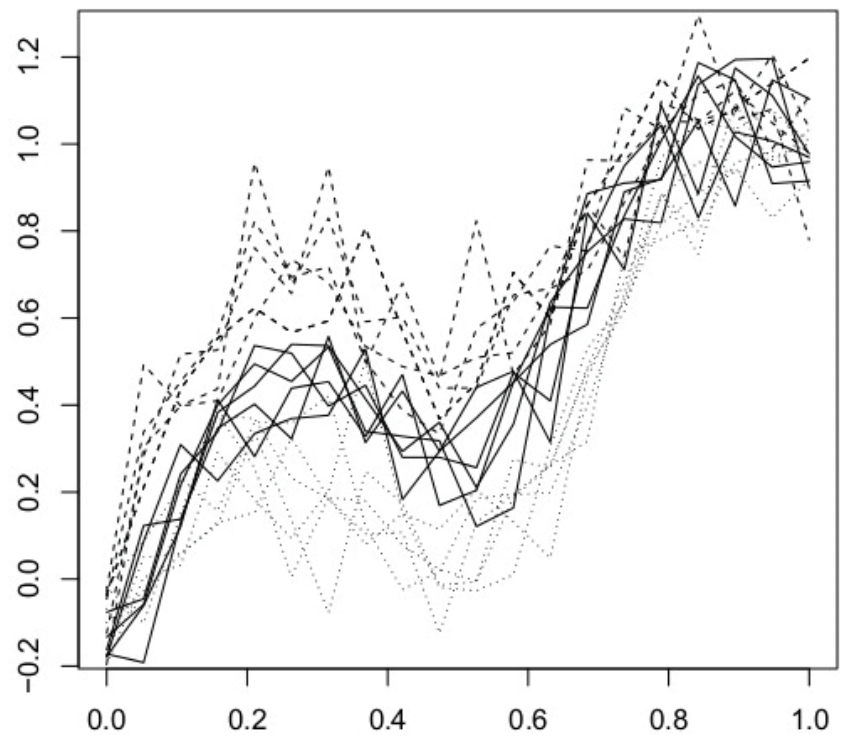

Notes: Functions based on $\sqrt{x}$ are on dashed lines, ones based on $x$ are on solid lines and ones based on $x^{3 / 4}$ are on dotted lines.

Table 5: Results for the Three Algorithms on the Functional Data

\begin{tabular}{|c|c|c|c|c|}
\hline Algorithm & $\begin{array}{c}\text { \% of Correct } \\
\text { Number of } \\
\text { Clusters }\end{array}$ & A.R.I. & $\begin{array}{c}\text { Dunn } \\
\text { Index }\end{array}$ & Time \\
\hline$X$-means & 26 & 0.75 & 0.43 & 2.4 \\
\hline$X$-means-R & 31 & 0.75 & 0.46 & 3.2 \\
\hline$X$-Alter & 40 & 0.77 & 0.46 & 28.7 \\
\hline
\end{tabular}


THOMAS LALOË \& REMI SERVIEN

Figure 7: Example of the Results of the Perturbation of $\sqrt{x}+\cos (10 x+\pi / 2-10) / 5$

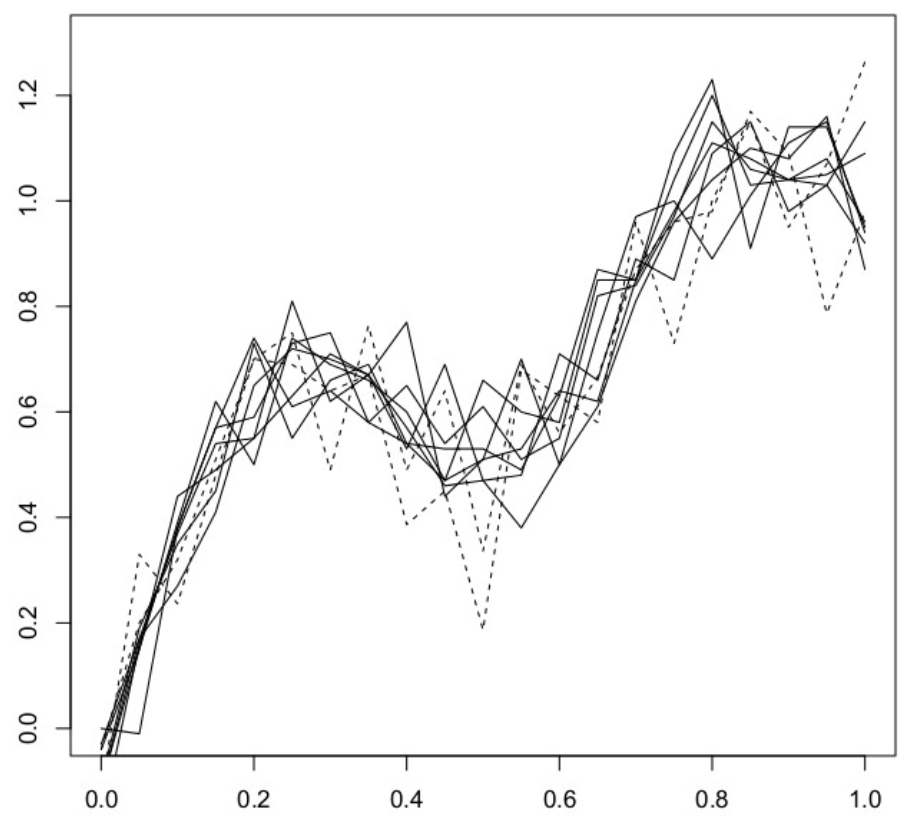

Note: Affected functions are on dashed lines.

Table 6: Results for the Three Algorithms on the Perturbated Functional Data Sets

\begin{tabular}{|c|c|c|c|c|}
\hline Algorithm & $\begin{array}{c}\text { \% of Correct } \\
\text { Number of } \\
\text { Clusters }\end{array}$ & A.R.I. & $\begin{array}{c}\text { Dunn } \\
\text { Index }\end{array}$ & Time \\
\hline$X$-means & 77 & 0.87 & 0.52 & 2.6 \\
\hline$X$-means-R & 79 & 0.87 & 0.52 & 3.8 \\
\hline$X$-Alter & 95 & 0.88 & 0.53 & 29.4 \\
\hline
\end{tabular}

\section{Real Data}

The proposed method is next used with two conventional data sets from the UCI Machine Learning Repository (Frank \& Asuncion, 2010); these are wine and iris data. In this case, the spherical Gaussian assumption of the BIC criterion cannot be assumed to be verified, therefore, it is important to test to ensure that this hypothesis is reasonable. The proposed method was compared to the $X$-means algorithm but also to the $K$-means algorithm with $K$ known to be 3 (the real number of clusters); thus, 3-means have a significant advantage over others methods by knowing the number of clusters. In these two real cases, as suggested in the description of the data sets, each variable is centered and standardized before performing clustering. Because $K$-means, $X$-means and $X$-means-R depend on the initialization, averaged results are given (over 50 runnings) for these methods.

\section{Wine Data Set}

The wine data set is composed of 178 instances and 13 variables found in each of three types of wines. These data are the results of a 


\section{X-ALTER ALGORITHM: A PARAMETER-FREE METHOD OF CLUSTERING}

chemical analysis of wines grown in the same region in Italy but derived from three different cultivars. In a classification context, this is a well posed problem with well-behaved class structures. The results for the 4 methods are presented in Table 7. The proposed method retrieves the real number of clusters and the same adjusted Rand index of 3-means is obtained, which is slightly less than the 2 others. Conversely, the method does not result in a good Dunn Index because one extreme instance is bad classified. X-Alter can also be compared to other methods used on this data set and listed on the UCI Machine Learning (Frank \& Asuncion, 2010). For example, it better estimates the number of clusters than Dy and Brodley (2004) with their different methods.
Iris Data Set

The iris data set is composed of 150 instances and 4 variables of 3 classes of 50 instances each, where each class refers to a type of iris plant. One class is linearly separable from the other two; the latter are not linearly separable from each other which makes it more difficult to classify. The results are gathered in Table 8.

The proposed method does not find the real number of clusters but it gets closer than other methods. Although the adjusted Rand Index was previously very close for all methods, the $X$-Alter is significantly better. Because the adjusted Rand Index is here considered - as opposed to the Rand Index - it does not indicate that the classification is perfect.

Table 7: Results for the Wine Data Set

\begin{tabular}{|c|c|c|c|}
\hline Algorithm & $\begin{array}{c}\text { Number of } \\
\text { Clusters }\end{array}$ & A.R.I. & Dunn \\
\hline$X$-means & $\begin{array}{c}8.67 \\
(\mathrm{var}=6.92)\end{array}$ & $\begin{array}{c}0.78 \\
(\mathrm{var}=0.03)\end{array}$ & $\begin{array}{c}0.162 \\
\left(\mathrm{var}=2.10^{-4}\right)\end{array}$ \\
\hline$X$-means-R & $\begin{array}{c}8.54 \\
(\mathrm{var}=6.01)\end{array}$ & $\begin{array}{c}0.78 \\
(\mathrm{var}=0.03)\end{array}$ & $\begin{array}{c}0.165 \\
\left(\mathrm{var}=10^{-4}\right)\end{array}$ \\
\hline 3-means & - & $\begin{array}{c}0.76 \\
(\mathrm{var}=0.03)\end{array}$ & $\begin{array}{c}0.163 \\
(\mathrm{var}=0.0002)\end{array}$ \\
\hline$X$-Alter & 3 & 0.76 & 0.142 \\
\hline
\end{tabular}

Table 8: Results for Iris Data Set

\begin{tabular}{|c|c|c|c|}
\hline Algorithm & $\begin{array}{c}\text { Number of } \\
\text { Clusters }\end{array}$ & A.R.I. & Dunn \\
\hline$X$-means & $\begin{array}{c}13.7 \\
(\mathrm{var}=6.2)\end{array}$ & $\begin{array}{c}0.46 \\
(\mathrm{var}=0.07)\end{array}$ & $\begin{array}{c}0.0405 \\
\left(\mathrm{var}=6.10^{-5}\right)\end{array}$ \\
\hline$X$-means-R & $\begin{array}{c}8 \\
(\mathrm{var}=1.56)\end{array}$ & $\begin{array}{c}0.57 \\
(\mathrm{var}=0.03)\end{array}$ & $\begin{array}{c}0.0398 \\
(\mathrm{var}=0)\end{array}$ \\
\hline 3-means & - & $\begin{array}{c}0.46 \\
(\mathrm{var}=0.0036)\end{array}$ & $\begin{array}{c}0.04 \\
(\mathrm{var}=0)\end{array}$ \\
\hline$X$-Alter & 6 & 1 & 0.402 \\
\hline
\end{tabular}




\section{THOMAS LALOË \& REMI SERVIEN}

However, the high value of the A.R.I. indicates that the great majority of iris plants are well-classified; the 3 additional clusters are very small and do not affect the A.R.I and the global quality of the obtained clustering. In Dy and Brodley (2004), the estimation of the number of clusters was slightly better but, as discussed, the quality of clustering seems (as different criterions are used) to be better. Moreover, the interest of the aggregation step in $X$-means- $\mathrm{R}$ is enlightened and it appears that the spherical Gaussian assumption required for the BIC is acceptable and the $X$-Alter can be used with every data set.

Finally, in all cases the proposed method performed better than the others to estimate the number of clusters. This confirms that the local convergence of $X$-means, inherited from $K$ means, is avoided. Further, according to the adjusted Rand and the Dunn Indexes the quality of clustering is either equal to or significantly better than the other methods considered.

\section{Conclusion}

A simple new algorithm to perform clustering was presented. The main advantage of this method is that it is parameter-free and, thus, it can be easily used without an expert knowledge of the data. This algorithm combines the Alter and $X$-means algorithms to benefit from the qualities of both (respectively the convergence and the automatic selection of the number of clusters). Moreover, combining the methods eliminates the main drawbacks of these two methods: the high complexity for Alter and the dependence on initials conditions for $X$-means.

Experiments using both simulated and real data sets show the relevance of the proposed method. However, even if complexity decreases (with respect to the Alter algorithm) it is too important for the method to be applied on very large data sets. A possible way to overcome this problem could be the utilization of the Alter-Fast algorithm (Laloë, 2010) as opposed to the Alter. Alter-Fast runs several times Alter in randomly chosen partitions of a data set and it can help save computational time but with some loss of efficiency. In future studies it would be interesting to look for others ways to accelerate Alter while preserving (as much as possible) its properties of convergence.
References

Dunn, J. (1974). Well separated clusters and fuzzy partitions. Journal on Cybernetics, 4, 95-104.

Dy, J., \& Brodley, C. (2004). Feature selection for unsupervised learning. Journal of Machine Learning Research, 5, 845-889.

Frank, A., \& Asuncion, A. (2010). UCI machine learning repository. Accessed at: $\mathrm{http}: / / \operatorname{archive.ics.uci.edu/ml}$.

Graf, S., \&Luschgy, H. (2000). Foundations of quantization for probability distributions: Lecture Notes in Mathematics, 1730. Berlin, Germany: Springer-Verlag.

Handl, J., Knowles, K., \& Kell, D. (2005), Computational cluster validation in postgenomic data analysis. Bioinformatics, 21, 32013212.

Hartigan, J., \& Wong, M. (1979). A $k$ means clustering algorithm. Journal of the Royal Statistical Society, 28, 100-108.

Hubert, L., \& Arabie, P. (1985). Comparing partitions. Journal of Classification, 2(1), 193-218.

Kass, R., \& Wasserman, L. (1995). A reference bayesian test for nested hypotheses and its relationship to the schwarz criterion. Journal of the American Statistical Association, 90, 928-934.

Kaufman, L., \& Rousseeuw, P. (1990). Finding groups in data: An introduction to cluster analysis. New York, NY: John Wiley \& Sons.

Kemperman, J. H. B. (1987). The median of a finite measure on a Banach space. In Statistical data analysis fased on the L1-norm and related methods, Neuchâtel, Ed., 217-230. Amsterdam: North-Holland.

Laloë, T. (2010). $L_{1}$ quantizationand clustering in Banach spaces. Mathematical Methods of Statistics, 19(2), 136-150.

Li, M., Ng, M., Cheung, Y. M., \& Huang, J. (2008). Agglomerative fuzzy k-means clustering algorithm with selection of number of clusters. IEEE Transactions on Knowledge and Data Engineering, 20, 1519-1534. 


\section{X-ALTER ALGORITHM: A PARAMETER-FREE METHOD OF CLUSTERING}

Linder, T. (2002). Learning-theoretic methods in vector quantization. In Principles of nonparametric learning (Udine, 2001), CISM Courses and Lectures, Vol. 434, 163-201. Vienna, Austria: Springer.

MacQueen, J. (1967). Some methods for classification and analysis of multivariate observations. In Proceedings of 5-th Berkeley symposium on mathematical statistics and probability, 281-297. Berkeley, CA: University of California Press.

Nguyen, X., Epps, J., \& Bailey, J. (2009). Information theoretic measures for clustering comparison: Is a correction for chance necessary? In ICML'09: Proceedings of the 26th Annual International Conference on Machine Learning, 1073-1080. San Francisco, CA: Morgan Kaufmann.
Pelleg, D., \& Moore, A. (2000). $X$ means: Extending $k$-means with efficient estimation of the number of clusters. In Proceedings of the Seventeenth International Conference on Machine Learning, 727-734. San Francisco, CA: Morgan Kaufmann.

Pham, T., Dimov, S., \& Nguyen, C. (2005). Selection of $K$ in $K$-means clustering. Proceedings of the Institution of Mechanical Engineers, Part C: Journal of Mechanical Engineering Science, 219, 103-119.

Rand, W. (1971). Objective criteria for the evaluation of clustering methods. Journal of the American Statistical Association, 66(336), 846-850. 


\title{
On The Gamma-Half Normal Distribution and Its Applications
}

\author{
Ayman Alzaatreh Kristen Knight \\ Austin Peay State University, \\ Clarksville, TN
}

A new distribution, the gamma-half normal distribution, is proposed and studied. Various structural properties of the gamma-half normal distribution are derived. The shape of the distribution may be unimodal or bimodal. Results for moments, limit behavior, mean deviations and Shannon entropy are provided. To estimate the model parameters, the method of maximum likelihood estimation is proposed. Three real-life data sets are used to illustrate the applicability of the gamma-half normal distribution.

Key words: $\quad T-X$ families; gamma- $X$ family; unimodal; bimodal; Shannon entropy.

Introduction

In recent years, advancements in technology and science have resulted in a wealth of information, which is expanding the level of knowledge across many disciplines. This information is gathered and analyzed by statisticians, who hold the responsibility of accurately assessing the data and making inferences about the population of interest. Without this precise evaluation of data, each field remains limited to its current state of knowledge. In the last decade, it has been discovered that many well-known distributions used to model data sets do not offer enough flexibility to provide an adequate fit. For this reason, new methods are being proposed and used to derive generalizations of wellknown distributions. With these distributions, strong applications have been made to real-life scenarios.

Alzaatreh, et al. (2013b) proposed the $T$ $X$ families of distributions. These families of distributions were used to generate a new class of distributions which offer more flexibility in modeling a variety of data sets. Several members of the $T$ - $X$ families have been studied

Ayman Alzaatreh is an Assistant Professor in the Department of Mathematics \& Statistics. Email him at: alzaatreha@apsu.edu. Kristen Knight is a student in the Department of Mathematics and Statistics. Email her at: kknight13@my.apsu.edu. in the literature (e.g., Alzaatreh, et al. (2013a); Alzaatreh, et al. (2013b); Alzaatreh, et al. (2012a); Alzaatreh, et al. (2012b); Lee, et al. (2013)).

One well-known distribution is the halfnormal distribution, which has been used in variety of applications. Previous work by Bland and Altman (1999) used the half-normal distribution to study the relationship between measurement error and magnitude. Bland (2005) extended the work of Bland and Altman (1999) by using the distribution to estimate the standard deviation as a function so that measurement error could be controlled. In his work, various exercise tests were analyzed and it was determined that variability of performance does decline with practice (Bland, 2005). Manufacturing industries have utilized the halfnormal distribution to model lifetime processes under fatigue. These industries often produce goods with a long lifetime need for customers, making the cost of the resources needed to analyze the product failure times very high. To save time and money the half normal distribution is used in this reliability analysis to study the probabilistic aspects of the product failure times (Castro, et al., 2012).

Due to the fact that the half-normal distribution has only one shape, various generalizations of the distribution have been derived. These generalizations include the generalized half-normal distribution (Cooray, et al., 2008), the beta-generalized half-normal (Pescrim, et al., 2010) and the Kumaraswamy 


\section{ON THE GAMMA-HALF NORMAL DISTRIBUTION AND ITS APPLICATIONS}

generalized half-normal (Cordeiro, et al., 2012). Several of the corresponding applications include the stress-rupture life of kevlar 49/epoxy strands placed under sustained pressure (Cooray, et al., 2008), failure times of mechanical components and flood data (Cordeiro, et al., 2012). In this article the gamma and half normal distributions are combined to propose a new generalization of the half-normal distribution, namely, the gamma half-normal distribution.

Let $F(x)$ be the cumulative distribution function (CDF) of any random variable $X$ and $r(t)$ be the probability density function (PDF) of a random variable $T$ defined on $[0, \infty)$. The CDF of the $T-X$ family of distributions defined by Alzaatreh, et al. (2013b) is given by

$$
G(x)=\int_{0}^{-\log (1-F(x))} r(t) d t=R\{-\log (1-F(x)\}
$$

When $X$ is a continuous random variable, the probability density function of the $T-X$ family is

$$
\begin{aligned}
g(x) & =\frac{f(x)}{1-F(x)} r(-\log (1-F(x))) \\
& =h(x) r(H(x)),
\end{aligned}
$$

where $h(x)$ and $H(x)$ are the hazard and the cumulative hazard functions of the random variable $X$ associated with $f(x)$.

If a random variable $T$ follows the gamma distribution with parameters $\alpha$ and $\beta$, $r(t)=\left(\beta^{\alpha} \Gamma(\alpha)\right)^{-1} t^{\alpha-1} e^{-t / \beta}, t \geq 0, \quad$ then the definition in (1.2) leads to the gamma- $X$ family with the PDF

$$
\begin{aligned}
& g(x)= \\
& \frac{1}{\Gamma(\alpha) \beta^{\alpha}} f(x)(-\log (1-F(x)))^{\alpha-1}(1-F(x))^{\frac{1}{\beta}-1} .
\end{aligned}
$$

When $\beta=1$, the gamma- $X$ family in (1.3) reduces to the gamma-generated distribution introduced by Zografos and Balakrishnan
(2009). When $\alpha=1$ and $1 / \beta=n \in \mathbb{N}$, the gamma- $X$ family reduces to the distribution of the first order statistics of the random variable $X$.

If $X$ is the half normal random variable with the density function

$$
\begin{aligned}
& f(x)=\frac{\sqrt{2}}{\theta \sqrt{\pi}} e^{-x^{2} / 2 \theta^{2}}, x>0, \text { then (1.3) gives } \\
& g(x)= \\
& \frac{\sqrt{2}}{\sqrt{\pi} \theta \Gamma(\alpha) \beta^{\alpha}} e^{\frac{-x^{2}}{2 \theta^{2}}}\left(-\log \left(2 \Phi\left(\frac{-x}{\theta}\right)\right)\right)^{\alpha-1}\left(2 \Phi\left(\frac{-x}{\theta}\right)\right)^{\frac{1}{\beta}-1}, \\
& \alpha, \beta, \theta>0 ; x>0
\end{aligned}
$$

where $\Phi$ is the CDF of the standard normal distribution.

A random variable $X$ with the PDF $g(x)$ in (1.4) is said to follow the gamma-half normal distribution with parameters $\alpha, \beta$ and $\theta$. From (1.1), the CDF of the gamma- half normal distribution is obtained as

$$
G(x)=\gamma\left\{\alpha,-\beta^{-1} \log (2 \Phi(-x / \theta))\right\} / \Gamma(\alpha),
$$

where $\gamma(\alpha, t)=\int_{0}^{t} u^{\alpha-1} e^{-u} d u$ is the incomplete gamma function.

A series representation of $G(x)$ in (1.5) can be obtained by using the series expansion of the incomplete gamma function from Nadarajah and Pal (2008) as

$$
\gamma(\alpha, x)=\sum_{k=0}^{\infty} \frac{(-1)^{k} x^{\alpha+k}}{k !(\alpha+k)} .
$$

From (1.6), the CDF of the gamma half-normal distribution can be written as

$$
G(x)=\frac{1}{\Gamma(\alpha)} \sum_{k=0}^{\infty} \frac{(-1)^{k}[-\log (2 \Phi(-x / \theta))]^{\alpha+k}}{k !(\alpha+k) \beta^{\alpha+k}} .
$$

The hazard function associated with the gammahalf normal distribution is 


$$
\begin{aligned}
h(x) & =\frac{g(x)}{1-G(x)} \\
& =\frac{\sqrt{2} e^{-x^{2} / 2 \theta^{2}}(-\log (2 \Phi(-x / \theta)))^{\alpha-1}(2 \Phi(-x / \theta))^{\frac{1}{\beta}-1}}{\sqrt{\pi} \theta \beta^{\alpha}\left[\Gamma(\alpha)-\gamma\left\{\alpha,-\beta^{-1} \log (2 \Phi(-x / \theta))\right\}\right]},
\end{aligned}
$$
$x>0$.

Some Properties of the Gamma-Half Normal Distribution

Lemma 1 gives the relation between the gamma-half normal distribution and the gamma distribution.

Lemma 1 (Transformation)

If a random variable $Y$ follows the gamma distribution with parameters $\alpha$ and $\beta$, then the random variable $X=\theta \Phi^{-1}\left(1-0.5 e^{-Y}\right)$ follows the gamma-half normal distribution with parameters $\alpha, \beta$ and $\theta$.

Lemma 1 (Transformation) Proof

The results follow by using the transformation technique.

The limiting behaviors of the gammahalf normal PDF and the hazard function are given in Lemma 2.

Lemma 2

The limit of the gamma-half normal density function as $x \rightarrow \infty$ is 0 and the limit of the gamma-half normal hazard function as $x \rightarrow \infty$ is $\infty$. Also, the limit of the gamma-half normal and hazard function as $x \rightarrow 0^{+}$is given by

$$
\lim _{x \rightarrow 0^{+}} g(x)=\lim _{x \rightarrow 0^{+}} h(x)= \begin{cases}0, & \alpha>1 \\ \frac{\sqrt{2}}{\sqrt{\pi} \theta \beta}, & \alpha=1 \\ \infty, & \alpha<1 .\end{cases}
$$

\section{Lemma 2 Proof}

Since the random variable $X$ is defined on $(0, \infty)$, this implies $\lim _{x \rightarrow \infty} g(x)=0$. Using L'Hôpital's rule it can be shown that $\lim _{x \rightarrow \infty} h(x)=\infty$. Now, $h(x)[1-G(x)]=g(x)$ implies that $\lim _{x \rightarrow 0^{+}} g(x)=\lim _{x \rightarrow 0^{+}} h(x)$. Results in (2.1) follow immediately from definition (1.4). The modes of the gamma-half normal distribution can be obtained by taking the derivative of $g(x)$. The derivative with respect to $x$ of (1.4) can be simplified to

$g^{\prime}(x)=$

$\frac{\sqrt{2}}{\sqrt{\pi} \theta \Gamma(\alpha) \beta^{\alpha}} e^{\frac{-x^{2}}{2 \theta^{2}}}\left(-\log \left(2 \Phi\left(\frac{-x}{\theta}\right)\right)\right)^{\alpha-2}\left[2 \Phi\left(\frac{-x}{\theta}\right)\right]^{\frac{1}{\beta}-2} k(x)$,

where

$$
\begin{aligned}
& k(x)= \\
& {\left[\begin{array}{l}
\frac{x}{\theta} \log \left(2 \Phi\left(\frac{-x}{\theta}\right)\right)+(\alpha-1) h_{z}\left(\frac{x}{\theta}\right) \\
+\left(\beta^{-1}-1\right) h_{z}\left(\frac{x}{\theta}\right) \log \left(2 \Phi\left(\frac{-x}{\theta}\right)\right.
\end{array}\right]}
\end{aligned}
$$

Setting (2.2) to 0 , the critical values of $g(x)$ are $x=0$ and the solution of the equation $k(x)=0$. The solution of $k(x)=0$ is equivalent to the equation

$$
x=\theta h_{z}(x / \theta)\left\{1-\frac{1}{\beta}-\frac{\alpha-1}{\log (2 \Phi(-x / \theta))}\right\},
$$

where $h_{z}(x / \theta)=\phi(x / \theta) /(1-\Phi(x / \theta))$.

Corollary 1

If $\alpha \leq 1$ and $\beta \leq 1$, the gamma-half normal distribution is unimodal and the mode is at $x=0$. 


\section{ON THE GAMMA-HALF NORMAL DISTRIBUTION AND ITS APPLICATIONS}

Corollary 1 Proof

If $\alpha<1$, then Lemma 2 implies that $x=0$ is a modal point. When $\alpha<1$ and $\beta \leq 1$, it follows from (2.3) that $x<0$, and hence equation (2.3) has no solution, thus, $x=0$ is a unique modal point. The proof is complete by noting that when $\alpha=1$ and $\beta \leq 1$, the PDF of gamma-half normal in (1.4) is a strictly decreasing function.

Figures 1-3 show various graphs of $g(x)$ and $h(x)$. These figures indicate that the gamma-half normal PDF may take on a variety of shapes for different values of $\alpha, \beta$ and $\theta$. The shapes range from reversed-J shape, bimodal, right-skewed and approximately symmetric. As $\beta$ decreases, the right tail of the gamma-half normal distribution becomes longer. Bimodality appears when $\alpha$ is less than 1 . Figure 3 indicates that the gamma-half normal hazard function is either a bathtub shape or increasing failure rate shape.
When $\alpha<1$, and for certain values of $\beta$, the gamma-half normal distribution becomes bimodal. It is difficult to find analytically the region where the distribution is bimodal. However, a numerical solution is obtained to determine the number of roots of the derivative of the gamma-half normal distribution. Figure 4 shows the boundary region of $\alpha$ and $\beta$ where the gamma-half normal distribution is bimodal.

Lemma 3

If $Q(\lambda), 0<\lambda<1$ denotes the quantile function for the gamma-half normal distribution, then

$$
Q(\lambda)=-\theta \Phi^{-1}\left(0.5 \exp \left\{-\beta \gamma^{-1}(\alpha, \lambda \Gamma(\alpha))\right\}\right) .
$$

Figure 1: The Gamma-Half Normal PDF for Various Values of $\alpha, \beta$ and $\theta$

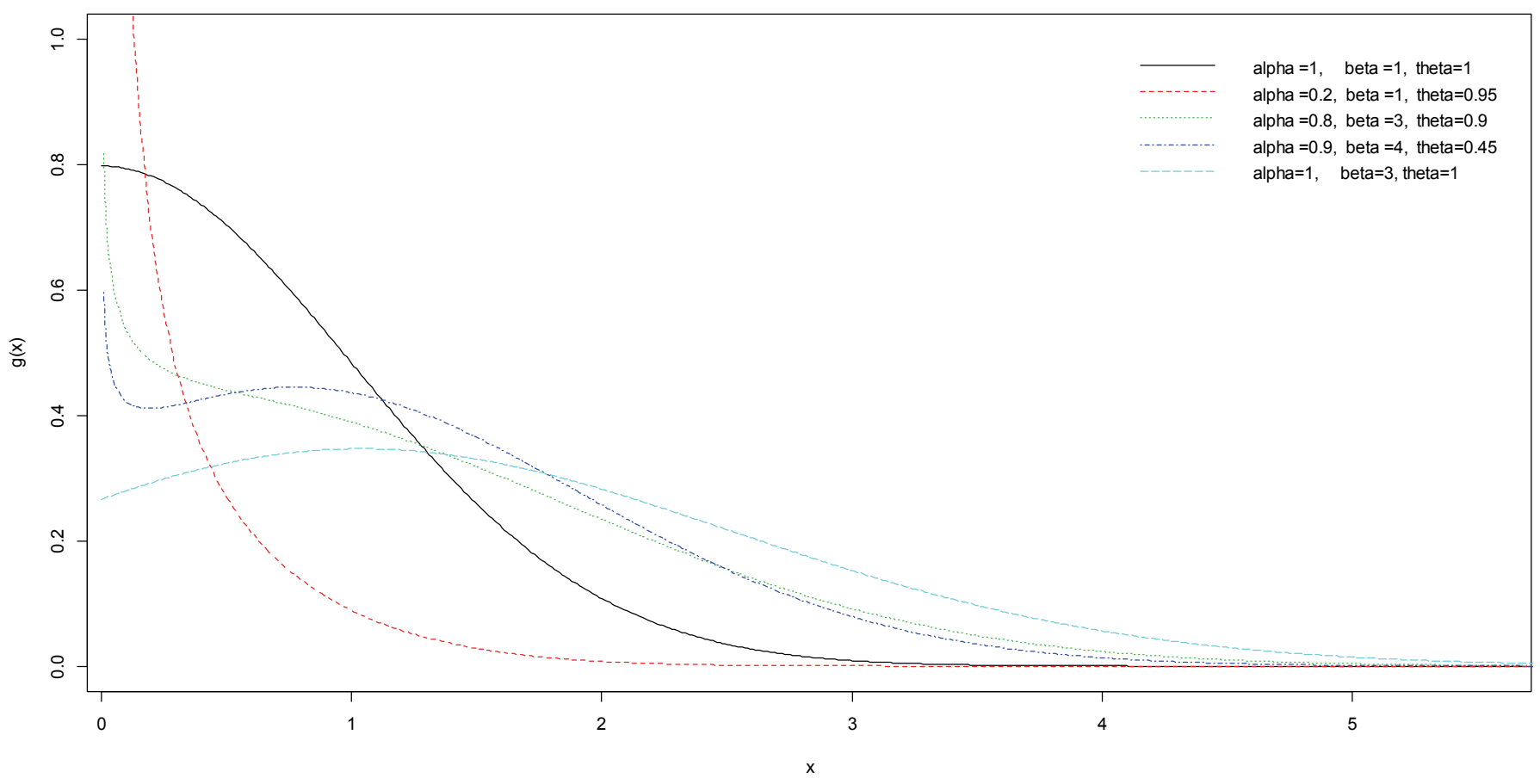


Figure 2: The Gamma-Half Normal PDF for Various Values of $\alpha, \beta$ and $\theta$

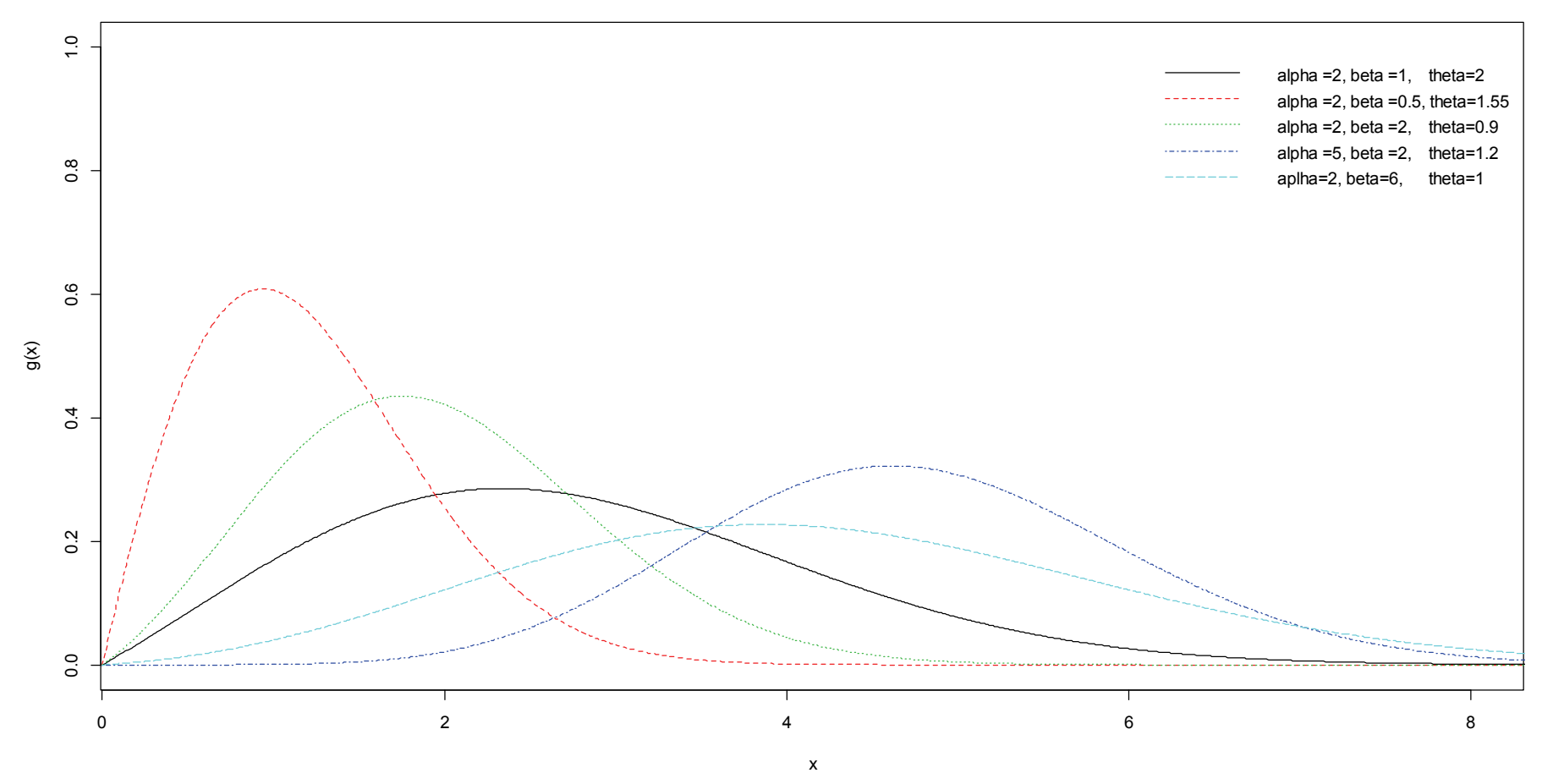

Figure 3: The Gamma-Half Normal Hazard Function for Various Values of $\alpha, \beta$ and $\theta$

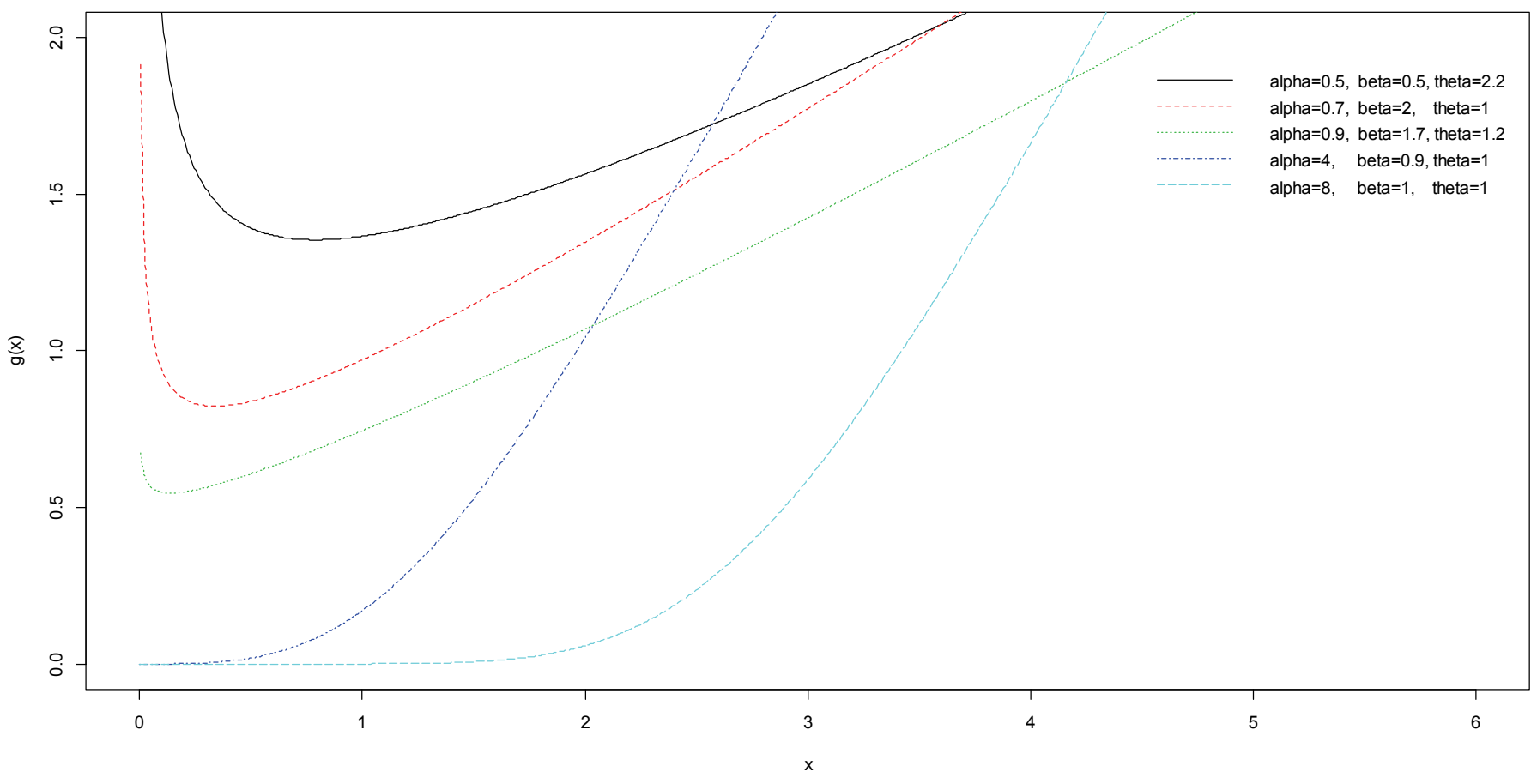




\section{ON THE GAMMA-HALF NORMAL DISTRIBUTION AND ITS APPLICATIONS}

\section{Lemma 3 Proof}

The proof follows by taking the inverse function of (1.5).

The entropy of a random variable $X$ is a measure of variation of uncertainty (Rényi, 1961). Shannon's entropy (Shannon, 1948), for a random variable $X$ with PDF $g(x)$ is defined as $E\{-\log (g(X))\}$. Since 1948 many applications have been used with Shannon's entropy in different areas, including engineering, physics, biology, economics and information theory.

According to Alzaatreh, et al. (2013b), the Shannon entropy of the gamma- $X$ family of distributions is given by

$$
\begin{aligned}
\eta_{X}= & -E\left\{\log f\left(F^{-1}\left(1-e^{-T}\right)\right)\right\}+\alpha(1-\beta), \\
& +\log \beta+\log \Gamma(\alpha)+(1-\alpha) \psi(\alpha)
\end{aligned}
$$

where $\psi$ is the digamma function and $T$ is the gamma random variable with parameters $\alpha$ and $\beta$.

Theorem 3

The Shannon entropy for the gammahalf normal distribution is given by

$$
\begin{aligned}
\eta_{X}= & -\log \sqrt{2}+\log \sqrt{\pi}+\log \theta \\
& +\frac{1}{2 \theta^{2}}\left(\sigma^{2}+\mu^{2}\right)+\alpha(1-\beta)+\log \beta \\
& +\log \Gamma(\alpha)+(1-\alpha) \psi(\alpha),
\end{aligned}
$$

where $\mu$ and $\sigma$ are the mean and variance of the gamma-half normal, respectively.

Figure 4: Bimodal Region for the Gamma-Half Normal Density Function where $\theta=1$

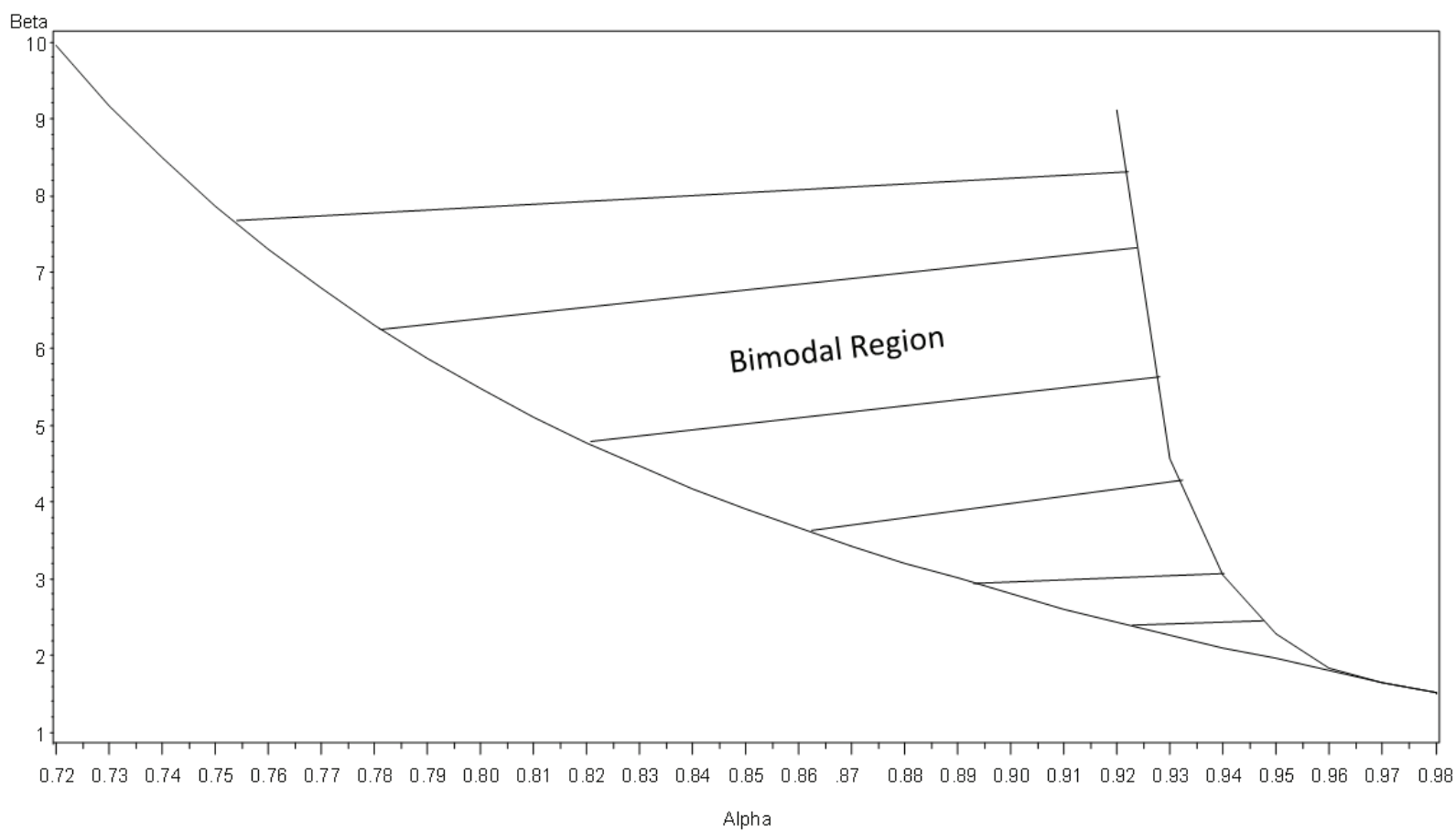


Theorem 3 Proof

First it is necessary to find $-E\left\{\log f\left(F^{-1}\left(1-0.5 e^{-T}\right)\right)\right\}$, where $f(x)$ and $F(x)$ are the PDF and CDF of the halfnormal distribution, respectively. From the CDF of the half-normal distribution it follows that $F^{-1}(x)=\theta \Phi^{-1}\left(\frac{x+1}{2}\right)$ and hence,

$$
\begin{aligned}
-E\left\{\log f\left(F^{-1}\left(1-e^{-T}\right)\right)\right\} \\
=-\log \sqrt{2}+\log \sqrt{\pi}+\log \theta, \\
\quad+\left(\frac{1}{2 \theta^{2}}\right) E\left(\theta \Phi^{-1}\left(1-0.5 e^{-T}\right)\right)^{2}
\end{aligned}
$$

where $T$ follows the gamma distribution. By Lemma $1, \quad \theta \Phi^{-1}\left(1-0.5 e^{-T}\right)$ follows the gamma-half normal with parameters $\alpha, \beta$ and $\theta$. Hence $E\left(\theta \Phi^{-1}\left(1-0.5 e^{-T}\right)\right)=\sigma^{2}+\mu^{2}$ where $\sigma^{2}$ and $\mu$ are the variance and the mean for the gamma-half normal distribution. The result in (2.5) follows from equation (2.4).

Moments and Mean Deviations

The $r^{\text {th }}$ moments for the gamma-half normal distribution in (1.4) can be written as

$$
\begin{aligned}
& E\left(X^{r}\right)= \\
& \frac{\sqrt{2}}{\sqrt{\pi} \theta \Gamma(\alpha) \beta^{\alpha}} \int_{\theta}^{\infty} x^{r} e^{\frac{-x^{2}}{2 \theta^{2}}}\left(-\log \left(2 \Phi\left(\frac{-x}{\theta}\right)\right)\right)^{\alpha-1}\left(2 \Phi\left(\frac{-x}{\theta}\right)\right)^{\frac{1}{-1}} d x .
\end{aligned}
$$

Using the substitution $u=-\log (2 \Phi(-x / \theta))$, (3.1) reduces to

$$
E\left(X^{r}\right)=\frac{(-1)^{r} \theta^{r}}{\Gamma(\alpha) \beta^{\alpha}} \int_{0}^{\infty}\left(\Phi^{-1}\left(0.5 e^{-u}\right)\right)^{r} u^{\alpha-1} e^{-u / \beta} d u
$$

Because no closed form is found for (3.2), numerical integration can be used to calculate the $r^{\text {th }}$ moments.
Table 1 provides the mode, mean, median and variance of the gamma-half normal distribution for various values of $\alpha$ and $\beta$ when $\theta=1$. Equations (2.2) and (3.2) are used for these calculations. For fixed $\alpha$ and $\theta$, the mode, mean, median and variance are increasing functions of $\beta$. Also, for fixed $\beta$ and $\theta$, the mode, mean, median and variance are increasing functions of $\alpha$. Figure 5 displays the skewness and kurtosis graphs of the gamma-half normal distribution for different values of $\alpha$ and $\beta$ with $\theta=1$. For fixed $\alpha$, the skewness and kurtosis are decreasing functions of $\beta$; for fixed $\beta$, the skewness and kurtosis are decreasing functions of $\alpha$.

Lemma 5

If the median is denoted by $M$, then the mean deviation from the mean, $D(\mu)$, and the mean deviation from the median, $D(M)$, for the gamma- $X$ distribution are given by

$$
D(\mu)=2 \mu \gamma\left\{\alpha, \beta^{-1} \log (2 \Phi(-\mu)\} / \Gamma(\alpha)-2 I_{\mu}\right.
$$

and

$$
D(M)=\mu-2 I_{M}
$$

where $\quad I_{m}=\frac{\sqrt{2} \theta}{\Gamma(\alpha)} \sum_{k=0}^{\infty} \sum_{i=0}^{2 k+1} a(k, i) \gamma\left(\alpha, \delta_{i}(m)\right)$,

$a(k, i)=\frac{c_{k}}{2 k+1}(\sqrt{\pi} / 2)^{2 k+1}\left(\frac{2 k+1}{i}\right) \frac{(-1)^{i}}{(1+i \beta)^{\alpha}}$ and $\delta_{i}(m)=-(i+1 / \beta) \log (2 \Phi(-m / \theta))$.

\section{Lemma 5 Proof}

If $g(x)$ and $G(x)$ are the PDF and the $\mathrm{CDF}$ of the gamma-half normal distribution, then the mean deviations from the mean and the median can be written as

$$
D(\mu)=2 \mu G(\mu)-2 \int_{0}^{\mu} x g(x) d x
$$

and

$$
D(M)=\mu-2 \int_{0}^{M} x g(x) d x
$$


Consider the integral:

$I_{m}=\int_{0}^{m} x g(x) d x$

$=\frac{\sqrt{2}}{\sqrt{\pi} \theta \Gamma(\alpha) \beta^{\alpha}} \int_{0}^{m} x e^{\frac{-x^{2}}{2 \theta^{2}}}\left(-\log \left(2 \Phi\left(\frac{-x}{\theta}\right)\right)\right)^{\alpha-1}\left(2 \Phi\left(\frac{-x}{\theta}\right)\right)^{(1 / \beta)-1} d x$

By substituting $u=-\log (2 \Phi(-x / \theta))$ and because $\quad \operatorname{erf}(x / \sqrt{2} \theta)=2 \Phi(x / \theta)-1$, equation (3.6) can be written as

$I_{m}=\frac{\sqrt{2} \theta}{\Gamma(\alpha) \beta^{\alpha}} \int_{0}^{-\log \left(2 \Phi\left(\frac{-m}{\theta}\right)\right)} \operatorname{erf}^{-1}\left(1-e^{-u}\right) u^{\alpha-1} e^{\frac{-u}{\beta}} d u$

Using the series representation for $\operatorname{erf}^{-1}\left(1-e^{-u}\right)$ (see Wolfram website), results in

$$
\operatorname{erf}^{-1}(x)=\sum_{k=0}^{\infty} \frac{c_{k}}{2 k+1}(\sqrt{\pi} / 2)^{2 k+1} x^{2 k+1}
$$

where $c_{k}=\sum_{m=0}^{k-1} \frac{c_{m} c_{k-1-m}}{(m+1)(2 m+1)}$

and $c_{0}=1$.

Using (3.8), equation (3.7) reduces to

$$
\begin{aligned}
I_{m}= & \frac{\sqrt{2} \theta}{\Gamma(\alpha) \beta^{\alpha}} \sum_{k=0}^{\infty} \frac{c_{k}}{2 k+1}\left(\frac{\sqrt{\pi}}{2}\right)^{2 k+1} \\
& \int_{0}^{-\log \left(2 \Phi\left(\frac{-m}{\theta}\right)\right)}\left(1-e^{-u}\right)^{2 k+1} u^{\alpha-1} e^{\frac{-u}{\beta}} d u .
\end{aligned}
$$

Using the series expansion of $\left(1-e^{-u}\right)^{2 k+1}$, (3.9) reduces to

$$
I_{m}=\frac{\sqrt{2} \theta}{\Gamma(\alpha)} \sum_{k=0}^{\infty} \sum_{i=0}^{2 k+1} a(k, i) \gamma\left(\alpha, \delta_{i}\right)
$$

where

$$
a(k, i)=\frac{c_{k}}{2 k+1}(\sqrt{\pi} / 2)^{2 k+1}\left(\begin{array}{c}
2 k+1 \\
i
\end{array}\right) \frac{(-1)^{i}}{(1+i \beta)^{\alpha}}
$$

and $\delta_{i}(m / \theta)=-(i+1 / \beta) \log (2 \Phi(-m / \theta))$.
The results follow by substituting $I_{m}$ in $D(\mu)$ and $D(M)$ in (3.3).

Order Statistics

The density function of the $r^{\text {th }}$ order statistic, $X_{r: n}$, for a random sample of size $n$ drawn from (1.3), is

$$
f(x)=\frac{1}{B(r, n-r+1)} g(x)(G(x))^{r-1}(1-G(x))^{n-r} .
$$

Using the binomial expansion, (4.1) can be written as

$$
\begin{aligned}
f_{r: n}(x)= & \frac{g(x)}{B(r, n-r+1)} \\
& \sum_{j=0}^{n-r}(-1)^{j}\left(\begin{array}{c}
n-r \\
j
\end{array}\right)(G(x))^{r+j-1} .
\end{aligned}
$$

From (1.7), equation (4.2) can be written as

$$
\begin{aligned}
& f_{r: n}(x)=\frac{g(x)}{B(r, n-r+1)} \sum_{j=0}^{n-r} \frac{(-1)^{j}}{\Gamma(\alpha)^{r+j-1}}\left(\begin{array}{c}
n-r \\
j
\end{array}\right) \\
& \times\left(\sum_{k=0}^{\infty} \frac{(-1)^{k}}{\beta^{k+\alpha}} \frac{(-\log (2 \Phi(-x / \theta)))^{\alpha+k}}{k !(k+\alpha)}\right)^{r+j-1} \\
& =\frac{g(x)}{B(r, n-r+1)} \sum_{j=0}^{n-r} \sum_{k_{1}=0}^{\infty} \sum_{k_{2}=0}^{\infty} \ldots . \\
& \sum_{k_{r+j-1}=0}^{\infty} \frac{1}{\beta^{s_{k}+(r+j-1) \alpha} P_{k}} \frac{(-1)^{s_{k}+j}}{\Gamma(\alpha)^{r+j-1}}\left(\begin{array}{c}
n-r \\
j
\end{array}\right) \\
& \left(-\log 2 \Phi\left(\frac{-x}{\theta}\right)\right)^{s_{k}+(r+j-1) \alpha} .
\end{aligned}
$$


Table 1: Mode, Mean, Median and Variance for Some Values of $\alpha$ and $\beta$ with $\theta=1$

\begin{tabular}{|c|c|c|c|c|c|}
\hline$\alpha$ & $\beta$ & Mode & Mean & Median & Variance \\
\hline \multirow{5}{*}{0.5} & 0.5 & 0 & 0.2482 & 0.1351 & 0.0887 \\
\hline & 1 & 0 & 0.4347 & 0.2578 & 0.23895 \\
\hline & 4 & 0 & 1.1785 & 0.8370 & 1.3385 \\
\hline & 7 & 0 & 1.6911 & 1.2718 & 2.4929 \\
\hline & 9 & 0 & 1.9761 & 1.5177 & 3.2649 \\
\hline \multirow{5}{*}{0.9} & 0.5 & 0 & 0.4255 & 0.3292 & 0.1355 \\
\hline & 1 & 0 & 0.7298 & 0.5969 & 0.3442 \\
\hline & 4 & $0,1.0373$ & 1.8914 & 1.6854 & 1.7215 \\
\hline & 7 & $0,1.7998$ & 2.6687 & 2.4242 & 3.0935 \\
\hline & 9 & $0,2.1818$ & 3.0968 & 2.8303 & 3.9973 \\
\hline \multirow{5}{*}{1} & 0.5 & 0 & 0.4674 & 0.3757 & 0.1449 \\
\hline & 1 & 0 & 0.7979 & 0.6745 & 0.3634 \\
\hline & 4 & 1.3744 & 2.0485 & 1.8627 & 1.7774 \\
\hline & 7 & 2.1441 & 2.8811 & 2.6601 & 3.1744 \\
\hline & 9 & 2.5481 & 3.3388 & 3.0973 & 4.0932 \\
\hline \multirow{5}{*}{4} & 0.5 & 1.3439 & 1.4376 & 1.4069 & 0.2516 \\
\hline & 1 & 2.1644 & 2.2701 & 2.2349 & 0.5257 \\
\hline & 4 & 4.9410 & 5.1202 & 5.0607 & 2.0628 \\
\hline & 7 & 6.7013 & 6.9392 & 6.8604 & 3.5516 \\
\hline & 9 & 7.6885 & 7.9315 & 7.8419 & 4.5361 \\
\hline \multirow{5}{*}{7} & 0.5 & 2.0642 & 2.1197 & 2.1012 & 0.2677 \\
\hline & 1 & 3.1775 & 3.2457 & 3.2229 & 0.5355 \\
\hline & 4 & 6.9108 & 7.0418 & 6.9982 & 2.0578 \\
\hline & 7 & 8.0469 & 9.4615 & 9.4032 & 3.5509 \\
\hline & 9 & 10.5824 & 10.7830 & 10.7164 & 4.5419 \\
\hline
\end{tabular}


Using (1.4),

$$
\begin{aligned}
f_{r: n}(x)= & \frac{1}{B(r, n-r+1)} \sum_{j=0}^{n-r} \sum_{k_{1}}^{\infty} \sum_{k_{2}}^{\infty} \ldots \\
& \sum_{k_{r+j-1}}^{\infty} \frac{1}{\beta^{s_{k}+(r+j) \alpha} P_{k}} \frac{\Gamma\left(s_{k}+(r+j) \alpha\right)}{\Gamma(\alpha)^{r+j-1}} \\
& \times\left(\begin{array}{c}
n-r \\
j
\end{array}\right) g\left(x \mid s_{k}+(r+j) \alpha, \beta, \theta\right),
\end{aligned}
$$

where $s_{k}=\sum_{i=1}^{r-j+1} k_{i}$ and $P_{k}=\prod_{i=1}^{r-j+1} k_{i}\left(k_{i}+\alpha\right)$
The result in (4.3) shows that the PDF of the $r^{\text {th }}$ order statistics of the gamma half-normal distribution can be expressed in terms of infinite sums of the gamma half-normal PDFs. Using the same technique as Ristic and Balakrishnan (2012), the asymptotic distribution of the sample minimum $X_{1: n}$ can be obtained by utilizing Theorem 8.3.6 in Arnold, et al. (1992), which states that if $\lim _{\varepsilon \rightarrow G^{-1}(0)} \frac{G(\varepsilon x)}{G(\varepsilon)}=x^{\gamma}$, then the asymptotic distribution of $X_{1: n}$ will be of Weibull type with shape parameter $\gamma$.

Figure 5: The Gamma-Half Normal Skewness and Kurtosis Graphs for Various Values of $\alpha$ and $\beta$ when $\theta=1$
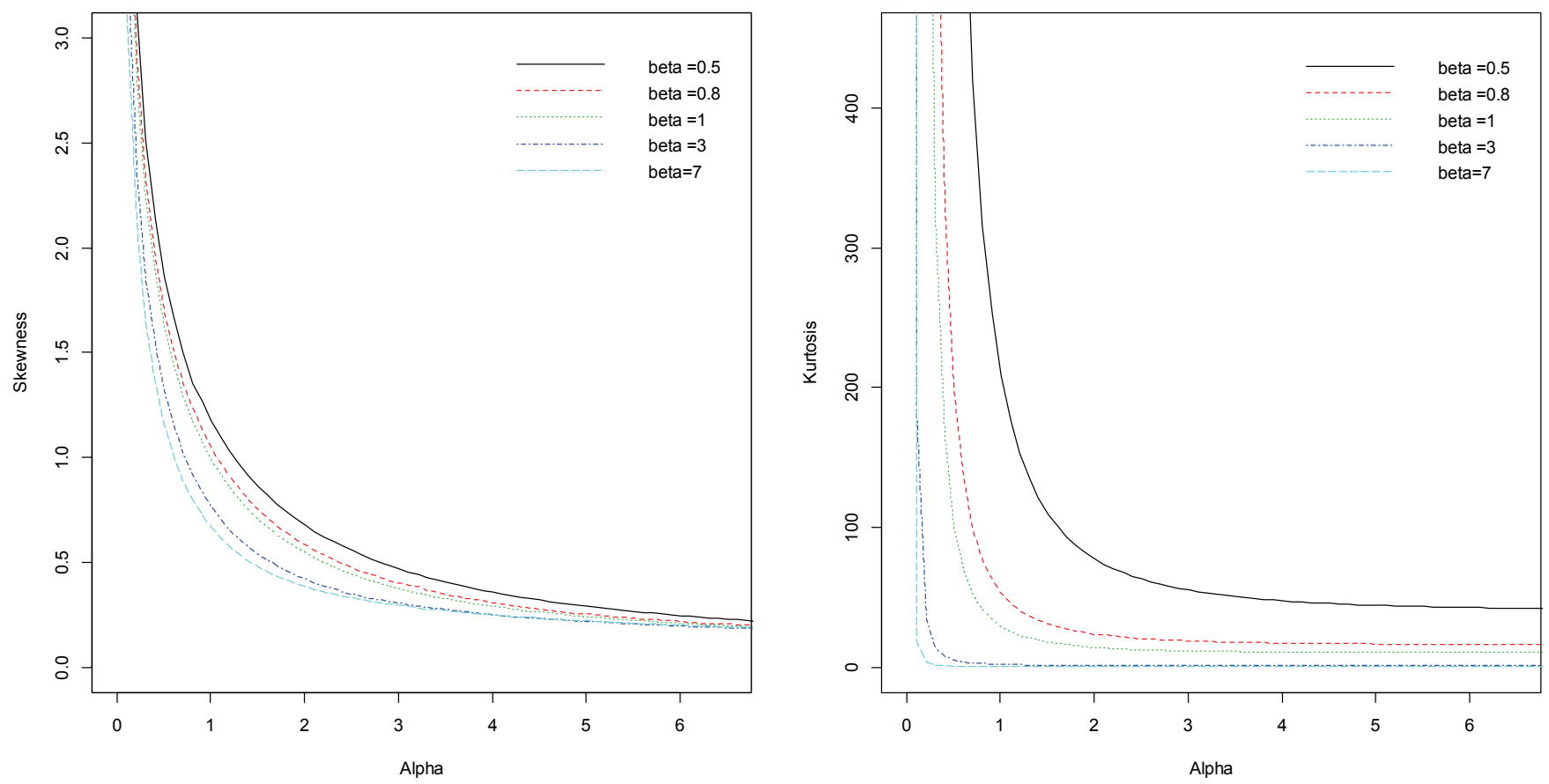
For the gamma half-normal distribution, $G^{-1}(0)=0$ and

$$
\begin{aligned}
\lim _{\varepsilon \rightarrow 0^{+}} \frac{G(\varepsilon x)}{G(\varepsilon)} & =x \lim _{\varepsilon \rightarrow 0^{+}} \frac{g(\varepsilon x)}{g(\varepsilon)} \\
& =x\left[\lim _{\varepsilon \rightarrow 0^{+}} \frac{\log (2 \Phi(-\varepsilon x / \theta))}{\log (2 \Phi(-\varepsilon / \theta))}\right]^{\alpha-1} \\
& =x\left[x \lim _{\varepsilon \rightarrow 0^{+}}\left(\frac{\phi(-\varepsilon x / \theta)}{\phi(-\varepsilon / \theta)}\right)\right]^{\alpha-1}=x^{\alpha} .
\end{aligned}
$$

Hence, the asymptotic distribution $X_{r: n}$ is of Weibull type with shape parameter $\alpha$. The asymptotic distribution of the sample maximum $X_{n: n}$ can be viewed as $G_{n}(x)$, where $G_{n}(x)=1-G_{1}(-x)$ and $G_{1}$ is the CDF of $X_{1: n}$.

\section{Parameter Estimation}

Let a random sample of size $n$ be taken from the gamma-half normal distribution. The log-likelihood function for the gamma-half normal distribution in (1.4) is given by

$$
\begin{aligned}
& \log L(\alpha, \beta)= \\
& \quad \frac{n}{2} \log 2-n \log \theta-\frac{n}{2} \log \pi \\
& -n \log \Gamma(\alpha)-\alpha n \log \beta-\frac{1}{2 \theta^{2}} \sum_{i=1}^{n} x_{i}^{2} \\
& +(\alpha-1) \sum_{i=1}^{n} \log \left(-\log \left(2 \Phi\left(-x_{i} / \theta\right)\right)\right) \\
& +\left(\frac{1}{\beta}-1\right) \sum_{i=1}^{n} \log \left(2 \Phi\left(-x_{i} / \theta\right) .\right.
\end{aligned}
$$

The derivatives of (5.1) with respect to $\alpha, \beta$ and $\theta$ respectively, are given by

$$
\begin{aligned}
& \frac{\partial \log L}{\partial \alpha}= \\
& -n \psi(\alpha)-n \log \beta+\sum_{i=1}^{n} \log \left(-\log \left(2 \Phi\left(-x_{i} / \theta\right)\right)\right) \\
& \frac{\partial \log L}{\partial \beta}=-\frac{\alpha n}{\beta}-\frac{1}{\beta^{2}} \sum_{i=1}^{n} \log \left(2 \Phi\left(-x_{i} / \theta\right)\right) \\
& \frac{\partial \log L}{\partial \theta}= \\
& \quad-\frac{n}{\theta}+\frac{1}{\theta^{3}} \sum_{i=1}^{n} x_{i}^{2} \\
& \quad+(\alpha-1) \sum_{i=1}^{n} \frac{x_{i} h_{z}\left(x_{i} / \theta\right)}{\log \left(2 \Phi\left(-x_{i} / \theta\right)\right)} \\
& \quad-\left(\frac{1}{\beta}-1\right) \sum_{i=1}^{n} x_{i} h_{z}\left(x_{i} / \theta\right)
\end{aligned}
$$

Setting (5.2), (5.3) and (5.4) to zero and solving them simultaneously results in $\hat{\alpha}, \hat{\beta}$ and $\hat{\theta}$.

The initial values for the parameters $\alpha$, $\beta$ and $\theta$ can be obtained by assuming the random sample, $x_{i}, i=1, \ldots, n$ is taken from the half-normal distribution with parameter $\theta$. By equating the population mean to the sample mean of $x_{i}, i=1, \ldots, n$ and solving for $\theta$, the initial value $\hat{\theta}=\sqrt{\pi / 2} \bar{x}$. Assuming $y_{i}=-\log \left(2 \Phi\left(-x_{i} / \hat{\theta}\right)\right), i=1, \ldots, n$ are taken from the gamma distribution with parameters $\alpha$ and $\beta$ (see Lemma 1). By equating the population mean and the population variance of gamma distribution (with parameters $\alpha$ and $\beta$ ) to the corresponding sample mean and sample variance of $y_{i}, i=1, \ldots, n$ and solving for $\alpha$ and $\beta$, the initial values are $\alpha_{0}=\bar{y}^{2} / s_{y}^{2}$ and $\beta_{0}=s_{y}^{2} / \bar{y}$, where $\bar{y}$ and $s_{y}^{2}$ are the sample mean and the sample variance for $y_{1}$, $y_{2}, \ldots, y_{n}$. 


\section{ON THE GAMMA-HALF NORMAL DISTRIBUTION AND ITS APPLICATIONS}

When $\alpha=\beta=1$, the gamma-half normal distribution reduces to the half-normal distribution; thus, the likelihood ratio test can be used to determine whether the gamma-half normal distribution or the half-normal distribution is the best model for fitting a given data set. The likelihood ratio test can be used for testing the hypothesis $H_{0}: \alpha=\beta=1$ against $H_{a}: \alpha \neq 1$ or $\beta \neq 1$, which is based on $\lambda=L_{0}(\tilde{\theta}) / L_{a}(\hat{\alpha}, \hat{\beta}, \hat{\theta})$, where $L_{0}$ and $L_{a}$ are the likelihood functions for the half-normal and the gamma-half normal distributions, respectively. The quantity $-2 \log \lambda$ follows the Chi-square distribution with 2 degrees of freedom asymptotically.

Application

Three data sets were applied to the gamma-half normal distribution, and compared with the half-normal, generalized half-normal, beta generalized half-normal and inverse Gaussian distributions. The first two data sets (see Tables 2 and 4), were analyzed by Raqab, et al. (2008). This data represents the tensile strength data measured in GPa for single-carbon fibers that were tested at gauge lengths of 20 $\mathrm{mm}$ and $10 \mathrm{~mm}$. The third data set (see Table 6) was analyzed by Cheng, et al. (1981) and represents the flood level for the Susquehanna River at Harrisburg, PA. The maximum likelihood estimates, KS (Kolmogorov-Smirnov) test-statistics and $p$-values for the fitted distributions are reported in Tables 3, 5 and 7.

The data in Tables 2 and 4 are fitted to the gamma-half normal, half-normal, generalized half-normal and beta generalizedhalf normal distributions. The half-normal distribution did not produce an adequate model for the data. However, the generalized halfnormal, beta generalized and gamma-half normal each provide a good fit for the two data sets. Among the three generalizations of the half-normal distribution, the gamma-half normal provides the best fit for the first data set, and generalized half-normal provides the best fit for the second. When graphing the first data set, an approximately symmetric distribution is obtained. The distribution of the second data set, however, is a right-skewed shape. This suggests that the gamma-half normal distribution is able to model data of both approximately symmetric and right-skewed shapes. Figures 6 and 7 display the empirical and fitted cumulative distribution functions; these figures support the results in Tables 3 and 5, respectively.

Table 2: Single Carbon Fibers at $20 \mathrm{~mm}$

\begin{tabular}{|l|l|l|l|}
\hline 0.312 & 0.314 & 0.479 & 0.552 \\
\hline 0.700 & 0.803 & 0.861 & 0.865 \\
\hline 0.944 & 0.958 & 0.966 & 0.997 \\
\hline 1.006 & 1.021 & 1.027 & 1.055 \\
\hline 1.063 & 1.098 & 1.140 & 1.179 \\
\hline 1.224 & 1.240 & 1.253 & 1.270 \\
\hline 1.272 & 1.274 & 1.301 & 1.301 \\
\hline 1.359 & 1.382 & 1.382 & 1.426 \\
\hline 1.434 & 1.435 & 1.478 & 1.490 \\
\hline 1.511 & 1.514 & 1.535 & 1.554 \\
\hline 1.566 & 1.570 & 1.586 & 1.629 \\
\hline 1.633 & 1.642 & 1.648 & 1.684 \\
\hline 1.697 & 1.726 & 1.770 & 1.773 \\
\hline 1.800 & 1.809 & 1.818 & 1.821 \\
\hline 1.848 & 1.880 & 1.954 & 2.012 \\
\hline 2.067 & 2.084 & 2.090 & 2.096 \\
\hline 2.128 & 2.233 & 2.433 & 2.585 \\
\hline 2.585 & \multicolumn{3}{|l}{} \\
\cline { 1 - 2 } & & &
\end{tabular}

The third data set (see Table 6) was analyzed by Cheng, et al. (1981) and fitted to the inverse Gaussian distribution. These results, as well as the comparisons made to the halfnormal, beta generalized half-normal and gamma-half normal distributions, are reported in Table 7. The generalized half-normal distribution was divergent for the third data set. In view of these results, the gamma half-normal and inverse Gaussian distributions give a moderate fit to the data. The half-normal distribution does not give an adequate fit to the data, while the generalized half-normal provided the best fit. In viewing the distribution of the third data set, another right-skewed distribution is observed. This confirms the fact that the gamma-half normal distribution can be used to fit data of a right-skewed shape. Figure 8 displays the empirical and fitted cumulative distribution functions. 
Table 3: Parameter Estimates for Single Carbon Fibers at $20 \mathrm{~mm}$

\begin{tabular}{|c|c|c|c|c|}
\hline Distribution & Half-Normal & $\begin{array}{c}\text { Generalized } \\
\text { Half-Normal }\end{array}$ & $\begin{array}{c}\text { Beta } \\
\text { Generalized } \\
\text { Half-Normal }\end{array}$ & $\begin{array}{c}\text { Gamma-Half } \\
\text { Normal }\end{array}$ \\
\hline $\begin{array}{c}\text { Parameter } \\
\text { Estimates }\end{array}$ & $\hat{\theta}=1.5323$ & $\begin{array}{c}\hat{\alpha}=2.2823 \\
\hat{\theta}=1.5879 \\
\hat{\mu}=0.1220\end{array}$ & $\begin{array}{c}\hat{a}=1.3742 \\
\hat{b}=0.2369 \\
\hat{\alpha}=1.8610 \\
\hat{\theta}=0.9766\end{array}$ & $\begin{array}{c}\hat{\alpha}=2.8794 \\
\hat{\beta}=3.1725 \\
\hat{\theta}=0.3934\end{array}$ \\
\hline KS & 0.3317 & 0.0548 & 0.0863 & 0.0425 \\
\hline P-value & 0.0000 & 0.9857 & 0.9985 & 0.9996 \\
\hline
\end{tabular}

Conclusion

The gamma-half normal distribution, a new generalization of the half-normal distribution, was derived using the method proposed by Alzaatreh, et al. (2013b). Various properties of the distribution were studied including the moments, mean deviations from the mean and median, hazard function, modality and Shannon entropy. The maximum likelihood method was proposed for the estimation of the gamma-half normal parameters. In order to demonstrate the applicability of the gamma-half normal distribution it was fitted to three real data sets and compared with the half-normal, generalizedhalf normal, inverse Gaussian and beta generalized half-normal distributions.

Results show that the gamma-half normal distribution provides an adequate fit for each data set. Because the distribution was fitted to data sets with right-skewed and approximately symmetric shapes, this indicates that the gamma-half normal distribution offers flexibility that extends beyond the half-normal distribution. Although the gamma-half normal distribution can be bimodal, it was difficult to find data in the literature with the specific form of bimodality. The maximum likelihood functions may be further studied under different types of censoring for future applications of the gamma-half normal distribution.

\section{References}

Alzaatreh, A., Famoye, F., \& Lee, C. (2013a).Weibull-Pareto distribution and its applications. Communications in Statistics: Theory \& Methods, 42(9), 1673-1691.

Alzaatreh, A., Lee, C., \& Famoye, F. (2013b). A new method for generating families of continuous distributions. To Appear Metron: International Journal of Statistics.

Alzaatreh, A., Famoye, F., \& Lee, C. (2012a). Gamma-Pareto distribution and its applications. Journal of Modern Applied Statistical Methods, 11(1), 78-94.

Alzaatreh, A., Lee, C., \& Famoye, F. (2012b). On the discrete analogues of continuous distributions. Statistical Methodology, 9, 589-603.

Arnold, B. C., Balakrish, N., \& Nagaraja, H. N. (1992). A first course in order statistics. New York, NY: Wiley.

Bland, J. M., \& Altman, D. G. (1999). Measuring agreement in method comparison studies. Statistical Methods in Medical Research, 8, 135-160.

Bland, J. M. (2005). The half-normal distribution method for measurement error: two case studies. Unpublished lecture available on http://wwwusers.york.ac.uk/ mb55/talks/halfnor .pdf. 


\section{ON THE GAMMA-HALF NORMAL DISTRIBUTION AND ITS APPLICATIONS}

Castro, L., Gomez, H., \& Valenzuela, M. (2012). Epsilon half-normal model: properties and inference. Computational Statistics \& Data Analysis, 56(12), 4338-4347.

Cheng, R. C. H., \& Amin, N. A. K., (1981). Maximum likelihood estimation of parameters in the inverse Gaussian distribution, with unknown origin. Technometrics, 23, 257263.

Cordeiro, G., Pescim, R., \& Ortega, E. (2012). The Kumaraswamy generalized halfnormal distribution for skewed positive data. Journal of Data Science, 10, 195-224.

Cooray, K., \& Anada, M. (2008). A Generalization of the Half-Normal distribution with applications to lifetime data. Communications in Statistics-Theory and Methods, 37, 1323-1337.

Lee, C., Famoye, F. \& Alzaatreh, A. (2013). Methods for generating families of continuous distribution. To Appear in WIREs Computational Statistics.

Nadarajah, S., \& Pal, M. (2008). Explicit expressions for moments of gamma order statistics. Bulletin of the Brazilian Mathematical Society, 39(1), 45-60.
Pescrim, R., et al. (2010). The beta generalized half- normal distribution. Computational Statistics and Data Analysis, 54, 945-957.

Raqab, M., Madi, M., \& Debasis, K. (2008). Estimation of $\mathrm{P}(\mathrm{Y}<\mathrm{X})$ for the 3parameter generalized exponential distribution. Communications in Statistics-Theory and Methods, 37(18), 2854-2864.

Renyi, A. (1961). On measures of entropy and information, In: Proceedings of the Fourth Berkeley Symposium on Mathematical Statistics and Probability, I, 547-561. Berkeley, CA: University of California Press.

Ristic, M., \& Balakrishnan, N. (2012). The gamma-exponentiated exponential distribution. Journal of Statistical Computation and Simulation, 82(8), 1191-1206.

Shannon, C. E. (1948). A mathematical theory of communication. Bell System Technical Journal, 27, 379-432.

Wolfram. http://functions.wolfram.com/ GammaBetaErf/InverseErf/06/01/02/0004/, retrieved on December 04, 2012.

Zografos, K., \& Balakrishnan, N. (2009). On families of beta and generalized gamma-generated distributions and associated inference. Statistical Methodology, 6, 344-362. 
Figure 6: CDF for Fitted Distributions for Gauge Length of 20mm Data

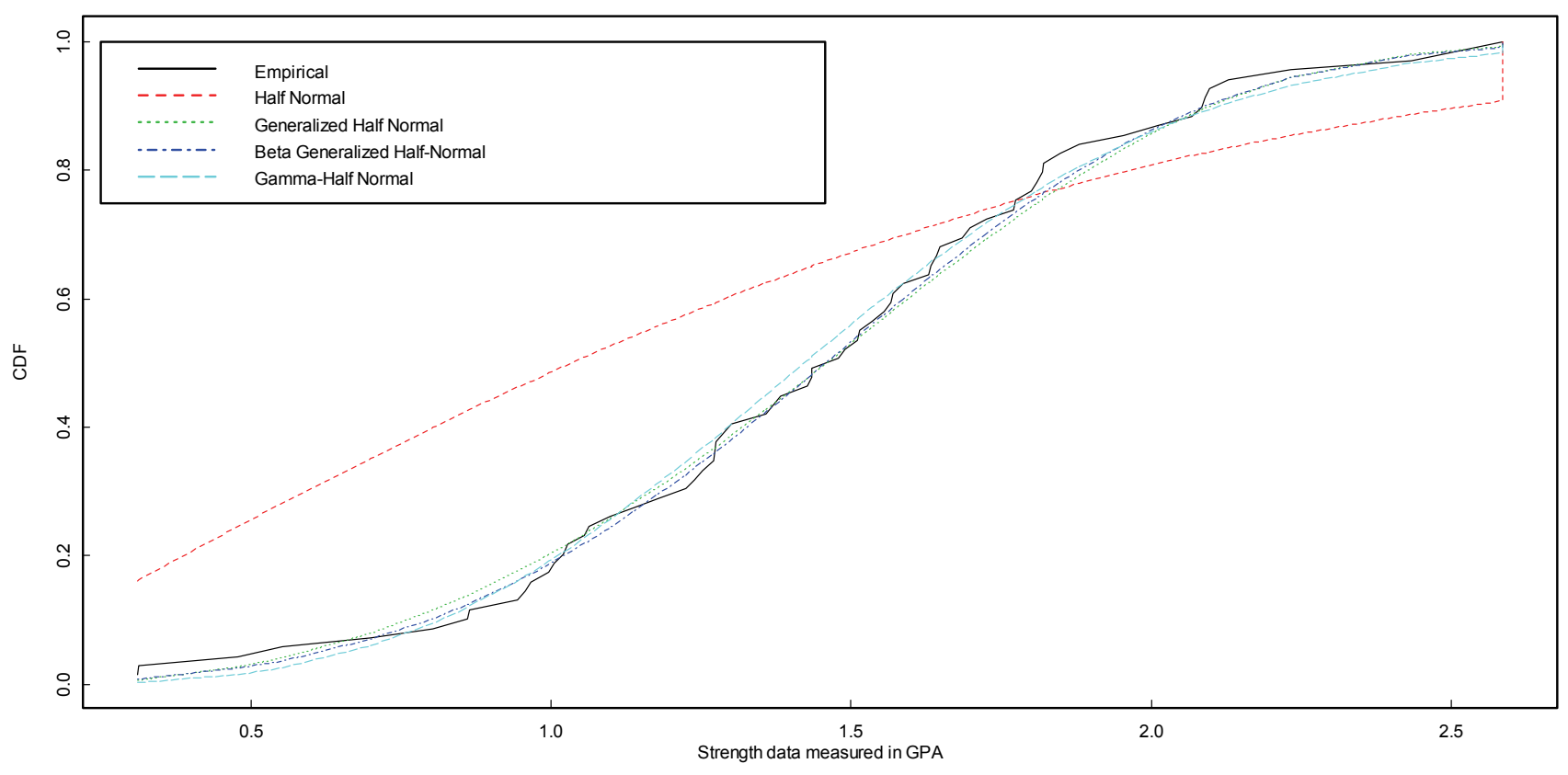

Table 4: Single Carbon Fibers at $10 \mathrm{~mm}$

\begin{tabular}{|l|l|l|l|l|l|l|l|}
\hline 0.101 & 0.332 & 0.403 & 0.428 & 0.457 & 0.550 & 0.561 & 0.596 \\
\hline 0.597 & 0.645 & 0.654 & 0.674 & 0.718 & 0.722 & 0.725 & 0.732 \\
\hline 0.775 & 0.814 & 0.816 & 0.818 & 0.824 & 0.859 & 0.875 & 0.938 \\
\hline 0.940 & 1.056 & 1.117 & 1.128 & 1.137 & 1.137 & 1.177 & 1.196 \\
\hline 1.230 & 1.325 & 1.339 & 1.345 & 1.420 & 1.423 & 1.435 & 1.443 \\
\hline 1.464 & 1.472 & 1.494 & 1.532 & 1.546 & 1.577 & 1.608 & 1.635 \\
\hline 1.693 & 1.701 & 1.737 & 1.754 & 1.762 & 1.828 & 2.052 & 2.071 \\
\hline 2.086 & 2.171 & 2.224 & 2.227 & 2.425 & 2.595 & 3.220 & \\
\hline
\end{tabular}

Table 5: Parameter Estimates for Single Carbon Fibers at 10mm

\begin{tabular}{|c|c|c|c|c|}
\hline Distribution & Half-Normal & $\begin{array}{c}\text { Generalized } \\
\text { Half-Normal }\end{array}$ & $\begin{array}{c}\text { Beta } \\
\text { Generalized } \\
\text { Half-Normal }\end{array}$ & $\begin{array}{c}\text { Gamma-Half } \\
\text { Normal }\end{array}$ \\
\hline Parameter & $\hat{\theta}=1.4019$ & $\begin{array}{c}\hat{\alpha}=1.5347 \\
\hat{\theta}=1.4798 \\
\hat{\mu}=0.0699\end{array}$ & $\begin{array}{c}\hat{a}=1.9544 \\
\hat{b}=0.1522 \\
\hat{\alpha}=1.0954 \\
\hat{\theta}=0.4722\end{array}$ & $\begin{array}{c}\hat{\alpha}=2.4260 \\
\hat{\beta}=0.4216 \\
\hat{\theta}=1.4529\end{array}$ \\
\hline KS & 0.2099 & 0.0606 & 0.0863 & 0.0678 \\
\hline P-value & 0.0078 & 0.9748 & 0.7361 & 0.9342 \\
\hline
\end{tabular}


ON THE GAMMA-HALF NORMAL DISTRIBUTION AND ITS APPLICATIONS

Figure 7: CDF for Fitted Distributions for Gauge Lengths of 10mm Data

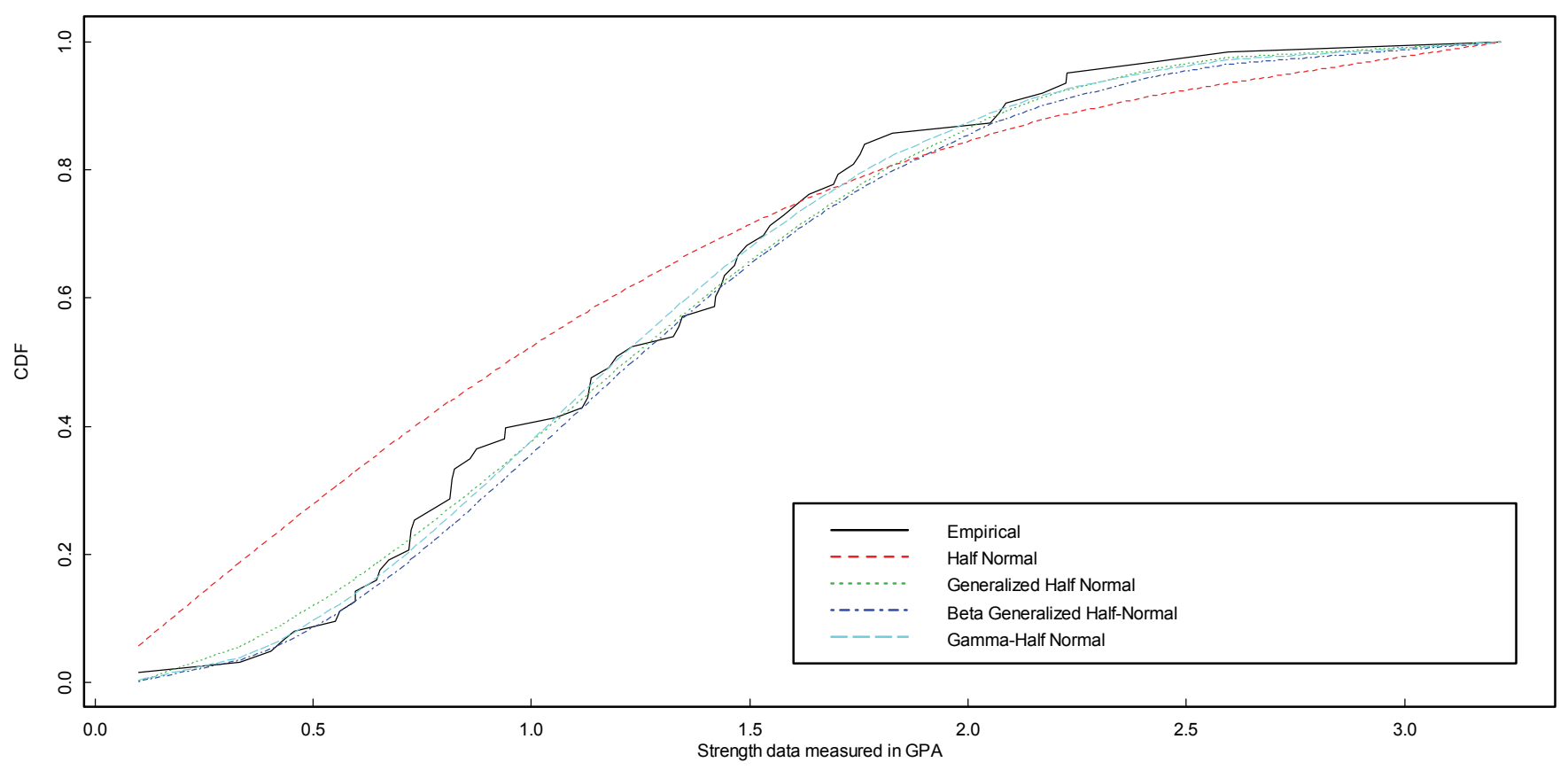


AYMAN ALZAATREH \& KRISTEN KNIGHT

Table 6: Maximum Flood Level for Susquehanna River

\begin{tabular}{|l|l|l|l|}
\hline .654 & .613 & .402 & .379 \\
\hline .269 & .740 & .416 & .338 \\
\hline .315 & .449 & .297 & .423 \\
\hline .379 & .324 & .418 & .412 \\
\hline .494 & .392 & .484 & .265 \\
\hline
\end{tabular}

Table 7: Parameter estimates for maximum flood levels

\begin{tabular}{|c|c|c|c|c|}
\hline Distribution & Half-Normal & $\begin{array}{c}\text { Generalized } \\
\text { Half-Normal }\end{array}$ & $\begin{array}{c}\text { Beta } \\
\text { Generalized } \\
\text { Half-Normal }\end{array}$ & $\begin{array}{c}\text { Gamma-Half } \\
\text { Normal }\end{array}$ \\
\hline $\begin{array}{c}\text { Parameter } \\
\text { Estimates }\end{array}$ & $\hat{\theta}=0.4404$ & Divergent & $\begin{array}{c}\hat{a}=244.48 \\
\hat{b}=176.11 \\
\hat{\alpha}=0.1901 \\
\hat{\theta}=1.2483\end{array}$ & $\begin{array}{c}\hat{\alpha}=0.1780 \\
\hat{\beta}=0.245 \\
\hat{\lambda}=0.9140\end{array}$ \\
\hline KS & 0.4026 & & 0.1507 & 0.1026 \\
\hline P-value & 0.0031 & & 0.7540 & 0.5638 \\
\hline
\end{tabular}

Figure 8: CDF for Fitted Distributions for Maximum Flood Level

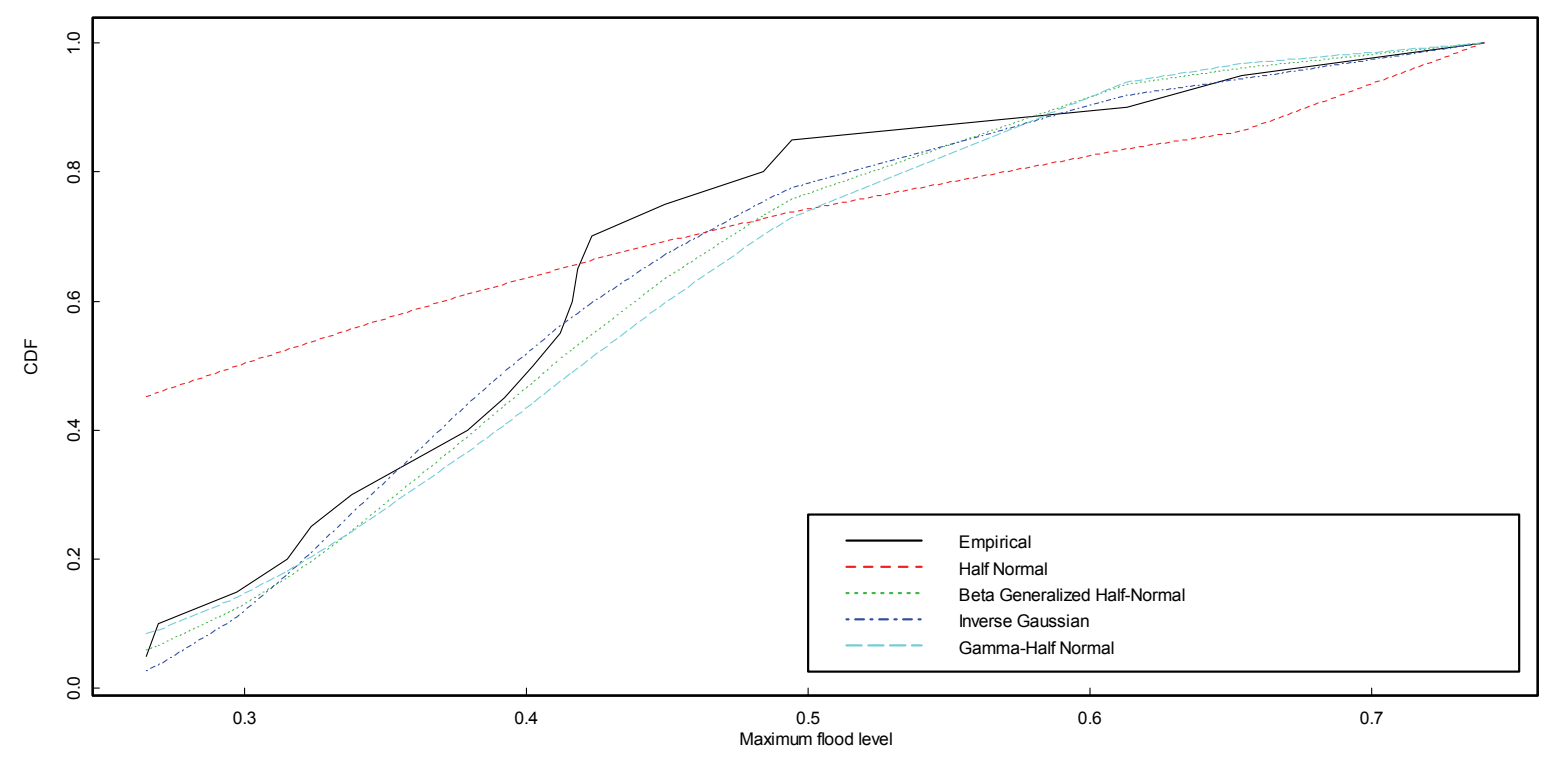




\title{
Improved Estimators in Finite Population Surveys: Theory and Applications
}

\author{
Sunil Kumar \\ SOSU, \\ Indian Statistical Institute - Kolkata
}

Improved estimators are proposed for estimating the population mean $\bar{Y}$ of the study variable $y$ using auxiliary variable $x$ in simple random sampling. Explicit expression for the bias and MSE of the proposed family are derived to the first order of approximation. The proposed estimators are compared with other estimators and theoretical findings are illustrated by two numerical examples.

Key words: Ratio estimator, product estimator, regression estimator, bias, mean square error.

Introduction

Consider a simple random sample of size $n$ drawn without replacement from a finite population $U=\left(U_{1}, U_{2}, \ldots, U_{N}\right)$ of $N$ units, let $\bar{y}$ and $\bar{x}$ be the sample mean estimates of $\bar{Y}$ and $\bar{X}$, respectively, the population means of the study variable $y$ and auxiliary variable $x$. To date in the literature of survey sampling, the efficiencies of estimators of unknown population means of a study variable $y$ have been increased by the use of known information on an auxiliary variable $x$ which is highly correlated with study variable $y$, the well-known ratio estimator is

$$
t_{R}=\bar{y} \frac{\bar{X}}{\bar{x}} .
$$

When the correlation between auxiliary variable $x$ and study variable $y$ is highly negative, then the conventional product estimator for $\bar{Y}$ is defined as

$$
t_{P}=\bar{y} \frac{\bar{x}}{\bar{X}} .
$$

Sunil Kumar is a Visiting Scientist in the SOSU, ISI Kolkata. Email him at: sunilbhougal06@gmail.com.
Theoretically, it has been established that, in general, the linear regression estimator is more efficient than the ratio and product estimators except in the case where the regression line passes through the neighborhood of the origin, thus the classical regression estimator is

$$
t_{g}=\bar{y}+b_{y x}(\bar{X}-\bar{x})
$$

Furthermore, to find more precise estimates several authors have used prior values of certain population parameter(s). Searls (1964) used known coefficient of variation (CV) of study variable at estimation stage. Sisodiya and Dwivedi (1981) extended the Searls (1964) work by using the known $\mathrm{CV}$ of the auxiliary variable for estimating population mean of study variable $y$ in a ratio method of estimation. Thus, use of prior value of coefficient of kurtosis in estimating the population variance of study variable $y$ was first conducted by Singh, et al. (1973). It was later, used by Sen (1978), Upadhyaya and Singh (1984) and Searls and Interpanich (1990) in the estimation of population mean of a study variable. Further, Singh and Tailor (2003) proposed a modified ratio estimator by using a known value of a correlation coefficient. This study suggests a new family of estimators to estimate a population mean $\bar{Y}$ of a study variable $y$ by using estimators from Khoshnevisan, et al. 


\section{SUNIL KUMAR}

(2007); the optimum cases of the suggested family of estimators are also obtained.

The Suggested Family of Estimators

A family of estimators proposed by Khoshnevisan, et al. (2007) for estimating the population mean is

$$
t=\bar{y}\left\{\frac{a \bar{X}+c}{\alpha(a \bar{x}+c)+(1-\alpha)(a \bar{X}+c)}\right\}^{g}
$$

where $a(\neq 0), c$ are either real numbers or functions of known parameters of an auxiliary variable $x$ such as Standard Deviation $\left(S_{x}\right)$, Coefficient of Variation $\left(C_{x}\right)$, Skewness $\left(\beta_{1}(x)\right)$, Kurtosis $\left(\beta_{2}(x)\right)$ and Correlation Coefficient $\left(\rho_{y x}\right)$ of the population, and $\alpha$ and $g$ are suitably chosen scalars.

The expressions of bias and MSE of the Khoshnevisan's estimator are respectively given as

$$
B(t)=\left(\frac{1-f}{n}\right) \bar{Y}\left\{\frac{g(g+1)}{2} \alpha^{2} v^{2} C_{x}^{2}-\alpha v g C_{y x}\right\}
$$

and

$$
\begin{aligned}
& \operatorname{MSE}(t)= \\
& \left(\frac{1-f}{n}\right)\left\{S_{y}^{2}+\alpha^{2} v^{2} g^{2} R^{2} S_{x}^{2}-2 \alpha v g R S_{y x}\right\}
\end{aligned}
$$

which is minimum, when $\alpha=\frac{K}{v g}$, then

$\min \cdot \operatorname{MSE}(t)=\left(\frac{1-f}{n}\right)\left(1-\rho_{y x}^{2}\right) S_{y}^{2}=\operatorname{MSE}\left(t_{g}\right)$.

Following Khoshnevisan, et al. (2007), a class of estimators for estimating the population mean $\bar{Y}$ of the study variable $y$ can be defined by combining regression estimator with $t$, the
Khoshnevisan, et al. (2007) estimator for full information case is

$T=$

$\left\{\bar{y}+b_{y x}(\bar{X}-\bar{x})\right\}\left\{\frac{a \bar{X}+c}{\alpha(a \bar{x}+c)+(1-\alpha)(a \bar{X}+c)}\right\}^{g}$,

where $\left\{\bar{y}+b_{y x}(\bar{X}-\bar{x})\right\} \quad$ is the classical regression estimator, $b_{y x}$ is the sample estimate

$$
\begin{aligned}
& \text { of } \quad \beta_{y x}, \bar{y}=(1 / n) \sum_{i=1}^{n} y_{i}, \bar{x}=(1 / n) \sum_{i=1}^{n} x_{i}, \\
& \bar{X}=(1 / N) \sum_{i=1}^{N} X_{i}, \bar{Y}=(1 / N) \sum_{i=1}^{N} Y_{i}, \\
& b_{y x}=\left(s_{y x} / s_{x}^{2}\right), \beta_{y x}=\left(S_{y x} / S_{x}^{2}\right), \\
& s_{x y}=(1 /(n-1)) \sum_{i=1}^{n}\left(y_{i}-\bar{y}\right)\left(x_{i}-\bar{x}\right), \\
& s_{x}^{2}=(1 /(n-1)) \sum_{i=1}^{n}\left(x_{i}-\bar{x}\right)^{2}, \\
& S_{x y}=(1 /(N-1)) \sum_{i=1}^{N}\left(y_{i}-\bar{Y}\right)\left(x_{i}-\bar{X}\right), \text { and } \\
& S_{x}^{2}=(1 /(N-1)) \sum_{i=1}^{N}\left(x_{i}-\bar{X}\right)^{2} .
\end{aligned}
$$

To obtain the bias and MSE of the class of estimators $T$,

$$
\begin{aligned}
\bar{y} & =\bar{Y}\left(1+\varepsilon_{0}\right), \\
\bar{x} & =\bar{X}\left(1+\varepsilon_{1}\right), \\
s_{x y} & =S_{x y}\left(1+\varepsilon_{2}\right),
\end{aligned}
$$

and

$$
s_{x}^{2}=S_{x}^{2}\left(1+\varepsilon_{3}\right)
$$

such that $E\left(\varepsilon_{i}\right)=0, \forall i=0$ to 3 ,

$$
\begin{aligned}
& E\left(\varepsilon_{0}^{2}\right)=\left(\frac{1-f}{n}\right) \frac{S_{y}^{2}}{\bar{Y}^{2}}, \quad E\left(\varepsilon_{1}^{2}\right)=\left(\frac{1-f}{n}\right) \frac{S_{x}^{2}}{\bar{X}}, \\
& E\left(\varepsilon_{0} \varepsilon_{1}\right)=\left(\frac{1-f}{n}\right) \frac{S_{y x}}{\bar{Y} \bar{X}}, \quad C_{x}=\frac{S_{x}}{\bar{X}}, C_{y}=\frac{S_{y}}{\bar{X}}, \\
& C_{y x}=\frac{S_{x y}}{\bar{Y} \bar{X}}, \\
& E\left(\varepsilon_{1} \varepsilon_{2}\right)=\left(\frac{1-f}{n}\right) \frac{\lambda_{12} S_{x}}{\bar{X} \rho_{y x}},
\end{aligned}
$$




\section{IMPROVED ESTIMATORS IN FINITE POPULATION SURVEYS}

$E\left(\varepsilon_{1} \varepsilon_{3}\right)=\left(\frac{1-f}{n}\right) \frac{\lambda_{03} S_{x}}{\bar{X}}, \lambda_{r s}=\frac{\mu_{r s}}{\mu_{20}^{r / 2} \mu_{02}^{s / 2}}$, and $\mu_{r s}=\frac{1}{N} \sum_{i=1}^{N}\left(y_{i}-\bar{Y}\right)^{r}\left(x_{i}-\bar{X}\right)^{s},(r, s)$ being non-negative integers.

Expanding $T$ in terms of $\varepsilon^{\prime} s$ results in

$T=$

$\bar{Y}\left\{1+\varepsilon_{0}-A \varepsilon_{1}\left(1+\varepsilon_{2}\right)\left(1+\varepsilon_{3}\right)^{-1}\right\}\left(1+\alpha v \varepsilon_{1}\right)^{-g}$,

where $A=\left(\beta_{y x} / R\right), R=(\bar{Y} / \bar{X}), v=\frac{a \bar{X}}{a \bar{X}+c}$.

It is assumed that $\left|\mathcal{E}_{3}\right|<1$ and $\left|\alpha v \varepsilon_{1}\right|<1$ so that $\left(1+\varepsilon_{3}\right)^{-1}$ and $\left(1+\alpha v \varepsilon_{1}\right)^{-g}$ are expandable in terms of $\mathcal{E}^{\prime} s$. Further, expanding the right hand side of (9), in terms of $\varepsilon^{\prime} s$ and neglecting terms of $\varepsilon^{\prime} s$ having power greater than two, results in

$$
(T-\bar{Y})=\bar{Y}\left\{\begin{array}{l}
\varepsilon_{0}-A \varepsilon_{1}-g \alpha v \varepsilon_{1}-g \alpha v \varepsilon_{0} \varepsilon_{1} \\
-A \varepsilon_{1} \varepsilon_{2}+A \varepsilon_{1} \varepsilon_{3}+g \alpha v \varepsilon_{1}^{2} \\
+\frac{g(g+1)}{2} \alpha^{2} v^{2} \varepsilon_{1}^{2}
\end{array}\right\} .
$$

Taking expectations of both sides on (10), give the bias of $T$ to the first degree of approximation, as

$$
\begin{aligned}
& B(T)= \\
& {\left[\frac{1}{\bar{X}}\left(\frac{1-f}{n}\right)\left\{\begin{array}{l}
\frac{g(g+1)}{2} \alpha^{2} v^{2} R S_{x}^{2} \\
+g \alpha v\left(A-\beta_{y x}\right) R S_{x}^{2}
\end{array}\right\}-\left(\frac{\lambda_{12}}{\rho_{y x}}-\lambda_{03}\right) S_{x}\right]}
\end{aligned}
$$

Squaring both sides of (10) and neglecting terms of $\varepsilon^{\prime} s$ having power greater than two, results in

$$
\begin{aligned}
(T-\bar{Y})^{2} & =\bar{Y}^{2}\left\{\varepsilon_{0}-(A+g \alpha v) \varepsilon_{1}\right\}^{2} \\
& =\bar{Y}^{2}\left\{\begin{array}{l}
\varepsilon_{0}^{2}+A^{2} \varepsilon_{1}^{2}+g^{2} \alpha^{2} v^{2} \varepsilon_{1}^{2}-2 A \varepsilon_{0} \varepsilon_{l} \\
-2 g \alpha v \varepsilon_{0} \varepsilon_{1}+2 A g \alpha v \varepsilon_{l}^{2}
\end{array}\right\}
\end{aligned}
$$

Taking expectations of both sides on (12), gives the MSE of $T$ to the first degree of approximation as

$$
\begin{aligned}
& \operatorname{MSE}(T)= \\
& \left(\frac{1-f}{n}\right)\left[S_{y}^{2}+R(A+g \alpha v)\left\{R(A+g \alpha v)-2 \beta_{y x}\right\} S_{x}^{2}\right]
\end{aligned}
$$

which is minimum when, for example, $\alpha=0=\alpha_{0}$. Thus, the resulting minimum MSE of $T$, is given by

$$
\begin{aligned}
\min \cdot \operatorname{MSE}(T) & =\left(\frac{1-f}{n}\right)\left(1-\rho_{y x}^{2}\right) S_{y}^{2} \\
& =\operatorname{MSE}\left(t_{g}\right) \\
& =\min \cdot \operatorname{MSE}(t)
\end{aligned} .
$$

The ratio-cum-regression estimators (see Table 1) are in the same family of (8) and the bias and mean squared error (MSE) in (13) for these estimators can be expressed as

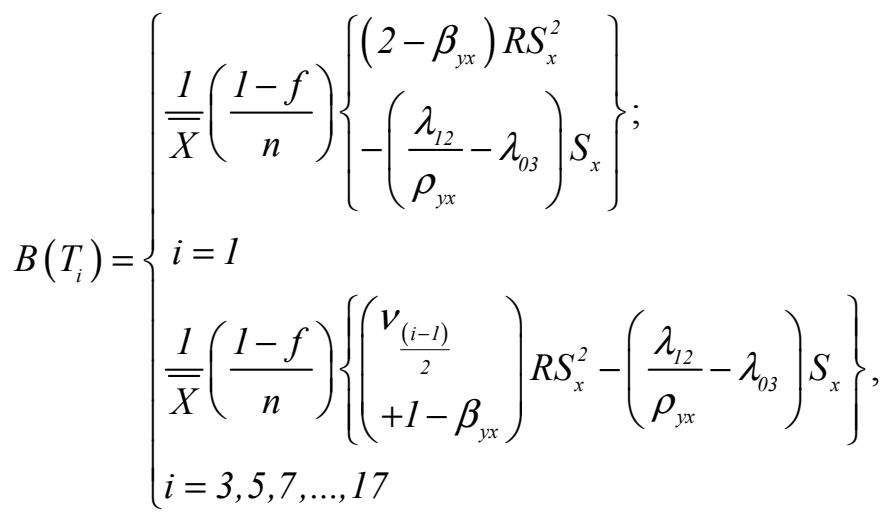

and 


\section{SUNIL KUMAR}

$$
\operatorname{MSE}\left(T_{i}\right)=\left\{\begin{array}{l}
\left(\frac{1-f}{n}\right)\left\{S_{y}^{2}-\left(\beta_{y x}^{2}-R^{2}\right) S_{x}^{2}\right\} ; \\
\left(\frac{1-f}{n}\right)\left\{S_{y}^{2}-\left(\beta_{y x}^{2}-v_{\frac{(i-I)}{2}} R^{2}\right) S_{x}^{2}\right\}, \\
i=3,5,7, \ldots, 17 .
\end{array}\right.
$$

For the product-cum-regression estimators given in Table 1, the bias and MSE of the estimators are

$B\left(T_{j}\right)=$

$\left\{\begin{array}{l}\frac{1}{\bar{X}}\left(\frac{1-f}{n}\right)\left\{\left(\beta_{y x}-1\right) R S_{x}^{2}-\left(\frac{\lambda_{12}}{\rho_{y x}}-\lambda_{03}\right) S_{x}\right\} ; \\ j=2 \\ \frac{1}{\bar{X}}\left(\frac{1-f}{n}\right)\left\{\left(\beta_{y x}-1\right) v_{(j / 2)-1} R S_{x}^{2}-\left(\frac{\lambda_{12}}{\rho_{y x}}-\lambda_{03}\right) S_{x}\right\}, \\ j=4,6,8, \ldots, 18\end{array}\right.$

$$
\operatorname{MSE}\left(T_{j}\right)=\left\{\begin{array}{l}
\left(\frac{1-f}{n}\right)\left\{S_{y}^{2}+\left(\beta_{y x}^{2}+R^{2}\right) S_{x}^{2}\right\} ; \\
j=2 \\
\left(\frac{1-f}{n}\right)\left\{S_{y}^{2}+\left(\beta_{y x}^{2}+v_{(j / 2)-l} R^{2}\right) S_{x}^{2}\right\}, \\
j=4,6,8, \ldots, 18
\end{array}\right.
$$

where $v_{1}=\frac{\bar{X}}{\bar{X}+C_{x}}, v_{2}=\frac{\beta_{2}(x) \bar{X}}{\beta_{2}(x) \bar{X}+C_{x}}$,

$$
v_{3}=\frac{C_{x} \bar{X}}{C_{x} \bar{X}+\beta_{2}(x)}, v_{4}=\frac{\bar{X}}{\bar{X}+S_{x}} \text {, }
$$

$$
\begin{aligned}
& v_{5}=\frac{\beta_{1}(x) \bar{X}}{\beta_{1}(x) \bar{X}+S_{x}}, v_{6}=\frac{\beta_{2}(x) \bar{X}}{\beta_{2}(x) \bar{X}+S_{x}}, \\
& v_{7}=\frac{\bar{X}}{\bar{X}+\rho_{y x}}, \text { and } v_{8}=\frac{\bar{X}}{\bar{X}+\beta_{2}(x)} .
\end{aligned}
$$

Many more estimators can be generated from the proposed estimator in (8) by substituting the different values of $\alpha, g, a$ and $c$.

Efficiency Comparisons

The expressions of MSE of various estimators to the first degree of approximation are

$$
\begin{aligned}
& \operatorname{Var}(\bar{y})=\left(\frac{1-f}{n}\right) S_{y}^{2} \\
& \operatorname{MSE}\left(t_{R}\right)=\left(\frac{1-f}{n}\right)\left(S_{y}^{2}+R^{2} S_{x}^{2}-2 R S_{y x}\right) \\
& \operatorname{MSE}\left(T_{1}\right)=\left(\frac{1-f}{n}\right)\left\{S_{y}^{2}-\left(\beta_{y x}^{2}-R^{2}\right) S_{x}^{2}\right\}
\end{aligned}
$$

$$
\operatorname{MSE}\left(T_{3}\right)=\left(\frac{1-f}{n}\right)\left\{S_{y}^{2}-\left(\beta_{y x}^{2}-v_{1} R^{2}\right) S_{x}^{2}\right\}
$$

$$
\operatorname{MSE}\left(T_{5}\right)=\left(\frac{1-f}{n}\right)\left\{S_{y}^{2}-\left(\beta_{y x}^{2}-v_{2} R^{2}\right) S_{x}^{2}\right\}
$$

$$
\operatorname{MSE}\left(T_{7}\right)=\left(\frac{1-f}{n}\right)\left\{S_{y}^{2}-\left(\beta_{y x}^{2}-v_{3} R^{2}\right) S_{x}^{2}\right\}
$$

$$
\operatorname{MSE}\left(T_{9}\right)=\left(\frac{1-f}{n}\right)\left\{S_{y}^{2}-\left(\beta_{y x}^{2}-v_{4} R^{2}\right) S_{x}^{2}\right\}
$$

$$
\operatorname{MSE}\left(T_{11}\right)=\left(\frac{1-f}{n}\right)\left\{S_{y}^{2}-\left(\beta_{y x}^{2}-v_{5} R^{2}\right) S_{x}^{2}\right\}
$$




\section{IMPROVED ESTIMATORS IN FINITE POPULATION SURVEYS}

Table 1: Some Members of the Family of Estimators of $T$

\begin{tabular}{|c|c|c|c|c|}
\hline $\begin{array}{l}\text { Ratio-Cum-Regression Estimators } \\
\qquad(g=1)\end{array}$ & $\begin{array}{l}\text { Product-Cum-Regression Estimators } \\
\qquad(g=-1)\end{array}$ & $\alpha$ & $a$ & $c$ \\
\hline$T_{1}=\left\{\bar{y}+b_{y x}(\bar{X}-\bar{x})\right\} \frac{\bar{X}}{\bar{x}}$ & $T_{2}=\left\{\bar{y}+b_{y x}(\bar{X}-\bar{x})\right\} \frac{\bar{x}}{\bar{X}}$ & 1 & 1 & 0 \\
\hline$T_{3}=\left\{\bar{y}+b_{y x}(\bar{X}-\bar{x})\right\}\left(\frac{\bar{X}+C_{x}}{\bar{x}+C_{x}}\right)$ & $T_{4}=\left\{\bar{y}+b_{y x}(\bar{X}-\bar{x})\right\}\left(\frac{\bar{x}+C_{x}}{\bar{X}+C_{x}}\right)$ & 1 & 1 & $C_{x}$ \\
\hline$T_{5}=\left\{\bar{y}+b_{y x}(\bar{X}-\bar{x})\right\}\left(\frac{\beta_{2}(x) \bar{X}+C_{x}}{\beta_{2}(x) \bar{x}+C_{x}}\right)$ & $T_{6}=\left\{\bar{y}+b_{y x}(\bar{X}-\bar{x})\right\}\left(\frac{\beta_{2}(x) \bar{x}+C_{x}}{\beta_{2}(x) \bar{X}+C_{x}}\right.$ & 1 & $\beta_{2}(x)$ & $C_{x}$ \\
\hline$T_{7}=\left\{\bar{y}+b_{y x}(\bar{X}-\bar{x})\right\}\left(\frac{C_{x} \bar{X}+\beta_{2}(x)}{C_{x} \bar{x}+\beta_{2}(x)}\right)$ & $T_{8}=\left\{\bar{y}+b_{y x}(\bar{X}-\bar{x})\right\}\left\{\frac{C_{x} \bar{x}+\beta_{2}(x)}{C_{x} \bar{X}+\beta_{2}(x)}\right.$ & 1 & $C_{x}$ & $\beta_{2}(x)$ \\
\hline$T_{9}=\left\{\bar{y}+b_{y x}(\bar{X}-\bar{x})\right\}\left(\frac{\bar{X}+S_{x}}{\bar{x}+S_{x}}\right)$ & $T_{10}=\left\{\bar{y}+b_{y x}(\bar{X}-\bar{x})\right\}\left(\frac{\bar{x}+S_{x}}{\bar{X}+S_{x}}\right)$ & 1 & 1 & $S_{x}$ \\
\hline$T_{11}=\left\{\bar{y}+b_{y x}(\bar{X}-\bar{x})\right\}\left(\frac{\beta_{1}(x) \bar{X}+S_{x}}{\beta_{1}(x) \bar{x}+S_{x}}\right)$ & $T_{12}=\left\{\bar{y}+b_{y x}(\bar{X}-\bar{x})\right\}$ & 1 & $\beta_{1}(x)$ & $S_{x}$ \\
\hline$T_{13}=\left\{\bar{y}+b_{y x}(\bar{X}-\bar{x})\right\}\left(\frac{\beta_{2}(x) \bar{X}+S_{x}}{\beta_{2}(x) \bar{x}+S_{x}}\right)$ & $T_{14}=\left\{\bar{y}+b_{y x}(\bar{X}-\bar{x})\right\}\left(\frac{\beta_{2}(x) \bar{x}+S_{x}}{\beta_{2}(x) \bar{X}+S_{x}}\right.$ & 1 & $\beta_{2}(x)$ & $S_{x}$ \\
\hline$T_{15}=\left\{\bar{y}+b_{y x}(\bar{X}-\bar{x})\right\}\left(\frac{\bar{X}+\rho_{y x}}{\bar{x}+\rho_{y x}}\right)$ & $T_{16}=\left\{\bar{y}+b_{y x}(\bar{X}-\bar{x})\right\}\left(\frac{\bar{x}+\rho_{y x}}{\bar{X}+\rho_{y x}}\right)$ & 1 & 1 & $\rho_{y x}$ \\
\hline$T_{17}=\left\{\bar{y}+b_{y x}(\bar{X}-\bar{x})\right\}\left(\frac{\bar{X}+\beta_{2}(x)}{\bar{x}+\beta_{2}(x)}\right)$ & $T_{18}=\left\{\bar{y}+b_{y x}(\bar{X}-\bar{x})\right\}\left(\frac{\bar{x}+\beta_{2}(x)}{\bar{X}+\beta_{2}(x)}\right.$ & 1 & 1 & $\beta_{2}(x)$ \\
\hline
\end{tabular}




\section{SUNIL KUMAR}

$$
\begin{aligned}
& \operatorname{MSE}\left(T_{13}\right)=\left(\frac{1-f}{n}\right)\left\{S_{y}^{2}-\left(\beta_{y x}^{2}-v_{6} R^{2}\right) S_{x}^{2}\right\} \\
& \operatorname{MSE}\left(T_{15}\right)=\left(\frac{1-f}{n}\right)\left\{S_{y}^{2}-\left(\beta_{y x}^{2}-v_{7} R^{2}\right) S_{x}^{2}\right\} \\
& \operatorname{MSE}\left(T_{17}\right)=\left(\frac{1-f}{n}\right)\left\{S_{y}^{2}-\left(\beta_{y x}^{2}-v_{8} R^{2}\right) S_{x}^{2}\right\} .
\end{aligned}
$$

The efficiency comparison of the proposed estimator $T$ at its optimum with the usual unbiased estimator $\bar{y}$, ratio estimator $t_{r}$ and $T_{i} ; i=1,3,5,7, \ldots, 17$, the results are:

$$
\begin{gathered}
\operatorname{Var}(\bar{y})-\min \cdot M S E(T)=\rho_{y x}^{2} S_{y}^{2}>0 \\
\operatorname{MSE}\left(t_{R}\right)-\min \cdot \operatorname{MSE}(T)=\left\{R^{2}+\beta_{y x}\left(\beta_{y x}-2 R\right)\right\} S_{x}^{2}>0 \\
\operatorname{MSE}\left(T_{1}\right)-\min \cdot \operatorname{MSE}(T)=R^{2} S_{x}^{2}>0 \\
\operatorname{MSE}\left(T_{3}\right)-\min \cdot \operatorname{MSE}(T)=v_{1} R^{2} S_{x}^{2}>0 \\
\operatorname{MSE}\left(T_{5}\right)-\min \cdot \operatorname{MSE}(T)=v_{2} R^{2} S_{x}^{2}>0 \\
\operatorname{MSE}\left(T_{7}\right)-\min \cdot \operatorname{MSE}(T)=v_{3} R^{2} S_{x}^{2}>0 \\
\operatorname{MSE}\left(T_{9}\right)-\min \cdot \operatorname{MSE}(T)=v_{4} R^{2} S_{x}^{2}>0 \\
\operatorname{MSE}\left(T_{11}\right)-\min \cdot \operatorname{MSE}(T)=v_{5} R^{2} S_{x}^{2}>0 \\
\operatorname{MSE}\left(T_{13}\right)-\min \cdot \operatorname{MSE}(T)=v_{6} R^{2} S_{x}^{2}>0 \\
\operatorname{MSE}\left(T_{15}\right)-\min \cdot \operatorname{MSE}(T)=v_{7} R^{2} S_{x}^{2}>0 \\
\operatorname{MSE}\left(T_{17}\right)-\min \cdot \operatorname{MSE}(T)=v_{8} R^{2} S_{x}^{2}>0 \\
\operatorname{MSE}\left(t_{R}\right)-\operatorname{Var}(\bar{y})<0 ; \text { if } R<2 \beta_{y x}
\end{gathered}
$$

$$
\begin{gathered}
\operatorname{MSE}\left(T_{1}\right)-\operatorname{Var}(\bar{y})<0 ; \text { if } R^{2}<\beta_{y x}^{2} \\
\operatorname{MSE}\left(T_{i}\right)-\operatorname{Var}(\bar{y})<0 ; \text { if } v_{j} R^{2}<\beta_{y x}^{2} ; \\
i=3,5,7,9,11,13,15,17 \text { and } j=1 \text { to } 8 .
\end{gathered}
$$

If these defined conditions are true, then the proposed estimator is more efficient than the usual unbiased estimator $\bar{y}$ and unbiased ratio estimator $t_{R}$.

Empirical Study

Population I (Koyuncu \& Kadilar, 2009)

Data concerning primary and secondary schools for 923 districts of Turkey in 2007 (Source: Ministry of Education, Republic of Turkey), taking the number of teachers as study variable and the number of students as auxiliary variable in both primary and secondary schools. A sample of size $n=180$ was selected from the dataset and the correlations observed between auxiliary and study variables are positive. Therefore, ratio estimators were used for the estimation of the population mean. The summary statistics about the population are: $N=923, \quad n=180, \quad S_{y}=749.9395$, $S_{x}=21331.1315, \quad \bar{Y}=436.4345$, $\bar{X}=11440.4984, \quad \rho_{y x}=0.9543$, $\beta_{2}(x)=18.7208$ and $\beta_{1}(x)=3.9365$.

Population II (Singh, 2001)

Consider the data used by Anderson (1958). The parameters of the population consist of 25 families as follows: $y$ : head length of second son; $x$ : head breadth of first son; $N=25, n=7, C_{y}=0.0546, C_{x}=0.0488$, $\bar{Y}=183.84, \quad \bar{X}=151.12, \quad \rho_{y x}=0.6932$, $\beta_{2}(x)=2.6519$, and $\beta_{1}(x)=0.0002$.

Here, the percent relative efficiencies (PRE) of different suggested estimators were computed with respect to the usual unbiased estimator $\bar{y}$ for both populations. The outcomes are shown in Table 2. 


\section{IMPROVED ESTIMATORS IN FINITE POPULATION SURVEYS}

Table 2: Percent Relative Efficiencies of Different Suggested Ratio Type

Estimators with Respect to the Usual Unbiased Estimator $\bar{y}$

\begin{tabular}{|c|c|c|c|}
\hline \multicolumn{2}{|c|}{ Population I } & \multicolumn{2}{c|}{ Population II } \\
\hline $\begin{array}{c}\text { Ratio Type } \\
\text { estimators }\end{array}$ & PRE(., $\bar{y})$ & $\begin{array}{c}\text { Ratio Type } \\
\text { estimators }\end{array}$ & PRE(., $\bar{y})$ \\
\hline $\bar{y}$ & 100.00 & $\bar{y}$ & 100.00 \\
\hline$t_{r}$ & 939.7177 & $t_{r}$ & 178.6661 \\
\hline$T_{1}$ & 78.94465 & $T_{1}$ & 75.85505 \\
\hline$T_{3}$ & 78.95661 & $T_{3}$ & 75.86713 \\
\hline$T_{5}$ & 78.94529 & $T_{5}$ & 75.85961 \\
\hline$T_{7}$ & 79.00904 & $T_{7}$ & 87.91403 \\
\hline$T_{9}$ & 199.8646 & $T_{9}$ & 78.05581 \\
\hline$T_{11}$ & 112.5773 & $T_{11}$ & 191.3018 \\
\hline$T_{13}$ & 86.20194 & $T_{13}$ & 76.6948 \\
\hline$T_{15}$ & 78.95077 & $T_{15}$ & 6.02636 \\
\hline$T_{17}$ & 79.06471 & $T_{17}$ & 76.50771 \\
\hline$T_{(\text {opt. })}$ & 1119.6777 & $T_{(o p t .)}$ & 192.5025 \\
\hline
\end{tabular}

\section{Conclusion}

It is envisaged from Table 2 that the proposed estimator at its optimum is more efficient than among all discussed estimators. Also, the estimators $T_{1}, T_{3}, T_{5}, T_{7}, T_{13}, T_{15}$ and $T_{17}$ are less efficient than the usual unbiased estimator because the condition $v_{j} R^{2}<\beta_{y x}^{2}$; $i=3,5,7,9,11,13,15,17$ and $j=1$ to 8 is not satisfied for both the populations.

\section{References}

Anderson, T. W. (1958). An introduction to multivariate statistical analysis. New York, NY: John Wiley \& Sons, Inc.

Khoshnevisan, M., et al. (2007). A general family of estimators for estimating population mean using known value of some population parameter(s). Far East Journal of Theoretical Statistics, 22(2), 181-191.
Koyuncu, N., \& Kadilar, C. (2009). Efficient estimators for the population mean. Hacettepe Journal of Mathematics and Statistics, 38(2), 217-225.

Searls, D. T. (1964). The utilization of known coefficient of variation in the estimation procedure. Journal of the American Statistics Association, 59, 1225-1226.

Searls, D. T., \& Intarapanich, P. (1990).A note on an estimator for the variance that utilizes the kurtosis. The American Statistician, 44(4), 295-296.

Sen, A. R. (1978). Estimation of the population mean when the coefficient of variation is known. Communication in Statistics, Theory and Methods, 7, 657-672.

Singh, G. N. (2001). On the use of transformed auxiliary variable in estimation of population mean in two phase sampling. Statistics in Transition, 5(3), 405-416. 


\section{SUNIL KUMAR}

Singh, J., Pandey, B. N., \& Hiran, K. (1973). On the utilization of a known coefficient of kurtosis in the estimation procedure of variance. Annals of the Institute of Statistical Mathematics, 25, 51-55.

Singh, H. P., \& Tailor, R. (2003). Use of known correlation coefficient in estimating the finite population mean. Statistics in Transition,6, 555-560.
Sisodia, B. V. S., \& Dwivedi, V. K. (1981).A modified ratio estimator using coefficient of variation of auxiliary variable. Journal of Indian Society Agricultural Statistics, 33, 13-18.

Upadhyaya, L. N., \& Singh, H. P. (1984).On the estimator of the population mean with known coefficient of variation. Biometrical Journal, 26(8), 915-922. 


\section{An Alternative Approach to Reduce Dimensionality in Data Envelopment Analysis}

\author{
Grace Lee Ching Yap \\ The University of Nottingham Malaysia Campus, \\ Selangor Darul Ehsan, Malaysia
}

\author{
Wan Rosmanira Ismail Zaidi Isa \\ Universiti Kebangsaan Malaysia, \\ Selangor Darul Ehsan, Malaysia
}

Principal component analysis reduces dimensionality; however, uncorrelated components imply the existence of variables with weights of opposite signs. This complicates the application in data envelopment analysis. To overcome problems due to signs, a modification to the component axes is proposed and was verified using Monte Carlo simulations.

Key words: Data envelopment analysis, principal component analysis, redundancy analysis, Monte Carlo simulation.

\begin{abstract}
Introduction
Data envelopment analysis (DEA), first introduced by Charnes, et al. (1978), serves as a tool for relative performance evaluation and benchmarking among decision making units (DMUs) with common inputs and outputs. In many circumstances, researchers may be faced with too many variables (inputs and outputs) involved in a performance measure: This will distort the discerning power of the analysis if the number of observations cannot be increased accordingly due to the curse of dimensionality (Daraio, et al., 2007). There are several approaches to increasing discrimination between observations. Based on reviews by AnguloMeza and Lins (2002) and Podinovski and Thanassoulis (2007), the most popular
\end{abstract}

Grace Yap is an Assistant Professor of Applied Mathematics at the University of Nottingham Malaysia. Her research interests include data analysis, efficiency analysis and time series. Email her at: grace.yap@nottingham.edu.my. Wan Rosmanira Ismail is a Senior Lecturer in the Faculty of Science and Technology in the School of Mathematical Sciences. Email her at: wrismail@ukm.my. Zaidi Isa is a Professor in the Faculty of Science and Technology in the School of Mathematical Sciences. Email him at: zaidiisa@ukm.my. approaches used are super efficiency (Andersen \& Petersen, 1993) and cross-efficiency (Doyle \& Green, 1994; Green, et al., 1996; Sexton, et al., 1986). These approaches do not attempt to reduce dimensionality but, by using complete information, they involve additional procedures to rank the observations. Conversely, to increase discrimination, researchers may consider keeping a reasonable dimensionality in a DEA model. Dyson, et al. (2001) indicated that the number of observations must be at least $2 p \times q$ where $p \times q$ is the product of the number of inputs and outputs; thus, practitioners should be parsimonious in numbers of inputs and outputs. Although it is tempting to omit correlated variables in order to increase discrimination, Dyson, et al. (2001) showed that omitting even highly correlated variables could have a significant effect on computed efficiency scores.

Several approaches address issues of determining relevant variables, including: aggregates (Simar \& Wilson, 2001), variable reduction (VR) (Jenkins \& Anderson, 2003), principal component analysis (PCA-DEA) (Alder \& Golany, 2001, 2002; Alder \& Yazhemsky, 2010; Ueda \& Hoshiai 1997), efficiency contribution measure (ECM) (Pastor, et al., 2002) and regression-based test (RB) (Ruggiero, 2005). These approaches were compared and reviewed by Sirvent, et al. (2005), Alder \& Yazhemsky (2010) and Nataraja \& Johnson (2011). Their analyses showed that the aggregates method requires the longest run time and its performance is not satisfactory. ECM 
performs moderately well under most scenarios, but it requires a long run time. The performance of RB is not as good as ECM, but its run time is significantly shorter than that of ECM. RB performs worst when variables are highly correlated; this is due to misspecification because the correlated variables would not be identified as part of the production process. Under such a scenario, PCA-DEA outperforms the other methods because it considers all original variables in the form of principal components. Most importantly, PCA-DEA involves the smallest run time due to its noniterative characteristic. Unfortunately, PCADEA may not work well when data are high dimensional, meaning that some variables with weak correlation are included in the dataset. Under such a condition, these variables may cloud the principal components' dominant attributes and, consequently, the efficiency estimation is corrupted. This problem becomes less severe as the correlation between variables increases. Thus, it may be concluded that PCADEA is preferable when all variables are known to be relevant, and performance improves as the correlation between variables increases. In addition, PCA-DEA is robust to sample size.

Alternative to principal components, Kao, et al. (2011) proposed independent components to be used as new variables in a DEA model. The independent components are generated from independent component analysis (ICA) which is viewed as an extension of PCA in the sense that it not only de-correlates the data, but it also reduces high order statistical dependencies (Lee, 1998). However, ICA does not overcome the problem of PCA-DEA. Because PCA is popular due to its undemanding nature to reduce the dimensionality, this study focuses on the use of principal components in DEA.

PCA reorients multivariate data so that the first few dimensions account for as much of the information as possible. To be uncorrelated to each other amongst the principal components, the underlying eigenvectors must be orthogonal. This implies the existence of variables with opposite signs within a principal component because the principal components are constructed based on a mixture of positive and negative weights due to the eigenvectors. This research finds that these principal components are not suitable to replace the original variables in a DEA model as they violate the disposability assumption, consequently, meaningful efficiency estimates may not be feasible. In addition, the existence of positive and negative weights within a principal component may give rise to the problem of unboundedness in the linear program of a DEA model that uses principal components as input and/or output variables.

Although available literature does not report such a problem caused by principal components, the possibility exists for obtaining an unbounded feasible region due to the effect of positive and negative weights in the constraints of a linear program. To avoid these problems, this article proposes modifying the weights to form the principal components. As modifications to the principal components may misrepresent the original dataset, a procedure that leads to a minimal alteration is sought. The viability of such modification will be justified via a redundancy analysis whereby the proportion of explained variation in an original dataset is examined. To ascertain the motivation of such modification, the accuracy of this proposed method will be compared with the results of the standard DEA.

Reviews on Data Envelopment Analysis Model and Principal Component Analysis: Data Envelopment Analysis (DEA) Model

Data envelopment analysis (DEA) is a non-parametric method of measuring the efficiency of a decision making unit (DMU) with multiple inputs and outputs without predefining a production function. Following standard economic theory, the production set must be a set that contains all the input-output correspondences that are feasible in principle. The framework is similar to that in Daraio and Simar (2007), Kneip, et al. (1998), Kneip, et al. (2008) and Simar and Wilson (1998; 2000a). To illustrate, let there be a vector of $p$ inputs, $x \in \mathrm{R}_{+}^{p}$ and a vector of $q$ outputs $y \in \mathrm{R}_{+}^{q}$. The production set may be defined as:

$$
\psi=\left\{(x, y) \in \mathrm{R}_{+}^{p+q} \mid x \text { that can produce } y\right\} .
$$




\section{APPROACH TO REDUCE DIMENSIONALITY IN DATA ENVELOPMENT ANALYSIS}

Specifically, the production set is assumed to be closed and strictly convex (Shephard, 1970; Fare, 1998), with the assumption of monotonicity of technology both inputs and outputs are strongly disposable. This can be described as:

$$
\begin{gathered}
\text { If }(x, y) \in \psi \text {, then for any } \\
\left(x^{\prime}, y^{\prime}\right) \text { such that } x^{\prime} \geq x \text { and } \\
y^{\prime} \leq y,\left(x^{\prime}, y^{\prime}\right) \in \psi
\end{gathered}
$$

Consequently, the DMUs that are relatively efficient will lie on the production frontier. In the input orientation, the production frontier $\partial X(y)$ is defined as:

$\partial X(y)=\{x \mid(x, y) \in \psi,(e x, y) \notin \psi, \forall 0<e<1\}$.

Based on the efficient front of the production set, the Debreu-Farrell input measure of efficiency can be computed in a radial direction orthogonal to $y$, defined as follows:

$$
e(x, y)=\inf \{e \mid(e x, y) \in \psi, e>0\}
$$

In practice, with the strong disposability and constant returns-to-scale assumptions, the DEA estimator of $\psi$ is the conical hull of the free disposal hull of an observed sample with inputs $\mathbf{X}=\left[x_{i}\right]$ and outputs $\mathbf{Y}=\left[y_{i}\right], i=1, \cdots, n, x_{i}$ where $y_{i}$ is the column vectors of $p$ inputs and $q$ outputs. The DEA estimator of $\psi$ is given by

$$
\widehat{\psi}=\{(x, y) \mid y \leq \mathbf{Y} \lambda, \quad x \geq \mathbf{X} \lambda, \quad \lambda \geq 0\}
$$

where $\lambda=$ column vector of $n$ non-negative variables.

The measure of efficiency is estimated using a linear programming model:

$$
\begin{aligned}
& \hat{e}(x, y)= \\
& \min \left\{\begin{array}{l}
e>0 \mid y=\mathbf{Y} \lambda-s_{y}, \text { ex }=\mathbf{X} \lambda+s_{x}, \\
\lambda, s_{y}, s_{x} \geq 0
\end{array}\right\}
\end{aligned}
$$

where $s_{y}=$ column $q$-vector of output slack variables and $s_{x}=$ column $p$-vector of input excess variables.

It is observed that the mechanism underlying this method depends largely on the constraints imposed on the model. When there are too many constraints, desirable solutions might be ruled out. In the context of DEA, this might lead to the problem of overestimating the efficiencies due to sparsity bias (Smith, 1997; Pedraja-Chaparro, et al. 1999). To avoid this problem, Simar and Wilson (2000b) suggested that the number of DMUs must increase exponentially with the addition of variables. Based on their bootstrap results, there must be at least 25 DMUs involved for the case of single input and output. For the same scenario, more than 100 DMUs are needed to have an almost exact confidence interval of the efficiency estimator. Unfortunately, this is almost impossible to achieve as large samples are generally not available in practice. This illustrates the need for discrimination improving methodologies. Because DEA is a nonparametric method, the principal component analysis (PCA) seems to be a good choice and this method has been proposed by some researchers (Ueda \& Hoshiai, 1997; Alder \& Golany, 2001, 2002; Alder \& Yazhemsky, 2010). However, noting that PCA might violate the assumption of non-negative data in DEA, possible approaches to improve the construct of principal components for the use in DEA must be sought.

Reviews on Data Envelopment Analysis Model and Principal Component Analysis: Principal Component Analysis (PCA)

Principal component analysis (PCA) is a statistical technique that reorients a dataset so that the first few dimensions account for as much information as possible. These dimensions are represented by the principal components, which are in the form of uncorrelated weighted linear combinations of the original variables that capture the maximum variance. The uncorrelated property is imposed in order to rule out the possibility of overlapped variation. These weights can be found by Eigen-decomposition, where the correlation matrix of the original set 
of variables is taken as the basis for PCA. To illustrate, let there be $p$ original standardized variables $\tilde{x}_{i}$ of size $n \times 1, i=1, \ldots, p$ with the matrix $\mathbf{X}=\left[\tilde{x}_{1}, \tilde{x}_{2}, \ldots, \tilde{x}_{p}\right]$. The correlation matrix of these variables is a $p \times p$ matrix $\mathbf{R}$. The decomposition of the correlation matrix $\mathbf{R}$ is

$$
\begin{aligned}
\mathbf{R} & =\mathbf{V} \mathbf{L} \mathbf{V}^{\mathrm{T}} \\
& =\left[\begin{array}{llll}
v_{1} & v_{2} \cdots & v_{p}
\end{array}\right]\left[\begin{array}{cccc}
\beta_{1} & 0 & \cdots & 0 \\
0 & \beta_{2} & & 0 \\
\vdots & & \ddots & \vdots \\
0 & 0 & \cdots & \beta_{p}
\end{array}\right]\left[v_{1} v_{2} \cdots v_{p}\right]^{T}
\end{aligned}
$$

where $v_{j}=j^{\text {th }}$ eigenvector of size $p \times 1, j=1$, $\ldots, p$ and $\beta_{j}=j^{\text {th }}$ eigenvalue that corresponds to $v_{j}$ eigenvector, $j=1, \ldots, p$.

Note that the eigenvalues represent the explained variation the principal components, thus, they are arranged such that $\beta_{1} \geq \beta_{2} \geq \ldots \geq$ $\beta_{p} \geq 0$. The corresponding principal components $\mathbf{K}=\left[\gamma_{j}\right]^{T}$, with $\gamma_{j}$ being the column vector of $j$ principal component, $j=1, \ldots, p$ are constructed based on the weights obtained from the eigenvectors:

$$
\begin{aligned}
& \mathbf{K}=\mathbf{V}^{T} \mathbf{X} \\
& \text { i.e., } \\
& \gamma_{1}=v_{11} \tilde{x}_{1}+v_{21} \tilde{x}_{2}+\cdots+v_{p 1} \tilde{x}_{p} \\
& \gamma_{2}=v_{12} \tilde{x}_{1}+v_{22} \tilde{x}_{2}+\cdots+v_{p 2} \tilde{x}_{p} \\
& \vdots \\
& \gamma_{p}=v_{1 p} \tilde{x}_{1}+v_{2 p} \tilde{x}_{2}+\cdots+v_{p p} \tilde{x}_{p}
\end{aligned}
$$

where $v_{i j}=i^{\text {th }}$ entry of $j^{\text {th }}$ eigenvector, $i, j=1, \ldots$, $p$.

For the purpose of dimension reduction, Kaiser's rule is typically followed to choose the principal components whose eigenvalues are greater than 1; otherwise, an elbow in the Scree plot may be identified to determine the number of components to be retained. In the context of DEA, Adler and Yazhemsky (2010) noted that it is ideal to drop the principal components oneby-one until a reasonable level of discrimination is achieved or until the principal components capture at least $80 \%$ of the variance of the original data. These principal components are then used to replace the targeted inputs or outputs in the DEA model. Adapting to the additive DEA model with constant returns-toscale (CRS) of Charnes, et al. (1985), a mixture of original data and principal components may be used to arrive at the additive model as described by Adler and Yazhemsky (2010). Equivalently, the model can be written in the form of input oriented, CRS, radial linear program as in equation (2.6).

\section{Contrast Variables in Principal Components}

Because the eigenvectors are orthogonal, there must be a mixture of positive and negative entries $v_{i j}, i, j=1, \ldots, p$ within them. To illustrate, consider the first eigenvector to be $v_{1}=\left\langle v_{11} v_{21} \cdots v_{p 1}\right\rangle^{T}$. Even if $v_{1}$ has all positive entries, note that in order to be orthogonal to $v_{1}$, the second eigenvector $v_{2}=\left\langle v_{12} v_{22} \cdots v_{p 2}\right\rangle^{T}$ must satisfy the equation:

$$
\begin{aligned}
& v_{1} \cdot v_{2}=0 \\
& \text { i.e., } \\
& v_{11} v_{12}+v_{21} v_{22}+\cdots+v_{p 1} v_{p 2}=0
\end{aligned}
$$

Thus, it is straightforward to conclude that $v_{2}=\left\langle v_{12} v_{22} \cdots v_{p 2}\right\rangle^{T}$ consists of a mixture of positive and negative entries, for example $v_{12}, v_{22}>0$ and $v_{32}, \cdots, v_{p 2}<0$.

For the corresponding principal component $\gamma_{2}=v_{12} \tilde{x}_{1}+v_{22} \tilde{x}_{2}+\cdots+v_{p 2} \tilde{x}_{p}$, the variables $\tilde{x}_{1}$ and $\tilde{x}_{2}$ are in contrast with the other variables $\tilde{x}_{3}, \ldots, \tilde{x}_{p}$ as $\tilde{x}_{1}$ and $\tilde{x}_{2}$ correlate positively with $\gamma_{2}$ but $\tilde{x}_{3}, \ldots, \tilde{x}_{p}$ correlate negatively with $\gamma_{2}$. To use principal components in a DEA model it is good to avoid variables with counter effect within a principal component. To simplify the label, the group of variables that capture a smaller portion of sum of squared loadings (SSL) of a principal 


\section{APPROACH TO REDUCE DIMENSIONALITY IN DATA ENVELOPMENT ANALYSIS}

component are called contrast variables. Particularly for this illustration, the proportion of SSL for $\left\{\tilde{x}_{1}, \tilde{x}_{2}\right\}$ in $\gamma_{2}$ is $\operatorname{SSL}_{\gamma_{2}(+)}=\frac{v_{12}^{2}+v_{22}^{2}}{v_{12}^{2}+v_{22}^{2}+\ldots+v_{p 2}^{2}}$. Thus, if $\operatorname{SSL}_{\gamma_{2}(+)}<\frac{1}{2}$, then $\tilde{x}_{1}$ and $\tilde{x}_{2}$ are the contrast variables in $\gamma_{2}$, and they are to be avoided in the construct of $\gamma_{2}$.

In a very unfortunate (and unlikely) case if $\mathrm{SSL}_{\gamma_{2}(+)}=\frac{1}{2}$, the contrast variables may be classified to the group $\left\{\tilde{x}_{1}, \tilde{x}_{2}\right\}$ or $\left\{\tilde{x}_{3}, \cdots, \tilde{x}_{p}\right\}$ that consists of the variables that have not been labeled as contrast variables in other principal components; this is to minimize the loss of information when the components are used to replace the original variables in a DEA model. To secure orthogonality, there must be contrast variables in the subsequent principal components $\gamma_{3}, \cdots, \gamma_{p}$, and the contrast variables may be any of the original variables $\left\{\tilde{x}_{1}, \cdots, \tilde{x}_{p}\right\}$. In other words, the contrast variables cannot be identified prior to PCA and they are not the same from one principal component to another; thus, the contrast variables are classified per principal component based on the sign of the entries in the eigenvector and they are not a cluster of variables that have diverse characteristics from the other variables in the dataset as a whole.

\section{Problems of Principal Components in DEA}

With the counter effect due to contrast variables, a component score can be minimized by increasing the variables that are assigned with negative weights. Hence, it cannot be interpreted that the bigger the values of the original variables, the bigger the principal component score or vice versa. This implies that the principal components violate the free disposability assumption of a DEA model as described in equation (2.2). As a result, efficiencies cannot be meaningfully estimated because the measures of efficiency rely on estimating maximum output levels for given input levels, or alternatively, minimum input levels for given output levels (Thanassoulis, 2001). In addition, the counter effect may lead to the problem of unboundedness in the linear program. To illustrate the problem, let there be $m$ principal components $\mathbf{K}^{*}=\left[\gamma_{j}\right]^{T}, j=1, \cdots, m$ replacing all $p$ original input variables, with the other conditions remains the same as in equation (2.6). The linear program for $\mathrm{DMU}_{0}$ with data $\left(x_{0}, y_{0}\right)$ is then in the form:

\section{Minimize $e$}

Subject to

$$
\begin{aligned}
& \mathbf{Y} \lambda-s_{y}=y_{0} \\
& \mathbf{K}^{*} \lambda+\mathbf{V}^{* T} s_{x}=e k_{0}^{*} \\
& \lambda, s_{y}, s_{x} \geq 0 \\
& \text { where } \\
& \mathbf{V}^{*}=\left[v_{1} v_{2} \cdots v_{m}\right] \\
& k_{0}^{*}=\mathbf{V}^{* T} x_{0}
\end{aligned}
$$

Note that the constraints in terms of the principal components can be restructured as follows:

$$
\begin{aligned}
& \mathbf{K}^{*} \lambda+\mathbf{V}^{*} T s_{x}=e k_{0}^{*} \\
\Rightarrow & \left(\mathbf{V}^{* T} \mathbf{X}\right) \lambda+\mathbf{V}^{* T}{ }_{s_{x}}=e\left(\mathbf{V}^{* T} x_{0}\right) \\
\Rightarrow & \mathbf{V}^{* T}\left(\mathbf{X} \lambda+s_{x}\right)=\mathbf{V}^{* T} e_{0}
\end{aligned}
$$

To simplify the notation, let $\mathbf{T}=\left[t_{1} t_{2} \cdots t_{p}\right]^{T}=\mathbf{X} \lambda+s_{x}$ and $x_{0}=\left[\begin{array}{llll}x_{1_{0}} & x_{2_{0}} \cdots x_{p_{0}}\end{array}\right]$. By using the notations in equation (2.8), constraints in equation (2.11) can be written as:

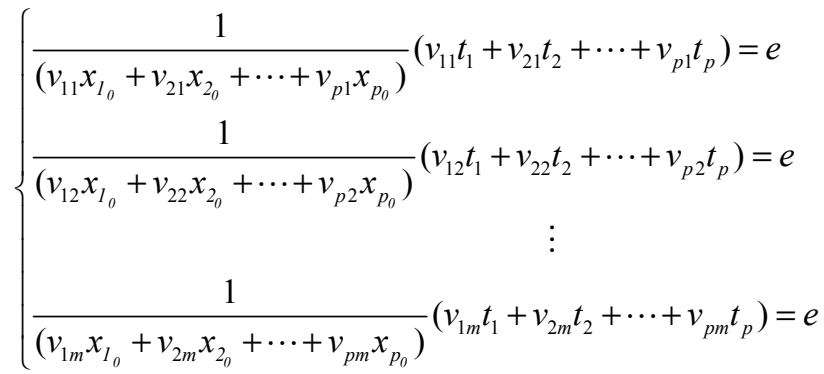

Based on equation (2.10) and the requirement that $x \in \mathbf{R}_{+}^{p}$, note that $t_{k} \geq 0, k=1$, $\ldots, p$. Thus, when all the weights $v_{i j}, i=1, \ldots, p$, $j=1, \ldots, m$ in equation (2.12) are the same sign (positive or negative), the linear program 
produces a meaningful solution because the feasible region is bounded $(\geq 0)$, and an optimal $e^{*}$ can be obtained to minimize the objective function in equation (2.10). However, when there are positive and negative weights within a constraint, the problem of unboundedness may arise. This problem occurs when there is at least a variable $x_{u}$ with moderately large weights $\left\langle v_{u 1}, v_{u 2}, \cdots, v_{u m}\right\rangle$ of which the weights are in the opposite sign with the weights of another variable $x_{s}$ that are moderately large $\left\langle v_{s 1}, v_{s 2}, \cdots, v_{s m}\right\rangle$ giving the product:

$$
\left(v_{u j}\right)\left(v_{s j}\right)<0 \quad \forall j=1, \cdots, m
$$

The effect on the constraints in equation (2.12) is illustrated by equation (2.14) shown in Figure 1 .

Note that when the weights vectors $\left\langle v_{u 1}, v_{u 2}, \cdots, v_{u m}\right\rangle$ and $\left\langle v_{s 1}, v_{s 2}, \cdots, v_{s m}\right\rangle$ are dominating, and equation (2.13) is met, the values on the left hand side of equation (2.14) can be made zeros (or even negative) by loading huge input excess for, $\tilde{x}_{s}$ and/or $\tilde{x}_{u}$, namely, $s_{x(s)}$ and $s_{x(u)}$. This will inflate the magnitudes of $t_{u}$ and/or $t_{s}$, and subsequently cause the feasible region to be unbounded, of which $e$ can be made as small as possible. In other words, this gives an unbounded solution to the objective function in equation (2.10). In order to meet the free disposability assumption and to avoid the problem of unboundedness in linear program, it is crucial to ensure that the weights assigned to the variables are non-negative.

\section{Methodology}

As weights are extracted from the eigenvectors, modifications to the eigenvectors are needed to avoid the problems of contrast variables. Nonetheless, changes made to the eigenvectors may hamper the components' potential to represent the original dataset. To provoke minimal alteration to the eigenvectors, it would be good to work on the simple structure produced by a varimax rotation; that is, an orthogonal rotation of the factor axes that maximizes the variance of the squared loadings on all the variables in a factor matrix (Kaiser, 1958). As a result, each factor tends to have a few high loadings with the rest of the loadings being zero or close to zero, leading to a simple structure, where ideally each item is loaded on only one axis (Kline, 2002). Traditionally, based

Figure 1: Effect on the Constraints in Equation (2.12)

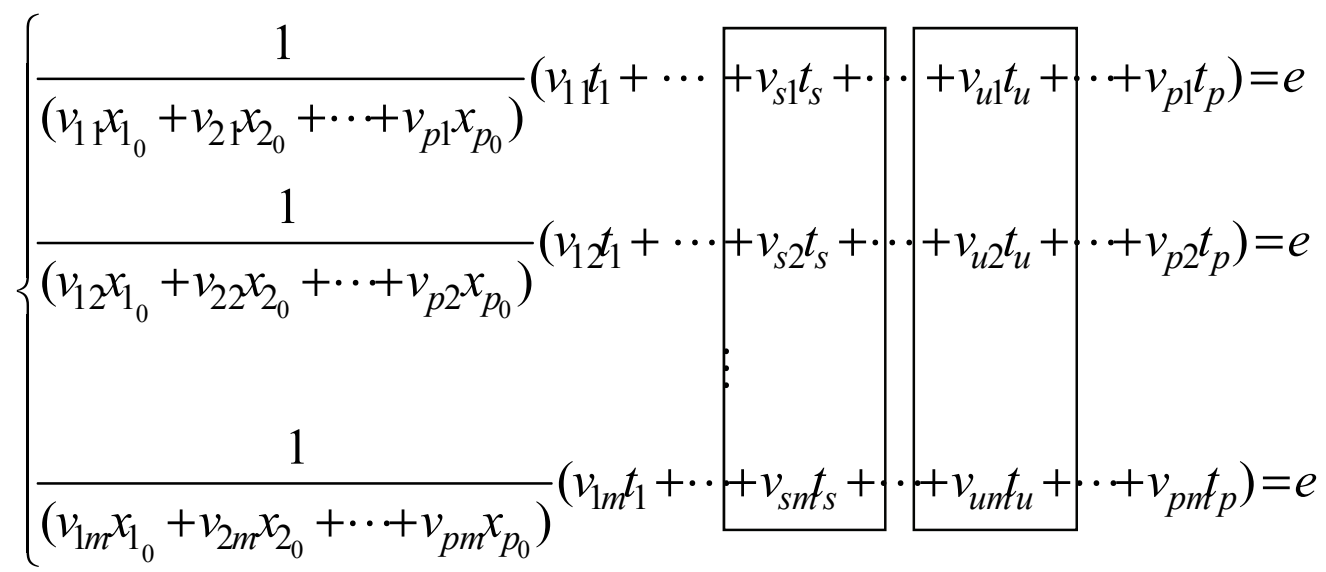




\section{APPROACH TO REDUCE DIMENSIONALITY IN DATA ENVELOPMENT ANALYSIS}

on the simple structure, only variables with loadings above a cutoff point (for example, 0.5) are interpreted (Jolliffe, 2002). Component scores computed with such simple weighting schemes often hold up better under crossvalidation compared to the exact component scores (Dunteman, 1989). By having the advantage to omit the variables with small loadings, it would be possible to restructure the weighting vectors with minimal perturbation.

To start, a varimax rotation is performed on the loadings matrix in order to obtain the simple structure $\mathbf{V}^{r}=\left[v_{1}^{r} v_{2}^{r} \cdots v_{m}^{r}\right]$. From the simple structure, dominating variables can be identified, whereby the variables with high loadings exhibit strong correlations with a principal component. In order to avoid counter effects within a component, for each component axis $v_{j}^{r}, j=1, \ldots, m$ the variables with positive loadings should be segregated from those with negative loadings. For illustrative purposes, the groups are labeled as positive group $\mathrm{v}_{\mathrm{j}}^{\mathrm{r}^{(+)}}$and negative group $v_{j}^{r^{(-)}}$. The explained variation associated to each group is depicted by the corresponding SSL, that is, $\operatorname{SSL}\left(v_{j}^{r(+)}\right)$ and $\operatorname{SSL}\left(v_{j}^{r(-)}\right)$.

To minimize deviations from the original principal components, the group of variables that capture a bigger portion of explained variation (the one with a larger SSL) will be extracted. Variables of another group with smaller SSL are labeled as the contrast variables. These variables are relatively less significant and are subject to be dropped: this is equivalent to assigning a zero weight to each of the contrast variables. To satisfy the requirement of unit vector (Hand, 2001), these vectors are then normalized, and hence are called the modified principal directions. The absolute values of these modified principal directions are taken to form the new weights for the construction of the modified components. The modifications can be performed with MATLAB, and the steps are described in algorithmic form as:

1. Launch varimax rotation, obtain the rotational matrix, $\boldsymbol{\Lambda}$.
2. Obtain the rotated component axes, that is $\mathbf{V}^{r}=\left[\begin{array}{llll}v_{1}^{r} & v_{2}^{r} \cdots v_{m}^{r}\end{array}\right]=\mathbf{V}^{*} \boldsymbol{\Lambda}$.

3. Divide the entries in each vector $v_{j}^{r}$ into two groups, one with positive sign $v_{j}^{r^{(+)}}$, and another with negative sign $v_{j}^{r^{(-)}}, j=1, \ldots, m$.

4. In each vector $v_{j}^{r}$, identify the group that has a bigger SSL, $v_{D_{j}}$ (e.g. $v_{D_{j}}=v_{j}^{r^{(-)}}$).

5. Normalize the vectors $v_{D_{j}}, j=1, \cdots, m$.

6. Take the absolute values on the principal directions formed in step (5), giving the modified axes matrix:

$$
\begin{aligned}
& \mathbf{W}=\left[\begin{array}{lll}
\omega_{11} & \cdots & \omega_{p 1} \\
\omega_{12} & \cdots & \omega_{p 2} \\
\vdots & & \vdots \\
\omega_{1 m} & \cdots & \omega_{p m}
\end{array}\right] \\
& \omega_{i j} \geq 0 \text { for } i=1, \cdots, m, j=1, \cdots, p
\end{aligned}
$$

7. Form the modified components $\mathbf{C}=\left[c_{1} c_{1} \cdots\right.$ $\left.c_{m}\right]^{T}$ based on the weights in equation (3.1):

$$
\begin{gathered}
c_{1}=\omega_{11} x_{1}+\omega_{21} x_{2}+\cdots+\omega_{p 1} x_{p} \\
c_{2}=\omega_{12} x_{1}+\omega_{22} x_{2}+\cdots+\omega_{p 2} x_{p} \\
\vdots \\
c_{m}=\omega_{1 m} x_{1}+\omega_{2 m} x_{2}+\cdots+\omega_{p m} x_{p}
\end{gathered}
$$

Simply stated, this modification only involves the exclusion of a less significant group of variables. Alternatively, to avoid negative weights, other options may be considered, such as: (1) taking the squared values on the eigenvectors, or (2) taking the absolute values of the eigenvectors. The option that best fits the original dataset should capture the most amount of explained variation in the original data.

To compare the options graphically, a specific case with 3 variables that can be 
explained by two principal components is used. Figure 2(a) shows how the eigenvectors capture the distribution of the data. Using the same set of data, the modified axes from the proposed model and the other options (1) squaring the entries of the eigenvectors and (2) taking absolute values of the eigenvectors are shown in Figures 2(b), 2(c) and 2(d) respectively. Note that the proposed model gives the nearest approximation to the original eigenvectors, hence capturing almost the same amount of explained variation in the original data. To consolidate the justification, the amount of explained variation will be verified via redundancy analysis.

\section{Justification of Modifications}

The aim of the proposed modifications is to avoid the contrast variables in principal components without much sacrifice to the ability to represent the original data. To examine this aspect, redundancy analysis (Van den Wollenberg, 1977) is used. This procedure aims to extract factors from the set of dependent variables $\tilde{\mathbf{Y}}$ that are the most predictive of the independent variables $\tilde{\mathbf{X}}$. Because interest lies in knowing how much of the variance in the original variables is explained by the modified components, let the modified components be the dependent variables, $\tilde{\mathbf{Y}}$, and the original

Figure 2: A Comparison between Eigenvectors and the Modified Directions

(a) Eigenvectors of Principal Components

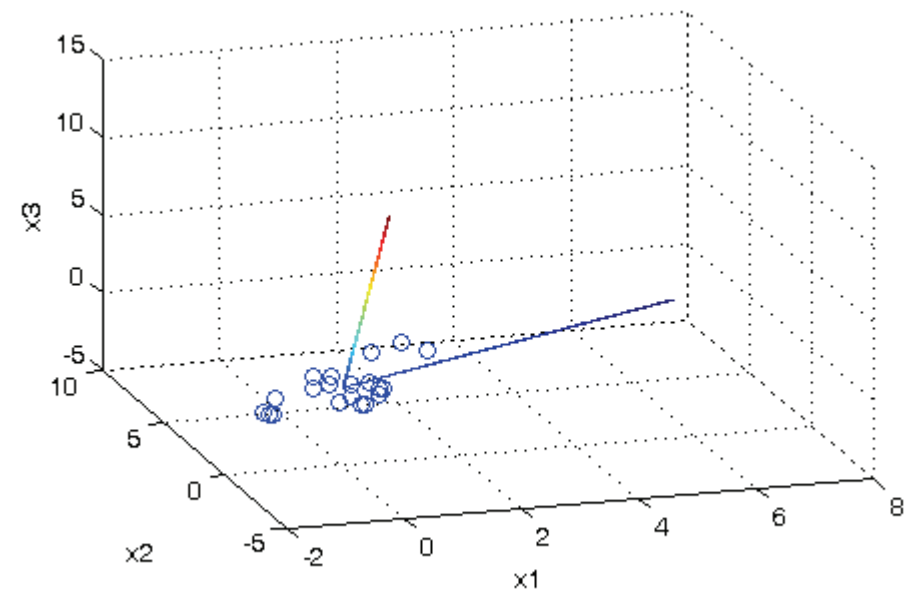

(c) Principal Directions of Squared Modification

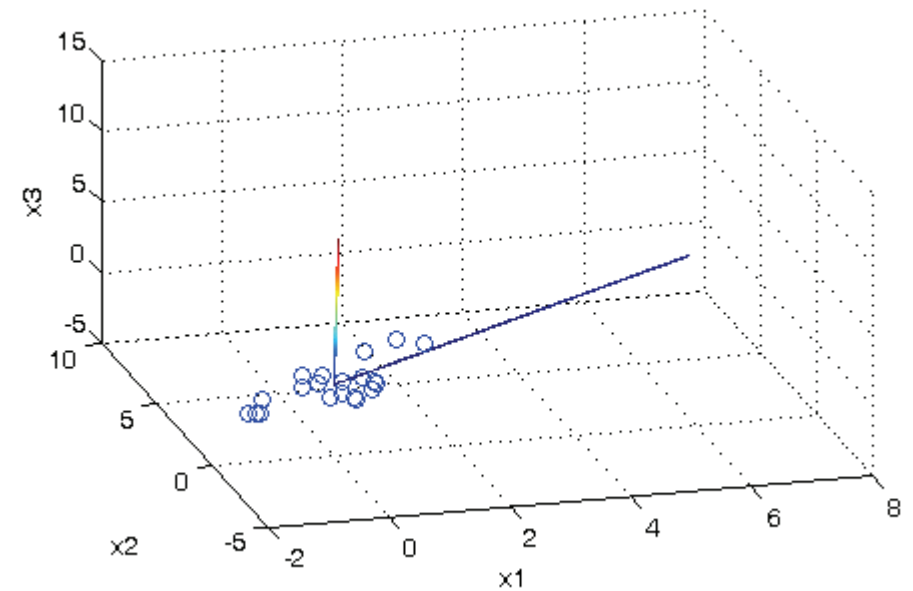

(b) Principal Directions of Proposed Modification

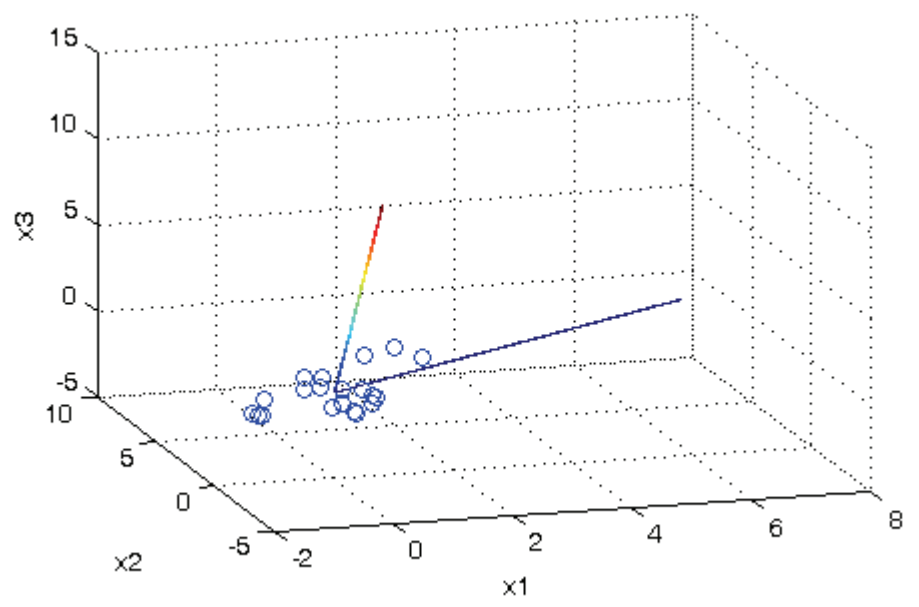

(d) Principal Directions of Absolute Value Modification

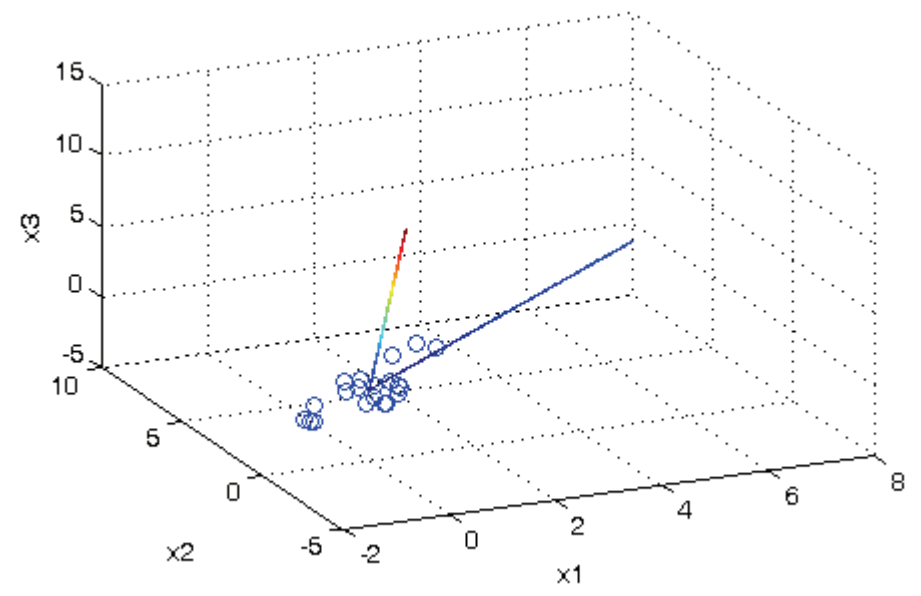


variables be the independent variables, $\tilde{\mathbf{X}}$. Based on the objective of canonical correlation analysis (Hotelling, 1936), two sets of canonical variates, $\mathbf{u}_{x}=\left[u_{x_{j}}\right]$ and $\mathbf{u}_{y}=\left[u_{y_{j}}\right], j=1, \cdots, m$ are constructed to represent $\tilde{\mathbf{X}}$ and $\tilde{\mathbf{Y}}$ respectively, such that the correlation between the canonical variates, $\quad r_{j}\left(u_{x j}, u_{y j}\right), j=1, \cdots, m$ is maximized. Based on the canonical correlations, the proportion of variation in $\widetilde{\mathbf{X}}$ being explained by $\tilde{\mathbf{Y}}$ can be computed using the redundancy index developed by (Stewart and Love 1968):

$$
r d_{y \rightarrow x}=\sum_{j=1}^{m}\left(\sum_{i=1}^{p} \frac{a_{x_{i, j}}^{2}}{p}\right) r_{j}^{2}
$$

where $a_{x_{i, j}}=$ canonical loadings.

To compare the proposed modification to the other two options, redundancy analysis will be carried out on all the methods. The option causing the least perturbations to the eigenvectors should largely retain the proportion of explained variation, which will then be indicated by a largest redundancy.

\section{Modified PC-DEA}

After the modification that captures the largest redundancy is identified, the modified PC-DEA model can be constructed based on the modified axes and the corresponding components. To simplify the notation, assume that the proposed modification gives the largest redundancy. Thus, the modified components $\mathbf{C}$ and the modified axes $\mathbf{W}$ will be used to replace the principal components and the eigenvectors in equation (2.10). In essence, the modified PCDEA model for $\mathrm{DMU}_{0}$ with data $\left(x_{0}, y_{0}\right)$ is as follows:

\section{Minimize $e$}

Subject to

$$
\begin{aligned}
& \mathbf{Y} \lambda-s_{y}=y_{0} \\
& \mathbf{C} \lambda+\mathbf{W}^{T} s_{x}=e c_{0} \\
& \lambda, s_{y}, s_{x} \geq 0 \\
& \text { where } c_{0}=\mathbf{W}^{T} x_{0}
\end{aligned}
$$

As shown in (3.4), the modified PCDEA is similar to PCA-DEA, except changing the eigenvectors to the modified axes. Thus, the modified PC-DEA is suitable for the scenarios that are favorable to PCA-DEA, particularly when all the variables are known to be relevant in the production function under study. The modification can be obtained by running MATLAB codes that execute steps 1-6 described earlier. Because these steps are not heavy, the inclusion of them in a computer program would not increase the run time, and hence would preserve the strength of having the shortest run time amongst the alternatives to reduce the dimensionality. In other words, by having a better data reconstruction that avoids the problem of unboundedness in a linear program, the modified PC-DEA improves the use of principal components in a DEA model, and it offers a convenient alternative to dimension reduction.

\section{Results}

To demonstrate the problem of contrast variables within principal components in the DEA framework, the data generation process (DGP) based on the idea of Kneip, et al. (1998) and Simar and Wilson (1998, 2000a, 2001) were followed where each DMU is attached with single output efficiency and no DMU is regarded as strictly efficient. However, DEA identifies the estimates of relative efficiency. By definition, at least one DMU will be identified as relatively efficient. To mitigate the need of large sample size, it is necessary to restrict to CRS because when the boundary of the production set displays constant returns-to-scale, the DEA estimators converge faster and, hence, introduce less noise (Daraio \& Simar, 2007). Each $\mathrm{DMU}_{k}$ is associated with an inefficiency index, $\tau_{k}$, which is drawn independently from an inefficiency distribution. Following the criteria set by Alder and Yazhemsky (2010), a DMU is deemed relatively efficient if the simulated $e^{-\tau}$ is greater than 0.9 .

To emphasize the problem of discriminatory power, consider cases with relatively many input variables compared to the number of DMUs and begin with a numerical illustration that consists of 20 DMUs that use 7 
inputs to produce an output. Correlated input variables $\tilde{x}_{j}, j=1, \cdots, 7$ are generated by postmultiplying a set of random numbers from a uniform distribution on the interval $(0,100)$ by the upper triangular Cholesky decomposition of a pre-assigned correlation matrix $\mathbf{R}_{1}$ with moderate pairwise correlation $(r<0.6)$. These input variables are used in a Cobb-Douglas production function $\tilde{y}=\prod_{j=1}^{7}\left(\tilde{x}_{j}\right)^{\frac{1}{7}}$. An inefficiency index is simulated for each DMU independently from a half normal distribution, that is, $\tau_{k} \sim$ $\mathrm{HN}(0,1)$. Under CRS, the inefficiency parameter can be assigned to either input side or the output side, as they produce the same efficiency score. In this example, the output values are calculated based on the equation $\tilde{y}=\prod_{j=1}^{7}\left(\tilde{x}_{j}\right)^{\frac{1}{7}} \cdot e^{-\tau}$, and the data for 20 DMUs are generated as shown in Table 1(a). The correlation matrix for the input variables is shown in Table 1(b).

To reduce dimensionality, PCA is applied to all the input variables. Four principal components were extracted in order to retain at least $80 \%$ explained variation. These components are then taken for efficiency estimations using equation (2.10). The component scores are shown in the first 4 columns of Table 2 and the eigenvectors are the first 4 rows of Table 3 . From the eigenvectors, observe that the weights attached to variables $\tilde{x}_{4}, \tilde{x}_{5}$ and $\tilde{x}_{7}$ are dominant and a combination of these weights will cause the feasible region to be unbounded. To illustrate, following equation (2.12), the constraints relating to the principal components for the efficiency estimation for DMU1 are:

Table 1: Simulated Data and Correlation Matrix for Input Variables

(a) Simulated Data for $\tilde{y}=\prod_{j=1}^{7}\left(\tilde{x}_{j}\right)^{\frac{1}{7}} \cdot e^{-\tau}$

\begin{tabular}{|c|c|c|c|c|c|c|c|c|c|}
\hline DMU & $\tilde{y}$ & $\tilde{x}_{1}$ & $\tilde{x}_{2}$ & $\tilde{x}_{3}$ & $\tilde{x}_{4}$ & $\tilde{x}_{5}$ & $\tilde{x}_{6}$ & $\tilde{x}_{7}$ & $e^{-\tau}$ \\
\hline DMU1 & 26.260 & 75.793 & 89.197 & 73.386 & 88.115 & 0.201 & 123.52 & 73.210 & 0.728 \\
\hline DMU2 & 9.977 & 93.371 & 67.702 & 101.92 & 91.872 & 19.778 & 74.313 & 10.790 & 0.194 \\
\hline DMU3 & 48.509 & 15.580 & 74.798 & 80.262 & 97.408 & 36.999 & 143.91 & 17.229 & 0.961 \\
\hline DMU4 & 18.868 & 14.366 & 65.858 & 94.079 & 64.095 & 17.039 & 84.701 & 66.670 & 0.397 \\
\hline DMU5 & 26.997 & 9.258 & 78.416 & 88.645 & 65.644 & 49.054 & 141.87 & 9.075 & 0.630 \\
\hline DMU6 & 17.032 & 34.792 & 40.767 & 47.370 & 86.329 & 9.530 & 97.046 & 3.994 & 0.569 \\
\hline DMU7 & 5.631 & 37.114 & 16.970 & 53.378 & 37.088 & 65.351 & 73.318 & 10.042 & 0.163 \\
\hline DMU8 & 16.999 & 41.154 & 90.177 & 87.628 & 53.287 & 15.536 & 116.66 & 69.836 & 0.293 \\
\hline DMU9 & 11.283 & 66.556 & 76.222 & 28.593 & 54.421 & 3.057 & 48.587 & 58.638 & 0.319 \\
\hline DMU10 & 10.045 & 80.534 & 36.821 & 78.626 & 135.93 & 5.326 & 135.96 & 15.306 & 0.225 \\
\hline DMU11 & 11.785 & 24.151 & 25.517 & 61.538 & 85.657 & 15.639 & 115.45 & 13.299 & 0.328 \\
\hline DMU12 & 15.525 & 64.394 & 33.103 & 105.25 & 102.50 & 41.122 & 84.464 & 54.637 & 0.243 \\
\hline DMU13 & 8.922 & 86.984 & 30.712 & 86.334 & 102.49 & 7.062 & 96.267 & 14.978 & 0.211 \\
\hline DMU14 & 7.937 & 32.015 & 51.944 & 60.220 & 103.44 & 15.248 & 129.60 & 23.887 & 0.170 \\
\hline DMU15 & 8.212 & 21.021 & 16.192 & 99.012 & 112.37 & 54.485 & 110.26 & 12.282 & 0.190 \\
\hline DMU16 & 5.920 & 33.092 & 38.637 & 40.868 & 51.107 & 17.277 & 45.590 & 30.691 & 0.169 \\
\hline DMU17 & 9.307 & 2.936 & 53.055 & 102.67 & 84.770 & 1.466 & 140.52 & 2.934 & 0.496 \\
\hline DMU18 & 13.535 & 70.179 & 87.534 & 120.91 & 77.687 & 5.979 & 109.71 & 4.156 & 0.340 \\
\hline DMU19 & 14.805 & 6.832 & 61.754 & 57.921 & 42.567 & 63.600 & 58.750 & 3.897 & 0.520 \\
\hline DMU20 & 12.697 & 29.560 & 9.457 & 38.286 & 104.42 & 39.826 & 78.962 & 37.364 & 0.328 \\
\hline
\end{tabular}




$$
\left\{\begin{array}{l}
\frac{1}{(-6.635)}\left(\begin{array}{l}
-0.342 t_{1}-0.272 t_{2}-0.448 t_{3}-0.44 t_{4} \\
+0.446 t_{5}-0.46 t_{6}-0.067 t_{7}
\end{array}\right)=e \\
\frac{1}{(0.751)}\left(\begin{array}{l}
0.305 t_{1}+0.421 t_{2}-0.193 t_{3}-0.36 t_{4} \\
-0.342 t_{5}-0.357 t_{6}+0.564 t_{7}
\end{array}\right)=e \\
\frac{1}{(1.345)}\left(\begin{array}{l}
-0.51 t_{1}+0.566 t_{2}+0.314 t_{3}-0.427 t_{4} \\
+0.183 t_{5}+0.325 t_{6}+0.004 t_{7}
\end{array}\right)=e \\
\frac{1}{(0.153)}\left(\begin{array}{l}
0.486 t_{1}+0.085 t_{2}+0.587 t_{3}-0.191 t_{4} \\
+0.365 t_{5}-0.405 t_{6}-0.281 t_{7}
\end{array}\right)=e
\end{array}\right.
$$

To emphasize the problem of unboundedness, choose a point within the feasible region, that is, $t_{1}=t_{2}=t_{3}=t_{6}=0$. At this point, equation (4.1) is simplified to

$$
\left\{\begin{array}{l}
0.066 t_{4}-0.067 t_{5}+0.01 t_{7}=e \\
-0.48 t_{4}-0.455 t_{5}+0.751 t_{7}=e \\
-0.317 t_{4}+0.136 t_{5}+0.003 t_{7}=e \\
-1.245 t_{4}+2.383 t_{5}-1.839 t_{7}=e
\end{array}\right.
$$

Observe that if the input excesses $s_{x(4)}, s_{x(5)}$ and $s_{x(7)}$ are loaded heavily, for example $s_{x(4)}=s_{x(5)}=s_{x(7)}=s_{b}$, where $s_{b}$ is a large number, the constraints will then be driven by

$$
\left\{\begin{array}{l}
0.009 s_{b}=e \\
-0.184 s_{b}=e \\
-0.178 s_{b}=e \\
-0.701 s_{b}=e
\end{array}\right.
$$

It can be observed from equation (4.3) that the constraints related to $\gamma_{2}, \gamma_{3}$ and $\gamma_{4}$ lead to unbounded feasible region for $e$ because $e$ can be made as small as possible in the linear program. In the constraint related to $\gamma_{1}$, the input excesses are weighted with a very small positive number. Thus, this constraint can easily be made zero or negative, if $v_{1}^{\mathrm{T}}(\mathbf{X} \lambda)$ is negative. As a result, the PCA-DEA estimator encounters the problem of unboundedness, and this is shown in the efficiency scores obtained in column 2 of Table 4 . These values are close to zero due to the setting of the lower bound of $e$ to a zero in the linear program.

To produce non-negative data that meet the free disposability assumption in a DEA model, modifications on the eigenvectors were performed on the same set of data following the procedure suggested herein. First, the eigenvectors are rotated with a varimax rotation, giving the rotated factor axes shown in rows 5-8 of Table 3. Note that the first rotated axis $v_{1}^{r}$ is dominated by the variables with negative

Table 1 (continued): Simulated Data and Correlation Matrix for Input Variables

(b) Correlation Matrix of Input Variables

\begin{tabular}{|c|c|c|c|c|c|c|c|}
\cline { 2 - 8 } \multicolumn{1}{c|}{} & $\tilde{x}_{1}$ & $\tilde{x}_{2}$ & $\tilde{x}_{3}$ & $\tilde{x}_{4}$ & $\tilde{x}_{5}$ & $\tilde{x}_{6}$ & $\tilde{x}_{7}$ \\
\hline$\tilde{x}_{1}$ & 1 & 0.110 & 0.154 & 0.323 & -0.454 & -0.164 & 0.198 \\
\hline$\tilde{x}_{2}$ & 0.110 & 1 & 0.290 & -0.320 & -0.346 & 0.220 & 0.318 \\
\hline$\tilde{x}_{3}$ & 0.154 & 0.290 & 1 & 0.295 & -0.065 & 0.471 & -0.106 \\
\hline$\tilde{x}_{4}$ & 0.323 & -0.320 & 0.295 & 1 & -0.254 & 0.505 & -0.142 \\
\hline$\tilde{x}_{5}$ & -0.454 & -0.346 & -0.065 & -0.254 & 1 & -0.199 & -0.256 \\
\hline$\tilde{x}_{6}$ & -0.164 & 0.220 & 0.471 & 0.505 & -0.199 & 1 & -0.180 \\
\hline$\tilde{x}_{7}$ & 0.198 & 0.318 & -0.106 & -0.142 & -0.256 & -0.180 & 1 \\
\hline
\end{tabular}


weights. Thus, variables $\tilde{x}_{1}, \tilde{x}_{2}, \tilde{x}_{5}$ and $\tilde{x}_{7}$ with positive weights that capture $17.1 \%$ of the SSL in $\gamma_{1}$ are classified as contrast variables in $\gamma_{1}$. To form an axis without the counter effect from the contrast variables, these variables are excluded, and the remaining variables $\tilde{x}_{3}, \tilde{x}_{4}$ and $\tilde{x}_{6}$ are used to form the normalized principal direction $\omega_{1}$. This procedure is repeated for the other rotated axes $v_{j}^{r}, j=2,3,4$ and the corresponding normalized principal directions $\omega_{j}, j=2,3,4$ are produced (refer to rows 10-12 of Table 3 ).

This example illustrates that the contrast variables differ from one component to the other, and they cannot be identified prior to PCA. To examine if the modifications made to the eigenvectors weaken the components' ability to represent the dataset, a redundancy analysis was performed on the modified components against the original dataset. Results show that the modified components retain $82.1 \%$ explained variation of the original dataset, compared to $84.8 \%$, captured by the principal components.

As described in the methodology, there are alternatives to avoid negative weights in eigenvectors, for example, (1) squaring the entries of the eigenvectors and (2) taking absolute values of the eigenvectors. To compare these alternatives, redundancy analyses were performed on the modified components corresponding to these methods against the original dataset using equation (3.3). The redundancy analyses show that there is a $69.0 \%$ redundancy from components obtained by option (1) and 69.5\% redundancy from components obtained by option (2). This means that, although there is a drop in the amount of retained variation, the proposed modification is still the best among the other options. Hence, the components from the proposed modifications are used to replace the original variables in the DEA model for the efficiency estimation.

To illustrate the benefit gained from the dimensionality reduction due to these modified components the efficiency scores of the proposed method (modified PC-DEA) was compared to the results of the standard DEA (columns 2 and 4 of Table 4). As expected, the standard DEA suffers from overestimation. Refer to the efficiencies pre-assigned, $e^{-\tau}$ (see column 10, Table 1), DMUs 1, 5, 6, 17, 18, 19 and 20 should not be classified as efficient as being identified by the standard DEA (see column 2, Table 4). This problem is overcome by the proposed method, whereby only DMU3 is identified as efficient, reflecting the scenario as portrayed in the pre-assigned efficiencies. As such, it may be said that there is no significant loss of information due to the modified components. This example shows that the efficiency estimates obtained from the modified PC-DEA is more accurate than that of the standard DEA.

It is known that DEA is sensitive towards the dimensionality relative to the sample size and PCA is best used for dimension reduction when data are highly correlated. To generalize the findings, Monte Carlo simulations that take 100 trials were designed for each of the cases classified by these factors, that is, the dimensionality, correlation levels and the sample sizes. The data generating process is the same as described above, whereby a production function $\tilde{y}=\prod_{j=1}^{p}\left(\tilde{x}_{j}\right)^{\frac{1}{p}}$, where $p$ is the number of inputs is used to simulate data with CRS. For the factor of correlation, two levels of correlations are examined; a case where variables are moderately correlated $(r<0.6)$; pre-assigned with a correlation matrix $\mathbf{R}_{1}$, and another case where variables are highly correlated $(r>0.6)$; preassigned with a correlation matrix $\mathbf{R}_{2}$. Random samples for both levels of correlation are generated based on the upper triangular Cholesky decomposition of $\mathbf{R}_{1}$ and $\mathbf{R}_{2}$ respectively. These cases were repeated for the sample sizes of 20, 50 and 100 (see Table 5).

Results shows that, on average, for the inputs that are highly correlated, there is 1 principal component returned for case of 4 inputs and 1.4 principal components returned for the case with 7 inputs for all the sample sizes. The sharp reduction in the dimensionality validates the use of PCA when the data that are highly correlated. For the inputs that are moderately correlated, more principal components are returned in order to capture at least $80 \%$ of explained variation. On average, 
Table 2: Principal Components $\left(\gamma_{j}\right)$ and Modified Components $\left(c_{j}\right)$

\begin{tabular}{|c|c|c|c|c|c|c|c|c|}
\hline DMU & $\gamma_{1}$ & $\gamma_{2}$ & $\gamma_{3}$ & $\gamma_{4}$ & $c_{1}$ & $c_{2}$ & $c_{3}$ & $c_{4}$ \\
\hline DMU1 & -6.635 & 0.751 & 1.345 & 0.153 & 5.420 & 4.536 & 5.439 & 4.488 \\
\hline DMU2 & -5.867 & -0.876 & 0.495 & 2.702 & 4.371 & 4.784 & 3.270 & 5.423 \\
\hline DMU3 & -5.417 & -2.427 & 2.552 & 0.189 & 6.172 & 2.328 & 3.900 & 4.853 \\
\hline DMU4 & -4.665 & -0.079 & 2.314 & 0.555 & 3.929 & 2.332 & 4.453 & 4.633 \\
\hline DMU5 & -4.680 & -2.417 & 3.450 & 0.847 & 5.370 & 1.692 & 3.865 & 5.311 \\
\hline DMU6 & -4.377 & -1.700 & 0.523 & 0.024 & 4.646 & 2.510 & 2.124 & 2.862 \\
\hline DMU7 & -1.916 & -1.910 & 1.080 & 1.683 & 2.874 & 2.024 & 1.408 & 3.591 \\
\hline DMU8 & -5.456 & 0.529 & 2.789 & 0.543 & 4.441 & 3.032 & 5.429 & 4.964 \\
\hline DMU9 & -3.829 & 1.724 & 0.475 & 0.372 & 2.613 & 3.448 & 4.091 & 2.459 \\
\hline DMU10 & -6.962 & -2.320 & -0.440 & 0.425 & 6.909 & 4.864 & 2.608 & 4.301 \\
\hline DMU11 & -4.496 & -2.252 & 0.809 & -0.120 & 5.126 & 2.315 & 2.014 & 3.302 \\
\hline DMU12 & -5.221 & -1.361 & 0.417 & 1.823 & 4.891 & 4.379 & 3.209 & 5.375 \\
\hline DMU13 & -5.917 & -1.524 & -0.443 & 1.486 & 5.131 & 4.672 & 2.234 & 4.381 \\
\hline DMU14 & -5.392 & -1.887 & 1.083 & -0.374 & 5.907 & 2.875 & 3.150 & 3.639 \\
\hline DMU15 & -4.570 & -3.680 & 0.958 & 1.202 & 5.755 & 2.754 & 1.853 & 5.039 \\
\hline DMU16 & -2.773 & -0.130 & 0.541 & 0.593 & 2.492 & 2.181 & 2.336 & 2.483 \\
\hline DMU17 & -5.872 & -2.627 & 2.441 & 0.107 & 5.843 & 1.765 & 2.992 & 4.746 \\
\hline DMU18 & -6.683 & -1.084 & 2.055 & 2.291 & 4.957 & 3.911 & 4.032 & 6.076 \\
\hline DMU19 & -2.000 & -1.562 & 2.392 & 1.611 & 2.659 & 1.109 & 2.632 & 3.910 \\
\hline DMU20 & -3.324 & -1.954 & -0.399 & -0.138 & 4.613 & 2.804 & 1.726 & 2.583 \\
\hline
\end{tabular}

Table 3: Eigenvectors $\left(v_{j}\right)$, Rotated Axes $\left(v_{j}^{r}\right)$ and Modified Principal Directions $\left(\omega_{j}\right)$

\begin{tabular}{|c|c|c|c|c|c|c|c|}
\cline { 2 - 8 } \multicolumn{1}{c|}{} & $\tilde{x}_{1}$ & $\tilde{x}_{2}$ & $\tilde{x}_{3}$ & $\tilde{x}_{4}$ & $\tilde{x}_{5}$ & $\tilde{x}_{6}$ & $\tilde{x}_{7}$ \\
\hline$v_{1}$ & -0.342 & -0.272 & -0.448 & -0.440 & 0.446 & -0.460 & -0.067 \\
\hline$v_{2}$ & 0.305 & 0.421 & -0.193 & -0.360 & -0.342 & -0.357 & 0.564 \\
\hline$v_{3}$ & -0.510 & 0.566 & 0.314 & -0.427 & 0.183 & 0.325 & 0.004 \\
\hline$v_{4}$ & 0.486 & 0.085 & 0.587 & -0.191 & 0.365 & -0.405 & -0.281 \\
\hline$v_{1}^{r}$ & 0.156 & 0.037 & -0.066 & -0.575 & 0.380 & -0.703 & 0.031 \\
\hline$v_{2}^{r}$ & 0.806 & -0.046 & 0.121 & 0.299 & -0.373 & -0.273 & 0.176 \\
\hline$v_{3}^{r}$ & -0.051 & 0.718 & 0.110 & -0.349 & -0.329 & 0.145 & 0.468 \\
\hline$v_{4}^{r}$ & 0.171 & 0.244 & 0.806 & -0.018 & 0.303 & 0.136 & -0.389 \\
\hline$\omega_{1}$ & 0 & 0 & 0.072 & 0.632 & 0 & 0.772 & 0 \\
\hline$\omega_{2}$ & 0.910 & 0 & 0.136 & 0.337 & 0 & 0 & 0.198 \\
\hline$\omega_{3}$ & 0 & 0.820 & 0.126 & 0 & 0 & 0.166 & 0.534 \\
\hline$\omega_{4}$ & 0.186 & 0.265 & 0.875 & 0 & 0.329 & 0.148 & 0 \\
\hline
\end{tabular}


Table 4: Estimated Efficiency Scores for DEA, PCA-DEA and Modified PC-DEA

\begin{tabular}{|c|c|c|c|}
\hline DMU & $\begin{array}{c}\hat{e} \\
(\text { DEA })\end{array}$ & $\begin{array}{c}\hat{e} \\
(\text { PCA-DEA })\end{array}$ & $\begin{array}{c}\hat{e} \\
(\mathrm{mPC}-\mathrm{DEA})\end{array}$ \\
\hline DMU1 & 1 & $5.4 \mathrm{E}-14$ & 0.616 \\
\hline DMU2 & 0.398 & $4.0 \mathrm{E}-15$ & 0.290 \\
\hline DMU3 & 1 & $3.1 \mathrm{E}-15$ & 1 \\
\hline DMU4 & 0.750 & $2.7 \mathrm{E}-15$ & 0.611 \\
\hline DMU5 & 1 & $2.0 \mathrm{E}-16$ & 0.766 \\
\hline DMU6 & 1 & $1.2 \mathrm{E}-16$ & 0.645 \\
\hline DMU7 & 0.466 & $9.4 \mathrm{E}-18$ & 0.321 \\
\hline DMU8 & 0.707 & $5.8 \mathrm{E}-14$ & 0.487 \\
\hline DMU9 & 0.958 & $1.1 \mathrm{E}-16$ & 0.549 \\
\hline DMU10 & 0.729 & $2.6 \mathrm{E}-15$ & 0.310 \\
\hline DMU11 & 0.689 & $1.2 \mathrm{E}-16$ & 0.470 \\
\hline DMU12 & 0.655 & $2.4 \mathrm{E}-15$ & 0.404 \\
\hline DMU13 & 0.642 & $2.0 \mathrm{E}-16$ & 0.321 \\
\hline DMU14 & 0.304 & $2.2 \mathrm{E}-15$ & 0.218 \\
\hline DMU15 & 0.679 & $1.2 \mathrm{E}-16$ & 0.356 \\
\hline DMU16 & 0.385 & $2.3 \mathrm{E}-17$ & 0.302 \\
\hline DMU17 & 1 & $4.2 \mathrm{E}-15$ & 0.253 \\
\hline DMU18 & 1 & $5.4 \mathrm{E}-15$ & 0.347 \\
\hline DMU19 & 1 & $6.3 \mathrm{E}-18$ & 0.708 \\
\hline DMU20 & 1 & $7.6 \mathrm{E}-17$ & 0.591 \\
\hline
\end{tabular}

Table 5: List of Monte Carlo Experiments

\begin{tabular}{|c|c|c|c|}
\hline Experiment & Sample Size & $n$ (inputs) & Pairwise Correlation Level \\
\hline 1 & 20 & 4 & High $(r>0.6)$ \\
\hline 2 & 20 & 4 & Moderate $(r<0.6)$ \\
\hline 3 & 20 & 7 & High $(r>0.6)$ \\
\hline 4 & 20 & 7 & Moderate $(r<0.6)$ \\
\hline 5 & 50 & 4 & High $(r>0.6)$ \\
\hline 6 & 50 & 4 & Moderate $(r<0.6)$ \\
\hline 7 & 50 & 7 & High $(r>0.6)$ \\
\hline 8 & 50 & 7 & Moderate $(r<0.6)$ \\
\hline 9 & 100 & 4 & High $(r>0.6)$ \\
\hline 10 & 100 & 4 & Moderate $(r<0.6)$ \\
\hline 11 & 100 & 7 & High $(r>0.6)$ \\
\hline 12 & 100 & 7 & Moderate $(r<0.6)$ \\
\hline
\end{tabular}




\section{APPROACH TO REDUCE DIMENSIONALITY IN DATA ENVELOPMENT ANALYSIS}

there are 2.7-3.0 principal components returned for the case with 4 inputs, and 3.9-4.4 principal components returned for the case with 7 inputs. To compare the information retention power, redundancy analyses between the original variables and the modified components were performed on these simulated dataset, comparing the redundancies due to the proposed method, taking squared value of eigenvectors (option 1) and taking absolute value of the eigenvectors (option 2). The results of the analyses are shown in Table 6 . Note that, when there is only 1 principal component returned, there is no difference between the three options because there is only one factor axis to be considered. However, when there is more than one principal component, the redundancies captured by these options differ. As the proposed method provokes the least perturbations to the eigenvectors, it captures the most explained variation in all the cases, with reasonably low standard deviation. Referring to column 2 of Table 6 , it is observed that the modified components obtained with the proposed method retain almost as much the information as in the principal components, that is, capturing at least $80 \%$ of explained variation. Thus, it may be concluded that the proposed method is the best alternative among these options to avoid negative weights in principal components because it causes the least information loss.

To compare the efficacy of the proposed method (modified PC-DEA) to the standard DEA, the efficiency estimates from the modified PC-DEA and the standard DEA were compared to the simulated efficiencies. Figure 3 illustrates the comparisons for two extreme cases, namely (a) the worst case with a sample size $n=20,1$ output and 7 moderately correlated inputs, and (b) the best case with a sample size $n=100,1$ output and 4 highly correlated inputs. Note that for both cases, the efficiency estimates from the modified PC-DEA are closer to the simulated efficiencies compared to the standard DEA.

Table 6: Results of the Redundancy Analyses

\begin{tabular}{|c|c|c|c|c|c|c|}
\hline \multirow{2}{*}{ Experiment } & \multicolumn{2}{|c|}{$\begin{array}{c}\text { Redundancy }^{\mathrm{a}} \\
\text { Proposed Method }\end{array}$} & \multicolumn{2}{c|}{$\begin{array}{c}\text { Redundancy } \\
\text { Option }^{\mathrm{b}}\end{array}$} & \multicolumn{2}{c|}{$\begin{array}{c}\text { Redundancy }^{\mathrm{a}} \\
\text { Option }^{\mathrm{c}}\end{array}$} \\
\cline { 2 - 7 } & Average & Std Dev & Average & Std Dev & Average & Std Dev \\
\hline 1 & 0.937 & 0.021 & 0.937 & 0.021 & 0.937 & 0.021 \\
\hline 2 & 0.883 & 0.046 & 0.833 & 0.069 & 0.822 & 0.070 \\
\hline 3 & 0.860 & 0.034 & 0.857 & 0.033 & 0.857 & 0.032 \\
\hline 4 & 0.846 & 0.031 & 0.770 & 0.060 & 0.760 & 0.058 \\
\hline 5 & 0.936 & 0.011 & 0.936 & 0.011 & 0.935 & 0.012 \\
\hline 6 & 0.905 & 0.033 & 0.845 & 0.042 & 0.838 & 0.041 \\
\hline 7 & 0.851 & 0.034 & 0.849 & 0.032 & 0.850 & 0.032 \\
\hline 8 & 0.831 & 0.034 & 0.773 & 0.052 & 0.759 & 0.053 \\
\hline 9 & 0.933 & 0.009 & 0.933 & 0.009 & 0.933 & 0.009 \\
\hline 10 & 0.910 & 0.020 & 0.834 & 0.032 & 0.827 & 0.030 \\
\hline 11 & 0.841 & 0.033 & 0.839 & 0.031 & 0.839 & 0.031 \\
\hline 12 & 0.834 & 0.043 & 0.780 & 0.058 & 0.762 & 0.059 \\
\hline
\end{tabular}

a: Redundancy between the original variables and the modified components

b: Option 1 represents the squared value of eigenvectors

c: Option 2 represents the absolute value of eigenvectors 
Figure 3: Comparison of Efficiency Estimates to the Simulated Efficiencies

(a) Efficiency Estimates for 20 DMUs with 1 Output and 7 Moderately Correlated Inputs

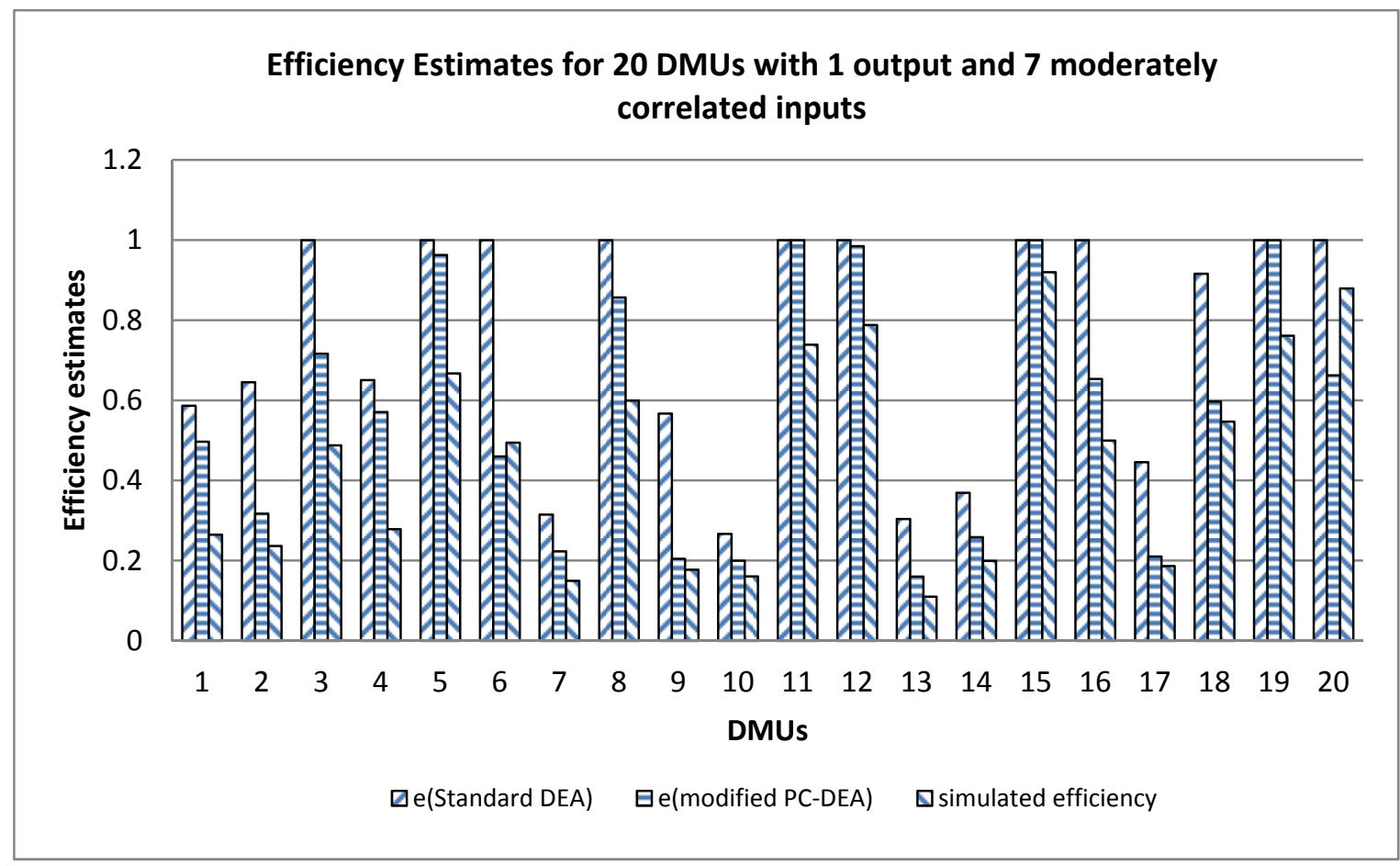

(b) Difference in Efficiency Estimates for 100 DMUs with 1 Output and 4 Highly Correlated Inputs

Difference in efficiency estimates for 100 DMUs with 1 output, 4 highly correlated inputs

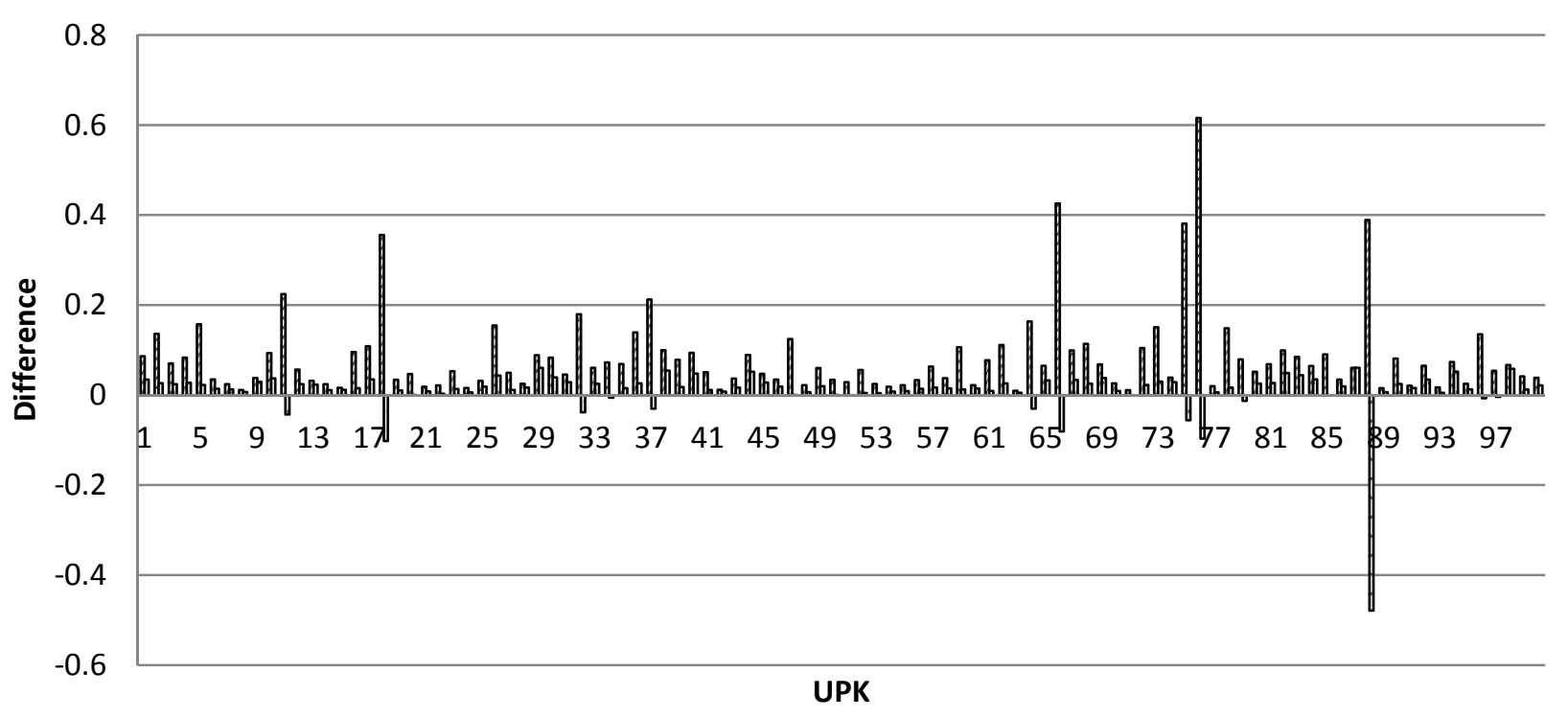

$\square \mathrm{e}($ Standard DEA) - simulated efficiency $\square \mathrm{e}($ modified PC-DEA) - simulated efficiency 


\section{APPROACH TO REDUCE DIMENSIONALITY IN DATA ENVELOPMENT ANALYSIS}

To further examine the discriminatory power of the estimators, the percentages of overestimation and underestimation of each model were reckoned. An overestimation is observed when an inefficient DMU $\left(e^{-\tau}<0.9\right)$ is identified as efficient $(\hat{e}=1)$, and an underestimation occurs when an efficient DMU $\left(e^{-\tau}>0.9\right)$ is identified as inefficient $(\hat{e}<1)$. The results of the Monte Carlo simulations are shown in Table 7. Note that the standard DEA suffers from the curse of dimensionality. As expected, the worst case (Experiment 4, of which $n=20$, with 1 output and 7 moderately correlated inputs) produces huge overestimation (42\%). Consistent with Simar and Wilson (2000b), the increase in the sample size (from $n$ $=20$ to $n=100$ ) does not give much ease to the overestimation problem (from $42 \%$ to $26.31 \%$ ). Conversely, note that by using the modified components to replace the original variables, the problem of overestimation is reduced sharply.
For this worst case (Experiment 4), the proposed method replaces all the 7 inputs with 4 modified components, thus reduces the overestimation to $17.8 \%$. Note also that both the modified PC-DEA and the standard DEA work better when data are highly correlated because the constraints attributable to the variables are rather similar to each other. Nonetheless, even in the best scenario (Experiment 9, of which $n=$ 100, with 4 highly correlated inputs), the modified PC-DEA is still better than the standard DEA by having a much slighter overestimation $(0.06 \%$ compared to $4.24 \%)$. The modified PC-DEA performs well in all cases to overcome the problem of overestimation. Although it produces underestimations $(0.24 \%-$ $2.11 \%$ ) due to the loss of information, the effect is deemed slender compared to the improvement in the discriminatory power.

Table 7: Results of Monte Carlo Simulations (100 trials) on the Percentages of Overestimation and Underestimation

\begin{tabular}{|c|c|c|c|c|}
\hline \multirow{2}{*}{ Experiment } & \multicolumn{2}{|c|}{ \% Overestimation } & \multicolumn{2}{c|}{ \% Underestimation } \\
\cline { 2 - 5 } & Std DEA & $\begin{array}{c}\text { Modified PC- } \\
\text { DEA }\end{array}$ & Std DEA & $\begin{array}{c}\text { Modified PC- } \\
\text { DEA }\end{array}$ \\
\hline 1 & 11.50 & 1.30 & 0 & 1.65 \\
\hline 2 & 22.05 & 11.05 & 0 & 0.20 \\
\hline 3 & 18.75 & 2.80 & 0 & 1.15 \\
\hline 4 & 42.00 & 17.80 & 0 & 0.30 \\
\hline 5 & 7.20 & 0.30 & 0 & 1.70 \\
\hline 6 & 14.58 & 6.98 & 0 & 0.16 \\
\hline 7 & 13.26 & 1.18 & 0 & 1.20 \\
\hline 8 & 33.16 & 12.32 & 0 & 0.28 \\
\hline 9 & 4.24 & 0.06 & 0 & 2.11 \\
\hline 10 & 10.05 & 4.32 & 0 & 0.28 \\
\hline 11 & 9.08 & 0.33 & 0 & 1.80 \\
\hline 12 & 26.31 & 8.91 & 0 & 0.24 \\
\hline
\end{tabular}




\section{Conclusion}

Literature shows that PCA-DEA outperforms other methods when all the variables under consideration are relevant. Furthermore, it is a convenient approach to reduce the dimensionality because it involves the least run time and estimation results are satisfactory. Principal components are the uncorrelated weighted linear combinations of original variables that capture the maximum variance. As the linear combinations are formed with a mixture of positive and negative weights, principal components could not meet the free disposability assumption in a DEA model. Consequently, the problem of unboundedness might arise in the linear program of the DEA model.

To overcome this problem, this study proposed that the eigenvectors be modified whereby each of the modified axes is constructed based on a set of variables that correlate in the same direction to the respective principal component. The modification involves the exclusion of contrast variables that capture a smaller portion of SSL, thus, there would not be significant information loss due to the modification. This was illustrated in redundancy analysis using Monte Carlo experiments. Compared to other possible alternatives to obtain non-negative weights for the principal components, the modified components due to proposed method captured the largest redundancy - in fact, they retained almost as much the explained variation as in the extracted principal components.

This study showed that the modified PC-DEA performs well to overcome the problem of overestimation, particularly when data are highly correlated. Because the modification can be obtained easily by adding programming codes to existing PCA-DEA its run time is not different from that of PCA-DEA. Better data reconstruction avoids the problem of unboundedness in a linear program, thus, the modified PC-DEA is a practical alternative to reduce dimensionality in a DEA model. In circumstances when there are many relevant variables, but not many comparable observations, researchers may consider applying the proposed method to aid meaningful benchmarking processes.
Acknowledgement

The authors would like to thank L. P. Teo for constructive advice.

$$
\text { References }
$$

Adler, N, \& Golany, B. (2001). Evaluation of deregulated airline networks using data envelopment analysis combined with principal component analysis with an application to Western Europe. European Journal of Operational Research, 132(2), 18-31.

Adler, N, \& Golany, B. (2002). Including principal component weights to improve discrimination in data envelopment analysis. Journal of the Operational Research Society, 53, 985-991.

Adler, N., \& Yazhemsky, E. (2010). Improving discrimination in data envelopment analysis: PCA-DEA or variable reduction. European Journal of Operational Research, 202, 273-284.

Andersen, P., \& Petersen, N. C. (1993). A procedure for ranking efficient units in data envelopment analysis. Management Science, 39(10), 1261-1264.

Angulo-Meza, L., \& Lins, M. R. E. (2002). Review of methods for increasing discrimination in data envelopment analysis. Annals of Operations Research, 116, 225-242.

Charnes, A., Cooper, W. W., Golany, B., Seiford, L. M., \& Stutz, J. (1985). Foundations of data envelopment analysis for Pareto-Koopmans efficient empirical production functions. Journal of Econometrics 30, 91-107.

Charnes, A., Cooper, W. W., \& Rhodes, E. (1978). Measuring the efficiency of decision making units. European Journal of Operational Research, 2, 429-444.

Daraio, C., \& Simar, L. (2007). Advanced robust and nonparametric methods in efficiency analysis: Methodology and applications. New York, NY: Springer.

Doyle, J. R., \& Green, R. H. (1994). Efficiency and cross-efficiency in DEA: derivations, meanings and uses. Journal of the Operational Research Society, 45(5), 567-578.

Dunteman, G. H. (1989). Principal components analysis. Sage University Paper Series on Quantitative Applications in the Social Sciences, Series no. 07-069. Newbury Park: Sage. 


\section{APPROACH TO REDUCE DIMENSIONALITY IN DATA ENVELOPMENT ANALYSIS}

Dyson, R., Allen, R., Camanho, A. S., Podinovski, V. V., Sarrico, C. S., \& Shale, E. A. (2001). Pitfalls and protocols in DEA. European Journal of Operational Research, 132, 245-259.

Fare, R. (1998). Fundamentals of production theory. Berlin, Germany: SpringerVerlag.

Green, R. H., Doyle, J. R., \& Cook, W. D. (1996). Preference voting and project ranking using DEA and cross-evaluation. European Journal of Operational Research, 90, 461-472.

Hand, D. J., Mannila, H., \& Smyth, P. (2001). Principles of data mining. Cambridge, MA: Massachusetts Institute of Technology.

Hotelling, H. (1936). Relations between two sets of variates. Biometrika, 28, 321-377.

Jenkins, L., \& Anderson, M. (2003). A multivariate statistical approach to reducing the number of variables in data envelopment analysis. European Journal of Operational Research, 147, 51-61.

Jolliffe, I. T. (2002). Principal component analysis, $2^{\text {nd }} E d$. New York, NY: Springer-Verlag.

Kaiser, H. F. (1958). The varimax criterion for analytic rotation in factor analysis. Psychometrika, 23, 187-200.

Kao, L. J., Lu, C. J., \& Chiu, C. C. (2011). Efficiency measurement using independent component analysis and data envelopment analysis. European Journal of Operational Research, 210, 310-317.

Kline, P. (2002). An easy guide to factor analysis. London, UK: Routledge.

Kneip, A., Park, B. U., \& Simar, L. (1998). A note on the convergence of nonparametric DEA estimators for production efficiency scores. Econometric Theory, 14, 183793.

Kneip, A., Simar, L., \& Wilson, P. W. (2008). Asymptotics and consistent bootstraps for DEA estimators in non-parametric frontier models. Econometric Theory, 24, 1663-1697.

Lee, T. W. (1998). Independent component analysis: Theory and application. Boston, MA: Kluwer Academic Publisher.
Nataraja, N. R., \& Johnson, A. L. (2011). Guidelines for using variable selection techniques in data envelopment analysis. European Journal of Operational Research, 215, 662-669.

Pastor, J. T., Ruiz, J. L., \& Sirvent, I. (2002). A statistical test for nested radial DEA models. Operations Research, 50(4), 728-735.

Pedraja-Chaparro, F., Salinas-Jimenez, J., \& Smith, P. (1999). On the quality of the data envelopment analysis model. Journal of the Operational Research Society, 50, 636-644.

Podinovski, V. V., \& Thanassoulis, E. (2007). Improving discrimination in data envelopment analysis: some practical suggestions. Journal of Productivity Analysis, 28, 117-126.

Ruggiero, J. (2005). Impact assessment of input omission on DEA. International Journal of Information Technology \& Decision Making, 4(3), 359-368.

Sexton, T. R., Silkman, R. H., \& Hogan, A. J. (1986). Data envelopment analysis: critique and extensions. In Measuring efficiency: An assessment of data envelopment analysis, R. H. Silkman, Ed. 32, 73-105. San Francisco, CA: Jossey-Bass.

Shephard, R. W. (1970). Theory of cost and production functions. Princeton, NJ: Princeton University Press.

Simar, L., \& Wilson, P. W. (1998). Sensitivity analysis of efficiency scores: How to bootstrap in nonparametric frontier models. Management Science, 44, 49-61.

Simar, L., \& Wilson, P. W. (2000a). A general methodology fir bootstrapping in nonparametric frontier models. Journal of Applied Statistics, 27, 779-802.

Simar, L., \& Wilson, P. W. (2000b). Statistical inference in nonparametric frontier models: the state of the art. Journal of Productivity Analysis, 13, 49-78.

Simar, L., \& Wilson, P. W. (2001). Testing restriction in nonparametric efficiency models. Communication in Statistics, 30, 159184. 
Sirvent, L., Ruiz, J. L., Borras, F., \& Pastor, J. T. (2005). A Monte Carlo evaluation of several tests for the selection of variables in DEA models. International Journal of Information Technology \& Decision Making, 4(3), 325-343.

Smith, P. (1997). Model misspecification in data envelopment analysis. Annals of Operations Research, 73, 233-252.

Stewart, D. K., \& Love, W. A. (1968). A general canonical correlation index. Psychological Bulletin, 70, 160-163.
Thanassoulis, E. (2001). Introduction to the theory and application of data envelopment analysis: A foundation text with integrated software. USA: Kluwer Academic Publishers.

Ueda, T., \& Hoshiai, Y. (1997) Application of principal component analysis for parsimonious summarization of DEA inputs and/or outputs. Journal of the Operational Research Society of Japan, 40, 466-478.

van den Wollenberg, A. L. (1977). Redundancy analysis: Alternative for canonical analysis. Psychometrika, 42, 207-219. 


\title{
Robustness of DEWMA versus EWMA Control Charts to Non-Normal Processes
}

\author{
Saad Saeed Alkahtani \\ Performance Measurement Center of Government Agencies, \\ Institute of Public Administration \\ Riyadh, Saudi Arabia
}

Exponentially weighted moving average (EWMA) and double EWMA (DEWMA) control charts were designed under the normality assumption. This study considers various skewed (Gamma) and symmetric non-normal (t) distributions to examine the effect of non-normality on the average run length (ARL) performance of EWMA and DEWMA. ARL performances were investigated and compared using Monte Carlo simulations. Results show that DEWMA charts can be designed to be robust to non-normality, that the ARL performances of EWMA and DEWMA charts were more robust to $t$ distributions and DEWMA was more robust to non-normality for larger values of the smoothing parameter.

Key words: Average run length, control charts, EWMA, DEWMA, robustness, non-normality.

Introduction

A popular control chart used to detect small shifts in a process mean is the EWMA (Roberts, 1959). In an effort to increase the sensitivity of EWMA control charts to detect small shifts and drifts in a process, a double EWMA (DEWMA) control chart was developed by Shamma and Shamma (1992). Zhang (2002) has conducted extensive studies on DEWMA control charts for the mean.

Like most commonly used control charts, the traditional EWMA and DEWMA control charts for monitoring process means were developed under the assumption of normality. The behavior of the EWMA control chart performance for non-normal distributions has been investigated. Borror, et al. (1999) used the Markov chain method and simulations to study the average run length (ARL) performance of the EWMA control charts for the mean of

Saad Saeed Alkahtani is an Assistant Professor of Applied Statistics. Dr. Alkahtani teaches courses in statistics such as SPC, regression, multivariate statistics, experimental design, SAS and SPSS. He also provides consultations in statistics and has published several articles. Email him at: alkahtanisas@yahoo.com. skewed (gamma) and heavy-tailed ( $\mathrm{t}$ ) symmetric non-normal distributions. They concluded that the ARL performance of a well-designed EWMA control chart was robust to violations of the normality assumption.

As a part of an extensive study of the effect of non-normality and auto-correlation on the performance of EWMA control charts, Stoumbos and Reynolds (2000) concluded that some combinations of EWMA control charts for detecting small shifts in a process mean and/or variance can be designed to be robust to the violation of normality assumption. Montgomery (2005) found that an appropriately designed EWMA chart will perform well even for nonnormal data.

Simulation studies on the robustness of an EWMA control chart for process mean monitoring have been conducted by Borror, et al. (1999) and by Koshti and Kalgonda (2011). In addition, Human, et al. (2011) ran an extensive simulation to study the robustness of an EWMA control chart for individual observations. They investigated the in-control robustness of the designs studied by Borror, et al. (1999) and found that, with some types of non-normal data, caution should be taken not to overuse EWMA charts.

Studies related to the concept of robustness to non-normality of the EWMA control statistic have also been conducted. For 
example, Lin and Chou (2010) investigated the robustness of EWMA and -EWMA control charts with variable sampling intervals to nonnormality; Shiau and Hsu (2005) studied the robustness of the EWMA control chart to nonnormality for auto-correlated process; and Calzada and Scariano (2003) investigated the robustness of the MaxEWMA control charts to the violation of normality. The robustness to non-normality of DEWMA control charts for detecting shifts in a process mean has not been investigated; thus, this study considers the robustness of DEWMA and compares it to EWMA using Monte Carlo simulations.

Background

If $X_{i}$, where $i=1,2, \ldots, m$, is a sequence of random variables taken from a normal distribution with mean $\mu_{0}$ and variance $\sigma^{2}$, then the EWMA control statistic is defined as:

$$
Z_{i}=\lambda X_{i}+(1-\lambda) Z_{i-1}
$$

where $0<\lambda \leq 1$ is a smoothing parameter and $Z_{0}=\mu_{0}$ (i.e., the in-control or target process mean). Typically, $\mu_{0}$ is unknown and is estimated from the preliminary sample by the sample mean $\bar{X}$. The control limits for the EWMA control chart are:

$$
\begin{aligned}
& U C L=\mu_{0}+L \sigma \sqrt{\left(\frac{\lambda}{2-\lambda}\right)\left[1-(1-\lambda)^{2 i}\right]} \\
& C L=\mu_{0} \\
& L C L=\mu_{0}-L \sigma \sqrt{\left(\frac{\lambda}{2-\lambda}\right)\left[1-(1-\lambda)^{2 i}\right]}
\end{aligned}
$$

where $\mathrm{L}$ is the distance between the control limits and the center line (CL) measured in $\sigma$ units. For large values of $i$, the control limits become:

$$
\begin{aligned}
& U C L=\mu_{0}+L \sigma \sqrt{\left(\frac{\lambda}{2-\lambda}\right)} \\
& C L=\mu_{0} \\
& L C L=\mu_{0}-L \sigma \sqrt{\left(\frac{\lambda}{2-\lambda}\right)}
\end{aligned}
$$

The DEWMA control statistic $Z_{i}$ is defined as:

$$
\begin{aligned}
& Y_{i}=\lambda X_{i}+(1-\lambda) Y_{i-1} \\
& Z_{i}=\lambda Y_{i}+(1-\lambda) Z_{i-1}
\end{aligned}
$$

such that $0<\lambda<1$ and $Y_{0}=Z_{0}=\mu_{0}$. It can be shown that (see Appendix A)

$$
\begin{gathered}
Z_{i}=\lambda^{2} \sum_{j=1}^{i}(i-j+1)(1-\lambda)^{i-j} X_{j} \\
+i \lambda(1-\lambda)^{i} Y_{0}+(1-\lambda)^{i} Z_{0} \\
E\left(Z_{i}\right)=\mu_{0}
\end{gathered}
$$

and

$$
\begin{gathered}
1+(1-\lambda)^{2}-\left(i^{2}+2 i+1\right)(1-\lambda)^{2 i} \\
\sigma_{Z_{i}}^{2}=\lambda^{4} \frac{+\left(2 i^{2}+2 i-1\right)(1-\lambda)^{2 i+2}-i^{2}(1-\lambda)^{2 i+4}}{\left(1-(1-\lambda)^{2}\right)^{3}} \sigma_{0}^{2}
\end{gathered}
$$

The control limits for the DEWMA control chart are: 
$U C L=$

$\mu_{0}+L \sigma \sqrt{\begin{array}{c}1+(1-\lambda)^{2}-\left(i^{2}+2 i+1\right)(1-\lambda)^{2 i} \\ \lambda^{4} \frac{+\left(2 i^{2}+2 i-1\right)(1-\lambda)^{2 i+2}-i^{2}(1-\lambda)^{2 i+4}}{\left(1-(1-\lambda)^{2}\right)^{3}}\end{array}}$

$C L=\mu_{0}$

$L C L=$

$\mu_{0}-L \sigma \sqrt{\begin{array}{c}1+(1-\lambda)^{2}-\left(i^{2}+2 i+1\right)(1-\lambda)^{2 i} \\ \lambda^{4} \frac{+\left(2 i^{2}+2 i-1\right)(1-\lambda)^{2 i+2}-i^{2}(1-\lambda)^{2 i+4}}{\left(1-(1-\lambda)^{2}\right)^{3}}\end{array}}$

where $\mathrm{L}$ is as defined. For large values of $i$, the control limits become:

$$
\begin{aligned}
& U C L=\mu_{0}+L \sigma \sqrt{\frac{\lambda\left(2-2 \lambda+\lambda^{2}\right)}{(2-\lambda)^{3}}} \\
& C L=\mu_{0} \\
& L C L=\mu_{0}-L \sigma \sqrt{\frac{\lambda\left(2-2 \lambda+\lambda^{2}\right)}{(2-\lambda)^{3}}}
\end{aligned}
$$

For both EWMA and DEWMA control charts, the control statistics in (1.1) and (1.4), respectively, are plotted on the control chart and the process is considered to be out of control if the plotted point lies outside the LCL and UCL.

Borror, et al. (1999) used various symmetric and skewed non-normal distributions to study the robustness of the EWMA control charts for process mean. They considered the $t$ distributions with different numbers of degrees of freedom (i.e., $d f=4,6,8,10,15,20,30,40$, 50). The mean and the variance of the $t$ distribution are: $\mu=0$ and $\sigma^{2}=\frac{d f}{d f-2}$ such that $d f>2$, respectively. For the skewed distribution, the authors considered Gamma distributions denoted $\operatorname{Gam}(\alpha, \beta)$ with $\alpha=4$,
$3,2,1,0.5$ and $\beta=1$. The mean and variance of the gamma distribution are: $\mu=\frac{\alpha}{\beta}$ and $\sigma^{2}=\frac{\alpha}{\beta^{2}}$, respectively

Average Run Length Performances of EWMA vs DEWMA

This study considers $t$ distributions with $d f=4,10,20,40$, and 50 and $\operatorname{Gam}(\alpha, \beta)$ with $\alpha=4,3,2,1,0.5$ and $\beta=1$ to compare the effect of non-normality on the RL performance of both EWMA and DEWMA control charts. For $\lambda=0.10,0.20,0.30,0.40,0.50$, the values of $\mathrm{L}$ for both control charts were chosen such that the in-control ARL $\cong 370.4$ (as used by Borror, et al. 1999) when the process followed a normal distribution. As shown herein, the robustness study of EWMA charts reproduces Borror's findings and they were considered here for the ease of comparison.

\section{Methodology}

All RL calculations were completed based on 10,000 Monte Carlo simulations for each scenario, using SAS ${ }^{\circledR}$ V. 9.2 RANNOR and RANGAM functions. The simulations were conducted as follows:

1. Pseudo random numbers from normal, gamma, and $t$ distributions were generated by SAS ${ }^{\circledR}$ V. 9.2 RANNOR and RANGAM functions.

2. The control statistics $Y_{i}$ for EWMA and $Z_{i}$ for DEWMA were computed.

3. The control statistic was compared with an experimental LCL and UCL and a run length was obtained and recorded.

4. After 10,000 simulation runs, the mean of the 10, 000 derived RL (ARL) and the standard error of ARL (SEARL) values were obtained. 
5. The values of $\mathrm{L}$ were chosen such that the computed in-control ALR is almost equal to 370.4 .

Steps from 1 to 4 were run for each scenario of the combination of the previously assigned different values of the parameters $\lambda, \alpha, \beta, d f$ and shifts.

Tables 1 and 2 summarize the results of the calculated $\mathrm{L}$ values and the in-control ARL along with their SEARL in parenthesis for EWMA and DEWMA control chart for Normal and Various Gamma and $t$ distributions. The following are noted:

i. For Gamma and $t$ distributions, the incontrol ARL for both EWMA and DEWMA were reasonably close to 370.4 for small values of $\lambda$ (i.e., $\lambda<0.20$ ) especially with larger $\alpha$ and $d f$ values of gamma and $t$ distributions, respectively (i.e., when both distributions approach normality). ii. The degree of in-control ARL deterioration for both EWMA and DEWMA was less for $t$ distribution than for gamma distribution.

In general, for gamma and $t$ distributions with larger parameters $\alpha, \beta$ and $d f$ respectively, the in-control ARL values for EWMA were better (larger) than those for DEWMA for small values of $\lambda(\lambda<0.20)$. Conversely, the in-control ARL values for DEWMA were better (i.e., larger) than those for EWMA for larger values of $\lambda(\lambda>0.40)$ particularly with gamma distribution.

Tables 3 and 4 show the out-of-control ARLs for the EWMA and DEWMA Control Charts for Various Gamma Distributions and shifts in the mean measured in standard deviation units. For small shifts in the process mean (shift $=0.25$ ) and $\lambda>0.20$, the out-ofcontrol ARLs for both EWMA and DEWMA are significantly less than the value that would be obtained if the process was normal; other than

Table 1: In-control ARL for EWMA and DEWMA Control Chart for Various Normal and Various Gamma Distributions

\begin{tabular}{|c|c|c|c|c|c|c|c|c|c|c|}
\cline { 2 - 11 } \multicolumn{1}{c|}{} & \multicolumn{5}{c|}{$\begin{array}{c}\text { EWMA } \\
\text { (SEARL) }\end{array}$} & \multicolumn{5}{c|}{$\begin{array}{c}\text { DEWMA } \\
\text { (SEARL) }\end{array}$} \\
\hline$\lambda$ & 0.1 & 0.2 & 0.3 & 0.4 & 0.5 & 0.1 & 0.2 & 0.3 & 0.4 & 0.5 \\
\hline L & 2.698 & 2.856 & 2.929 & 2.956 & 2.977 & 3.796 & 4.018 & 4.069 & 4.059 & 3.991 \\
\hline \multirow{2}{*}{ Normal } & 369.9 & 370.3 & 370.2 & 370.2 & 368.9 & 370.2 & 370.6 & 370.9 & 369.8 & 369.9 \\
& $(3.62)$ & $(3.64)$ & $(3.67)$ & $(3.71)$ & $(3.71)$ & $(3.65)$ & $(3.75)$ & $(3.68)$ & $(3.60)$ & $(3.66)$ \\
\hline \multirow{2}{*}{$\operatorname{Gam}(4,1)$} & 339.8 & 261.5 & 202.7 & 159.9 & 135.8 & 330.2 & $\begin{array}{c}259.4 \\
(2.59)\end{array}$ & $\begin{array}{c}200.2 \\
(2.00)\end{array}$ & $\begin{array}{c}162.8 \\
(1.64)\end{array}$ & $\begin{array}{c}139.7 \\
(1.40)\end{array}$ \\
\hline \multirow{2}{*}{$\operatorname{Gam}(3,1)$} & $(3.32)$ & $(2.56)$ & $(2.02)$ & $(1.57)$ & $(1.37)$ & $(3.31)$ & $(2.5)$ & \\
& 332.6 & 238.9 & 182.6 & 143.3 & 121.8 & 326.5 & 239.2 & 176.0 & 140.2 & 121.3 \\
& $(3.21)$ & $(2.38)$ & $(1.82)$ & $(1.41)$ & $(1.21)$ & $(3.22)$ & $(2.39)$ & $(1.74)$ & $(1.38)$ & $(1.18)$ \\
\hline \multirow{2}{*}{$\operatorname{Gam}(2,1)$} & 316.4 & 206.3 & 149.7 & 123.2 & 101.4 & 297.3 & 205.7 & 150.0 & 120.4 & 103.1 \\
& $(3.06)$ & $(2.02)$ & $(1.51)$ & $(1.23)$ & $(1.01)$ & $(2.91)$ & $(2.00)$ & $(1.48)$ & $(1.19)$ & $(1.04)$ \\
\hline \multirow{2}{*}{$\operatorname{Gam}(1,1)$} & 271.4 & 163.2 & 117.6 & 92.8 & 77.2 & 264.0 & 162.7 & 117.3 & 91.0 & 77.8 \\
& $(2.69)$ & $(1.61)$ & $(1.17)$ & $(0.91)$ & $(0.78)$ & $(2.63)$ & $(1.63)$ & $(1.17)$ & $(0.91)$ & $(0.78)$ \\
\hline \multirow{2}{*}{$\operatorname{Gam}(0.5,1)$} & 228.9 & 133.9 & 94.6 & 75.8 & 62.6 & 228.0 & 133.6 & 93.7 & 74.9 & 64.1 \\
& $(2.21)$ & $(1.32)$ & $(0.92)$ & $(0.75)$ & $(0.63)$ & $(2.32)$ & $(1.30)$ & $(0.95)$ & $(0.72)$ & $(0.64)$ \\
\hline
\end{tabular}


that, the ARLs are comparable. Generally, the behavior of robustness to gamma distributions of both charts was similar.

Tables 5 and 6 show out-of-control ARL's for the EWMA and DEWMA Control Charts for Various $t$ Distributions and shifts. The ARL performance of EWMA and DEWMA for both $t$ and normal distributions are comparable except for the case that shift $=0.25$ and $\mathrm{df}<20$; that is, the difference between the ALRs of both normal and $t$ distribution is considerable. For the in-control case, the degree of out-of-control ARL deterioration for both EWMA and DEWMA is less for $t$ distribution than for gamma distribution.

\section{Conclusion}

The effect of non-normality on the ARL performances for EWMA and DEWMA was investigated using Monte Carlo simulations. SAS ${ }^{8} \quad$ V. 9.2 RANNOR and RANGAM functions were used to generate data from various normal, gamma, and $t$ distributions and to perform the calculations for all scenarios.
Results show that, in general, the incontrol ARL performances of both EWMA and DEWMA control charts were more robust for the $t$ distribution than for gamma. The degree of robustness of the EWMA and DEWMA control charts to non-normality increased for smaller values of smoothing parameter and as the $t$ and gamma distributions approach normality. In addition, for gamma and $t$ distributions, the incontrol ARL values for EWMA were more robust than those for DEWMA for small values of $\lambda(\lambda<0.20)$; however, the in-control ARL values for DEWMA were more robust than those for EWMA for large values of $\lambda(\lambda>0.40)$ specifically with gamma distribution with larger parameters

Similarly to the in-control case, the outof-control ARLs of EWMA and DEWMA were more robust for $t$ distribution than for gamma. However, some details needed to be considered. It was noticed that the out-of-control ARL for EWMA and DEWMA was significantly less for gamma comparing to normal-theory ARL for small shift (shift $=0.25)$ and large smoothing

Table 2: In-control ARL for EWMA and DEWMA Control Chart for Various Normal and Various $t$ Distributions

\begin{tabular}{|c|c|c|c|c|c|c|c|c|c|c|}
\cline { 2 - 11 } \multicolumn{1}{c|}{} & \multicolumn{5}{c|}{$\begin{array}{c}\text { EWMA } \\
\text { (SEARL) }\end{array}$} & \multicolumn{5}{c|}{$\begin{array}{c}\text { DEWMA } \\
\text { (SEARL) }\end{array}$} \\
\hline$\lambda$ & 0.1 & 0.2 & 0.3 & 0.4 & 0.5 & 0.1 & 0.2 & 0.3 & 0.4 & 0.5 \\
\hline $\mathrm{L}$ & 2.698 & 2.856 & 2.929 & 2.956 & 2.977 & 3.796 & 4.018 & 4.069 & 4.059 & 3.991 \\
\hline \multirow{2}{*}{ Normal } & 369.9 & 370.3 & 370.2 & 370.2 & 368.9 & 370.2 & 370.6 & 370.9 & 369.8 & 369.9 \\
& $(3.62)$ & $(3.64)$ & $(3.67)$ & $(3.71)$ & $(3.71)$ & $(3.65)$ & $(3.75)$ & $(3.68)$ & $(3.60)$ & $(3.66)$ \\
\hline \multirow{2}{*}{$t_{50}$} & 365.3 & 340.3 & 354.3 & 329.6 & 314.6 & 349.1 & 348.6 & 332.8 & 336.0 & 307.2 \\
& $(3.48)$ & $(3.31)$ & $(3.46)$ & $(3.25)$ & $(3.06)$ & $(3.46)$ & $(3.51)$ & $(3.33)$ & $(3.36)$ & $(3.06)$ \\
\hline \multirow{2}{*}{$t_{40}$} & 358.3 & 349.8 & 338.6 & 310.8 & 306.4 & 344.6 & 350.7 & 328.7 & 314.0 & 302.4 \\
& $(3.46)$ & $(3.44)$ & $(3.31)$ & $(3.09)$ & $(3.09)$ & $(3.40)$ & $(3.48)$ & $(3.21)$ & $(3.21)$ & $(3.02)$ \\
\hline \multirow{2}{*}{$t_{20}$} & 351.8 & 330.7 & 303.5 & 266.9 & 251.8 & 340.9 & 322.4 & 298.3 & 268.0 & 251.5 \\
& $(3.47)$ & $(3.19)$ & $(2.97)$ & $(2.62)$ & $(2.58)$ & $(3.32)$ & $(3.19)$ & $(2.92)$ & $(2.65)$ & $(2.58)$ \\
\hline \multirow{2}{*}{$t_{10}$} & 331.2 & 283.7 & 244.4 & 201.7 & 175.2 & 327.1 & 276.0 & 233.7 & 204.5 & 180.0 \\
& $(3.24)$ & $(2.82)$ & $(2.43)$ & $(2.00)$ & $(1.75)$ & $(3.19)$ & $(2.72)$ & $(2.29)$ & $(2.05)$ & $(1.75)$ \\
\hline \multirow{2}{*}{$t_{4}$} & 268.6 & 187.5 & 148.2 & 117.4 & 102.2 & 264.3 & 191.6 & 141.6 & 118.1 & 101.5 \\
& $(2.62)$ & $(1.82)$ & $(1.47)$ & $(1.16)$ & $(1.02)$ & $(2.60)$ & $(1.90)$ & $(1.38)$ & $(1.19)$ & $(1.01)$ \\
\hline
\end{tabular}


value $(\lambda>0.20)$ and comparable otherwise. For the $t$ distribution, the out-of-control ARL for EWMA and DEWMA were comparable to the normal-theory ARL except for shift $=0.25$ and $d f<20$. In addition, for larger values of $\lambda$ the out-of-control ARLs for DEWMA were similar or slightly better than those for EWMA. Shamma and Shamma (1992) stated:

Baxley (1990) reported EWMA results for a simulated industrial process requiring a larger $\lambda(\lambda=0.35)$ but the optimal EWMA chart requires that $\lambda=0.05$. dEWMA charts will be more sensitive to cases which can be best modeled by dEWMA models with larger values as compared to EWMA since such values will be non-optimal for EWMA charts (p. 21).

Based on both the results of this study and Shamma and Shamma's report, DEWMA control charts should be considered in practice because the EWMA is non-optimal for larger values of $\lambda$. Also, the variability of the simulated average run length for DEWMA is generally smaller than that for EWMA. These properties should motivate the use of DEWMA in industrial process.

\section{References}

Baxley, R. V. (1990). Discussion. Technometrics, 32, 13-16.

Borror, C. M., Montgomery, D. C., \& Runger, G. C. (1999). Robustness of the EWMA control chart to non-normality. Journal of Quality Technology, 10, 139-149.

Calzada, M. E., \& Scariano, S. M. (2003). The robustness of the MaxEWMA chart to non-normality. Communications in StatisticsSimulations and Computations, 32(2), 573-590.

Gradshteyn, I. S., \& Ryzhik, I. M. (1979). Table of integrals, series, and products. Waltham, MA: Academic Press.
Human, S. W., Kritzinger, P., \& Chakraborti, S. (2011). Robustness of the EWMA control chart for individual observations. Journal of Applied Statistics, 38(10), 2071-2087.

Koshti, V. V., \& Kalgonda, A. A. (2011). A study of robustness of the exponentially weighted moving average control chart: a simulation approach. International Journal of Advanced Scientific and technical research, 2(1), 519-525.

Lin, Y. C., \& Chou, C. Y. (2010). Robustness of EWMA and combined $\bar{X}$ EWMA control charts with variable sampling intervals to non-normality. Journal of Applied Statistics, 38(3), 553-570.

Montgomery, D. C. (2005). Introduction to statistical quality control $\left(5^{\text {th }} E d\right.$.). New York, NY: John Wiley \& Sons, Inc.

Roberts, S. W. (1959). Control charts tests based on geometric moving average. Technimetrics, 1(3), 239-250.

Shamma, S. E., \& Shamma, A. K. (1992). Development and evaluation of control charts using double exponentially weighted moving averages. International Journal of Quality \& Reliability Management, $9(6), 18-25$.

Shiau, J. H., \& Hsu, Y. C. (2005). Robustness of the EWMA control chart to nonnormality for autocorrelated processes. Quality Technology \& Quantitative Management, 2(2), 125-146.

Stoumbos, Z. G., \& Reynolds, M. R. (2000). Robustness to non-normality and autocorrelation of individuals control charts.

Journal of Statistical Computation and Simulation, 66(2), 145-187.

Zhang, L. Y. (2002). EWMA control charts and extended EWMA control charts. Unpublished doctoral dissertation. University of Regina, Saskatchewan, Canada. 
Table 3: Out-of-control ARL's for the EWMA Control Charts for Normal and Various Gamma Distributions

\begin{tabular}{|c|c|c|c|c|c|c|c|c|}
\hline & \multirow[b]{2}{*}{ Distribution } & \multicolumn{7}{|c|}{$\begin{array}{c}\text { Shift } \\
\text { (Number of Standard Deviations) }\end{array}$} \\
\hline & & 0.25 & 0.50 & 1.00 & 1.50 & 2.00 & 2.50 & 3.00 \\
\hline \multirow{6}{*}{$\begin{array}{c}\text { EWMA } \\
\lambda=0.1 \\
L= \\
2.698\end{array}$} & Normal & $\begin{array}{c}90.4 \\
(0.80)\end{array}$ & $\begin{array}{c}27.9 \\
(0.19)\end{array}$ & $\begin{array}{c}9.6 \\
(0.04)\end{array}$ & $\begin{array}{c}5.8 \\
(0.02)\end{array}$ & $\begin{array}{c}4.2 \\
(0.01)\end{array}$ & $\begin{array}{c}3.3 \\
(0.01)\end{array}$ & $\begin{array}{c}2.8 \\
(0.01)\end{array}$ \\
\hline & $\operatorname{Gam}(4,1)$ & $\begin{array}{l}78.8 \\
(0.72)\end{array}$ & $\begin{array}{c}27.9 \\
(0.20)\end{array}$ & $\begin{array}{l}10.0 \\
(0.05)\end{array}$ & $\begin{array}{c}5.9 \\
(0.02)\end{array}$ & $\begin{array}{c}4.2 \\
(0.01)\end{array}$ & $\begin{array}{c}3.3 \\
(0.01)\end{array}$ & $\begin{array}{c}2.8 \\
(0.01)\end{array}$ \\
\hline & $\operatorname{Gam}(3,1)$ & $\begin{array}{c}76.6 \\
(0.69)\end{array}$ & $\begin{array}{l}28.5 \\
(0.21)\end{array}$ & $\begin{array}{l}10.0 \\
(0.05)\end{array}$ & $\begin{array}{c}5.9 \\
(0.02)\end{array}$ & $\begin{array}{c}4.2 \\
(0.01)\end{array}$ & $\begin{array}{c}3.3 \\
(0.01)\end{array}$ & $\begin{array}{c}2.8 \\
(0.01)\end{array}$ \\
\hline & $\operatorname{Gam}(2,1)$ & $\begin{array}{c}76.2 \\
(0.67)\end{array}$ & $\begin{array}{c}29.2 \\
(0.22)\end{array}$ & $\begin{array}{c}10.1 \\
(0.05)\end{array}$ & $\begin{array}{c}5.9 \\
(0.02)\end{array}$ & $\begin{array}{c}4.2 \\
(0.01) \\
\end{array}$ & $\begin{array}{c}3.3 \\
(0.01)\end{array}$ & $\begin{array}{c}2.8 \\
(0.01)\end{array}$ \\
\hline & $\operatorname{Gam}(1,1)$ & $\begin{array}{l}74.5 \\
(0.68)\end{array}$ & $\begin{array}{c}30.0 \\
(0.23)\end{array}$ & $\begin{array}{c}10.4 \\
(0.05)\end{array}$ & $\begin{array}{c}5.9 \\
(0.02)\end{array}$ & $\begin{array}{c}4.2 \\
(0.01)\end{array}$ & $\begin{array}{c}3.3 \\
(0.01)\end{array}$ & $\begin{array}{c}2.8 \\
(0.01)\end{array}$ \\
\hline & $\operatorname{Gam}(.5,1)$ & $\begin{array}{c}73.4 \\
(0.67)\end{array}$ & $\begin{array}{l}30.5 \\
(0.24)\end{array}$ & $\begin{array}{c}10.7 \\
(0.05)\end{array}$ & $\begin{array}{c}6.0 \\
(0.02)\end{array}$ & $\begin{array}{c}4.2 \\
(0.01)\end{array}$ & $\begin{array}{c}3.3 \\
(0.01)\end{array}$ & $\begin{array}{c}2.7 \\
(0.01)\end{array}$ \\
\hline \multirow{6}{*}{$\begin{array}{c}\text { EWMA } \\
\lambda=0.2 \\
L= \\
2.856\end{array}$} & Normal & $\begin{array}{c}119.3 \\
(1.17)\end{array}$ & $\begin{array}{c}36.0 \\
(0.30)\end{array}$ & $\begin{array}{c}9.8 \\
(0.06)\end{array}$ & $\begin{array}{c}5.2 \\
(0.02)\end{array}$ & $\begin{array}{c}3.6 \\
(0.01)\end{array}$ & $\begin{array}{c}2.8 \\
(0.01)\end{array}$ & $\begin{array}{c}2.3 \\
(0.01)\end{array}$ \\
\hline & $\operatorname{Gam}(4,1)$ & $\begin{array}{c}82.0 \\
(0.80)\end{array}$ & $\begin{array}{c}33.2 \\
(0.29)\end{array}$ & $\begin{array}{c}10.2 \\
(0.06)\end{array}$ & $\begin{array}{c}5.4 \\
(0.02)\end{array}$ & $\begin{array}{c}3.7 \\
(0.01)\end{array}$ & $\begin{array}{c}2.8 \\
(0.01)\end{array}$ & $\begin{array}{c}2.3 \\
(0.01)\end{array}$ \\
\hline & $\operatorname{Gam}(3,1)$ & $\begin{array}{c}79.7 \\
(0.77)\end{array}$ & $\begin{array}{c}32.8 \\
(0.29)\end{array}$ & $\begin{array}{c}10.2 \\
(0.07)\end{array}$ & $\begin{array}{c}5.4 \\
(0.02)\end{array}$ & $\begin{array}{c}3.6 \\
(0.01)\end{array}$ & $\begin{array}{c}2.8 \\
(0.01)\end{array}$ & $\begin{array}{c}2.3 \\
(0.01)\end{array}$ \\
\hline & $\operatorname{Gam}(2,1)$ & $\begin{array}{c}74.7 \\
(0.71) \\
\end{array}$ & $\begin{array}{c}32.4 \\
(0.29) \\
\end{array}$ & $\begin{array}{c}10.3 \\
(0.29) \\
\end{array}$ & $\begin{array}{c}5.5 \\
(0.07) \\
\end{array}$ & $\begin{array}{c}3.7 \\
(0.03) \\
\end{array}$ & $\begin{array}{c}2.8 \\
(0.01)\end{array}$ & $\begin{array}{c}2.3 \\
(0.01)\end{array}$ \\
\hline & $\operatorname{Gam}(1,1)$ & $\begin{array}{c}67.4 \\
(0.65)\end{array}$ & $\begin{array}{c}32.0 \\
(0.28)\end{array}$ & $\begin{array}{c}10.8 \\
(0.07)\end{array}$ & $\begin{array}{c}5.7 \\
(0.03) \\
\end{array}$ & $\begin{array}{c}3.7 \\
(0.01)\end{array}$ & $\begin{array}{c}2.8 \\
(0.01)\end{array}$ & $\begin{array}{c}2.3 \\
(0.01)\end{array}$ \\
\hline & $\operatorname{Gam}(.5,1)$ & $\begin{array}{c}62.7 \\
(0.60)\end{array}$ & $\begin{array}{c}32.8 \\
(0.29)\end{array}$ & $\begin{array}{c}11.8 \\
(0.08)\end{array}$ & $\begin{array}{c}5.8 \\
(0.03)\end{array}$ & $\begin{array}{c}3.6 \\
(0.01)\end{array}$ & $\begin{array}{c}2.8 \\
(0.01)\end{array}$ & $\begin{array}{c}2.3 \\
(0.01)\end{array}$ \\
\hline \multirow{6}{*}{$\begin{array}{c}\text { EWMA } \\
\lambda=0.3 \\
\mathrm{~L}= \\
2.929\end{array}$} & Normal & $\begin{array}{c}149.4 \\
(1.46)\end{array}$ & $\begin{array}{c}46.8 \\
(0.44)\end{array}$ & $\begin{array}{c}10.9 \\
(0.08)\end{array}$ & $\begin{array}{c}5.2 \\
(0.03)\end{array}$ & $\begin{array}{c}3.4 \\
(0.01)\end{array}$ & $\begin{array}{c}2.6 \\
(0.01)\end{array}$ & $\begin{array}{c}2.1 \\
(0.01)\end{array}$ \\
\hline & $\operatorname{Gam}(4,1)$ & $\begin{array}{c}79.1 \\
(0.76)\end{array}$ & $\begin{array}{c}36.1 \\
(0.34)\end{array}$ & $\begin{array}{c}11.2 \\
(0.08)\end{array}$ & $\begin{array}{c}5.5 \\
(0.03)\end{array}$ & $\begin{array}{c}3.5 \\
(0.01)\end{array}$ & $\begin{array}{c}2.6 \\
(0.01)\end{array}$ & $\begin{array}{c}2.1 \\
(0.01)\end{array}$ \\
\hline & $\operatorname{Gam}(3,1)$ & $\begin{array}{c}72.9 \\
(0.72) \\
\end{array}$ & $\begin{array}{c}35.9 \\
(0.32)\end{array}$ & $\begin{array}{c}11.4 \\
(0.09)\end{array}$ & $\begin{array}{c}5.6 \\
(0.03)\end{array}$ & $\begin{array}{c}3.5 \\
(0.01) \\
\end{array}$ & $\begin{array}{c}2.6 \\
(0.01) \\
\end{array}$ & $\begin{array}{c}2.1 \\
(0.01)\end{array}$ \\
\hline & $\operatorname{Gam}(2,1)$ & $\begin{array}{c}68.7 \\
(0.67)\end{array}$ & $\begin{array}{c}33.5 \\
(0.31)\end{array}$ & $\begin{array}{c}11.6 \\
(0.09)\end{array}$ & $\begin{array}{c}5.7 \\
(0.03)\end{array}$ & $\begin{array}{c}3.6 \\
(0.01)\end{array}$ & $\begin{array}{c}2.6 \\
(0.01)\end{array}$ & $\begin{array}{c}2.1 \\
(0.01)\end{array}$ \\
\hline & $\operatorname{Gam}(1,1)$ & $\begin{array}{c}60.4 \\
(0.59)\end{array}$ & $\begin{array}{c}33.1 \\
(0.32)\end{array}$ & $\begin{array}{c}12.2 \\
(0.10)\end{array}$ & $\begin{array}{c}5.9 \\
(0.03)\end{array}$ & $\begin{array}{c}3.6 \\
(0.01)\end{array}$ & $\begin{array}{c}2.6 \\
(0.01)\end{array}$ & $\begin{array}{c}2.1 \\
(0.01)\end{array}$ \\
\hline & $\operatorname{Gam}(.5,1)$ & $\begin{array}{c}56.3 \\
(0.55)\end{array}$ & $\begin{array}{c}32.4 \\
(0.30)\end{array}$ & $\begin{array}{c}13.1 \\
(0.10)\end{array}$ & $\begin{array}{c}6.4 \\
(0.04)\end{array}$ & $\begin{array}{c}3.6 \\
(0.01)\end{array}$ & $\begin{array}{c}2.6 \\
(0.01)\end{array}$ & $\begin{array}{c}2.1 \\
(0.01)\end{array}$ \\
\hline
\end{tabular}


Table 3 (continued): Out-of-control ARL's for the EWMA Control Charts for Normal and Various Gamma Distributions

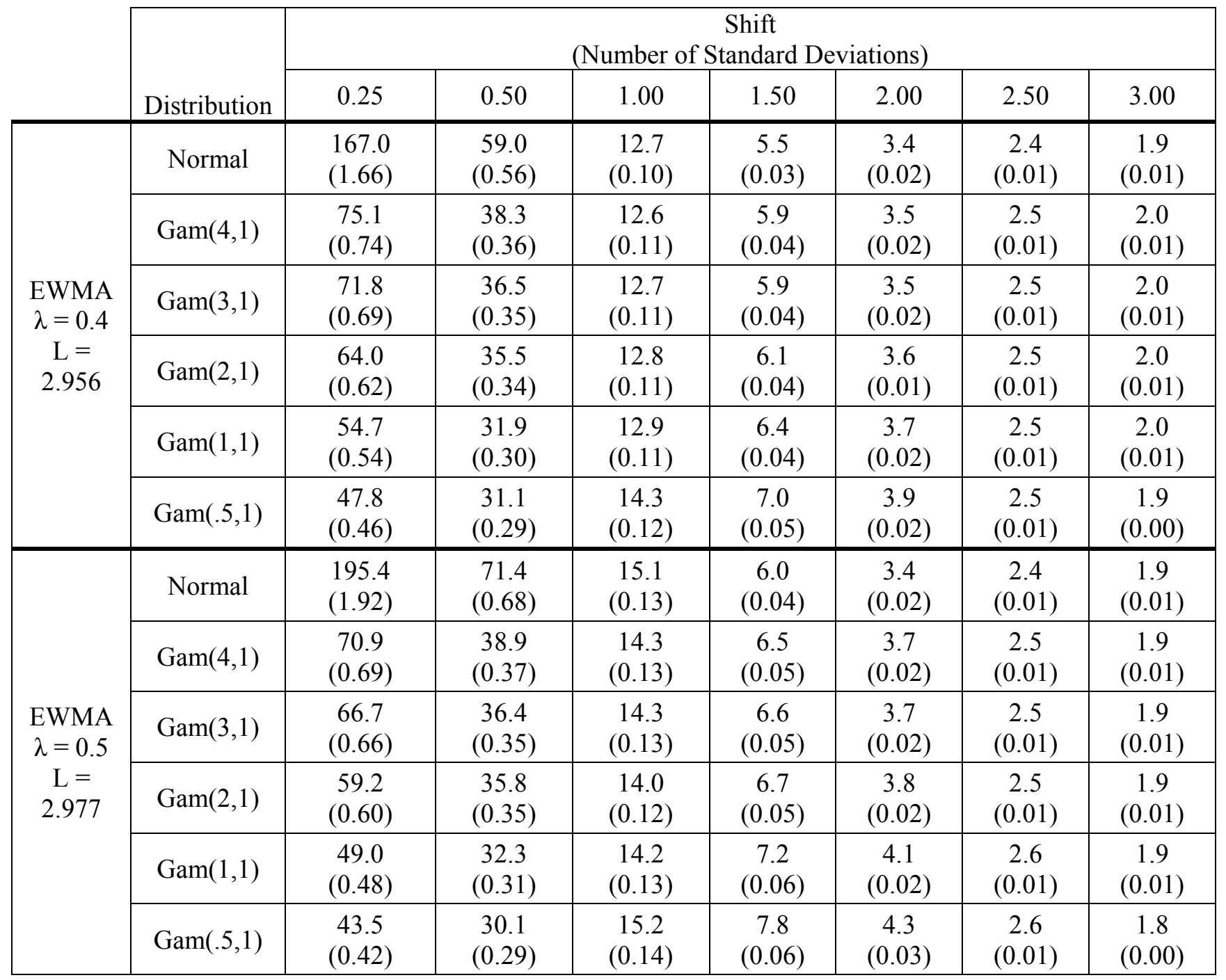


Table 4: Out-of-control ARL's for the DEWMA Control Charts for Normal and Various Gamma Distributions

\begin{tabular}{|c|c|c|c|c|c|c|c|c|}
\hline & \multirow[b]{2}{*}{ Distribution } & \multicolumn{7}{|c|}{$\begin{array}{c}\text { Shift } \\
\text { (Number of Standard Deviations) }\end{array}$} \\
\hline & & 0.25 & 0.50 & 1.00 & 1.50 & 2.00 & 2.50 & 3.00 \\
\hline \multirow{6}{*}{$\begin{array}{c}\text { DEWMA } \\
\lambda=0.1 \\
\mathrm{~L}=3.796\end{array}$} & Normal & $\begin{array}{c}88.2 \\
(0.80) \\
\end{array}$ & $\begin{array}{c}27.5 \\
(0.20) \\
\end{array}$ & $\begin{array}{c}9.7 \\
(0.04) \\
\end{array}$ & $\begin{array}{c}5.7 \\
(0.02) \\
\end{array}$ & $\begin{array}{c}4.2 \\
(0.01) \\
\end{array}$ & $\begin{array}{c}3.3 \\
(0.01)\end{array}$ & $\begin{array}{c}2.7 \\
(0.01) \\
\end{array}$ \\
\hline & $\operatorname{Gam}(4,1)$ & $\begin{array}{l}78.3 \\
(0.69)\end{array}$ & $\begin{array}{c}28.4 \\
(0.20)\end{array}$ & $\begin{array}{l}10.0 \\
(0.05)\end{array}$ & $\begin{array}{c}5.9 \\
(0.02)\end{array}$ & $\begin{array}{c}4.2 \\
(0.01)\end{array}$ & $\begin{array}{c}3.3 \\
(0.01)\end{array}$ & $\begin{array}{c}2.8 \\
(0.01)\end{array}$ \\
\hline & $\operatorname{Gam}(3,1)$ & $\begin{array}{l}75.3 \\
(0.66)\end{array}$ & $\begin{array}{c}28.8 \\
(0.21)\end{array}$ & $\begin{array}{c}10.1 \\
(0.05)\end{array}$ & $\begin{array}{c}5.9 \\
(0.02)\end{array}$ & $\begin{array}{c}4.2 \\
(0.01)\end{array}$ & $\begin{array}{c}3.3 \\
(0.01)\end{array}$ & $\begin{array}{c}2.8 \\
(0.01)\end{array}$ \\
\hline & $\operatorname{Gam}(2,1)$ & $\begin{array}{l}75.3 \\
(0.69)\end{array}$ & $\begin{array}{c}28.7 \\
(0.22)\end{array}$ & $\begin{array}{c}10.1 \\
(0.05)\end{array}$ & $\begin{array}{c}5.9 \\
(0.02)\end{array}$ & $\begin{array}{c}4.2 \\
(0.01)\end{array}$ & $\begin{array}{c}3.3 \\
(0.01)\end{array}$ & $\begin{array}{c}2.7 \\
(0.01)\end{array}$ \\
\hline & $\operatorname{Gam}(1,1)$ & $\begin{array}{c}73.9 \\
(0.68) \\
\end{array}$ & $\begin{array}{r}29.9 \\
(0.23) \\
\end{array}$ & $\begin{array}{c}10.4 \\
(0.05)\end{array}$ & $\begin{array}{c}5.9 \\
(0.02) \\
\end{array}$ & $\begin{array}{c}4.2 \\
(0.01) \\
\end{array}$ & $\begin{array}{c}3.3 \\
(0.01) \\
\end{array}$ & $\begin{array}{c}2.7 \\
(0.01)\end{array}$ \\
\hline & $\operatorname{Gam}(.5,1)$ & $\begin{array}{c}71.6 \\
(0.67)\end{array}$ & $\begin{array}{c}30.4 \\
(0.24)\end{array}$ & $\begin{array}{c}10.7 \\
(0.05)\end{array}$ & $\begin{array}{c}5.9 \\
(0.02)\end{array}$ & $\begin{array}{c}4.2 \\
(0.01)\end{array}$ & $\begin{array}{c}3.3 \\
(0.01)\end{array}$ & $\begin{array}{c}2.7 \\
(0.00)\end{array}$ \\
\hline \multirow{6}{*}{$\begin{array}{c}\text { DEWMA } \\
\lambda=0.2 \\
L=4.018\end{array}$} & Normal & $\begin{array}{l}122.7 \\
(1.16)\end{array}$ & $\begin{array}{c}36.6 \\
(0.31)\end{array}$ & $\begin{array}{c}9.7 \\
(0.06)\end{array}$ & $\begin{array}{c}5.3 \\
(0.02)\end{array}$ & $\begin{array}{c}3.6 \\
(0.01)\end{array}$ & $\begin{array}{c}2.8 \\
(0.01)\end{array}$ & $\begin{array}{c}2.3 \\
(0.01)\end{array}$ \\
\hline & $\operatorname{Gam}(4,1)$ & $\begin{array}{c}81.1 \\
(0.78) \\
\end{array}$ & $\begin{array}{c}33.1 \\
(0.29) \\
\end{array}$ & $\begin{array}{c}10.4 \\
(0.06) \\
\end{array}$ & $\begin{array}{c}5.4 \\
(0.02)\end{array}$ & $\begin{array}{c}3.6 \\
(0.01)\end{array}$ & $\begin{array}{c}2.8 \\
(0.01)\end{array}$ & $\begin{array}{c}2.3 \\
(0.01)\end{array}$ \\
\hline & $\operatorname{Gam}(3,1)$ & $\begin{array}{c}79.9 \\
(0.77)\end{array}$ & $\begin{array}{c}33.0 \\
(0.29)\end{array}$ & $\begin{array}{c}10.3 \\
(0.06)\end{array}$ & $\begin{array}{c}5.4 \\
(0.03)\end{array}$ & $\begin{array}{c}3.7 \\
(0.01)\end{array}$ & $\begin{array}{c}2.8 \\
(0.01)\end{array}$ & $\begin{array}{c}2.3 \\
(0.01)\end{array}$ \\
\hline & $\operatorname{Gam}(2,1)$ & $\begin{array}{c}74.6 \\
(0.71) \\
\end{array}$ & $\begin{array}{c}32.5 \\
(0.29) \\
\end{array}$ & $\begin{array}{c}10.6 \\
(0.07) \\
\end{array}$ & $\begin{array}{c}5.5 \\
(0.02) \\
\end{array}$ & $\begin{array}{c}3.7 \\
(0.01) \\
\end{array}$ & $\begin{array}{c}2.8 \\
(0.01) \\
\end{array}$ & $\begin{array}{c}2.3 \\
(0.01) \\
\end{array}$ \\
\hline & $\operatorname{Gam}(1,1)$ & $\begin{array}{c}67.9 \\
(0.64)\end{array}$ & $\begin{array}{c}32.9 \\
(0.29)\end{array}$ & $\begin{array}{c}11.0 \\
(0.07)\end{array}$ & $\begin{array}{c}5.7 \\
(0.03)\end{array}$ & $\begin{array}{c}3.7 \\
(0.01)\end{array}$ & $\begin{array}{c}2.8 \\
(0.01)\end{array}$ & $\begin{array}{c}2.3 \\
(0.01)\end{array}$ \\
\hline & $\operatorname{Gam}(.5,1)$ & $\begin{array}{c}63.6 \\
(0.60)\end{array}$ & $\begin{array}{c}32.6 \\
(0.30)\end{array}$ & $\begin{array}{c}11.6 \\
(0.08)\end{array}$ & $\begin{array}{c}5.9 \\
(0.03)\end{array}$ & $\begin{array}{c}3.7 \\
(0.01)\end{array}$ & $\begin{array}{c}2.8 \\
(0.01)\end{array}$ & $\begin{array}{c}2.3 \\
(0.01)\end{array}$ \\
\hline \multirow{6}{*}{$\begin{array}{c}\text { DEWMA } \\
\lambda=0.3 \\
L=4.069\end{array}$} & Normal & $\begin{array}{l}149.2 \\
(1.44)\end{array}$ & $\begin{array}{c}46.0 \\
(0.42)\end{array}$ & $\begin{array}{c}10.9 \\
(0.08)\end{array}$ & $\begin{array}{c}5.2 \\
(0.03)\end{array}$ & $\begin{array}{c}3.4 \\
(0.01)\end{array}$ & $\begin{array}{c}2.5 \\
(0.01)\end{array}$ & $\begin{array}{c}2.1 \\
(0.01)\end{array}$ \\
\hline & $\operatorname{Gam}(4,1)$ & $\begin{array}{c}78.8 \\
(0.76)\end{array}$ & $\begin{array}{c}36.7 \\
(0.33)\end{array}$ & $\begin{array}{c}11.4 \\
(0.09)\end{array}$ & $\begin{array}{c}5.5 \\
(0.3)\end{array}$ & $\begin{array}{c}3.5 \\
(0.01)\end{array}$ & $\begin{array}{c}2.6 \\
(0.01)\end{array}$ & $\begin{array}{c}2.1 \\
(0.01)\end{array}$ \\
\hline & $\operatorname{Gam}(3,1)$ & $\begin{array}{c}74.2 \\
(0.71)\end{array}$ & $\begin{array}{c}35.4 \\
(0.32)\end{array}$ & $\begin{array}{c}11.3 \\
(0.08)\end{array}$ & $\begin{array}{c}5.5 \\
(0.03)\end{array}$ & $\begin{array}{c}3.5 \\
(0.01)\end{array}$ & $\begin{array}{c}2.6 \\
(0.01)\end{array}$ & $\begin{array}{c}2.1 \\
(0.01)\end{array}$ \\
\hline & $\operatorname{Gam}(2,1)$ & $\begin{array}{c}68.5 \\
(0.65) \\
\end{array}$ & $\begin{array}{c}33.9 \\
(0.32) \\
\end{array}$ & $\begin{array}{c}11.4 \\
(0.09) \\
\end{array}$ & $\begin{array}{c}5.6 \\
(0.03) \\
\end{array}$ & $\begin{array}{c}3.5 \\
(0.01) \\
\end{array}$ & $\begin{array}{c}2.6 \\
(0.01) \\
\end{array}$ & $\begin{array}{c}2.1 \\
(0.01) \\
\end{array}$ \\
\hline & $\operatorname{Gam}(1,1)$ & $\begin{array}{c}60.1 \\
(0.57)\end{array}$ & $\begin{array}{c}32.8 \\
(0.31)\end{array}$ & $\begin{array}{c}12.1 \\
(0.09)\end{array}$ & $\begin{array}{c}5.9 \\
(0.03)\end{array}$ & $\begin{array}{c}3.6 \\
(0.01)\end{array}$ & $\begin{array}{c}2.6 \\
(0.01)\end{array}$ & $\begin{array}{c}2.1 \\
(0.01)\end{array}$ \\
\hline & $\operatorname{Gam}(.5,1)$ & $\begin{array}{c}54.7 \\
(0.54) \\
\end{array}$ & $\begin{array}{c}32.9 \\
(0.32) \\
\end{array}$ & $\begin{array}{c}13.2 \\
(0.11) \\
\end{array}$ & $\begin{array}{c}6.3 \\
(0.04) \\
\end{array}$ & $\begin{array}{c}3.6 \\
(0.01) \\
\end{array}$ & $\begin{array}{c}2.6 \\
(0.01) \\
\end{array}$ & $\begin{array}{c}2.0 \\
(0.01) \\
\end{array}$ \\
\hline
\end{tabular}


Table 4 (continued): Out-of-control ARL's for the DEWMA Control Charts for Normal and Various Gamma Distributions

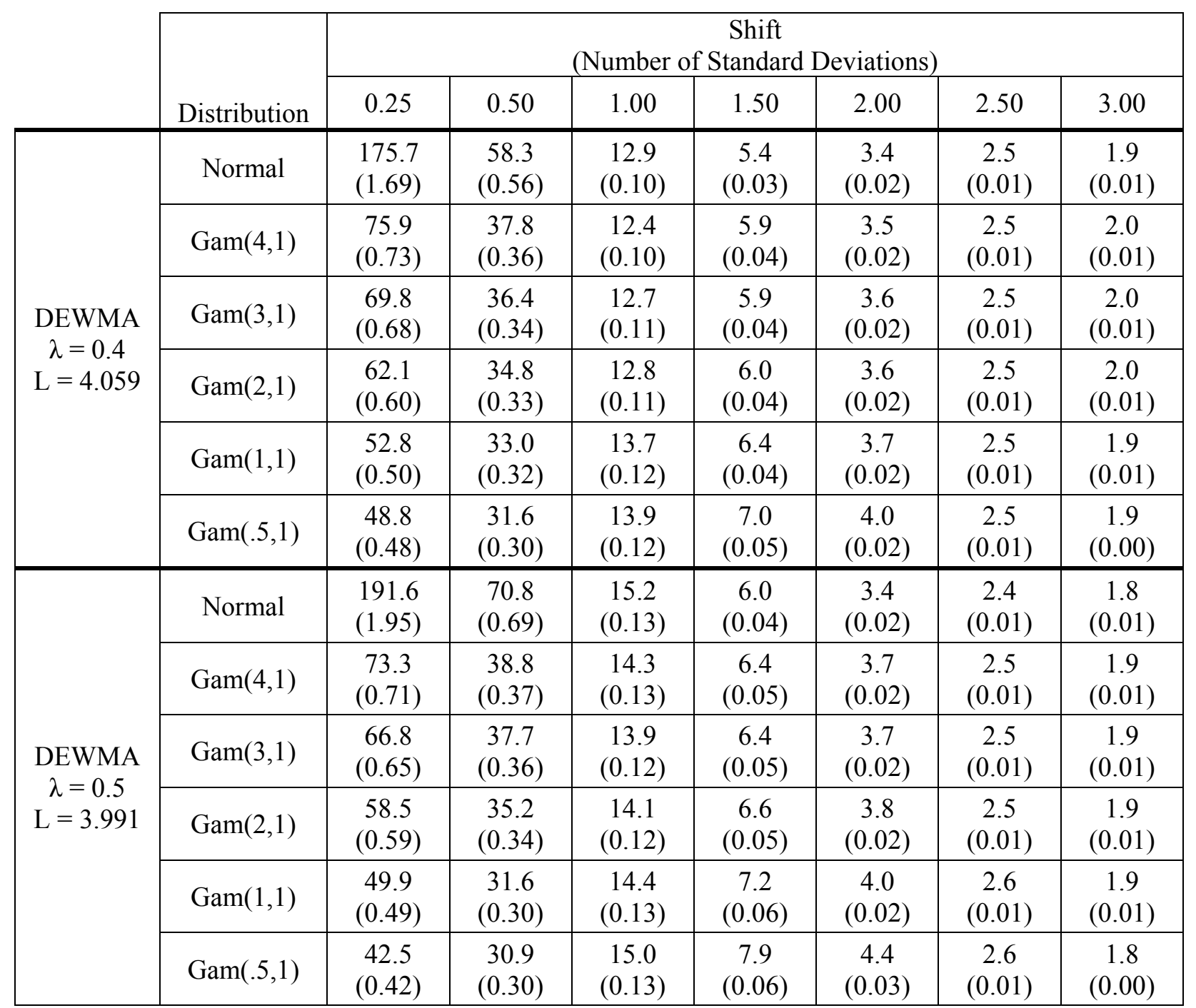


DEWMA VS. EWMA CONTROL CHART ROBUSTNESS TO NON-NORMAL PROCESSES

Table 5: Out-of-control ARL's for the EWMA Control Charts for Normal and Various t Distributions

\begin{tabular}{|c|c|c|c|c|c|c|c|c|}
\hline & \multirow[b]{2}{*}{ Distribution } & \multicolumn{7}{|c|}{$\begin{array}{c}\text { Shift } \\
\text { (Number of Standard Deviations) }\end{array}$} \\
\hline & & 0.25 & 0.50 & 1.00 & 1.50 & 2.00 & 2.50 & 3.00 \\
\hline \multirow{6}{*}{$\begin{array}{c}\text { EWMA } \\
\lambda=0.1 \\
L= \\
2.698\end{array}$} & Normal & $\begin{array}{c}90.4 \\
(0.80)\end{array}$ & $\begin{array}{l}27.9 \\
(0.19)\end{array}$ & $\begin{array}{c}9.6 \\
(0.04)\end{array}$ & $\begin{array}{c}9.6 \\
(0.04)\end{array}$ & $\begin{array}{c}4.2 \\
(0.01)\end{array}$ & $\begin{array}{c}3.3 \\
(0.01)\end{array}$ & $\begin{array}{c}2.8 \\
(0.01)\end{array}$ \\
\hline & $t_{50}$ & $\begin{array}{c}87.4 \\
(0.78)\end{array}$ & $\begin{array}{c}27.9 \\
(0.19)\end{array}$ & $\begin{array}{c}9.8 \\
(0.04)\end{array}$ & $\begin{array}{c}9.8 \\
(0.04)\end{array}$ & $\begin{array}{c}4.2 \\
(0.01)\end{array}$ & $\begin{array}{c}3.3 \\
(0.01)\end{array}$ & $\begin{array}{c}2.8 \\
(0.01)\end{array}$ \\
\hline & $t_{40}$ & $\begin{array}{c}88.9 \\
(0.78)\end{array}$ & $\begin{array}{c}28.5 \\
(0.20)\end{array}$ & $\begin{array}{c}9.7 \\
(0.04)\end{array}$ & $\begin{array}{c}9.7 \\
(0.04)\end{array}$ & $\begin{array}{c}4.2 \\
(0.01)\end{array}$ & $\begin{array}{c}3.3 \\
(0.01)\end{array}$ & $\begin{array}{c}2.8 \\
(0.01)\end{array}$ \\
\hline & $t_{20}$ & $\begin{array}{c}90.7 \\
(0.81)\end{array}$ & $\begin{array}{c}28.5 \\
(0.20)\end{array}$ & $\begin{array}{c}9.7 \\
(0.04)\end{array}$ & $\begin{array}{c}9.7 \\
(0.04)\end{array}$ & $\begin{array}{c}4.2 \\
(0.01)\end{array}$ & $\begin{array}{c}3.3 \\
(0.01)\end{array}$ & $\begin{array}{c}2.8 \\
(0.01)\end{array}$ \\
\hline & $t_{10}$ & $\begin{array}{c}90.4 \\
(0.80)\end{array}$ & $\begin{array}{c}28.3 \\
(0.20)\end{array}$ & $\begin{array}{c}9.7 \\
(0.05)\end{array}$ & $\begin{array}{c}9.7 \\
(0.05)\end{array}$ & $\begin{array}{c}4.2 \\
(0.01)\end{array}$ & $\begin{array}{c}3.3 \\
(0.01)\end{array}$ & $\begin{array}{c}2.8 \\
(0.01)\end{array}$ \\
\hline & $t_{4}$ & $\begin{array}{l}94.6 \\
(0.86)\end{array}$ & $\begin{array}{c}30.4 \\
(0.22)\end{array}$ & $\begin{array}{c}9.8 \\
(0.04)\end{array}$ & $\begin{array}{c}9.8 \\
(0.04)\end{array}$ & $\begin{array}{c}4.2 \\
(0.01)\end{array}$ & $\begin{array}{c}3.3 \\
(0.01)\end{array}$ & $\begin{array}{c}2.8 \\
(0.01)\end{array}$ \\
\hline \multirow{6}{*}{$\begin{array}{c}\text { EWMA } \\
\lambda=0.2 \\
L= \\
2.856\end{array}$} & Normal & $\begin{array}{l}119.3 \\
(1.17)\end{array}$ & $\begin{array}{c}36.0 \\
(0.30)\end{array}$ & $\begin{array}{c}9.8 \\
(0.06)\end{array}$ & $\begin{array}{c}9.8 \\
(0.06)\end{array}$ & $\begin{array}{c}3.6 \\
(0.01)\end{array}$ & $\begin{array}{c}2.8 \\
(0.01)\end{array}$ & $\begin{array}{c}2.3 \\
(0.01)\end{array}$ \\
\hline & $t_{50}$ & $\begin{array}{c}117.4 \\
(1.10)\end{array}$ & $\begin{array}{c}35.9 \\
(0.30)\end{array}$ & $\begin{array}{c}9.8 \\
(0.06)\end{array}$ & $\begin{array}{c}9.8 \\
(0.06)\end{array}$ & $\begin{array}{c}3.6 \\
(0.01)\end{array}$ & $\begin{array}{c}2.8 \\
(0.01)\end{array}$ & $\begin{array}{c}2.3 \\
(0.01)\end{array}$ \\
\hline & $t_{40}$ & $\begin{array}{l}120.8 \\
(1.16)\end{array}$ & $\begin{array}{c}36.6 \\
(0.31)\end{array}$ & $\begin{array}{c}9.7 \\
(0.06)\end{array}$ & $\begin{array}{c}9.7 \\
(0.06)\end{array}$ & $\begin{array}{c}3.6 \\
(0.01)\end{array}$ & $\begin{array}{c}2.8 \\
(0.01)\end{array}$ & $\begin{array}{c}2.3 \\
(0.01)\end{array}$ \\
\hline & $t_{20}$ & $\begin{array}{l}119.6 \\
(1.13)\end{array}$ & $\begin{array}{c}36.3 \\
(0.31)\end{array}$ & $\begin{array}{c}9.8 \\
(0.06)\end{array}$ & $\begin{array}{c}9.8 \\
(0.06)\end{array}$ & $\begin{array}{c}3.6 \\
(0.01)\end{array}$ & $\begin{array}{c}2.8 \\
(0.01)\end{array}$ & $\begin{array}{c}2.3 \\
(0.01)\end{array}$ \\
\hline & $t_{10}$ & $\begin{array}{l}113.3 \\
(1.07)\end{array}$ & $\begin{array}{c}37.2 \\
(0.32)\end{array}$ & $\begin{array}{c}10.0 \\
(0.06)\end{array}$ & $\begin{array}{c}10.0 \\
(0.06)\end{array}$ & $\begin{array}{c}3.6 \\
(0.01)\end{array}$ & $\begin{array}{c}2.8 \\
(0.01)\end{array}$ & $\begin{array}{c}2.3 \\
(0.01)\end{array}$ \\
\hline & $t_{4}$ & $\begin{array}{c}104.1 \\
(1.00)\end{array}$ & $\begin{array}{c}40.3 \\
(0.35)\end{array}$ & $\begin{array}{c}10.2 \\
(0.06)\end{array}$ & $\begin{array}{c}10.2 \\
(0.06)\end{array}$ & $\begin{array}{c}3.6 \\
(0.01)\end{array}$ & $\begin{array}{c}2.8 \\
(0.01)\end{array}$ & $\begin{array}{c}2.3 \\
(0.01)\end{array}$ \\
\hline \multirow{6}{*}{$\begin{array}{c}\text { EWMA } \\
\lambda=0.3 \\
L= \\
2.929\end{array}$} & Normal & $\begin{array}{l}149.4 \\
(1.46)\end{array}$ & $\begin{array}{c}46.8 \\
(0.44)\end{array}$ & $\begin{array}{c}10.9 \\
(0.08)\end{array}$ & $\begin{array}{c}10.9 \\
(0.08)\end{array}$ & $\begin{array}{c}3.4 \\
(0.01)\end{array}$ & $\begin{array}{c}2.6 \\
(0.01)\end{array}$ & $\begin{array}{c}2.1 \\
(0.01)\end{array}$ \\
\hline & $t_{50}$ & $\begin{array}{l}143.7 \\
(1.43)\end{array}$ & $\begin{array}{c}47.1 \\
(0.43)\end{array}$ & $\begin{array}{c}11.0 \\
(0.08)\end{array}$ & $\begin{array}{c}11.0 \\
(0.08)\end{array}$ & $\begin{array}{c}3.4 \\
(0.01)\end{array}$ & $\begin{array}{c}2.6 \\
(0.01)\end{array}$ & $\begin{array}{c}2.1 \\
(0.01)\end{array}$ \\
\hline & $t_{40}$ & $\begin{array}{l}143.9 \\
(1.42)\end{array}$ & $\begin{array}{c}46.5 \\
(0.43)\end{array}$ & $\begin{array}{c}11.0 \\
(0.08)\end{array}$ & $\begin{array}{c}11.0 \\
(0.08)\end{array}$ & $\begin{array}{c}3.4 \\
(0.01)\end{array}$ & $\begin{array}{c}2.6 \\
(0.01)\end{array}$ & $\begin{array}{c}2.1 \\
(0.01)\end{array}$ \\
\hline & $t_{20}$ & $\begin{array}{l}141.7 \\
(1.39) \\
\end{array}$ & $\begin{array}{c}46.6 \\
(0.43) \\
\end{array}$ & $\begin{array}{c}11.0 \\
(0.08) \\
\end{array}$ & $\begin{array}{c}11.0 \\
(0.08) \\
\end{array}$ & $\begin{array}{c}3.4 \\
(0.01) \\
\end{array}$ & $\begin{array}{c}2.6 \\
(0.01) \\
\end{array}$ & $\begin{array}{c}2.1 \\
(0.01) \\
\end{array}$ \\
\hline & $t_{10}$ & $\begin{array}{l}126.5 \\
(1.24) \\
\end{array}$ & $\begin{array}{c}47.6 \\
(0.45) \\
\end{array}$ & $\begin{array}{c}11.2 \\
(0.08) \\
\end{array}$ & $\begin{array}{c}11.2 \\
(0.08)\end{array}$ & $\begin{array}{c}3.4 \\
(0.01)\end{array}$ & $\begin{array}{c}2.6 \\
(0.01)\end{array}$ & $\begin{array}{c}2.1 \\
(0.01)\end{array}$ \\
\hline & $t_{4}$ & $\begin{array}{l}105.5 \\
(1.03)\end{array}$ & $\begin{array}{c}50.8 \\
(0.47)\end{array}$ & $\begin{array}{c}12.3 \\
(0.09)\end{array}$ & $\begin{array}{c}12.3 \\
(0.09)\end{array}$ & $\begin{array}{c}3.4 \\
(0.01)\end{array}$ & $\begin{array}{c}2.5 \\
(0.01)\end{array}$ & $\begin{array}{c}2.1 \\
(0.01)\end{array}$ \\
\hline
\end{tabular}


SAAD SAEED ALKAHTANI

Table 5 (continued): Out-of-control ARL's for the EWMA Control Charts for Normal and Various t Distributions

\begin{tabular}{|c|c|c|c|c|c|c|c|c|}
\hline & \multirow[b]{2}{*}{ Distribution } & \multicolumn{7}{|c|}{$\begin{array}{c}\text { Shift } \\
\text { (Number of Standard Deviations) }\end{array}$} \\
\hline & & 0.25 & 0.50 & 1.00 & 1.50 & 2.00 & 2.50 & 3.00 \\
\hline \multirow{6}{*}{$\begin{array}{c}\text { EWMA } \\
\lambda=0.4 \\
L= \\
2.956\end{array}$} & Normal & $\begin{array}{r}167.0 \\
(1.66)\end{array}$ & $\begin{array}{c}59.0 \\
(0.56)\end{array}$ & $\begin{array}{c}12.7 \\
(0.10)\end{array}$ & $\begin{array}{c}12.7 \\
(0.10)\end{array}$ & $\begin{array}{c}3.4 \\
(0.02)\end{array}$ & $\begin{array}{c}2.4 \\
(0.01)\end{array}$ & $\begin{array}{c}1.9 \\
(0.01)\end{array}$ \\
\hline & $t_{50}$ & $\begin{array}{l}163.5 \\
(1.59) \\
\end{array}$ & $\begin{array}{c}58.2 \\
(0.55) \\
\end{array}$ & $\begin{array}{c}12.7 \\
(0.10)\end{array}$ & $\begin{array}{c}12.7 \\
(0.10)\end{array}$ & $\begin{array}{c}3.3 \\
(0.02)\end{array}$ & $\begin{array}{c}2.4 \\
(0.01)\end{array}$ & $\begin{array}{c}1.9 \\
(0.01)\end{array}$ \\
\hline & $t_{40}$ & $\begin{array}{l}160.9 \\
(1.60)\end{array}$ & $\begin{array}{l}56.7 \\
(0.55)\end{array}$ & $\begin{array}{c}12.8 \\
(0.10)\end{array}$ & $\begin{array}{c}12.8 \\
(0.10)\end{array}$ & $\begin{array}{c}3.3 \\
(0.02)\end{array}$ & $\begin{array}{c}2.4 \\
(0.01)\end{array}$ & $\begin{array}{c}2.0 \\
(0.01)\end{array}$ \\
\hline & $t_{20}$ & $\begin{array}{l}149.5 \\
(1.46)\end{array}$ & $\begin{array}{c}56.4 \\
(0.52)\end{array}$ & $\begin{array}{c}13.1 \\
(0.10)\end{array}$ & $\begin{array}{c}13.1 \\
(0.10)\end{array}$ & $\begin{array}{c}3.3 \\
(0.02)\end{array}$ & $\begin{array}{c}2.4 \\
(0.01)\end{array}$ & $\begin{array}{c}2.0 \\
(0.01)\end{array}$ \\
\hline & $t_{10}$ & $\begin{array}{c}128.4 \\
(1.28)\end{array}$ & $\begin{array}{c}54.4 \\
(0.51) \\
\end{array}$ & $\begin{array}{c}13.1 \\
(0.10)\end{array}$ & $\begin{array}{c}13.1 \\
(0.10)\end{array}$ & $\begin{array}{c}3.4 \\
(0.02)\end{array}$ & $\begin{array}{c}2.4 \\
(0.01)\end{array}$ & $\begin{array}{c}1.9 \\
(0.01)\end{array}$ \\
\hline & $t_{4}$ & $\begin{array}{r}93.3 \\
(0.93) \\
\end{array}$ & $\begin{array}{c}56.0 \\
(0.53) \\
\end{array}$ & $\begin{array}{c}15.1 \\
(0.13) \\
\end{array}$ & $\begin{array}{c}15.1 \\
(0.13) \\
\end{array}$ & $\begin{array}{r}3.3 \\
(0.01) \\
\end{array}$ & $\begin{array}{c}2.4 \\
(0.01) \\
\end{array}$ & $\begin{array}{c}2.0 \\
(0.01) \\
\end{array}$ \\
\hline \multirow{6}{*}{$\begin{array}{c}\text { EWMA } \\
\lambda=0.5 \\
L= \\
2.977\end{array}$} & Normal & $\begin{array}{l}195.4 \\
(1.92) \\
\end{array}$ & $\begin{array}{c}71.4 \\
(0.68) \\
\end{array}$ & $\begin{array}{c}15.1 \\
(0.13) \\
\end{array}$ & $\begin{array}{c}15.1 \\
(0.13) \\
\end{array}$ & $\begin{array}{r}3.4 \\
(0.02) \\
\end{array}$ & $\begin{array}{c}2.4 \\
(0.01) \\
\end{array}$ & $\begin{array}{c}1.9 \\
(0.01) \\
\end{array}$ \\
\hline & $t_{50}$ & $\begin{array}{r}182.2 \\
(1.77)\end{array}$ & $\begin{array}{c}69.0 \\
(0.67)\end{array}$ & $\begin{array}{c}15.2 \\
(0.13)\end{array}$ & $\begin{array}{c}15.2 \\
(0.13)\end{array}$ & $\begin{array}{c}3.4 \\
(0.02)\end{array}$ & $\begin{array}{c}2.4 \\
(0.01)\end{array}$ & $\begin{array}{c}1.9 \\
(0.01)\end{array}$ \\
\hline & $t_{40}$ & $\begin{array}{c}170.4 \\
(1.69)\end{array}$ & $\begin{array}{c}70.5 \\
(0.68)\end{array}$ & $\begin{array}{c}15.3 \\
(0.13)\end{array}$ & $\begin{array}{c}15.3 \\
(0.13)\end{array}$ & $\begin{array}{c}3.5 \\
(0.02)\end{array}$ & $\begin{array}{c}2.4 \\
(0.01)\end{array}$ & $\begin{array}{c}1.8 \\
(0.01)\end{array}$ \\
\hline & $t_{20}$ & $\begin{array}{c}157.1 \\
(1.54) \\
\end{array}$ & $\begin{array}{c}66.7 \\
(0.65) \\
\end{array}$ & $\begin{array}{c}15.6 \\
(0.14) \\
\end{array}$ & $\begin{array}{c}15.6 \\
(0.14) \\
\end{array}$ & $\begin{array}{c}3.5 \\
(0.02) \\
\end{array}$ & $\begin{array}{c}2.4 \\
(0.01) \\
\end{array}$ & $\begin{array}{c}1.9 \\
(0.01) \\
\end{array}$ \\
\hline & $t_{10}$ & $\begin{array}{r}131.0 \\
(1.34) \\
\end{array}$ & $\begin{array}{c}62.5 \\
(0.60) \\
\end{array}$ & $\begin{array}{c}16.0 \\
(0.14)\end{array}$ & $\begin{array}{c}16.0 \\
(0.14)\end{array}$ & $\begin{array}{c}3.4 \\
(0.02)\end{array}$ & $\begin{array}{c}2.4 \\
(0.01)\end{array}$ & $\begin{array}{c}1.9 \\
(0.01)\end{array}$ \\
\hline & $t_{4}$ & $\begin{array}{c}87.1 \\
(0.88)\end{array}$ & $\begin{array}{c}58.8 \\
(0.57)\end{array}$ & $\begin{array}{c}18.6 \\
(0.17)\end{array}$ & $\begin{array}{c}18.6 \\
(0.17)\end{array}$ & $\begin{array}{c}3.5 \\
(0.02)\end{array}$ & $\begin{array}{c}2.4 \\
(0.01)\end{array}$ & $\begin{array}{c}1.9 \\
(0.01)\end{array}$ \\
\hline
\end{tabular}


DEWMA VS. EWMA CONTROL CHART ROBUSTNESS TO NON-NORMAL PROCESSES

Table 6: Out-of-control ARL's for the DEWMA Control Charts for Normal and Various t Distributions

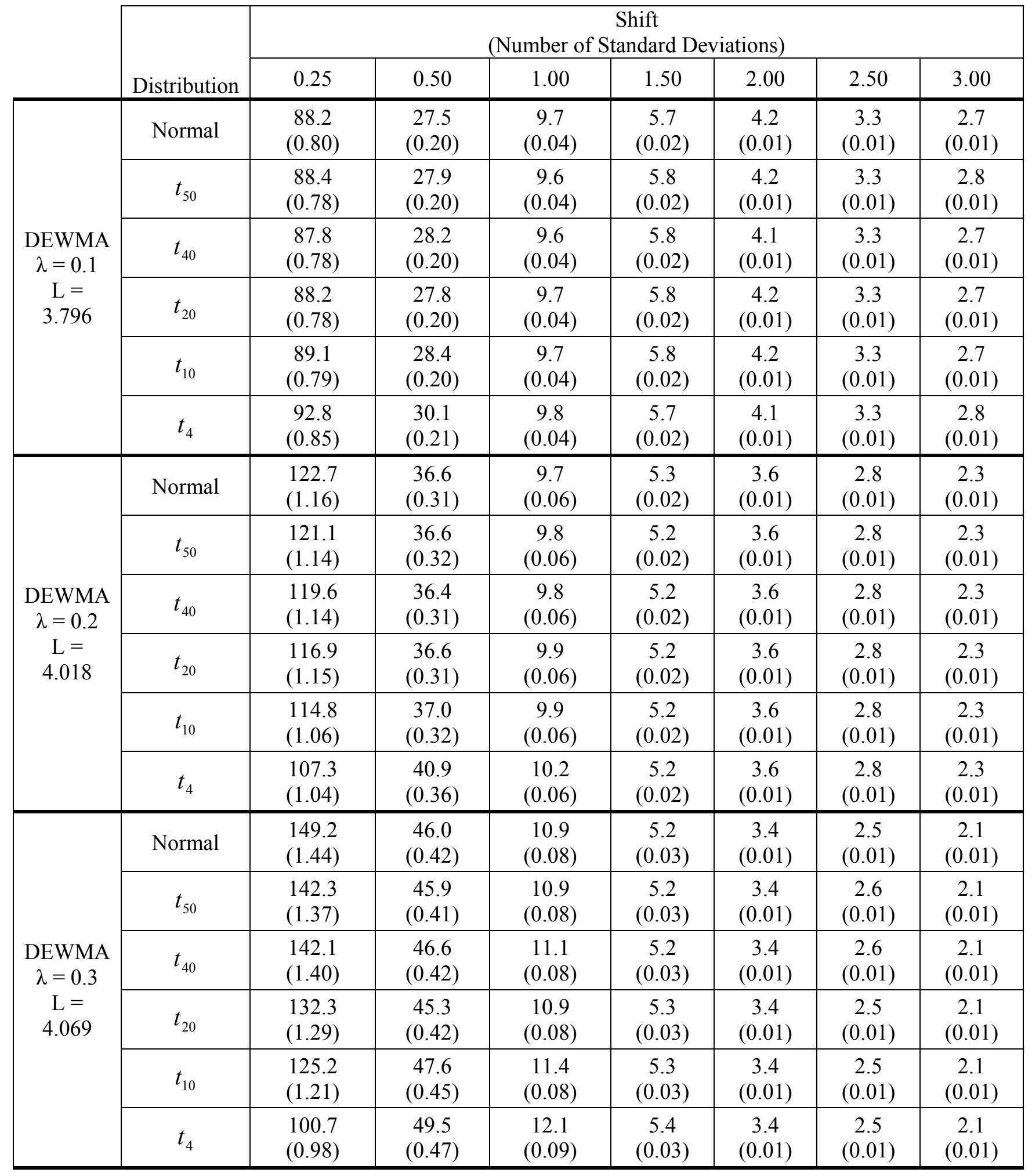


SAAD SAEED ALKAHTANI

Table 6 (continued): Out-of-control ARL's for the DEWMA Control Charts for Normal and Various t Distributions

\begin{tabular}{|c|c|c|c|c|c|c|c|c|}
\hline & \multirow[b]{2}{*}{ Distribution } & \multicolumn{7}{|c|}{$\begin{array}{c}\text { Shift } \\
\text { (Number of Standard Deviations) }\end{array}$} \\
\hline & & 0.25 & 0.50 & 1.00 & 1.50 & 2.00 & 2.50 & 3.00 \\
\hline \multirow{6}{*}{$\begin{array}{c}\text { DEWMA } \\
\lambda=0.4 \\
\mathrm{~L}= \\
4.059\end{array}$} & Normal & $\begin{array}{l}175.7 \\
(1.69)\end{array}$ & $\begin{array}{c}58.3 \\
(0.56)\end{array}$ & $\begin{array}{c}12.9 \\
(0.10)\end{array}$ & $\begin{array}{c}5.4 \\
(0.03)\end{array}$ & $\begin{array}{c}3.4 \\
(0.02)\end{array}$ & $\begin{array}{c}2.5 \\
(0.01)\end{array}$ & $\begin{array}{c}1.9 \\
(0.01)\end{array}$ \\
\hline & $t_{50}$ & $\begin{array}{l}163.7 \\
(1.62) \\
\end{array}$ & $\begin{array}{c}56.1 \\
(0.54) \\
\end{array}$ & $\begin{array}{c}12.8 \\
(0.10) \\
\end{array}$ & $\begin{array}{c}5.5 \\
(0.03) \\
\end{array}$ & $\begin{array}{c}3.4 \\
(0.02) \\
\end{array}$ & $\begin{array}{c}2.4 \\
(0.01)\end{array}$ & $\begin{array}{c}2.0 \\
(0.01)\end{array}$ \\
\hline & $t_{40}$ & $\begin{array}{l}164.9 \\
(1.62)\end{array}$ & $\begin{array}{c}58.3 \\
(0.57)\end{array}$ & $\begin{array}{c}12.8 \\
(0.10)\end{array}$ & $\begin{array}{c}5.5 \\
(0.03)\end{array}$ & $\begin{array}{c}3.4 \\
(0.02)\end{array}$ & $\begin{array}{c}2.4 \\
(0.01)\end{array}$ & $\begin{array}{c}1.9 \\
(0.02)\end{array}$ \\
\hline & $t_{20}$ & $\begin{array}{l}146.9 \\
(1.45)\end{array}$ & $\begin{array}{l}58.0 \\
(0.55)\end{array}$ & $\begin{array}{c}12.9 \\
(0.10)\end{array}$ & $\begin{array}{c}5.5 \\
(0.03)\end{array}$ & $\begin{array}{c}3.4 \\
(0.02)\end{array}$ & $\begin{array}{c}2.4 \\
(0.01)\end{array}$ & $\begin{array}{c}2.0 \\
(0.01)\end{array}$ \\
\hline & $t_{10}$ & $\begin{array}{l}130.0 \\
(1.29)\end{array}$ & $\begin{array}{c}55.2 \\
(0.52) \\
\end{array}$ & $\begin{array}{l}13.0 \\
(0.10)\end{array}$ & $\begin{array}{c}5.5 \\
(0.03) \\
\end{array}$ & $\begin{array}{c}3.4 \\
(0.02) \\
\end{array}$ & $\begin{array}{c}2.4 \\
(0.01) \\
\end{array}$ & $\begin{array}{c}1.9 \\
(0.01)\end{array}$ \\
\hline & $t_{4}$ & $\begin{array}{c}93.0 \\
(0.90)\end{array}$ & $\begin{array}{c}54.6 \\
(0.53)\end{array}$ & $\begin{array}{c}15.2 \\
(0.13)\end{array}$ & $\begin{array}{c}5.8 \\
(0.04)\end{array}$ & $\begin{array}{c}3.4 \\
(0.02)\end{array}$ & $\begin{array}{c}2.4 \\
(0.01)\end{array}$ & $\begin{array}{c}2.0 \\
(0.01)\end{array}$ \\
\hline \multirow{6}{*}{$\begin{array}{c}\text { DEWMA } \\
\lambda=0.5 \\
\mathrm{~L}= \\
3.991\end{array}$} & Normal & $\begin{array}{l}191.6 \\
(1.95)\end{array}$ & $\begin{array}{c}70.8 \\
(0.69) \\
\end{array}$ & $\begin{array}{c}15.2 \\
(0.13)\end{array}$ & $\begin{array}{c}6.0 \\
(0.04) \\
\end{array}$ & $\begin{array}{c}3.4 \\
(0.02) \\
\end{array}$ & $\begin{array}{c}2.4 \\
(0.01) \\
\end{array}$ & $\begin{array}{c}1.8 \\
(0.01) \\
\end{array}$ \\
\hline & $t_{50}$ & $\begin{array}{l}173.0 \\
(1.75)\end{array}$ & $\begin{array}{c}68.0 \\
(0.65)\end{array}$ & $\begin{array}{c}15.6 \\
(0.13)\end{array}$ & $\begin{array}{c}6.0 \\
(0.04)\end{array}$ & $\begin{array}{c}3.4 \\
(0.02)\end{array}$ & $\begin{array}{c}2.4 \\
(0.01)\end{array}$ & $\begin{array}{c}1.9 \\
(0.01)\end{array}$ \\
\hline & $t_{40}$ & $\begin{array}{l}169.7 \\
(1.67)\end{array}$ & $\begin{array}{c}69.9 \\
(0.68) \\
\end{array}$ & $\begin{array}{c}15.3 \\
(0.13)\end{array}$ & $\begin{array}{c}6.1 \\
(0.04) \\
\end{array}$ & $\begin{array}{c}3.4 \\
(0.02) \\
\end{array}$ & $\begin{array}{c}2.4 \\
(0.01) \\
\end{array}$ & $\begin{array}{c}1.8 \\
(0.01)\end{array}$ \\
\hline & $t_{20}$ & $\begin{array}{l}158.7 \\
(1.57)\end{array}$ & $\begin{array}{c}66.1 \\
(0.65) \\
\end{array}$ & $\begin{array}{c}15.6 \\
(0.13) \\
\end{array}$ & $\begin{array}{c}6.1 \\
(0.04) \\
\end{array}$ & $\begin{array}{c}3.4 \\
(0.02) \\
\end{array}$ & $\begin{array}{c}2.4 \\
(0.01)\end{array}$ & $\begin{array}{c}1.9 \\
(0.01)\end{array}$ \\
\hline & $t_{10}$ & $\begin{array}{l}125.8 \\
(1.22)\end{array}$ & $\begin{array}{c}61.7 \\
(0.60)\end{array}$ & $\begin{array}{c}15.8 \\
(0.14)\end{array}$ & $\begin{array}{c}6.2 \\
(0.04)\end{array}$ & $\begin{array}{c}3.5 \\
(0.02)\end{array}$ & $\begin{array}{c}2.4 \\
(0.01)\end{array}$ & $\begin{array}{c}1.9 \\
(0.01)\end{array}$ \\
\hline & $t_{4}$ & $\begin{array}{c}85.7 \\
(0.86)\end{array}$ & $\begin{array}{c}57.2 \\
(0.56)\end{array}$ & $\begin{array}{c}18.3 \\
(0.17)\end{array}$ & $\begin{array}{c}6.8 \\
(0.05)\end{array}$ & $\begin{array}{c}3.4 \\
(0.02)\end{array}$ & $\begin{array}{c}2.4 \\
(0.01)\end{array}$ & $\begin{array}{c}1.8 \\
(0.01)\end{array}$ \\
\hline
\end{tabular}




\section{Appendix A}

To obtain the DEWMA statistic $z_{i}$ defined in equation (1.5), repeated substitutions were applied to equations in (1.4) to obtain $y_{i}$ and $z_{i}$ rewritten as:

$$
\begin{aligned}
y_{i} & =\lambda x_{i}+(1-\lambda) y_{i-1} \\
& =\lambda \sum_{j=0}^{i-1}(1-\lambda)^{j} x_{i-j}+(1-\lambda)^{i} y_{0}
\end{aligned}
$$

and

$$
\begin{aligned}
z_{i} & =\lambda y_{i}+(1-\lambda) z_{i-1} \\
& =\lambda \sum_{j=0}^{i-1}(1-\lambda)^{j} y_{i-j}+(1-\lambda)^{i} z_{0}
\end{aligned}
$$

Substituting (A1) into (A2) results in:

$$
\begin{aligned}
z_{i} & =\lambda \sum_{j=0}^{i-1}(1-\lambda)^{j}\left[\lambda \sum_{k=0}^{i-j-1}(1-\lambda)^{k} x_{i-j-k}+(1-\lambda)^{i-j} y_{0}\right]+(1-\lambda)^{i} z_{0} \\
& =\lambda^{2} \sum_{j=0}^{i-1}(1-\lambda)^{j} \sum_{k=0}^{i-j-1}(1-\lambda)^{k} x_{i-j-k}+i \lambda(1-\lambda)^{i} y_{0}+(1-\lambda)^{i} z_{0} \\
& =\lambda^{2} \sum_{j=0}^{i-1}(1-\lambda)^{j} \sum_{l=1}^{i-j}(1-\lambda)^{i-j-l} x_{l}+i \lambda(1-\lambda)^{i} y_{0}+(1-\lambda)^{i} z_{0} \\
& =\lambda^{2} \sum_{l=1}^{i}(i-l+1)(1-\lambda)^{i-l} x_{l}+i \lambda(1-\lambda)^{i} y_{0}+(1-\lambda)^{i} z_{0}
\end{aligned}
$$

Replacing $l$ with $j,(\mathrm{~A} 3)$ is:

$$
\mathbf{z}_{i}=\lambda^{2} \sum_{j=1}^{i}(i-j+1)(1-\lambda)^{i-j} \mathbf{x}_{j}+i \lambda(1-\lambda)^{i} \mathbf{y}_{0}+(1-\lambda)^{i} \mathbf{z}_{0}
$$

where it was assumed, without loss of generality, that $y_{0}=z_{0}=\mu_{0}$.

The following quantities were presented by Gradshteyn and Ryzhik (1979).

For $a \neq 0$

$$
\sum_{k=1}^{n} k a^{k}=\frac{a\left(1-a^{n}\right)}{(1-a)^{2}}-\frac{n a^{n+1}}{1-a}
$$

and

$$
\sum_{k=1}^{n} k^{2} a^{k}=\frac{a+a^{2}-(n+1)^{2} a^{n+1}+\left(2 n^{2}+2 n-1\right) a^{n+2}-n^{2} a^{n+3}}{(1-a)^{3}}
$$

are needed to prove the quations (1.6) and (1.7). 


\section{SAAD SAEED ALKAHTANI}

Equation (1.6) Proof

$$
\text { Appendix A (continued) }
$$

$$
\begin{aligned}
\mu_{z_{i}} & =E\left(z_{i}\right)=E\left[\lambda^{2} \sum_{j=1}^{i}(i-j+1)(1-\lambda)^{i-j} x_{j}+i \lambda(1-\lambda)^{i} y_{0}+(1-\lambda)^{i} z_{0}\right] \\
& =\lambda^{2} \sum_{j=1}^{i}(i-j+1)(1-\lambda)^{i-j} E\left(x_{j}\right)+i \lambda(1-\lambda)^{i} E\left(y_{0}\right)+(1-\lambda)^{i} E\left(z_{0}\right) \\
& =\frac{\lambda^{2}}{1-\lambda} \sum_{j=1}^{i}(i-j+1)(1-\lambda)^{i-j+1} \mu_{0}+i \lambda(1-\lambda)^{i} \mu_{0}+(1-\lambda)^{i} \mu_{0}
\end{aligned}
$$

Letting $k=i-j+1$ in the first term

$$
\mu_{z_{i}}=\frac{\lambda^{2}}{1-\lambda} \sum_{k=1}^{i} k(1-\lambda)^{k} \mu_{0}+i \lambda(1-\lambda)^{i} \mu_{0}+(1-\lambda)^{i} \mu_{0}
$$

and applying equation (A.5) to the first term with $a=1-\lambda$ and $n=i$ results in

$$
\begin{aligned}
\mu_{z_{i}} & =\frac{\lambda^{2}}{1-\lambda}\left[\frac{(1-\lambda)\left[1-(1-\lambda)^{i}\right]}{[1-(1-\lambda)]^{2}}-\frac{i(1-\lambda)^{i+1}}{1-(1-\lambda)}\right] \mu_{0}+i \lambda(1-\lambda)^{i} \mu_{o}+(1-\lambda)^{i} \mu_{o} \\
& =\lambda^{2}\left[\frac{1-(1-\lambda)^{i}}{\lambda^{2}}-\frac{i(1-\lambda)^{i}}{\lambda}\right] \mu_{0}+i \lambda(1-\lambda)^{i} \mu_{0}+(1-\lambda)^{i} \mu_{0} \\
& =\left[1-(1-\lambda)^{i}-i \lambda(1-\lambda)^{i}+i \lambda(1-\lambda)^{i}+(1-\lambda)^{i}\right] \mu_{0} \\
& =\mu_{0}
\end{aligned}
$$

Equation (1.7) Proof

$$
\begin{aligned}
\sigma_{z_{i}}^{2} & =\operatorname{Var}\left[z_{i}\right]=\operatorname{Var}\left[\lambda^{2} \sum_{j=1}^{i}(i-j+1)(1-\lambda)^{i-j} x_{j}+i \lambda(1-\lambda)^{i} y_{0}+(1-\lambda)^{i} z_{0}\right] \\
& =\lambda^{4} \sum_{j=1}^{i}(i-j+1)^{2}\left[(1-\lambda)^{2}\right]^{i-j} \operatorname{Var}\left(x_{j}\right)+0 \\
& =\frac{\lambda^{4}}{(1-\lambda)^{2}} \sum_{j=1}^{i}(i-j+1)^{2}\left[(1-\lambda)^{2}\right]^{i-j+1} \sigma^{2}
\end{aligned}
$$

Applying equation (A.6) with $a=(1-\lambda)^{2}, k=i-j+1$ and $n=i$, results in:

$$
\begin{aligned}
\sigma_{z_{i}}^{2} & =\frac{\lambda^{4}}{(1-\lambda)^{2}} \frac{(1-\lambda)^{2}+(1-\lambda)^{4}-(i+1)^{2}(1-\lambda)^{2 i+2}+\left(2 i^{2}+2 i-1\right)(1-\lambda)^{2 i+4}-i^{2}(1-\lambda)^{2 i+6}}{\left[1-(1-\lambda)^{2}\right]^{3}} \sigma^{2} \\
& =\lambda^{4} \frac{1+(1-\lambda)^{2}-(i+1)^{2}(1-\lambda)^{2 i}+\left(2 i^{2}+2 i-1\right)(1-\lambda)^{2 i+2}-i^{2}(1-\lambda)^{2 i+4}}{\left[1-(1-\lambda)^{2}\right]^{3}} \sigma^{2}
\end{aligned}
$$




\title{
The Probit Link Function in Generalized Linear Models for Data Mining Applications
}

\author{
Mehdi Razzaghi \\ Bloomsburg University, \\ Bloomsburg, PA
}

The use of logistic regression for outcome classification of dichotomous variables is well known in data mining applications. The estimated probability of the logit transformation belongs to the class of canonical link functions that follow from particular probability distribution functions. A closely related model is the probit link which can be used for binary responses. Although the probit link is not canonical, in some cases the overall fit of the model can be improved by using non-canonical link functions. This article reviews the properties of the probit link function and discusses its applications in data mining problems. Contrasts and comparisons are made with the logistic link function and an example provides further illustration.

Key words: Probit, logistic, linear models, data mining, link functions.

\section{Introduction}

The problem of outcome classification of qualitative data is a major task in data mining. The goal of classification is to accurately predict the target class for each case in the data. Specifically, in binary classification, the target attribute has only two possible outcomes and fast and accurate classifiers are highly desirable. Several predictive models such as naïve Bayes, classification trees, support vector machine and k-nearest neighbor have traditionally been used with some success. However, recently the use of logistic regression has found more widespread popularity and the method has attracted the attention of several practitioners. The advantage of such a model is that it transforms information about the binary dependent variable into an

Mehdi Razzaghi is a Professor in the Department of Mathematics. He holds a Ph.D. in statistics from the University of London, England and has over 30 years of teaching and research experience. His primary research interests are in distribution theory and application of statistics in environmental sciences. Email him at: mrazzagh@bloomu.edu. unbounded continuous variable and estimates a regular multivariate model. Komarek and Moore (2005) present a simple parameter-free implementation of the logistic regression and demonstrate that the model is sufficiently fast and accurate for classification of binary outcomes in large real-world datasets. Maalouf (2011) presented an overview of various aspects of logistic regression, calling it one of the most important and one of the most widely used data mining techniques. Comparatively less attention has been focused on a similar, but slightly different probit model. The difference between the two models is that the logistic model is based on the logit transformation while the probit model uses the inverse Gaussian link. In most cases, the classification outcome is similar for the two models even though the underlying distributions are different.

\section{Generalized Linear Models}

In linear regression analysis there is a random component $\boldsymbol{Y}$ which identifies the response variable and several explanatory variables (features or attributes) $X_{1}, X_{2}, \ldots, X_{p}$. The response variable is expressed as a linear predictor of the explanatory variables, 


\section{MEHDI RAZZAGHI}

$\boldsymbol{Y}=\beta_{0}+\beta_{1} \boldsymbol{X}_{1}+\beta_{2} \boldsymbol{X}_{2}+\cdots+\beta_{p} \boldsymbol{X}_{\boldsymbol{p}}+\boldsymbol{\varepsilon}$

where $\varepsilon$ is the error term and $\boldsymbol{Y}, X_{1}, X_{2}, \ldots X_{p}$ and $\boldsymbol{\varepsilon}$ are all $\mathrm{n} \times 1$ vectors where $\mathrm{n}$ is the number of instances or the sample size. In matrix form, the model can be expressed as

$$
\boldsymbol{Y}=\boldsymbol{X} \boldsymbol{\beta}+\boldsymbol{\varepsilon}
$$

where $\boldsymbol{\beta}=\left(\beta_{1}, \beta_{2}, \ldots, \beta_{p}\right)^{T}$ and $\boldsymbol{X}$ is the $\mathrm{n} \times \mathrm{p}$ data matrix often referred to as the design matrix. The assumption of linearity in this model can be too restrictive and in many cases is unrealistic. In addition, the model assumes that response variable $\boldsymbol{Y}$ has a normal distribution with a constant variance; that is, if $\boldsymbol{Y}=$ $\left(y_{1}, y_{2}, \ldots, y_{n}\right)^{T}$ then it is assumed that $E(\boldsymbol{Y})=$ $\boldsymbol{\mu}=\boldsymbol{X} \boldsymbol{\beta}, \boldsymbol{\mu}=\left(\mu_{1}, \mu_{2}, \ldots, \mu_{p}\right)^{\boldsymbol{T}}$ and that $y_{i}$ has a normal distribution with mean $\mu_{i}=E\left(y_{i}\right)$ and variance $\sigma^{2}$ for $\mathrm{i}=1,2, \ldots, \mathrm{n}$. These assumptions, in many instances, may not be justified. To overcome this problem, the assumptions are relaxed by allowing $\boldsymbol{\mu}$ and $\boldsymbol{X} \boldsymbol{\beta}$ to be related by a so-called link function, $g$, so that

$$
g(\boldsymbol{\mu})=\boldsymbol{X} \boldsymbol{\beta} .
$$

In this way, the normal model becomes a special case of generalized linear model in which the link function is the identity function. Therefore, in general, for distributions other than normal that fit the data, a suitable link function can be determined. Specifically, if responses are binary as in the binomial distribution, the two popular link functions are the logit transformation, $\log \left(\frac{\mu_{i}}{1-\mu_{i}}\right)$ and the probit transformation, $\Phi^{-1}\left(\mu_{i}\right)$ where $\Phi$ is the cumulative distribution function of the standard normal distribution. In the case of logit transformation, the outcome probability is assumed to have the logistic distribution, whereas in the case of the probit link, the distribution of the outcome probability does not have an easily interpretable form. This is why the logit transformation belongs to the canonical family of link functions while the probit link is not canonical.

\section{Logistic Regression Model}

Assume that response variable $\boldsymbol{Y}$ is binary and let $\mathrm{P}\left(y_{i}=1\right)=\pi_{i}$ be the success probability for the $i^{\text {th }}$ measurement. Then, it can be shown that $E\left(y_{i}\right)=\pi_{i}$ and $V\left(y_{i}\right)=\pi_{i}\left(1-\pi_{i}\right)$. In ordinary regression, $\pi=\left(\pi_{1}, \pi_{2}, \ldots \pi_{n}\right)^{T}$ is modeled as a linear function $\boldsymbol{X}$ with a constant variance. However, because the expected value and variance of the response variable are not constant, ordinary linear regression does not apply. Moreover, it can be shown that the relationship between $\boldsymbol{\pi}$ and $\boldsymbol{X}$ is generally not linear. Constant changes in the explanatory variables usually have less impact in the success probability $\pi_{i}$ especially when $\pi_{i}$ is close to 0 or 1. Sigmoid shaped curves are often more realistic for such relationships, and among these, the most commonly used is the logistic function defined as

$$
\pi_{i}=\left(1+e^{-x_{i} \boldsymbol{\beta}}\right)^{-1}
$$

where $\boldsymbol{x}_{\boldsymbol{i}}$ is the $i^{\text {th }}$ row of the $\boldsymbol{X}$ matrix, that is, the $i^{\text {th }}$ record in the dataset. Taking the logarithm of the odds ratio, called the logit transformation, from equation (4) results in the logistic regression model

$$
\operatorname{logit}\left(\pi_{i}\right)=\log \left(\frac{\pi_{i}}{1-\pi_{i}}\right)=\boldsymbol{x}_{i} \boldsymbol{\beta}+\epsilon_{i}
$$

where $\epsilon_{i}$ is the error term. A vast body of literature exists regarding methods for fitting a logistic regression model. The popular maximum likelihood method is used to estimate model parameters. Note that if the responses are independent, then by applying the Bernoulli distribution, the likelihood for $n$ binary observations as a function of the parameters is

$$
L(\boldsymbol{\beta})=\prod_{i=1}^{n} \pi_{i}^{y_{i}}\left(1-\pi_{i}\right)^{1-y_{i}}
$$

and the log-likelihood is given by

$$
\log L(\boldsymbol{\beta})=\sum_{i=1}^{n}\left(y_{i} \log \pi_{i}+\left(1-y_{i}\right) \log \left(1-\pi_{i}\right)\right)
$$

which must be maximized in order to derive the maximum likelihood estimates of the model 


\section{USE OF THE PROBIT LINK FUNCTION IN THE GENERALIZED LINEAR MODELS}

parameters. The first derivative of equation (7) is referred to as the score function (McCullagh $\&$ Nelder, 1989). Because no analytic solution for the maximum likelihood estimates can be derived, numerical methods are used. The most popular method for numerical derivation of the parameter estimates is an adaptation of the Newton-Raphson method, called the Iteratively Re-Weighted Least Squares (IRLS). In this method, a new set of weights are estimated at each iteration (Hosmer \& Lemeshow, 2000; Hilbe, 2009; Maalouf, 2011).

\section{Probit Regression Model}

The probit model is another sigmoidshaped curve used in modeling dichotomous outcome variables. For this model, the link function, called the probit link, uses the inverse of the cumulative distribution function of the standard normal distribution to transform probabilities to the standard normal variable. Thus

$$
\Phi^{-1}\left(\pi_{i}\right)=\boldsymbol{x}_{i} \boldsymbol{\beta}+\epsilon_{i}
$$

where

$$
\Phi(z)=\int_{-\infty}^{z} \frac{1}{\sqrt{2 \pi}} e^{-\frac{1}{2} t^{2}} d t .
$$

The use of the probit regression model dates back to Bliss (1934). Bliss was interested in finding an effective pesticide to control insects that fed on grape leaves (Greenberg, 1980). He found that the relationship between response to a dose of pesticide was sigmoid and he applied the probit transformation to transform the sigmoid shape dose-response curve to a linear relationship. His ideas were later generalized in a book by Finney (1985) where the applications of probit analysis in toxicological experiments were explored. According to some sources, probit analysis remains the preferred method in understanding dose-response relationships. In data mining, however, this application remains fairly unknown even though most popular statistical software such as SPSS, SAS and R carry functions for probit regression.

The probit model has also found popularity in economics. Cramer (2003) provides a survey of the early origins of the model. In comparing the probit model to the logistic model, many authors believe that there is little theoretical justification in choosing one formulation over the other in most circumstances involving binary responses. The logit model is considered to be computationally simpler but it is based on a more restrictive assumption of error independence, although many other generalizations have dealt with that assumption as well. By contrast, the probit model assumes that random errors have a multivariate normal distribution. This assumption makes the probit model attractive because the normal distribution provides a good approximation to many other distributions. The model does not rely on the assumption of error independence and econometricians utilize a general random utility model to describe the correlation. Hausman and Wise (1978) defined the covariance probit model and used it in economic applications. The model parameters are estimated by using the generalized least squares

$$
\widehat{\boldsymbol{\beta}}=\left(\boldsymbol{X}^{\prime} \boldsymbol{\Sigma}^{-1} \boldsymbol{X}\right)^{-1} \boldsymbol{X}^{\prime} \boldsymbol{\Sigma}^{-1} \boldsymbol{Z}
$$

where $\boldsymbol{\Sigma}$ is the covariance matrix of the errors and $\boldsymbol{Z}$ is the vector of observed probits:

$$
\boldsymbol{Z}=\left(\Phi^{-1}\left(\pi_{1}\right), \Phi^{-1}\left(\pi_{2}\right), \ldots, \Phi^{-1}\left(\pi_{n}\right)\right)^{T}
$$

In practice, $\boldsymbol{\Sigma}$ is replaced by some consistent estimate of the covariance matix. This makes the probit model computationally more complicated; however, as argued by Judge, et al. (1980) the probit model is more flexible. The use of noncanonical link functions is not prohibited by the fact that they are more computationally complex. Czado and Munk (2000) argued that, in some applications, the overall fit of the model as measured by the p-value of the goodness-offit statistics can be improved significantly by the use of a noncanonical link.

\section{Applications in Data Mining}

Now consider a data set with binary responses and consider analyzing the data using both logistic regression and probit regression models. This study used a data set regarding credit reliability of individuals. If a financial institution gives a loan based on a credit to a person, they clearly would be interested in 


\section{MEHDI RAZZAGHI}

estimating the person's ability to pay the loan back, thus, the aim is to model credit reliability as a function of the person's risk factors (covariates). The data set consists of 1,000 customers from a bank in southern Germany. The response variable is in dichotomous form using 0 for a reliable client and 1 for not reliable. The data set, which is described in Fahrmeir and Hamerele (1994), consists of the following 20 covariates that were considered to be important in credit evaluation of a client:

$\mathrm{X}_{1}$ : Running account (trichotomous)

$\mathrm{X}_{2}$ : Duration of credit (month)

$\mathrm{X}_{3}$ : Payment of previous credits

$\mathrm{X}_{4}$ : Purpose of Credit

$\mathrm{X}_{5}$ : Amount of credit

$\mathrm{X}_{6}$ : Value of savings or stocks

$\mathrm{X}_{7}$ : Employment history

$\mathrm{X}_{8}$ : Credit payment as percentage of income

$\mathrm{X}_{9}$ : Marital status

$\mathrm{X}_{10}$ : Further debtors/guarantors

$\mathrm{X}_{11}$ : Number of years in current household

$\mathrm{X}_{12:}$ Most valuable assets

$\mathrm{X}_{13}$ : Age

$\mathrm{X}_{14}$ : Running credits in other institutions

$\mathrm{X}_{15}$ : Own/rent

$\mathrm{X}_{16}$ : Number of previous credits at this bank

$\mathrm{X}_{17}$ : Occupation

$\mathrm{X}_{18}$ : Number of persons entitled to maintenance

$\mathrm{X}_{19}$ : Telephone

$\mathrm{X}_{20}$ : Foreign or national worker

Fahrmeir and Hamerele (1994) used a logit model to analyze a subset of the data with seven covariates; later Fahrmeir and Tutz (1997) used the data set to illustrate an example in logistic regression. The data set was further analyzed in depth as a case study by Giudici (2003) where a descriptive analysis of the data set was also included. PROC LOGISTIC and PROC PROBIT in SAS were used to analyze the data. Table 1 provides the parameter estimates for each covariate under the two modeling structures together with standard error, the value of the Wald Chi square and the significance level as measured by the p-value. It can be observed that, although the values of the parameter estimates are different - as they should be, both models produce very similar results and point to the same set of parameters as significant. The standard error of the estimates appears to be smaller for some variables in the logistic model and larger in others. The predicted values and other standard statistics were computed and again very similar results were obtained under both models.

Discussion

In data mining, there is a strong urge to use logistic regression as one of the main approaches for classification of binary responses. Komarek and Moore (2005) presented an argument in introducing the logistic regression as a core in data mining tools. A large body of literature exists on the use of logistic regression in data mining applications. Comparatively less is known about a similar, but intrinsically different approach of probit regression. This article introduced this model as another powerful and useful approach for modeling binary data. Many authors have used the probit model in other applications with success; for example, Shariff, et al. (2009) compared the two models for estimating the strength of gear teeth. The goal herein was to present the probit regression to the data mining community; it was not to introduce the probit model as a rival to the logistic model, but rather as an alternative. Experience shows that in most situations the two approaches produce similar results although some differences exist. This similarity is not necessarily sustained when multivariate responses are used. Further research is needed to investigate the advantages or disadvantages in using one model over the other in data mining applications. 
USE OF THE PROBIT LINK FUNCTION IN THE GENERALIZED LINEAR MODELS

Table 1: Maximum Likelihood Estimate of Model Parameters

\begin{tabular}{|c|c|c|c|c|c|c|c|c|}
\hline & \multicolumn{4}{|c|}{ Logistic Regression Parameters } & \multicolumn{4}{|c|}{ Probit Regression Parameters } \\
\hline & Estimate & SE & Chi-Square & $\operatorname{Pr}>$ ChiSq & Estimate & SE & Chi-Square & $\operatorname{Pr}>$ ChiSq \\
\hline Intercept & 3.9940 & 1.0238 & 15.2178 & $<.0001$ & 2.2004 & 0.5729 & 14.7500 & 0.0001 \\
\hline $\mathrm{X}_{1}$ & -0.5799 & 0.0700 & 68.5630 & $<.0001$ & -0.3424 & 0.0400 & 73.4300 & $<.0001$ \\
\hline $\mathrm{X}_{2}$ & 0.0246 & 0.0087 & 7.9300 & 0.0049 & 0.0140 & 0.0051 & 7.4600 & 0.0063 \\
\hline $\mathrm{X}_{3}$ & -0.3822 & 0.0874 & 19.1204 & $<.0001$ & -0.2220 & 0.0501 & 19.6300 & $<.0001$ \\
\hline $\mathrm{X}_{4}$ & -0.0315 & 0.0301 & 1.0980 & 0.2947 & -0.0173 & 0.0173 & 1.0000 & 0.3180 \\
\hline $\mathrm{X}_{5}$ & 0.0001 & 0.0000 & 5.4199 & 0.0199 & 0.0001 & 0.0000 & 5.7000 & 0.0170 \\
\hline $\mathrm{X}_{6}$ & -0.2391 & 0.0583 & 16.8395 & $<.0001$ & -0.1312 & 0.0326 & 16.2000 & $<.0001$ \\
\hline $\mathrm{X}_{7}$ & -0.1517 & 0.0712 & 4.5444 & 0.0330 & -0.0876 & 0.0413 & 4.4800 & 0.0342 \\
\hline $\mathrm{X}_{8}$ & 0.2983 & 0.0828 & 12.9949 & 0.0003 & 0.1748 & 0.0478 & 13.3800 & 0.0003 \\
\hline $\mathrm{X}_{9}$ & -0.2574 & 0.1157 & 4.9473 & 0.0261 & -0.1477 & 0.0668 & 4.8800 & 0.0271 \\
\hline $\mathrm{X}_{10}$ & -0.3473 & 0.1777 & 3.8188 & 0.0507 & -0.1804 & 0.1011 & 3.1800 & 0.0744 \\
\hline $\mathrm{X}_{11}$ & 0.0141 & 0.0774 & 0.0332 & 0.8553 & 0.0076 & 0.0453 & 0.0300 & 0.8660 \\
\hline $\mathrm{X}_{12}$ & 0.1828 & 0.0910 & 4.0367 & 0.0445 & 0.1102 & 0.0528 & 4.3500 & 0.0369 \\
\hline $\mathrm{X}_{13}$ & -0.0089 & 0.0082 & 1.1807 & 0.2772 & -0.0055 & 0.0047 & 1.3500 & 0.2457 \\
\hline $\mathrm{X}_{14}$ & -0.2419 & 0.1111 & 4.7428 & 0.0294 & -0.1381 & 0.0650 & 4.5100 & 0.0337 \\
\hline $\mathrm{X}_{15}$ & -0.2931 & 0.1677 & 3.0542 & 0.0805 & -0.1629 & 0.0981 & 2.7600 & 0.0969 \\
\hline $\mathrm{X}_{16}$ & 0.2436 & 0.1610 & 2.2894 & 0.1303 & 0.1460 & 0.0921 & 2.5100 & 0.1130 \\
\hline $\mathrm{X}_{17}$ & -0.0189 & 0.1367 & 0.0191 & 0.8901 & -0.0101 & 0.0805 & 0.0200 & 0.8996 \\
\hline $\mathrm{X}_{18}$ & 0.1708 & 0.2319 & 0.5421 & 0.4616 & 0.1133 & 0.1348 & 0.7100 & 0.4007 \\
\hline $\mathrm{X}_{19}$ & -0.2947 & 0.1880 & 2.4567 & 0.1170 & -0.1692 & 0.1089 & 2.4100 & 0.1204 \\
\hline $\mathrm{X}_{20}$ & -1.1582 & 0.6078 & 3.6317 & 0.0567 & -0.6389 & 0.3292 & 3.7700 & 0.0523 \\
\hline
\end{tabular}




\section{References}

Bliss, C. L. (1934). Methods of probits. Science, 79, 38-39.

Cramer, J. S. (2003). Logit models from economics and other fields. Cambridge, MA: Cambridge University Press.

Czado, C., \& Munk, A. (2000). Noncanonical links in generalized linear models: When is the effort justified? Journal of Statistical Planning and Inference, 87, 317-345.

Fahrmeir, L., \& Hamerele, A. (1984). Multivariate statistische verfahren. Berlin: Springer Verlag.

Fahrmeir, L., \& Tutz, G. (2001). Multivariate statistical modeling based on generalized linear models. Berlin: Springer Verlag.

Finney, D. J. (1971). Probit analysis. Cambridge, MA: Cambridge University Press.

Giudici, P. (2003). Applied data mining, statistical methods for business and industry. Chichester, MA: Wiley.

Greenberg, B. G. (1980). Chester I. Bliss, 1899-1979. International Statistical Review, 8, 135-136.

Hausman, J. A., \& Weis, D. A. (1978). A conditional probit model for qualitative choice: Discrete decisions recognizing interdependence and heterogeneous preferences. Econometrica, 46, 403-426.
Hilbe, J. M. (2009). Logistic regression models. Boca Raton, FL: Chapman \& Hall/CRC Press.

Hosmer, D. W., \& Lemeshow, S. (2000). Applied logistic regression. New York, NY: Wiley.

Judge, G. G., Griffith, W. E., Hill, R. C., $\&$ Lee, T. C. (1985). The theory and practice of econometrics. New York, NY: Wiley.

Komarek, P., \& Moore, A. (2005). Making logistic regression a core data mining tool: A practical investigation of accuracy, speed, and simplicity. Technical Report, Carnegie-Mellon University.

Maalouf, M. (2011). Logistic regression in data analysis: An overview. International Journal of data Analysis Techniques and Strategies, 3, 281-299.

McCullagh, P., \& Nelder, J. A. (1989). Generalized linear models. London: Chapman and Hall.

Shariff, A. A., Zaharim, A., \& Sopian, K. (2009). The comparison of logit and probit regression analyses in estimating the strength of gear teeth. European Journal of Scientific Research, 4, 548-553. 


\title{
An Approximate Approach to the Economic Design of $\bar{x}$ Charts By Considering the Cost of Quality
}

\author{
M. A. A. Cox \\ Newcastle University \\ Newcastle upon Tyne, United Kingdom
}

The selection of three parameters $\{h, k, n\}$ is necessary to design a $\bar{x}$ control chart. A cost model employing a Burr distribution is examined. Previously employed methods are refined and extended. A series of approximations are proposed that enable a rapid parameter selection. It is hoped that reducing the computational complexity of previous approaches will lead to wider utilization of $\bar{x}$ control charts.

Key words: Burr distribution, control charts, cost of quality, generalized charts.

Introduction

When designing a $\bar{x}$ control chart to monitor a process three parameters must be selected, sample size $(n)$, interval between successive subgroups $(h)$ and control limits $(k)$. A cost model is employed to assist in the selection of these parameters (Duncan 1956, Alexander, et al. 1995, Chou, et al. 2000). (A summary of notations adopted, which closely follows that of Chou, et al. (2000), is listed in the Appendix.)

Chou, et al. (2000) did not fully describe their numerical approach: They used tables presented in Burr (1942) to estimate the parameters of the eponymous distribution given the first four central moments for a data set; the mean and standard deviation of this distribution corresponding to the parameters from tables presented in Burr (1942) were then introduced. Finally a computer program, which employs a grid search to minimize the cost model, is mentioned and finally key parameters $\{h, k, n\}$ are estimated. No technical details were provided of this procedure, for example, which interval was scanned and what step length was employed.

Dr. Cox is a lecturer in Psychology specializing in numerical disciplines. Email him at: mike.cox@ncl.ac.uk.
In this study, neither Burr's (1942) tables nor a grid search is employed. Instead, numerical procedures are employed to address estimation of the parameters for the Burr distribution. The key estimation step is divided into three sub-problems, one for each parameter $\{h, k, n\}$, to make estimation more efficient and accurate. Chou, et als. (2000) original work presented two key equations and a related table (see equations (9) and (12) and Table 1). Although the equations are correct, their range of application is limited, as noted when the density function is defined in equation 5 of the original work; thus the equations are not strictly consistent with the results presented in their table. This shortcoming will be addressed, which is essentially notational. In addition a four-step procedure for parameter selection is developed and is further refined as a series of approximate methods to allow more rapid calculation of these parameters.

The Key Equations

The Burr (1942) distribution density function (1) is defined as

$$
\begin{aligned}
& f(y)=\frac{q c y^{c-1}}{\left(1+y^{c}\right)^{q+1}} \\
& \text { for } y \geq 0,
\end{aligned}
$$

with cumulative density 


$$
F(y)=1-\frac{1}{\left(1+y^{c}\right)^{q}}, \text { for } y \geq 0
$$

This is particularly useful in modeling data that does not follow a normal distribution; note that the argument $(y)$ is non-negative. The Burr distribution density function is frequently used to model data that has a slight positive skewness, such as for the size of insurance claims. To avoid the problem of the argument straying outside the region of interest the Heaviside step function (3) is employed.

$$
\begin{aligned}
& H(y)=1 \\
& \text { for } y \geq 0, \text { or } 0 \text { otherwise. }
\end{aligned}
$$

The expected cost ( $E$, equation 4) is from equation 4 in Chou, et al. (2000). All parameters are defined in the Appendix and the elements of the equation $\left(\mathrm{B}, \mathrm{v}_{1}, \mathrm{v}_{2}, \mathrm{~L}_{1}\right.$ and $\left.\mathrm{L}_{2}\right)$ are presented in (5), (6) and (7).

$$
\begin{gathered}
E=\frac{a_{1}+a_{2} n}{h}+\frac{a_{3} \lambda+\frac{a_{5} \alpha}{h}+L_{1} P+L_{2} P \lambda B}{1+\lambda B}, \\
B=\left(\frac{1}{1-\beta}-\frac{1}{2}+\frac{\lambda h}{12}\right) h+D+g n \\
v_{1}=\sigma \\
v_{2} \sigma \sqrt{1+\delta^{2}} \\
L_{i}=\frac{A}{\Delta^{2}} v_{i}^{2} \text { for } i=1,2 .
\end{gathered}
$$

The probabilities associated with Type I errors $(\alpha)$ and Type II errors $(\beta)$ are of key interest. The Type I error, or producers risk, is defined in terms of the probability of deviation of the sample mean outside the upper and lower confidence limits $(U C L, L C L)$. It is essential, at this stage, that the Heaviside function be associated with the density to correctly restrict its range. This was omitted, although tacitly assumed, in the original work.

$$
\begin{gathered}
\alpha=\operatorname{Pr}(\bar{x}>U C L)+\operatorname{Pr}(\bar{x}<L C L) \\
\alpha=\operatorname{Pr}(y>M+k S)+\operatorname{Pr}(y<M-k S) \\
\alpha= \\
\left(\begin{array}{l}
(1-F(M+k S)) H(M+k S) \\
+F(M-k S) H(M-k S)
\end{array}\right)
\end{gathered}
$$

The Type II error, or consumer's risk, is defined in terms of the probability of deviation of the sample mean outside the upper and lower confidence limits (UCL, LCL) when the deviation from the target mean $(\delta)$ is known.

$$
\begin{gathered}
\beta=\operatorname{Pr}(L C L \leq \bar{x} \leq U C L \mid \mu=T+\delta \sigma) \\
\beta=\operatorname{Pr}(M-k S-S \delta \sqrt{n} \leq y \leq M+k S-S \delta \sqrt{n}) \\
\beta=F(M+k S-S \delta \sqrt{n}) H(M+k S-S \delta \sqrt{n}) \\
-F(M-k S-S \delta \sqrt{n}) H(M-k S-S \delta \sqrt{n})
\end{gathered}
$$

Equations (8) and (9) may be combined with that for the cumulative density (2) into a more appropriate form than those previously presented (Chou, et al., 2000). A four-step procedure was developed to derive the parameters from equations (4), (8) and (9) and the steps described will be used consistently throughout this article; the numerical methodology will be also be discussed. Because these procedures are eliminated from the final approach, they are included as an indication of the massive computational effort associated with the problem; they are also necessary to indicate the reliability of the approximate methods employed. 


\section{APPROXIMATE APPROACH TO THE ECONOMIC DESIGN OF $\bar{x}$ CHARTS}

Step 1

\section{Methodology}

Previous data is used to evaluate the skewness and kurtosis coefficients of the underlying process and these are then used to estimate the parameters $\{c, q\}$ of the corresponding Burr distribution. The moments of the distribution are estimated by numerical integration.

Step 2

Burr parameters $\{c, q\}$ are used to estimate the mean and standard deviation $\{M, S\}$ of the distribution by numerical integration and equation 8 is then employed to estimate $k$ for given $\alpha$.

Step 3

The available estimates $\{M, S, k\}$ and equation (9) are used to estimate $n$ (for consumer's risk $\beta$ ), and absolute mean shift $(\delta)$ for an out of control process.

\section{Step 4}

The parameters, plus the estimates $\{k$, $n\}$ and the minimization of equation (4) are used to estimate $h$.

This completes the procedure; the three parameters $\{h, k, n\}$ for an ideal $\bar{x}$ control chart are now available. It is now appropriate to address the lengthy numerical procedures required to complete these steps.

Numerical Method

Step 1

The Powell (1970) hybrid method is used to simultaneously solve the equations for skewness and kurtosis. For each evaluation, four calls to a numerical integration procedure (Piessensl, 1983) are required to evaluate the first four moments, providing two function estimates.

\section{Step 2}

A procedure is required to find the zero of a continuous function introduced in Step 2; the method of Bus and Dekker (1975) is employed. This procedure incorporates interpolation, extrapolation plus bisection.

Step 3

To find the zero of a continuous function employed in Step 3; the method of Bus and Dekker (1975) is employed.

Step 4

Applying simple algebra this step reduces to finding the roots of a quartic equation. Prior to substituting $B$ defined in equation (5), it is written as a quadratic function of $h$ :

$$
B=c_{0}+c_{1} h+c_{2} h^{2}
$$

$$
\left.\begin{array}{c}
c_{0}=D+g n \\
c_{1}=\frac{1}{1-\beta}-\frac{1}{2} \\
c_{2}=\frac{\lambda}{12}
\end{array}\right\}
$$

The expected cost (4) may also be represented as a function of $h$.

$$
E=\frac{n_{0}+n_{1} h+n_{2} h^{2}+n_{3} h^{3}}{d_{0}+d_{1} h+d_{2} h^{2}}
$$

The numerator coefficients are

$$
\left.\begin{array}{c}
n_{0}=\left(a_{1}+a_{2} n\right)\left(1+\lambda c_{0}\right)+a_{5} \alpha \\
n_{1}=\left(a_{1}+a_{2} n\right) \lambda c_{1}+a_{3} \lambda+L_{1} P+L_{2} \lambda P c_{0} \\
n_{2}=\left(a_{1}+a_{2} n\right) \lambda c_{2}+L_{2} \lambda P c_{1} \\
n_{3}=L_{2} \lambda P c_{2}
\end{array}\right\}
$$

and the denominator coefficients are

$$
\left.\begin{array}{c}
d_{0}=1+\lambda c_{0} \\
d_{1}=\lambda c_{1} \\
d_{2}=\lambda c_{2}
\end{array}\right\}
$$


To minimize the cost, a turning point is required. This corresponds to a zero in the numerator of the derivative of $E$ (11) with respect to $h$. This derivative polynomial is denoted by $d E$.

$$
d E=d E_{0}+d E_{1} h+d E_{2} h^{2}+d E_{3} h^{3}+d E_{4} h^{4}
$$

with coefficients

$$
\left.\begin{array}{c}
d E_{0}=-n_{0} d_{1} \\
d E_{1}=-2 n_{0} d_{2} \\
d E_{2}=-n_{1} d_{2}-3 n_{0} d_{3}+n_{1} d_{2} \\
d E_{3}=-2 n_{1} d_{3}+2 n_{1} d_{3} \\
d E_{4}=-n_{2} d_{3}+n_{3} d_{2}
\end{array}\right\}
$$

A variant of Laguerre's method may be employed (Smith, 1967) to solve this quartic equation. It should be noted that, as $h$ increases from the minimum, the gradient is relatively small. Thus, a large increase in $h$ will lead to only a small increase in $E$. This completes the outline of the numerical approach. For given $\{\alpha, \beta\}$ the procedure may be repeated and Table 1 from the original work (Chou, et al., 2000) reproduced.

A Numerical Example for an Exact Solution

To illustrate the numerical methods procedure, consider an example employed by Chou, et al. (2000), which corresponds to the first row of Table 1 (Alexander, et al., 1995).

\section{Step 1}

The first four moments of a given data set, from the process for which an $\bar{x}$ chart is required, are calculated. The required measures are: skewness $=0.4836$ and kurtosis $=3.3801$. Resulting in $c=3.0003$ and $q=5.9989$. The exact measures for $c=3$ and $q=6$ correspond to a skewness of 0.48364038 and a kurtosis of 3.38009234 .

Step 2

Calculating the moments corresponding to $\{c, q\}$ results in: $M=0.5109$ and $S=0.2022$.
Selecting the Type I error probability as $\alpha=0.005$ gives $k=3.0299$.

\section{Step 3}

The Type II error probability and the magnitude of the mean shift for an out of control process are now selected as $\beta=0.08114$ and $\delta=1$, where $\beta=1$-power. These result in $n=18.9998$. Because $n$ is the sample size it is adjusted to 19; in general estimates of $n$ are rounded up to the nearest integer.

Step 4

All additional parameters (see Table 1), reflecting details of the cost and frequency of the measurement process, are now required. These result in numerical values (see Table 2) where the estimates are obtained from the equations described.

Table 1: Input Coefficients for the Numerical Example

\begin{tabular}{|c|l|}
\hline Coefficient & \multicolumn{1}{|c|}{ Description } \\
\hline$a_{1}=1$ & fixed cost of taking a sample \\
\hline$a_{2}=0.1$ & variable cost of sampling \\
\hline$a_{3}=50$ & $\begin{array}{l}\text { cost of eliminating an } \\
\text { assignable cause }\end{array}$ \\
\hline$a_{5}=50$ & $\begin{array}{l}\text { cost of investigating a false } \\
\text { alarm }\end{array}$ \\
\hline$A=5$ & $\begin{array}{l}\text { cost to rework or scrap a } \\
\text { faulty item }\end{array}$ \\
\hline$D=2$ & $\begin{array}{l}\text { time required to investigate } \\
\text { an out-of-control signal }\end{array}$ \\
\hline$g=0.01$ & $\begin{array}{l}\text { time to measure and record } \\
\text { the quantity of interest }\end{array}$ \\
\hline$\Delta=0.3$ & tolerance \\
\hline$\lambda=0.25$ & frequency of process shifts \\
\hline$\sigma=0.1$ & $\begin{array}{l}\text { process standard deviation, } \\
\text { from past data }\end{array}$ \\
\hline$P=100$ & \begin{tabular}{l} 
production rate \\
\hline
\end{tabular} \\
\hline
\end{tabular}


APPROXIMATE APPROACH TO THE ECONOMIC DESIGN OF $\bar{x}$ CHARTS

Table 2: Calculated Coefficients for the Numerical Example

\begin{tabular}{|c|c|c|c|c|c|}
\hline \multicolumn{6}{|c|}{ Source Equation } \\
\hline 10 & 6 & 7 & 12 & 13 & 15 \\
\hline$c_{0}=2.19$ & $v_{1}=0.01$ & $L_{1}=0.5555$ & $n_{0}=4.7378$ & $d_{1}=1.5475$ & $d E_{0}=7.3317$ \\
\hline$c_{1}=0.5883$ & $v_{2}=0.1414$ & $L_{2}=1.1111$ & $n_{1}=129.3569$ & $d_{2}=0.1471$ & $d E_{1}=1.3936$ \\
\hline$c_{2}=0.0208$ & & & $n_{2}=16.3569$ & $d_{3}=0.0052$ & $d E_{2}=-6.2191$ \\
\hline & & & $n_{3}=0.5787$ & & $d E_{3}=-0.4441$ \\
\hline & & & & & $d E_{4}=0.00008$ \\
\hline
\end{tabular}

For the final parameter (Step 4) the root of interest (14) is $h=1.1523$. These estimates agree with those previously reported (Chou, et al., 2000) and result in an expected cost: $E=88.7779$.

A great deal of computer code was required to reach this point. To encourage the adoption of this approach approximate methods for each step of the procedure are proposed. These eliminate the computational complexity; however this results in the introduction of additional notation and the consideration of certain special cases.

\section{Approximate Methods}

The computational complexity of the numerical methods described may discourage some users. In view of this, a series of approximations are proposed. Although care must be taken in creating the various terms required it is not necessary to employ the complex numerical procedures utilized previously.

\section{Step 1}

The proposed method is entirely different from that previously employed. The tail areas $\left\{\beta_{1}, \beta_{2}\right\}$ associated with the upper/lower cut-off $\left\{y_{U}, y_{L}\right\}$ for the raw data are selected. The values adopted would be $\{0.75,0.25\}$ for quartiles. Employing (2) with some manipulation results in a function that should vanish for $c$.

$$
Z(c)=\left(1+y_{U}^{c}\right)-\left(1+y_{L}^{c}\right)^{\frac{\ln \left(1-\beta_{1}\right)}{\ln \left(1-\beta_{2}\right)}}
$$

To obtain the zero the Newton-Raphson method, which requires evaluation of the first derivative of $Z$, may be employed.

$$
Z^{\prime}(c)=\ln \left(y_{U}\right) y_{U}^{c}-\frac{\ln \left(1-\beta_{1}\right)}{\ln \left(1-\beta_{2}\right)} \ln \left(y_{L}\right) y_{L}^{c}\left(1+y_{L}^{c}\right)^{\frac{\ln \left(1-\beta_{1}\right)}{\ln \left(1-\beta_{2}\right)}-1}
$$

Then successive estimates $\left\{c_{0}, c_{1}, \ldots, c_{n}\right\}$ for $c$ are then obtained

$$
c_{i+1}=c_{i}-\frac{Z\left(c_{i}\right)}{Z^{\prime}\left(c_{i}\right)}
$$

based on an initial estimate for $c_{0}$, for example, 2.

The simplest choice for the exponent in equation 16 is 2 . If $\beta_{1}+\beta_{2}=1$ is set - which is a reasonable choice for the tail areas - then the corresponding estimates are

$$
\beta_{1}=\frac{-1+\sqrt{5}}{2}=0.6180
$$

and

$$
\beta_{2}=1-\frac{-1+\sqrt{5}}{2}=0.3820 \text {. }
$$


This leads to associated simplifications in (17). Recognizing that the expected value of $y^{N c}$ takes the form

$$
\varepsilon\left(y^{N c}\right)=\frac{N !}{\prod_{i=1}^{N}(q-i)} \text { for } N<q .
$$

(Note that the above expression may be proved inductively employing the following result: $\varepsilon\left(\left(1+y^{c}\right)^{N}\right)=\frac{q}{q-N}$ for $\left.N<q.\right)$ In particular for $N=1, \varepsilon\left(y^{c}\right)=\frac{1}{q-1}\left(q=\frac{1}{\varepsilon\left(y^{c}\right)}+1\right)$, thus using $c$ and the raw data $\left(\varepsilon\left(y^{c}\right)\right)$ the parameter $q$ may be immediately estimated. Alternately employing the median $\left(y_{M}\right)$ for the sample

$$
q=\frac{\ln (2)}{\ln \left(1+y_{M}^{c}\right)}
$$

the parameter $q$ may again be immediately estimated.

Step 2

The program employed for Step 1 was used to generate 5,041 examples of the mean and standard deviation for given $c$ and $q$ $(c: 2.5,2.75, \ldots, 20, q: 2.5,2.75, \ldots, 20)$. This data was used to train a neural network (Goodman, 2001) to provide estimates $\{M, S\}$. A simple topology was employed with 2 inputs, 2 outputs and a hidden layer of width 2 . The inputs are $x_{1}=c$ and $x_{2}=q$ and the transfer functions are $\phi_{0}(x)=x$ and $\phi_{1}(x)=\frac{1-e^{-x}}{2\left(1+e^{-x}\right)}$. The activation level of the neurons in the hidden layer is $x_{j}=\phi_{1}\left(u_{j}+\sum_{i=1}^{2} x_{i} a_{i j}\right) j=3,4$ and the activation of the output neurons is $x_{j}=\phi_{0}\left(u_{j}+\sum_{i=3}^{4} x_{i} a_{i j}\right) j=5,6$. Which result in the required outputs: $M=x_{5}$ and $S=x_{6}$. In this case the fitted values from the software
(Goodman, 2001) are: $u_{3}=-1.06871$, $u_{4}=0.00297517, \quad u_{5}=0.344999 \quad$ and $u_{6}=0.254552$. The weights representing the links between neurons are: $a_{13}=0.120744$, $a_{23}=0.185339, \quad a_{14}=-0.22152$, $a_{24}=-0.00139201, \quad a_{35}=-0.544563$, $a_{36}=-0.157016, \quad a_{45}=-1.55586$ and $a_{46}=0.252306$.

Alternately the integrals may be evaluated numerically employing Simpson's rule or other quadrature formulas. Given the mean and standard deviation two cases must be considered when estimating $k$.

Case 1: $\mathrm{M}-\mathrm{kS}>0$

In this case both tails of equation (8) contribute, a Taylor expansion of $\ln (\alpha)$ as a function of $k$, because $k$ is small, is generated.

$$
\ln (\alpha) \approx-\frac{2 q M^{c-1} c S}{\left(1+M^{c}\right)^{q+1}} k
$$

$$
k=-\ln (\alpha) \frac{\left(1+M^{c}\right)^{q+1}}{2 q M^{c-1} c S} .
$$

It is necessary to ensure that the expected criterion, $M-k S>0$, is satisfied.

Case 2: $\mathrm{M}-\mathrm{kS} \leq 0$

In this case only one tail of equation (8) contributes, a Maclaurin expansion of $\ln (\alpha)$ as a function of $k$ about $z$ is generated.

$$
\ln (\alpha) \approx \ln \left(\frac{1}{\left(1+(M+z S)^{c}\right)^{q}}\right)-\frac{q(M+z S)^{c-1} c S}{1+(M+z S)^{c}}(k-z)
$$

$$
k=z+\frac{1+(M+z S)^{c}}{q(M+z S)^{c-1} c S}\left(\ln \left(\frac{1}{\left(1+(M+z S)^{c}\right)^{q}}\right)-\ln (\alpha)\right)
$$


Because $k \geq \frac{M}{S}, \quad z$ is chosen to satisfy this criterion, typically $z=\left(\frac{M}{S}\right)(1.4-40 \alpha)$. It is necessary to ensure that the expected criterion, $M-k S \leq 0$, is satisfied.

Step 3

Two cases arise when evaluating $n$.

Case 1: $\sqrt{n} \leq \frac{M+k S}{S \delta}$

In this case only one tail of equation (9) contributes, a Maclaurin expansion of $\beta$ as a function of $\sqrt{n}$, about $z$ is generated.

$$
\begin{aligned}
& \beta \approx \\
& 1-\frac{1}{\left(1+(M+k S-S \delta z)^{c}\right)^{q}}-\frac{c S \delta q(M+k S-S \delta z)^{c-1}}{\left(1+(M+k S-S \delta z)^{c}\right)^{q+1}}(\sqrt{n}-z) \\
& n= \\
& \text { floor }\left(\left(z+\frac{\left(1+(M+k S-S \delta z)^{c}\right)^{q+1}}{c S \delta q(M+k S-S \delta z)^{c-1}}\left(1-\frac{1}{\left(1+(M+k S-S \delta z)^{c}\right)^{q}}-\beta\right)\right)^{2}+1\right)
\end{aligned}
$$

Where the floor function returns the largest previous integer. Because $\sqrt{n} \leq \frac{M+k S}{S \delta}, z$ is chosen to satisfy this criterion, typically $z=\left(\frac{M+k S}{S \delta}\right)(0.9-1.9 \beta) . \quad$ It is necessary to ensure that the expected criterion, $\sqrt{n} \leq \frac{M+k S}{S \delta}$, is satisfied.

Case 2: $\sqrt{n} \leq \frac{M-k S}{S \delta}$

This case is extremely complex requiring examination of the two-tail situation for (9). It is unlikely to be satisfied because

$$
n \leq\left(\frac{M-k S}{S \delta}\right)^{2}
$$

for the parameters generally considered, as is the case here, leads to $n=0$. Thus this case is not considered.
Step 4

It is straightforward to employ Step 4 from the numerical methods, however, if desired, a slight variant on the procedure may be adopted. Noticing that $d E_{4} \approx 0$, the problem reduces to solving a cubic equation. To derive a solution the following coefficients (recall equation 14) are useful:

$$
\begin{gathered}
p_{3}=\frac{3 d E_{1} d E_{3}-d E_{2}^{2}}{3 d E_{3}^{2}} \\
q_{3}=\frac{2 d E_{2}^{3}-9 d E_{1} d E_{2} d E_{3}+27 d E_{0} d E_{3}^{2}}{27 d E_{3}^{3}} \\
u_{3}=\left(-\frac{q_{3}}{2}+\sqrt{\left(\frac{q_{3}}{2}\right)^{2}+\left(\frac{p_{3}}{3}\right)^{3}}\right)^{\frac{1}{3}}
\end{gathered}
$$

and

$$
v_{3}=\left(-\frac{q_{3}}{2}-\sqrt{\left(\frac{q_{3}}{2}\right)^{2}+\left(\frac{p_{3}}{3}\right)^{3}}\right)^{\frac{1}{3}}
$$

result in:

$$
h=u_{3}+v_{3}-\frac{d E_{2}}{3 d E_{3}}
$$

A Numerical Example for the Approximate Solution

The approximation procedure outlined may be used to reproduce the results presented in Table 1 of Chou, et al. (2000); bearing in mind that in this case the parameters and moments of the Burr distribution are already available. Thus, $c=3, q=6, M=0.5109$, $S=0.2022, \quad$ skewness $=0.4836 \quad$ and kurtosis $=3.3768$.

The values presented in Table 3 are in exact agreement to the precision given with those earlier reported (Chou, et al., 2000). This suggests that the numerical approximations described are reliable and may safely replace the previously described complex computational approach. This leaves the accuracy of two of the numerical procedures undemonstrated. To check 


\section{A. A. COX}

Table 3: Results of the Numerical Approximation

\begin{tabular}{|c|c|c|c|c|c|}
\hline$\alpha$ & Power $(1-\beta)$ & $n$ & $h$ & $k$ & Cost \\
\hline 0.00500 & 0.918860 & 19 & 1.15 & 3.03 & 88.78 \\
\hline 0.00500 & 0.939008 & 20 & 1.19 & 3.03 & 88.80 \\
\hline 0.00500 & 0.955365 & 21 & 1.23 & 3.03 & 88.84 \\
\hline 0.00500 & 0.968362 & 22 & 1.26 & 3.03 & 88.89 \\
\hline 0.00500 & 0.978435 & 23 & 1.30 & 3.03 & 88.96 \\
\hline 0.00500 & 0.986008 & 24 & 1.33 & 3.03 & 89.03 \\
\hline 0.00500 & 0.991489 & 25 & 1.35 & 3.03 & 89.12 \\
\hline 0.00455 & 0.993538 & 26 & 1.37 & 3.08 & 89.21 \\
\hline 0.00406 & 0.994851 & 27 & 1.39 & 3.14 & 89.30 \\
\hline 0.00362 & 0.995924 & 28 & 1.41 & 3.20 & 89.39 \\
\hline 0.00322 & 0.996797 & 29 & 1.43 & 3.26 & 89.48 \\
\hline 0.00282 & 0.997296 & 30 & 1.45 & 3.33 & 89.57 \\
\hline
\end{tabular}

the estimation of $c$ and $q, 1,000$ observations from a Burr distribution $(c=3, q=6)$ were produced. Initially uniform random numbers in the interval $[0,1]$ were generated, and then transformed to $\left\{y_{i}\right\}$ using equation (2). The quartiles for this data were $(0.375,0.655)$ which should be compared with the exact values $(0.366,0.638)$. Employing the measured values with tail values $(0.25,0.75)$, the iterative scheme (equation 18) $\begin{array}{lll}\text { results in: } & c_{0}=2.000, & c_{1}=2.497, \\ c_{2}=2.843, & c_{3}=2.982, & c_{4}=3.000 .\end{array}$ Successive estimates converge to the expected result for $c$, then $q=\frac{1}{1000}+1$ because $\sum_{i=1}^{1000} y_{i}^{c}$

$\varepsilon\left(\mathrm{y}^{\mathrm{c}}\right)=\frac{1}{q-1}$, which results in 5.68, a reasonable estimate for $q$.
Alternately employing the median for the sample, the value $\left(y_{M}\right)=0.504$, which is close to the exact value 0.497 . This results in $q=5.986$, again very close to the true value. A detailed analysis would be necessary to assess which of the methods to estimate $q$ is superior and whether selection depends on the parameters of the underlying distribution.

The final procedure requiring verification is to use a relatively small neural network to estimate the mean and standard deviation. Using the same test example, the inputs are $x_{1}=3=c$ and $x_{2}=6=q$. These generate the following potentials in the hidden layer $x_{3}=0.1000$ and $x_{4}=-0.1615$. which result in the output $x_{5}=0.5418=M$ and $x_{6}=0.1881=S$.

The results exhibit reasonable agreement with the exact values $(M=0.5109$ and $S=0.2022$ ), if improved accuracy is desirable a larger network could be developed or a quadrature procedure might be employed to evaluate the required moments. 


\section{APPROXIMATE APPROACH TO THE ECONOMIC DESIGN OF $\bar{x}$ CHARTS}

\section{Conclusion}

To help popularize the economic design of $\bar{x}$ control charts, this study employed the Burr distribution for non-normal data. Slight shortcomings in an earlier work (Chou, et al., 2000) were corrected and a series of approximations were used to reduce computational complexity. It is hoped that the reduction of the computational effort involved in the approach will encourage wider adoption.

\section{References}

Alexander, S. M., Dillman, M. A., Usher, J. S., \& Damodaran, B. (1995). Economic design of control charts using the Taguchi loss function. Computers and Industrial Engineering, 28(3), 671-679.

Burr, I. W. (1942). Cumulative frequency distribution. Annals of Mathematical Statistics, 13, 215-232.

Bus, J. C. P., \& Dekker, T. J. (1975). Two efficient algorithms with guaranteed convergence for finding a zero of a function. ACM Transactions in Mathematical Software, 1, 330-345.
Chou, C. Y., Chen, C. H., \& Liu, H. R. (2000). Economic-statistical design of $\bar{x}$ charts for non-normal data by considering quality loss. Journal of Applied Statistics, 27(8), 939-951.

Duncan, A. J. (1956). The economic design of $\bar{x}$ charts used to maintain current control of process. Journal of the American Statistical Association, 51, 228-242.

Goodman P. H. (2001). NevProp software: Version 4. Reno, NV: University of Nevada.

Piessens, R., De Doncker-Kapenga, E., Uberhuber, C., \& Kahaner D. (1983). QUADPACK: A subroutine package for automatic integration. Berlin, Germany: Springer-Verlag.

Powell, M. J. D. (1970). A hybrid method for nonlinear algebraic equations. In Numerical Methods for Nonlinear Algebraic Equations, P. Rabinowitz (Ed.), 87-114, New York, NY: Gordon and Breach Science Publishers.

Smith, B. T. (1967). ZERPOL: A zero finding algorithm for polynomials using Laguerre's Method. Technical Report, Department of Computer Science, Toronto, Canada: University Toronto.

$$
\begin{aligned}
& \text { Appendix: Notation and Definitions } \\
a_{1}: & \text { fixed cost of taking a sample } \\
a_{2}: & \text { variable cost of sampling } \\
a_{3}: & \text { cost of eliminating an assignable cause } \\
a_{5}: & \text { cost of investigating a false alarm } \\
a_{i j}: & \text { weights in neural networks } \\
A: & \text { cost to rework or scrap a faulty item } \\
B: & \text { sub-equation of the expected cost } \\
c: & \text { first parameter of the Burr distribution } \\
\left\{c_{0}, c_{1}, c_{2}\right\}: & \text { coefficients for } B \text { as a function of } h \\
\left\{c_{0}, c_{1}, \ldots, c_{n}\right\}: & \text { successive numerical estimates for } c \\
\left\{d_{0}, d_{1}, d_{2}\right\}: & \text { coefficients for the denominator of } E \text { as a function of } h
\end{aligned}
$$




\section{A. A. COX}

Appendix (continued): Notation and Definitions

$d E:$ the numerator of the derivative of $E$

$\left\{d E_{0}, d E_{1}, d E_{2}, d E_{3}, d E_{4}\right\}:$ coefficients for $d E$ as a function of $h$

$D$ : time required to investigate an out-of-control signal

$E:$ expected cost

$f:$ density function of the Burr distribution

$F:$ cumulative density function of the Burr distribution

$g:$ time to measure and record the quantity of interest

$h$ : parameter of the $\bar{x}$ control chart, interval between successive subgroups

$H$ : Heaviside step function

$k$ : parameter of the $\bar{x}$ control chart, control limits

$\left\{L_{1}, L_{2}\right\}:$ sub-equation of the expected cost

$L C L$ : lower control limit

$M:$ the mean of the fitted Burr distribution

$n$ : parameter of the $\bar{x}$ control chart, sample size

$\left\{n_{0}, n_{1}, n_{2}, n_{3}\right\}:$ coefficients for the numerator of $E$ as a function of $h$

$P$ : production rate

$p_{3}:$ factor in the cubic solution

$q: \quad$ second parameter of the Burr distribution

$q_{3}:$ factor in the cubic solution

$S$ : the standard deviation of the fitted Burr distribution

$T$ : target mean 
Appendix (continued): Notation and Definitions

$u_{3}:$ factor in the cubic solution

$u_{i}:$ potentials for the neural networks

$U C L$ : upper control limit

$\left\{v_{1}, v_{2}\right\}:$ sub-equation of the expected cost

$v_{3}:$ factor in the cubic solution

$x$ : random variable

$x_{i}:$ inputs/outputs for the neural networks

$y,\left\{y_{i}\right\}:$ Burr random variables

$y_{M}:$ the median of the raw data

$\left\{y_{U}, y_{L}\right\}:$ upper/lower cut offs of the raw data corresponding to $\left\{\beta_{1}, \beta_{2}\right\}$

$z:$ point about which the Maclaurin expansion is performed

$Z, Z^{\prime}:$ the function (and its derivative) with a zero at $\mathrm{c}$

$\alpha$ : probability of a Type I error

$\beta$ : probability of a Type II error

$\left\{\beta_{1}, \beta_{2}\right\}:$ tail areas associated with estimating $\mathrm{c}$

$\delta: \quad$ magnitude of the mean shift for an out of control process

$\varepsilon$ : expectation (average) over the observed data

$\Delta:$ tolerance

$\left\{\phi_{0}, \phi_{1}\right\}:$ decision function for the neural networks

$\lambda: \quad$ frequency of process shifts

$\mu:$ process mean

$\sigma:$ process standard deviation (from past data) 


\title{
Parameter Estimation of a Class of Hidden Markov Model with Diagnostics
}

\author{
E. B. Nkemnole O. Abass R. A. Kasumu \\ University of Lagos, \\ Nigeria, Africa
}

A stochastic volatility (SV) problem is formulated as a state space form of a Hidden Markov model (HMM). The SV model assumes that the distribution of asset returns conditional on the latent volatility is normal. This article analyzes the SV model with the student-t distribution and the generalized error distribution (GED) and compares these distributions with a mixture of normal distributions from Kim and Stoffer (2008). A Sequential Monte Carlo with Expectation Maximization (SMCEM) algorithm technique was used to estimate parameters for the extended volatility model; the Akaike Information Criteria (AIC) and forecast statistics were calculated to compare distribution fit. Distribution performance was assessed using simulation study and real data. Results show that, although comparable to the normal mixture SV model, the Student-t and GED were empirically more successful.

Key words: Hidden Markov Model, sequential Monte Carlo, expectation maximization, Student-t distribution, state-space model, stochastic volatility, likelihood, stock exchange.

\section{Introduction}

The Hidden Markov Model (HMM), originally introduced in 1957, (MacDonald \& Zucchini, 1997, Cappe, et al., 2005) has many applications in fields such as signal processing, medicine, engineering and management. The HMM is a doubly stochastic process, $\left(X_{t}, Y_{t}\right)_{t \geq 0}$, with an underlying stochastic process, $X_{t}$, that is not directly observable but can be observed through another process, $Y_{t}$, that produces a sequence of independent random observations.HMM are equivalently defined via a functional representation known as a state space model. The state space model (Doucet \& Johansen, 2009) of a HMM is represented by two equations: state (1) and observation(2) as

E. B. Nkemnole is a Lecturer in the Department of Mathematics. Email her at: enkemnole@unilag.edu.ng. O. Abass is a Professor in the Department of Computer Science. Email him at: olabass@unilag.edu.ng. R. A. Kasumu is a Lecturer in the Department of Mathematics. Email him at: bkasumu@yahoo.com.

$$
\begin{gathered}
x_{t}=f\left(x_{t-1}\right)+w_{t} \\
y_{t}=g\left(x_{t}\right)+v_{t}
\end{gathered}
$$

where $f$ and $g$ are linear or nonlinear function sand $w_{t}$ and $v_{t}$ are white noise processes. Models represented by (1) and (2) are referred to as state space models and include a class of HMMs with non-linear Gaussian state-space model such as the stochastic volatility (SV) model. The SV model (Taylor, 1982), accounts for time-varying and persistent volatility and the leptokurtosis in financial return series. The SV model has become popular for explaining the behavior of financial variables, such as stock prices and exchange rates (Durbin \& Koopman, 2000; Doucet \& Tadic, 2003) and its popularity has resulted in several different proposed approaches for estimating model parameters. Though theoretically attractive, the SV model is empirically challenging due to the fact that the unobserved volatility process enters the model in a non-linear fashion which leads to the likelihood function depending upon highdimensional integrals.

Estimation procedures, such as the Generalized Method of Moments (GMM) 


\section{PARAMETER ESTIMATION OF A HIDDEN MARKOV MODEL WITH DIAGNOSTICS}

(Mellino \& Turnbull, 1990) and the Efficient Method of Moments (EMM) (Gallant, et al., 1997) have been proposed for the SV model. Other proposed estimation procedures include the method of moments and the quasi maximum likelihood approach methodology to approximate the SV model to a linear Gaussian model (Harvey, et al, 1994; Ruiz, 1994).

Durbin and Koopman (2000) used the idea of linearization of general state-space models and matched terms in the likelihood of a linearized model to those of a linear Gaussian model. Several studies (Jacquier, et al., 1994; Chib, et al., 2002; Kim, et al., 1998) adopted the Gibbs sampling scheme, and Shephard and Pitt (1997) applied the Metropolis-Hastings scheme for the analysis of the SV.Kim and Stoffer (2008) showed how the incorporation of the EM algorithm and SMC (particle filters and smoothers) forms a basic idea to handle the parameter estimation problem in the SV model. Estimation can be accomplished by applying a filtering algorithm. (Kitagawa\& Sato, 2001) combined particle filtering methods and gradient algorithms. This article expands the scope of application of SV models, by extending SMC techniques with the EM algorithm developed by Kim and Stoffer (2008) to estimate SV model parameters with the student-t distribution.

The SV model usually assumes that the distribution of asset returns conditional on the latent volatility is normal. However, financial data often have heavier tails than can be captured by the standard SV model: This has led to the use of non-normal distributions to bettermodel and to deal with the heavy tails (Shephard, 1996; Kim, et al., 1998; Bai, et al., 2003; Sadorsky, 2005; Kim \& Stoffer, 2008). Liesenfeld and Jung (2000) fit a Student-t distribution to the error distribution in the SV model using the simulated maximum likelihood method developed by Danielsson and Richard (1993) and Danielsson (1994). A promising distribution that models both skewness and kurtosis is the Skewed Student-t (Fernandez \& Steel, 1998). Hence, it is necessary to determine the best-fitted model out of a potentially huge class of candidates; it has become pertinent to develop efficient model selection criteria. As this background illustrates there is an evergrowing literature on time-varying financial market volatility; it is abound with empirical studies in which competing models are evaluated and compared on the basis of their forecast performance (Andersen, et al., 2005).

Stochastic Volatility (SV) Models

$\mathrm{SV}$ models belong to class of Hidden Markov model and account for volatility of data. The SV model can be expressed as an autoregressive (AR) process:

$$
\begin{aligned}
& x_{t}=\phi x_{t-1}+w_{t} \\
& y_{t}=\beta \exp \left(\frac{x_{t}}{2}\right) v_{t}
\end{aligned}
$$

where $w_{t} \sim N(0, \tau), x_{0} \sim N\left(\mu_{0}, \sigma_{0}^{2}\right)$, $v_{t} \sim N(0,1),\left\{y_{t}\right\}_{t \geq 0}$ is the log-return on day $\mathrm{t}$, and $\beta$ is the constant scaling factor so that $\left\{x_{t}\right\}_{t \geq 0}$ represents the $\log$ of volatility of $y_{t}$ (Taylor, 1982).To ensure stationarity of $y_{t}$ it is assumed that $\phi_{1}<1$. Squaring (4) and taking its logarithm results in the linear equation

$$
y_{t}=\alpha+x_{t}+z_{t} \text {. }
$$

Equations (3) and (5) form a version of the SV model that can be modified in many ways; together they form a linear, non-Gaussian, statespace model for which (5) is the observation equation and (3) is the state equation.

Stochastic Volatility with Heavy-Tailed Distribution

The standard form of the SV modelwas shown in equations (3) and (4); in (4) $v_{t}$ follows a normal distribution. Various authors have argued that real data may have heavier tails than can be captured by the standard SV model.

The Stochastic Volatility Model with Normal Mixture

The observational noise process (Kim \& Stoffer, 2008) is a mixture of two normal's with unknown parameters given as 


$$
y_{k}=x_{t}+z_{t}
$$

with $z_{t}=I_{t} z_{t 1}+\left(1-I_{t}\right) z_{t 0}, z_{t 0} \sim N\left(m_{0}, R_{0}\right)$, $z_{t 1} \sim N\left(m_{1}, R_{1}\right), \quad m_{0}=\alpha-\mu \pi$, $m_{1}=\alpha+(1-\pi) \mu$ and $I_{t} \sim \operatorname{Bernuolli}(\pi)$. $I_{t}$ is an indicator variable, where $\pi$ is an unknown mixing probability, that is, $p\left(I_{t}=1\right)=\pi=1-p\left(I_{t} \sim \operatorname{Bernuolli}(\pi)\right.$. The likelihood of $\left\{x_{0}, \cdots, x_{n}, y_{1}, \cdots, y_{n}, I_{1}, \cdots, I_{n}\right\}$ is shown in Figure 1 where

$R_{t}^{*}=I_{t} R_{1}+\left(1-I_{t}\right) R_{0}, \mu_{t}^{*}=I_{t} q_{1}+\left(1-I_{t}\right) q_{0}$.

In the SV-normal mixture model defined by (6), the vector of the model parameter is denoted by $\left\{q_{0}, q_{1}, R_{0}, R_{1}, \pi\right\}$. These parameters are estimated along with the other parameters, $\{\phi, \tau\}$ (see Kim \& Stoffer, 2008 for details).

Student-t as an Observation Noise

Equations (3) and (5) are an extension of the linearized version of the SV model wherein it is assumed that the observational noise process, $z_{t}$ is a student-t distribution. The model, first presented in Shumway and Stoffer (2006), retains the state equation for the volatility as: $x_{t}=\phi x_{t-1}+w_{t}$.

However, the proposed Student-t distribution with degrees of freedom, $v$, for the observation error term, $z_{t}$, effects a change in the observation equation:

$$
\begin{gathered}
y_{t}=\alpha+x_{t}+z_{t} \\
z_{t} \sim t_{v}, t=1, \cdots, n
\end{gathered}
$$

The distribution of the error term for this specification according to Shimada \& Tsukuda (2005) takes the form

$$
\begin{aligned}
& f\left(y_{t} \mid x_{t}\right)= \\
& \frac{1}{\sqrt{\pi(v-2)}} \frac{\Gamma\left(\frac{v+1}{2}\right)}{\Gamma\left(\frac{v}{2}\right)} e^{-\frac{x_{t}}{2}}\left(1+\frac{y_{t}^{2} e^{-x_{t}}}{v-2}\right)^{-\frac{v+1}{2}}
\end{aligned}
$$

where $v$ represents a parameter of degree of freedom and $\Gamma$ stands for the Gamma function. The likelihood function of $\left\{x_{0}, x_{1}, \cdots, x_{n}, y_{1}, \cdots, y_{n}\right\}$ is

$f(X, Y)=$

$\left(\frac{1}{\sqrt{2 \pi \sigma_{0}^{2}}} \exp \left(-\frac{1}{2}\left(\frac{x_{0}-\mu_{0}}{\sigma_{0}}\right)^{2}\right)\right.$
$\left.\times \prod_{t=1}^{n} \frac{1}{\sqrt{2 \pi \tau}} \exp \left(-\frac{1}{2}\left(\frac{x_{t}-\varphi x_{t-1}}{\tau}\right)^{2}\right)\right)$
$\times \prod_{t=1}^{n} \frac{1}{\sqrt{\pi(v-2)}} \frac{\Gamma\left(\frac{v+1}{2}\right)}{\Gamma\left(\frac{v}{2}\right)} e^{-\frac{x_{t}}{2}}\left(1+\frac{y_{t}^{2} e^{-x_{t}}}{v-2}\right)^{-\frac{v+1}{2}}$

Figure 1: Likelihood of $\left\{x_{0}, \cdots, x_{n}, y_{1}, \cdots, y_{n}, I_{1}, \cdots, I_{n}\right\}$

where $R_{t}^{*}=I_{t} R_{1}+\left(1-I_{t}\right) R_{0}, \mu_{t}^{*}=I_{t} q_{1}+\left(1-I_{t}\right) q_{0}$

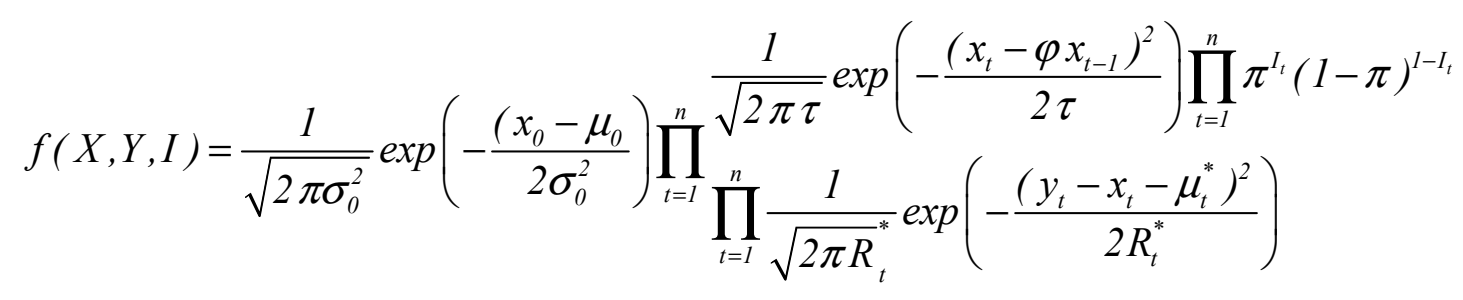




\section{PARAMETER ESTIMATION OF A HIDDEN MARKOV MODEL WITH DIAGNOSTICS}

Generalized Error Distribution as an Observation Noise

The distribution of the GED according to Bao, et al. (2006) takes the form

$$
f\left(y_{t} \mid x_{t}\right)=\frac{v \exp \left[-\frac{1}{2}\left(\left(y_{t}-\alpha-v_{t}\right) / \psi\right)^{v}\right]}{\psi \Gamma\left(\frac{1}{v}\right) 2^{\left(1+\frac{1}{v}\right)}}
$$

where

$$
\psi=\left[2^{-\frac{2}{v}} \frac{\Gamma\left(\frac{1}{v}\right)}{\Gamma\left(\frac{3}{v}\right)}\right]^{\frac{1}{2}}
$$

The log-likelihood function for the GEDmodel is:

$$
\begin{aligned}
\log f\left(y_{t} \mid x_{t}\right)= & n \log v-\frac{1}{2} \sum_{t=1}^{n}\left(\frac{y_{t}-\alpha-v_{t}}{\psi}\right)^{v} \\
& -n \log \psi-n \log \Gamma\left(\frac{1}{v}\right) \\
& -n \log 2^{\left(1+\frac{I}{v}\right)} .
\end{aligned}
$$

The EM Algorithm

The paramount parameter estimation toolto achieve maximum likelihood estimator is the EM algorithm and it has been widely applied to the cases where the data is considered to be incomplete in the sense that it is not fully observable. It is comprised of the two following steps:

E-step: Compute the expected likelihood,

$$
\begin{gathered}
Q\left(\theta \mid \theta^{(k)}\right) \\
Q\left(\theta \mid \theta^{\prime}\right)=E\left(\log f\left(x \mid \theta^{\prime}\right) \mid \mathrm{y}, \theta\right)
\end{gathered}
$$

M-step: Choose $\theta^{(k+1)}$ the parameter values that maximize the function, $Q\left(\theta \mid \theta^{(\mathrm{k})}\right)$ (for details see Baum, et al, 1970; Dempster, et al., 1977; Rabiner, 1989).
The E- and M- steps are repeated until some stopping criteria is met, such as $\left|\hat{\theta}^{n+1}-\hat{\theta}^{n}\right|<Q$, for some specified $Q$, obtaining suitable initial parameters inclusive. An online EM algorithm recently proposed for discrete HMM can be extended to more general settings, including non-linear non-Gaussian state-space models that necessitate the use of Sequential Monte Carlo (SMC) filtering approximations.

Sequential Monte Carlo Methods (SMC)

After its introduction in the 1960's, SMC has become an emerging methodology for the nonlinear or non-Gaussian state-space models. The chief initiative is to represent the interested density function $p\left(x_{0: k-1}, y_{0: k-1}\right)$ at time $k-1$ by a set of random samples with associated weights, $\left\{x_{0: k-1}^{(i)}, w_{0: k-1}^{(i)} i=1, \cdots, N\right\}$ and compute estimates based on these samples and associated weights. As the number of samples becomes very large, this Monte Carlo characterization develops into an equivalent representation to the functional description of the probability density function (Arulampalam, et al., 2002).

$$
\text { If } \quad\left\{x_{0: k-1}^{(i)}, w_{0: k-1}^{(i)} i=1, \cdots, N\right\} \quad \text { are }
$$

samples and associated weights approximating the density function $p\left(x_{0: k-1}, y_{0: k-1}\right)$, with $\sum_{i=1: N} w^{(i)}=1$, then the density function is approximated by

$$
p\left(x_{0: k-1}: y_{0: k-1}\right) \approx \sum_{i=1}^{N} w_{k-1}^{(i)} \delta\left(x_{k-1}-x_{k-1}^{(i)}\right)
$$

where $\delta(x)$ signifies the Dirac delta role. The particle approximation $\quad\left\{w_{k}^{(i)}, x_{k}^{(i)}\right\}_{i=1}^{N}$ is transformed into an equally weighted random sample from $p\left(x_{0: k-1}, y_{0: k-1}\right)$ by sampling, with replacement from the discrete distribution $\left\{w_{k}^{(i)}, x_{k}^{(i)}\right\}_{i=1}^{N}$. This procedure, also called resampling, produces a new sample with uniformly distributed weights so that $w_{k}^{(i)}=N^{-1}$. 


\section{NKEMNOLE, ABASS \& KASUMU}

Particle filters and smoothers are SMC methods grounded in particle representations and are considered generalizations of Kalman filters and smoothers for general state-space models. The fundamental approach used to obtain particles from the desired density is based on sequential importance sampling (SIS) and resampling. SIS, a Monte Carlo method, forms the basis for most particle filtering methods. To approximate the conditional density of $x_{t}$ given previous states, $x_{t-1}$, and past and present data, $y_{t}, p\left(x_{t} \mid x_{t-1}, y_{t}\right), \quad$ SIS introduces a importance sampling density, $q\left(x_{t} \mid x_{t-1}, y_{t}\right)$ where it is easier to sample from $\pi\left(x_{t} \mid x_{t-1}, y_{t}\right)$ than $p\left(x_{t} \mid x_{t-1}, y_{t}\right)$ (Doucet, et al., 2001).

\section{Particle Filter Algorithm}

If at time $t$ weighted particles $\left\{f_{t}^{(i)}, w_{t}^{(i)}\right\}$ drawn from $f\left(x_{t}, y_{t}\right), f_{t}^{(i)}$ is a set of particle filter with associated weight $w_{t}^{(i)}$, then this is considered an empirical approximation for the density comprised of point masses, $f\left(x_{t}, y_{t}\right) \approx \sum_{i=1}^{M} w_{t}^{(i)} \delta\left(x_{t}-f_{t}^{(i)}\right)$.

Kitagawa \& Sato (2001) and Kitagawa (1996) provide an algorithm for filtering in general state space model. This is a Monte Carlo filtering for general state-space models:

1. For $i=1, \cdots, N$, generate a random number $f_{0}^{(i)} \sim p\left(x_{0}\right)$

2. Repeat the following steps for $t=1, \cdots, T$.

a. For $i=1, \cdots, N$, generate a random number $w_{t}^{(i)} \sim q(w)$.

b. For $\quad i=1, \cdots, N, \quad$ compute $p_{t}^{(i)}=F\left(f_{t-1}^{(i)}, w_{t}^{(i)}\right)$

c. For $\quad i=1, \cdots, N, \quad$ compute $w_{t}^{(i)}=p\left(y_{t} \mid p_{t}^{(i)}\right)$

d. Generate $f_{t}^{(i)}, i=1, \cdots N \quad$ by resampling $p_{t}^{(i)}, \cdots, p_{t}^{(N)}$
The Monte Carlo filter returns $\left\{f_{t}^{(i)}, i=1, \cdots, N, t=1, \cdots, m\right\} \quad$ so that $\sum_{i=1}^{N} \frac{1}{N} \delta\left(x_{t}-f_{t}^{(i)}\right) \approx f\left(x_{t \mid} \mid Y_{t}\right)$.

Particle Smoothing Algorithm

If $\left\{s_{t}^{(i)}, w_{t}^{(i)}\right\}_{i=1}^{M}$ is a set of particle smoothers and associated weights approximating the density function $f\left(x_{t} \mid Y_{n}\right)$, then the density function is approximated by:

$$
f\left(x_{t} \mid Y_{n}\right) \approx \sum_{j=1}^{M} w_{t}^{(i)} \delta\left(x_{t}-s_{t}^{(i)}\right) .
$$

The problem with smoothed estimates is degeneracy. Godsill, et al. (2004) suggested a new smoothing method (particle smoother using backwards simulation). The method assumes that filtering has already been performed, thus, the particles and associated weights, $\left\{f_{t}^{(i)}\right\}_{i=1}^{M}$, $\left\{w_{t}^{(i)}\right\}_{i=1}^{M}$ can approximate the filtering density,

$$
f\left(x_{t} \mid Y_{t}\right), \text { by }=\frac{\sum w_{t}^{(i)} \delta\left(x_{t}-f_{t}^{(i)}\right)}{\sum_{i=1}^{N} w_{t}^{(i)}}
$$

The algorithm from Godsill, et al. (2004) supposes that weighted particles $\left\{f_{t}^{(i)}, w_{t}^{(i)} ; i=1,2, \cdots, M\right\}$ are available for $t=1,2, \cdots, n$. The algorithm for $i=1,2, \cdots, M$ is:

1. Choose $s_{n}^{(i)}=f_{n}^{(j)}$ with probability $w_{n}^{(j)}$

2. For $n-1$ to 1

a. Calculate $w_{t \mid t+1}^{(j)} \propto w_{t}^{(j)} f\left(s_{t+1}^{(i)} \mid f_{t}^{(j)}\right)$ for each $j$.

b. Choose $s_{t}^{(i)}=f_{t}^{(j)}$ with probability $w_{t \mid t+1}^{(j)}$. 


\section{PARAMETER ESTIMATION OF A HIDDEN MARKOV MODEL WITH DIAGNOSTICS}

3. $s_{1: n}^{(i)}=\left(s_{1}^{(i)}, \cdots, s_{n}^{(i)}\right)$ is an approximate realization from $p\left(X_{n} \mid Y_{n}\right)$.

Sequential Monte Carlo Expectation Maximization (SMCEM) Algorithm Analysis

Parameter Estimation

The SMCEM estimation procedure consists of three main steps: filtering, smoothing and estimation. Parameter estimation for the Student-t and GED SV model were considered. A basic approach for the Student-t SV model, equation (7), is to apply the EM algorithm; with the output of filtering and a smoothing step an approximate expected likelihood is calculated.

Filtering Step

The algorithm for the filtering and smoothing steps shows a slight modification of Godsill, et al. (2004) and Kim and Stoffer (2008). $M$ samples from $f\left(x_{t}, \mid Y_{t}\right)$ for each $t$ were obtained as:

1. Generate $f_{0}^{(i)} \sim N\left(\mu_{0}, \sigma_{0}^{2}\right)$

2. For $t=1, \cdots, n$

a. Generate a random number $w_{t}^{(i)} \sim N(0, \tau), \quad j=1, \cdots, M$

b. Compute $p_{t}^{(i)}=\phi f_{t-1}^{(i)}+w_{t}^{(i)}$

c. Compute

$$
w_{t}^{(i)}=p\left(y_{t} \mid p_{t}^{(i)},\right) \propto e^{-\frac{x_{t}}{2}}\left(1+\frac{y_{t}^{2} e^{-x_{t}}}{v-2}\right)^{-\frac{v+1}{2}}
$$

d. Generate $f_{t}^{(i)}$ by resampling with weights, $w_{t}^{(j)}$

\section{Smoothing Step}

In the smoothing step, particle smoothers that are needed to acquire the expected likelihood in the expectation step of the EM algorithm were obtained. Suppose that equally weighted particles $\left\{f_{t}^{(i)}\right\}, i=1, \cdots, M$ from $f\left(x_{t}, \mid Y_{t}\right)$ are available for $t=1, \cdots, n$, from the filtering step.

1. Choose $\left[s_{n}^{(i)}\right]=\left[f_{n}^{(j)}\right]$ with probability $\frac{1}{M}$

2. For $n-1$ to 0 calculate

$$
\begin{aligned}
& w_{t \mid t+1}^{(i)} \propto f\left(s_{t+1}^{(i)} \mid f_{t}^{(j)}\right) \propto \exp \left(-\frac{\left(s_{t+1}^{(i)}-\varphi f_{t}^{(j)}\right)^{2}}{2 \tau}\right) \\
& \frac{1}{\sqrt{\pi(v-2)}} \frac{\Gamma\left[\frac{v+1}{2}\right]}{\Gamma \frac{v}{2}} \exp ^{-\frac{\tilde{s}_{t+1}}{2}}\left(1+\frac{y_{t}^{2} e^{-\tilde{s}_{t+1}^{(j)}}}{v-2}\right)^{-\frac{v+1}{2}}
\end{aligned}
$$

for each $j$

a. Choose $\left[s_{t}^{(i)}\right]=\left[f_{t}^{(j)}\right]$ with probability $w_{t \mid t+1}^{j}$.

3. $\left(s_{0: n}^{(i)}\right)=\left\{\left(s_{0}^{(i)}, \cdots, s_{n}^{(i)}\right)\right\}$ is the random sample from $f\left(x_{0}, \cdots, x_{n} \mid Y_{n}\right)$

4. Repeat $1-3$, for $i=1, \cdots, M$ and calculate

$$
\hat{x}_{t}^{n}=\frac{\sum_{i=1}^{M} s_{t}^{(i)}}{M}, \hat{p}_{t}^{n}=\frac{\sum_{i=1}^{M}\left(s_{t}^{(i)}-\hat{x}_{t}^{n}\right)^{2}}{M-1},
$$

$$
\hat{p}_{t, t-1}^{n}=\frac{\sum_{i=1}^{M}\left(s_{t}^{(i)}-\hat{x}_{t}^{n}\right)\left(s_{t-1}^{(i)}-\hat{x}_{t-1}^{n}\right)}{M},
$$

$$
\begin{aligned}
& \frac{E\left[1+\frac{y_{t}^{2} e^{x_{t}}}{v-2}\right]^{-\frac{v+1}{2}}=}{n(v-2)} \\
& (v+1) \sum_{t=1}^{n} y_{t}^{2} e^{-y_{t}+v_{t}}\left[1+\frac{y_{t}^{2} e^{x_{t}}}{v-2}\right]^{-l}
\end{aligned}
$$




\section{Estimation Step}

This step consists of obtaining parameter estimates by setting the derivative of the expected likelihood, of the complete data $\left\{x_{0}, \cdots, x_{n}, y_{1}, \cdots, y_{n}\right\}$ given $\left\{x_{0}, \cdots, x_{n}\right\}$, with respect to each parameter to zero and solving for $\hat{\phi}, \hat{\tau}$, and $\hat{\alpha}$. The complete likelihood of $\left\{x_{0}, x_{1}, \cdots, x_{n}, y_{1}, \cdots, y_{n}\right\}$ is

$\log f(X, Y)=$

$$
\begin{aligned}
& \log \frac{1}{\sqrt{2 \pi}} \frac{1}{\sigma_{0}}+\log \exp \left(-\frac{\left(x_{0}-\mu_{0}\right)^{2}}{2 \sigma_{0}^{2}}\right) \\
& +\log \prod_{t=1}^{n} \frac{1}{\sqrt{2 \pi \tau}} \exp \left(\frac{-\left(x_{t}-\varphi x_{t-1}\right)^{2}}{2 \tau}\right) \\
& +\left(\begin{array}{l}
\log \prod_{t=1}^{n} \frac{1}{\sqrt{\pi(v-2)}} \frac{\Gamma\left(\frac{v+1}{2}\right)}{\Gamma\left(\frac{v}{2}\right)} e^{-\frac{\left(y_{t}-\alpha-v_{t}\right)}{2}} \\
\left(1+\frac{y_{t}^{2} e^{-\left(y_{t}-\alpha-v_{t}\right.}}{v-2}\right)^{-\frac{v+1}{2}}
\end{array}\right)
\end{aligned}
$$

This method results in the estimates:

$$
\begin{gathered}
\hat{\phi}=\frac{S_{10}}{S_{00}} \hat{\tau}=\frac{1}{n}\left[S_{11}-\frac{S_{10}^{2}}{S_{00}}\right], \\
\hat{\alpha}=\log \frac{n(v-2)}{(v+1) \sum_{t=1}^{n} y_{t}^{2} e^{-y_{t}+v_{t}}\left[1+\frac{y_{t}^{2} e^{x_{t}}}{v-2}\right]^{-1}} \\
\hat{\alpha}=\left[n \sum_{t=1}^{n}\left(y_{t}-v_{t}\right)^{v-1}\right]^{\frac{1}{v-1}}
\end{gathered}
$$

where

$$
\begin{gathered}
S_{00}=\sum_{t=1}^{n}\left(x_{t-1}^{n}\right)^{2}+p_{t-1}^{n}, \\
S_{I 1}=\sum_{t=1}^{n}\left(x_{t}^{n}\right)^{2}-p_{t}^{n}, S_{10}=\sum_{t=1}^{n} x_{t}^{n} x_{t-1}^{n}+p_{t, t-1}^{n}
\end{gathered}
$$

When $z_{t}$ follows the GED, it is not possible to represent it as equation (3.2). Hence, for the SV-
GED model, the parameter $v$, as well as the other parameters, and $x_{t}$ were sampled from their full conditional distributions using SMCEM techniques.

\section{Methodology}

The proposed method to compare the fit of the distributions is illustrated using three simulated data sets and daily exchange rates of the Nigerian Naira, Ghana Cedi, British Pound and Euro compared to the U. S. Dollar, from March 3, 2009 to March 3, 2011. Figures 1-3 show the plots and histograms of data generated from the normal mixture, Student-t and GED SV model respectively and Tables 1-4 show the results of the estimation for the models.

\section{Simulation 1}

Data were generated from the normal mixture SV model $x_{t}=0.7 x_{t-1}+w_{t}$, $y_{t}=-2.75+x_{t}+v_{t} \quad$ where $w_{t} \sim N(0,0.96)$,

$v_{t} \sim I_{t} N(-2,6)+\left(1-I_{t}\right) N(-3.5,4) \quad$ and $I_{t} \sim \operatorname{Bernoulli}(0.5)$ with true parameter set $\left(\phi, \tau, q_{0}, q_{1}, R_{0}, R_{1}, \pi\right)=(0.7,0.96,-3.5,-2$, $4,6,0.5)$. The technique based on mixture and Student-t SV was applied to this data to examine the performance of the proposed model. To make the process stationary, 11,000 samples were generated and the first 10,000 values were discarded. Figures $2 \mathrm{a}$ and $2 \mathrm{~b}$ show the plot and histogram for Simulation 1.

Simulation 2

Data were generated from the Student-t SV model with true parameter set $(\phi, \tau, \alpha, v)=$ $(0.81,1.45,-3.01,8)$. The technique based on the mixture and Student-t SV models was applied to this data to examine the merit of the Student-t idea; the length of the data, $\left\{y_{t}\right\}$, was 1,000. Figures $3 \mathrm{a}$ and $3 \mathrm{~b}$ show the plot and histogram for Simulation 2. The second data set was used to observe the behavior of the estimation procedure when a departure from the normal mixture observational error assumption exists. 
Figure2a: Representation of SMCEM SequenceSimulated from the Normal Mixture SV Model

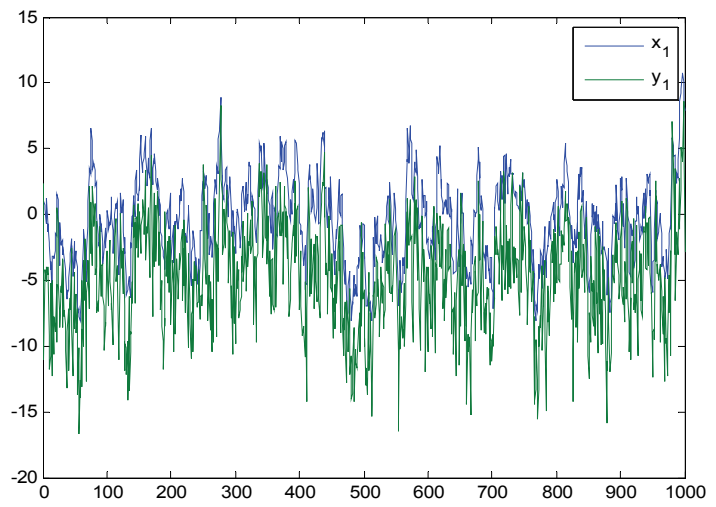

Figure3a: Representation of SMCEM Sequence Simulated from the Student-t SV Model

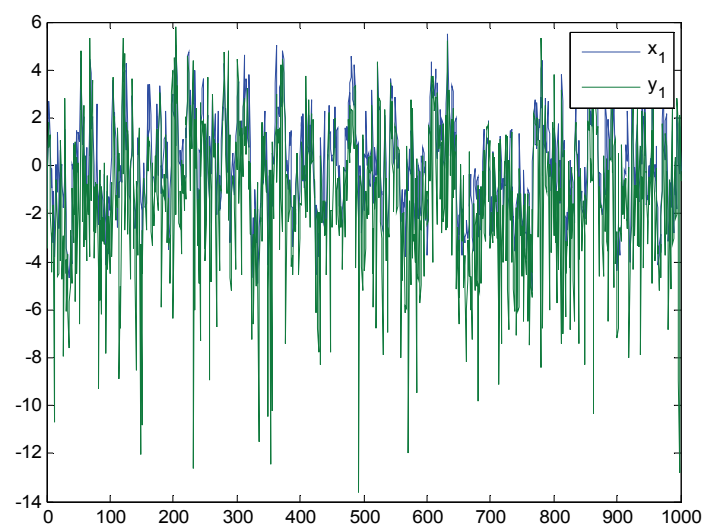

Figure4a: Representation of SMCEM Sequence Simulated from the Normal Mixture SV Model

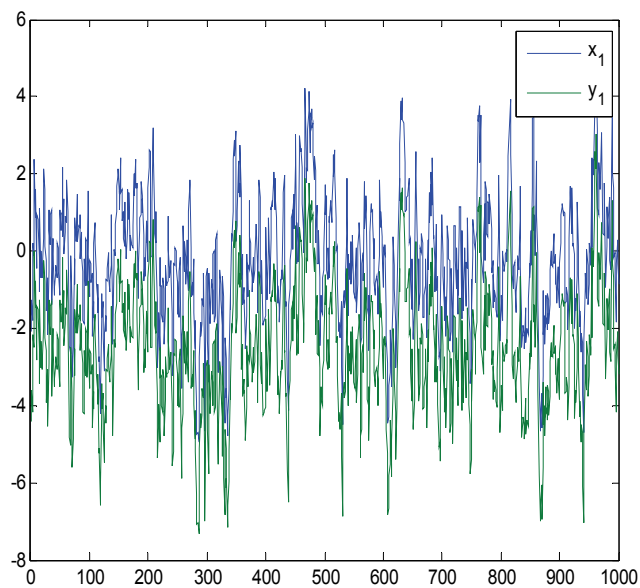

Figure 2b: Histogram of Final Values of Parameters of the Normal Mixture SV Model

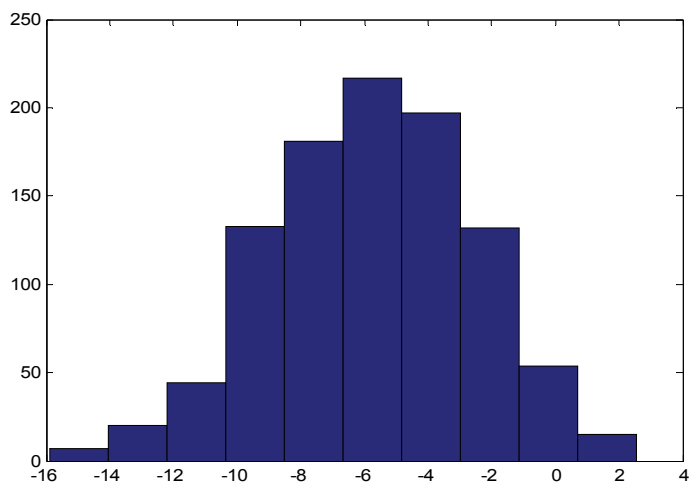

Figure 3b: Histogram of Final Values of the Parameters of the Student-t SV Model

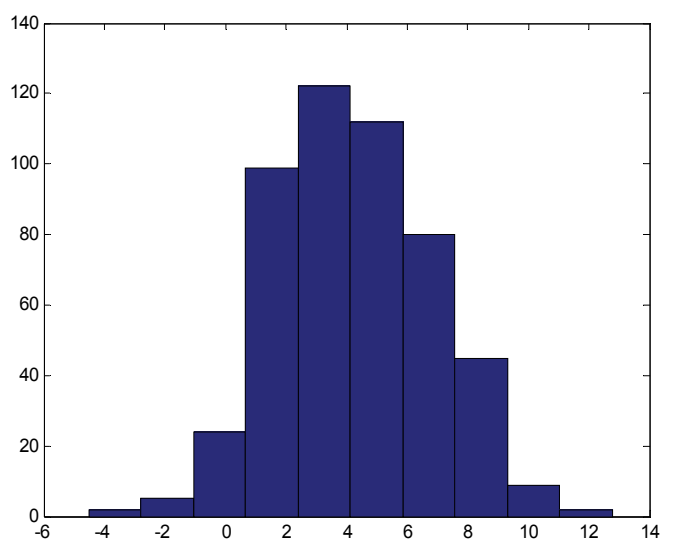

Figure 4b: Histogram of Final Values of Parameters of the Normal Mixture SV Model

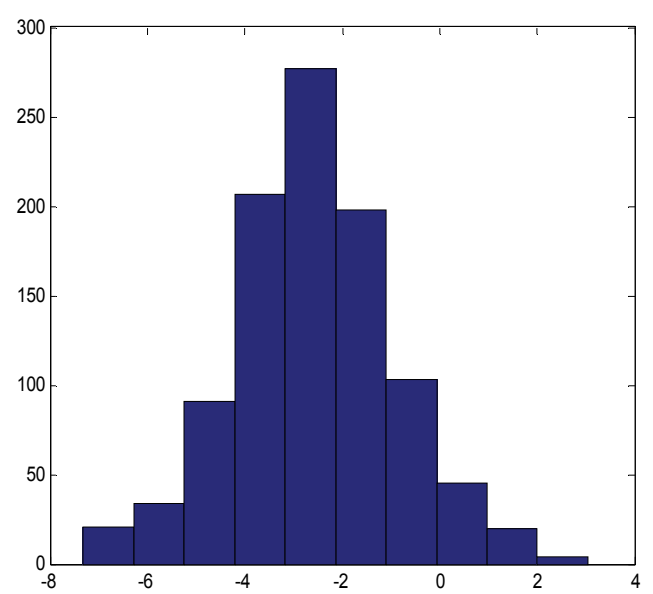


Simulation 3

Data were generated from the GED SV model with true parameter set $(\phi, \tau, \alpha, v)=$ $(0.9,1.6,0.7)$. Techniques based on mixture and the GED SV model were applied to this data to examine the merit of the GED idea; the length of the data, $\left\{y_{t}\right\}$, is 1,000 . Figures $4 \mathrm{a}$ and $4 \mathrm{~b}$ show the plot and histogram for Simulation 3.

\section{Results}

Using the procedures described $[0.9500,1.0729$, $-0.6794,-3.6794,4.000,4.000,0.5000]$ were selected for the initial parameters for $\left(\phi, \tau, q_{0}, q_{1}, R_{0}, R_{1}, \pi\right)$. Table 1 shows final estimates with their standard error (in parenthesis) for Simulation 1. The final estimates, along with their standard deviations (in parentheses), were: $\hat{\phi}=0.7568$ (0.027826), $\hat{\tau}=0.3466 \quad(0.00931), \quad \hat{q}_{0}=-1.9486$ (0.10989), $\quad \hat{q}_{1}=-3.7620 \quad(0.08690)$, $\hat{R}_{0}=2.3169$ (0.20936), $\hat{R}_{1}=7.5643$ (0.67241), $\hat{\pi}=0.3854(0.02635)$ where the true parameters are $(0.7,1.06,-3.5,-2,4,6,0.5)$. In this approach, $\hat{\alpha}=\hat{\pi} \hat{q}_{1}+(1-\hat{\pi}) \hat{q}_{0}=-2.6475$; $(\hat{\phi}, \hat{\tau}, \hat{\alpha})=(0.7568,0.3466,-2.6475)$; based on results, the estimation procedure based on the normal mixture model works well because that the estimates are close to the true parameters.

Based on the Student- $t$ technique, $(0.9500,1.0729,-2.1496)$ were used as the initial values for parameters $(\phi, \tau, \alpha)$; the process was stopped when the value of relative likelihood was less than 0.001 . The final estimates, along with their standard deviations (in parentheses) were: $\hat{\phi}=0.6913$ (0.037981), $\hat{\tau}=1.0336$ (0.14839), $\hat{\alpha}=-2.9009$ (0.024501).

These results show that the model provides good estimates despite the fact that the true observation noise is not a normal mixture distribution.A similar simulation study was performed using the data from simulation 2 (see Table 2).

The initial parameter set [0.8214, $1.3359,-2.7823,-5.7823,4.000,4.000,0.5000]$ was selected for parameters $\left(\phi, \tau, q_{0}, q_{1}, R_{0}, R_{1}, \pi\right)$. Table 2 shows the results of the parameter estimation procedure based on the normal mixture. The final estimates, along with their standard deviations (in parentheses) were: $\hat{\phi}=0.6547$ (0.005272), $\hat{\tau}=1.2930 \quad(0.002473), \quad \hat{q}_{0}=-3.0180$ (0.0.035241), $\quad \hat{q}_{1}=-5.8536 \quad(0.012445)$, $\hat{R}_{0}=3.3275 \quad(0.15), \quad \hat{R}_{1}=5.2663 \quad(0.32564)$, $\hat{\pi}=0.4806 \quad(0.004338) \quad$ where the true parameters are $(0.81,1.45,-3.01)$ for the parameters, $\quad(\hat{\varphi}, \hat{\tau}, \hat{\alpha}) ; \quad$ where $\hat{\alpha}=\hat{\pi} \hat{q}_{1}+(1-\hat{\pi}) \hat{q}_{0}=-4.3808$.

When the data from simulation 2 was fitted with the techniques based on the Student- $t$ $(0.8214,1.3359,-2.2823)$ were used as initial parameters of $(\phi, \tau, \alpha)$. At the $11^{\text {th }}$ iteration the relative likelihood was less than 0.001 and the process was considered converged. The final estimates, along with their standard deviations were: $\hat{\phi}=0.8383 \quad(0.008552), \quad \hat{\tau}=1.5357$ (0.12403), $\hat{\alpha}=-3.0912 \quad(0.005302)$. These estimates are similar to the true parameters $(0.81,1.45,-3.01)$, while Normal mixture returns $(0.6547,1.2930,-4.3808)$ as $(\hat{\phi}, \hat{\tau}, \hat{\alpha})$. The method based on the Student-t SV model worked well in both cases. When the estimation procedure based on the normal mixture SV model was applied, the estimates were distant to the true parameter. Conversely, the application of the technique based on Student-t model indicated a better proximity to the true parameters; therefore, extension of the SV model by adopting Student-t is meaningful.

Table 3 shows the results of the parameter estimation procedure on technique based on the normal mixture SV and GED on data generated from the normal mixture model; $[0.8699,3.6899,-4.8897,-7.8897,4.000$, $4.000,0.5000]$ were selected for the initial parameter for the parameters $\left(\phi, \tau, q_{0}, q_{1}, R_{0}, R_{1}, \pi\right)$. The final estimates, along with their standard deviations (in parentheses) were: $\hat{\phi}=0.9869 \quad(0.0013341)$, $\hat{\tau}=4.1936 \quad(0.51468), \quad \hat{q}_{0}=-4.3900$ 


\section{PARAMETER ESTIMATION OF A HIDDEN MARKOV MODEL WITH DIAGNOSTICS}

$(0.021891), \quad \hat{q}_{1}=-6.4554 \quad(0.026309)$, $\hat{R}_{0}=3.7342 \quad(0.069579), \quad \hat{R}_{1}=4.3517$ (0.16268), $\hat{\pi}=0.4895(0.0032833)$ where the true parameters are $(0.8,3.5,-5,-8,3,4.1,0.5)$. In this approach, $\hat{\alpha}=\hat{\pi} \hat{q}_{1}+(1-\hat{\pi}) \hat{q}_{0}=-5.4010 ; \quad(\hat{\phi}, \hat{\tau}, \hat{\alpha})=$ $(0.9869,4.1936,-5.4010)$. Results show that the estimation procedure based on the normal mixture model worked well in the sense that the estimates are close to the true parameters.

For the GED technique, $(0.8699,3.6899$, -4.4365 ,) were used as the initial values for parameters $(\phi, \tau, \alpha)$. Table 3 shows the results of the estimation procedure. The process was stopped when the value of relative likelihood was less than 0.001 . The final estimates, along with their standard deviations (in parentheses) were: $\hat{\phi}=0.8127$ (0.0027854), $\hat{\tau}=4.2368$ (0.020652), $\hat{\alpha}=-4.7144$ (0.0083309). Results show that the GED model gives good estimates even though the true observation noise is not a normal mixture distribution.

Table 4 shows results of the parameter estimation procedure on technique based on the normal mixture SV and GED on data generated from the GED SV model. The method based on the GED model works well in both cases. When the estimation procedure based on the normal mixture SV model was applied, the estimates were far from the true parameters. By contrast, the application of the technique based on GED model indicated a better proximity to the true parameters. $(0.9500,1.3288,0.6309)$ were used as initial parameters $(\phi, \tau, \alpha)$. The final estimates, along with their standard deviations were: $\hat{\phi}=0.9749 \quad(0.0026845), \quad \hat{\tau}=2.3496$ (0.2678), $\hat{\alpha}=0.6821 \quad(0.014247) . \quad$ These estimates are similar to the true parameters $(0.9$, $1.6,0.7)$ while the normal mixture returns $(0.7515,2.3496,1.3259732)$ as $(\phi, \tau, \alpha)$. Thus, the method based on the GED works well in both cases.

Table 1: Parameter Estimates and Standard Errors (in parenthesis) on Technique Based on Normal Mixture and Student-t on Data Generated from Normal Mixture Model

\begin{tabular}{|c|c|c|c|c|c|c|c|}
\hline & & $\begin{array}{c}\text { Normal } \\
\text { Mixture SV }\end{array}$ & Student-t SV & $\begin{array}{c}\text { Normal } \\
\text { Mixture SV }\end{array}$ & Student-t SV & $\begin{array}{c}\text { Normal } \\
\text { Mixture SV }\end{array}$ & Student-t SV \\
\hline & $\begin{array}{c}\text { True } \\
\text { Parameter }\end{array}$ & $M=500$ & $\mathcal{E}=0.1$ & $M=1000$ & $\mathcal{E}=0.01$ & $M=1000$ & $\varepsilon=0.001$ \\
\hline$\phi^{(i)}$ & 0.7 & $\begin{array}{c}0.7368 \\
(0.028438)\end{array}$ & $\begin{array}{c}0.6976 \\
(0.01475)\end{array}$ & $\begin{array}{c}0.8677 \\
(0.048671)\end{array}$ & $\begin{array}{c}0.7308 \\
(0.049383)\end{array}$ & $\begin{array}{c}0.7568 \\
(0.027826)\end{array}$ & $\begin{array}{c}0.6913 \\
(0.037981)\end{array}$ \\
\hline$\tau^{(i)}$ & 1.06 & $\begin{array}{c}0.9408 \\
(0.014796)\end{array}$ & $\begin{array}{c}1.3654 \\
(0.0029492)\end{array}$ & $\begin{array}{c}0.1186 \\
(0.0059242)\end{array}$ & $\begin{array}{c}0.7625 \\
(0.20677)\end{array}$ & $\begin{array}{c}0.3466 \\
(0.00931)\end{array}$ & $\begin{array}{c}1.0336 \\
(0.14839)\end{array}$ \\
\hline$q_{0}^{(i)}$ & -3.5 & $\begin{array}{c}-0.9826 \\
(0.085676)\end{array}$ & & $\begin{array}{c}-0.8746 \\
(0.084237)\end{array}$ & & $\begin{array}{c}-1.0486 \\
(0.10989)\end{array}$ & \\
\hline$q_{1}{ }^{(i)}$ & -2 & $\begin{array}{c}-3.5081 \\
(0.037916) \\
\end{array}$ & & $\begin{array}{c}-3.4744 \\
(0.00879)\end{array}$ & & $\begin{array}{c}-3.7620 \\
(0.08690) \\
\end{array}$ & \\
\hline$R_{0}{ }^{(i)}$ & 4 & $\begin{array}{c}2.2144 \\
(0.38686)\end{array}$ & & $\begin{array}{c}1.8883 \\
(0.27135) \\
\end{array}$ & & $\begin{array}{c}2.3169 \\
(0.20936)\end{array}$ & \\
\hline$R_{1}^{(i)}$ & 6 & $\begin{array}{c}7.5758 \\
(1.0062)\end{array}$ & & $\begin{array}{c}7.9490 \\
(0.59001)\end{array}$ & & $\begin{array}{c}7.5643 \\
(0.67241)\end{array}$ & \\
\hline$\pi^{(i)}$ & 0.5 & $\begin{array}{c}0.4330 \\
(0.017748)\end{array}$ & & $\begin{array}{c}0.4267 \\
(0.014455)\end{array}$ & & $\begin{array}{c}0.3854 \\
(0.02635)\end{array}$ & \\
\hline$\alpha^{(i)}$ & -2.75 & -2.0761 & $\begin{array}{c}-2.1465 \\
(0.0038743)\end{array}$ & -1.983935 & $\begin{array}{c}-1.6703 \\
(0.10626)\end{array}$ & -2.094344 & $\begin{array}{c}-2.9009 \\
(0.024501)\end{array}$ \\
\hline $\begin{array}{l}\text { Rel. } \\
\text { Lik }\end{array}$ & & 0.0865 & 0.0250 & 0.0025 & -0.0021 & -0.0004 & -0.000 \\
\hline
\end{tabular}


Table 2: Parameter Estimation on Technique Based on the Normal Mixture and Student-ton Data Generated from the Student-t Model

\begin{tabular}{|c|c|c|c|c|c|c|c|}
\hline & & $\begin{array}{c}\text { Normal } \\
\text { Mixture SV }\end{array}$ & Student-t SV & $\begin{array}{c}\text { Normal } \\
\text { Mixture SV }\end{array}$ & Student-t SV & $\begin{array}{c}\text { Normal } \\
\text { Mixture SV }\end{array}$ & Student-t SV \\
\hline & Parameter & $M=500$ & $\varepsilon=0.1$ & $M=1000$ & $\varepsilon=0.01$ & $M=1000$ & $\varepsilon=0.001$ \\
\hline$\phi^{(i)}$ & 0.81 & $\begin{array}{c}0.6388 \\
(0.021863)\end{array}$ & $\begin{array}{c}0.8439 \\
(0.005971)\end{array}$ & $\begin{array}{c}0.5601 \\
(0.052847)\end{array}$ & $\begin{array}{c}0.8693 \\
(0.002036)\end{array}$ & $\begin{array}{c}0.6547 \\
(0.005272)\end{array}$ & $\begin{array}{c}0.8383 \\
(0.008552)\end{array}$ \\
\hline$\tau^{(i)}$ & 1.45 & $\begin{array}{c}1.2585 \\
(0.029249)\end{array}$ & $\begin{array}{c}1.2696 \\
(0.0029492)\end{array}$ & $\begin{array}{c}1.5805 \\
(0.092768)\end{array}$ & $\begin{array}{c}1.4500 \\
(0.037812)\end{array}$ & $\begin{array}{c}1.2930 \\
(0.002473)\end{array}$ & $\begin{array}{c}1.5357 \\
(0.12403)\end{array}$ \\
\hline$q_{0}^{(i)}$ & & $\begin{array}{c}-2.8256 \\
(0.035245)\end{array}$ & & $\begin{array}{c}-2.8979 \\
(0.02815)\end{array}$ & & $\begin{array}{c}-3.0180 \\
(0.035241)\end{array}$ & \\
\hline$q_{1}^{(i)}$ & & $\begin{array}{c}-5.7964 \\
(0.011406)\end{array}$ & & $\begin{array}{c}-5.7544 \\
(0.002826)\end{array}$ & & $\begin{array}{c}-5.8536 \\
(0.012445)\end{array}$ & \\
\hline$R_{0}{ }^{(i)}$ & & $\begin{array}{c}3.8225 \\
(0.075907)\end{array}$ & & $\begin{array}{c}3.8111 \\
(0.057768)\end{array}$ & & $\begin{array}{c}3.3275 \\
(0.15)\end{array}$ & \\
\hline$R_{1}^{(i)}$ & & $\begin{array}{c}4.6134 \\
(0.14959)\end{array}$ & & $\begin{array}{c}4.2066 \\
(0.060831)\end{array}$ & & $\begin{array}{c}5.2663 \\
(0.32564) \\
\end{array}$ & \\
\hline$\pi^{(i)}$ & & $\begin{array}{c}0.4911 \\
(0.0025628)\end{array}$ & & $\begin{array}{c}0.4911 \\
(0.001607)\end{array}$ & & $\begin{array}{c}0.4806 \\
(0.004338)\end{array}$ & \\
\hline$\alpha^{(i)}$ & -3.01 & -4.28456 & $\begin{array}{c}-3.1243 \\
(0.000596)\end{array}$ & -4.300727 & $\begin{array}{c}-3.1645 \\
(0.005662) \\
\end{array}$ & -4.3808 & $\begin{array}{c}-3.0912 \\
(0.005302)\end{array}$ \\
\hline $\begin{array}{l}\text { Rel. } \\
\text { Lik }\end{array}$ & & 0.0643 & 0.0045 & 0.0057 & 0.0042 & 0.0009 & -0.0010 \\
\hline
\end{tabular}

Table 3: Parameter Estimation on Technique Based On the Normal Mixture SV and GED on Data Generated from the Normal Mixture Model

\begin{tabular}{|c|c|c|c|c|c|c|c|}
\hline & & $\begin{array}{c}\text { Normal } \\
\text { Mixture SV }\end{array}$ & GED SV & $\begin{array}{c}\text { Normal } \\
\text { Mixture SV }\end{array}$ & GED SV & $\begin{array}{c}\text { Normal } \\
\text { Mixture SV }\end{array}$ & GED SV \\
\hline & $\begin{array}{l}\text { Irue } \\
\text { Parameter }\end{array}$ & $M=500$ & $\varepsilon=0.1$ & $M=1000$ & & $M=1000$ & \\
\hline$\phi^{(i)}$ & 0.8 & $\begin{array}{c}0.7875 \\
(0.0045855)\end{array}$ & $\begin{array}{c}0.8485 \\
(0.0059856)\end{array}$ & $\begin{array}{c}0.8425 \\
(0.0046651)\end{array}$ & $\begin{array}{c}0.8788 \\
(0.0037027)\end{array}$ & $\begin{array}{c}0.9869 \\
(0.0013341)\end{array}$ & $\begin{array}{c}0.8127 \\
(0.0027854)\end{array}$ \\
\hline$\tau^{(i)}$ & 3.5 & $\begin{array}{c}3.4771 \\
(0.17068) \\
\end{array}$ & $\begin{array}{c}4.0273 \\
(0.13077) \\
\end{array}$ & $\begin{array}{c}2.9591 \\
(0.17631) \\
\end{array}$ & $\begin{array}{c}3.0502 \\
(0.052579)\end{array}$ & $\begin{array}{c}4.1936 \\
(0.51468) \\
\end{array}$ & $\begin{array}{c}4.2368 \\
(0.020652)\end{array}$ \\
\hline$q_{0}^{(i)}$ & -5 & $\begin{array}{c}-5.5322 \\
(0.0059081)\end{array}$ & & $\begin{array}{c}-4.8080 \\
(0.026411)\end{array}$ & & $\begin{array}{c}-4.3900 \\
(0.021891)\end{array}$ & \\
\hline$q_{1}^{(i)}$ & -8 & $\begin{array}{c}-8.5269 \\
(0.00932) \\
\end{array}$ & & $\begin{array}{c}\text { v7.9658 } \\
(0.032108)\end{array}$ & & $\begin{array}{c}-6.4554 \\
(0.026309)\end{array}$ & \\
\hline$R_{0}{ }^{(i)}$ & 3 & $\begin{array}{c}3.6937 \\
(0.10267)\end{array}$ & & $\begin{array}{c}3.7054 \\
(0.081339)\end{array}$ & & $\begin{array}{c}3.7342 \\
(0.069579)\end{array}$ & \\
\hline$R_{1}^{(i)}$ & 4.1 & $\begin{array}{c}4.4847 \\
(0.16612)\end{array}$ & & $\begin{array}{c}4.1121 \\
(0.057281)\end{array}$ & & $\begin{array}{c}4.3517 \\
(0.16268)\end{array}$ & \\
\hline$\pi^{(i)}$ & 0.5 & $\begin{array}{c}0.4895 \\
(0.003056)\end{array}$ & & $\begin{array}{c}0.4930 \\
(0.0025453)\end{array}$ & & $\begin{array}{c}0.4805 \\
(0.0032833)\end{array}$ & \\
\hline$\alpha^{(i)}$ & -5.55 & -6.9980 & $\begin{array}{c}-4.3205 \\
(0.035379)\end{array}$ & -6.3648 & $\begin{array}{c}-4.2576 \\
(0.003807)\end{array}$ & -5.3824 & $\begin{array}{c}-4.7144 \\
(0.0083309)\end{array}$ \\
\hline $\begin{array}{l}\text { Rel. } \\
\text { Lik }\end{array}$ & & 0.0248 & 0.0853 & -0.0148 & 0.0064 & -0.0684 & 0.0007 \\
\hline
\end{tabular}




\section{PARAMETER ESTIMATION OF A HIDDEN MARKOV MODEL WITH DIAGNOSTICS}

Table 4: Parameter Estimation on Technique Based On the Normal Mixture SV and GED on Data Generated from the GED Model

\begin{tabular}{|c|c|c|c|c|c|c|c|}
\hline & & $\begin{array}{c}\text { Normal } \\
\text { Mixture SV }\end{array}$ & GED SV & $\begin{array}{c}\text { Normal } \\
\text { Mixture SV }\end{array}$ & GED SV & $\begin{array}{c}\text { Normal } \\
\text { Mixture SV }\end{array}$ & GED SV \\
\hline & $\begin{array}{c}\text { True } \\
\text { Parameter }\end{array}$ & $M=500$ & $\mathcal{E}=0.1$ & $M=1000$ & & $M=1000$ & \\
\hline$\phi^{(i)}$ & 0.9 & $\begin{array}{c}0.9050 \\
(0.015127)\end{array}$ & $\begin{array}{c}0.9770 \\
(0.0021463)\end{array}$ & $\begin{array}{c}0.8871 \\
(0.026021)\end{array}$ & $\begin{array}{c}0.9754 \\
(0.0025267)\end{array}$ & $\begin{array}{c}0.7515 \\
(0.015215)\end{array}$ & $\begin{array}{c}0.9749 \\
(0.0026845)\end{array}$ \\
\hline$\tau^{(i)}$ & 1.6 & $\begin{array}{c}0.3136 \\
(0.01557)\end{array}$ & $\begin{array}{c}2.1311 \\
(0.2265)\end{array}$ & $\begin{array}{c}0.4605 \\
(0.025703)\end{array}$ & $\begin{array}{c}2.3108 \\
(0.26119)\end{array}$ & $\begin{array}{c}1.2777 \\
(0.069801)\end{array}$ & $\begin{array}{c}2.3496 \\
(0.2678)\end{array}$ \\
\hline$q_{0}{ }^{(i)}$ & & $\begin{array}{c}0.1491 \\
(0.08715)\end{array}$ & & $\begin{array}{c}-0.3568 \\
(0.11521)\end{array}$ & & $\begin{array}{c}-0.3193 \\
(0.088286)\end{array}$ & \\
\hline$q_{1}^{(i)}$ & & $\begin{array}{l}-2.4081 \\
(0.1269)\end{array}$ & & $\begin{array}{c}-2.6609 \\
(0.13263)\end{array}$ & & $\begin{array}{c}-3.1988 \\
(0.15449)\end{array}$ & \\
\hline$R_{0}{ }^{(i)}$ & & $\begin{array}{c}2.9473 \\
(0.2282)\end{array}$ & & $\begin{array}{c}2.5108 \\
(0.28846)\end{array}$ & & $\begin{array}{c}1.6365 \\
(0.47054)\end{array}$ & \\
\hline$R_{1}^{(i)}$ & & $\begin{array}{c}7.8467 \\
(0.84311)\end{array}$ & & $\begin{array}{c}9.4387 \\
(1.1097)\end{array}$ & & $\begin{array}{c}8.7981 \\
(1.1994)\end{array}$ & \\
\hline$\pi^{(i)}$ & & $\begin{array}{c}0.4448 \\
(0.015076)\end{array}$ & & $\begin{array}{c}0.3916 \\
(0.028448)\end{array}$ & & $\begin{array}{c}0.3496 \\
(0.041351)\end{array}$ & \\
\hline$\alpha^{(i)}$ & 0.7 & -0.9883 & $\begin{array}{c}0.7654 \\
(0.018455)\end{array}$ & 1.2590 & $\begin{array}{c}0.7627 \\
(0.021224)\end{array}$ & -1.3259732 & $\begin{array}{c}0.6821 \\
(0.014247)\end{array}$ \\
\hline $\begin{array}{l}\text { Rel. } \\
\text { Lik }\end{array}$ & & 0.0052 & 0.0248 & -0.0125 & 0.0057 & 0.0003 & 0.0007 \\
\hline
\end{tabular}

Application to Real Life Financial Data

The normal mixture, Student-t and GED SV model were applied to analyze daily rates on the Naira/Dollar, Cedi/Dollar, Pound/Dollar and Euro/Dollar exchange rates from March 3, 2009 to March 3, 2011. Figures 5-8 show the plots of the daily exchange rates and log returns of the data.Patterns of behavior are evident in the second plots in Figures 5-8: the data experience a small variance for some periods of time, and for other periods they show a large variance. For this reason, it cannot be assumed that the data have a constant variance.

Table 6 presents the estimation results along with their standard deviations for the Student- $t$, normal mixture and the GED SV models. These distributions produce comparable maximum likelihood values, indicating an acceptable overall fit. The values (ranging from 0.927 to 0.988 ) suggest high persistence of the volatility of the series indicatingthat volatility clustering is observed in all the exchange rates return series.
The Akaike values and the evaluation statistics using the all data are shown in Table 7. The AIC and the log-likelihood values highlight the fact that (GED) Student-t distribution better estimates the series than the normal mixture distribution for the SV model. In fact, the loglikelihood function increases, leading to AIC criteria of 2.805, 3.4593, 3.9989 and 9.6632 with the normal mixture versus (2.7814304) 2.776433, (3.4223827) 3.391374, (3.9749741) 3.969968 and (9.6513786) 9.646376 with the non-normal densities, for the Naira/Dollar, Cedi/Dollar, Pound/Dollar and Euro/Dollar rate respectively. The statistics from the volatility forecasts (Sadorsky, 2005) are presented. In terms of MSE, the Student-t performs better than the normal mixture for the Naira/Dollars and the Euro/Dollar exchange rate while the opposite is true for the Cedi/Dollars and Pound/Dollar exchange rate. Generally, the MAE results are not different from the MSE results. In terms of MAPE, the Student-t SV model is preferred in three cases and the GED SV model once. 
NKEMNOLE, ABASS \& KASUMU

Table 5: Descriptive Statistics of Daily Returns for the Exchange Rate

\begin{tabular}{|c|c|c|c|c|}
\hline Statistics & Naira/Dollar Rate & Cedi/Dollar & Pound/Dollar & Euro/Dollar \\
\hline Mean & -0.001385 & -0.006258 & 0.020803 & 0.001676 \\
\hline $\begin{array}{c}\text { Standard } \\
\text { Deviation }\end{array}$ & 0.708650 & 0.536507 & 0.506541 & 0.488392 \\
\hline Skewness & -0.074139 & 0.966923 & 0.022958 & 0.434943 \\
\hline Kurtosis & 8.805879 & 13.11769 & 4.262290 & 7.993814 \\
\hline Jarque-Bera & 735.0376 & 2312.255 & 34.76827 & 559.9343 \\
\hline
\end{tabular}

Figure5: Naira/Dollar Daily Exchange Rate and Log Returns
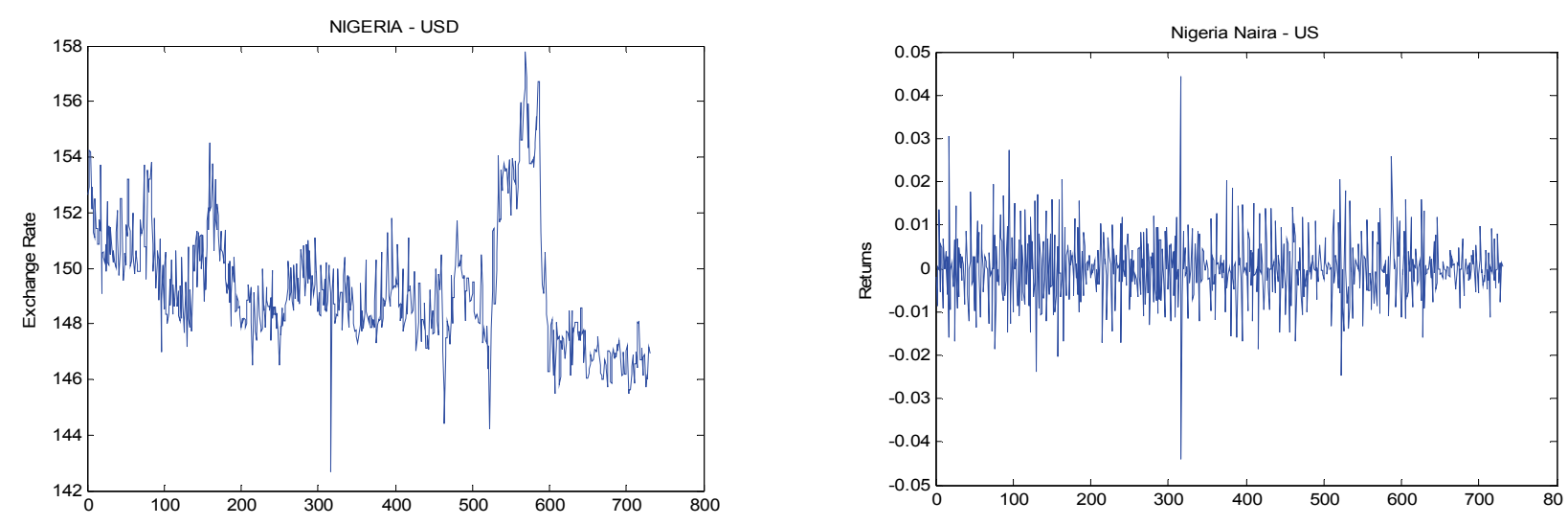

Figure 6: Cedi/Dollar Exchange Rate and Log Returns
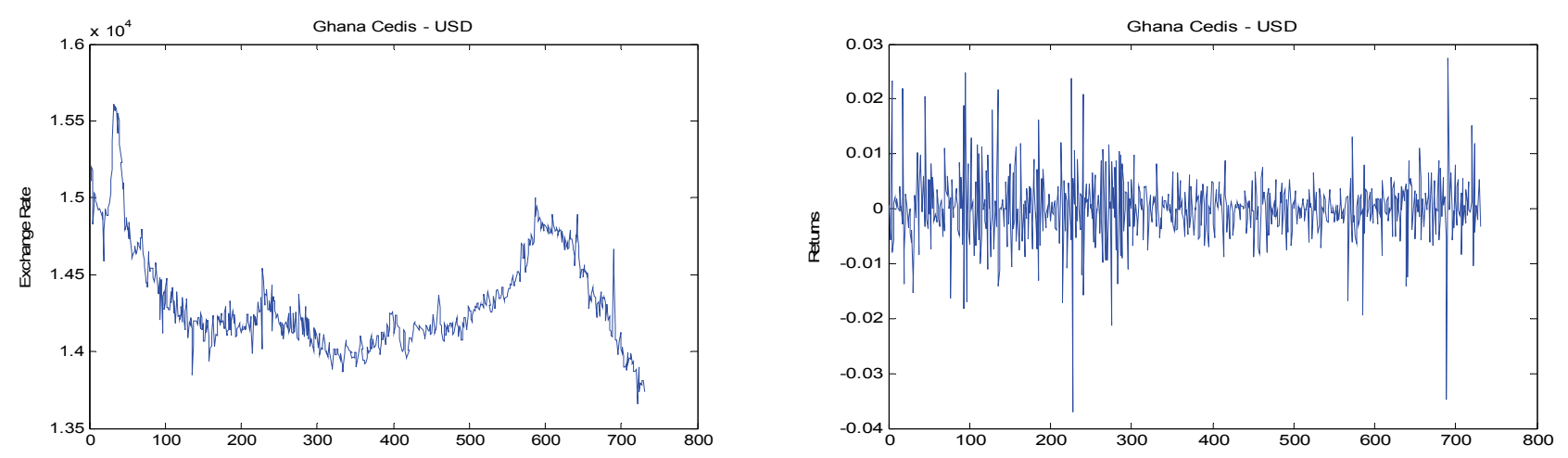


\section{PARAMETER ESTIMATION OF A HIDDEN MARKOV MODEL WITH DIAGNOSTICS}

Figure 7: Euro/Dollar Daily Exchange Rate and Log Returns
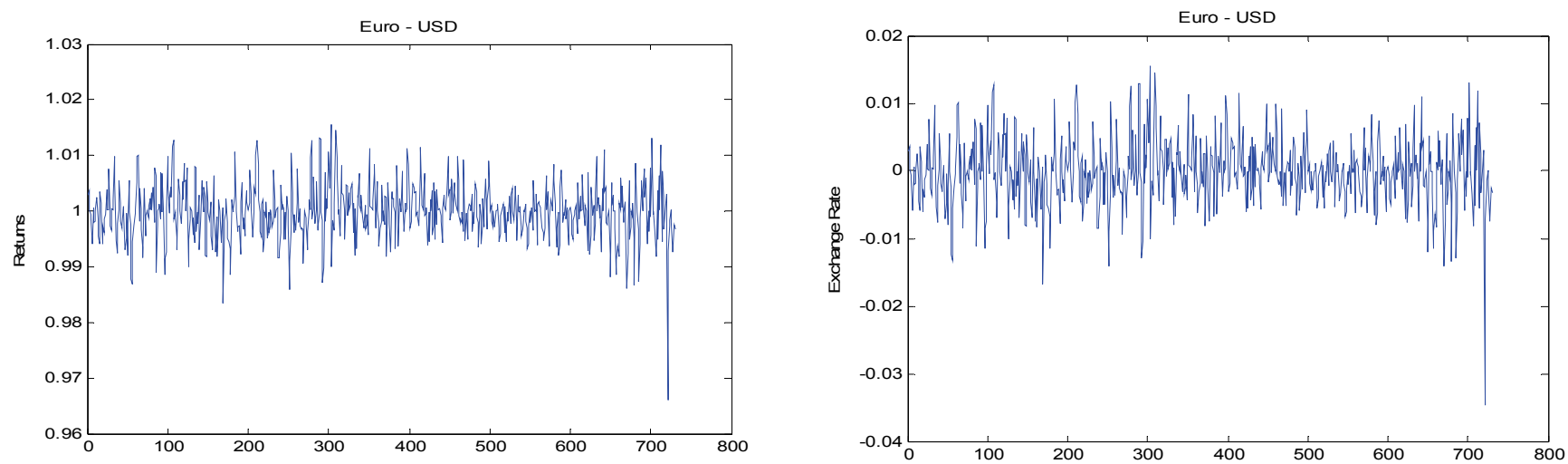

Figure 8: Pound/Dollar Daily Exchange Rate and Log Returns
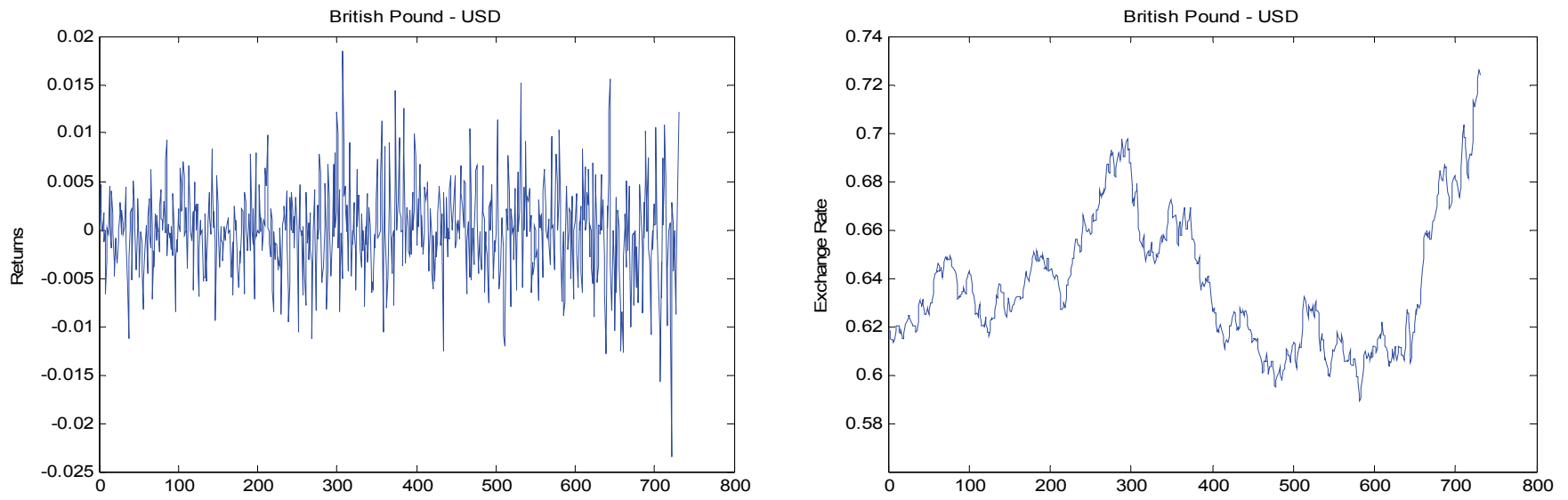

\section{Conclusion}

An extension of the observation error in the SV model from normal mixture to Student-t and GED distributions was presented. A sequential Monte-Carlo expectation maximization experiment was used to estimate parameters for the extended SV model. Functions provided by MATLAB enabled techniques based on the Student-t and GED SV model to be developed along with a strategy for fitting a model that combines the EM algorithm and SMC; this change to the proposed model allowed for a more robust fit, providing a new tool to explore the tail fit. The Student-t and GED SV model was compared with the normal mixture. The EM algorithm makes it possible to obtain maximum likelihood estimators. The estimation
Algorithm was completed by applying the Godsill, et al. (2004) particle smoothing algorithm to the SV model with (3) and (5) as the observation and state equations. The outcome of the simulation and real data analyses confirm the viability of the proposed method. Results show that the proposed estimation algorithm yields acceptable results when the normal assumption is violated as well as when it holds, thus widening the range of application of the SV model.Statistics were calculated to compare the fit of distributions. Results, based on data from the Naira/Dollar, Cedi/Dollar, Pound/Dollar and Euro/Dollar exchange rates, reveal that the Student-t is comparable to the normal mixture SV model but is empirically more successful. 
NKEMNOLE, ABASS \& KASUMU

Table 6a: Estimation Results - Distribution Comparison

\begin{tabular}{|c|c|c|c|c|c|c|}
\hline & \multicolumn{3}{|c|}{ Naira/Dollar } & \multicolumn{3}{|c|}{ Cedi/Dollar } \\
\hline & $\begin{array}{c}\text { Normal } \\
\text { Mixture SV } \\
\text { (SD) }\end{array}$ & $\begin{array}{l}\text { Student-t SV } \\
\text { (SD) }\end{array}$ & $\begin{array}{l}\text { GED SV } \\
\text { (SD) }\end{array}$ & $\begin{array}{c}\text { Normal } \\
\text { Mixture SV } \\
\text { (SD) }\end{array}$ & $\begin{array}{l}\text { Student-t SV } \\
\text { (SD) }\end{array}$ & $\begin{array}{l}\text { GED SV } \\
\text { (SD) }\end{array}$ \\
\hline$\phi^{(i)}$ & $\begin{array}{c}0.9759 \\
(0.0041766)\end{array}$ & $\begin{array}{c}0.9769 \\
(0.0029595)\end{array}$ & $\begin{array}{c}0.9684 \\
(0.015815)\end{array}$ & $\begin{array}{c}0.983 \\
(0.0049939)\end{array}$ & $\begin{array}{c}0.9887 \\
(0.00078342)\end{array}$ & $\begin{array}{c}0.9741 \\
(0.01001)\end{array}$ \\
\hline$\tau^{(i)}$ & $\begin{array}{c}0.0988 \\
(0.019774)\end{array}$ & $\begin{array}{c}0.1300 \\
(0.16604)\end{array}$ & $\begin{array}{c}0.3227 \\
(0.030638)\end{array}$ & $\begin{array}{c}0.0902 \\
(0.033484)\end{array}$ & $\begin{array}{c}0.0854 \\
(0.0057923)\end{array}$ & $\begin{array}{c}0.09831 \\
(0.048961)\end{array}$ \\
\hline$q_{0}{ }^{(i)}$ & $\begin{array}{c}3.0673 \\
(0.043267) \\
\end{array}$ & & & $\begin{array}{c}3.4686 \\
(0.015039) \\
\end{array}$ & & \\
\hline$q_{1}^{(i)}$ & $\begin{array}{c}1.6633 \\
(0.65851) \\
\end{array}$ & & & $\begin{array}{c}0.9826 \\
(0.22583)\end{array}$ & & \\
\hline$R_{0}{ }^{(i)}$ & $\begin{array}{c}0.6954 \\
(0.11387)\end{array}$ & & & $\begin{array}{c}0.1865 \\
(0.015231)\end{array}$ & & \\
\hline$R_{1}^{(i)}$ & $\begin{array}{c}1.9713 \\
(0.88689) \\
\end{array}$ & & & $\begin{array}{c}2.3618 \\
(0.10685) \\
\end{array}$ & & \\
\hline$\pi^{(i)}$ & $\begin{array}{c}0.0546 \\
(0.017691)\end{array}$ & & & $\begin{array}{c}0.0705 \\
(0.006188)\end{array}$ & & \\
\hline \multirow[t]{3}{*}{$\alpha^{(i)}$} & -2.0731 & $\begin{array}{l}-2.1245 \\
(0.5369) \\
\end{array}$ & $\begin{array}{c}-2.8836 \\
(0.35001) \\
\end{array}$ & -4.28356 & $\begin{array}{c}-3.2243 \\
(0.0019277) \\
\end{array}$ & $\begin{array}{c}-4.9834 \\
(0.0022972) \\
\end{array}$ \\
\hline & \multicolumn{3}{|c|}{ Pound/Dollar } & \multicolumn{3}{|c|}{ Euro/Dollar } \\
\hline & $\begin{array}{c}\text { Normal } \\
\text { Mixture SV } \\
\text { (SD) }\end{array}$ & $\begin{array}{l}\text { Student-t SV } \\
\text { (SD) }\end{array}$ & $\begin{array}{l}\text { GED SV } \\
\text { (SD) }\end{array}$ & $\begin{array}{c}\text { Normal } \\
\text { Mixture SV } \\
\text { (SD) }\end{array}$ & $\begin{array}{l}\text { Student-t SV } \\
\text { (SD) }\end{array}$ & $\begin{array}{l}\text { GED SV } \\
\text { (SD) }\end{array}$ \\
\hline$\phi^{(i)}$ & $\begin{array}{c}0.9895 \\
(0.0013354) \\
\end{array}$ & $\begin{array}{c}0.9754 \\
(0.0025267) \\
\end{array}$ & $\begin{array}{c}0.9697 \\
(0.0084966) \\
\end{array}$ & $\begin{array}{c}0.9579 \\
(0.0090241) \\
\end{array}$ & $\begin{array}{c}0.9113 \\
(0.022502)\end{array}$ & $\begin{array}{c}0.9763 \\
(0.0021063) \\
\end{array}$ \\
\hline$\tau^{(i)}$ & $\begin{array}{c}0.7114 \\
(0.36943) \\
\end{array}$ & $\begin{array}{c}0.7627 \\
(0.26119) \\
\end{array}$ & $\begin{array}{c}0.9018 \\
(0.183815) \\
\end{array}$ & $\begin{array}{c}0.4170 \\
(0.24553) \\
\end{array}$ & $\begin{array}{c}0.4731 \\
(0.45638)\end{array}$ & $\begin{array}{c}0.7258 \\
(0.361172) \\
\end{array}$ \\
\hline$q_{0}{ }^{(i)}$ & $\begin{array}{c}3.3388 \\
(0.022015)\end{array}$ & & & $\begin{array}{c}-1.9638 \\
(0.21412)\end{array}$ & & \\
\hline$q_{1}^{(i)}$ & $\begin{array}{c}0.4351 \\
(0.023938) \\
\end{array}$ & & & $\begin{array}{c}-4.3710 \\
(0.54791)\end{array}$ & & \\
\hline$R_{0}{ }^{(i)}$ & $\begin{array}{c}0.7835 \\
(0.056033)\end{array}$ & & & $\begin{array}{c}4.1677 \\
(1.1257)\end{array}$ & & \\
\hline$R_{1}^{(i)}$ & $\begin{array}{c}4.4129 \\
(0.14603) \\
\end{array}$ & & & $\begin{array}{c}8.1096 \\
(0.4471) \\
\end{array}$ & & \\
\hline$\pi^{(i)}$ & $\begin{array}{c}0.4941 \\
(0.0028666) \\
\end{array}$ & & & $\begin{array}{c}0.4404 \\
(0.03083) \\
\end{array}$ & & \\
\hline$\alpha^{(i)}$ & -4.28356 & $\begin{array}{c}2.3108 \\
(0.021224)\end{array}$ & $\begin{array}{c}1.9588 \\
0.0082487 \\
\end{array}$ & -4.28356 & $\begin{array}{c}-2.2692 \\
(0.17681)\end{array}$ & $\begin{array}{c}-3.5033 \\
(0.058033)\end{array}$ \\
\hline
\end{tabular}




\section{PARAMETER ESTIMATION OF A HIDDEN MARKOV MODEL WITH DIAGNOSTICS}

Table 7: Evaluation Statistics - Distribution Comparison

\begin{tabular}{|c|c|c|c|c|c|}
\hline & & & & & \\
\hline & AIC & Log-like & MSE & MAE & MAPE \\
\hline \multicolumn{6}{|l|}{ Naira/Dollar } \\
\hline Normal Mixture SV & 2.805 & 2537.403 & 0.2601914 & 0.210278 & 0.133428 \\
\hline Student-t SV & 2.776433 & 2565.97 & 0.1111914 & 0.075379 & 0.053437 \\
\hline GED SV & 2.7814304 & 2563.16 & 0.1131914 & 0.081256 & 0.064035 \\
\hline \multicolumn{6}{|l|}{ Cedi/Dollar } \\
\hline Normal Mixture SV & 3.4593 & 2652.836 & 0.037074 & 0.059524 & 0.147437 \\
\hline Student-t SV & 3.391374 & 2769.66 & 0.121086 & 0.171621 & 0.126563 \\
\hline GED SV & 3.4223827 & 2760.56 & 0.16377 & 0.188346 & 0.131424 \\
\hline \multicolumn{6}{|l|}{ Pound/Dollar } \\
\hline Normal Mixture SV & 3.9989 & 2801.144 & 0.151623 & 0.072823 & 1.192232 \\
\hline Student-t SV & 3.969968 & 2869.07 & 0.178377 & 0.098361 & 0.045245 \\
\hline GED SV & 3.9749741 & 2857.27 & 0.184677 & 0.103932 & 0.034675 \\
\hline \multicolumn{6}{|l|}{ Euro/Dollar } \\
\hline Normal Mixture SV & 9.6632 & 2874.968 & 0.170612 & 0.260176 & 0.0988 \\
\hline Student-t SV & 9.646376 & 2903.9 & 0.095612 & 0.194364 & 0.0578 \\
\hline GED SV & 9.6513786 & 2897.8 & 0.101414 & 0.196355 & 0.0634 \\
\hline
\end{tabular}

References

Andersen, T. G., Bollerslev, T., \& Diebold, F. X. (2005). Parametric and nonparametric financial econometrics. Amsterdam: North-Holland.

Arulampalam, M. S., Maskell, S., Gordon, N., \& Clapp, T. (2002). A tutorial on particle filter for on-line non-linear/nonGaussian Bayesian tracking. IEEE Transaction on Signal Processing, 50, 174-188.

Bai, X., Russell, J. R., \& Tiao, G. C. (2003). Kurtosis of GARCH and Stochastic Volatility Models with Non-normal Innovations. Journal of Econometrics, 114, 349-360.

Bao, Y., Tae-Hwy, L., \& Burak, S. (2006). Comparing density forecast models. Journal of Forecasting, 26(3), 203-225.

Baum, L. E., Peterie, T., Souled, G., \& Weiss, N. (1970). A maximization technique occurring in the statistical analysis of probabilistic functions of Markov chains. Annals of Mathematical Statistics, 41(1), 164-171.

Cappe, O., Moulines, E., \& Ryden, T. (2005). Inference in hidden Markov models. New York, NY: Springer.
Chib, S., Nardari, F., \& Shephard, N. (2002). Markov chain Monte Carlo methods for stochastic volatility models. Journal of Econometrics, 108, 281-316.

Danielsson, J., \& Richard, J. F. (1993). Accelerated Gaussian importance sampler with application to dynamic latent variable models. Journal of Applied Econometrics, 8, 153-173.

Danielson, J. (1994). Stochastic volatility in asset prices: Estimation with simulated maximum likelihood.Journal of Econometrics, 61, 375-400.

Dempster, A. P., Laird, N. M., \& Rubin, D. B. (1977). Maximum likelihood from incomplete data via the EM algorithm. Journal of the Royal Statistical Society, 39(1), 1-38.

Doucet, A., De Freitas, J. F. G., \& Gordon, N. (2001). Sequential Monte Carlo methods in practice. New York, NY: SpringerVerlag.

Doucet, A., \& Tadic, B. B. (2003). Parameter estimation in general state-space models using particle methods. Annals of Institute of Statistical Mathematics, 55, 409-422. 
Doucet A., \& Johansen, A. M. (2009). A tutorial on particle filtering and smoothing: Fifteen years later. In Oxford handbook of nonlinear giltering, D. Crisan \& B. Rozovsky (Eds.), 231-260. Oxford: Oxford University Press.

Durbin, J., \& Koopman, S. J. (2000). Time series analysis of non-Gaussian observations based on state space models from both classical and Bayesian perspectives. Journal of the Royal Statistical Society, 62, 356.

Fernandez, C., \& Steel, M. (1998). On Bayesian modelling of fat tails and skewness. Journal of the American Statistical Association, 93, 359-371.

Gallant, A. R., Hsieh, D., \& Tauchen, G. (1995). Estimation of stochastic volatility model with diagnostics. Journal of Econometrics, 81, 159-192.

Godsill, S., Doucet, A., \& West, M. (2004). Monte Carlo smoothing for non-linear time series. Journal of the American Statistical Association, 199, 156-168.

Harvey, A. C., Ruiz, E., \& Shephard, N. (1994). Multivariate stochastic variance models. Review of Economic Studies, 61, 247-264.

Jacquier, E., Polson, N. G., \& Rossi, P. E. (1994). Bayesian analysis of stochastic volatility models. Journal of Business and Economic Statistics, 12(4), 371-417.

MacDonald, I. L., \& Zucchini, W. (1997). Hidden Markov and other models for discrete-valued time series, Vol. 70: Monographs on statistics and applied probability. London: Chapman \& Hall.

Kim, S., Shephard, N., \& Chib, S. (1998). Stochastic volatility: Likelihood inference and comparison with ARCH models. The review of economic studies, 65(3), 361-393.

Kim, J., \& Stoffer, D. S. (2008). Fitting stochastic volatility models in the presence of irregular sampling via particle methods and the EM algorithm. Journal of Time Series Analysis, 29(5), 811-833.

Kitagawa, G. (1996). Monte Carlo filter and smoother for non-Gaussian nonlinear state space models. Journal of Computational and Graphical Statistics, 5, 1-25.
Kitagawa, G., \& Sato, S. (2001). Monte Carlo smoothing and self-organising state space model. In Sequential Monte Carlo methods in practice, A. Doucet, N. de Freitas \& N. Gordon, Eds.,177-195. New York, NY: Springer-Verlag.

Liesenfeld, R., \& Jung, R. C. (2000). Stochastic volatility models: conditional normality versus heavy-tailed distributions. Journal of Applied Econometrics, 15, 137-160.

MacDonald, I. L., \& Zucchini, W. (1997). Hidden Markov and other models for discrete-valued time series, Vol. 70: Monographs on statistics and applied probability. London: Chapman and Hall.

Mellino, A., \& Turnbull, S. (1990). Pricing foreign currency options with stochastic volatility. Journal of Econometrics, 45, 7-39.

Rabiner, L. R. (1989). A tutorial on hidden Markov Models and selected applications in speech recognition. Proceedings of the IEEE, 77(2), 257-286.

Ruiz, E. (1994). Quasi-maximum likelihood estimation of stochastic volatility models. Journal of Econometrics, 63, 289-306.

Sadorsky, P. (2005). Stochastic volatility forecasting and risk management. Applied Financial Economics, 15, 121-135.

Shephard, N. (1996). Statistical aspects of ARCH and stochastic volatility. In Time series models in econometrics, finance and other fields, D. R. Cox, D. V. Hinkley \& O. E. Barndorff-Nielsen (Eds.), 1-67. London: Chapman and Hall.

Shephard, N., \& Pitt, M. K. (1997). Likelihood analysis of non-Gaussian measurement time series. Biometrika, 84, 653667.

Shimada, J., \& Tsukuda, Y. (2005). Estimation of stochastic volatility models: Anapproximation to the nonlinear state space representation. Communications in Statistics Simulation and Computation, 34, 429-450.

Shumway, R. H., \& Stoffer, D. S. (2006). Time series analysis and its applications. New York, NY: Springer.

Taylor, S. J. (1982). Financial returns modelled by the product of two stochastic processes: A study of daily sugar prices, 19611979. In Time series analysis: Theory and practice, O. D. Anderson (Ed.), 203-226. New York, NY: Elsevier Science Publishing Co. 


\section{Estimation and Testing in Type I Generalized Half Logistic Distribution}

\author{
R. R. L. Kantam \\ AcharyaNagarjuna University, \\ Nagarjunanagar, India
}

\author{
V. Ramakrishna \\ K. L. University, \\ Vaddeswaram, India
}

\author{
M. S. Ravikumar \\ AcharyaNagarjuna University, \\ Nagarjunanagar, India
}

A generalization of the half logistic distribution is developed through exponentiation of its cumulative distribution function and termed the Type I Generalized Half Logistic Distribution (GHLD). GHLD's distributional characteristics and parameter estimation using maximum likelihood and modified maximum likelihood methods are presented with comparisons. Comparison of Type I GHLD and the exponential distribution is conducted via likelihood ratio criterion.

Key words: Generalized half logistic distribution, ML estimation, modified ML estimation, testing, likelihood ratio criterion.

\section{Introduction}

In life testing and reliability studies a combination of monotone and constant failure rates over various segments of the range of lifetime of a random variable is known as a bathtub or a non-monotone failure rate. In the biological and engineering sciences there are situations of non-monotone failure rates available to model such data; a comprehensive narration of the models is given in Rajarshi \& Rajarshi (1988). Mudholkar, et al. (1995) presented an extension of the Weibull family that contains unimodel distributions with bathtub failure rates and also allows for a broader class of monotone hazard rates; they named their extended version the Exponentiated Weibull Family. Gupta and Kundu (1999) also proposed a new model called generalized exponential distribution. If $\theta$ is a positive real number and $\mathrm{F}(\mathrm{x})$ is the cumulative distribution

R. R. L. Kantam is a Professor in the Department of Statistics. Email him at: kantam.rrl@gmail.com. V. Ramakrishna is an Associate Professor in the Department of Computer Science and Engineering. Email him at: vramakrishna2006@gmail.com. M. S. Ravikumar is a UGC Research Fellow in the Department of Statistics. Email him at: msrk.raama@gmail.com. function (cdf) of a continuous positive random variable, then $[\mathrm{F}(\mathrm{x})]^{\ominus}$ and the corresponding probability distribution may be termed exponentiated or generalized versions of $\mathrm{F}(\mathrm{x})$.This generalization is adapted to the half logistic distribution and the resulting model is considered in this study.

A half logistic model obtained as the distribution of an absolute standard logistic variate is a probability model of recent origin (Balakrishnan, 1985). Its standard probability density function (pdf), cdf and hazard functions are given by:

$$
\begin{gathered}
f(x)=\frac{2 e^{-x}}{\left(1+e^{-x}\right)^{2}}, \mathrm{x} \geq 0, \\
F(x)=\left[\frac{1-e^{-x}}{1+e^{-x}}\right], \mathrm{x} \geq 0, \\
h(x)=\frac{1}{1+e^{-x}}, \mathrm{x} \geq 0 .
\end{gathered}
$$

The pdf, cdf and hazard functions of the generalized half logistic distribution (GHLD-I) are given by

$$
f(x)=\frac{2 \theta e^{-x}\left(1-e^{-x}\right)^{\theta-1}}{\left(1+e^{-x}\right)^{\theta+1}}, \mathrm{x}>0, \theta>0,
$$




$$
\begin{gathered}
F(x)=\left[\frac{1-e^{-x}}{1+e^{-x}}\right]^{\theta}, \\
\mathrm{x}>0, \theta>0, \\
h(x)=\frac{2 \theta e^{-x}\left(1-e^{-x}\right)^{\theta-1}}{\left(1+e^{-x}\right)\left[\left(1+e^{-x}\right)^{\theta}-\left(1-e^{-x}\right)^{\theta}\right]}, \\
\mathrm{x}>0, \theta>0
\end{gathered}
$$

Balakrishnan and Sandhu (1995) have suggested a new probability model with a standard pdf and cdf given by the following equations

$$
\begin{gathered}
f(x)=\frac{2(1-k x)^{(1 / k)-1}}{\left[1+(1-k x)^{1 / k}\right]^{2}}, 0 \leq x \leq \frac{1}{k}, k \geq 0, \\
F(x)=\frac{1-(1-k x)^{1 / k}}{1+(1-k x)^{1 / k}}, 0 \leq x \leq \frac{1}{k}, k \geq 0 .
\end{gathered}
$$

The limits of (1.7) and (1.8) as $k \rightarrow \infty$ are respectively (1.1) and (1.2), the pdf and cdf of HLD; for this reason Balakrishnan and Sandhu (1995) called the distribution (1.7) and (1.8) a generalized HLD.

Olapade (2007, 2008) considered two distributions and discussed their distributional properties and order statistics in samples from these distributions; he termed these two distributions type-I and type-III GHLD respectively. The generalized HLD of Olapade $(2007,2008)$ are obtained via truncation of the type-I and type-III generalized logistic distributions of Balakrishnan and Leung (1988). Thus, the proposed type-I GHLD is conceptually different from the GHLDs of Balakrishnan and Sandhu (1995) and Olapade (2007, 2008).

Estimation

The pdf and distribution function of GHLD-I with scale parameter $\sigma$ and shape parameter $\theta$ are:

$$
\begin{aligned}
& f(x)=\frac{2 \theta\left(1-e^{-x / \sigma}\right)^{\theta-1} e^{-x / \sigma}}{\sigma\left(1+e^{-x / \sigma}\right)^{\theta+1}} \\
& 0<\mathrm{x}<\infty, \sigma>0, \theta>0
\end{aligned}
$$

and

$$
\begin{aligned}
& F(x)=\left[\frac{1-e^{-x / \sigma}}{1+e^{-x / \sigma}}\right]^{\theta} \\
& 0<\mathrm{x}<\infty, \sigma>0, \theta>0 .
\end{aligned}
$$

Let $\mathrm{x}_{1}<\mathrm{x}_{2}<\ldots<\mathrm{x}_{\mathrm{n}}$ be an ordered sample of size $n$ from GHLD-I (because the theory of order statistics is required in the estimation, an ordered sample is itself first considered). The $\log$ likelihood equations to estimate the parameters $\theta$ and $\sigma$ are given by

$$
\frac{\partial \log L}{\partial \sigma}=0
$$

and

$$
\frac{\partial \log L}{\partial \theta}=0
$$

where $\mathrm{L}$ is the likelihood function of the considered sample:

$$
\begin{aligned}
& \log L=\left[\begin{array}{l}
n \log \frac{2 \theta}{\sigma}+(\theta-1) \sum_{i=1}^{n} \log \left(1-e^{-x_{i} / \sigma}\right) \\
-(\theta+1) \sum_{i=1}^{n} \log \left(1+e^{-x_{i} / \sigma}\right)-\sum_{i=1}^{n} \frac{x_{i}}{\sigma}
\end{array}\right] \\
& \frac{\partial \log L}{\partial \sigma}=0 \Rightarrow\left[\begin{array}{l}
n-\sum_{i=1}^{n} z_{i}+(\theta-1) \sum_{i=1}^{n} \frac{z_{i} e^{-z_{i}}}{1-e^{-z_{i}}} \\
-(\theta+1) \sum_{i=1}^{n} \frac{z_{i} e^{-z_{i}}}{1+e^{-z_{i}}}
\end{array}\right]=0
\end{aligned}
$$

where $z_{i}=\frac{x_{i}}{\sigma}$

$$
\frac{\partial \log L}{\partial \theta}=0 \Rightarrow \hat{\theta}=\frac{n}{\sum_{i=1}^{n} \log \left(\frac{1+e^{-z_{i}}}{1-e^{-z_{i}}}\right)}
$$




\section{ESTIMATION AND TESTING IN TYPE I GHLD}

These two equations must be solved iteratively for $\theta$ and $\sigma$ given a sample. The asymptotic variances and covariance of MLEs of $\theta$ and $\sigma$ can be obtained by inverting the information matrix whose elements are the mathematical expectations of the following expressions.

$$
\begin{aligned}
& -\left(\frac{\partial^{2} \log L}{\partial \sigma^{2}}\right)= \\
& \sum_{i=1}^{n} \frac{2 z_{i}}{\sigma^{2}}+(\theta-1) \sum_{i=1}^{n} \frac{z_{i} e^{-2 z_{i}}+z_{i}^{2} e^{-2 z_{i}}-z_{i} e^{-z_{i}}}{\left(1-e^{-z_{i}}\right)^{2}} \\
& +(\theta+1) \sum_{i=1}^{n} \frac{\frac{z_{i}}{\sigma} e^{-z_{i}}+\frac{2 z_{i}^{2}}{\sigma} e^{-x_{i} / \sigma}-\frac{2 z_{i}}{\sigma^{2}} e^{-2 z_{i}}}{\left(1+e^{-z_{i}}\right)^{2}}-\frac{n}{\sigma^{2}} \\
& \quad-\left(\frac{\partial^{2} \log L}{\partial \theta^{2}}\right)=\frac{n}{\theta^{2}},(2.6)
\end{aligned}
$$

and

$$
-\left(\frac{\partial^{2} \log L}{\partial \sigma \partial \theta}\right)=\frac{1}{\sigma}\left[\sum_{i=1}^{n} \frac{z_{i} e^{-z_{i}}}{1-e^{-z_{i}}}+\sum_{i=1}^{n} \frac{z_{i} e^{-z_{i}}}{1+e^{-z_{i}}}\right] .
$$

Expressions (2.5), (2.6) and (2.7) evaluated at estimates of $\theta$, oresult in an estimated dispersion matrix. This process is iterative in nature; to obtain analytical estimators the estimating equations can be reasonably approximated by some admissible expressions. The solutions of resulting approximating equations are termed approximate MLEs or modified MLEs. Such modifications have been proposed and studied by many researchers in various models, including: Tiku (1967); Mehrotra \& Nanda (1974); Pearson \& Rootzen (1977); Tiku\& Suresh (1992); Rosaiah, et al. (1993a); Rosaiah, et al. (1993b); Kantam \& Srinivasa Rao (1993); Kantam \& Srinivasa Rao (2002); Kantam \& Sri Ram (2003. These works generally estimate the scale parameter of the model and the shape parameter (if any), is either assumed to be known or estimated by another method and the resulting estimator of the shape parameter is used to for modified ML estimation of the scale parameter. The former is the most usual situation and the latter is occasional. This study attempts to estimate $\sigma$ when $\theta$ is known. In order to obtain an analytical expression for $\sigma$, the expressions in Equation (2.3) are approximated by some linear function in the respective population quantiles:

$$
\begin{aligned}
& G\left(z_{i}\right)=\frac{Z_{i} e^{-z_{i}}}{1-e^{-z_{i}}} \cong \gamma_{i}+\delta_{i} z_{i}, \\
& K\left(z_{i}\right)=\frac{Z_{i} e^{-z_{i}}}{1+e^{-z_{i}}} \cong \alpha_{i}+\beta_{i} z_{i},
\end{aligned}
$$

where $\alpha_{\mathrm{i}}, \beta_{\mathrm{i}}, \gamma_{\mathrm{i}}$ and $\delta_{\mathrm{i}}$ are to be found. After using the approximations in (2.3) the solution for $\sigma$ is:

$$
\hat{\sigma}=\frac{\sum_{i=1}^{n} x_{i}-(\theta-1) \sum_{i=1}^{n} \delta_{i} x_{i}-(\theta+1) \sum_{i=1}^{n} \beta_{i} x_{i}}{n+(\theta-1) \sum_{i=1}^{n} \gamma_{i}+(\theta+1) \sum_{i=1}^{n} \alpha_{i}} .
$$

This estimator is named the MMLE of $\sigma$, which is a linear estimator in $\mathrm{x}_{\mathrm{i}}{ }^{\prime} \mathrm{s}$. Now consider a method to obtain $\alpha_{\mathrm{i}}, \beta_{\mathrm{i}}, \gamma_{\mathrm{I}}$ and $\delta_{\mathrm{i}}$.

$$
\text { Let } p_{i}=\frac{i}{n+1} ; \mathrm{i}=1,2, \ldots, \mathrm{n} \text {, and let }
$$

$t_{i}, t_{i}^{*}$ be the solutions of the following equations, for example:

$$
\begin{aligned}
& F\left(t_{i}\right)=p_{j}-\sqrt{\frac{p_{i} q_{i}}{n}}=p_{i}^{\prime}, \\
& F\left(t_{i}^{*}\right)=p_{i}+\sqrt{\frac{p_{i} q_{i}}{n}}=p_{i}^{\prime \prime},
\end{aligned}
$$

with $q_{i}=1-p_{i}$ and where F(.) is cdf of GHLDI.

The intercepts $\gamma_{\mathrm{i}}, \alpha_{\mathrm{i}}$ and slopes $\delta_{\mathrm{I}}$ and $\beta_{\mathrm{i}}$, of linear approximations (2.8) and (2.9) are respectively given by 


\section{KANTAM, RAMAKRISHNA \& RAVIKUMAR}

$$
\begin{gathered}
\delta_{i}=\frac{G\left(t_{i}^{*}\right)-G\left(t_{i}\right)}{t_{i}^{*}-t_{i}} ; \\
\beta_{i}=\frac{K\left(t_{i}^{*}\right)-K\left(t_{i}\right)}{t_{i}^{*}-t_{i}}, \\
\gamma=G\left(t_{i}^{*}\right)-\delta_{i} t_{i}^{*},
\end{gathered}
$$

and

$$
\alpha_{i}=K\left(t_{i}^{*}\right)-\beta_{i} t_{i}^{*},
$$

Using the cdf of GHLD-I, the expressions for $t_{i}, t_{i}^{*}$ are given by

$$
t_{i}=\log \left[\frac{1+\left(p_{i}^{\prime}\right)^{1 / \theta}}{1-\left(p_{i}^{\prime}\right)^{1 / \theta}}\right] ; t_{i}^{*}=\log \left[\frac{1+\left(p_{i}^{\prime \prime}\right)^{1 / \theta}}{1-\left(p_{i}^{\prime \prime}\right)^{1 / \theta}}\right] .
$$

The values of $\gamma_{i}, \alpha_{i}, \beta_{i}$ and $\delta_{i}$ for various $\theta$ and $n$ are shown in Tables $2.1 \mathrm{a}, 2.1 \mathrm{~b}$ and $2.1 \mathrm{c}$.

In this modified method, the basic principle is that certain expressions in the log likelihood equation are linearized in the neighborhood of the population quantile, which also depends on the size of the sample: The larger the sample, the closer the approximation, that is, the exactness of the approximation becomes finer for large values of $n$. Hence the approximate log likelihood equation and the exact log likelihood equation tend to each other as $\mathrm{n} \rightarrow \infty$. Thus, the exact and modified MLEs are asymptotically identical (Tiku, et al., 1986). The same may not be true in small samples. Due to non-availability of analytical sampling variances, the modified ML method was compared with the exact ML method via Monte Carlo simulation. The empirical bias, variance, mean square error (MSE) of the exact and modified MLEs for samples of sizes 5(5) 20 and $\theta=2$ (1) 4 are given in Table 2.2. The following observations are made based on results in Table 2.2: The MMLE over estimates the bias compared to the exact MLE for some sizes of samples and the exact MLE is slightly less biased than the MMLE. The empirical variance, MSE of MLE, MMLE corrected to third decimal place are nearly equal in most combinations of $n$ and $\theta$, indicating that exact MLE and MMLE are almost equally efficient in small samples.

\section{GHLD-I vs. Exponential Models}

The comparison between the GHLD-I and Exponential model is made with the help of the likelihood ratio (LR) criterion. GHLD-I is specified as the null population $\left(\mathrm{P}_{\mathrm{o}}\right)$ and the Exponential model as alternative population $\left(\mathrm{P}_{1}\right)$. The test hypotheses proposed are:

$$
\begin{gathered}
\mathrm{H}_{0} \text { :a given sample belongs to GHLD-I }\left(\mathrm{P}_{0}\right) \\
\text { vs. } \\
\mathrm{H}_{1} \text { : the sample belongs to the population } \\
\text { Exponential model }\left(\mathrm{P}_{1}\right) \text {. }
\end{gathered}
$$

Let $\mathrm{L}_{1}, \mathrm{~L}_{0}$ respectively represent the likelihood function of a sample with population $\mathrm{P}_{1}$ and $\mathrm{P}_{0}$. The LR criterion $\mathrm{L}_{1} / \mathrm{L}_{0}$ percentiles are obtained by simulation: 10,000 random samples sized $n=5,10,15,20$ are generated from the null population $\mathrm{P}_{0}$ and its parameters are estimated using each sample. The value of the likelihood function of the null population is computed at the generated sample observations and the corresponding parameter estimates; this value is denoted by $\mathrm{L}_{0}$.

Using the same sample generated from $\mathrm{P}_{0}$, the parameters and likelihood function value of the alternative population are calculated and called $\mathrm{L}_{1}$. The values of $\mathrm{L}_{1} / \mathrm{L}_{0}$ over 10,000 runs are sorted and selected percentiles are identified for a given $n$ and $\theta$ (see Table 3.1). The entries under the column headings 0.95 in Table 3.1 may be considered the $5 \%$ level of significance critical values for discriminating between the GHLD-I and Exponential models. The powers of the test statistic $\mathrm{L}_{1} / \mathrm{L}_{0}$ are also evaluated through simulation by calculating $\mathrm{L}_{1} / \mathrm{L}_{0}$ with samples generated from exponential population $\left(\mathrm{P}_{1}\right)$ and estimating, the parameters calculating the values of the likelihood functions $\mathrm{L}_{1}, \mathrm{~L}_{0}$ with sample from $\mathrm{P}_{1}$. The proportion of $\mathrm{L}_{1} / \mathrm{L}_{0}$ values falling above $95^{\text {th }}$ percentile of $\mathrm{L}_{1} / \mathrm{L}_{0}$ become power of the LR test criterion are shown in Table 3.2, which reveals the following: (1) The GHLD-I and Exponential model are indistinguishable with 0 power or negligible power of 0.02 for $n=$ 5; (2) as $n$ increases, the power increases specifically at $\theta=4$ and $n=20$, resulting in significant discrimination between two populations with respect to the LR criterion. 


\section{ESTIMATION AND TESTING IN TYPE I GHLD}

Table 2.1a: Intercept and Slope of the Approximations $\mathrm{G}\left(\mathrm{Z}_{\mathrm{i}}\right)=\gamma_{\mathrm{i}}+\delta_{\mathrm{i}} \mathrm{Z}_{\mathrm{i}}$ and $\mathrm{K}\left(\mathrm{Z}_{\mathrm{i}}\right)=\alpha_{\mathrm{i}}+\beta_{\mathrm{i}} \mathrm{Z}_{\mathrm{i}}$ when $\theta=2$

\begin{tabular}{|c|c|c|c|c|c|}
\hline$n$ & $\mathrm{i}$ & $\gamma_{i}$ & $\delta_{\mathrm{i}}$ & $\alpha_{\mathrm{i}}$ & $\beta_{\mathrm{i}}$ \\
\hline \multirow{5}{*}{5} & 1 & 0.0000 & 0.3660 & 0.0000 & 0.2113 \\
\hline & 2 & 0.8945 & -0.2953 & 0.2309 & 0.0090 \\
\hline & 3 & 0.7922 & -0.2269 & 0.3543 & -0.0660 \\
\hline & 4 & 0.6516 & -0.1566 & 0.4121 & -0.0888 \\
\hline & 5 & 0.2576 & 0.0000 & 0.2103 & 0.0000 \\
\hline \multirow{10}{*}{10} & 1 & 0.0000 & 0.6726 & 0.0000 & 0.2867 \\
\hline & 2 & 0.9503 & -0.3592 & 0.1281 & 0.1210 \\
\hline & 3 & 0.9093 & -0.3180 & 0.2123 & 0.0393 \\
\hline & 4 & 0.8640 & -0.2817 & 0.2853 & -0.0169 \\
\hline & 5 & 0.8127 & -0.2474 & 0.3465 & -0.0559 \\
\hline & 6 & 0.7532 & -0.2134 & 0.3933 & -0.0809 \\
\hline & 7 & 0.6821 & -0.1784 & 0.4215 & -0.0932 \\
\hline & 8 & 0.5937 & -0.1409 & 0.4236 & -0.0925 \\
\hline & 9 & 0.4745 & -0.0980 & 0.3839 & -0.0768 \\
\hline & 10 & 0.1579 & 0.0000 & 0.1428 & 0.0000 \\
\hline \multirow{15}{*}{15} & 1 & 0.0000 & 0.9142 & 0.0000 & 0.3232 \\
\hline & 2 & 0.9675 & -0.3859 & 0.0883 & 0.1815 \\
\hline & 3 & 0.9417 & -0.3538 & 0.1489 & 0.1074 \\
\hline & 4 & 0.9146 & -0.3263 & 0.2045 & 0.0522 \\
\hline & 5 & 0.8855 & -0.3011 & 0.2556 & 0.0091 \\
\hline & 6 & 0.8540 & -0.2773 & 0.3019 & -0.0248 \\
\hline & 7 & 0.8196 & -0.2543 & 0.3428 & -0.0513 \\
\hline & 8 & 0.7816 & -0.2315 & 0.3775 & -0.0714 \\
\hline & 9 & 0.7393 & -0.2086 & 0.4049 & -0.0854 \\
\hline & 10 & 0.6915 & -0.1852 & 0.4234 & -0.0937 \\
\hline & 11 & 0.6365 & -0.1608 & 0.4309 & -0.0963 \\
\hline & 12 & 0.5716 & -0.1350 & 0.4239 & -0.0929 \\
\hline & 13 & 0.4919 & -0.1067 & 0.3966 & -0.0825 \\
\hline & 14 & 0.3868 & -0.0739 & 0.3368 & -0.0633 \\
\hline & 15 & 0.1173 & 0.0000 & 0.1098 & 0.0000 \\
\hline \multirow{20}{*}{20} & 1 & 0.0000 & 1.1201 & 0.0000 & 0.3456 \\
\hline & 2 & 0.9758 & -0.4015 & 0.0673 & 0.2202 \\
\hline & 3 & 0.9571 & -0.3743 & 0.1143 & 0.1528 \\
\hline & 4 & 0.9377 & -0.3513 & 0.1585 & 0.1011 \\
\hline & 5 & 0.9174 & -0.3306 & 0.2002 & 0.0593 \\
\hline & 6 & 0.8960 & -0.3113 & 0.2394 & 0.0246 \\
\hline & 7 & 0.8732 & -0.2929 & 0.2761 & -0.0044 \\
\hline & 8 & 0.8490 & -0.2752 & 0.3100 & -0.0286 \\
\hline & 9 & 0.8230 & -0.2578 & 0.3407 & -0.0488 \\
\hline & 10 & 0.7951 & -0.2406 & 0.3681 & -0.0651 \\
\hline & 11 & 0.7650 & -0.2234 & 0.3915 & -0.0780 \\
\hline & 12 & 0.7321 & -0.2061 & 0.4104 & -0.0876 \\
\hline & 13 & 0.6961 & -0.1885 & 0.4242 & -0.0939 \\
\hline & 14 & 0.6561 & -0.1704 & 0.4319 & -0.0969 \\
\hline & 15 & 0.6112 & -0.1516 & 0.4321 & -0.0967 \\
\hline & 16 & 0.5598 & -0.1318 & 0.4232 & -0.0928 \\
\hline & 17 & 0.4998 & -0.1106 & 0.4022 & -0.0850 \\
\hline & 18 & 0.4268 & -0.0873 & 0.3643 & -0.0726 \\
\hline & 19 & 0.3318 & -0.0602 & 0.2994 & -0.0537 \\
\hline & 20 & 0.0946 & 0.0000 & 0.0900 & 0.0000 \\
\hline
\end{tabular}


KANTAM, RAMAKRISHNA \& RAVIKUMAR

Table 2.1b: Intercept and Slope of the Approximations $\mathrm{G}\left(\mathrm{Z}_{\mathrm{i}}\right)=\gamma_{\mathrm{i}}+\delta_{\mathrm{i}} \mathrm{Z}_{\mathrm{i}}$ and $\mathrm{K}\left(\mathrm{Z}_{\mathrm{i}}\right)=\alpha_{\mathrm{i}}+\beta_{\mathrm{i}} \mathrm{Z}_{\mathrm{i}}$ when $\theta=3$

\begin{tabular}{|c|c|c|c|c|c|}
\hline$n$ & $\mathrm{i}$ & $\gamma_{i}$ & $\delta_{\mathrm{i}}$ & $\alpha_{i}$ & $\beta_{\mathrm{i}}$ \\
\hline \multirow{5}{*}{5} & 1 & 0.0000 & 0.2211 & 0.0000 & 0.1533 \\
\hline & 2 & 0.8191 & -0.2449 & 0.3321 & -0.0531 \\
\hline & 3 & 0.7007 & -0.1829 & 0.4098 & -0.0891 \\
\hline & 4 & 0.5559 & -0.1230 & 0.4096 & -0.0857 \\
\hline & 5 & 0.1950 & 0.0000 & 0.1704 & 0.0000 \\
\hline \multirow{10}{*}{10} & 1 & 0.0000 & 0.3825 & 0.0000 & 0.2167 \\
\hline & 2 & 0.8959 & -0.3060 & 0.2355 & 0.0193 \\
\hline & 3 & 0.8393 & -0.2652 & 0.3183 & -0.0380 \\
\hline & 4 & 0.7834 & -0.2313 & 0.3743 & -0.0705 \\
\hline & 5 & 0.7247 & -0.2003 & 0.4105 & -0.0883 \\
\hline & 6 & 0.6605 & -0.1705 & 0.4283 & -0.0956 \\
\hline & 7 & 0.5877 & -0.1407 & 0.4264 & -0.0939 \\
\hline & 8 & 0.5014 & -0.1096 & 0.4003 & -0.0838 \\
\hline & 9 & 0.3908 & -0.0749 & 0.3393 & -0.0639 \\
\hline & 10 & 0.1175 & 0.0000 & 0.1099 & 0.0000 \\
\hline \multirow{15}{*}{15} & 1 & 0.0000 & 0.5000 & 0.0000 & 0.2500 \\
\hline & 2 & 0.9232 & -0.3332 & 0.1871 & 0.0659 \\
\hline & 3 & 0.8838 & -0.2997 & 0.2584 & 0.0069 \\
\hline & 4 & 0.8467 & -0.2727 & 0.3119 & -0.0310 \\
\hline & 5 & 0.8098 & -0.2489 & 0.3537 & -0.0572 \\
\hline & 6 & 0.7721 & -0.2270 & 0.3860 & -0.0753 \\
\hline & 7 & 0.7328 & -0.2062 & 0.4098 & -0.0873 \\
\hline & 8 & 0.6911 & -0.1861 & 0.4255 & -0.0944 \\
\hline & 9 & 0.6464 & -0.1663 & 0.4327 & -0.0972 \\
\hline & 10 & 0.5975 & -0.1463 & 0.4309 & -0.0961 \\
\hline & 11 & 0.5431 & -0.1259 & 0.4187 & -0.0911 \\
\hline & 12 & 0.4809 & -0.1046 & 0.3940 & -0.0823 \\
\hline & 13 & 0.4071 & -0.0817 & 0.3526 & -0.0692 \\
\hline & 14 & 0.3132 & -0.0558 & 0.2856 & -0.0504 \\
\hline & 15 & 0.0866 & 0.0000 & 0.0828 & 0.0000 \\
\hline \multirow{20}{*}{20} & 1 & 0.0000 & 0.5948 & 0.0000 & 0.2716 \\
\hline & 2 & 0.9377 & -0.3498 & 0.1577 & 0.0986 \\
\hline & 3 & 0.9068 & -0.3203 & 0.2200 & 0.0406 \\
\hline & 4 & 0.8782 & -0.2969 & 0.2685 & 0.0015 \\
\hline & 5 & 0.8506 & -0.2766 & 0.3083 & -0.0271 \\
\hline & 6 & 0.8230 & -0.2582 & 0.3414 & -0.0487 \\
\hline & 7 & 0.7950 & -0.2411 & 0.3690 & -0.0652 \\
\hline & 8 & 0.7664 & -0.2249 & 0.3915 & -0.0776 \\
\hline & 9 & 0.7369 & -0.2093 & 0.4093 & -0.0867 \\
\hline & 10 & 0.7060 & -0.1940 & 0.4225 & -0.0929 \\
\hline & 11 & 0.6736 & -0.1790 & 0.4310 & -0.0966 \\
\hline & 12 & 0.6392 & -0.1641 & 0.4346 & -0.0979 \\
\hline & 13 & 0.6024 & -0.1491 & 0.4330 & -0.0970 \\
\hline & 14 & 0.5625 & -0.1338 & 0.4257 & -0.0940 \\
\hline & 15 & 0.5188 & -0.1182 & 0.4118 & -0.0889 \\
\hline & 16 & 0.4701 & -0.1020 & 0.3901 & -0.0814 \\
\hline & 17 & 0.4145 & -0.0848 & 0.3586 & -0.0716 \\
\hline & 18 & 0.3488 & -0.0662 & 0.3138 & -0.0587 \\
\hline & 19 & 0.2659 & -0.0451 & 0.2483 & -0.0419 \\
\hline & 20 & 0.0694 & 0.0000 & 0.0671 & 0.0000 \\
\hline
\end{tabular}




\section{ESTIMATION AND TESTING IN TYPE I GHLD}

Table 2.1c: Intercept and Slope of the Approximations

$\mathrm{G}\left(\mathrm{Z}_{\mathrm{i}}\right)=\gamma_{\mathrm{i}}+\delta_{\mathrm{i}} \mathrm{Z}_{\mathrm{i}}$ and $\mathrm{K}\left(\mathrm{Z}_{\mathrm{i}}\right)=\alpha_{\mathrm{i}}+\beta_{\mathrm{i}} \mathrm{Z}_{\mathrm{i}}$ when $\theta=4$

\begin{tabular}{|c|c|c|c|c|c|}
\hline$n$ & $\mathrm{i}$ & $\gamma_{\mathrm{i}}$ & $\delta_{\mathrm{i}}$ & $\alpha_{i}$ & $\beta_{\mathrm{i}}$ \\
\hline \multirow{5}{*}{5} & 1 & 0.0000 & 0.1580 & 0.0000 & 0.1200 \\
\hline & 2 & 0.7573 & -0.2116 & 0.3848 & -0.0787 \\
\hline & 3 & 0.6333 & -0.1552 & 0.4235 & -0.0931 \\
\hline & 4 & 0.4907 & -0.1026 & 0.3903 & -0.0787 \\
\hline & 5 & 0.1591 & 0.0000 & 0.1437 & 0.0000 \\
\hline \multirow{10}{*}{10} & 1 & 0.0000 & 0.2657 & 0.0000 & 0.1735 \\
\hline & 2 & 0.8453 & -0.2687 & 0.3104 & -0.0335 \\
\hline & 3 & 0.7805 & -0.2299 & 0.3771 & -0.0717 \\
\hline & 4 & 0.7198 & -0.1986 & 0.4137 & -0.0896 \\
\hline & 5 & 0.6585 & -0.1705 & 0.4300 & -0.0962 \\
\hline & 6 & 0.5936 & -0.1440 & 0.4290 & -0.0952 \\
\hline & 7 & 0.5222 & -0.1178 & 0.4105 & -0.0878 \\
\hline & 8 & 0.4397 & -0.0909 & 0.3714 & -0.0746 \\
\hline & 9 & 0.3371 & -0.0615 & 0.3032 & -0.0546 \\
\hline & 10 & 0.0948 & 0.0000 & 0.0901 & 0.0000 \\
\hline \multirow{15}{*}{15} & 1 & 0.0000 & 0.3408 & 0.0000 & 0.2026 \\
\hline & 2 & 0.8788 & -0.2949 & 0.2655 & 0.0002 \\
\hline & 3 & 0.8313 & -0.2623 & 0.3305 & -0.0432 \\
\hline & 4 & 0.7890 & -0.2367 & 0.3731 & -0.0681 \\
\hline & 5 & 0.7484 & -0.2146 & 0.4021 & -0.0834 \\
\hline & 6 & 0.7081 & -0.1946 & 0.4210 & -0.0924 \\
\hline & 7 & 0.6671 & -0.1758 & 0.4314 & -0.0968 \\
\hline & 8 & 0.6246 & -0.1577 & 0.4341 & -0.0976 \\
\hline & 9 & 0.5799 & -0.1401 & 0.4292 & -0.0954 \\
\hline & 10 & 0.5320 & -0.1226 & 0.4163 & -0.0904 \\
\hline & 11 & 0.4795 & -0.1049 & 0.3946 & -0.0829 \\
\hline & 12 & 0.4208 & -0.0866 & 0.3623 & -0.0726 \\
\hline & 13 & 0.3524 & -0.0672 & 0.3164 & -0.0594 \\
\hline & 14 & 0.2673 & -0.0454 & 0.2495 & -0.0421 \\
\hline & 15 & 0.0694 & 0.0000 & 0.0671 & 0.0000 \\
\hline \multirow{20}{*}{20} & 1 & 0.0000 & 0.4000 & 0.0000 & 0.2222 \\
\hline & 2 & 0.8975 & -0.3113 & 0.2357 & 0.0255 \\
\hline & 3 & 0.8588 & -0.2821 & 0.2969 & -0.0196 \\
\hline & 4 & 0.8250 & -0.2595 & 0.3392 & -0.0473 \\
\hline & 5 & 0.7934 & -0.2403 & 0.3706 & -0.0660 \\
\hline & 6 & 0.7628 & -0.2232 & 0.3943 & -0.0789 \\
\hline & 7 & 0.7326 & -0.2074 & 0.4119 & -0.0878 \\
\hline & 8 & 0.7023 & -0.1926 & 0.4243 & -0.0937 \\
\hline & 9 & 0.6714 & -0.1784 & 0.4320 & -0.0970 \\
\hline & 10 & 0.6398 & -0.1648 & 0.4353 & -0.0982 \\
\hline & 11 & 0.6071 & -0.1514 & 0.4343 & -0.0976 \\
\hline & 12 & 0.5729 & -0.1382 & 0.4289 & -0.0954 \\
\hline & 13 & 0.5368 & -0.1250 & 0.4190 & -0.0917 \\
\hline & 14 & 0.4982 & -0.1117 & 0.4042 & -0.0864 \\
\hline & 15 & 0.4566 & -0.0982 & 0.3839 & -0.0797 \\
\hline & 16 & 0.4107 & -0.0843 & 0.3571 & -0.0715 \\
\hline & 17 & 0.2994 & -0.0541 & 0.2766 & -0.0495 \\
\hline & 18 & 0.2254 & -0.0366 & 0.2141 & -0.0346 \\
\hline & 19 & 0.0555 & 0.0000 & 0.0541 & 0.0000 \\
\hline & 20 & 0.0000 & 0.1580 & 0.0000 & 0.1200 \\
\hline
\end{tabular}




\section{KANTAM, RAMAKRISHNA \& RAVIKUMAR}

Table 3.1: Percentiles of $\mathrm{L}_{1} / \mathrm{L}_{0}$ $\left(\mathrm{P}_{0}\right.$ : GHLD-I; $\mathrm{P}_{1}$ : Exponential $)$

\begin{tabular}{|c|c|c|c|c|c|c|c|c|}
\hline$\theta$ & $\mathrm{n} p$ & 0.00135 & 0.001 & 0.025 & 0.05 & 0.9500 & 9750 & 0.99865 \\
\hline \multirow{4}{*}{2} & 5 & 0.0295 & 0.0493 & 0.0743 & 0.1126 & 3.5 & 4.6 & 8.8 \\
\cline { 2 - 8 } & 10 & 0.0072 & 0.0303 & 0.0663 & 0.1241 & 11.0 & 15.3 & 58.6 \\
\cline { 2 - 9 } & 15 & 0.0068 & 0.0337 & 0.0744 & 0.1657 & 32.2 & 46.0 & 153.6 \\
\cline { 2 - 9 } & 20 & 0.0061 & 0.0308 & 0.0885 & 0.2034 & 88.1 & 133.9 & 575.4 \\
\hline \multirow{3}{*}{3} & 5 & 0.0166 & 0.0308 & 0.0515 & 0.0860 & 10.5 & 15.9 & 44.7 \\
\cline { 2 - 9 } & 10 & 0.0037 & 0.0222 & 0.0613 & 0.1408 & 104.0 & 170.6 & 1217.2 \\
\cline { 2 - 9 } & 15 & 0.0045 & 0.0367 & 0.1059 & 0.3192 & 899.8 & 1595.0 & 10736.5 \\
\hline \multirow{3}{*}{4} & 20 & 0.0055 & 0.0518 & 0.2048 & 0.6646 & 7345.6 & 14907.5 & 110093.4 \\
\cline { 2 - 8 } & 10 & 0.0026 & 0.0207 & 0.0714 & 0.1927 & 879.7 & 1698.5 & 20257.6 \\
\hline & 15 & 0.0043 & 0.0551 & 0.2066 & 0.7839 & 21578.0 & 47370.6 & 587772.50 \\
\hline & 20 & 0.0079 & 0.1255 & 0.6597 & 2.9169 & 496183.4 & 1314785.0 & 19609204.91 \\
\hline
\end{tabular}

Table 3.2: Powers of LR Test Criterion at $\alpha=0.05:$ GHLD vs Exponential

\begin{tabular}{|c|c|c|}
\hline$\theta$ & $n$ & Power \\
\hline \multirow{4}{*}{2} & 5 & 0.0207 \\
& 10 & 0.7786 \\
& 15 & 0.8919 \\
& 20 & 0.9422 \\
\hline \multirow{4}{*}{3} & 5 & 0 \\
& 10 & 0.9135 \\
& 15 & 0.9772 \\
& 20 & 0.9938 \\
\hline \multirow{4}{*}{4} & 5 & 0 \\
& 10 & 0.9552 \\
& 15 & 0.9940 \\
& 20 & 0.9941 \\
\hline
\end{tabular}




\section{ESTIMATION AND TESTING IN TYPE I GHLD}

\section{References}

Rajarshi, S., \& Rajarshi, M. B. (1988). Bathtub distributions: A review. Communications in Statistics - Theory \& Methods, 17, 2597-2621.

Mudholkar, G. S., Srivastava, D., \& Freimer, M. (1995). Exponentiated Weibull family: A reanalysis of the bus-motor failure data. Technometrics, 37(4), 436-445.

Gupta, R. D., \& Kundu, D. (1999).Generalized exponential distributions. Australian and New Zealand Journal of Statistics, 41, 173-188.

Balakrishnan, N. (1985). Order statistics from the half logistic distribution. Journal of Statistical Computation and Simulation, 20, 287-309.

Olapade, A. K. (2007). On Type-I generalized half logistic distribution. International Journal of Pure and Applied Mathematics, http://arxiv.org/PS_cache/arxiv/pdf/0806/0806.1 580v1.pdf.

Olapade, A. K. (2008). On Type III generalized half logistic distribution. arXiv:0806.1580v1 [math.ST] 10 Jun 2008.

Balakrishnan, N., \& Sandhu, R. A. (1995). Recurrence relations for single and product moments of order Statistics from a generalized half logistic distribution with applications to inference. Journal of Statistical Computation and Simulation, 52(4), 385-398.

Balakrishnan, N., \& Leung, M. Y. (1988). Order statistics from type-I generalized logistic distribution. Communications in Statistics - Simulation and Computation, 17(1), 25-50.

Tiku, M. L. (1967). Estimating the mean and standard deviation from a censored normal sample. Biometrika, 54, 155-165.
Mehrotra, K. G., \& Nanda, P. (1974). Unbiased estimation of parameters by order statistics in the case of censored samples. Biometrika, 61, 601-606.

Pearson, T., \& Rootzen, H. (1977). Simple highly efficient estimators for a type-I censored normal sample. Biometrika, 64, 123128.

Tiku, M. L., \& Suresh, R. P. (1992).A new method of estimation for location and scale parameters. Journal of Statistical Planning and Inference, 30, 281-92.

Rosaiah, K., Kantam, R. R. L., \& Narasimham, V. L. 1993a). ML and Modified ML Estimation in gamma distribution with known prior relation among the Parameters. Pakistan Journal of Statistics, 9(3)B, 37-48.

Rosaiah, K., Kantam, R. R. L., \& Narasimham, V. L. (1993b).On modified maximum likelihood estimation of gamma parameters. Journal of Statistical Research, Bangladesh, 27(1), 15-28.

Kantam, R. R. L., \& Srinivasa Rao, G. (1993). Reliability estimation in Rayleigh distribution with censoring some approximations to ML Method. In Proceedings of II Annual Conference of Society for Development of Statistics, Acharya Nagarjuna University, 56-63.

Kantam, R. R. L., \& Srinivasa Rao, G. (2002). Log-logistic distribution: Modified maximum likelihood estimation. Gujarat Statistical Review, 29(1), 25-36.

Kantam, R. R. L., \& Sriram, B. (2003). Maximum likelihood estimation from censored samples: Some modifications in length biased version of exponential model. Statistical Methods, 5(1), 63-78. 


\title{
Estimating Heterogeneous Intra-class Correlation Coefficients in Dyadic Ecological Momentary Assessment
}

\author{
Emily A. Blood Leslie A. Kalish Lydia A. Shrier \\ Boston Children's Hospital \\ Department of Pediatrics Harvard Medical School, Boston, MA
}

A method is described for estimating and testing predictors for influence on the variance of momentary behaviors in dyadic ecological momentary assessment data. Results show that the method allows intraclass correlations of momentary observations from two members of the same couple to vary by observation-level, individual-level and couple-level predictors.

Key words: Dyadic data, intra-class correlation coefficient, ecological momentary assessment.

Introduction

Human emotions and behaviors are difficult to measure and can vary greatly with time, company and context. Testing hypotheses about variable relationship dynamics often requires collecting data in a manner that accounts for this variability and records information about the many factors that can influence it, such as, social context, individual-level and dyad-level characteristics. Ecological momentary assessment (EMA) is a technique that permits data to be collected in-the-moment on emotion, behavior and other related factors at several points over the time during which the measured emotion or behavior is expected to vary.

When studying people in close relationships, such as romantic partnerships, one of the factors that has the most potential to influence an individual's emotion is that of the

Emily A. Blood is a Senior Biostatistician at Boston Children's Hospital and Assistant Professor. Email her at: emily.blood@childrens.harvard.edu. Leslie A. Kalish is a Principal Biostatistician at Boston Children's Hospital and Associate Professor. Email him at: Les.kalish@childrens.harvard.edu Lydia A. Shrier is a Senior Associate in Medicine at Boston Children's Hospital and Associate Professor of Pediatrics. Email her at: lydia.shrier@childrens.harvard.edu. partner. Thus, when studying characteristics of a partnership, such as sexual behavior or individual mood, collecting data on both members of a couple is important (Bolger, Davis \& Rafaeli, 2003; Harvey, et al., 2004). Data collected in this way are often referred to as dyadic data. Momentary information from both members of a dyad is likely to be associated, but there are certain factors that may influence the dyad-level intra-class correlation (ICC) or degree of association of these momentary measures (Newsom, 2002). This study focuses on research questions related to factors associated with the dyad-level ICC.

Ecological Momentary Assessment

Ecological momentary assessment (EMA) is a data collection technique in which research study participants complete questionnaires via a handheld computer signaled repeatedly throughout a day. This method allows many behavioral research questions to be answered (Schwatz \& Stone, 2007). As opposed to only obtaining one or a few datapoints from each individual in a study, as in traditional crosssectional or longitudinal studies, several datapoints are collected per day, thus, each participant typically provides many datapoints; this permits a researcher to gain near real-time assessment of behavioral measures of interest. EMA is particularly useful in behavioral studies because it reduces recall bias associated with self-report data (Stone \& Shiffman, 2002). In addition, the amount of data collected from each 
individual allows a researcher to study not only the mean of the desired outcome, but also how an individual's response changes over time and in response to momentary influences. This provides a level of data not previously available - even from long-term longitudinal studies. The most commonly used method for analysis of these data is a mixed effects model (Laird \& Ware, 1982), which provides a flexible approach to account for correlation due to multiple observations from the same individual, while not requiring each individual to have the same number of observations.

\section{Dyadic Data}

The study of behavior and emotion naturally benefits from gathering information about the most influential factors. For many individuals, characteristics of their close relationships may extensively influence their behavior and affect (Burleson, Trevathan \& Todd, 2007; Widman, Welsh, McNulty \& Little, 2006). For this reason, it is desirable to study couples or dyads together. Methodologically, these data are more complete in terms of potentially influential factors, because interactions between partners (such as disagreements or sexual intercourse), couplelevel characteristics (such as relationship duration) and individual-level characteristics (such as age) may all play a role in determining behavior and mood (Burleson, et al., 2007; Fortenberry, et al., 2005). Dyadic data, therefore, can answer complex questions about behavior and mood.

Analytically, dyadic data presents challenges when compared with data from independent individuals. Several methods have been proposed for analyzing dyadic data (Kenny, Kashy \& Cook, 2006), among them are methods based on mixed effects or multi-level models and structural equation models (Laurenceau \& Bolger, 2005). Many of these methods, however, require each observation to have a measure from one member of the dyad paired with a measure from the other member of the dyad so that the data consists of multiple paired observations; this is not always the case depending on how data is collected. Laurenceau and Bolger (2005) emphasized that predicting causes of variability among dyads and covariability between members of a dyad are of distinct interest. A method proposed by Raudenbush, Brennan and Barnett (1995) for distinguishable dyads does not require observations to be paired and proposes a multilevel approach to analyzing dyadic data; their model fits a two-intercept model (one for males, one for females) and individual-level random effects for each partner. The covariance between partners is captured as the covariance of the male and female random effect.

\section{Dyadic EMA Data}

EMA can be used in observational behavioral studies of dyads where information is collected from both members of a dyad electronically on a momentary basis. This technique provides rich data that allow the study of both mean and variability within and between individuals as well as within and between dyads, thus providing the advantages of both EMA data and dyadic data. Data collection is aimed at providing a random sample of moments throughout the day for every individual, each of whom may have differing schedules. Individuals in the dyad are therefore not signaled at exactly the same time.

These data, however, increase the complexity of the analysis; there is a more complicated correlation structure than in typical EMA data and more repeated measures than a usual dyadic diary data. Unlike diary dyadic data, the measurements for each member of a dyad are not distinctly paired to an observation from the other member (because each individual is signaled randomly within a day); therefore, methods specific to dyadic diary data (Laurenceau \& Bolger, 2005) cannot always be applied. The Raudenbush, et al. (1995) method can be applied to dyadic EMA data with distinguishable dyads.

A mixed effect regression model with a random individual intercept to account for the correlation of repeated observations from the same individual and a random dyad intercept to account for the correlation of repeated observations from the same dyad. The model can incorporate momentary observation-level predictors, individual-level predictors and couple-level predictors. In addition to modeling means based on all levels of predictors, variance 
components can also be modeled on multiple levels of predictors.

Hedeker and Mermelstein (2007) described methods for estimating heterogeneous variances determined by subject-level characteristics in individual EMA data and note the importance of characterizing individual-level variability. The method of estimating heterogeneous variances is presently applied to estimate heterogeneous variances at the levels of the dyad, the observation and the individual and the way in which heterogeneous variances determined by dyad-level predictors contribute to heterogeneous dyad-level ICCs is illustrated herein. Thus, by estimating heterogeneous dyadlevel ICCs, questions about differences in within-couple similarity of responses for different types of couples can be answered.

The multi-level model proposed is most similar to that introduced by Raudenbush, et al. (1995), but does not require distinguishable dyads and does not fit separate fixed and random intercepts for each member of the couple. The proposed model fits a single individual-level random effect in addition to a dyad-level random effect, which allows for testing dyad-level variance heterogeneity. The variance of the individual-level random effects is thus the same for both members of the couple, not specific to gender. The proposed model also does not fit separate effects of individual-level predictors for each member of the couple, although such effects could be estimated in the current model by adding interaction terms with gender.

It is of interest whether momentary observations from the two members of a dyad in one group are closely related while observations from the two members of a dyad in another group tend to be more disparate. Specifically, do dyads with a given dyad-level characteristic tend to have individual momentary responses that are similar to one another more often (high covariance, large dyad-level ICC) than dyads with a different value of that couple-level characteristic? The advantage of having data that is both dyadic and EMA in nature is that these types of research questions can be answered. The way in which couple-level predictors affect both mean level of momentary individual outcomes as well as the interplay between the members of the couple can be determined.

\section{Young Adult Couples Study}

Shrier and colleagues performed a study designed to assess affective states, emotional intimacy, relationship qualities and sexual behaviors in heterosexual young adult couples (Sunner et al. 2012). Primary research questions of this study included exploring affective patterns and affective concordance and discordance within a couple related to intimacy, communication and behaviors during the daily course of a relationship. Secondary research questions investigated whether couple-level characteristics influenced the degree to which momentary measures from a couple were related. For example, do couples who have been in a relationship for longer periods of time tend to have momentary measures of affect that are more similar while couples with shorter relationship duration tend to have momentary measures of affect that are more dissimilar? When the male partner of a heterosexual couple rates relationship conflict higher than the female, does that couple tend to have more similar momentary affect measures than couples in which the female rates relationship conflict higher? To assess these research questions, analytic techniques are needed to test for couplelevel heterogeneity in the association of momentary measures.

Dyadic EMA data were collected from both members of a dyad over time. Data from each member is not necessarily collected at the same moment and each member of the dyad is not required to have the same number of observations. However, observations from different members of the same dyad should be assumed to be correlated, as should the repeated observations from each individual. Dyadic EMA data can be represented with a mixed effects regression model, with a random effect of the couple, a random effect of the individual and an observation-level error term. The model can also include fixed effects at the observation, individual, or couple level. The model is:

$Y_{i p j}=\beta_{0}+\beta_{1} x_{i p j}+\beta_{2} x_{i p}+\beta_{3} x_{p}+v_{i p}+v_{p}+\varepsilon_{i p j}$ 
where $i(i=1, \ldots, N)$ indexes the individual, $p=$ $1, \ldots, P$ indexes the dyad and $j\left(j=1, \ldots, J_{i}\right)$ indexes the observation. Observation, individual and couple-level predictors are represented by $x_{i p j}, x_{i p}$, and $x_{p}$, respectively. Conditional on the random individual and random dyad effects, the observations are assumed independent from each other and errors are assumed to be normally distributed; therefore the covariance matrix for $\varepsilon$ is $\sigma_{\varepsilon}^{2} I$. If the individual and dyad random effects ( $v_{i p}$ and $v_{p}$, respectively) are assumed to be independent, then the covariance matrix of the random effects is:

$$
\left[\begin{array}{cc}
\sigma_{i p}^{2} & 0 \\
0 & \sigma_{p}^{2}
\end{array}\right]
$$

The resulting variance-covariance matrix of all observations is block diagonal with observations from different dyads being independent and observations from the same dyad having the following variance-covariance block matrix (see Figure 1). The upper left and lower right sections of this matrix give the within-person covariance matrix for each member of the dyad and the lower left and upper right blocks give the between-person covariance matrix between the members of the dyad. The variance of a given observation $Y_{i p j}$ is $\sigma_{i p}^{2}+\sigma_{p}^{2}+$ $\sigma_{\varepsilon}^{2}$. This quantity is made up of the variance in individuals $\left(\sigma_{i p}^{2}\right)$, the variance in dyads $\left(\sigma_{p}^{2}\right)$ and the degree of residual variability in an observation $\left(\sigma_{\varepsilon}^{2}\right)$. The covariance between two observations from the same individual at different times is $\sigma_{i p}^{2}+\sigma_{p}^{2}$, and the covariance between two observations from different individuals is $\sigma_{p}^{2}$.

In dyadic data, several research questions focus on the similarity of momentary responses among members of the same dyad. Because the observations from each member of the dyad are collected randomly throughout the day, each datapoint from one member does not match up to the time and date of a datapoint from the other member. The correlation between paired observations from the same dyad can therefore not be computed (which is possible with non-EMA dyadic data): the ICC of a dyad must be computed to evaluate this phenomenon. This is accomplished by examining the covariance between any given observation from one member of the dyad and any given observation from the other member of the dyad. The variance-covariance matrix (Figure 1) from one dyad shows that this quantity is expressed as $\sigma_{p}^{2}$ and, when scaled to the total variance of all observations, represents the dyad-level ICC. A dyad-level ICC is generally defined as the variance of a dyad divided by the total variance and this quantity represents the degree of association among observations from the same pair (Newsom, 2002). With this model specification, the dyad-level ICC is the ratio of the dyad-level variance to the total variance of an observation:

Figure 1: Block Diagonal Variance-Covariance Matrix of All Observations

$\left[\begin{array}{cccccccc}\sigma_{i p}^{2}+\sigma_{p}^{2}+\sigma_{\varepsilon}^{2} & \sigma_{i p}^{2}+\sigma_{p}^{2} & \cdots & \sigma_{i p}^{2}+\sigma_{p}^{2} & \sigma_{p}^{2} & \sigma_{p}^{2} & \cdots & \sigma_{p}^{2} \\ \vdots & \sigma_{i p}^{2}+\sigma_{p}^{2}+\sigma_{\varepsilon}^{2} & & \sigma_{i p}^{2}+\sigma_{p}^{2} & \sigma_{p}^{2} & \sigma_{p}^{2} & \cdots & \sigma_{p}^{2} \\ \vdots & & \ddots & \sigma_{i p}^{2}+\sigma_{p}^{2} & \vdots & \vdots & & \vdots \\ \sigma_{i p}^{2}+\sigma_{p}^{2} & & & \sigma_{i p}^{2}+\sigma_{p}^{2}+\sigma_{\varepsilon}^{2} & \sigma_{p}^{2} & \sigma_{p}^{2} & \cdots & \sigma_{p}^{2} \\ \sigma_{p}^{2} & \sigma_{p}^{2} & \cdots & \sigma_{p}^{2} & \sigma_{i p}^{2}+\sigma_{p}^{2}+\sigma_{\varepsilon}^{2} & \sigma_{i p}^{2}+\sigma_{p}^{2} & \cdots & \sigma_{i p}^{2}+\sigma_{p}^{2} \\ \sigma_{p}^{2} & \sigma_{p}^{2} & \cdots & \sigma_{p}^{2} & \sigma_{i p}^{2}+\sigma_{p}^{2} & \sigma_{i p}^{2}+\sigma_{p}^{2}+\sigma_{\varepsilon}^{2} & & \sigma_{i p}^{2}+\sigma_{p}^{2} \\ \vdots & & & \vdots & \vdots & & \ddots & \sigma_{i p}^{2}+\sigma_{p}^{2} \\ \sigma_{p}^{2} & \cdots & \cdots & \sigma_{p}^{2} & \sigma_{i p}^{2}+\sigma_{p}^{2} & & & \sigma_{i p}^{2}+\sigma_{p}^{2}+\sigma_{\varepsilon}^{2}\end{array}\right]$




$$
\frac{\sigma_{p}^{2}}{\sigma_{i p}^{2}+\sigma_{p}^{2}+\sigma_{\varepsilon}^{2}} .
$$

This result is highlighted because, if heterogeneous variance components for the dyad-level random effect are estimated, heterogeneous covariance between two observations from the same dyad $\left(\sigma_{p}^{2}\right)$ and therefore heterogeneous dyad-level ICCs will be modeled. However, heterogeneous dyad-level ICCs can also result from heterogeneity in $\sigma_{i p}^{2}$ and/or $\sigma_{\varepsilon}^{2}$, because these terms appear in the denominator of the ICC. The dyad-level ICC is of interest in describing EMA data from a couple because it represents the degree of association between momentary responses of the two members of a dyad. Although in some longitudinal studies, correlation between observations are sometimes considered a nuisance rather than an object of study, in individual EMA studies, Hedeker and Mermelstein (2007) have shown the variances and covariances of observations can themselves be informative. This is particularly true with dyadic EMA data where there is interest in the degree of association of momentary response from dyad members.

Suppose there is a couple-level characteristic such as relationship duration that is believed influences the dyad-level ICC. A model of heterogeneous dyad-level random effects can be described where one dyad-level variance is specified for the dyads with short relationship duration and another dyad-level variance is specified for the dyads with a longer relationship duration:

Short Duration Group $Y_{i p j}=\beta_{0}+\beta_{1} x_{i p j}+\beta_{2} x_{i p}+\beta_{3} x_{p}+v_{i p}+v_{p^{*}}+\varepsilon_{i p j}$

Long Duration Group

$Y_{i p j}=\beta_{0}+\beta_{1} x_{i p j}+\beta_{2} x_{i p}+\beta_{3} x_{p}+v_{i p}+v_{p}+\varepsilon_{i p j}$

The dyad-level ICC for dyads in the short relationship duration group is

$$
\frac{\sigma_{p^{*}}^{2}}{\sigma_{i p}^{2}+\sigma_{p^{*}}^{2}+\sigma_{\varepsilon}^{2}} .
$$

Similarly, the dyad-level ICC for dyads with longer relationship duration is

$$
\frac{\sigma_{p^{\prime}}^{2}}{\sigma_{i p}^{2}+\sigma_{p^{\prime}}^{2}+\sigma_{\varepsilon}^{2}} .
$$

Heterogenous variance models can be estimated using the GROUP option in SAS PROC MIXED RANDOM and REPEATED statements (see Appendix A for example SAS code).

In addition to fitting this model, the hypothesis that there is a difference in dyadlevel variance can be tested, similar to the tests on the individual-level variance performed by Hedeker and Mermelstein (2007) in individual EMA data. If all other aspects of the model remain the same, a model with homogeneous dyad-level variance is nested within a model with heterogeneous variance at this level, thus, differences in deviances ( $-2 \log$ likelihood) between the two models can be computed and compared to a Chi-square critical value with 1 degree of freedom.

Because the dyad-level ICC is composed of several variance components, however, heterogeneity in dyad-level ICC due to a dyad-level characteristic is possible in several ways. If, for example, the observation-level variance differs between dyads with a longer versus shorter relationship durations, but all other variance components are homogeneous, the dyad-level ICC will still differ by relationship duration. Specifically, the dyadlevel ICC in the long duration group would be:

$$
\frac{\sigma_{p}^{2}}{\sigma_{i p}^{2}+\sigma_{p}^{2}+\sigma_{\varepsilon^{\prime}}^{2}}
$$

and the dyad-level ICC in the short duration group would be:

$$
\frac{\sigma_{p}^{2}}{\sigma_{i p}^{2}+\sigma_{p}^{2}+\sigma_{\varepsilon^{*}}^{2}} .
$$


This heterogeneity can be modeled and tested. With dyadic data, there are three levels of variability, each of which could be affected by a dyad-level characteristic. Table 1 shows all combinations of homogeneous and heterogeneous variance in a dyadic multilevel model. If no level of variance differs by a dyadlevel characteristic then Model (1) is appropriate. If there is variance heterogeneity only at the dyad-level then Model (2) is appropriate. If there is variance heterogeneity at all levels of variance then Model (8) is appropriate. Appropriate variance models can be selected by comparing $-2 \log$ likelihood values for nested models or comparing AIC and BIC for non-nested models (Hedeker \& Mermelstein, 2007). After the appropriate model is selected, heterogeneity in dyad-level ICC $(\hat{\rho})$ can be estimated and tested.

Using the delta method (Casella \& Berger, 2002) the variance of each ICC can be estimated and these variances can be used to create confidence intervals and to construct a test of differences in ICCs between dyad groups. The delta-method variance of an ICC estimate is:

$$
\begin{aligned}
& \operatorname{var}(\hat{\rho})= \\
& {[\hat{\rho}(1-\hat{\rho})]^{2}\left[\begin{array}{c}
\frac{\operatorname{var}\left(\hat{\sigma}_{p}^{2}\right)}{\left(\hat{\sigma}_{p}^{2}\right)^{2}}+\frac{\operatorname{var}\left(\hat{\sigma}_{i p}^{2}\right)}{\left(\hat{\sigma}_{i p}^{2}+\sigma_{\varepsilon}^{2}\right)^{2}} \\
+\frac{\operatorname{var}\left(\hat{\sigma}_{\varepsilon}^{2}\right)}{\left(\hat{\sigma}_{i p}^{2}+\sigma_{\varepsilon}^{2}\right)^{2}}
\end{array}\right]} \\
& -2 \frac{[\hat{\rho}(1-\hat{\rho})]^{2}}{\hat{\sigma}_{i p}^{2}+\sigma_{\varepsilon}^{2}}\left[\begin{array}{c}
\frac{\operatorname{cov}\left(\hat{\sigma}_{p}^{2}, \hat{\sigma}_{i p}^{2}\right)}{\hat{\sigma}_{p}^{2}}+\frac{\operatorname{cov}\left(\hat{\sigma}_{p}^{2}, \hat{\sigma}_{\varepsilon}^{2}\right)}{\hat{\sigma}_{p}^{2}} \\
-\frac{\operatorname{cov}\left(\hat{\sigma}_{i p}^{2}, \hat{\sigma}_{\varepsilon}^{2}\right)}{\hat{\sigma}_{p}^{2}+\hat{\sigma}_{\varepsilon}^{2}}
\end{array}\right]
\end{aligned}
$$

which leads to confidence intervals of the form $\hat{\rho} \pm z_{\alpha / 2} \sqrt{\operatorname{var}(\hat{\rho})}$ and a test statistic for comparing two dyad-level ICCs, $\frac{\hat{\rho}_{1}-\hat{\rho}_{2}}{\sqrt{\operatorname{var}\left(\hat{\rho}_{1}-\hat{\rho}_{2}\right)}}$, with a standard normal distribution.

\section{Young Adult Couples Study}

The technique of selecting the appropriate model and testing for heterogeneity in dyad-level ICC is illustrated using data collected in the Young Adult Couples Study conducted by Shrier, et al. (Sunner et al. 2012). A total of 2,089 observations were obtained from 36 participants (18 heterosexual couples) aged 18-25 years. To be eligible for the study, couples had to have been in a relationship for at least 3 weeks. Ecological momentary assessment data was gathered from participants, with each individual contributing between 15 and 107 observations. Each member of the couple was asked several baseline questions regarding demographics, relationship duration and quality, emotional and physical intimacy, sexual behavior and substance use. Each individual was given a handheld computer and was randomly signaled several times within a day to complete questionnaires on affect, disagreements, sexual behavior and substance use. Individuals carried the handheld computer for up to two weeks.

Momentary affective states were measured using an abbreviated version of the Positive and Negative Affect Scale (PANAS; Watson, Clark \& Tellegen, 1988) consisting of 5-point Likert scale ratings for 6 positive and 6 negative affective states. A composite positive score and a composite negative score were computed by summing item ratings for each type. Baseline measures included: relationship duration was dichotomized to shorter duration, defined as $<3$ months, and longer duration, defined as $\geq 3$ months and Quality of Relationship Inventory (QRI; Pierce, Sarason \& Sarason, 1991) measuring relationship quality. The QRI included a depth subscale (6 items, Cronbach's $\alpha=0.77)$, a conflict subscale (12 items, Cronbach's $\alpha=0.89$ ) and a social support subscale ( 7 items, Cronbach's $\alpha=0.66$ ). Dyadic data from 18 of 20 couples were available.

To explore methods for secondary research questions specific to the effect of dyadlevel predictors on the dyad-level ICC in EMA data, two example hypotheses were examined. These analyses represent a range of possible applications of the heterogeneous ICC technique in dyadic EMA data. Whether couples in longerterm relationships tended to have momentary affect measures that were more similar to their 
Table 1: Dyadic Data Models of Variance Heterogeneity

\begin{tabular}{|c|l|l|l|c|}
\hline Model & Dyad Variance & $\begin{array}{c}\text { Individual } \\
\text { Variance }\end{array}$ & $\begin{array}{c}\text { Observation } \\
\text { Variance }\end{array}$ & $\begin{array}{c}\text { Variance } \\
\text { Parameter } \\
\text { Estimates }\end{array}$ \\
\hline$(1)$ & Homogeneous & Homogeneous & Homogeneous & 3 \\
\hline$(2)$ & Heterogeneous & Homogeneous & Homogeneous & 4 \\
\hline$(3)$ & Homogeneous & Heterogeneous & Homogeneous & 4 \\
\hline$(4)$ & Homogeneous & Homogeneous & Heterogeneous & 4 \\
\hline$(5)$ & Heterogeneous & Heterogeneous & Homogeneous & 5 \\
\hline$(6)$ & Heterogeneous & Homogeneous & Heterogeneous & 5 \\
\hline$(7)$ & Homogeneous & Heterogeneous & Heterogeneous & 5 \\
\hline$(8)$ & Heterogeneous & Heterogeneous & Heterogeneous & 6 \\
\hline
\end{tabular}

Table 2: Fit statistics From Models of Relationship Duration Associated with Momentary Negative Affect

\begin{tabular}{|c|c|c|c|c|c|c|}
\hline Model & Dyad Variance & $\begin{array}{c}\text { Individual } \\
\text { Variance }\end{array}$ & $\begin{array}{c}\text { Observation } \\
\text { Variance }\end{array}$ & $-2 L^{1}$ & AIC $^{2}$ & BIC $^{3}$ \\
\hline$(1)$ & Homogeneous & Homogeneous & Homogeneous & 9950.6 & 9956.6 & 9959.2 \\
\hline$(2)$ & Heterogeneous & Homogeneous & Homogeneous & 9941.9 & 9949.9 & 9953.5 \\
\hline$(3)$ & Homogeneous & Heterogeneous & Homogeneous & 9941.7 & 9949.7 & 9953.3 \\
\hline$(4)$ & Homogeneous & Homogeneous & Heterogeneous & 9807.9 & 9815.9 & 9819.5 \\
\hline$(5)$ & Heterogeneous & Heterogeneous & Homogeneous & 9934.5 & 9944.5 & 9948.9 \\
\hline$(6)$ & Heterogeneous & Homogeneous & Heterogeneous & 9799.3 & 9808.3 & 9813.8 \\
\hline$(7)$ & Homogeneous & Heterogeneous & Heterogeneous & 9799.5 & 9809.5 & 9813.9 \\
\hline$(8)$ & Heterogeneous & Heterogeneous & Heterogeneous & 9792.2 & 9804.2 & 9809.6 \\
\hline
\end{tabular}

1: -2 log-likelihood

2: Akaike information criterion

3: Bayesian information criterion 
partner versus couples who had not been in a relationship for as long was examined first. Next, because the study of young adult couples consisted only of heterosexual couples, the members of the dyads are distinguishable by gender. This distinguishability can therefore be used to create directional couple-level variables. As an example, a couple-level characteristic indicating whether the male rated the relationship conflict higher than the female is created. In the second research question, this directional couple-level predictor is used to test whether those couples in which males rated the relationship conflict higher than the female tended to have more or less similar momentary negative affect. In all models, fixed effects of dyad-level predictors are included in the models to test whether the dyad-level predictors affects the mean outcome in addition to the variability.

\section{Results}

Relationship Duration and Negative Affect

In the Young Adult Couples Study data, two hypotheses related to heterogeneous ICCs were tested. The first was related to the effect of relationship duration on similarity in negative affect within a couple. A first step in testing this hypothesis was determining whether heterogeneity exists at the dyad-level, individual-level or observation-level. Therefore, all possible models allowing for heterogeneity of variance component were fit. The fit statistics from these models are provided in Table 2.

First, the fit statistics from each of Model (2), Model (3) and Model (4) respectively, were compared to Model (1), the completely homogeneous model. The homogeneous model is nested within each of these models with only one degree of freedom difference and a Chi-square likelihood ratio test was performed. To test dyad-level variance heterogeneity, Model (2), with a $\quad-2$ log likelihood value of 9941.9, was compared to Model (1), with a -2 log likelihood of 9950.6. The difference between these likelihoods is 8.7 indicating significant dyad-level heterogeneity $\left(\mathrm{X}^{2}(1)=8.7, \mathrm{p}=0.003\right)$. Likewise, $\mathrm{a}$ comparison of likelihoods from Model (3) to (1) $(9950.5-9941.7=8.9)$ shows significant individual-level heterogeneity $\left(\mathrm{X}^{2}(1)=8.9, \mathrm{p}=\right.$ 0.003 ). Finally, a comparison of Models (4) and
(1) indicates heterogeneity at the observation level as well $\left(\mathrm{X}^{2}(1)=142.7, \mathrm{p}<0.001\right)$. Because there is evidence of heterogeneity at all three levels of variance, the full Model (8) was considered. Nesting Models (5), (6) and (7) within Model (8) and conducting likelihood ratio tests confirmed that all three levels of heterogeneity were statistically significant within the full model. Model (8), therefore, appears to be the best model; this model has the lowest AIC and BIC values (9804.2 and 9809.6) indicating best fit. Model (8) was fit to the data and the dyad-level ICCs for short and long duration were computed. Model (8)'s fit to the adolescent couples study is displayed in Table 3.

Examining the effects of relationship duration, a shorter relationship duration was associated with more similarity in momentary negative affect. Couples with a shorter relationship duration $(\mathrm{n}=9)$ had a dyad-level ICC $(95 \%$ CI) of $0.49(0.20,0.77)$; dyad-level ICC in the group of couples with longer relationship duration was $0.19(0.01,0.38)$. To test whether this difference in similarity within couple was significant, a significance test based on the delta-method was performed. The value of the test statistic was 1.71 ( $\mathrm{p}$-value $=0.09$ ). Despite the significant variability at all levels of variance, the heterogeneity in the dyad-level ICC's for short and longer duration couples was not statistically significant.

Relationship Conflict and Negative Affect Working with distinguishable dyads, directional differences in dyad-level characteristics can also be examined. This is illustrated by categorizing couples by which partner rated level of relationship conflict higher, the male or the female, and whether this influenced the similarity of within-couple momentary negative affect was explored. First, it is necessary to determine if heterogeneity in variance components occurs at any of the three levels. To do this, likelihood values for Models (2), (3) and (4) were compared to Model (1), the homogeneous model. Fit statistics from Models (1) through (8) are shown in Table 4. Comparing Model (2) to (1), shows significant heterogeneity at the dyad-level $\left(\mathrm{X}^{2}(1)=8.9, \mathrm{p}=0.003\right)$; comparing Model (3) to (1) and Model (4) to (1). 
Table 3: Model of Relationship Duration Affecting Momentary Negative Affect Allowing Heterogeneity of Variance at All Levels (Model (8))

\begin{tabular}{|c|c|c|}
\hline & Estimate & $(\mathrm{SE})$ \\
\hline \multicolumn{3}{|l|}{ Fixed Effect } \\
\hline Short duration & 9.4024 & $(1.1706)$ \\
\hline Long duration & 78.542 & $(0.3780)$ \\
\hline p-value & .23 & \\
\hline \multicolumn{3}{|l|}{ Random Dyad Effect } \\
\hline Short duration & 11.0387 & $(6.2010)$ \\
\hline Long duration & 1.0992 & $(0.6490)$ \\
\hline \multicolumn{3}{|l|}{ Individual Effect } \\
\hline Short duration & 2.4066 & $(1.2202)$ \\
\hline Long duration & 0.2765 & $(0.1708)$ \\
\hline \multicolumn{3}{|l|}{ Observation Effect } \\
\hline Short duration & 9.1568 & $(0.4093)$ \\
\hline Long duration & 4.3069 & $(0.1891)$ \\
\hline \multicolumn{3}{|c|}{ Dyad-level Intra-class Correlation Coefficient (ICC) } \\
\hline Short duration & 0.4884 & $(0.1455)$ \\
\hline Long duration & 0.1934 & $(0.0933)$ \\
\hline \multicolumn{3}{|c|}{ Test of Heterogeneity of Dyad-level ICC } \\
\hline p-value & .09 & \\
\hline
\end{tabular}

Table 4: Fit Statistics from Models of Ratings of Relationship Conflict Affecting Momentary Negative Affect

\begin{tabular}{|c|c|c|c|c|c|c|}
\hline Model & Dyad Variance & $\begin{array}{c}\text { Individual } \\
\text { Variance }\end{array}$ & $\begin{array}{c}\text { Observation } \\
\text { Variance }\end{array}$ & $-2 L^{1}$ & AIC $^{2}$ & BIC $^{3}$ \\
\hline$(1)$ & Homogeneous & Homogeneous & Homogeneous & 9949.9 & 9955.9 & 9958.6 \\
\hline$(2)$ & Heterogeneous & Homogeneous & Homogeneous & 9941.0 & 9949.0 & 9952.6 \\
\hline$(3)$ & Homogeneous & Heterogeneous & Homogeneous & 9949.6 & 9957.6 & 9961.2 \\
\hline$(4)$ & Homogeneous & Homogeneous & Heterogeneous & 9949.8 & 9957.8 & 9961.4 \\
\hline$(5)$ & Heterogeneous & Heterogeneous & Homogeneous & 9940.9 & 9950.9 & 9955.3 \\
\hline$(6)$ & Heterogeneous & Homogeneous & Heterogeneous & 9940.9 & 9950.9 & 9955.4 \\
\hline$(7)$ & Homogeneous & Heterogeneous & Heterogeneous & 9949.5 & 9959.5 & 9964.0 \\
\hline$(8)$ & Heterogeneous & Heterogeneous & Heterogeneous & 9940.8 & 9952.8 & 9958.1 \\
\hline
\end{tabular}

1:-2 log-likelihood

2: Akaike information criterion

3: Bayesian information criterion 
show that no significant heterogeneity occurs at the individual-level $\left(\mathrm{X}^{2}(1)=0.3, \mathrm{p}=0.58\right)$ or the observation-level $\left(\mathrm{X}^{2}(1)=0.1, \mathrm{p}=0.75\right)$. Model (2) appears to be the appropriate model for these data; fitting this model allowed estimation of the heterogeneity of the dyad-level ICC. Results from Model (2) are displayed in Table 5.

In dyads where females reported higher relationship conflict, momentary negative affect was more similar between members of the dyad compared to dyads in which males reported higher relationship conflict (dyad-level ICC, 95\% CI, where females reported higher relationship conflict than males was $0.75(0.44$, 1.1) versus $0.18(0.01,0.35))$. Testing whether these two ICC's were statistically different, yields a test statistic of $3.16(p$-value $=0.002)$ indicating that these values differ significantly. This significant heterogeneity in dyad-level ICC is observed despite no significant effect of relationship conflict measures on mean negative affect.

\section{Conclusion}

It was demonstrated that the use of heterogeneous variance terms in a mixed effect model of dyadic EMA data effectively estimates and allows for testing of heterogeneous dyadlevel ICCs, which are often the focus of dyadic data research questions. Thus, this technique fills a methodologic void in dyadic EMA data analysis.

Many techniques available for dyadic data are not applicable to unpaired EMA data and require paired observations from members of a dyad. Analyzing unpaired EMA data with these techniques requires aggregation to obtain paired observations from members of a dyad (Laurenceau \& Bolger, 2005). Although aggregating these data across a period of time allows questions to be asked regarding the influence of one dyad member's response on the other dyad member's response, it also results in a loss of the momentary aspect of this data so the EMA data are not used to their fullest extent.

Table 5: Model of Ratings of Relationship Conflict Associated With Momentary Negative Affect Allowing Heterogeneity of Variance at the Dyad-Level (Model (2))

\begin{tabular}{|c|c|c|}
\hline & \\
\hline & Estimate & (SE) \\
\hline \multicolumn{3}{|l|}{ Fixed Effect } \\
\hline Male $\geq$ Female & 8.1837 & $(0.4227)$ \\
\hline Male $<$ Female & 10.1931 & $(2.4936)$ \\
\hline p-value & .44 & \\
\hline \multicolumn{3}{|l|}{ Random Dyad Effect } \\
\hline Male $\geq$ Female & 1.7594 & $(1.0050)$ \\
\hline Male $<$ Female & 24.1525 & $(20.3225)$ \\
\hline \multicolumn{3}{|l|}{ Individual Effect } \\
\hline & 1.3316 & $(0.4889)$ \\
\hline \multicolumn{3}{|l|}{ Observation Effect } \\
\hline & 6.6898 & $(0.2096)$ \\
\hline \multicolumn{3}{|c|}{ Dyad-level Intra-class Correlation Coefficient (ICC) } \\
\hline Male $\geq$ Female & 0.1799 & $(0.0869)$ \\
\hline Male $<$ Female & 0.7507 & $(0.1581)$ \\
\hline \multicolumn{3}{|c|}{ Test of Heterogeneity of Dyad-level ICC } \\
\hline p-value & .002 & \\
\hline
\end{tabular}




\section{BLOOD, KALISH \& SHRIER}

Answering research questions using mixed effect models of momentary data capitalizes on EMA data benefits, such as reduction of recall bias and in-the-moment information about behavior and emotions, while also being able to answer questions that take the dyadic nature of the data into account.

With dyadic EMA data, research questions often focus on the degree to which momentary responses of individuals in a dyad are related (as measured by a dyad-level ICC). To answer such questions, the application of a technique for estimating heterogeneous variance components to the random effects in EMA data was proposed with the result of actually estimating heterogeneous dyad-level ICCs. With this method dyad-level characteristics can be tested for their influence on dyad-level ICCs.

Data from the Young Adult Couples Study was used to demonstrate that couple-level characteristics can influence the dyad-level ICC despite not directly influencing the mean of the measure itself. For example, the variability at the observation, individual and dyad-level was significant by relationship duration, however, the associated effect on the dyad-level ICC was tested and no significant difference was found. With the gender-distinguishable dyads the direction - not only the degree - of the discrepancy between couples was related to the magnitude of the dyad-level ICC. For example, when couples rated a difference in relationship conflict, momentary affect measures were more similar when the female rated the relationship conflict higher than the male. Together these results show the possible research questions that can be answered by applying this technique to dyadic EMA data.

One limitation of this study was the small sample size available to demonstrate this methodology. Although data from only 36 participants (18 couples) was used, a large amount of data within participant was available providing adequate data for evaluating momentary measures. Additionally, this study was limited to a basic application of heterogeneous variance estimation technique to dyadic EMA data. The relationship between dyad-level predictor and degree of association between momentary assessments of members of the dyad was limited to categorical dyad-level predictors. Hedeker, et al. (2008) proposed more complex log-linear models estimated via nonlinear mixed effects models for individual-level EMA data that can incorporate continuous predictors as well as categorical predictors into the variance models. These models, however, have not been extended to dyadic data or used for computing heterogeneous ICCs. More complex associations between dyad-level characteristics and momentary measures may require more complex variance models. Finally, the model proposed does not account for time between measurements. For a dyadic EMA analysis, it is possible to incorporate random slope terms in addition to random intercept terms, however, this will make the estimation of dyad-level ICC much more complex and dependent on time. The model proposed examines, over the course of the study, the similarity in measurements between members of a couple.

The proposed analysis technique allows for testing the influence of dyad-level characteristics on degree of association among momentary responses of members of a dyad. The set of analyses performed on the Young Adult Couples Study illustrates that important insights about behavior and affect of dyads can be gained by testing such hypotheses. By examining the influences on the couple's emotion and behavior, as measured by ICC, in addition to the individual's behavior, as measured by mean and variance, it is possible to study the couple as a unit as opposed to solely as two individuals.

\section{Acknowledgements}

The authors are grateful to Al Ozonoff for helpful discussions of the problem and review of the article. This research was supported by a grant from the Aerosmith Endowment Fund, Boston Children's Hospital, Boston, MA.

\section{References}

Bolger, N., Davis, A., \& Rafaeli, E. (2003). Diary methods: Capturing life as it is lived. Annual Review of Psychology, 54, 579616. 
Burleson, M., Trevathan, W., \& Todd, M. (2007). In the mood for love or vice versa? Exploring the relations among sexual activity, physical affection, affect, and stress in the daily lives of mid-aged women. Archives of Sexual Behavior, 36, 357-368.

Casella, G., \& Berger, R. (2002). Statistical Inference $\left(2^{\text {nd }} E d\right.$.). Pacific Grove, CA: Duxbury.

Fortenberry, J., et al. (2005). Daily mood, partner support, sexual interest, and sexual activity among adolescent women. Health Psychology, 24, 252-257.

Harvey, S., et al. (2004). He said, she said: Concordance between sexual partners. Sexually Transmitted Diseases, 31(3), 185-191.

Hedeker, D., \& Mermelstein, R. (2007). Mixed-effects regression models with heterogeneous variance: Analyzing ecological momentary assessment (EMA) data of smoking Modeling contextual effects in longitudinal studies. Mahwah, NJ: Erlbaum.

Hedeker, D., \& Mermelstein, R. (2008). An application of a mixed-effects location scale model for analysis of ecological momentary assessment (EMA) data. Biometrics, 64, 627634.

Kenny, D., Kashy, D., \& Cook, W. (2006). Dyadic data analysis. New York, NY: The Guilford Press.

Laird, N., \& Ware, J. (1982). Random effects models for longitudinal data. Biometrics, 38, 963-974.

Laurenceau, J-P., \& Bolger, N. (2005). Using diary methods to study marital and family process. Journal of Family Psychology, 19, 8697.
Newsom, J. (2002). A multilevel structural equation model for dyadic data. Structural Equation Modeling, 9, 431-447.

Pierce, G., Sarason, I., \& Sarason, B. (1991). General and relationship-based perceptions of social support: Are two contructs better than one? Journal of Personality and Social Psychology, 61, 1028-1039.

Raudenbush, S., Brennan, R., \& Barnett, R. (1995). A multivariate hierarchical model for studying psychological change within married couples. Journal of Family Psychology, 9(2), 161-174.

Schwatz, J., \& Stone, A. (2007). The science of real-time data capture: Self-reports in health research. New York, NY: Oxford University Press.

Stone, A., \& Shiffman, S. (2002). Capturing momentary, self-report data: A proposal for reporting guidelines. Annals of Behavioral Medicine, 24(3), 236-243.

Sunner, L., et al. (2012). Feasibility and utility of momentary sampling of sex events in young couples. Journal of Sex Research. Accessed from PubMed, PMID: 22823546.

Watson, D., Clark, L., \& Tellegen, A. (1988). Development and validation of brief measures of positive and negative affect: The PANAS scales. Journal of Personality and Social Psychology, 54, 1063-1070.

Widman, L., Welsh, D., McNulty, J., \& Little, K. (2006). Sexual communication and contraceptive use in adolescent dating couples. Journal of Adolescent Health, 39, 893-899. 


\section{BLOOD, KALISH \& SHRIER}

Appendix A: SAS Code for Estimating Heterogeneity at All Levels of Variance

This code is based on the negative affect model (na) examining the effect of relationship duration (longduration).

PROC MIXED DATA=couples COVTEST ASYCOV;

CLASS subid pairid longduration;

MODEL na = longduration / SOLUTION;

RANDOM intercept / SUBJECT=pairid GROUP=longduration;

RANDOM intercept / SUBJECT=subid (pairid) GROUP=longduration;

REPEATED / SUBJECT=subid (pairid) GROUP=longduration;

TITLE 'Model 8';

RUN ;

A RANDOM statement is given to specify heterogeneity at each of: the random effect at the dyad level (pairid) and the random effect at the individual level (subid (pairid)).

A REPEATED statement is given to specify heterogeneity in the residual error.

The ASYCOV option is used to output the covariance components of the covariance parameter estimates needed for calculation of the delta-method variance. 


\title{
An Approach for Dealing with Statuses of Non-Statistically Significant Interactions between Treatments
}

\author{
Zakaria M. Sawan \\ Cotton Research Institute, \\ Giza, Egypt
}

\begin{abstract}
A field experiment on cotton yield resulted in a non-statistically significant interaction. An approach for follow-up examination between treatments based on least significant difference values was suggested to identify the effect regardless of insignificance. It was found that the classical formula used in calculating the significance of interactions suffers a possible shortage that can be eliminated by applying a suggested revision.
\end{abstract}

Key words: Cotton yield, Mepiquat Chloride, Nitrogen, non-significant interactions, Potassium.

\section{Introduction}

Managing the balance of vegetative and reproductive growth is the essence of managing a cotton crop. It is known from numerous fertilizer experiments that the yield of field crop is strongly dependent on the supply of mineral nutrients (Gormus, 2002; Ansari \& Mahey, 2003; Pervez, et al., 2004). Excess of vegetative growth, poor bud development, shedding of fruiting forms and growth imbalance between the source and sink are responsible for unpredictable behavior of a crop. Several approaches have been used in an attempt to break this yield plateau, among them the application of plant growth regulators (PGR's), particularly Mepiquat Chloride (MC) has received much attention recent years (Kumar, et al., 2004; Nuti, et al., 2004).

This study evaluates the effects of $\mathrm{N}$ fertilization rate, foliar $\mathrm{K}$ application, and $\mathrm{MC}$ application on the cotton yield with the goal of identifying production treatments that may improve yield. Also, a statistical approach for

Dr. Zakaria M. Sawan is a Professor in the Cotton Research Institute at the Agricultural Research Center, Ministry of Agriculture and Land Reclamation, 9 Gamaa Street, 12619, Giza, Egypt. Email him at: zmsawan@hotmail.com. dealing with non-significant interactions between treatments depending on least significant differences, regardless of statistical insignificance is suggested (Sawan, 2011).

\section{Methodology}

A field experiment was conducted at the Agricultural Research Center, Ministry of Agriculture in Giza $\left(30^{\circ} \mathrm{N}, 31^{\circ}: 28^{\prime} \mathrm{E}\right.$ and $19 \mathrm{~m}$ altitude), Egypt using the cotton cultivar Giza 86 (Gossypium barbadense L.) in I and II seasons. The soil texture in both seasons was a clay loam with an alluvial substratum $(\mathrm{pH}=8.10,44.75 \%$ clay, $27.40 \%$ silt, $20.00 \%$ fine sand, $3.00 \%$ coarse sand, $2.85 \%$ calcium carbonate and $1.85 \%$ organic matter). Each experiment included 16 treatment combinations of:

(i) Two $\mathrm{N}$ rates (95 and $143 \mathrm{~kg} \mathrm{~N}$ per hectare), which were applied as ammonium nitrate $\left(\mathrm{NH}_{4} \mathrm{NO}_{3}, 33.5 \% \mathrm{~N}\right)$ at two equal doses, 6 and 8 weeks after planting. Each application (in the form of pinches beside each hill) was followed immediately by irrigation.

(ii) Four K rates $(0,319,638$ and $957 \mathrm{~g} \mathrm{~K}$ per hectare) were applied as potassium sulfate $\left(\mathrm{K}_{2} \mathrm{SO}_{4}\right.$ ' $40 \% \mathrm{~K}$ ') as a foliar spray, 70 and 95 days after planting (during square initiation and boll development stage). The solution volume applied was $960 \mathrm{~L}$ per hectare. 
(iii) Two rates from the PGR, 1,1dimethylpiperidinium chloride (Mepiquat Chloride, MC or Pix) were foliar applied (75 days after planting at 0 or $48 \mathrm{~g}$ active ingredient per hectare, 90 days after planting at 0 and $24 \mathrm{~g}$ active ingredient per hectare) where the solution volume applied was also $960 \mathrm{~L}$ per hectare. The $\mathrm{K}$ and $\mathrm{MC}$ were applied to the leaves with uniform coverage using a knapsack sprayer. The pressure used was $0.4 \mathrm{~kg}$ per $\mathrm{cm}^{2}$, resulting in a nozzle output of $1.43 \mathrm{~L}$ per min. The application was carried out between 9.0 and $11.0 \mathrm{~h}$ (Sawan, 2011).

A randomized complete block design with four replications was used for both experiments. Seeds were planted on 3 April in season I and 20 April in season II. Plot size was $1.95 \times 4 \mathrm{~m}$ including three ridges (beds) after the precaution of border effect was taken into consideration. Hills were spaced $25 \mathrm{~cm}$ apart on one side of the ridge, with seedlings thinned to two plants hill ${ }^{-1}$ six weeks after planting. This provided a plant density of 123,000 plants per hectare. The total amount of surface irrigation applied during the growing season was about $6,000-\mathrm{m}^{3}$ per hectare. The first irrigation was applied three weeks after planting and the second three weeks later. Thereafter, plots were irrigated every two weeks until the end of the season (October 11, in season I and October 17 in season II), for a total of nine irrigations. On the basis of soil test results, phosphorus (P) fertilizer was applied at the rate of $24 \mathrm{~kg}$ P per hectare as calcium super phosphate during land preparation. The $\mathrm{K}$ fertilizer was applied at the rate of $47 \mathrm{~kg} \mathrm{~K}$ per hectare as potassium sulfate before the first irrigation (the recommended level for semi-fertile soil). Fertilization ( $\mathrm{P}$ and $\mathrm{K})$, along with pest and weed management was carried out during the growing season according to the local practice performed at the experimental station (Sawan, 2011).

In both seasons, ten plants were randomly taken from the center ridge of each plot to determine the seed cotton yield in $g$ per plant. Hand picking was conducted on 20 and 26 September and final picking on 11 and 17 October in seasons I and II, respectively. Total seed cotton yield of each plot (including ten plant sub samples) was used to determine seed cotton and lint yield (kg per hectare) (Sawan, 2011).

Following the procedure outlined by Snedecor \& Cochran (1980), results were analyzed as factorial experiments in a randomized complete block design for the studied characters each season and the combined statistical analysis for the two seasons. The least significant difference (LSD) test method at 5\% level of significance was used to verify the significance of differences among treatment means and the interactions to determine the optimum combination of $\mathrm{N}, \mathrm{K}$ and $\mathrm{MC}$ (Sawan, 2011).

\section{Results}

Results from the analysis of variance for yield (combined data of the two seasons) are presented in Table 1 (Sawan, 2011).

\section{Effects of Main Treatments on Yield}

Seed cotton yield per plant, as well as seed cotton and lint yield per hectare, were increased by as much as $12.8,12.8$, and $12.3 \%$, respectively, when the nitrogen rate was increased (see Table 2) (Sawan, 2011). Both boll numbers and boll weight increased, which was attributed to the fact that $\mathrm{N}$ is an important nutrient for control of new growth and preventing abscission of squares and bolls and is also essential for photosynthetic activity (McConnell \& Mozaffari, 2004; Wiatrak, et al., 2006). When $\mathrm{K}$ was applied at all three rates (319, 638 and $957 \mathrm{~g} \mathrm{~K}$ per hectare), seed cotton yield per plant and seed cotton and lint yield per hectare also increased (Sawan, 2011). These increases could be attributed to the favorable effects of $\mathrm{K}$ on yield components, that is, the number of opened bolls per plant and boll weight leading consequently to higher cotton yield (Pettigrew, et al., 2005; Sharma \& Sundar, 2007). Mepiquat Chloride (MC) significantly increased seed cotton yield per plant, as well as seed cotton and lint yield per hectare (by 9.5, 9.6 and $9.3 \%$, respectively), compared to the untreated control (Sawan, 2011). These results may be attributed to the beneficial promoting and supplemental effects of this substance that lead to yield enhancements of both boll retention and boll weight (Sharma \& Sundar, 2007). 
APPROACH FOR NON-SIGNIFICANT INTERACTIONS BETWEEN TREATMENTS

Table 1: Mean Squares for Combined Analysis of Variance for Cotton Yield during Seasons I and II

\begin{tabular}{|c|c|c|c|c|}
\hline \multicolumn{1}{|c|}{ Source } & d.f. & $\begin{array}{c}\text { Seed Cotton Yield } \\
\text { (g per plant) }\end{array}$ & $\begin{array}{c}\text { Seed Cotton Yield } \\
\text { (kg per hectare) }\end{array}$ & $\begin{array}{c}\text { Lint Yield } \\
\text { (kg per hectare) }\end{array}$ \\
\hline Year & 1 & $147.21^{* *}$ & $1415571.4^{* *}$ & $332917.8^{* *}$ \\
\hline Replicates within Years & 6 & $40.27^{*}$ & $404859.0^{*}$ & $50458.4^{*}$ \\
\hline Treatments & 15 & $75.94^{* *}$ & $714189.8^{* *}$ & $83868.9^{* *}$ \\
\hline Nitrogen $(\mathrm{N})$ & 1 & $456.74^{* *}$ & $4325402.3^{* *}$ & $500162.5^{* *}$ \\
\hline Potassium (K) & 3 & $132.53^{* *}$ & $1223590.9^{* *}$ & $145491.8^{* *}$ \\
\hline Mepiquat Chloride (MC) & 1 & $261.15^{* *}$ & $2504937.5^{* *}$ & $294768.0^{* *}$ \\
\hline $\mathrm{N} \times \mathrm{K}$ & 3 & 3.47 & 31778.5 & 3934.8 \\
\hline $\mathrm{N} \times \mathrm{MC}$ & 1 & 0.17 & 1463.4 & 298.6 \\
\hline $\mathrm{K} \times \mathrm{MC}$ & 3 & 4.19 & 36432.4 & 4632.6 \\
\hline $\mathrm{N} \times \mathrm{K} \times \mathrm{MC}$ & 3 & 0.18 & 1879.3 & 209.1 \\
\hline Treatments $\times \mathrm{Year}$ & 15 & 2.50 & 24239.8 & 3070.9 \\
\hline Error $\mathrm{SD}$ & 90 & 14.36 & 135377.4 & 16752.8 \\
\hline & & 3.79 & 367.9 & 129.4 \\
\hline & & 12.04 & 12.0 & 12.0 \\
\hline
\end{tabular}

*Significant at $P=0.05 ; * *$ Significant at $P=0.01$ (Sawan, 2011)

Effects of Interactions between Treatments on Yield

No significant interactions were identified among the variables in this study $(\mathrm{N}$ rates, $\mathrm{K}$ rates and $\mathrm{MC}$ ) with respect to the characters under investigation. Generally, interactions indicated that the favorable effects accompanied the application of $\mathrm{N}$, spraying cotton plants with $\mathrm{K}$ combined with $\mathrm{MC}$ on cotton productivity, was more obvious by applying $\mathrm{N}$ at $143 \mathrm{~kg}$ per hectare and combined with spraying cotton plants with $\mathrm{K}$ at $957 \mathrm{~g}$ per hectare and also with $\mathrm{MC}$ at $48+24 \mathrm{~g}$ active ingredient per hectare.
Regarding the non-significant interaction effects, increases were observed in seed cotton yield per hectare (about $40 \%$ ) as a result of applying the same combination (Sawan, 2011).

Differences were observed between the interactions in this study, that is, the first order (see Tables 3-5) and the second order (see Table 6); however, these interactions were not statistically significant. Because it is possible that experimental error could mask the pronounced effects of the interactions (Sawan, 2011) a statistical approach for dealing with the non-significant interactions between treatments is suggested. 
Table 2: Effect of N-Rate and Foliar Application of K and MC on Cotton Yield Combined Over Seasons I and II*

\begin{tabular}{|c|c|c|c|}
\hline Treatment & $\begin{array}{l}\text { Seed Cotton Yield } \\
\quad \text { (g per plant) }\end{array}$ & $\begin{array}{l}\text { Seed Cotton Yield } \\
\text { (kg per hectare) }\end{array}$ & $\begin{array}{c}\text { Lint Yield } \\
\text { (kg per hectare) }\end{array}$ \\
\hline \multicolumn{4}{|c|}{$\mathrm{N}$ rate (kg per hectare) } \\
\hline 95 & $29.58^{\mathrm{b}}$ & $2882.3^{\mathrm{b}}$ & $1020.0^{\mathrm{b}}$ \\
\hline 143 & $33.36^{\mathrm{a}}$ & $3250.0^{\mathrm{a}}$ & $1145.0^{\mathrm{a}}$ \\
\hline $\operatorname{LSD}(0.05)$ & 1.33 & 128.9 & 45.4 \\
\hline \multicolumn{4}{|c|}{$\mathrm{K}$ rate (g per hectare) } \\
\hline 0 & $28.61^{\mathrm{b}}$ & $2792.5^{\mathrm{b}}$ & $988.2^{\mathrm{b}}$ \\
\hline 319 & $31.51^{\mathrm{a}}$ & $3068.6^{\mathrm{a}}$ & $1083.4^{\mathrm{a}}$ \\
\hline 638 & $32.51^{\mathrm{a}}$ & $3163.0^{\mathrm{a}}$ & $1115.2^{\mathrm{a}}$ \\
\hline 957 & $33.25^{\mathrm{a}}$ & $3240.7^{\mathrm{a}}$ & $1143.1^{\mathrm{a}}$ \\
\hline $\operatorname{LSD}(0.05)$ & 1.88 & 182.3 & 64.1 \\
\hline \multicolumn{4}{|c|}{$\mathrm{MC}$ rate (g per hectare) } \\
\hline 0 & $30.04^{\mathrm{b}}$ & $2926.3^{b}$ & $1034.5^{\mathrm{b}}$ \\
\hline $48+24$ & $32.90^{\mathrm{a}}$ & $3206.1^{\mathrm{a}}$ & $1130.5^{\mathrm{a}}$ \\
\hline $\operatorname{LSD}(0.05)$ & 1.33 & 128.9 & 45.4 \\
\hline $\mathrm{SD}$ & 3.79 & 367.9 & 129.4 \\
\hline $\mathrm{CV} \%$ & 12.04 & 12.0 & 12.0 \\
\hline
\end{tabular}

*Values followed by the same letter in a column are not significantly different at $P=0.05$ (Sawan, 2011)

Table 3: Effect of Interaction between N Rate and Foliar Application of K on Cotton Yield Combined Over Seasons I and II*

\begin{tabular}{|c|c|c|c|c|c|c|}
\hline \multirow{2}{*}{ Character } & \multicolumn{2}{|c|}{$\begin{array}{c}\text { Seed Cotton Yield } \\
\text { (g per plant) }\end{array}$} & \multicolumn{7}{c|}{$\begin{array}{c}\text { Seed Cotton Yield } \\
\text { (kg per hectare) }\end{array}$} & \multicolumn{2}{c|}{$\begin{array}{c}\text { Lint Yield } \\
\text { (kg per hectare) }\end{array}$} \\
\cline { 2 - 8 } & \multicolumn{7}{|c|}{$\begin{array}{c}\text { N Rate } \\
\text { (kg per hectare) }\end{array}$} \\
\hline $\begin{array}{c}\text { K Rate } \\
\text { (g per hectare) }\end{array}$ & 95 & 143 & 95 & 143 & 95 & 143 \\
\hline 0 & $27.04^{\mathrm{d}}$ & $30.18^{\mathrm{c}}$ & $2639.2^{\mathrm{d}}$ & $2945.8^{\mathrm{c}}$ & $936.0^{\mathrm{d}}$ & $1040.3^{\mathrm{c}}$ \\
\hline 319 & $29.73^{\mathrm{c}}$ & $33.28^{\mathrm{ab}}$ & $2896.6^{\mathrm{c}}$ & $3240.5^{\mathrm{ab}}$ & $1025.3^{\mathrm{c}}$ & $1141.5^{\mathrm{ab}}$ \\
\hline 638 & $30.16^{\mathrm{c}}$ & $34.86^{\mathrm{a}}$ & $2935.5^{\mathrm{c}}$ & $3390.4^{\mathrm{a}}$ & $1037.2^{\mathrm{c}}$ & $1193.3^{\mathrm{a}}$ \\
\hline 957 & $31.38^{\mathrm{bc}}$ & $35.11^{\mathrm{a}}$ & $3058.0^{\mathrm{bc}}$ & $3423.3^{\mathrm{a}}$ & $1081.4^{\mathrm{bc}}$ & $1204.7^{\mathrm{a}}$ \\
\hline${ }^{\dagger} L S D(0.05)$ & \multicolumn{2}{|c|}{2.66} & 257.8 & & 90.7 \\
\hline
\end{tabular}

*Values followed by the same letter in columns under every character head are not significantly different at $P=0.05 ;{ }^{\dagger}$ LSD, Least Significant Difference; (Sawan, 2011) 
Table 4: Effect of Interaction between N Rate and Foliar Application of MC on Cotton Yield Combined Over Seasons I and II*

\begin{tabular}{|c|c|c|c|c|c|c|}
\hline \multirow{2}{*}{ Character } & \multicolumn{2}{|c|}{$\begin{array}{c}\text { Seed Cotton Yield } \\
\text { (g per plant) }\end{array}$} & \multicolumn{2}{c|}{$\begin{array}{c}\text { Seed Cotton Yield } \\
\text { (kg per hectare) }\end{array}$} & \multicolumn{2}{c|}{$\begin{array}{c}\text { Lint Yield } \\
\text { (kg per hectare) }\end{array}$} \\
\cline { 2 - 7 } & \multicolumn{7}{c|}{$\begin{array}{c}\text { MC Rate } \\
\text { (g per hectare) }\end{array}$} \\
\hline $\begin{array}{c}\text { N Rate } \\
\text { (kg per hectare) }\end{array}$ & 0 & $48+24$ & 0 & $48+24$ & 0 & $48+24$ \\
\hline 95 & $28.11^{\mathrm{c}}$ & $31.04^{\mathrm{b}}$ & $2739.1^{\mathrm{c}}$ & $3025.6^{\mathrm{b}}$ & $970.4^{\mathrm{c}}$ & $1069.5^{\mathrm{b}}$ \\
\hline 143 & $31.96^{\mathrm{b}}$ & $34.75^{\mathrm{a}}$ & $3113.5^{\mathrm{b}}$ & $3386.5^{\mathrm{a}}$ & $1098.5^{\mathrm{b}}$ & $1191.4^{\mathrm{a}}$ \\
\hline${ }^{\dagger}$ LSD $(0.05)$ & \multicolumn{2}{|c|}{1.88} & 182.3 & \multicolumn{2}{c|}{64.1} \\
\hline
\end{tabular}

*Values followed by the same letter in columns under every character head are not significantly different at $P=0.05 ;{ }^{\dagger}$ LSD, Least Significant Difference; (Sawan, 2011)

Table 5: Effect of Interaction between K Rate and Foliar Application of MC on Cotton Yield Combined Over Seasons I and II*

\begin{tabular}{|c|c|c|c|c|c|c|}
\hline \multirow{2}{*}{ Character } & \multicolumn{2}{|c|}{$\begin{array}{l}\text { Seed Cotton Yield } \\
\quad \text { (g per plant) }\end{array}$} & \multicolumn{2}{|c|}{$\begin{array}{l}\text { Seed Cotton Yield } \\
\text { (kg per hectare) }\end{array}$} & \multicolumn{2}{|c|}{$\begin{array}{c}\text { Lint Yield } \\
\text { (kg per hectare) }\end{array}$} \\
\hline & \multicolumn{6}{|c|}{$\begin{array}{c}\text { MC Rate } \\
\text { (g per hectare) }\end{array}$} \\
\hline $\begin{array}{c}\text { K Rate } \\
\text { (g per hectare) }\end{array}$ & 0 & $48+24$ & 0 & $48+24$ & 0 & $48+24$ \\
\hline 0 & $27.22^{\mathrm{c}}$ & $29.99^{\mathrm{b}}$ & $2655.0^{\mathrm{c}}$ & $2930.0^{\mathrm{b}}$ & $941.1^{\mathrm{c}}$ & $1035.3^{\mathrm{b}}$ \\
\hline 319 & $29.66^{b c}$ & $33.35^{\mathrm{a}}$ & $2891.3^{\mathrm{bc}}$ & $3245.8^{\mathrm{a}}$ & $1022.0^{\mathrm{bc}}$ & $1144.9^{\mathrm{a}}$ \\
\hline 638 & $31.00^{\mathrm{b}}$ & $34.03^{\mathrm{a}}$ & $3014.1^{b}$ & $3311.8^{\mathrm{a}}$ & $1064.2^{b}$ & $1166.3^{\mathrm{a}}$ \\
\hline 957 & $32.28^{\mathrm{ab}}$ & $34.21^{\mathrm{a}}$ & $3144.7^{\mathrm{ab}}$ & $3336.6^{\mathrm{a}}$ & $1110.7^{\mathrm{ab}}$ & $1175.5^{\mathrm{a}}$ \\
\hline${ }^{\dagger} \mathrm{LSD}(0.05)$ & \multicolumn{2}{|c|}{2.66} & \multicolumn{2}{|c|}{257.8} & \multicolumn{2}{|c|}{90.7} \\
\hline
\end{tabular}

*Values followed by the same letter in columns under every character head are not significantly different at $P=0.05 ;{ }^{\dagger}$ LSD, Least Significant Difference; (Sawan, 2011) 
Table 6: Effect of Interactions between N Rate, Foliar Application of K and MC on Cotton Yield Combined Over Seasons I and II*

\begin{tabular}{|c|c|c|c|c|c|}
\hline \multicolumn{3}{|c|}{ Treatment } & \multirow{2}{*}{$\begin{array}{l}\text { Seed Cotton } \\
\text { Yield } \\
\text { (g per plant) }\end{array}$} & \multirow{2}{*}{$\begin{array}{c}\text { Seed Cotton } \\
\text { Yield } \\
\text { (kg per hectare) }\end{array}$} & \multirow{2}{*}{$\begin{array}{l}\text { Lint Yield } \\
\text { (kg per } \\
\text { hectare) }\end{array}$} \\
\hline $\begin{array}{c}\text { N Rate } \\
\text { (kg per hectare) }\end{array}$ & $\begin{array}{c}\text { K Rate } \\
\text { (g per hectare) }\end{array}$ & $\begin{array}{c}\text { MC Rate } \\
\text { (g per hectare) }\end{array}$ & & & \\
\hline \multirow{8}{*}{95} & \multirow{2}{*}{0} & 0 & $25.54^{\mathrm{e}}$ & $2490.4^{\mathrm{e}}$ & $884.4^{\mathrm{e}}$ \\
\hline & & $48+24$ & $27.85^{\mathrm{de}}$ & $2716.3^{\mathrm{de}}$ & $963.2^{\mathrm{de}}$ \\
\hline & \multirow[b]{2}{*}{319} & 0 & $28.71^{\mathrm{de}}$ & $2793.6^{\mathrm{de}}$ & $987.6^{\mathrm{de}}$ \\
\hline & & $48+24$ & $30.36^{\text {cd }}$ & $2956.1^{\mathrm{cd}}$ & $1046.7^{\text {cd }}$ \\
\hline & \multirow{2}{*}{638} & 0 & $28.54^{\mathrm{de}}$ & $2788.0^{\mathrm{de}}$ & $987.6^{\mathrm{de}}$ \\
\hline & & $48+24$ & $31.62^{\mathrm{bcd}}$ & $3077.0^{\mathrm{bcd}}$ & $1087.4^{\mathrm{bcd}}$ \\
\hline & \multirow{2}{*}{957} & 0 & $31.62^{\mathrm{bcd}}$ & $3077.4^{\mathrm{bcd}}$ & $1086.7^{\mathrm{bcd}}$ \\
\hline & & $48+24$ & $32.40^{\mathrm{bc}}$ & $3160.0^{\mathrm{bc}}$ & $1116.2^{\mathrm{bc}}$ \\
\hline \multirow{8}{*}{143} & \multirow{2}{*}{0} & 0 & $28.91^{\mathrm{cd}}$ & $2819.7^{\mathrm{cd}}$ & $997.8^{\mathrm{cd}}$ \\
\hline & & $48+24$ & $31.48^{\mathrm{bcd}}$ & $3066.3^{\mathrm{bcd}}$ & $1080.8^{\mathrm{bcd}}$ \\
\hline & \multirow{2}{*}{319} & 0 & $33.28^{\mathrm{ab}}$ & $3234.7^{\mathrm{ab}}$ & $1140.8^{\mathrm{ab}}$ \\
\hline & & $48+24$ & $34.20^{\mathrm{ab}}$ & $3333.4^{\mathrm{ab}}$ & $1174.7^{\mathrm{ab}}$ \\
\hline & \multirow{2}{*}{638} & 0 & $31.45^{\mathrm{bc}}$ & $3072.0^{\mathrm{bc}}$ & $1082.9^{\mathrm{bc}}$ \\
\hline & & $48+24$ & $35.08^{\mathrm{ab}}$ & $3414.7^{\mathrm{ab}}$ & $1202.3^{\mathrm{ab}}$ \\
\hline & \multirow{2}{*}{957} & 0 & $36.44^{\mathrm{a}}$ & $3546.2^{\mathrm{a}}$ & $1245.8^{\mathrm{a}}$ \\
\hline & & $48+24$ & $36.03^{\mathrm{a}}$ & $3513.2^{\mathrm{a}}$ & $1234.8^{\mathrm{a}}$ \\
\hline \multicolumn{3}{|c|}{${ }^{\dagger} \operatorname{LSD}(0.05)$} & 3.76 & 364.6 & 128.3 \\
\hline
\end{tabular}

*Means followed by the same letter in a column are not significantly different at $P=0.05 ;{ }^{\dagger} \mathrm{LSD}$, Least Significant Difference; (Sawan, 2011)

This approach depends on the least significant difference (LSD) values to verify significant differences between treatment combinations regardless of the non-significance of the interaction effects from the ANOVA.

Results show that, if no significant differences are identified between the different levels of any main factor $(\mathrm{N}, \mathrm{K}$ or $\mathrm{MC}$ ) when the LSD is calculated, then the significance does not exist. Conversely, if the significance of the interactions between the main factors (first \& second order interactions) are not identified, then the estimation of the LSD of the interactions between the main factors could provide a significant result (Sawan, 2011). For these reasons, the formula used in calculating the significance of interactions suffers a possible shortage.
Study results indicate that it could be useful to modify or add to the original formula used for calculating $F$ values of interactions (Sawan, 2011) via:

$$
F=\frac{\text { Mean Square for Interaction }}{\text { Mean Square for Error }} .
$$

In this connection, calculating the significance of interactions could proceed as:

$$
F=\frac{\text { Mean square for int eraction } \times n}{\text { Root of mean square for error }}
$$

where $n=$ number of main factors in the interaction. Based on findings from this study, it 


\section{APPROACH FOR NON-SIGNIFICANT INTERACTIONS BETWEEN TREATMENTS}

may be concluded that the use of the suggested formula could secure the disclosure of any significant effects among interactions regardless of experimental error (Sawan, 2011).

\section{References}

Ansari, M. S., \& Mahey, R. K. (2003). Growth and yield of cotton species as affected by sowing dates and nitrogen levels. Journal of Research, Punjab Agricultural University, 40, 8-11.

Gormus, O. (2002). Effects of rate and time of potassium application on cotton yield and quality in Turkey. Journal of Agronomy and Crop Science, 188, 382-388.

Kumar, K. A. K., Patil, B. C., \& Chetti, M. B. (2004). Effect of plant growth regulators on biophysical, biochemical parameters and yield of hybrid cotton. Karnataka Journal of Agricultural Science, 16, 591-594.

McConnell, J. S., \& Mozaffari, M. (2004) Yield, petiole nitrate, and node development responses of cotton to early season nitrogen fertilization. Journal of Plant Nutrition, 27, 1183-1197.

Nuti, R. C., Witten, T. K., Jost, P. H., \& Cothren, J. T. (2000). Comparisons of Pix Plus and additional foliar Bacillus cereus in cotton. In Proceedings Beltwide Cotton Production Research Conference, San Antonio, TX, USA, January 4-8, 684-687. Memphis, TN: National Cotton Council.
Pervez, H., Ashraf, M., \& Makhdum, M. I. (2004). Influence of potassium rates and sources on seed cotton yield and yield components of some elite cotton cultivars. Journal of Plant Nutrition, 27, 1295-1317.

Pettigrew, W. T., Meredith, W. R. Jr., \& Young, L. D. (2005). Potassium fertilization effects on cotton lint yield, yield components and reniform nematode populations. Agronomy Journal, 97, 1245-1251.

Sawan, Z. M. (2011). A suggested statistical approach for dealing with the nonsignificant interactions between treatments. Natural Science, 3, 365-370.

Sharma, S. K., \& Sundar, S. (2007). Yield, yield attributes and quality of cotton as influenced by foliar application of potassium. Journal of Cotton Research and Development, 21, 51-54.

Snedecor, G. W., \& Cochran, W. G. (1980). Statistical Methods, $7^{\text {th }}$ Ed. Ames, Iowa: Iowa State University Press.

Wiatrak, P. J., Wright, D. L., \& Marois, J. J. (2006). Development and yields of cotton under two tillage systems and nitrogen application following white lupine grain crop. Journal Cotton Science, 10, 1-8. 


\title{
Bayesian Inference of Pair-Copula Constriction for Multivariate Dependency Modeling of Iran's Macroeconomic Variables
}

\author{
M. R. Zadkarami O. Chatrabgoun \\ ShahidChamran University, \\ Ahvaz, Iran
}

Bayesian inference of pair-copula constriction (PCC) is used for multivariate dependency modeling of Iran's macroeconomics variables: oil revenue, economic growth, total consumption and investment. These constructions are based on bivariate t-copulas as building blocks and can model the nature of extreme events in bivariate margins individually. The model parameter was estimated based on Markov chain Monte Carlo (MCMC) methods. A MCMC algorithm reveals unconditional as well as conditional independence in Iran's macroeconomic variables, which can simplify resulting PCC's for these data.

Key words: Monte Carlo Markov Chain Method, pair-copula construction, vine.

\section{Introduction}

Multivariate data usually exhibit a complex pattern of dependency. Methods such as graphical model and Bayesian networks are available to investigate dependency structures in multivariate data. One increasingly popular approach for constructing high dimensional dependency is based on copulas. Copulas are multivariate distribution functions with uniform margins which allow representation of joint distribution functions as a function of marginal distributions and a copula (Sklar, 1959). Copulas are used in various fields of applied sciences, but are most widely used in economics, finance and risk management (Embrechts, et al., 2003; Patton, 2004; Nolte, 2008). The class of copulas for bivariate data is rich in comparison to the one for $d$-dimensional data with $d \geq 3$. Until recently, Gaussian and t-copulas or, more

M. R. Zadkarami is a associated professor on the Faculty of Mathematics and Computer Sciences in the Statistics Department. Email him at: zadkarami@yahoo.co.uk. O. Chatrabgoun is a $\mathrm{PhD}$ student on the Faculty of Mathematics and Computer Sciences in the Statistics Department. Email him at: o-chatrabgoun@phdstu.scu.ac.ir. generally, elliptical copulas, have been used for multivariate data (Frahm, et al., 2003). The generalization of bivariate copulas to multivariate copulas of dimensions larger than 2 is not straightforward, however there is one simple generalization for Archimedean copulas known as exchangeable Archimedean copulas (Frey \& McNeil, 2003). It should be noted that not all bivariate Archimedean copulas have a corresponding multivariate exchangeable version (Nelsen, 1999).

Approaches for constructing multivariate Archimedean copulas of more than 2 have dimensions been developed by Joe (1997), Embrechts, et al. (2003), Whelan, (2004), McNeil, et al. (2006), Savu and Trede (2006) and McNeil (2007). Joe (1996) and Bedford and Cooke (2001, 2002) constructed flexible higher-dimensional copulas by using only bivariate copulas as building blocks, which they termed vines. Kurowicka and Cooke (2006) discussed Gaussian vine constructions in details. Aas, et al. (2007) first recognized the general construction principle for deriving multivariate copulas; they used more general bivariate copulas than the Gaussian copula and applied these construction methods to financial risk data using more appropriate pair-copulas such as the bivariate $t$ Clayton and Gumbel copulas. According to recent empirical investigations of Berg and Aas (2007) and Fischer, et al. (2007), the vine constructions based on bivariate $t$ - 


\section{BAYESIAN INFERENCE OF PAIR-COPULA CONSTRICTION FOR MODELING}

copulas provide a better fit to multivariate financial data.

Estimating copula parameters is generally based on classical maximum likelihood (ML) and its variations. The most common approach is semi-parametric where the margins are fitted empirically and the dependence parameters are fitted by ML. The asymptotic properties of these semi-parametric estimates have been rigorously investigated by Genest, et al. (1995); however, confidence intervals for dependence parameters are difficult to obtain because determination of the asymptotic variance is not a simple task. Due to this, data analyses often are exclusively based on point estimates of copula parameters. Bayesian inference, or Markov chain Monte Carlo (MCMC) estimation of the parameters, provides a solution for this problem - which is not simple to solve in a classical ML framework.

Using MCMC, interval estimation of parameters can be achieved by credible interval. This is due to the MCMC algorithm introduced by Metropolis, et al. (1953) and Hastings (1970). Credible intervals for parameters of a paircopula constriction (PCC) can simplify the PCC if they detect conditional and unconditional independency between pairs of variables. However, Bayesian literature on copulas is poor. Pitt, et al. (2006) investigated Gaussian copula regression, the main difficulty they encountered was sampling a positive definite correlation matrix. They solved the problem by employing a covariance selection prior that was introduced by Wong, et al. (2003).

Dalla Valle (2007) proposed Bayesian inference based on MCMC for multivariate Gaussian and t-copulas using the inverse Wishart distribution as a prior for the correlation matrix. The study used Bayesian inference for pair-copula constructions (PPC's) of Iran's macroeconomic variables based on bivariate $t-$ copulas using a method similar to that used by Min and Czado (2011) for a Norwegian financial data set. Min and Czado's method allows modeling of tail dependency between two chosen margins individually, while multivariate Gaussian and t-copulas have the same tail dependency structure for any two chosen margins. A tail dependence coefficient (see Embrechts, et al., 2002) accounts for extreme events of margins occurring simultaneously, this is one of the most important characteristics of financial data because it contains information on heavy-tailedness of multivariate financial data. PCC parameters considered are association and degrees of freedom (df) parameters of bivariate t-copulas.

Copulas

Copulas are d-dimensional multivariate distributions with uniformly distributed marginal distributions on $[0,1]$ and are very useful for modeling a dependence structure of multivariate data. Let $X=\left(X_{1}, X_{2}, \ldots, X_{d}\right)^{\prime}$ be a $\mathrm{d}$-dimensional random vector with joint distribution function $F\left(x_{1}, x_{2}, \ldots, x_{d}\right)$ and marginal distributions

$$
\mathrm{F}_{1}\left(x_{1}\right), \mathrm{F}_{2}\left(x_{2}\right), \ldots, \mathrm{F}_{\mathrm{d}}\left(x_{d}\right)
$$

According to Sklar's (1959) theorem a copula $C$ exists such that

$$
F\left(x_{1}, x_{2}, \ldots, x_{d}\right)=C\left(F_{1}\left(x_{1}\right), F_{2}\left(x_{2}\right), \ldots, F_{d}\left(x_{d}\right)\right)
$$

and the copula $C\left(u_{1}, u_{2}, \ldots, u_{d}\right)$ is unique if the marginal distributions are continuous. (See Joe, 1997 and Nelsen, 1999 for additional.)

The copula $C\left(u_{1}, u_{2}, \ldots, u_{d}\right)$ of a multivariate distribution $F\left(x_{1}, x_{2}, \ldots, x_{d}\right)$ with margins $\mathrm{F}_{1}\left(x_{1}\right), \mathrm{F}_{2}\left(x_{2}\right), \ldots, F_{d}\left(x_{d}\right)$ is given by

$$
C\left(u_{1}, u_{2}, \ldots, u_{d}\right)=C\left(F_{1}^{-1}\left(u_{1}\right), F_{2}^{-1}\left(u_{2}\right), \ldots, F_{d}^{-1}\left(u_{d}\right)\right)
$$

and the copula density is given by

$$
c\left(u_{1}, u_{2}, \ldots, u_{d}\right)=\frac{f\left(F_{1}^{-1}\left(u_{1}\right), F_{2}^{-1}\left(u_{2}\right), \ldots, F_{d}^{-1}\left(u_{d}\right)\right)}{f_{l}\left(F_{1}^{-1}\left(u_{1}\right)\right) \ldots f_{d}\left(F_{d}^{-1}\left(u_{d}\right)\right)}
$$

where $F_{i}^{-1}\left(u_{i}\right)$ is the inverse of the margins $F_{i}\left(x_{i}\right)$ for $i=1,2, \ldots, d$. Using (1), the multivariate density $f\left(x_{1}, x_{2}, \ldots, x_{d}\right)$ is a product of the corresponding copula density with marginal densities $f_{i}\left(x_{i}\right), i=1,2, \ldots, d$ and is given by 


\section{ZADKARAMI \& CHATRABGOUN}

$$
\begin{aligned}
& f\left(x_{1}, x_{2}, \ldots, x_{d}\right)= \\
& c\left(F_{1}\left(x_{1}\right), F_{2}\left(x_{2}\right), \ldots, F_{d}\left(x_{d}\right)\right) . f_{1}\left(x_{1}\right) f_{2}\left(x_{2}\right) \ldots f_{d}\left(x_{d}\right)
\end{aligned}
$$

thus separating the dependence structure from the marginal structure.

\section{PCC's for Multivariate Distributions}

$$
\text { Pair-copula constriction }
$$

(PCC)

modeling structure is based on a decomposition of a multivariate density into a cascade of bivariate copulae. In other words, a vine associated with $n$ variables is a nested set of trees where the edges of the tree, $j$, are the nodes of the tree $j+1 ; j=1, \ldots, n-2$, and each tree has the maximum number of edges. A regular vine on $n$ variables is a vine in which two edges in tree $j$ are joined by an edge in tree $j+1$ only if these edges share a common node, $j=$ $1, \ldots, n-2$. There are $n(n-1) / 2$ edges in a regular vine on $n$ variables (Kurowicka\& Cooke, 2006).

\section{Bedford and Cooke Theorem}

Bedford and Cooke (2001) presented the following theorem. Let $V=\left(T_{1}, \ldots, T_{n-1}\right)$ be a regular vine on $\mathrm{n}$ elements, where $T_{1}$ is a connected tree with nodes $N_{1}=\{1, \ldots, n\}$ and edges $E_{1}$; for $i=2, \ldots, n-1, T_{i}$ is a connected tree with nodes $N_{i}=E_{i-1}$. For each edge $e(j, k) \epsilon T_{i} ; i=1, \ldots, n-1$ with conditioned set $\{j, k\}$ and conditioning set $D_{e}$, let the conditional copula and copula density be $C_{j k \mid D_{e}}$ and $c_{j k \mid D_{e}}$ respectively. If the marginal distributions $F_{i}$ with densities $f_{i} ; i=1, \ldots, n$ are given, then the vine-dependent distribution is uniquely determined and has a density given by

$$
\begin{aligned}
& f\left(x_{1}, \ldots, x_{n}\right)= \\
& \prod_{i=1}^{n} f\left(x_{i}\right) \prod_{e(j, k) \in E_{i}} c_{j k \mid D_{e}}\left(F_{j \mid D_{e}\left(x_{j}\right)}, F_{j k \mid D_{e}\left(x_{k}\right)}\right)
\end{aligned}
$$

The density decomposition associated with 4 random variables $X=\left(X_{1}, \ldots, X_{4}\right)$ with a joint density function $f\left(x_{1}, \ldots, x_{4}\right)$ satisfying a copula-vine structure (this structure is called Dvine, see Kurowicka and Cooke, 2006, p. 93) as shown in Figure 1 with the marginal densities $f_{1}, \ldots, f_{4}$ is illustrated as:

$$
\begin{aligned}
& f_{1234}= \\
& \prod_{i=1}^{4} f\left(x_{i}\right) \times c_{12}\left(F\left(x_{1}\right), F\left(x_{2}\right)\right) \\
& \quad c_{23}\left(F\left(x_{2}\right), F\left(x_{3}\right)\right) c_{34}\left(F\left(x_{3}\right), F\left(x_{4}\right)\right) \\
& \quad \times c_{13}\left(F\left(x_{1} \mid x_{2}\right), F\left(x_{3} \mid x_{2}\right)\right) \\
& \quad c_{24}\left(F\left(x_{2} \mid x_{3}\right), F\left(x_{4} \mid x_{3}\right)\right) \\
& \quad c_{14}\left(F\left(x_{1} \mid x_{2}, x_{3}\right), F\left(x_{4} \mid x_{2}, x_{3}\right)\right)
\end{aligned}
$$

Joe (1996, p. 125) showed that the conditional distribution function $F_{U \mid V}(u \mid v)$ appearing in the PCC are partial derivatives with respect to the second argument of the conditional copula given by

$$
F_{U \mid \boldsymbol{V}}(u \mid \boldsymbol{v})=\frac{\partial C_{x, v_{j} \mid V_{-j}}\left(F\left(x \mid V_{-j}\right), F\left(v_{4} \mid V_{-j}\right)\right)}{\partial F\left(v_{4} \mid V_{-j}\right)}
$$

where $C_{x, v_{j} \mid V_{-j}}(.,$.$) is a bivariate copula$ distribution function.

Data Adjustment and MCMC Estimation

Data analyzed in this study are four time-series data related to the Iran's macroeconomics variables: (A) oil revenue, (B) economic growth, (C) total consumption and (D) investment. These data were collected from the Islamic republic of Iran's Central Bank.

First it is necessary to remove serial correlation of the four time series, that is, the observation of each variable must be independent over time. Hence, the serial correlation in the conditional mean and the conditional variance are modeled by an AR(1) and a GARCH(1,1) model (Bollerslev, 1986), respectively. For time series $i$, the model for logreturn $x_{i}$ is $x_{i, t}=c_{i}+\alpha_{i} x_{i, t-1}+\sigma_{i, t} z_{i, t}$, $E\left[z_{i, t}\right]=0, \sigma_{i, t}^{2}=\alpha_{i, 0}+a_{i} \varepsilon_{i, t-1}^{2}+b_{i} \sigma_{i, t-1}^{2}$ where $\varepsilon_{i, t-1}=\sigma_{i, t}+z_{i, t}$ (Aas, et al., 2009). Table 1 shows the analyses performed on the standard residuals, $z_{i}$. 
Figure 1: Vine Structure with 4 Elements

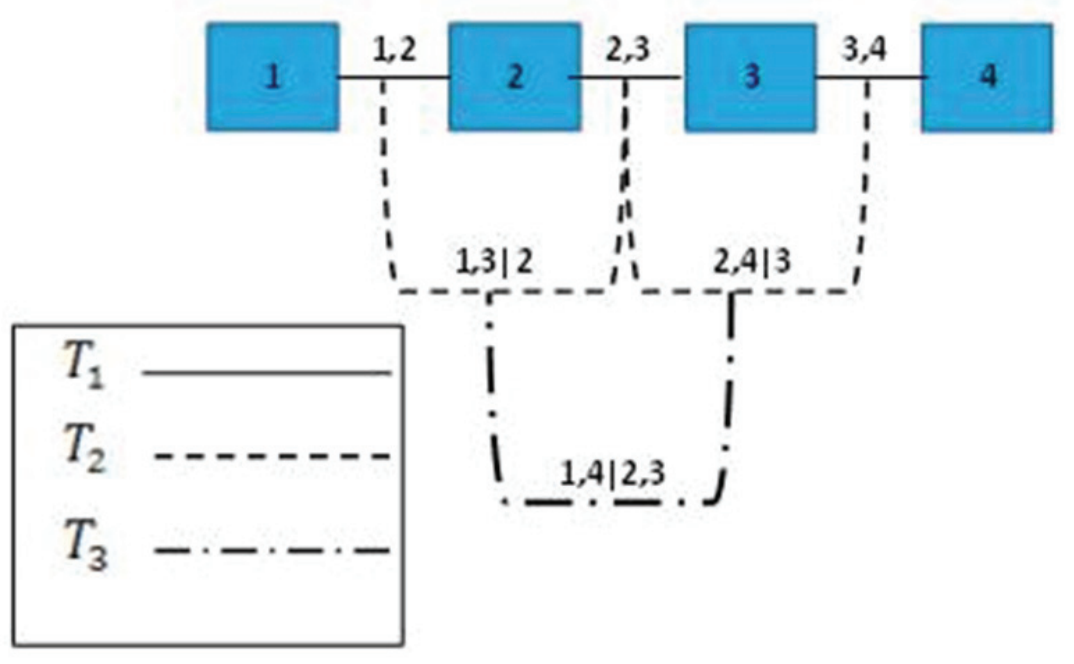

Table 1: Data Adjusted after Removing Serial Correlation from Data

\begin{tabular}{|c|c|c|}
\hline Data & Model & Distribution Error \\
\hline Oil Revenue & $\operatorname{ARMA}(1,1)-G A R C H(1,1)$ & Skewed Normal \\
\hline Economic Growth & GARCH(1,1) & Skewed t-student \\
\hline Total Consumption & $\operatorname{ARMA}(1,1)-G A R C H(1,1)$ & t-student \\
\hline Investment & $\operatorname{AR}(1)-G A R C H(1,1)$ & Student \\
\hline
\end{tabular}

Considering a regular vine (as shown in Figure 1), the Bayesian inference for these variables can be carried out. According the vine structure, the formula for these variables is

$c\left(u_{A}, u_{B}, u_{C}, u_{D}\right)=c_{A B} \cdot c_{B C} \cdot c_{C D} \cdot c_{A C \mid B} \cdot c_{B D \mid C} \cdot c_{A D \mid B C}$

The building pair-copulas of the PCC model (2) are now specified as bivariate $t$ copulas; however, the methodology is generic and applies more widely. Further it is assumed that the margins of $\mathrm{X}$ are uniform. This is motivated by the standard semi-parametric copula estimation procedure suggested by Genest, et al. (1995) where approximate uniform margins are obtained by applying the empirical probability integral transformation to multivariate data.

The bivariate t-copula (Embrechts, et al., 2003) has 2 parameters: the association parameter $\rho \in(-1,1)$ and the df parameter $\vartheta \in$ $(0, \infty)$ and its density is given by 


\section{ZADKARAMI \& CHATRABGOUN}

$c\left(u_{1}, u_{2} \mid \vartheta, \rho\right)=$

$\frac{\Gamma\left(\frac{\vartheta+1}{2}\right) \Gamma\left(\frac{\vartheta}{2}\right)}{\sqrt{1-\rho^{2}}\left[\Gamma\left(\frac{\vartheta+1}{2}\right)\right]^{2}}$
$\frac{\left.\left(1+\frac{\left(t_{\vartheta}^{-1}\left(u_{1}\right)\right)^{2}}{\vartheta}\right]\left[1+\frac{\left(t_{\vartheta}^{-1}\left(u_{2}\right)\right)^{2}}{\vartheta}\right]\right)^{\frac{\vartheta+1}{2}}}{\left(1+\frac{\left(t_{\vartheta}^{-1}\left(u_{1}\right)\right)^{2}+\left(t_{\vartheta}^{-l}\left(u_{2}\right)\right)^{2}-2 \rho t_{\vartheta}^{-1}\left(u_{1}\right) \cdot t_{\vartheta}^{-1}\left(u_{2}\right)}{\vartheta\left(1-\rho^{2}\right)}\right)^{\frac{\vartheta+1}{2}}}$

wheret $\vartheta^{\wedge}(-1)$ (.) is a quantile function of a $t-$ distribution with $\vartheta$ degrees of freedom.

The conditional distribution function for $\mathrm{x}=\mathrm{u} \_1$ and a scalar $\mathrm{v}=\mathrm{u} \_2$ takes the form

$$
\begin{aligned}
& h\left(u_{1} \mid u_{2}, \rho, \vartheta\right)= \\
& t_{\vartheta+1}\left(\frac{t_{\vartheta}^{-1}\left(u_{1}\right)-\rho t_{\vartheta}^{-1}\left(u_{2}\right)}{\sqrt{\frac{\left(\vartheta+\left(t_{\vartheta}^{-1}\left(u_{2}\right)\right)^{2}\right)\left(1-\rho^{2}\right)}{\vartheta+1}}}\right)
\end{aligned}
$$

and is called the h-function for the t-copula with parameters $\rho$ and $\vartheta$ (Aas, et al. 2007). The parameters of the model used for this study are:

$$
\begin{aligned}
\theta= & \left(\rho_{1,2}, \vartheta_{1,2}, \rho_{2,3}, \vartheta_{2,3}, \rho_{3,4}, \vartheta_{3,4}, \rho_{1,3 \mid 2},\right. \\
& \left.\vartheta_{1,3 \mid 2}, \rho_{2,4 \mid 3}, \vartheta_{2,4 \mid 3}, \rho_{1,4 \mid 2,3}, \vartheta_{1,4 \mid 2,3}\right)
\end{aligned}
$$

Because a Bayesian approach was followed, the statistical model must be completed by specifying the prior distributions for all model parameters. A uniform $(-1,1)$ prior is specified for the association parameter $\rho$ of a t-copula pair and a uniform $(1, U)$ prior for the corresponding df parameter $\vartheta$ because, in general, little prior information is available. Here the lower cut value 1 was chosen instead of 0 to avoid numerical instabilities in evaluating a quantile function of the bivariate t-distribution. The upper cut value $U$ can be chosen by the data analyst to assess the closeness to the bivariate Gaussian copula. Finally, it was assumed that prior distributions for $\rho$ and $\vartheta$ are independent within each pair and independent over all pairs.
For estimating the MCMC parameters the package bivariate $t$ distribution in Winbugs 14 software was used (in other tree of the vine structure this is conducting using an hfunction). By pre-specified prior distribution for $\rho$ and prior distribution $U(1,100)$ for $\vartheta$ and 80,000 a Metropolis-Hasting iteration algorithm MCMC estimation of the parameter can be obtained. The results of the Bayesian estimation and MlE are summarized in Table 2.

Based on results (see Table 2 and Figure 2) it can be concluded that the association parameter is unimodal and symmetric, the difference among mode, mean and median is negligible, and the degree of freedom is asymmetric. Based on the $95 \%$ credible interval, the dependency structure among variables can be simplified as:

$$
c\left(u_{A}, u_{B}, u_{C}, u_{D}\right)=c_{A B} \cdot c_{B C} \cdot c_{C D} \cdot c_{B D \mid C} \cdot c_{A D \mid B C}
$$

Conclusion

Bayesian inference provides solutions for many difficult problems that are not simple to solve in a classical ML framework. This study shows how identifying unconditional as well as conditional independence in macroeconomic variables can simplify resulting PCC's. Results show that the independence between oil revenue and total consumption given economic growth in these data is significant.

\section{Acknowledgement}

The authors wish to acknowledge the reviewer for his/her helpful and insightful comments to the manuscript. The authors also wish to acknowledge discussions with Dr. Gholamali Parham and Dr. Alireza Daneshkhah.

\section{References}

Aas, K., Czado, C., Frigessi, A., \& Bakken, H. (2007). Pair-copula constructions of multiple dependence. To appear in Insurance: Mathematics and Economics. DOI 10.1016/j.insmatheco.2007.02.001.

Bedford, T., \& Cooke, R. M. (2001). Probability density decomposition for conditionally dependent random variables modeled by vines. Annals of Mathematics and Artificial Intelligence, 32(1-4), 245-268. 


\section{BAYESIAN INFERENCE OF PAIR-COPULA CONSTRICTION FOR MODELING}

Table (2): Bayesian Estimation of Parameters

\begin{tabular}{|c|c|c|c|c|c|c|}
\hline Parameter & $2.5 \%$ Quantile & $\begin{array}{c}\text { Estimated } \\
\text { Median }\end{array}$ & $\begin{array}{c}97.5 \% \\
\text { Quantile }\end{array}$ & $\begin{array}{c}\text { Estimated } \\
\text { Post. Mean }\end{array}$ & $\begin{array}{c}\text { Estimated } \\
\text { Post. Mode }\end{array}$ & MLE \\
\hline$\rho_{1,2}$ & 0.1849 & 0.4431 & 0.7 & 0.4431 & 0.4422 & 0.4465 \\
\hline$\vartheta_{1,2}$ & 0.2475 & 0.3743 & 0.6568 & 0.3941 & 0.3655 & 0.3422 \\
\hline$\rho_{2,3}$ & 3.415 & 4.315 & 5.222 & 4.316 & 4.315 & 4.316 \\
\hline$\vartheta_{2,3}$ & 1.014 & 1.476 & 2.42 & 1.538 & 1.451 & 1.443 \\
\hline$\rho_{3,4}$ & 0.7843 & 1.398 & 2.016 & 1.399 & 1.389 & 1.401 \\
\hline$\vartheta_{3,4}$ & 0.6903 & 1.006 & 1.648 & 1.047 & 1.001 & 1.017 \\
\hline$\rho_{1,3 \mid 2}$ & -0.9819 & -0.01785 & 0.9422 & -0.01772 & -0.0187 & -0.0188 \\
\hline$\vartheta_{1,3 \mid 2}$ & 0.1661 & 0.5109 & 1.168 & 0.5518 & 0.491 & 0.489 \\
\hline$\rho_{2,4 \mid 3}$ & 0.8218 & 1.223 & 1.623 & 1.223 & 1.24 & 1.24 \\
\hline$\vartheta_{2,4 \mid 3}$ & 0.9594 & 2.954 & 6.757 & 3.191 & 2.02 & 2.08 \\
\hline$\rho_{1,4 \mid 2,3}$ & -0.7009 & -0.4408 & -0.1818 & -0.4408 & -0.441 & -0.441 \\
\hline$\vartheta_{1,4 \mid 2,3}$ & 2.285 & 7.031 & 16.09 & 7.596 & 6.642 & 6.831 \\
\hline
\end{tabular}

Bedford, T., \& Cooke, R. M. (2002). Vines: A new graphical model for dependent random variables. Annals of Statistics, 30(4), 1031-1068.

Berg, D., \& Aas, K. (2007). Models for construction of multivariate dependence. Discussion Paper SAMBA/23/07.

Bollerslev, T. (1986). Generalized autoregressive conditional heteroscedasticity. Journal of Econometrics, 31, 307-327.

Dalla Valle, L. (2007). Bayesian copulae distributions with application to operational risk management. To appear in Methodology and Computing in Applied Probability. DOI 10.1007/s11009-007- 9067-x.
Embrechts, P., Lindskog, F., \& McNeil, A. J. (2003). Modelling dependence with copulas and applications to risk management. In Handbook of heavy tailed distributions in finance, 31, 307-327. Amsterdam: Elsevier/North-Holland.

Embrechts, P., McNeil, A. J., \& Straumann, D. (2002). Correlation and dependence in risk management: Properties and pitfalls. In Risk management: Value at risk and beyond, M. Dempster (Ed.), 176-223. Cambridge, MA: Cambridge University Press. 


\section{ZADKARAMI \& CHATRABGOUN}

Figure 2: Plot of the Bayesian Estimation of the Parameters

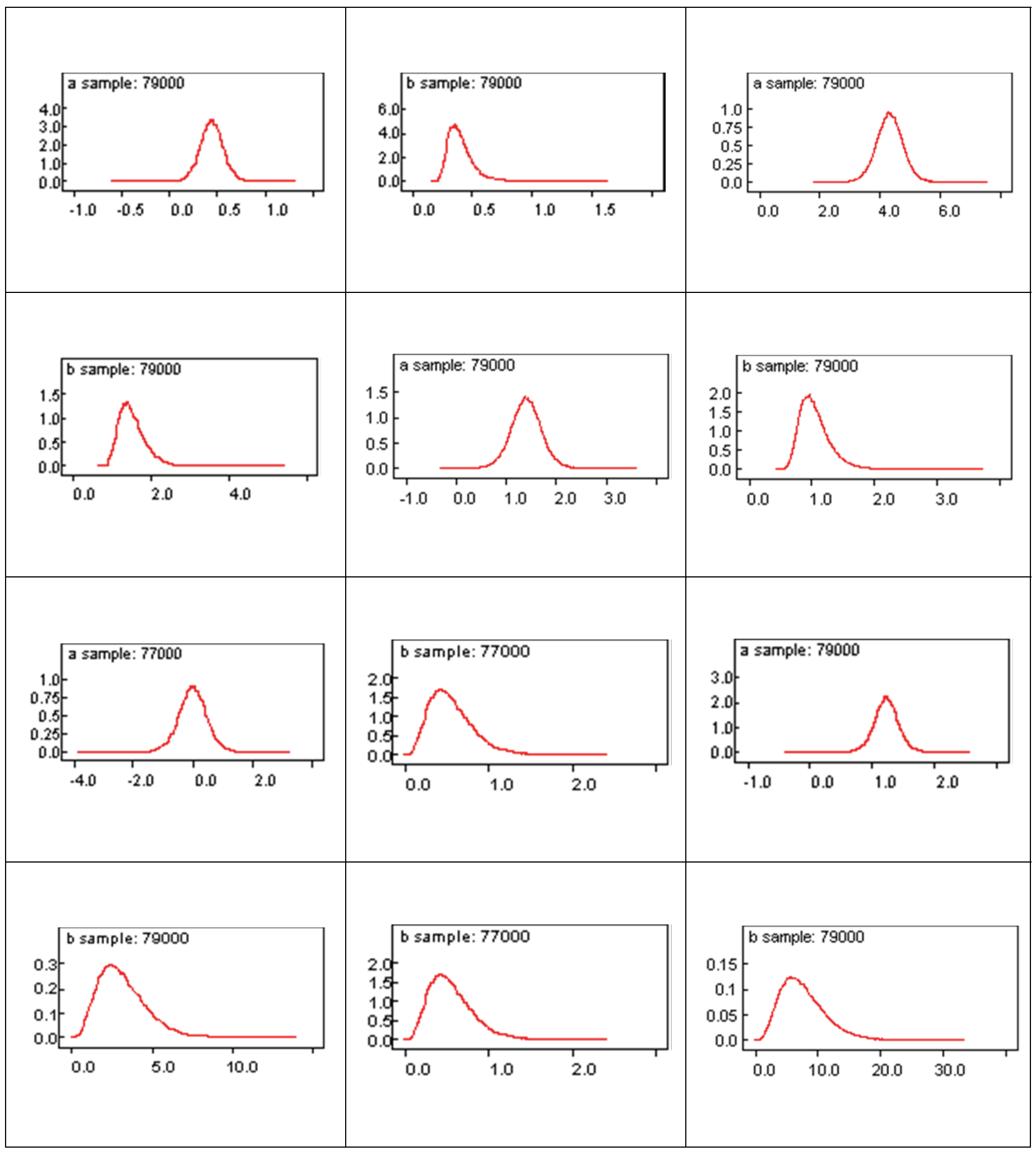




\section{BAYESIAN INFERENCE OF PAIR-COPULA CONSTRICTION FOR MODELING}

Fischer, M., Kock, C., Schluter, S., \& Weigert, F. (2007).An empirical analysis of multivariate copula models.Discussion Paper, http://www.statistik.wiso.unierlangen.de/

lehrstuhl/mfischer/mfwork.html.

Frahm, G., Junker, M., \&Szimayer, A. (2003). Elliptical copulas: Applicability and limitations. Statistics \& Probability Letters, 63(3), 275-286.

Frey, R., \& McNeil, A. J. (2003). Dependent defaults in models of portfolio credit risk. Journal of Risk, 6(1), 59-92.

Genest, C., Ghoudi, K., \&Rivest, L-P. (1995). A semiparametric estimation procedure of dependence parameters in multivariate families of distributions. Biometrika, 82(3), 543552.

Hastings, W. (1970). Monte Carlo sampling methods using Markov chains and their applications. Biometrika, 57, 97-109.

Joe, H. (1996). Families of m-variate distributions with given margins and $m(m-1) / 2$ bivariate dependence parameters. In Distributions with fixed marginals and related topics, L. Ruschendorf, B. Schweizer \& M. D. Taylor (Eds.), 120-141. Hayward: Institute of Mathematical Statistics.

Joe, H. (1997). Multivariate models and dependence concepts, volume 73: Monographs on statistics and applied probability. London: Chapman \& Hall.

Kurowicka, D., \& Cooke, R. (2006). Uncertainty analysis with high dimensional dependence modelling. Chichester: John Wiley $\&$ Sons.

McNeil, A. J. (2007). Sampling nested Archimedean copulas. To appear in Journal of Statistical Computation and Simulation.

McNeil, A. J., Frey, R., \& Embrechts, P. (2006). Quantitative risk management: Concepts, techniques and tools. Princeton, NJ: Princeton University Press.
Metropolis, N., et al. (1953). Equations of state calculations by fast computing machines. Journal of Chemical Physics, 21, 1087-1092.

Min, A., \& Czado, C. (2011). Bayesian inference for multivariate copulas using paircopula constructions. Journal of Financial Econometrics, 8(4), 511-546.

Nelsen, R. B. (1999). An introduction to copulas, volume 139: Lecture notes in statistics. New York, NY: Springer-Verlag.

Nolte, I. (2008). Modeling a multivariate transaction process.Journal of Financial Econometrics, 6, 143-170.

Patton, A. J. (2004). On the out-ofsample importance of skewness and asymmetric dependence for asset allocation. Journal of Financial Econometrics, 2, 130-168.

Pitt, M., Chan, D., \& Kohn, R. (2006). Efficient Bayesian inference for Gaussian copula regression models. Biometrika, 93(3), 537-554.

Savu, C., \& Trede, M. (2006). Hierarchical Archimedean copulas. In International Conference on High Frequency Finance, 109-116. Konstanz, Germany, May.

Sklar, M. (1959). Fonctions de $\mathrm{r}^{\prime}$ epartition 'a $\mathrm{n}$ dimensions et leursmarges. Publications de l'Institut de Statistique de l'Université de Paris, 8, 229-231.

Whelan, N. (2004). Sampling from Archimedean copulas. Quantitative Finance, 4(3), 339-352.

Wong, F., Carter, C. K., \& Kohn, R. (2003). Efficient estimation of covariance selection models. Biometrika, 90(4), 809-830. 


\title{
Fitting Proportional Odds Models to Educational Data with Complex Sampling Designs in Ordinal Logistic Regression
}

\author{
Xing Liu Hari Koirala \\ Eastern Connecticut State University, \\ Willimantic, CT
}

The conventional proportional odds (PO) model assumes that data are collected using simple random sampling by which each sampling unit has the equal probability of being selected from a population. However, when complex survey sampling designs are used, such as stratified sampling, clustered sampling or unequal selection probabilities, it is inappropriate to conduct ordinal logistic regression analyses without taking sampling design into account. Failing to do so may lead to biased estimates of parameters and incorrect corresponding variances. This study illustrates the use of PO models with complex survey data to predict mathematics proficiency levels using Stata and compare the results of PO models accommodating and not accommodating survey sampling features.

Key words: Ordinal logistic regression, PO models, complex survey designs, linearization, standard errors, single sampling unit, Stata.

\section{Introduction}

Ordinal logistic regression is an extension, or a special case, of binary logistic regression when an ordinal outcome variable has more than two levels. The three commonly known models for an ordinal outcome variable include the proportional odds (PO) model, the continuation ratio $(\mathrm{CR})$ model and the adjacent category $(\mathrm{AC})$ logistic regression model, depending on different comparisons among the categories of the response variable. Compared to the other two models, the PO model is the most popular

Xing Liu, Ph.D., is an Associate Professor of Research and Assessment in the Education Department at Eastern Connecticut State University. His research interests include educational assessment, categorical data analysis, longitudinal data analysis and multilevel modeling. Email him at: liux@easternct.edu. Hari Koirala, Ph.D., is Professor in the Education Department at Eastern Connecticut State University. His research interests include mathematics education and assessment. Email him at: koiralah@easternct.edu.
(Agresti, 1996, 2002, 2007, 2010; Anath \& Kleinbaum, 1997; Armstrong \& Sloan, 1989; Hardin \& Hilbe, 2007; Hilbe, 2009; Liu, 2009; Long, 1997; Long \& Freese, 2006; McCullagh, 1980; McCullagh \& Nelder, 1989; O'Connell, 2000, 2006; O'Connell \& Liu, 2011; Powers \& Xie, 2000). The PO model is widely implemented as the default for ordinal regression analysis in general-purpose statistical software packages, such as SAS, SPSS, Stata, SPlus and R. The PO model estimates the relationship between a set of predictor variables and an ordinal outcome variable via a logit link function. It is also known as the cumulative logit model, because it estimates the cumulative odds of being at or below a particular level of the response variable. In addition, for each predictor variable the estimated cumulative odds are assumed to be the same across all the ordinal categories, thus it is known as the proportional odds assumption.

The conventional PO model assumes that data are collected using simple random sampling by which each sampling unit has an equal probability of being selected from a population. In addition to simple random sampling, researchers also use more complex sampling techniques, such as stratified sampling and multistage cluster sampling. The National 


\section{PO MODELS WITH COMPLEX SURVEY DATA}

Center for Education Statistics (NCES) sponsored and conducted a series of studies, such as the Early Childhood Longitudinal StudyKindergarten (ECLS-K), National Educational Longitudinal Study of 1988 (NELS:88), and Educational Longitudinal Study of 2002 (ELS:2002), all of which applied complex sampling survey designs. These designs had the common features of using strata, clusters and unequal probability of selection in data collection.

Because complex survey sampling designs involve the use of different strata (e.g., geographic areas), clustered sampling techniques and unequal selection probabilities, it is inappropriate to conduct the PO model analysis for the ordinal response variable without taking the survey sampling designs into account. Failing to do so may lead to biased estimates of parameters, incorrect variance estimates and misleading results. Therefore, it is critical for researchers to understand techniques for analyzing data with complex sampling designs.

Although multilevel modeling is a valuable tool for analyzing complex sampling survey data, it is mainly used for model-based analysis (Hahs-Vaughn, 2005; Thomas \& Heck, 2001) when data structures are nested or hierarchical (O'Connell \& McCoach, 2008; Raudenbush \& Bryk, 2002). When the data structure only has a single level, researchers need to identify other appropriate methods which take complex sampling design features into account. Analysis that considers these design features is termed the design-based analysis (Lee \& Forthofer, 2006; Levy \& Lemeshow, 2008), compared to the model-based analysis (e.g., multilevel analysis). Although several strategies were commonly used for design-based analysis of survey data, using specialized software to accommodate complex sampling designs was the most desirable choice (Hahs-Vaughn, 2005; Thomas \& Heck, 2001). Among a few statistical software packages that can perform statistical analyses within the context of survey sampling designs, Stata is a well-known statistical package and is capable of performing numerous statistical analyses for complex survey data with its svy prefix command (StataCorp, 2007, 2009, 2011).
Various methods of incorporating weights and design effects in statistical models, such as multiple regression (Hahs-Vaughn, 2005, 2006; Thomas \& Heck, 2001), and structural equation modeling (Hahs-Vaughn \& Lomax, 2006; Muthen \& Setorra, 1995; Stapleton, 2002, 2006, 2008) have been proposed and examples were illustrated. However, the use of complex sampling designs in ordinal logistic regression analysis is scarce. In addition, although researchers are increasingly interested in conducting secondary data analysis using large-scale datasets, the lack of analytic skills makes the task intimidating. Therefore, it is imperative to help educational researchers better understand the ordinal logistic regression model with complex sampling data and utilize it in practice. This study illustrates the use of ordinal logistic regression models with complex survey data to predict mathematics proficiency levels using Stata and compares the results of PO models accommodating and not accommodating survey sampling features, such as stratification, clustering and weights. This article extends previous research which focused on different types of conventional ordinal logistic regression models (Liu, 2009; Liu, O'Connell, \& Koirala, 2011, Liu \& Koirala, 2012).

For demonstration purposes, ordinal regression analyses were based on data from the Educational Longitudinal Study (ELS): 2002, in which the ordinal outcome of students' mathematics proficiency was predicted from a set of variables in students' effort, such as, students can get no bad grades if they decide not to, they keep studying even if material is difficult, and they do best to learn.

Theoretical Framework: The Proportional Odds Model

In binary logistic regression, the outcome variable is dichotomous, with $1=$ success or experiencing an event and $0=$ failure or not experiencing the event. This model estimates the log odds of the outcome or the probability of success from a set of predictors. The logistic regression model (Menard, 1995) is: 


\section{XING LIU \& HARI KOIRALA}

$$
\begin{aligned}
\ln \left(Y^{\prime}\right) & =\operatorname{logit}[\pi(x)] \\
& =\ln \left(\frac{\pi(\underline{x})}{1-\pi(\underline{x})}\right) \\
& =\alpha+\beta_{1} \mathrm{X}_{1}+\beta_{2} \mathrm{X}_{2}+\ldots+\beta_{\mathrm{p}} \mathrm{X}_{\mathrm{p}}
\end{aligned}
$$

where logit $[\pi(\underline{x})]$ is the log odds of success, and the odds is a ratio between the probability of having an event and the probability of not having that event.

When the outcome variable has more than two levels and is ordinal, the ordinal logistic regression model estimates the odds and the probabilities of being at or below a particular category. The ordinal regression model can be expressed on the logit scale as follows (Liu, 2009):

$$
\begin{aligned}
\ln \left(\mathrm{Y}_{\mathrm{j}}^{\prime}\right) & =\operatorname{logit}\left[\pi_{\mathrm{j}}(\mathrm{x})\right] \\
& =\ln \left(\frac{\pi_{\mathrm{j}}(\mathrm{x})}{1-\pi_{\mathrm{j}}(\mathrm{x})}\right) \\
& =\alpha_{\mathrm{j}}+\left(-\beta_{1} \mathrm{X}_{1}-\beta_{2} \mathrm{X}_{2}-\ldots-\beta_{\mathrm{p}} \mathrm{X}_{\mathrm{p}}\right),
\end{aligned}
$$

where $\pi_{\mathrm{j}}(\underline{\mathrm{x}})=\pi\left(\mathrm{Y} \leq \mathrm{j} \mid \mathrm{x}_{1}, \mathrm{x}_{2}, \ldots \mathrm{x}_{\mathrm{p}}\right)$, which is the probability of being at or below category $j$, given a set of predictors. $\mathrm{j}=1,2, \ldots, \mathrm{J}-1 . \alpha_{\mathrm{j}}$ are the cut points, and $\beta_{1}, \beta_{2}, \ldots, \beta_{\mathrm{p}}$ are logit coefficients. This PO model estimates different cut points, but the effect of any predictor is assumed to be the same across these cut points. Therefore, for each predictor only one logit coefficient is estimated. The proportional odds assumption can be assessed by the Brant test (Brant, 1990), which provides the omnibus test for the overall models and the univariate test for each predictor. To estimate the cumulative odds of being at or below the $\mathrm{j}^{\text {th }}$ category, this model can be rewritten as:

$$
\begin{aligned}
\operatorname{logit}\left[\pi\left(Y \leq j \mid x_{1}, x_{2}, \ldots, X_{p}\right)\right] & =\ln \left(\frac{\pi\left(Y \leq j \mid x_{1}, x_{2}, \ldots x_{p}\right)}{\pi\left(Y>j \mid x_{1}, x_{2}, \ldots x_{p}\right)}\right) \\
& =\alpha_{j}+\left(-\beta_{1} X_{1}-\beta_{2} X_{2}-\ldots-\beta_{p} X_{p}\right)
\end{aligned}
$$

where logit is the log odds of being at or below a particular category relative to being beyond that category. By exponentiating the cumulative logits, we obtain the cumulative odds of being at or below the $j^{\text {th }}$ category. The PO model includes a series of binary logistic regression models where the ordinal response variable is dichotomized while assuming the estimated logit coefficients are the same across these binary models.

Researchers should be aware that software packages may use different forms to express the ordinal logistic regression model and parameterize it differently (Liu, 2009). For example, unlike Stata and SPSS, which both follow the above equation; SAS does not negate the signs before logit coefficients in the equation. To estimate cumulative odds of being at or below a particular category, the SAS PROC LOGISTIC procedure can be used with the ascending option, while the descending option can be applied to the same procedure to estimate the odds of being beyond a particular category.

Theoretical Framework: Variance Estimation in Complex Survey Sampling

Two techniques are widely used for unbiased variance estimation in complex sampling survey designs, including linearization and replicated sampling methods (Lee \& Forthofer, 2006; Levy \& Lemeshow, 2008; Lohr, 1999). The linearization method is the Taylor series approximation, also known as the delta method (Kalton, 1983); while the replicated methods estimate variance of a parameter by generating replicated subsamples and examining the variability of the subsample estimates. The replicated methods, also referred to as resampling methods, include the balanced repeated replication (BRR), the jackknife repeated replication (JRR) and the bootstrap method (Lee \& Forthofer, 2006; Levy \& Lemeshow, 2008). This article focuses on the Taylor series approximation method because it is implemented as the default in general purpose software packages, such as Stata, SAS, SPSS (Complex Samples Add-on Module) and in specialized software, such as SUDAAN, and AM. 


\section{PO MODELS WITH COMPLEX SURVEY DATA}

The general Taylor series linearization is expressed as (Lee \& Forthofer, 2006; Levy \& Lemeshow, 2008):

$f(x)=f(a)+f^{\prime}(a)(x-a)+\frac{f^{\prime \prime}(a)(x-a)^{2}}{2 !}+\ldots$

where $f^{\prime}$ and $f^{\prime \prime}$ are the first and second derivatives of the function, $f(x)$ at $a$. In statistics, the Taylor series linearization is used to obtain a linear approximation to the nonlinear function or statistic and then the variance of the function or statistic can be derived from the Taylor series approximation.

Specifically, the variance estimation in complex survey sampling using the Taylor series expansion follows two steps. First, use the firstorder Taylor series to obtain a linear approximation of the function. Second, estimate the variance of the parameter including complex survey features, such as strata, cluster and weight variables. Thus, the variance estimate is a weighted combination of the variance across primary sampling units (PSUs) within a stratum (Lee \& Forthofer, 2006). In statistical software packages it is necessary to specify strata, cluster and weights before fitting a statistical model.

To estimate sampling variance of a parameter estimate in ordinal logistic regression, Binder (1983) developed a general formula for linear regression and generalized linear models with the complex survey data using the Taylor series, which is widely used and known as the sandwich variance estimator. In the sandwich form, the middle variance-covariance matrix of the weighted score function is multiplied at both the left and right sides by the inverse of the matrix of second derivatives with respect to the parameter estimate (Binder, 1983; Heeringa, West, \& Berglund, 2010).

Sample

$$
\text { Methodology }
$$

The base-year data from the Educational Longitudinal Study (ELS) of 2002 was used for the analyses. This study, conducted by the National Center for Education Statistics (NCES), longitudinally followed students from $10^{\text {th }}$ grade to their postsecondary school education and/or even in their work. ELS used a two-stage sampling design (Ingels, et al., 2004; 2005). First, using a stratified sampling strategy, 1,221 eligible public and private schools were selected from a population of approximately 25,000 schools with $10^{\text {th }}$ grade students: of the eligible schools (clusters), 752 agreed to participate in the study. Second, in each of the schools, approximately 25 students in $10^{\text {th }}$ grade were randomly selected from the enrollment list.

The outcome variable of interest was students' mathematics proficiency levels in high school, which was an ordinal categorical variable with five levels $(1=$ capable of doing simple arithmetical operations on whole numbers; 2 = capable of doing simple operations with decimals, fractions, powers and root; $3=$ capable of doing simple problem solving; $4=$ understanding intermediate-level mathematical concepts and/or finding multi-step solutions to word problems; and 5 = capable of solving complex multiple-step word problems and/or understanding advanced mathematical material) (Ingels, et al., 2004, 2005). Those students who failed to pass through level 1 were assigned to level 0 . Table 1 provides the frequency of six mathematics proficiency levels (Liu, \& Koirala, 2012).

Data Analysis

First the PO model was fitted without considering the complex sampling designs using the Stata ologit command. Stata SPost (Long \& Freese, 2006) package was used to examine the fit statistics and the PO assumption. The same PO model was then fitted with Stata ologit with weights. Finally, svy, the Stata's survey data command was used to fit the PO model taking all the elements of survey design features, such as strata, cluster and weight variables into account. Before using the svy prefix command, the svyset command was employed to specify the complex sampling design features; the svy: ologit command was then used to conduct the subsequent ordinal regression analysis.

When a stratum contains only a single sampling unit, standard errors of the parameters are estimated to be missing. To deal with this issue, three singleunit() options (i.e., certainty, scaled and centered) were specified separately in the svyset command and the estimated standard 
XING LIU \& HARI KOIRALA

Table 1: Proficiency Categories and Frequencies (Proportions)

\begin{tabular}{|c|c|c|}
\hline \multicolumn{2}{|c|}{ for the Study Sample, ELS (2002) (N =15,976) } \\
\hline Proficiency & Description & $\begin{array}{c}\text { Frequency } \\
(\%)\end{array}$ \\
\hline 0 & Did not reach level 1 & $\begin{array}{c}842 \\
(5.27 \%)\end{array}$ \\
\hline 1 & Capable of doing simple arithmetical \\
operations on whole numbers & $\begin{array}{c}3,882 \\
(24.30 \%)\end{array}$ \\
\hline 2 & $\begin{array}{c}\text { Capable of doing simple operations with } \\
\text { decimals, fractions, powers and root }\end{array}$ & $\begin{array}{c}3,422 \\
(21.42 \%)\end{array}$ \\
\hline 3 & \begin{tabular}{c} 
Capable of doing simple problem solving \\
\multirow{2}{*}{3}
\end{tabular} & $\begin{array}{c}4,521 \\
(28.30 \%)\end{array}$ \\
\hline 5 & $\begin{array}{c}\text { Understanding intermediate-level } \\
\text { mathematical concepts and/or finding }\end{array}$ & $\begin{array}{c}3,196 \\
(20.01 \%)\end{array}$ \\
\hline \multirow{2}{*}{ multi-step solutions to word problems } & $\begin{array}{c}\text { Capable of solving complex multiple-step } \\
\text { word problems and/or understanding } \\
\text { advanced mathematical material }\end{array}$ & $\begin{array}{c}113 \\
(0.71 \%)\end{array}$ \\
\hline
\end{tabular}

errors from each of the three PO models were examined. The Taylor series approximation linearization method, which is the default method in Stata, was used to estimate the sampling variance. The results of the PO models accommodating and ignoring complex sampling designs were compared.

\section{Results}

Proportional Odds Model with Three Explanatory Variables without Weights

A PO model with all three predictor variables was fitted first. Stata ologit command was used for model fitting. Figures 1 and 2 show the results for the PO model without weights (Unweighted).

The log likelihood ratio chi-square test, LR $\chi_{(3)}^{2}=1102.83, \mathrm{p}<0.001$, indicated that the model with three predictors provides a better fit than the null model with no independent variables. The likelihood ratio, $R_{L}^{2}=0.034$, suggested that the relationship between the response variable, mathematics proficiency and the predictors was small.

All logit effects of the three predictors on the mathematics proficiency level were significant. The estimated logit regression coefficient for getting no bad grades if deciding to (decide), $\beta=0.509, \mathrm{z}=20.79, \mathrm{p}<0.001$; the logit coefficient for keeping studying if material is difficult (keeplrn), $\beta=0.060, \mathrm{z}=2.15, \mathrm{p}=$ 0.0311 ; and finally, for doing best to learn (dobest), $\beta=0.184, \mathrm{z}=6.60, \mathrm{p}<0.001$.

To estimate the cumulative odds of being at or below a certain mathematics proficiency level, it is only necessary to substitute the values of the estimated logit coefficients into the equation (3). For the first predictor, decide, logit $\left[\pi\left(\mathrm{Y} \leq \mathrm{j} \mid \mathrm{X}_{1}\right)\right]=$ $\alpha_{\mathrm{j}}+\left(-.509 \mathrm{X}_{1}\right)$. OR $=\mathrm{e}^{(-.509)}=.601$, suggesting that the odds of being at or below a particular proficiency level decreased by a factor of 0.601 with a one unit increase in the value of the predictor variable, getting no bad grades if 


\section{PO MODELS WITH COMPLEX SURVEY DATA}

Figure 1: Stata Proportional Odds Model with Three Explanatory Variables without Weights

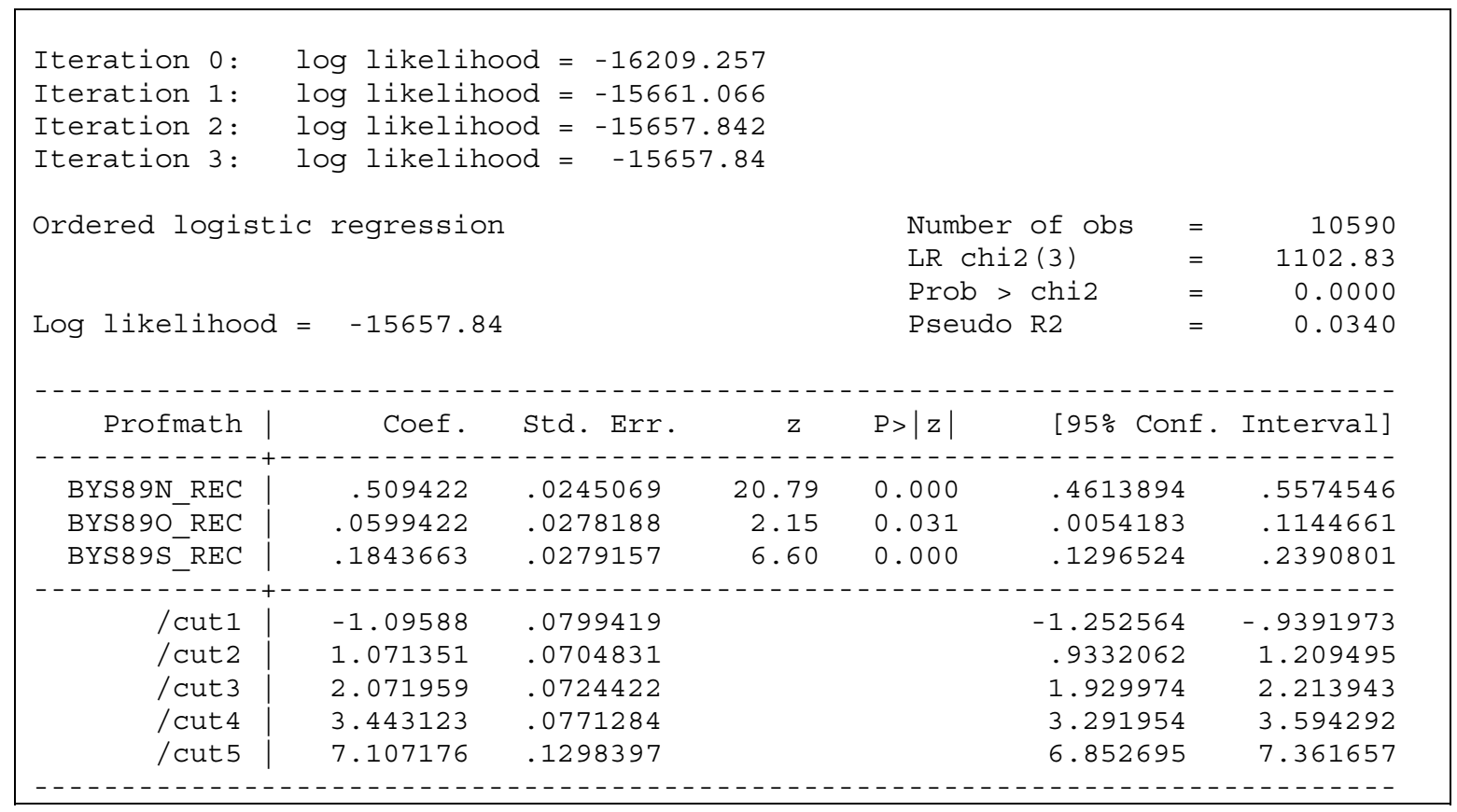

Figure 2: Measures of Fit Statistics Using Stata SPost package

\begin{tabular}{|lrlr|}
\hline fitstat & & & \\
Measures of Fit for ologit of Profmath & & \\
& & & \\
Log-Lik Intercept Only: & -16209.257 & Log-Lik Full Model: & -15657.840 \\
D(10582): & 31315.680 & LR(3): & 1102.833 \\
& & Prob > LR: & 0.000 \\
MCFadden's R2: & 0.034 & MCFadden's Adj R2: & 0.034 \\
ML (Cox-Snell) R2: & 0.099 & Cragg-Uhler(Nagelkerke) R2: & 0.104 \\
MCKelvey \& Zavoina's R2: & 0.097 & & 3.290 \\
Variance of Y*: & 3.643 & Variance of error: & 0.059 \\
Count R2: & 0.333 & Adj Count R2: & 31331.680 \\
AIC: & 2.959 & AIC*n: & -1075.030 \\
BIC: & -66754.755 & BIC': & 31331.680 \\
BIC used by Stata: & 31389.822 & AIC used by Stata: & \\
\end{tabular}

deciding to, holding others constant. In other words, students were more likely to be in a higher proficiency level with the increase of the frequency in the predictor, getting no bad grades if deciding to. The odds of being at or below a proficiency level for the other two predictors, keeplrn and dobest, were computed in the same way and they were 0.942 and 0.832 , respectively.

The odds of being beyond a category of mathematics proficiency are the inverse of those of being at or below a category. In equation (3), it is necessary to reverse the sign before the logit coefficients and take the exponential of the positive coefficients. All three predictors were positively associated with the odds of being beyond a proficiency level. In terms of odds ratio (OR), the odds of being beyond a proficiency level were 1.664 times greater with one unit increase in the frequency of getting no bad grades if deciding to, 1.062 times greater with one unit increase in the frequency of 


\section{XING LIU \& HARI KOIRALA}

keeping studying if material is difficult, and 1.202 times greater with a one-unit increase in the frequency of doing best to learn.

\section{Brant Test of the Proportional Odds Assumption}

The PO assumption of the ordinal logistic regression was tested using the brant command of the Stata SPost package (Long \& Freese, 2006). The Brant test provides results of a series of underlying binary logistic regression models across different category comparisons, the univariate test for each predictor, and the omnibus test for the overall model. Table 2 shows five associated binary logistic regression models for the full PO model where the ordinal response variable is dichotomized and each split compares $\mathrm{Y}>$ cat. $j$ to $\mathrm{Y} \leq$ cat. $j$. The effects of all three variables were similar across these five binary models. Among them, the logit coefficient of doing best to learn was the most stable across these five binary logistic regression models.

The Brant test was used to identify whether the effects of each predictor were the same across five splits after the visual examination of the above models. Table 3 presents $\chi^{2}$ tests and $\mathrm{p}$ values for the full PO model and separate predictors. The omnibus Brant test for the full model, $\chi_{12}^{2}=20.51, p=$ 0.058 , indicating that the proportional odds assumption for the full model was upheld. In addition, the univariate tests revealed that the PO assumptions were also tenable for the individual predictors.

Proportional Odds Model with Three Explanatory Variables with Weights

Next, the same PO model with weights was fitted. To fit this model, Stata ologit command with sampling weights was used. The probability weight, BYSTUWT, which was the student weights for the base year data, was specified in the model as [pweight = BYSTUWT]. Table 4 shows the result for the PO model with the estimation of weights.

The PO model with sampling weights used the pseudolikelihood instead of the true likelihood in the maximum likelihood estimation. The Wald Chi-Square test, $\chi_{(3)}^{2}=$ $744.25, \mathrm{p}<0.001$, indicated that the model with the three predictors provided a better fit than the null model with no independent variables. The pseudo $R^{2}=0.035$.

Table 4 presents a comparison of $\mathrm{PO}$ model results with and without weighted estimation. Compared to the unweighted PO model; all but the first cutpoints/intercepts slightly increased when sampling weights were specified in the PO model. Regarding the logit coefficients, the effect of one predictor (decide) increased and the other two, keeplrn and dobest, decreased. In addition, the standard errors of all three predictors increased. Specifically, when weights were applied to the PO model the estimated logit regression coefficient for getting no bad grades if deciding to (decide) increased by $4.1 \%$, and its standard error increased by $24 \%$, compared to those in the unweighted PO model; the logit coefficient for keeping studying if material is difficult (keeplrn) decreased by $15.4 \%$, and its standard error increased by $25 \%$; and the logit coefficient for doing best to learn (dobest) decreased by $14.3 \%$, with its standard error increased by $25 \%$.

In the unweighted PO model, the effects of all three predictors were significant. However, when weights were applied to the PO model, surprisingly only the first and last predictors were significant, and the second predictor (keeplrn) became insignificant, since the standard error was underestimated when weights were not applied.

Proportional Odds Model for Complex Survey Data Using Stata svy command

Finally, Stata's survey data svy prefix command was used to fit the PO model, taking all the elements of survey design features, such as strata, cluster and weight variables into account. Before fitting the model, the svyset command needed to be employed by specifying the complex sampling design variables and weights. In this example, the design features were specified as: svyset PSU [pweight = BYSTUWT], strata (STRAT_ID). In the svyset command, the variable name for the primary sampling units or clusters in the data was PSU; the probability weight, pweight, was the student weight for the based year data (BYSTUWT), and the strata was START_ID. Figure 3 presents the result of the specified sampling design information. 
Table 2: A Series (j-1=5) of Associated Binary Logistic Regression Models for the Full PO Model, where Each Split Compares $\mathrm{Y}>$ cat. $j$ to $\mathrm{Y} \leq$ cat. $j$

\begin{tabular}{|c|c|c|c|c|c|c|}
\cline { 2 - 7 } \multicolumn{1}{c|}{} & $\mathrm{Y}>0$ & $\mathrm{Y}>1$ & $\mathrm{Y}>2$ & $\mathrm{Y}>3$ & $\mathrm{Y}>4$ & $\begin{array}{c}\text { Brant Test } \\
\text { P Value }\end{array}$ \\
\hline Variable & Logit (b) & Logit (b) & Logit (b) & Logit (b) & Logit (b) & \\
\hline Constant & 1.413 & -.989 & -2.042 & -3.639 & -8.854 & \\
\hline decide & .502 & .494 & .507 & .533 & .910 & .309 \\
\hline keeplrn & -.017 & .018 & .060 & .096 & .296 & .263 \\
\hline dobest & .138 & .208 & .173 & .187 & .054 & .607 \\
\hline
\end{tabular}

Table 3: Brant Tests of the PO Assumption for Each Predictor and the Overall Model

\begin{tabular}{|c|c|c|}
\hline Variable & Test & P Value \\
\hline decide & $\chi^{2}{ }_{4}=4.79$ & .309 \\
\hline keeplrn & $\chi^{2}{ }_{4}=5.24$ & .263 \\
\hline dobest & $\chi^{2}{ }_{4}=2.71$ & .607 \\
\hline All (Full-model) & $\chi^{2}{ }_{12}=20.51$ & .058 \\
\hline
\end{tabular}

Table 4: Comparison of the PO Models with and without Weighted Estimation

\begin{tabular}{|c|c|c|c|c|c|c|}
\hline \multirow[b]{2}{*}{ Variable } & \multicolumn{3}{|c|}{ PO Model-Unweighted } & \multicolumn{3}{|c|}{ PO Model with Weights } \\
\hline & $\mathrm{b}(\mathrm{se}(\mathrm{b}))$ & OR & $\mathrm{P}$ & $\mathrm{b}(\mathrm{se}(\mathrm{b}))$ & OR & $\mathrm{P}$ \\
\hline$\alpha_{1}$ & -1.096 & & & -.955 & & \\
\hline$\alpha_{2}$ & 1.071 & & & 1.153 & & \\
\hline$\alpha_{3}$ & 2.072 & & & 2.153 & & \\
\hline$\alpha_{4}$ & 3.443 & & & 3.490 & & \\
\hline$\alpha_{5}$ & 7.107 & & & 7.245 & & \\
\hline decide & $\begin{array}{l}.509 * * \\
(.025)\end{array}$ & 1.664 & $<.001$ & $\begin{array}{l}.530 * * \\
(.031)\end{array}$ & 1.699 & $<.001$ \\
\hline keeplrn & $\begin{array}{l}.060^{*} \\
(.028)\end{array}$ & 1.062 & .031 & $\begin{array}{c}.052 \\
(.035)\end{array}$ & 1.054 & .131 \\
\hline dobest & $\begin{array}{c}.184 * * \\
(.028)\end{array}$ & 1.202 & $<.001$ & $\begin{array}{l}.161^{* *} \\
(.035)\end{array}$ & 1.175 & $<.001$ \\
\hline$L R R^{2}$ & .034 & & & .035 & & \\
\hline $\begin{array}{c}\text { Brant Test } \\
\text { (Omnibus } \\
\text { Test) }\end{array}$ & $\chi_{12}^{2}=20.51$ & & & $\mathrm{~N} / \mathrm{A}$ & & \\
\hline Model Fit & $\begin{array}{c}\chi^{2}{ }_{3}= \\
1102.83 * *\end{array}$ & & & $\begin{array}{c}\chi_{3}^{2}= \\
744.25 * *\end{array}$ & & \\
\hline
\end{tabular}

$* \mathrm{p}<0.05 ; * * \mathrm{p}<0.01$ 
Figure 3: Identifying the Sampling Design Variables and Weights Using the svyset Command

\begin{tabular}{|l|}
\hline . svyset PSU $[$ pweight $=$ BYSTUWT] , strata (STRAT_ID) \\
pweight: BYSTUWT \\
VCE: linearized \\
Single unit: missing \\
Strata 1: STRAT_ID \\
SU 1: PSU \\
FPC 1: < zero>
\end{tabular}

Table 5: Estimated Standard Errors from the PO Models for Complex Survey Data with Four Singleunit() Options

\begin{tabular}{|c|c|c|c|c|c|}
\hline Variable & $\mathrm{b}$ & $\begin{array}{c}\text { singleunit(missing) } \\
\mathrm{se}(\mathrm{b})\end{array}$ & $\begin{array}{c}\text { (certainty) } \\
\mathrm{se}(\mathrm{b})\end{array}$ & $\begin{array}{c}\text { (scaled) } \\
\mathrm{se}(\mathrm{b})\end{array}$ & $\begin{array}{c}\text { (centered) } \\
\text { se(b) }\end{array}$ \\
\hline$\alpha_{1}$ & -.955 &. & .1070 & .1072 & .1070 \\
\hline$\alpha_{2}$ & 1.153 &. & .0967 & .0968 & .0967 \\
\hline$\alpha_{3}$ & 2.153 &. & .1003 & .1004 & .1003 \\
\hline$\alpha_{4}$ & 3.490 &. & .1069 & .1070 & .1069 \\
\hline$\alpha_{5}$ & 7.245 &. & .1849 & .1851 & .1849 \\
\hline decide & $.530^{* *}$ &. & .0335 & .0336 & .0335 \\
\hline keeplrn & .052 &. & .0332 & .0333 & .0332 \\
\hline dobest & $.161^{* *}$ &. & .0396 & .0397 & .0396 \\
\hline
\end{tabular}

The result of the svyset output also indicated that by default missing values for the standard errors would be created when a stratum only contained a single sampling unit (single unit: missing). To deal with this singleton PSU issue, the svyset command provides the other three options (StataCorp, 2007), including certainty, scaled and centered. The first option, singleunit(certainty) recognizes the single sampling unit in a stratum as a certainty unit (sampling unit chosen with 100\% certainty), which contributes nothing to variance estimation across sampling units. The second option, singleunit(scaled) is a scaled version of the first one, which uses the average variance of the strata with multiple PSUs for the stratum with a single sampling unit. The third option, singleunit(centered) uses the grand mean across sampling units for variance estimation.
Each of these three options for the single unit was used separately in the svyset command because single sampling units resulted in missing standard errors in the model. Stata svy: ologit was then used to conduct for each survey ordinal logistic regression analysis. The results of the estimated standard errors using all singleunit options are shown in Table 5 and, because the singltunit(missing) is the default option, the missing values for standard error estimations are also provided.

The results of the standard errors estimated from all PO models were nearly the same. Therefore, only the result with the singleunit(certainty) option was reported in the following analysis. Figure 4 and Table 6 display the PO model result for complex survey data using svy: ologit. 


\section{PO MODELS WITH COMPLEX SURVEY DATA}

Figure 4: PO Model for Complex Survey Data Using Stata svy: ologit

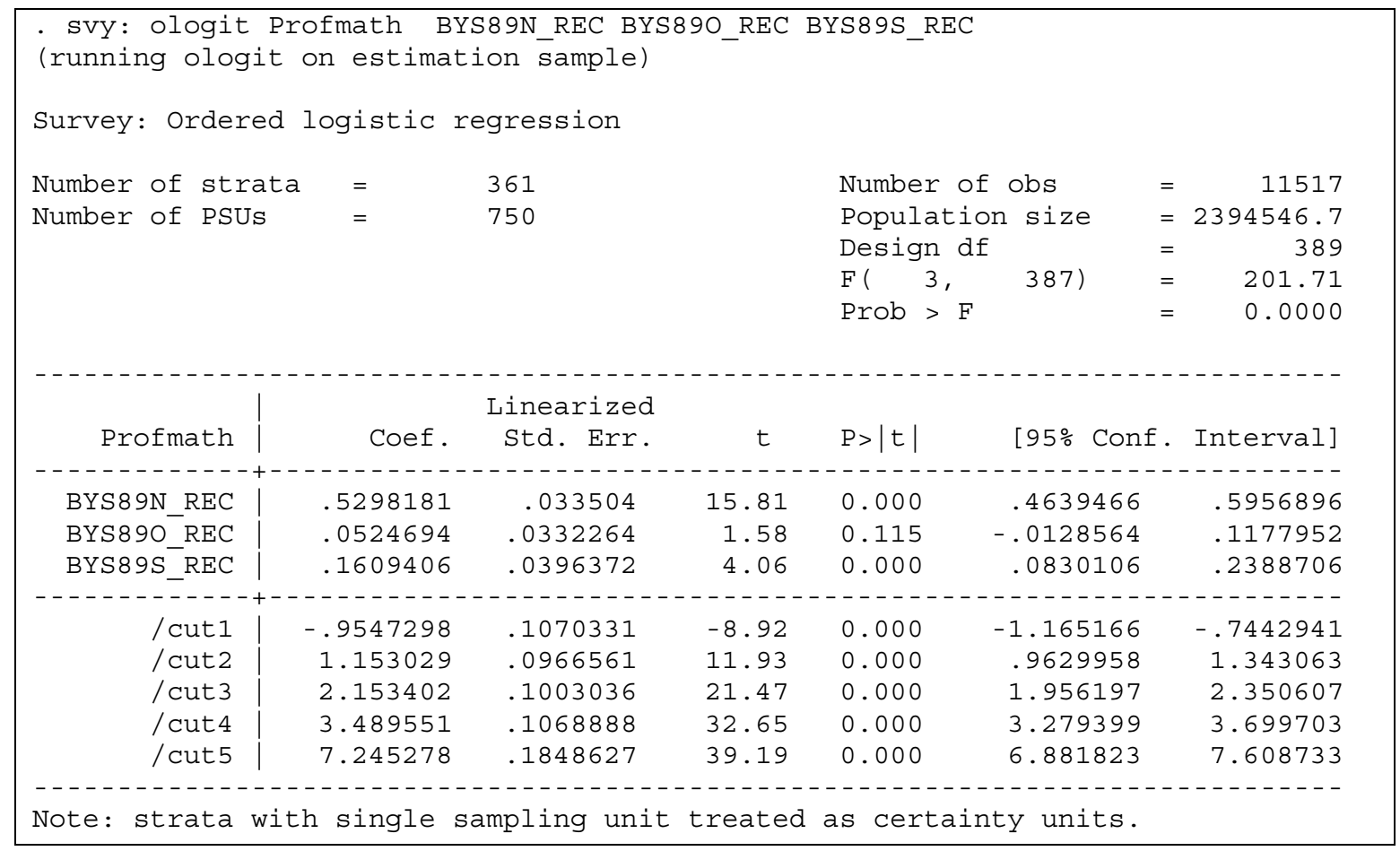

In the final PO model which accommodated sampling designs, Stata reports the the adjusted Wald test for all parameters rather than the log likelihood ratio Chi-Square test for the conventional PO model. $\mathrm{F}(3,387)=$ $201.71, p<0.001$, suggested that the full model with three predictors was significant in predicting odds of being at or below a particular mathematics proficiency level.

The logit effects of decide and dobest were significant. For the predictor, decide, $\beta=$ $0.530, \mathrm{t}=15.81, \mathrm{p}<0.001$; and for the predictor, dobest, $\beta=0.161, \mathrm{t}=4.06, \mathrm{p}<0.001$. However, the effect of keeplrn was not significantly different from zero. $\beta=0.052, \mathrm{t}=1.58, \mathrm{p}=$ 0.115 .

Substituting the values of the estimated logit coefficients into the equation (3) resulted in logit $\left[\begin{array}{lllll}\pi(\mathrm{Y} \leq \mathrm{j} & \mathrm{X}_{1}, & \mathrm{X}_{2}, & \left.\mathrm{X}_{3}\right)\end{array}\right]=\alpha_{\mathrm{j}}+\left(-.530 \mathrm{X}_{1}\right.$ $\left.-.052 \mathrm{X}_{2}-.161 \mathrm{X}_{3}\right)$. By exponentiating the negative logit coefficients $\left(\mathrm{e}^{(-\beta)}\right)$ the odds of being at or below a particular proficiency level were obtained. Therefore, the odds of being at or below a particular proficiency level as opposed to being beyond that level for the three predictors, decide, keeplrn, and dobest, were $0.589,0.949$ and 0.930 , respectively.

When estimating the odds of being at or below a proficiency level, five cutpoints were used to differentiate adjacent categories of the mathematics proficiency. $\alpha_{1}=-0.955$, which was the cutpoint for the cumulative logit model for Y $\leq 0$ (i.e., level 0 versus levels $1,2,3,4$, and 5); $\alpha_{2}$ was the cutpoint for the cumulative logit model for $\mathrm{Y} \leq 1$ (i.e., levels 0 and 1 versus levels 2, 3, 4, and 5); and the final $\alpha_{5}$ was used as the cutpoint for the logit model when $\mathrm{Y} \leq 4$.

To estimate the odds of being beyond a proficiency level, equation (3) can be transformed to logit $\left[\pi\left(\mathrm{Y}>\mathrm{j} \mid \mathrm{X}_{1}, \mathrm{X}_{2}, \mathrm{X}_{3}\right)\right]=$ $\alpha_{\mathrm{j}}+.530 \mathrm{X}_{1}+.052 \mathrm{X}_{2}+.161 \mathrm{X}_{3}$. Odds ratios can be calculated in the same way as above (see Table 6). In terms of odds ratio, getting no bad grades if deciding to $(\mathrm{OR}=1.699)$, and doing best to learn $(\mathrm{OR}=1.175)$ was positively associated with the odds of being above a particular mathematics proficiency level, rather than being at or below that level. The OR for keeping learning when the material is difficult was 1.054, which was not significant. 


\section{XING LIU \& HARI KOIRALA}

Comparison of Parameter and Standard Error Estimates from the PO model for Complex Survey Data and the Conventional Unweighted PO Model

Table 6 provides the parameter and standard error estimates obtained from the PO model for complex sampling data using Stata svy: ologit and those from the unweighted PO model with Stata ologit. After sampling design variables and probability weights were applied to the PO model, the estimated logit coefficients and their standard errors were different from those in the unweighted PO model. The logit coefficient of the first predictor (decide) increased and those of the last two predictors (keeplrn and dobest) decreased. Further, the standard errors of all three coefficients increased tremendously.

Compared to the unweighted PO model, the estimated logit coefficient for getting no bad grades if deciding to (decide) in the PO model for complex survey data increased by $4.1 \%$, and its standard error increased by $36 \%$; the logit coefficient for keeping studying if material is difficult (keeplrn) decreased by $15.4 \%$, and its standard error increased by $17.9 \%$; and the logit coeffecit for doing best to learn (dobest) decreased by $14.3 \%$, with its standard error increased by $42.9 \%$.

The change of the parameter and linearized standard error estimates impacted significance tests. In the unweighted PO model, the effects of all three predictors were significant. However, when the sampling design variables and weights were applied to the PO model, only the first and last predictors were significant, and the second predictor (keeplrn) turned to be nonsignificant $(\mathrm{p}=0.115)$.

\section{Conclusion}

This article explicated the use of the proportional odds models with complex survey sampling to estimate the ordinal response variable. Model fitting started from the conventional PO model without sampling weights, then the PO model with weights, and finally to the PO model for complex survey data with both weights and sampling design variables. Results of all three models were interpreted and compared. In addition, methods of dealing with a single sampling unit within strata were illustrated and the estimated standard errors from the PO models with different single unit options were compared.

After sampling design variables and probability weights were applied to the conventional PO model, the estimated logit coefficients and their standard errors were more accurate than those in the unweighted PO model and the PO model with weights only. Specifically, first, compared to the unweighted PO model, the PO model with sampling weights impacts the accuracy of both parameter estimates and standard errors, and thus, the test statistics and the p-values; second, applying both the sampling weights and design variables to the PO model produced more accurate standard errors than the PO model with weights only, although these two models had the same parameter estimates.

This article demonstrated that ignoring weights, clusters and strata leads to biased parameter estimates and erroneous standard errors in ordinal logistic regression analysis. It extends the work by Hahs-Vaughn $(2005,2006)$, and Thomas and Heck (2001), which focused on the survey data analysis in multiple regression. Theories and mathematical details on how to estimate unbiased parameters and standard errors for complex survey data are well documented in literature and are beyond the scope of this article. Interested readers should refer to Binder (1983), Heeringa, West and Berglund (2010), Levy and Lemeshow (2008) and Lohr (1999) for details.

The logit coefficients in the PO model for complex survey data can be interpreted in the same way as those in the standard PO model. However, these two models may have different parameter estimates and standard errors, or even different levels of statistical significance (pvalue). For example, the effect of one predictor in the above example became nonsignificant when weights and sampling design variables were applied to the conventional PO model.

In large-scale survey data, it is common to encounter a single sampling unit in a stratum, which results in the missing values of estimated standard errors in model fitting. This study suggests that any of the three single unit options, including certainty, scaled and centered, could 
PO MODELS WITH COMPLEX SURVEY DATA

Table 6: The PO Model Result for Complex Survey Data Using Stata svy: ologit Command (A

Comparison with the Unweighted PO Model)

\begin{tabular}{|c|c|c|c|c|c|c|}
\hline \multirow[b]{2}{*}{ Variable } & \multicolumn{3}{|c|}{ PO Model-Unweighted } & \multicolumn{3}{|c|}{$\begin{array}{l}\text { PO Model for Complex Survey } \\
\text { Sampling }\end{array}$} \\
\hline & $\mathrm{b}(\mathrm{se}(\mathrm{b}))$ & OR & $\mathrm{P}$ & $\mathrm{b}(\mathrm{se}(\mathrm{b}))$ & OR & $\mathrm{P}$ \\
\hline$\alpha_{1}$ & -1.096 & & & -.955 & & \\
\hline$\alpha_{2}$ & 1.071 & & & 1.153 & & \\
\hline$\alpha_{3}$ & 2.072 & & & 2.153 & & \\
\hline$\alpha_{4}$ & 3.443 & & & 3.490 & & \\
\hline$\alpha_{5}$ & 7.107 & & & 7.245 & & \\
\hline decide & $\begin{array}{l}.509 * * \\
(.025)\end{array}$ & 1.664 & $<.001$ & $\begin{array}{l}.530 * * \\
(.034)\end{array}$ & 1.699 & $<.001$ \\
\hline keeplrn & $\begin{array}{l}.060^{*} \\
(.028) \\
\end{array}$ & 1.062 & .031 & $\begin{array}{c}.052 \\
(.033) \\
\end{array}$ & 1.054 & .115 \\
\hline dobest & $\begin{array}{c}.184 * * \\
(.028) \\
\end{array}$ & 1.202 & $<.001$ & $\begin{array}{l}.161^{* *} \\
(.040) \\
\end{array}$ & 1.175 & $<.001$ \\
\hline$L R R^{2}$ & .034 & & & $\mathrm{~N} / \mathrm{A}$ & & \\
\hline $\begin{array}{c}\text { Brant Test } \\
\text { (Omnibus } \\
\text { Test) } \\
\end{array}$ & $\chi_{12}^{2}=20.51$ & & & N/A & & \\
\hline $\begin{array}{l}\text { Model Fit/F } \\
\text { test }\end{array}$ & $\begin{array}{c}\chi_{3}^{2}= \\
1102.83 * *\end{array}$ & & & $\begin{array}{c}\mathrm{F}(3,387)= \\
201.71 * *\end{array}$ & & \\
\hline
\end{tabular}

be used in the PO model to estimate standard errors.

This article focused on the Taylor series approximation method for variance estimation. For future research, other variance estimation methods, such as the balanced repeated replication (BRR), the jackknife repeated replication (JRR) and the bootstrap method should be examined for ordinal logistic regression analysis. In addition, other general purpose statistical software packages, such as SPSS and SAS, may use different procedures or parameterizations in fitting PO models with complex survey data, which warrants further investigation. It is hoped that researchers will use the most appropriate models to analyze ordinal categorical dependent variables when data are collected using complex sampling designs.
Notes

Previous versions of this paper were presented at the Modern Modeling Methods Conference in Storrs, CT (May, 2012), the Northeastern Educational Research Association Annual Conference in Rocky Hill, CT (Oct., 2012), and 2013 Annual Meeting of American Educational Research Association (AERA), San Francisco, CA (April, 2013).

\section{References}

Agresti, A. (1996). An introduction to categorical data analysis. New York, NY: John Wiley \& Sons.

Agresti, A. (2002). Categorical data analysis $\left(2^{\text {nd }} E d\right.$.). New York, NY: John Wiley $\&$ Sons. 


\section{XING LIU \& HARI KOIRALA}

Agresti, A. (2007). An introduction to categorical data analysis $\left(2^{\text {nd }} E d\right.$. $)$. New York, NY: John Wiley \& Sons.

Agresti, A. (2010). Analysis of ordinal categorical data $\left(2^{\text {nd }} E d\right.$.). Hoboken, NJ: John Wiley \& Sons.

Allison, P.D. (1999). Logistic regression using the SAS system: Theory and application. Cary, NC: SAS Institute, Inc.

Ananth, C. V., \& Kleinbaum, D. G. (1997). Regression models for ordinal responses: A review of methods and applications. International Journal of Epidemiology, 26, 1323-1333.

Armstrong, B. B., \& Sloan, M. (1989). Ordinal regression models for epidemiological data. American Journal of Epidemiology, 129(1), 191-204.

Binder, D. A. (1983). On the variances of asymptotically normal estimators from complex surveys, International Statistical Review, 51, 279-292.

Brant (1990). Assessing proportionality in the proportional odds model for ordinal logistic regression. Biometrics, 46, 1171-1178.

Fienberg, S. E. (1980). The analysis of cross-classified categorical data. Cambridge, MA: The MIT Press.

Hardin, J. W., \& Hilbe, J. M. (2007). Generalized linear models and extensions $\left(2^{\text {nd }}\right.$ $E d$.). Texas: Stata Press.

Heeringa, S. G., West, B. T., \& Berglund, P. A. (2010). Applied survey data analysis. Boca Raton, FL: Chapman \& Hall/CRC.

Hahs-Vaughn, D. L. (2005). A primer for understanding and using weights with national datasets. Journal of Experimental Education, 73(3), 221-240.

Hahs-Vaughn, D. L., \& Lomax, R. G. (2006). Utilization of sample weights in single level structural equation modeling. Journal of Experimental Education, 74(2), 163-190.

Hahs-Vaughn, D. L. (2006). Analysis of data from complex samples. International Journal of Research and Method in Education, 29(2), 163-181.

Hilbe, J. M. (2009). Logistic regression models. Boca Raton, FL: Chapman \& Hall/CRC.
Hosmer, D. W., \& Lemeshow, S. (2000). Applied logistic regression (2 $2^{\text {nd }} E d$.). New York, NY: John Wiley \& Sons.

Ingels, S. J., Pratt, D. J., Roger, J., Siegel, P. H., \& Stutts, E. (2004). ELS: 2002 Base Year Data File User's Manual. Washington, DC: NCES (NCES 2004-405).

Ingels, S. J., Pratt, D. J., Roger, J., Siegel, P. H., \& Stutts, E. (2005). Education Longitudinal Study: 2002/04 Public Use BaseYear to First Follow-up Data Files and Electronic Codebook System. Washington DC: NCES (NCES 2006-346).

Kalton, G. (1983). Introduction to survey sampling. Beverly Hills, CA: Sage.

Lee, E. S., \& Forthofer, R. N. (2006). Analyzing complex survey data $\left(2^{\text {nd }} E d\right.$. $)$. Thousand Oaks, CA: Sage.

Levy, P. S., \& Lemeshow, S. (2008). Sampling of populations: Methods and application $\left(4^{\text {th }}\right.$ Ed.). New York, NY: John Wiley.

Liu, X. (2009). Ordinal regression analysis: Fitting the proportional odds model using Stata, SAS and SPSS. Journal of Modern Applied Statistical Methods, 8(2), 632-645.

Liu, X., O’Connell, A.A., \& Koirala, H. (2011). Ordinal regression analysis: Predicting mathematics proficiency using the continuation ratio model. Journal of Modern Applied Statistical Methods, 10(2), 513-527.

Liu, X., \& Koirala, H. (2012). Ordinal regression analysis: Using generalized ordinal logistic regression models to estimate educational data. Journal of Modern Applied Statistical Methods, 11(1), 242-254.

Lohr, S. L. (1999). Sampling: Design and analysis. Pacific Grove, CA: Duxbury Press.

Long, J. S. (1997). Regression models for categorical and limited dependent variables. Thousand Oaks, CA: Sage.

Long, J. S. \& Freese, J. (2006). Regression models for categorical dependent variables using Stata $\left(2^{\text {nd }} E d\right.$.). Texas: Stata Press.

McCullagh, P. (1980). Regression models for ordinal data (with discussion). Journal of the Royal Statistical Society Series B, $42,109-142$. 


\section{PO MODELS WITH COMPLEX SURVEY DATA}

McCullagh, P. \& Nelder, J. A. (1989). Generalized linear models $\left(2^{\text {nd }} E d\right.$.). London: Chapman and Hall.

Menard, S. (1995). Applied logistic regression analysis. Thousand Oaks, CA: Sage.

Muthén, B. O., \& Satorra, A. (1995). Complex sample data in structural equation modeling. Sociological Methodology, 25, 267316.

O'Connell, A.A. (2000). Methods for modeling ordinal outcome variables. Measurement and Evaluation in Counseling and Development, 33(3), 170-193.

O'Connell, A. A. (2006). Logistic regression models for ordinal response variables. Thousand Oaks, CA: SAGE.

O'Connell, A. A., \& McCoach, D. B. (2008). Multilevel modeling of educational data. Charlotte, NC: IAP.

O'Connell, A.A., \& Liu, X. (2011). Model diagnostics for proportional and partial proportional odds models. Journal of Modern Applied Statistical Methods, 10(1), 139-175.

Powers D. A., \& Xie, Y. (2000). Statistical models for categorical data analysis. San Diego, CA: Academic Press.

Raudenbush, S. W., \& Bryk, A. S. (2002). Hierarchical linear models: Applications and data analysis methods $\left(2^{\text {nd }}\right.$ $E d$.). Thousand Oaks, CA: Sage.
Stokes, M. E., Davis, C. S., \& Koch, G. G. (2000). Categorical data analysis using the SAS system. Cary, NC: SAS Institute Inc.

Stapleton, L. M. (2002). The incorporation of sample weights into multilevel structural equation models. Structural Equation Modeling, 9(4), 475-502.

Stapleton, L. M. (2006). An assessment of practical solutions for structural equation modeling with complex sample data. Structural Equation Modeling, 13(1), 28-58.

Stapleton, L. M. (2008). Variance estimation using replication methods in structural equation modeling with complex sample data. Structural Equation Modeling, 15(2), 183-210.

StataCorp. (2007). Stata survey data reference manual. College Station, TX: Stata Press.

StataCorp. (2009). Stata survey data reference manual: Release 11. College Station, TX: Stata Press.

StataCorp. (2011). Stata survey data reference manual: Release 12. College Station, TX: Stata Press.

Thomas, S. L., \& Heck, R. H. (2001). Analysis of large-scale secondary data in higher education research: Potential perils associated with complex sampling designs. Research in Higher Education, 42(5), 517-540. 


\title{
Brief Report \\ A Note on $\alpha$-Curvature of the Manifolds of the Length-biased Lognormal and Gamma Distributions in View of Related Applications in Data Analysis
}

\author{
Makarand V. Ratnaparkhi \\ Wright State University, \\ Dayton, $\mathrm{OH}$
}

\author{
Uttara V. Naik-Nimbalkar \\ Pune University, \\ Pune, India
}

The $\alpha$-curvature tensors of the statistical manifolds of the length-biased versions of the log-normal and gamma distributions are derived and discussed. This study was designed to investigate observations related to the parameter estimation for the length-biased lognormal distribution as a model for the lengthbiased data from oil field exploration.

Key words: Statistical manifolds, $\alpha$-curvature tensor, duality and symmetry, length-biased lognormal distribution, estimation.

\section{Introduction}

Data are referred to as length-biased when the probability of inclusion of the observation in the sample is proportional to its magnitude. It has been documented in a number of research articles that the presence of length-biasedness in data arising in fields such as ecology, economics, geology, bioinformatics and medical science. Some examples of such data sets can be found in Ratnaparkhi and Naik-Nimbalkar (2012). The applications of concepts of differential geometry, such as the curvature tensor of the statistical manifolds, have been investigated with reference to statistical inference (Amari, 1985). Recently, Ratnaparkhi and Naik-Nimalkar (2012) observed that certain structural properties of parameter estimators of the length-biased lognormal distribution are significantly different from the corresponding properties of the original lognormal distribution. This article

Makarand V. Ratnaparkhi is a Professor of Statistics at Wright State University in Dayton, Ohio, USA. Email him at: makarand.ratnaparkhi@wright.edu. Uttara V. Naik-Nimbalkar is a Professor of Statistics at University of Pune, Pune, India. Email her at: uvnaik@stats.unipune.ac.in. investigates the properties of the manifold of the length-biased lognormal distribution for tracking changes in structural properties, such as the $\alpha$ curvature of the original manifold, of a usual lognormal distribution.

$$
\text { If } X \sim f(x ; \theta) \text { where } E[X]<\infty \text { and }
$$

$\theta$ is a scalar or a vector of parameters, then, the length-biased version of the pdf $f(x ; \theta)$ (referred to as the original distribution) is defined as:

$$
g(x ; \theta)=x f(x ; \theta) / E[X]
$$

The generalization of (1) (referred to as the weighted distribution) is given by:

$$
\begin{gathered}
(x ; \theta)=w(x) f(x ; \theta) / E[w(X)], \\
w(x)>0, E[w(X)]<\infty .
\end{gathered}
$$

The special cases of (1) and (1a) for commonly used statistical distributions such as Gamma, Poisson, etc., are useful in data analysis. For example, the survival or failure time data, the probability of inclusion of the observation is proportional to the magnitude, for example $x$, of the observation. The resulting data is referred to as length-biased data. For this type of data the pdf (1) is used for modeling the data where 


\section{$\alpha$-CURVATURE OF LENGTH-BIASED LOGNORMAL AND GAMMA DISTRIBUTIONS}

$f(x ; \theta)$ is the model that would have been used if the data were not length-biased. Because, for the estimation of $\theta$ (as a parameter of the original distribution), the length-biased version (1) is substituted for the original distribution it is necessary to study the pdf $g(x ; \theta)$ for the desired properties of the estimator of $\theta$.

In many situations, the pdf $g(x ; \theta)$

behaves as expected; hence the estimation problem is solvable without complexities. However, this may not be the case for every pdf $f(x ; \theta)$; therefore, it may be necessary to study the properties of $g(x ; \theta)$ in further details. For example, in a recent length-biased data analysis study arising in oil field exploration (Ratnaparkhi \& Naik-Nimbalkar, 2012) it was observed that the properties of the estimators of the parameters of the original lognormal distribution - when replaced by its length-biased version - undergo a considerable change. Therefore, a need for further detailed study of the family of length-biased lognormal distributions was identified; in particular, studying the properties of the manifold of the length-biased lognormal distributions is likely to lead to the root cause of the above mentioned somewhat curious situation.

The differential geometric properties, such as the symmetry and duality of $\alpha$-curvature tensor of a statistical manifold (Amari, 1985), are known to be related to the statistical inference for the parameters of any distribution belonging to the manifold. In particular, in this article the $\alpha$ - curvature tensors for the original lognormal and gamma distributions and their corresponding lengthbiased versions are derived and their properties are compared in view of related applications in the analysis of length-biased data.
Methodology

A random variable $X$ is said to have a lognormal (LN) distribution with parameters $(\mu, \sigma)$, if its pdf is

$$
f(x ; \mu, \sigma)=\frac{1}{x \sigma \sqrt{2 \pi}} \exp \left[-\frac{1}{2 \sigma^{2}}(\log x-\mu)^{2}\right],
$$$$
\text { where, } x>0,-\infty<\mu<\infty, \sigma>0 \text {. }
$$

Using (1) the pdf of the length-biased lognormal (LBLN) distribution is given by

$g(x ; \mu, \sigma)=\frac{1}{x \sigma \sqrt{2 \pi}} \exp \left[-\frac{1}{2 \sigma^{2}}\left(\log x-\left(\mu+\sigma^{2}\right)\right)^{2}\right]$,

where, $x>0,-\infty<\mu<\infty, \sigma>0$.

The Fisher information matrices for the LN $(\mu, \sigma)$ and $\operatorname{LBLN}(\mu, \sigma)$ distributions are provided in Table 1. Note that the information matrices have different structures. These matrices play an important role in deriving the differential geometrical properties of the corresponding manifolds.

Table 1: Fisher Information of $\operatorname{LN}(\mu, \sigma)$ and $\operatorname{LBLN}(\mu, \sigma)$

\begin{tabular}{|c|c|}
\cline { 2 - 2 } \multicolumn{1}{c|}{} & Fisher Information \\
\hline $\operatorname{LN}(\mu, \sigma)$ & {$\left[\begin{array}{cc}1 / \sigma^{2} & 0 \\
0 & 2 / \sigma^{2}\end{array}\right]$} \\
\hline $\operatorname{LBLN}(\mu, \sigma)$ & {$\left[\begin{array}{cc}1 / \sigma^{2} & 2 / \sigma \\
2 / \sigma & 4+2 / \sigma^{2}\end{array}\right]$} \\
\hline
\end{tabular}




\section{RATNAPARKHI \& NAIK-NIMBALKAR}

The Presence of Curvature Component in the Manifold of LBLN $(\mu, \sigma)$

Because, the derivation for pdf (3) is straightforward the detailed proof is omitted here for the sake of brevity. Let $U_{1} \sim \mathrm{LN}(\mu$, $\sigma / \sqrt{ } 2)$ and $V_{l} \sim \mathrm{L} \mathrm{N}\left(\sigma^{2}, \sigma / \sqrt{ } 2\right)$. If $U_{l}$ and $V_{l}$ are independent, then, $X=\left(U_{1} V_{l}\right) \sim \operatorname{LBLN}(\mu, \sigma)$ with pdf (3). However, it is notable that the distribution of $V_{l}$ belongs to the curved family of distributions, hence, the statistical manifold of the LBLN distributions must be investigated for the nature of its curvature. Note that, in the derivation, using the logarithmic transformation on $U_{1}$ and $V_{1}$ results in the distribution of $Y=\log (X)$ as a convolution of two normal random variables with respective distributions. Clearly, the manifold of the distributions of $Y$ has the corresponding curvature property.

\section{Statistical Manifolds}

A family of probability distributions $\mathcal{F}_{\theta}=\{f(x, \underset{\sim}{\theta})\} \quad$ with $p$-dimensional parameter vector $\underset{\sim}{\theta}$ having values over some open subset of $R^{p}$ is a manifold (denoted by $\mathscr{M}$ ), in which the pdf $f(x ; \underset{\sim}{\theta})^{\prime} s$ are the points and the parameters $\underset{\sim}{\theta}$ provide the necessary coordinate functions (for details, refer to Amari, 1985).

In this case, let $\boldsymbol{M}_{l}=\{f(x ; \mu, \sigma)\}$ denote the manifold of $\operatorname{LN}(\mu, \sigma)$ and let $\boldsymbol{M}_{2}=\{$ $g(x ; \mu, \sigma)\}$ denote the manifold of $\operatorname{LBLN}(\mu$, $\sigma)$. The regularity conditions (Amari, 1985) needed for deriving the differential geometric properties of $\boldsymbol{M}_{1}$ and $\boldsymbol{M}_{2}$ are satisfied and hence the derivation of the $\alpha$-curvature tensors for $\boldsymbol{M}_{1}$ and $\boldsymbol{M}_{2}$ can proceed. For these derivations the following are needed:

1. The Fisher information matrix provides a Riemannian metric for the manifold $\mathcal{M}$ of pdfs.

2. The Fisher score functions are the vectors that span the tangent space at a point in $\mathscr{M}$.

3. For application of differential geometric concepts in statistics what are known as the $\alpha$-connections are necessary. Such connections for $\mathscr{M}=\{f(x ; \mu, \sigma)\} \quad$ are defined by

$\Gamma_{i j k}^{(\alpha)}=\Gamma_{i j k}^{(1)}+\frac{(1-\alpha)}{2} T_{i j k}$

where

$\Gamma_{i j k}^{(1)}=\mathrm{E}\left[\partial_{i} \partial_{j} l(x ; \mu, \sigma) \partial_{k} l(x ; \mu, \sigma)\right]$

and

$T_{i j k}=\mathrm{E}\left[\partial_{i} l(x ; \mu, \sigma) \partial_{j} l(x ; \mu, \sigma) \partial_{k} l(x ; \mu, \sigma)\right]$

where $l(x ; \mu, \sigma)$ is the log-likelihood function. For this study, $l(x ; \mu, \sigma)$ is the likelihood function of $f(x ; \mu, \sigma)$ or $g(x ; \mu, \sigma)$ and the subscripts $i=1,2, j=1,2$ and $k=1,2$ correspond to the derivatives with respect to $\mu$ and $\sigma$ respectively. Thus, there are eight $\alpha$-connections for each manifold. The $\alpha$-curvature tensor, using the Einstein summation notation, in terms of the $\alpha$-connections is given by the formula

$$
\begin{aligned}
& R_{i j k m}^{(\alpha)}= \\
& \left(\partial_{i} \Gamma_{j k}^{(\alpha) s}-\partial_{j} \Gamma_{i k}^{(\alpha) s}\right) h_{s m}+\left(\Gamma_{i r m}^{(\alpha)} \Gamma_{j k}^{(\alpha) r}-\Gamma_{j r m}^{(\alpha)} \Gamma_{i k}^{(\alpha) r}\right),
\end{aligned}
$$

where $h_{s m}$ is the $(s, m)^{\text {th }}$ element of the inverse of the Fisher information matrix and using Einstein summation notation:

$$
\Gamma_{j k}^{(\alpha) s}=h^{s m} \Gamma_{j k m}^{(\alpha)},
$$

where $h^{s m}$ is the $(s, m)^{\text {th }}$ element of the inverse of Fisher information matrix. This formula is used for other subscripts and superscripts in (5). The values of the subscripts $(i, j, k, m)$ have the same meaning as in (4), thus, there are sixteen components of $\alpha$-curvatures for each manifold.

Statistical Applications of $\alpha$-Curvature

Three properties of a manifold related to the concepts of $\alpha$-connections and $\alpha$-curvature relevant to this study are: 


\section{$\propto$-CURVATURE OF LENGTH-BIASED LOGNORMAL AND GAMMA DISTRIBUTIONS}

1. If all components of $R_{i j k m}^{(\alpha)}=0$, then the manifold is said to be flat as opposed to curved. The efficiency of the parameter estimators depend on the curvature of the manifold. (See Amari (1985) for a discussion of $\alpha$-connections and the curvature tensors with reference to the exponential family of distributions.)

2. If the curvature vanishes at $\alpha= \pm 1$ then the $\alpha$-affine coordinate system exists for $\alpha=$ \pm 1 . In such cases 1 -affine coordinate system is called the natural parameter system and the -1 -affine coordinate system is called the expectation parameter system. For example, for the Gaussian manifold it can be shown that such coordinate systems exist. For $\alpha=0$ the curvature becomes the Riemannian curvature.

3. If $R$ denotes the curvature tensor, and if $R^{\alpha}=R^{-\alpha}$ for all real values of $\alpha$, then the manifold is said to be conjugate symmetric. This is considered to be one of the desirable properties in statistical applications of differential geometry, for, example, the Gaussian manifold is conjugate symmetric.

\section{Results}

The maximum likelihood estimators $\left(\hat{\mu}, \hat{\sigma}^{2}\right)$ of the parameters $\mu$ and $\sigma^{2}$ of the LBLN distribution and their properties are discussed in Ratnaparkhi and Naik-Nimbalkar (2012). These estimators, for reference, are:

$$
\begin{gathered}
\hat{\mu}=\left(\sum \log x_{i} / n\right)-\hat{\sigma}^{2} \\
\hat{\sigma}^{2}=\frac{1}{n}\left[\sum\left(\log x_{i}\right)^{2}-\left(\sum \log x_{i}\right)^{2} / n\right]
\end{gathered}
$$

Table 2 shows that the $\alpha$-connections for $\mathrm{LN}$ and LBLN do not have the same structure and that the $\alpha$-curvature tensors are expected to be different for these distributions, however, interest lies in the nature of the structure of the difference between the curvatures.

There are total of sixteen components of the $\alpha$-curvature tensor for each of the manifolds of the LN and LBLN distributions. The derivation of all such components is lengthy and tedious; for the objective of this article - and to draw conclusions - all sixteen components are unnecessary. For brevity, only some of the components are reported and serve the purpose of illustrating the difference between the behaviors of these two manifolds.

The $\alpha$-curvature of $\boldsymbol{M}_{l}$ is given by

$$
R_{1212}^{(\alpha)}=\left(1-\alpha^{2}\right) / \sigma^{4}
$$

and the Riemannian curvature, at $\alpha=0$ is

$$
\kappa=-R_{1212}^{(\alpha)} \cdot h^{11} h^{22} / 2=-1 / 2
$$

For $\boldsymbol{M}_{2}$ the scalar curvature $\kappa$ is a function of $\sigma^{2}$ (i.e., not constant as in case of $\boldsymbol{M}_{1}$ ).

The $\alpha$-Curvature of the Manifolds of Gamma $(\mu, \beta)$ and Length-Biased Gamma $(\mu, \beta)$

The gamma distribution is sometimes considered an alternate model for the lognormal distribution. Let $\mathrm{G}(\mu, \beta)$ and $\operatorname{LBG}(\mu, \beta)$ denote the gamma and its length-biased version respectively where parameter $\mu$ is the mean of the gamma distribution. The pdf of $\mathrm{G}(\mu, \beta)$ distribution is given by

$$
\begin{aligned}
& f_{2}(x ; \mu, \beta)=(\beta / \mu)^{\beta} x^{\beta-1} \exp (-x \beta / \mu) / \Gamma(\beta), \\
& x>0, \mu>0, \beta>0 .
\end{aligned}
$$

The pdf of LBG $(\mu, \beta)$ is given by

$g_{3}(x ; \mu, \beta)=(\beta / \mu)^{\beta+1} x^{\beta+1-1} \exp (-x \beta / \mu) / \Gamma(\beta+1)$, $x>0, \mu>0, \beta>0$.

Using formulae (4) and (5), the $\alpha$-curvature of the manifold of $\mathrm{G}(\mu, \beta)$ distributions is given by

$$
R_{1212}^{(\alpha)}=\left(\alpha^{2}-1\right)\left[\phi(\beta)+\beta \phi^{\prime}(\beta)\right] / 4 \mu^{2} \beta \phi(\beta)
$$




\section{RATNAPARKHI \& NAIK-NIMBALKAR}

Table 2: $\alpha$-Connections $\Gamma_{i j k}^{(\alpha)}$ for the $\operatorname{LN}(\mu, \sigma)$ and $\operatorname{LBLN}(\mu, \sigma)$

\begin{tabular}{|c|c|c|}
\hline \multicolumn{3}{|c|}{ Distributions } \\
\hline$\Gamma_{i j k}^{(\alpha)}$ & $\operatorname{LN}(\mu, \sigma)$ & $\operatorname{LBLN}(\mu, \sigma)$ \\
\hline$\Gamma_{111}^{(\alpha)}$ & 0 & $(1-\alpha) / \sigma^{3}$ \\
\hline$\Gamma_{112}^{(\alpha)}$ & $(1-\alpha) / \sigma^{3}$ & $-(1+\alpha) / \sigma^{3}$ \\
\hline$\Gamma_{121}^{(\alpha)}$ & $-(1+\alpha) / \sigma^{3}$ & $-4 \alpha / \sigma^{2}$ \\
\hline$\Gamma_{122}^{(\alpha)}$ & 0 & $-(1+\alpha) / \sigma^{3}$ \\
\hline$\Gamma_{211}^{(\alpha)}$ & $-(1+\alpha) / \sigma^{3}$ & $-4 \alpha / \sigma^{2}$ \\
\hline$\Gamma_{212}^{(\alpha)}$ & 0 & $-2(1+2 \alpha) / \sigma^{2}$ \\
\hline$\Gamma_{221}^{(\alpha)}$ & 0 & $-2\left(1+2 \alpha+6 \alpha \sigma^{2}\right) / \sigma^{3}$ \\
\hline$\Gamma_{222}^{(\alpha)}$ & $-2(1+2 \alpha) / \sigma^{3}$ & \\
\hline
\end{tabular}

The $\alpha$-curvature of the manifold of LBG $(\mu, \beta)$ is of the same form as (10) where $\beta$ is replaced by $(\beta+1)$.

\section{Conclusion}

Previous research investigated estimation of the parameters $(\mu, \sigma)$ of the original lognormal distribution (LN) using the length-biased lognormal (LBLN) distribution based on the known length-biased nature of data arising from oil field exploration (Ratnaparkhi \& NaikNimbalkar, 2012). In this estimation process, the $\operatorname{MLE}\left(\hat{\mu}, \hat{\sigma}^{2}\right)$ shown in (6) was obtained. Examination of (6) shows that the properties of these estimators are different from the properties of the corresponding estimators of the original lognormal distribution, thus, to better understand the structure of LBLN distribution the differential geometric properties of the manifolds of the LN and LBLN distributions were studied. In addition, this research was undertaken because the respective pdf's (1) and
(2) of the LN and LBLN distributions are considered to be of the same form. Although pdf's (1) and (2) appear similar it was thought that there must be some other criterion for referring to these two pdf's as of the same form. Finally, for comparison purposes the differential geometric properties of the gamma and lengthbiased distributions were derived.

Based on the above results the following conclusions are put forth:

1. It is known that all information about the curvature tensor of the manifold of the LN distribution can be extracted from the single component $R_{1212}^{(\alpha)}$ given in equations (7) and (8). This component vanishes at $\alpha= \pm 1$, hence the manifold has the three properties previously noted. Equation (8) shows that the scalar curvature and in particular the Riemannian curvature for $\boldsymbol{M}_{l}$ is constant. This property does not hold for $\boldsymbol{M}_{2}$, the manifold of LBLN distributions. Thus, the 
curvature is not constant and in particular is a function of $\sigma^{2}$. The properties of the estimators the parameters are known to be associated with the curvature of the manifold (Amari, 1985). Therefore, if the LBLN is the model that is used for the estimation of the parameters of the original LN distribution the properties of the estimators will be distorted non-uniformly depending on $\sigma$. In fact, the manifold of the LBLN distributions is a unique example that we have identified in real data analysis. The systematic theoretical analysis of the LBLN manifold is needed. The details are beyond the scope of this brief report and hence are not included here. However, this observation indicates that due caution is necessary if the LBLN distribution is to be considered as a model for length-biased data.

2. The gamma distribution can be used in certain data analysis problems in lieu of the lognormal distribution investigated herein, the differential geometrical properties of the gamma manifold. Equation (11) verifies that $R_{1212}^{(\alpha)}$ vanishes at $\alpha= \pm 1$ for the manifolds of the gamma and the length-biased version of gamma distribution and, therefore, the three properties presented herein hold equally for both manifolds. Hence, the parameter estimators of the original gamma distribution based on its length-biased version should not pose problems either locally or globally.

Thus it may be concluded that, in data analysis, a researcher should either use the LBLN distribution with due caution considering the unusual behavior of the LBLN manifold or, whenever possible, consider the LBG distribution as an alternative to the LBLN distribution.

\section{References}

Amari, S. I. (1985). Differentialgeometrical methods in statistics. New York: Springer-Verlag.

Ratnaparkhi, M. V., \& Naik-Nimbalkar, U. V. (2012). Length-biased lognormal distribution and its application in the analysis of data from the oil field exploration studies. Journal of Modern Applied Statistical Methods, $11(1), 255-260$. 


\title{
Emerging Scholars \\ Modeling and Handling Overdispersion Health Science Data with Zero-Inflated Poisson Model
}

\author{
Nur Syabiha Zafakali Wan Muhamad Amir W Ahmad \\ Universiti Malaysia Terengganu, \\ Kuala Terengganu, Malaysia
}

Health sciences research often involves analyses of repeated measurement or longitudinal count data analyses that exhibit excess zeros. Overdispersion occurs when count data measurements have greater variability than allowed. This phenomenon can be carried over to zero-inflated count data modeling. Referred to as zero-inflation, the Zero-Inflated Poisson (ZIP) model can be used to model such data. The Zero-Inflated Negative Binomial (ZINB) model is used to account for overdispersion detected in count data. The ZINB model is considered as an alternative for the Zero-Inflated Generalized Poisson (ZIGP) model for zero-inflated overdispersed count data. Consequently, zero-inflated models have been proposed for the situations where the data generating process results are overdispersed. This study considers modeling and handling overdispersion data among children with Thalassemia disease using the ZIP, ZINB and ZIGP models.

Key words: Count data; zero-inflation models; overdispersion; Thalassemia.

Introduction

Count data with too many zeros are common in a number of applications. Ridout, et al. (1998) cited examples of data with too many zeros from various disciplines including agriculture, econometrics, species abundance, medicine and recreational facility use. Several models have been proposed to handle count data with too many zeros. Lambert (1992) described ZeroInflated Poisson (ZIP) models with an

Nur Syabiha binti Zafakali is a final year postgraduate student in the Department of Mathematics within the Faculty of Science and Technology. She holds a Bachelor's of Computational Mathematics. Her research interests include Thalassemia disease among children in East Coast States of Malaysia. Email her at: syabiha_89@yahoo.com. Wan Muhamad Amir bin W Ahmad is a lecturer of Statistics in the Department of Mathematics within the Faculty of Science and Technology. He received his doctorate in Medical Statistics. Email him at: wmamir@umt.edu.my. application to defect in manufacturing. Lee, et al. (2001) generalized the ZIP model to accommodate the extent of individual exposure and Hall (2000) described the Zero-Inflated Negative Binomial (ZINB) model and incorporated random effects into ZIP and ZINB models.

The Poisson model emphasizes count data. Overdispersion implies that there is more variability around a model's fitted values than is consistent with a Poisson formulation and Poisson regression can be useful in the analyses of such data. Tsou (2006) demonstrated that a Poisson regression model could be adjusted to become asymptotically valid for inference about regression parameters, even if the Poisson assumption fails. Because positive counts may still be overdispersed with respect to the zerotruncated Poisson distribution, in the last decade Zero-Inflated Generalized Poisson (ZIGP) models have been found useful for the analyses of count data with a large amount of zerooutcomes (Famoye \& Singh, 2003). The generalized Poisson model has been used to model dispersed count data. It is a good competitor to the negative binomial model when 
the count data is over-dispersed. ZIP and ZINB models have been proposed for the situations where the data generating process results into too many zeros (Famoye \& Singh, 2006).

\section{Methodology \\ Zero-Inflated Poisson Model}

Consider the ZIP model, which is denoted by $\operatorname{Pr}\left(Y_{i}=y_{i}\right)$, in which the response variable $Y_{i}=(1,2, \ldots, n)$ has a probability mass function (pmf) given by

$$
\operatorname{Pr}\left(Y_{i}=y_{i}\right)=\left\{\begin{array}{cc}
\omega_{i}+\left(1-\omega_{i}\right) e^{-\theta_{i}}, & y_{i}=0, \\
\left(1-\omega_{i}\right) \frac{\theta_{i}^{y_{i}} e^{-0_{i}}}{y_{i} !}, & y_{i}>0,
\end{array}\right.
$$

Where $0 \leq \omega_{i}<1$ and $\theta_{i}>0$. The random variable $Y_{i}$ has a Poisson $\left(\theta_{i}\right)$ distribution when $\left(\omega_{i}\right)=0$. The parameters $\theta_{i}$ and $\omega_{i}$ depend on vectors of covariates $x_{i}$ and $z_{i}$, respectively. The ZIP model is given by

$$
\log \left(\theta_{i}\right)=x_{i}^{t} \beta, \log \left(\frac{\omega_{i}}{1-\omega_{i}}\right)=z_{i}^{t} \gamma
$$

and the mean and variance ZIP model are given by

$$
\begin{gathered}
E\left(Y_{i}\right)=\left(1-\omega_{i}\right) \theta_{i}, \\
\operatorname{Var}\left(Y_{i}\right)=\left(1-\omega_{i}\right) \theta_{i}\left(1+\omega_{i} \theta_{i}\right)
\end{gathered}
$$

\section{Zero-Inflated Negative Binomial Model}

For zero-inflated and overdispersed data a frequent modeling choice is the Zero-Inflated Negative Binomial (ZINB) model. The response variable $Y_{i}(i=1,2, \ldots, n)$ has a pmf given by

$$
\begin{aligned}
& \operatorname{Pr}\left(Y_{i}=y_{i}\right)= \\
& \left\{\begin{array}{l}
\omega_{i}+\left(1-\omega_{i}\right)\left(1+\kappa \theta_{i}^{c}\right)^{-\theta_{i}^{l-c / \kappa},} \\
y_{i}=0, \\
\left(1-\omega_{i}\right) \frac{\Gamma\left(y_{i}+\theta_{i}^{l-c} / \kappa\right)}{y_{i} ! \Gamma\left(\theta_{i}^{l-c} / \kappa\right)}\left(1+\kappa \theta_{i}^{c}\right)^{-\theta_{i}^{l-c} / x}\left(1+\theta_{i}^{-c} / \kappa\right)^{-y_{i}}, \\
y_{i}>0
\end{array}\right.
\end{aligned}
$$

Where $0 \leq \omega_{i} \leq 1$ and $\theta_{i}>0, \kappa$ is the dispersion parameter with $\kappa>0$ and $\Gamma($.$) is$ the gamma function. The mean and the variance of the model are defined as

$$
\begin{gathered}
E\left(Y_{i}\right)=\left(1-\omega_{i}\right) \theta_{i} \\
\operatorname{Var}\left(Y_{i}\right)=\left(1-\omega_{i}\right) \theta_{i}\left(1+\theta_{i} \kappa^{-1}+\omega_{i} \theta_{i}\right)
\end{gathered}
$$

The response variable $Y_{i}$ has a negative binomial distribution with mean $\theta_{i}$ and dispersion parameter $\kappa$ when $\omega_{i}=0$ (Garay, et al., 2011). Ridout, et al. (2001) fitted various models to these data on the basis of the Poisson and negative binomial distributions and their zero-inflated counterpart.

Zero-Inflated Generalized Poisson Model

The generalized Poisson model (ZIGP) was proposed by Consul \& Famoye (1992) and Famoye (1993). They emphasized the model to count data that are affected by a number of known predictor variables. Because positive counts may still be overdispersed with respect to the zero-truncated Poisson distribution, ZIGP models have been found to be useful for the analyses of count data with a large number of zero-outcomes. The generalized Poisson model has been used to model a household fertility data set (Wang \& Famoye, 1997) and to model injury data (Wulu, et al., 2002). The generalized Poisson distribution for random variable $Y_{i}$ takes the pmf given by 


$$
\begin{gathered}
f\left(y_{i} ; \lambda_{i}, \kappa\right)=\frac{\lambda_{i}\left(\lambda_{i}+\kappa y_{i}\right)^{y_{i}-1} e^{-\lambda_{i}-\kappa y_{i}}}{y_{i} !}, \\
y_{i}=0,1,2, \ldots,
\end{gathered}
$$

where $\lambda_{i}>0$ and $\max \left(-1,-\lambda_{i} / 4\right)<\kappa<1$. The mean and variance of $Y_{i}$ are

$$
\begin{gathered}
\mu_{i}=E\left(Y_{i}\right)=\frac{\lambda_{i}}{1-\kappa} \\
\operatorname{Var}\left(Y_{i}\right)=\frac{\lambda_{i}}{(1-\kappa)^{3}}=\frac{1}{(1-\kappa)^{2}} E\left(Y_{i}\right)=\varphi
\end{gathered}
$$

The term $\phi=1 /(1-\kappa)^{2}$ is a dispersion parameter. When $\kappa=0$, the generalized Poisson distribution reduces to a Poisson distribution with parameter $\lambda_{i}$ and is a case of equidispersion in the model, if $\kappa<0$, the generalized Poisson model represents count data with underdispersion, and if $\kappa>0$, generalized Poisson model represents count data with overdispersion. When there are more zero observations than expected, the generalized Poisson model will not provide good fit in general. Sampling zeros can be fitted into the ZIGP model, but not structural zeros. A good alternative to fit zero-inflated count data is a ZIGP model. The ZIGP model is defined as

$$
\begin{aligned}
& \operatorname{Pr}\left(Y_{i}=y_{i}\right)= \\
& \left\{\begin{array}{l}
\omega_{i}+\left(1-\omega_{i}\right) e^{-(1-\kappa)^{\theta_{i}}}, y_{i}=0, \\
\left(1-\omega_{i}\right)\left((1-\kappa) \theta_{i}+\kappa y_{i}\right)^{y_{i}-1} \frac{(1-\kappa) \theta_{i}}{y_{i} !} \exp \left[\begin{array}{c}
(-(1-\kappa) \\
\left.\times \theta_{i}-\kappa y_{i}\right)
\end{array}\right], \\
y_{i}>0
\end{array}\right.
\end{aligned}
$$

where $\theta_{i}>0$. The mean and variance of the ZIGP distribution are given by

$$
\begin{gathered}
E\left(Y_{i}\right)=\left(1-\omega_{i}\right) \theta_{i} \\
\operatorname{Var}\left(Y_{i}\right)=\left(1-\omega_{i}\right) \theta_{i}\left[1 /(1-\kappa)^{2}+\omega_{i} \theta_{i}\right]
\end{gathered}
$$

The parameters $\theta_{i}$ and $\omega_{i}$ depend on vectors of covariates $x_{i}$ and $z_{i}$, respectively. $\omega_{i}$ specifies the probability of structural zero status and can be modeled using a logit link function in which

$$
\log i t\left(\omega_{i}\right)=\log \omega_{i} /\left(1-\omega_{i}\right)=z_{i}^{\prime} \gamma
$$

where $z_{i}$ is the $i^{\text {th }}$ row vector of the covariate matrix and $\gamma$ is the parameter vector.

\section{Results}

To show the utility of the developed approach, the Zero-Inflated count models was applied to a real data set of underlying Thalassemia disease among children. Thalassemia is a genetic blood disorder in which the body makes an abnormal form of hemoglobin, the protein in red blood cells that carries oxygen. Thalassemia are common autosomal recessive disorders (Thursz, 2007). This study involved a sample of 930 for children age between 1-12 years. To build this dataset, the numbers of diagnoses among children aged 1-12 years who suffer from Thalassemia were counted. The data were collected at the Medical Record Unit in Hospital Universiti Sains Malaysia (HUSM), Kubang Kerian, Kelantan in north-east Malaysia 2005 to 2010. For the purpose of this study, the count data is used. The diagnosis is considered as the response variable and the selected variables are: disease of blood, health services, heart failure, Influenza, Anemia, Pneumonia, acute bronchitis, Asthma, Acute Tonsillitis, Jaundice and Tuberculosis.

To handle overdispersion in a zeroinflated dataset, a one-sided test was used and the level of significance was set as $\alpha=0.05$. The data analyses were performed in SAS 9.3, using proc genmod and proc nlmixed. In counting the number of responses to an exposure a patient may have no diagnosis response because of their immunity or resistance to a disease.

In the dataset, there were 930 patients and among these, 635 patients had no diagnosis (see Table 1); thus, there are $68 \%$ zero counts in the data. The overdispersion might have been 
due to many excess zeros for the case when $y=0$ because $68 \%$ of observed counts are zeros. Frequency (percent) of patients who received a different type of diagnosis is a total of $125(13.4 \%)$, while patients who received two different types of diagnosis was $95(10.2 \%)$. For patients who received three different types of diagnosis the frequency (percent) was 58 (6.2\%) and patients who received four different types of diagnosis was $17(1.8 \%)$. Mean (standard deviation) of the variables diagnosis showed a value of 600 (1019), while the variance of 1039 . Because the data has too many zeros and is overdispersed the zero-inflation model can be applied.

The dispersion parameters of all models correspond to 0.599 for the dispersion index. This value indicates that the dispersion in the data is not large and could be corrected by the use of a model that incorporates a dispersion parameter such as ZIP, ZINB or ZIGP. The models of ZIP, ZINB and ZIGP are positively associated $(p<0.0001)$ by implementing each of the models to the data using Proc Nlmixed (see Table 2). The analyses of fitting zeroinflated models variable include: Disease of Blood, Health Services, heart failure, Influenza, Anemia, Pneumonia, acute bronchitis, Asthma, Acute Tonsillitis, Jaundice and Tuberculosis.

A fit of these models gives a deviance of 1137.4476 on 930 d.f. The deviance (D) is equivalent to the likelihood-ratio test statistic $G^{2}$ that is defined as:

$$
G^{2}=2 \sum_{i=1}^{n} y_{i} \log \left(\frac{y_{i}}{\mu_{i}}\right)
$$

Consequently, a test of overdispersion of the data, which is measured by the ratio of deviance/d.f. $=1.233$. Because this value is greater than 1 , there is strong evidence that the data is overdispersed and therefore $E\left(Y_{i}\right) \neq \operatorname{Var}\left(Y_{i}\right)$. All three models fit the data well with corresponding log-likelihoods of: -559.8034 (ZIP), -568.7238 (ZINB) and -558.3192 (ZIGP) obtained from output ProcGenmod (see Table 3). Clearly, the ZIGP model seems to fit the data best as it has the smallest Akaike Information Criterion $(\mathrm{AIC}=1148.6384)$. Although the ZIP, ZINB and ZIGP all fit the data well, the effect of having a diagnosis in patients does not appear to have any significant effect on the number of diagnoses under these models.

\section{Conclusion}

This article focused on handling overdispersion data that involves zero-inflation models. Overdispersion can be modeled when counts show more variability than previously assumed models. However, the consideration of zeroinflation for sample size less than 50 is not encouraged, it is recommended to evaluate scores for sample sizes $\geq 100$. The parametric bootstrap method is recommended for sample size between 50 and 100 for a reliable performance (Jung, Jhun \& Lee, 2005).

Three different methods were used in this study: (i) ZIP model, (ii) ZINB model and (iii) ZIGP model with covariate dependence. The ZIP model described in Lambert's (1992) seminal work provides a sufficient fit to data when overdispersion in raw data is caused by zero-inflation. The ZINB model should be considered if data continue to suggest additional overdispersion (overdispersion can be the result of excess zeroes): The ZIP model is not appropriate for these data, because the Poisson model does not accommodate the remaining overdispersion and not accounted for through zero-inflation. The ZIGP model is applied in different fields to model zero-inflated and overdispersed data. The ZIGP could provide a better fit than the ZINB when there is a large zero-fraction; this implies that the ZIGP model could be a reasonable alternative to the ZINB model.

It is surprising that in all these models, it appears that the ZIGP model provided a good fit to the data because it had the smallest value of Akaike Information Criterion (AIC). Although the ZIGP model appears to be a good competitor to the ZIP and ZINB models, it is unknown under what conditions, if any, which model would perform best. 


\section{ZAFAKALI \& AHMAD}

Table 1: Diagnosis Count from 930 Thalassemia Patients in HUSM

\begin{tabular}{|c|c|c|}
\hline \multirow{2}{*}{ Diagnosis } & Frequency & Percent (\%) \\
\cline { 2 - 3 } & 635 & 68.3 \\
\hline 0 & 125 & 13.4 \\
\hline 1 & 95 & 10.2 \\
\hline 2 & 58 & 6.2 \\
\hline 3 & 17 & 1.8 \\
\hline 4 & 930 & 100 \\
\hline Total &
\end{tabular}

Table 2: Summary of Zero-Inflated Model Fit

\begin{tabular}{|c|c|c|c|}
\hline \multirow{2}{*}{ Variable } & \multicolumn{3}{|c|}{ Estimate (Standard Error) } \\
\cline { 2 - 4 } & ZIP & ZINB & ZIGP \\
\hline Disease of Blood & $1.0144(0.3594)$ & $0.4763(0.3546)$ & $0.4737(0.3594)$ \\
\hline Health Services & $1.2116(0.0883)$ & $0.5075(0.0722)$ & $0.5075(0.0925)$ \\
\hline Heart failure & $0.9702(0.1048)$ & $0.5222(0.1009)$ & $0.5222(0.1012)$ \\
\hline Influenza & $0.9429(0.1852)$ & $0.5852(0.1781)$ & $0.5852(0.1783)$ \\
\hline Anemia & $0.7778(0.1004)$ & $0.4996(0.0873)$ & $0.4997(0.0944)$ \\
\hline Pneumonia & $0.9062(0.1147)$ & $0.5174(0.1142)$ & $0.5173(0.1143)$ \\
\hline Acute bronchitis & $0.9116(0.0913)$ & $0.5072(0.0713)$ & $0.5071(0.0904)$ \\
\hline Asthma & $1.1563(0.0939)$ & $0.5869(0.0910)$ & $0.5869(0.0963)$ \\
\hline Acute Tonsillitis & $0.7234(0.1743)$ & $0.3794(0.1743)$ & $0.3794(0.1746)$ \\
\hline Jaundice & $1.2890(0.3675)$ & $0.5771(0.3823)$ & $0.5767(0.3888)$ \\
\hline Tuberculosis & $0.6550(0.3857)$ & $0.3791(0.3702)$ & $0.3789(0.3702)$ \\
\hline
\end{tabular}

\section{References}

Famoye, F., \& Singh, K. P. (2003). On inflated generalized Poisson models. Advances in Applied Statistics, 2, 145-158.

Ridout, M., Hinde, J., \& Dem'etrio, C. G. B. (2001). A score test for testing a ZIP model against ZINB alternatives. Journal of Biometrics, 57, 219-223.
Ridout, M., Demetrio, C. G. B., \& Hinde, J. (1998). Models for count data with many zeros. Invited paper presented at the Nineteenth International Biometric Conference, Cape Town, South Africa, 179-190.

Lambert, D. (1992). ZIP with an application to defects in manufacturing. Journal of Technometrics, 34, 1-14. 
Table 3: Parameter Estimates, Standard Errors (in parentheses) for All Models

\begin{tabular}{|c|c|c|c|}
\hline Parameters & ZIP & ZINB & ZIGP \\
\hline$a_{0}$ & $-17.4510\left(246.90^{*}\right)$ & $3.9808\left(0.4553^{*}\right)$ & $3.9811\left(0.4615^{*}\right)$ \\
\hline$b_{0}$ & $-1.9236\left(0.08624^{*}\right)$ & $-0.5294\left(0.0517^{*}\right)$ & $-0.5294\left(0.1125^{*}\right)$ \\
\hline Log likelihood & -569.8034 & -568.7238 & -558.3192 \\
\hline AIC & 1151.6069 & 1153.6069 & 1148.6384 \\
\hline BIC $^{\mathrm{b}}$ & 1228.9698 & 1235.8050 & 1226.0013 \\
\hline Pearson Chi-Square $\chi^{2}$ & 416.0162 & & \\
\hline Deviance & 393.0901 & & \\
\hline d.f & 918 & & \\
\hline
\end{tabular}

*significant at level 0.05, a = Akaike's information criteria (AIC), b = Bayesian information criterion

Hall, D. B. (2000). ZIP and binomial with random effects: A case study. Journal of Biometrics, 56, 1030-1039.

Lee, A. H., Wang, K., \& Yau, K. K. W. (2001).Analyses of ZIP data incorporating extent of exposure. Journal of Biometrics, 43, 963-975.

Famoye, F. (1993). Restricted generalized Poisson model. Communications in Statistics, Theory and Methods, 22, 1335-1354.

Consul, P. C., \& Famoye, F. (1992). Generalized Poisson model. Communications in Statistics, Theory and Methods, 21, 89-109.

Wang, W., \& Famoye, F. (1997). Modeling household fertility decisions with generalized Poisson. Journal of Population Economics, 10, 273-283.
Wulu, J. T., Singh, K. P., Famoye, F., \& McGwin, G. (2002). Analyses of count data. Journal of the Indian Society of Agricultural Statistics, 55, 220-231.

Garay, A. M., Hashimoto, E. M., Ortega, E. M. M., \& Lachos, V. H. (2011). On estimation and influence diagnostics for ZINB models. Journal of Computational Statistics and Data Analyses, 55, 1304-1318.

Thursz M. (2007). Iron, hemochromatosis and thalassaemia as risk factors for fibrosis in hepatitis $\mathrm{C}$ virus infection. Journal of Gut, 56, 613-614.

Jung, B. C., Jhun, M., \& Lee, J. W. (2005). Bootstrap tests for overdispersion in a zero-inflated Poisson model. Journal of Biometrics, 61, 626-629.

Tsou, T. S. (2006). Robust Poisson regression. Journal of Statistical Planning and Inference, 136, 3173-3186. 


\section{Algorithms \& Code \\ JMASM 32: Multiple Imputation of Missing Multilevel, Longitudinal Data: A Case When Practical Considerations Trump Best Practices?}

\author{
Jennifer E. V. Lloyd \\ University of British \\ Columbia, \\ Vancouver, Canada
}

\author{
Jelena Obradović \\ Stanford University, \\ Stanford, CA, USA
}

\author{
Richard M. Carpiano \\ University of British \\ Columbia, \\ Vancouver, Canada
}

\author{
Frosso Motti-Stefanidi \\ University of Athens, \\ Athens, Greece
}

A pedagogical tool is presented for applied researchers dealing with incomplete multilevel, longitudinal data. It explains why such data pose special challenges regarding missingness. Syntax created to perform a multiply-imputed growth modeling procedure in Stata Version 11 (StataCorp, 2009) is also described.

Key words: Missing data, longitudinal data, multilevel data, multiple imputation, growth modeling, Stata.

\section{Introduction}

One research challenge faced when conducting a longitudinal study is selecting a method for handling missing data. Incomplete assessment histories for longitudinal study participants are ubiquitous (Allison, 2002; Jeličić, Phelps \& Lerner, 2009), and are due to multiple factors, such as participants' attrition, illness, unwillingness or inability to answer certain questions, and problems related to the methods of data collection.

When considering how longitudinal data are inherently structured - with repeated measurements (at level-one) clustered or nested within individual participants (at level-two) such data are in effect multilevel or hierarchical

Jennifer E. V. Lloyd is a Research Associate at the Human Early Learning Partnership (HELP). Email her at: jennifer.lloyd@ubc.ca. Jelena Obradović is an Assistant Professor in the Graduate School of Education. Email her at: jelena.obradovic@stanford.edu. Richard M. Carpiano is an Associate Professor in the Department of Sociology. Email him at: richard.carpiano@ubc.ca. Frosso Motti-Stefanidi is a Professor in the Department of Psychology. Email her at: frmotti@psych.uoa.gr. (complex) (Zaidman-Zait \& Zumbo, 2013). Hence, incomplete assessment histories may affect the availability of values for both timevarying and time-invariant variables.

Ignoring missing data can significantly bias estimates of coefficients and standard errors, inflate Type I and II error rates, degrade confidence intervals and/or distort statistical power (Acock, 2005; Allison, 2002; Collins, Schafer, \& Kam, 2011; Little \& Rubin, 2002; Schafer \& Graham, 2002; Zaidman-Zait \& Zumbo, 2013). Therefore, missing data should be a focus of any longitudinal study, rather than being sidelined as a bother (Allison, 2002; Little \& Rubin, 2002; Schafer \& Graham, 2002).

Unfortunately, many of the strategies proposed to handle missingness tend to be primarily implementable in relatively rudimentary research contexts in which data lack the intricacy and "messiness" of real-life data (Carpenter, Goldstein \& Kenward, 2011; Graham, 2009; Johnson \& Young, 2011). To complicate matters, some best-practice studies of missing data imputation provide such stringent technical requirements for filling-in missing data that their recommendations cannot realistically be met in many real-life research contexts. Some of these requirements were generated from elementary simulated, singlelevel data sets (von Hippel, 2007, 2009). Furthermore, large percentages of longitudinal 


\section{MULTIPLE IMPUTATION OF MISSING MULTILEVEL, LONGITUDINAL DATA}

researchers either did not comment on their studies' missingness or they utilized outdated and even incorrect methods to handle missingness. As a result, the longitudinal research literature is scattered with examples of bad missing data practices (Jeličić, et al., 2009).

Given the lack of a robust empirical research base centered on this problem, the lack of conclusive recommendations about precisely how to deal with the problem, and the dearth of statistical software resources that allow users to resolve this problem (Allison, 2002; Carpenter, et al., 2011; Graham, 2009), many longitudinal researchers find themselves at an impasse. To further complicate matters, graduate training in statistics, measurement and research methodology in North American universities has declined significantly in recent years, while there has been an increasing trend toward doctoral-level researchers with minimal knowledge of statistics who nonetheless conduct analyses (Aiken, West \& Millsap, 2008; Aiken, et al., 1990; Merenda, 2003). For these reasons, dealing with incomplete complex data is a good idea in theory, but a challenging one in practice.

\section{Objectives}

The goal of this article is to provide a pedagogical tool for applied longitudinal researchers dealing with incomplete complex data. The first objective is to explain and illustrate why complex data pose special challenges when it comes to missingness. Inspired by the work of the UCLA Academic Technology Services' Statistical Consulting Group (n.d.a), the second objective is to provide a step-by-step description of syntax created to perform a multiply-imputed individual growth modeling procedure in a real-life longitudinal research context (Obradović, Lloyd \& MottiStefanidi, manuscript in preparation), using Stata Version 11 (StataCorp, 2009).

Strategies for handling missing complex data will be presented, although it is not claimed that they are a perfect solution to the problem. The complex structure of data in this study was not amenable to certain imputation-related recommendations offered in the general missing data literature. In short, missingness was dealt with in the best way possible given the unanswered questions that surround missing complex data. But it is precisely because of these unanswered questions that the implementation of a modern procedure, such as multiple imputation, to fill in incomplete multilevel, longitudinal data is, in principal, justifiable until the missing data literature provides conclusive recommendations for handling missing complex data in broadlydefined longitudinal research contexts.

Different types of missing data and missing data mechanisms were discussed by Allison (2002), Collins, et al. (2011), Graham (2009), Little (1995), Little and Rubin (2002), Schafer and Graham (2002) and Zaidman-Zait and Zumbo (2013). Applications, strengths, and limitations of assorted traditional and modern methods by which to handle missing data were discussed by Acock (2005), Allison (2002), Collins, et al. ( 2011), Little and Rubin (2002), Schafer and Graham (2002). Working knowledge of how to run individual growth models (multilevel models of change) is assumed (Raudenbush \& Bryk, 2002; Singer \& Willett, 2003), as is familiarity with Stata's programming language. That said, step-by-step syntax descriptions facilitate translating the Stata commands into other programming languages or platforms.

Why Complex Data Pose Special Challenges Multiple imputation involves four steps: (1) replication, wherein multiple copies of an incomplete data set are created; (2) imputation, wherein missing values in each data set are replaced with plausible versions of the complete data derived from multivariate data; (3) analysis, wherein each imputed data set is analyzed separately using standard methods of statistical analysis; and (4) recombination, wherein the results of the separate analyses are combined or pooled (Rubin, 1987; Schafer, 1999; Schafer \& Graham, 2002; von Hippel, 2007). The process of combining results of parameter estimates and their respective standard errors from several imputed data sets has been shown to yield valid statistical inferences that reflect the uncertainty due to the missingness (Yuan, 2011).

Unfortunately, it is challenging to begin the multiple imputation process when dealing with complex data. For example, von Hippel (2009) and Allison (2002) recommended 


\section{LLOYD, OBRADOVIĆ, CARPIANO \& MOTTI-STEFANIDI}

calculating transformations such as interactions and squared terms using the incomplete data and, in turn, imputing the transformations alongside the other regular variables. This transform-then-impute approach has been shown to yield better, less-biased regression estimates than when variables are imputed in their raw form and, in turn, transformations are calculated from the imputed data (the impute-thentransform approach; von Hippel, 2009). Although the transform-then-impute approach may be possible to heed in certain single-level or simulated contexts, it is difficult to implement when dealing with complex data.

Consider a hypothetical example in which a longitudinal study involves data collected across three waves, in which $Y_{t i}$ is the observed score at time or wave $t$ for individual participant $i$. Consider further that there is one time-varying predictor, $X_{t i}$, and two timeinvariant predictors, $V_{i}$ and $W_{i}$. Then imagine that the data have been entered into a spreadsheet in person or wide format - wherein all of the records collected for an individual participant are entered along one row of the spreadsheet. As indicated in Table 1, when data are formatted this way, there is no time or wave variable (i.e., a variable that explicitly denotes the particular period of data collection). Instead, individuals' scores for time-varying variables are represented by as many separate variable names (i.e., columns in the spreadsheet) as there are waves. For example, $X_{i}$ for waves 0 through 2 are respectively denoted by variables $X_{0}, X_{1}$, and $X_{2}$.

Although the lack of a wave variable makes it possible to create single-level interactions between time-invariant variables (e.g., $V_{i}^{*} W_{i}$ at level-two), it is computationally difficult to automate the inclusion of cross-level interactions in the imputation model. This difficulty is lamentable because the ability to explore cross-level interactions is one of the primary advantages of performing an individual growth modeling analysis (Holt, 2008). A crosslevel interaction refers the interaction between level-two variables and level-one variables, "that is, to modification of the effects of lower level variables by characteristics of the higher level units to which the lower level units belong (or vice versa)" (Diez-Roux, 2002, p. 589).
For example, suppose a study is designed to explore the cross-level interaction between time or wave (at level-one) and the time-invariant variable $W_{i}$ (at level-two). In the absence of an explicit time or wave variable, the interaction cannot be computed. Similarly, suppose a study is designed to explore the crosslevel interaction between the time-varying variable $X_{t i}$ (at level-one) and the time-invariant variable $V_{i}$ (at level-two). Because $X_{t i}$ 's timevarying values are represented by as many variable names (columns) as there are waves, there is no way of creating a cross-level product term that takes into account the temporal nature of $X_{t i}$ while also taking into account the constant nature of $V_{i}$.

Alternatively, imagine that the same data have been entered into a spreadsheet in person-period or long format - wherein each individual's records are entered into as many rows as there are waves of data collection (in the case of the example presented, three rows per individual).

Although it is shown in Table 2 that there is a wave variable, most statistical software programs, including Stata, require data to be in person format during imputation. If not, the software erroneously views separate rows as representing separate individuals. Hence, when exploring cross-level interactions, it is not possible to take into account the withinindividual covariance - the inherently nested or clustered structure of the data - whether the data are entered in person format or in person-period format (Han, 2008).

von Hippel (2009) recommended any centering of the scores of a given variable - a practice aimed at reducing collinearity and improving interpretation of the intercept (Raudenbush \& Bryk, 2002) - be carried out prior to imputation. What is unclear is which problems are introduced if the mean that is being subtracted is being skewed by the variable's missing values. The benefit of centering preimputation is also unclear, given that centering simply linearly transforms a variable's scores into those different metric. This problem is not endemic to complex data sets alone, but highlights the questions surrounding multiple imputation and the transform-then-impute approach specifically. 


\section{MULTIPLE IMPUTATION OF MISSING MULTILEVEL, LONGITUDINAL DATA}

Table 1: Hypothetical Example of Data Entered into a Spreadsheet in Person (Wide) Format

\begin{tabular}{|c|c|c|c|c|c|c|c|c|}
\hline$I D$ & $Y_{0}$ & $Y_{1}$ & $Y_{2}$ & $X_{0}$ & $X_{1}$ & $X_{2}$ & $V$ & $W$ \\
\hline 1 & 4 & 7 & 10 & 13 & 16 & 19 & 22 & 25 \\
\hline 2 & 5 & & 11 & 14 & & 20 & 23 & 26 \\
\hline 3 & 6 & 9 & 12 & 15 & 18 & 21 & & 27 \\
\hline
\end{tabular}

Table 2: Hypothetical Example of Data Entered into a Spreadsheet in Person-Period (Long) Format

\begin{tabular}{|c|c|c|c|c|c|}
\hline$I D$ & $Y$ & $X$ & Wave & $V$ & $W$ \\
\hline 1 & 4 & 13 & 0 & 22 & 25 \\
\hline 1 & 7 & 16 & 1 & 22 & 25 \\
\hline 1 & 10 & 19 & 2 & 22 & 25 \\
\hline 2 & 5 & 14 & 0 & 23 & 26 \\
\hline 2 & & & 1 & 23 & 26 \\
\hline 2 & 11 & 20 & 2 & 23 & 26 \\
\hline 3 & 6 & 15 & 0 & & 27 \\
\hline 3 & 9 & 18 & 1 & & 27 \\
\hline 3 & 12 & 21 & 2 & & 27 \\
\hline
\end{tabular}

Stata Tutorial

A step-by-step description of syntax used to perform a multiply-imputed growth modeling procedure in a longitudinal research context is presented (Obradović, et al., manuscript in preparation). Although this tutorial describes specific imputation and analytic choices made with respect to the data at hand, there is no 'one size fits all' approach to addressing the problem of missing data (Johnson \& Young, 2011; Yuan, 2011).

\section{Software}

Stata Version 11 (StataCorp, 2009) was used for the tutorial due to its versatile ability to perform data management tasks, multiple imputation and complex analyses. Schafer (2001) developed a statistical program, PAN, which accounts for the clustered nature of longitudinal data as part of S-Plus (Schafer, 2001; Schafer \& Yucel, 2002). An imputation macro for MLwiN, REALCOM-IMPUTE, was developed for multilevel data (Carpenter, et al., 2011). Although these are exciting advancements, PAN's limited availability and accessibility (Graham, 2009) and REALCOMIMPUTE's relative newness (with documentation focused only on non-growth model examples of nested data) indicate neither has made its way into routine use by applied longitudinal researchers.

\section{Missing Data Procedure}

Two choices of modern missing data procedures were available to implement in this study: full information maximum likelihood (FIML) or multiple imputation (MI). As Collins, et al. (2001) wrote, FIML "chooses parameter values that assign the highest possible probability or probability density to the data values actually seen, under a well-defined family of parametric probability models" (p. 334). 


\section{LLOYD, OBRADOVIĆ, CARPIANO \& MOTTI-STEFANIDI}

FIML treats the missing data as random variables to be removed from the likelihood function. This, in a sense, treats the missing data as if they were never sampled, rather than deleting or filling in the missing cases (Schafer \& Graham, 2002). Little and Rubin (2002) provided detail on FIML estimation. By contrast, MI "attempts to handle the missing data aspect in advance of the substantive analysis" (Collins, et al., p. 335) by combining results of parameter estimates and their respective standard errors from several imputed data sets, pre-analysis.

According to Carpenter, et al. (2011), $\mathrm{MI}$ is the leading approach to handling data that are missing at random (MAR). It is implementable with a larger variety of data and statistical models than FIML (Allison, 2002; Johnson \& Young, 2011). Although FIML yields more efficient estimates, it is a more casespecific (less general) approach to missingness, and is computationally more difficult (Allison, 2002; Schafer, 1999). Allison (2002) and Johnson and Young (2011) provided overviews of the advantages, disadvantages, and applications of both types of modern missing data procedures.

Approach to Multiple Imputation

The syntax features commands related to Stata 11's Imputation by Chained Equations (ICE) add-on program. Consider a dataset in which some or all of the variables, $X_{1}, \ldots, X_{k}$, have missing data:

Initially, all missing values are filled in at random. The first variable with at least one missing value, $X_{1}$ say, is then regressed on the other variables, $X_{2}, \ldots$, $X_{k}$. The estimation is restricted to individuals with observed $X_{1}$. Missing values in $X_{1}$ are replaced by simulated draws from the posterior predictive distribution of $X_{1}$, an important step known as proper imputation. The next variable with missing values, say $X_{2}$, is regressed on all the other variables, $X_{1}$; $X_{3} ; \ldots, X_{k}$. Estimation is restricted to individuals with observed $X_{2}$ and uses the imputed values of $X_{1}$. Again, missing values in $X_{2}$ are replaced by draws from the posterior predictive distribution of $X_{2}$. The process is repeated for all other variables with missing values in turn: one such round is called a cycle. To stabilize the results, the procedure is [repeated] to produce a single imputed dataset. (Royston \& White, 2011, p. 2)

The posterior predictive distribution refers to the predictive distribution of unobserved scores, conditional on the observed data (Kelly \& Smith, 2011). The process begins with each variable with missing values being imputed using a univariate regression model conditional on all of the other variables. The process cycles iteratively through the variables containing missing values until the procedure is stable - a process called regression switching (UCLA Academic Technology Services' Statistical Consulting Group, n.d.b).

Generally, ten to twenty repetitions of this cycle are required to produce an imputed data set. The procedure is repeated $m$ times to yield $m$ imputed data sets (White, Royston \& Wood, 2011). Because variables may be of different types (binary, continuous, etc.), a suitable model must be identified for each variable. For example, logistic regression is used to predict a binary variable's values and ordinary least squares (OLS) regression is used if the variable is continuous (Johnson \& Young, 2011). ICE has been lauded for its widereaching capabilities and different estimation methods depending on the type of variable (e.g., Acock, 2005; White, et al., 2011). In fact, ICE is now available by default in the multiple imputation module in Stata Version 12 (StataCorp, 2011). It is described by Royston (2004, 2005) and Royston and White (2011). White, et al. (2011) provided a tutorial using real and simulated datasets.

The chained equations approach is one of two multiple imputation approaches for handling missingness. In the second approach, called the multivariate normal model approach, the joint distribution of all variables in the imputation model is assumed to be multivariate normal (Little \& Rubin, 2002). Information from the variables is used to impute all other variables based on a single model. In contrast, the chained 


\section{MULTIPLE IMPUTATION OF MISSING MULTILEVEL, LONGITUDINAL DATA}

equation approach is based on each conditional density of a variable given other variables. It is the multivariate normal model approach used in Stata's mi impute mvn command (UCLA Academic Technology Services' Statistical Consulting Group, n.d.b).

In the context of this study, the chained equations approach was implemented because it does not assume a multivariate joint distribution and, therefore, can accommodate variables of different types. It also has lower sample size requirements than the multivariate normal approach (UCLA Academic Technology Services' Statistical Consulting Group, n.d.b).

\section{Data}

The data in the tutorial are a subset of the Athena Studies of Resilient Adaptation (AStRA) project, which focuses on the adaptation of immigrant youth living in Greece (Motti-Stefanidi, et al., 2008ab; Motti-Stefanidi \& Asendorpf, 2012; Motti-Stefanidi, Asendorpf $\&$ Masten, 2012). This subset contained records for 793 youth participants across nine schools in Athens, Greece. Participants either were of Albanian origin (306 or $38.6 \%$ ) or were native Greek youth (487 or $61.4 \%$ ).

As shown in Table 3, participants were measured on five outcomes across three annual waves: self-esteem, self-efficacy, behavior problems, school grades, and school engagement - all of which are continuous variables. Also collected were five time-invariant predictors: participants' immigrant status $(0=$ nonimmigrant, $1=$ immigrant $)$, sex $(0=$ male, $1=$ female), initial adversity, initial socioeconomic risk, and initial adaptability - the latter three of which were continuously scored. In addition, information for a time-varying variable, adaptability, which was also continuously scored, was collected. Note that in some of the growth models, adaptability was treated as a time-invariant predictor (initial adaptability at Wave 0), whereas, in other analyses it was treated as time-varying predictor (adaptability). Because the interest in the study was in exploring differences between immigrants and their native peers, growth models were stratified by immigrant status. The three waves were coded as 0,1 and 2, respectively.
Three waves of data were obtained for most of the 793 participants. For 165 participants $(20.8 \%)$ in two schools, however, data collection stopped after Wave 0; therefore, 68 immigrants $(22.2 \%$ of 306$)$ and 97 nonimmigrants (19.9\% of 487) were missing data for Waves 1 and 2.

Because the missingness had to do with administrative reasons and not with the participants themselves, the missingness was treated as MAR - which is an assumption of MI (Schafer \& Graham, 2002). Rather than deleting the participants missing Waves 1 and 2 from the sample, MI was performed to fill in the missingness, first, in order to avoid selection bias and, second, because these participants were considered to be a part of the population of interest (J. W. Graham, personal communication, January 14, 2011).

\section{Methodology}

Data were analyzed using two linear individual growth models. Although growth models allow for time-unstructured data (different data collection schedules for different individuals) and unbalanced data (different numbers of waves for each individual) (Holt, 2008), if it is suspected that growth curves are non-linear, large amounts of missing data may prohibit departures from linearity - even though intercepts and slopes can still be estimated (Bickel, 2007). Either complete or imputed data are required at higher levels of the analyses (Holt, 2008). An assumption underlying growth analyses is that there is a correctly specified level-one submodel.

Model specification refers to the process of choosing an appropriate functional form for, and variables to include in, the growth models. If the model is not correctly specified, growth models lose their ability to handle missing data well. A growth model's ability to handle incomplete data rests, in part, on the model's being correctly specified (B. D. Zumbo, personal communication, March 18, 2012). If the model is not correctly specified, conclusions may be distorted by the various missing data mechanisms (Zaidman-Zait \& Zumbo, 2013). As with any type of analysis, "the nature and number of missing data may badly compromise 


\section{LLOYD, OBRADOVIĆ, CARPIANO \& MOTTI-STEFANIDI}

Table 3: Variable Descriptions and Names

\begin{tabular}{|l|c|c|c|}
\hline \multicolumn{1}{|c|}{ Variable Description } & $\begin{array}{c}\text { Outcome } \\
\text { Variable Name }\end{array}$ & $\begin{array}{c}\text { Time-Invariant } \\
\text { Variable Name }\end{array}$ & $\begin{array}{c}\text { Time-Varying } \\
\text { Variable Name }\end{array}$ \\
\hline Self-esteem & Esteem & & \\
\hline Self-efficacy & Efficacy & & \\
\hline Behavior & Behavior & & \\
\hline School grades & Grades & & \\
\hline School engagement & Engage & & \\
\hline Immigrant status $(0=$ non-immigrant, $1=$ immigrant) & & Immigrant & \\
\hline Sex $(0=$ male, $1=$ female) & & Female & \\
\hline Initial adversity (Adversity at Wave 0$)$ & & Advers0 & \\
\hline Initial socioeconomic risk (SES risk at Wave 0) & & SESRisk0 & \\
\hline Initial adaptability (Adaptability at Wave 0) & & Adapt0 & \\
\hline Adaptability (Adaptability across Waves 0, 1,2) & & & Adapt \\
\hline Period of data collection & & Wave \\
\hline Participant identification number & & \\
\hline
\end{tabular}

[the] analysis, so that inferences from sample to population become dubious" (Bickel, 2007, p. 301). It is therefore necessary to pay heed to data missingness even when using methods of analysis that otherwise allow for some degree of time-unstructured and unbalanced data.

Models

Each of the growth models in this study was stratified by immigrant/non-immigrant status to allow for comparisons between immigrants and their native peers. Two growth models per outcome (esteem, efficacy, behavior, grades, engage) were run. Model 1 was designed to examine main effects of adaptability on initial levels and rate of change of adaptation, over and above sex, initial adversity, and initial SES risk. Model 2 was designed to examine whether changes in adaptability across the three annual waves were associated with changes in the participants' adaptation, which is known as dynamic covariation (Long \& Pellegrini, 2003; Murray-Close, Ostrov \& Crick, 2007).

Model 1, Level 1:

$$
Y_{t i}=\pi_{0 i}+\pi_{1}(\text { Wave })_{t i}+e_{t i}
$$

Model 1, Level 2:

$$
\begin{aligned}
\pi_{0 i}= & \beta_{00}+\beta_{01}(\text { Female })_{i}+\beta_{02}(\text { Adapt } 0)_{i} \\
& +\beta_{03}(\text { Advers } 0)_{i}+\beta_{04}(\text { SESRisk } 0)_{i}+r_{0 i}
\end{aligned}
$$

and

$$
\begin{aligned}
\pi_{1 i}= & \beta_{10}+\beta_{11}(\text { Adapt })_{i}+\beta_{12}(\text { Advers } 0)_{i} \\
& +\beta_{13}(\operatorname{SESRisk0})_{i}+r_{1 i}
\end{aligned}
$$

Model 2, Level 1:

$$
Y_{t i}=\pi_{0 i}+\pi_{1}(\text { Wave })_{t i}+\pi_{2}(\text { Adapt })_{t i}+e_{t i}
$$




\section{MULTIPLE IMPUTATION OF MISSING MULTILEVEL, LONGITUDINAL DATA}

Model 2, Level 2:

$$
\begin{aligned}
& \pi_{0 i}=\beta_{00}+r_{0 i}, \\
& \pi_{1 i}=\beta_{10}+r_{1 i}, \\
& \text { and } \\
& \pi_{2 i}=\beta_{20}
\end{aligned}
$$

Tutorial

A step-by-step description of syntax used to perform a multiply-imputed growth modeling procedure is provided in panels $1-5$. Syntax commands conveniently outlined by UCLA Academic Technology Services' Statistical Consulting Group (n.d.a) for longitudinal data are used as a framework around which to organize the syntax used in this study. Stata command language is identified in bold face.

\section{Conclusion}

Due to the well-documented problems associated with missing data, researchers have long been cautioned to investigate data missingness closely and to carefully select a missing data technique that will assist in filling in their data's missing values. Even so, there continues to be uncertainty about how to deal with the problem of incomplete complex data.

There is a paucity of empirical studies centered on this problem, a lack of conclusive recommendations about precisely how to deal with the problem, and limited statistical software resources that allow users to resolve the problem (Allison, 2002). These factors, combined with recent decline in statistics, measurement and research methodology training in North American universities (Aiken, et al., 2008; Aiken, et al., 1990; Merenda, 2003) means that finding a solution to dealing with the problem of incomplete complex data is not an easy task.

This article served as a pedagogical tool for applied longitudinal researchers who are dealing with this problem in their own research contexts. By explaining why complex data pose special challenges with respect to missingness, as well as providing readers with a step-by-step description of syntax created to perform a multiply-imputed individual growth modeling procedure in a real-life longitudinal research context, it is hoped that readers have a clearer sense of the methodological challenges and realities posed by incomplete complex data.

Once again, it is not claimed that the syntax outlined herein fully takes into account the within-individual dependencies among the study variables; however, the conspicuous lack of information available on this topic means that, from a practical perspective, researchers have little choice but to simply deal with missingness as best they can with available resources (Collins, et al., 2001). After all, it is likely better to fill in missing complex data using a modern missing data technique than it is to do nothing at all.

Acknowledgements

Lloyd acknowledges the Djavad Mowafaghian Foundation for supporting her research. Obradović acknowledges the Canadian Institute for Advanced Research (CIFAR) and the Jacobs Foundation for supporting her research. Carpiano acknowledges investigator award funding from the Canadian Institutes of Health Research (CIHR) and the Michael Smith Foundation for Health Research (MSFHR). The data included in the tutorial are a subset of those collected as part of the Athena Studies of Resilient Adaptation (AStRA) project, which is supported by a grant to Motti-Stefanidi, cofunded by the European Social Fund and Greek National Resources (EPEAEK IIPYTHAGORAS), and, partially, by the Special Account for Research Grants of the University of Athens, Greece. We also thank Michelle Frisco, John Graham, Jason Houle, Jeremy Miles, Margaret Weden, Ian White, Rebekah Young, and Bruno Zumbo for their feedback on earlier drafts.

\section{References}

Acock, A. C. (2005). Working with missing values. Journal of Marriage and Family, 67(4), 1012-1028.

Aiken, L. S., West, S. G., \& Millsap, R. E. (2008). Doctoral training in statistics, measurement, and methodology in psychology: Replication and extension of Aiken, West, Sechrest, and Reno's (1990) survey of PhD programs in North America. American Psychologist, 63, 32-50. 
set seed 123

\section{$\underline{\text { PANEL } 1}$}

Data were first entered in person (wide) format. Records for both immigrant and non-immigrant participants were included in the one dataset so that Stata had the fullest information possible about the data prior to imputation.

A command that set the seed for the random number generator was then written, so that the results of the imputation could be replicated if needed.

ice immigrant sex esteem0 esteem1 esteem2 efficacy0 efficacyl efficacy 2 behavior0 behavior1 behavior2 grades0 grades1 grades 2 engage0 engagel engage2 adapt0 adapt1 adapt2 advers0 sesrisk0, saving(imputed_dataset) $\mathrm{m}(5)$ cmd(sesrisk0:regress)

The ICE procedure began with this step. All time-varying and time-invariant predictors and outcomes in the two growth models were included in this imputation. To ensure that the imputation model had the most information possible, participants' immigrant flag was also included, rather than running separate imputations for each of the two groups.

Although the within-individual covariation among the variables could not be accounted for perfectly, as previously noted, an attempt was made to partially deal with the collinearity of the repeated measures nested within individuals by including all variables in the analytic models in the imputation model. This decision was made in an effort to account for as much variation as possible within and between individuals. A similar approach was taken by Han (2008).

With respect to certain segments of this command:

- $\mathbf{m}(5)=$ the number of imputations

- $\quad$ saving (imputed_dataset) = the name for the final outputted data set (containing all five imputation datasets, plus the original data, merged into one master file)

- $\quad$ cmd (sesrisk0:regress) = ICE automatically decides what type a variable is, based on the variable's number of values it takes on. Stata's default was overridden to treat SESRisk0 as an ordinal variable, so it could instead be treated as continuous. 


\section{MULTIPLE IMPUTATION OF MISSING MULTILEVEL, LONGITUDINAL DATA}

use imputed dataset, clear

\section{PANEL 2}

After imputation, ice saved a copy of the new dataset (imputed dataset) in the current working directory. This command told Stata to open the new file. The new dataset contained all of the variables, plus two new variables: $\_m i$, an identifier for each observation, and $\_m j$, which indicated which imputed data file each row of the data belongs to ( 0 for the original data, and 1-5, respectively for each of the five new imputed data sets).

drop if $m j==0$

This command instructed Stata to drop the original data that still contained missing values ( $m j$ $==0$ ), keeping only the five newly-imputed data sets. Before running this command, check the descriptive statistics generated for the imputed data sets against the original data. Doing so will ensure that the imputed data indeed have no missing cases and that the descriptive statistics for the each of the variables in the imputed datasets make sense.

\section{gen adaptob $=$ adapt 0}

Because a later step involved restructuring the data from person (wide) format to person-period (long) format, a copy (adapt $0 b$ ) of the initial adaptability variable (adapt0) was created.

The adaptation variables were tricky in that they served either as time-invariant or time-varying variables, depending on the growth model. It was therefore necessary to ensure that, during the restructuring, the initial adaptability would be preserved and, in turn, treated as a time-invariant predictor alongside the time-varying adaptability variables.

reshape long adapt esteem efficacy behavior grades engage, i(id mj )

The data were restructured to person-period (long) format, because such a format is required for the growth modeling analyses (described in a later step).

With respect to a certain segment of this command:

- $\quad \mathrm{i}\left(\mathrm{id} \_\mathrm{mj}\right)=$ here, $i d$ and $m j$ served as our index variables. As UCLA Academic Technology Services' Statistical Consulting Group (n.d.a) notes, "Returning the data to long format has an added complication: we already have [multiple] rows of data for each [participant], one for each of the imputations. As a result, the variable $i d$ no longer uniquely identifies an observation. However, including both $i d$ and $\_m j$ as identifiers will uniquely identify each case."

recode j $(3=2) \quad(2=1) \quad(1=0)$

In restructuring the data to person-period (long) format, Stata automatically assigned the codes 1, 2, and 3 to represent each of the waves of data collection. This command allowed the recoding of waves as 0,1 , and 2 , respectively. 


\section{LLOYD, OBRADOVIĆ, CARPIANO \& MOTTI-STEFANIDI}

\section{rename _j wave}

\section{$\underline{\text { PANEL } 3}$}

In restructuring the data to person-period (long) format, Stata automatically named the wave variable $j$; however, the variable name wave was used for this study.

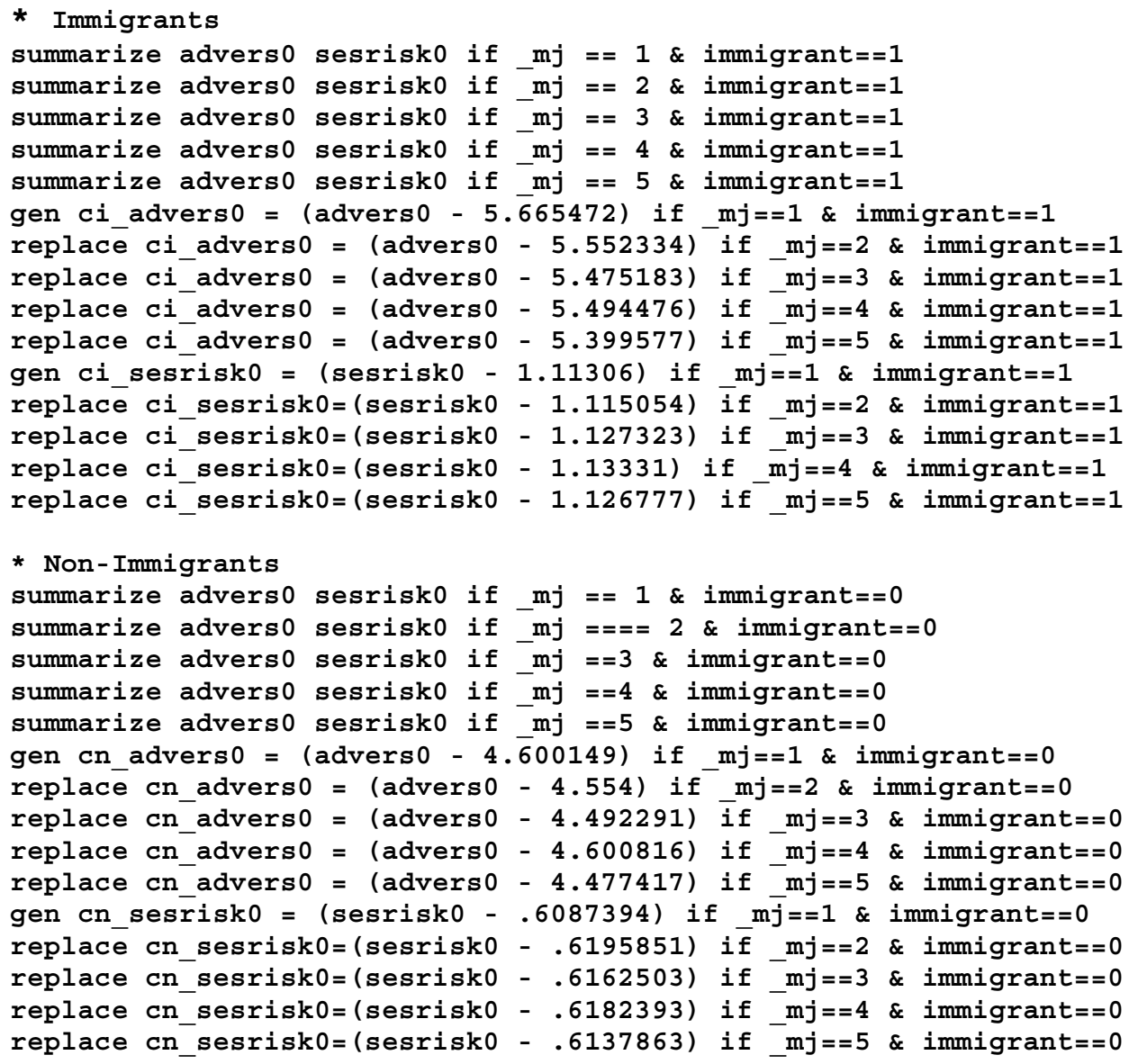

von Hippel (2009) recommended that centering of scores for a given variable be conducted prior to imputation in order to reduce collinearity and improve interpretation of the intercept. What is unclear is which problems, if any, are introduced if the mean that is being subtracted from the given value of a variable is being skewed by the variable's missing values.

For this reason, the scores of the moderating variables (initial adversity and initial SES risk) were grand-mean centered post-imputation, rather than pre-imputation. For brevity, specifics of each line of command in this step are not presented; the commands demonstrate that the respective variables' scores were centered for each immigrant group (x 2) and each imputed data file (x 5), separately. 
gen waveadaptob = wave*adaptob

\section{$\underline{\text { PANEL } 4}$}

gen waveadvers 0 = wave*advers 0

gen wavesesrisk0 = wave*sesrisk0

Cross-level product terms were computed for subsequent growth modeling (i.e., Model 1).

* Model 1 / IMMIGRANTS

mim: xtmixed esteem wave female adaptob adverso sesrisk0 waveadapt0b waveadvers 0 wavesesrisk0 if immigrant==1, || id: wave, covariance (un) variance mim: xtmixed efficacy wave female adaptob adverso sesrisko waveadapt $0 \mathrm{~b}$ waveadvers 0 wavesesrisk0 if immigrant $==1,||$ id: wave, covariance (un) variance mim: xtmixed behavior wave female adaptob adverso sesrisk0 waveadaptob waveadvers 0 wavesesrisk0 if immigrant==1, || id: wave, covariance (un) variance mim: xtmixed grades wave female adaptob adverso sesrisk0 waveadapt $0 \mathrm{~b}$ waveadvers 0 wavesesrisk0 if immigrant $==1,||$ id: wave, covariance (un) variance mim: xtmixed engage wave female adaptob adverso sesrisk0 waveadapt $0 \mathrm{~b}$ waveadvers 0 wavesesrisk0 if immigrant $==1,||$ id: wave, covariance (un) variance

* Model 2 / IMMIGRANTS

mim: xtmixed esteem wave adapt if immigrant==1, || id: wave, covariance (un) variance

mim: xtmixed efficacy wave adapt if immigrant $==1,||$ id: wave, covariance (un) variance

mim: xtmixed behavior wave adapt if immigrant $==1,||$ id: wave, covariance (un) variance

mim: xtmixed grades wave adapt if immigrant $==1,||$ id: wave, covariance (un) variance mim: xtmixed engage wave adapt if immigrant $==1,||$ id: wave, covariance(un) variance

* Model 1 / NON-IMMIGRANTS

mim: xtmixed esteem wave female adaptob advers0 sesrisk0 waveadapt0b waveadvers 0 wavesesrisk0 if immigrant==0, || id: wave, covariance(un) variance mim: xtmixed efficacy wave female adaptob adverso sesrisk0 waveadapt0b waveadvers 0 wavesesrisk0 if immigrant==0, || id: wave, covariance(un) variance mim: xtmixed behavior wave female adaptob advers0 sesrisk0 waveadapt0b waveadvers 0 wavesesrisk0 if immigrant==0, || id: wave, covariance(un) variance mim: xtmixed grades wave female adaptob advers0 sesrisk0 waveadapt0b waveadvers 0 wavesesrisk0 if immigrant==0, || id: wave, covariance(un) variance mim: xtmixed engage wave female adaptob advers0 sesrisk0 waveadapt $0 \mathrm{~b}$ waveadvers 0 wavesesrisk0 if immigrant==0, || id: wave, covariance(un) variance 


\section{LLOYD, OBRADOVIĆ, CARPIANO \& MOTTI-STEFANIDI}

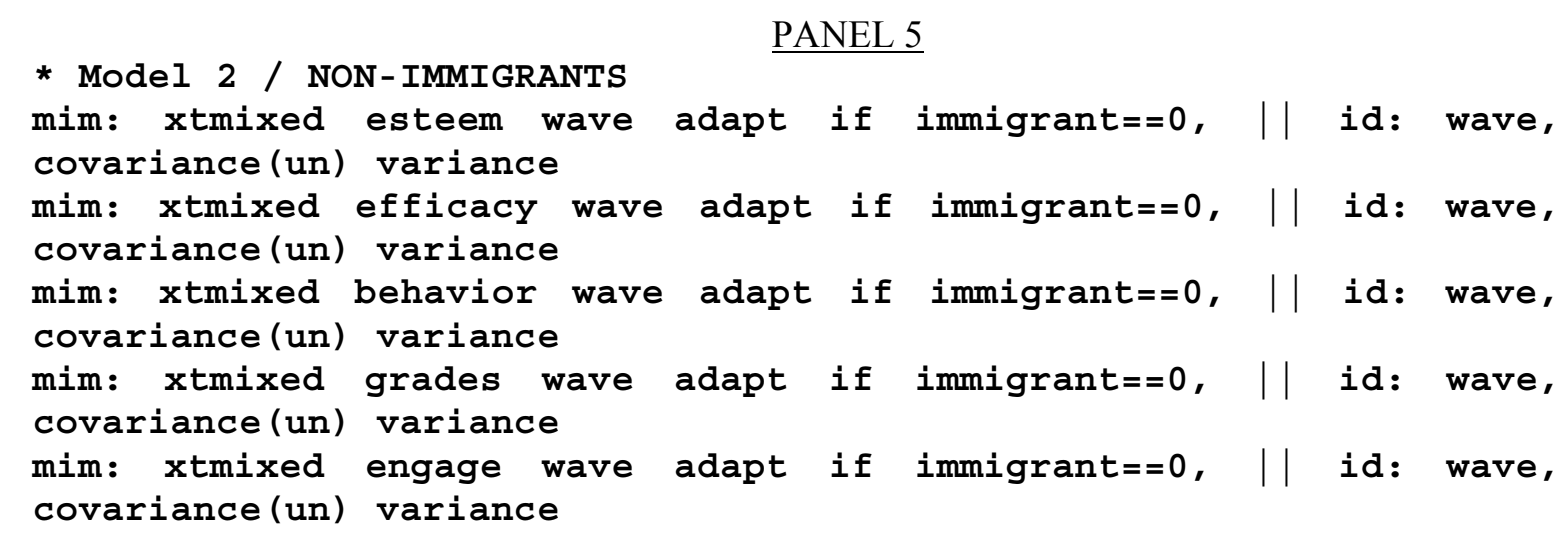

These commands pertain to the two growth models run for each of the outcomes (x 5), stratified by immigrant status (x 2).

With respect to certain segments of this command:

- $\operatorname{mim}=$ a Stata prefix that pools the results of the five imputed data files

- $\quad$ xtmixed = linear mixed-effect module of Stata

- id: wave $=i d$ is the clustering variable; adding wave immediately afterwards indicated an associated random effect

Aiken, L. S., West, S. G., Sechrest, L., \& Reno, R. R. (1990). Graduate training in statistics, methodology, and measurement in psychology: A survey of $\mathrm{PhD}$ programs in North America. American Psychologist, 45, 721-734.

Allison, P. D. (2002). Missing data. Thousand Oaks, CA: Sage.

Bickel, R. (2007). Multilevel analysis for applied research: It's just regression. New York, NY: Guilford Press.

Carpenter, J. R., Goldstein, H., Kenward, M. G. (2011). REALCOM-IMPUTE Software for multilevel multiple imputation with mixed response types. Journal of Statistical Software, 45(5), 1-14.

Collins, L. M., Schafer, J. L., \& Kam, C-M. (2001). A comparison inclusive and restrictive strategies in modern missing data procedures. Psychological Methods, 6(4), 330351.

Diez-Roux, A. V. (2002). A glossary for multilevel analysis. Journal of Epidemiology and Community Health, 56, 588-594.
Graham, J. W. (2009). Missing data analysis: Making it work in the real world. Annual Review of Psychology, 60, 549-576.

Han, W. J. (2008). The academic trajectories of children of immigrants and their school environments. Developmental Psychology, 44(6), 1572-1590.

Holt, J. K. (2008). Modeling growth using multilevel and alternative approaches. In Multilevel Analysis of Educational Data. Volume 3 of the Quantitative Methods in Education and the Behavioral Sciences: Issues, Research and Teaching Series, A. A. O'Connell \& D. B. McCoach (Eds.), 111-159. Charlotte, NC: Information Age Publishing.

Jeličić, H., Phelps, E., \& Lerner, R. M. (2009). Use of missing data methods in longitudinal studies: The persistence of bad practices in developmental psychology. Developmental Psychology, 45(4), 1195-1199.

Johnson, D. R., \& Young, R. (2011). Toward best practices in analyzing datasets with missing data: Comparisons and recommendations. Journal of Marriage and Family, 73, 926-945. 


\section{MULTIPLE IMPUTATION OF MISSING MULTILEVEL, LONGITUDINAL DATA}

Kelly, D., \& Smith, C. (2011). Bayesian Inference for Probabilistic Risk Assessment: A Practitioner's Guidebook. London: SpringerVerlag.

Little, R. J. A. (1995). Modeling the drop-out mechanism in repeated-measures studies. Journal of the American Statistical Association, 90, 1112-1121.

Little, R. J. A., \& Rubin, D. B. (2002). Statistical analysis with missing data, $2^{\text {nd }} \mathrm{Ed}$. New York, NY: Wiley.

Long, J. D., \& Pellegrini, A. D. (2003). Studying change in dominance and bullying with linear mixed models. School Psychology Review, 32, 401-417.

Merenda, P. F. (2003). Measurements in the future: Beyond the $20^{\text {th }}$ century. Psychological Reports, 92, 209-217.

Motti-Stefanidi, F., \& Asendorpf, J. B. (2012). Perceived discrimination of immigrant youth living in Greece: How does group discrimination translate into personal discrimination? [Special Issue], European Psychologist, 17(2), 93-104.

Motti-Stefanidi, F., Pavlopoulos, V., Obradović, J., Dalla, M., Takis, N., Papathanasiou, A., \& Masten, A. (2008a). Immigration as a risk factor for adolescent adaptation in Greek urban schools. European Journal of Developmental Psychology, 5(2), 235-261.

Motti-Stefanidi, F., Pavlopoulos, V., Obradović, J., \& Masten, A. S. (2008b). Acculturation and adaptation of immigrant adolescents in Greek urban schools. International Journal of Psychology, 43(1), 4558.

Motti-Stefanidi, F., Asendorpf, J. B., \& Masten, A. S. (2012). The adaptation and psychological well-being of adolescent immigrants in Greek schools: A multilevel, longitudinal study of risks and resources. [Special Issue], Development and Psychopathology. 24(2), 451-473.

Murray-Close, D., Ostrov, J. M., Crick, N. R. (2007). A short-term longitudinal study of growth of relational aggression during middle childhood: Associations with gender, friendship intimacy, and internalizing problems. Development and Psychopathology, 19, 187203.
Obradović, J., Lloyd, J. E. V., \& MottiStefanidi, F. (in preparation). Adaptation of immigrant and non-immigrant youth living in Greece: The role of family adaptability.

Raudenbush, S. W., \& Bryk, A. S. (2002). Hierarchical linear models: Applications and data analysis methods $\left(2^{\text {nd }}\right.$ Ed.). Newbury Park, CA: Sage.

Royston, P. (2004). Multiple imputation of missing values: update. Stata Journal, 5(2), 188-201.

Royston, P. (2005). Multiple imputation of missing values: Update of ICE. Stata Journal 5(4), 527-536.

Royston P., \& White, I. R. (2011). Multiple Imputation by Chained Equations (MICE): Implementation in Stata. Journal of Statistical Software, 45(4), 1-20.

Rubin, D. B. (1987). Multiple imputation for nonresponse in surveys. New York, NY: Wiley,

Schafer, J. L. (1999). Multiple imputation: A primer. Statistical Methods in Medical Research, 8, 3-15.

Schafer, J. L. (2001). Multiple imputation with PAN. In New methods for the analysis of change, A. G. Sayer and L. M. Collins (Eds.), 355-377. Washington, DC: American Psychological Association.

Schafer, J. L., \& Graham, J. W. (2002) Missing data: our view of the state of the art. Psychological Methods, 7, 147-177.

Schafer, J. L., Yucel, R. M. (2002). Computational strategies for multivariate linear mixed-effects models with missing values. Journal of Computational and Graphical Statistics, 11, 437-457.

Singer, J. D., \& Willett, J. B. (2003). Applied longitudinal data analysis: Modeling change and event occurrence. New York: Oxford University Press.

StataCorp. (2009). Stata Statistical Software: Release 11. College Station, TX: StataCorp LP.

StataCorp. (2011). Stata statistical software: Release 12. College Station, TX: StataCorp LP. 


\section{LLOYD, OBRADOVIĆ, CARPIANO \& MOTTI-STEFANIDI}

UCLA Academic Technology Services' Statistical Consulting Group (n.d.a). Stata FAQ: How can I perform multiple imputation on longitudinal data using ICE? Retrieved March 12, 2012, from http:// www.ats.ucla.edu/stat/stata/faq/mi_longitudinal. htm.

UCLA Academic Technology Services' Statistical Consulting Group (n.d.b). Stata library: Multiple imputation using ICE introduction. Retrieved March 12, 2012, from http://www.ats.ucla.edu/stat/stata/library/ice.htm von Hippel, P. T. (2007). Regression with missing Ys: An improved strategy for analyzing multiply-imputed data. Sociological Methodology, 37(1), 83-117. von Hippel, P. T. (2009). How to impute interactions, squares, and other transformed variables. Sociological Methodology, 39, 265291.

White, I.R., Royston, P., \& Wood, A. M. (2011). Multiple imputation using chained equations: Issues and guidance for practice. Statistics in Medicine, 30, 377-399

Yuan, Y. (2011). Multiple imputation using SAS software. Journal of Statistical Software, 45(6), 1-25.

Zaidman-Zait, A., \& Zumbo, B. D. (2013). Can multilevel (HLM) models of change over time adequately handle missing data? Journal of Educational Research and Policy Studies, 13(1), 18-31. 


\section{Pro Fortran 2013}

\section{Faster Code - Easier to Use - More Complete - Better Support}

\section{Free World Class Technical Assistance Includes LIVE Tech Support via Phone!}

\section{Absoft Pro Fortran - A Complete Fortran Solution}

\begin{tabular}{|c|c|c|c|c|}
\hline Fortran & IDE/GUI & SMP Vector & HPC & Graphics \\
\hline $\begin{array}{l}\text { Compiler } \\
\text { Suite }\end{array}$ & $\begin{array}{c}\text { Fortran /C } \\
\text { Tools }\end{array}$ & $\begin{array}{l}\text { Graphical } \\
\text { Analyzer }\end{array}$ & $\begin{array}{l}\text { Numerical } \\
\text { Libraries }\end{array}$ & $\begin{array}{c}\text { 2D/3D } \\
\text { HDF }\end{array}$ \\
\hline
\end{tabular}

F2008 extensions, AVX, OpenMP, auto parallel and vectorization features generate industry leading performance on both AMD and Intel based systems. The Absoft GUI/IDE for Fortran/C includes Fx3 graphical debugger, SMP/ Vector tools, AWE, HPC numerical libraries plus graphics which simplify application development and data display.

Absoft Pro Fortran includes cpu specific optimizers for both AMD \& Intel processors The Polyhedron Fortran Benchmark Suite results below demonstrate Absoft Pro Fortran's auto-parallel code runs $\mathbf{1 8 \%}$ FASTER than Intel's auto-parallel code on AMD.

\section{Polyhedron Fortran Benchmark Suite* - Shorter is Faster}

Absoft Pro Fortran Auto-Parallel runs 18\% FASTER than Intel on AMD

Absoft 2013 With Auto-Parallelization $34.64 \mathrm{sec}$

Intel 2013 With Auto-Parallelization

$42.22 \mathrm{sec}$

Tests run by Absoft on AMD/Piledriver/Linux using Polyhedron recommended options - See Reverse for details.

\section{Absoft IDE/GUI - Build Your Fortran/C Applications Faster}

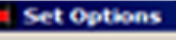

Intuitive, graphical environment means faster build, optimize, analyze, and debug cycles for threaded applications. OpenMP and MPI control features included. Same look, feel and functionality on Windows, Mac OS and Linux.

\section{Graphical SMP \& Vector Analyzer - Visual Tuning in Real Time}

- Aomeiponterse

O 17 locol 1 at

17 nisloegs

20 intenslu

20 istond L th

- 20 nis 1009 ,

Performance tuning using visual feedback and context specific advice on loop parallelization and vector optimizations. Visual

feedback at compile time, not runtime, speeds development.

\section{Absoft HPC Scientific Enginering Numerical Library}

Speed application development using pre-built, highly optimized, well tested industry standard core routines, threaded and vectorized math functions and FFTs designed for workstation and cluster environments. Royalty free.

\section{Absoft Visualization Libraries - 2D/3D and HDF4 \& HDF5}

Easily display your scientific data using a wide variety of 2D/3D full-color display formats and extended character sets. HDF, developed by NCSA for very large data sets includes Fortran and $C$ calling interfaces and is a standard used world-wide.
Free 3rd Party Plug-Ins

\begin{tabular}{|c|c|}
\hline GPU & $\mathrm{C} / \mathrm{C}++$ \\
\hline nVidia & Windows \\
CUDA & Mac \\
Libraries & Linux \\
\hline
\end{tabular}

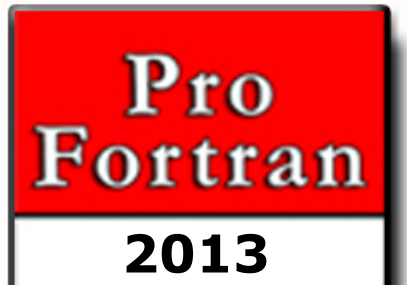

Software Development Tools

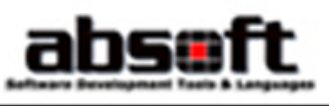

What do Absoft customers say about Pro Fortran?

I used Pro Fortran to write, debug, test and apply about 45,000 lines of code from the MS environment and it is, in a word: terrific. It is fast, has good diagnostics, builds great executable code and the IDE has a load of extra features that are invaluable. Beyond that, your support has been timely, helpful and detailed.

\section{D.J. Chadwick, President,} SAFE, Inc.

I prefer the Absoft interface because it is simple and easy to use. MS Visual Studio is HUGE and contains so many things that I don't need.

Troy Feese

EDI 


\title{
Classical FORTRAN \\ Programming for Engineering and Scientific Applications SECOND EDITION
}

\author{
by Michael Kupferschmid
}

CRC Press, 2009, ISBN 978-1-4200-5907-6, 562+xvi pages, hard covers, acid-free paper www.amazon.com/Classical-Fortran-Programming-Engineering-Applications/dp/1420059076

This book is a college text, self-study guide, and reference about computer programming in Classical ForTRAN, a simple subset language carefully chosen for its utility in numerical computing. In Chapters 1-6 (87 pages) the reader gets a working introduction; Chapters 7-10 (82 pages) cover the remaining topics that most statisticians need for casual programming. Succeeding chapters provide in-depth treatment of traditional ForTRAN memory management techniques; program design, documentation, and coding style; archaic, unusual, and dangerous usages (including the parts of FORTRAN-77 left out of the subset); program development in the Unix environment; performance measurement and optimization; vector processing; parallel computation with the Message Passing Interface library; and selected features of Fortran-90 and High Performance Fortran. The book concludes with a collection of handy utility routines, an extensive bibliography, and a 36-page concept-driven index. The emphasis throughout is on writing real programs for actual applications, with special attention to logical correctness, numerical accuracy, and run-time performance.

\section{OTHER FEATURES}

a conversational, classroom-proven style that is easy to read and understand

numerous case studies and examples

$\square$ unusually detailed coverage of floating-point arithmetic

$\square$ illustration of several debugging techniques

description of how to build and use Unix code libraries

illustration of how to write custom Unix man pages

software and techniques for the precise measurement of CPU time

expert advice on dealing with troublesome legacy codes

577 widely-varied end-of-chapter Exercises

Solutions Manual with extra Exercises available to instructors

TARGET AUDIENCE

statisticians, mathematicians, and economists who also write programs

graduate students whose research involves scientific programming

undergraduates studying or using numerical methods

$\square$ advanced high-school students who know some science and mathematics

$\square$ practicing engineers and scientists who need to do numerical calculations

programmers who need to maintain or translate legacy ForTRAN codes 


\section{The Rhyme of An Ancient Programmer}

by

Mike Kupferschmid

Data, data everywhere, but not a thought to think; truth is in the numbers, hidden neat.

Some model might enlighten us if we could make the link; statistics to the rescue would be sweet.

A simple calculation, that is all we need to do, arithmetic enough to lay us low.

Too much by far for this poor head, but if not me then who? Computing is the only way I know.

Fancy programs, slow and slick, priced on every rung; we might just even make our problem fit.

Or code ourselves from scratch in some arcane but stylish tongue. Computer science needed? Quite a bit!

But some of us are simple souls and only want to see how we can write a useful program quick.

Then we can complete our work and find that secret key. Classic ForTRAN might just do the trick. 


\section{Instructions For Authors}

Follow these guidelines when submitting a manuscript:

1. JMASM uses a modified American Psychological Association style guideline.

2. Submissions are accepted via e-mail only. Send them to the Editorial Assistant at ea@jmasm.com. Provide name, affiliation, address, e-mail address, and 30 word biographical statements for all authors in the body of the email message.

3. There should be no material identifying authorship except on the title page. A statement should be included in the body of the e-mail that, where applicable, indicating proper human subjects protocols were followed, including informed consent. A statement should be included in the body of the e-mail indicating the manuscript is not under consideration at another journal.

4. Provide the manuscript as an external e-mail attachment in MS Word for the PC format only. (Wordperfect and .rtf formats may be acceptable - please inquire.) Please note that Tex (in its various versions), Exp, and Adobe .pdf formats are designed to produce the final presentation of text. They are not amenable to the editing process, and are NOT acceptable for manuscript submission.

5. The text maximum is 20 pages double spaced, not including tables, figures, graphs, and references. Use 11 point Times New Roman font.

6. Create tables without boxes or vertical lines. Place tables, figures, and graphs "in-line", not at the end of the manuscript. Figures may be in .jpg, tif, .png, and other formats readable by Adobe Photoshop.

7. The manuscript should contain an Abstract with a 50 word maximum, following by a list of key words or phrases. Major headings are Introduction, Methodology, Results, Conclusion, and References. Center headings. Subheadings are left justified; capitalize only the first letter of each word. Sub-subheadings are left-justified, indent optional. Do not number headings or subheadings.

8. Number all formulas, tables, figures, and graphs, but do not use italics, bold, or underline.

9. Do not use underlining in the manuscript. Do not use bold, except for (a) matrices, or (b) emphasis within a table, figure, or graph. Do not number references. Do not use footnotes or endnotes.

10. In the References section, do not put quotation marks around titles of articles or books. Capitalize only the first letter of books. Italicize journal or book titles, and volume numbers. Use " $\&$ " instead of "and" in multiple author listings.

11. Suggestions for style: Instead of "I drew a sample of 40 " write "A sample of 40 was selected". Use "although" instead of "while", unless the meaning is "at the same time". Use "because" instead of "since", unless the meaning is "after". Instead of "Smith (1990) notes" write "Smith (1990) noted". Do not strike spacebar twice after a period.

\section{Print Subscriptions}

Print subscriptions including postage for professionals are US \$95 per year; for graduate students are US $\$ 47.50$ per year; and for libraries, universities and corporations are US \$195 per year. Subscribers outside of the US and Canada pay a US \$10 surcharge for additional postage. Online access is currently free at http://www.jmasm.com/. Mail subscription requests with remittances to JMASM, P. O. Box 48023, Oak Park, MI, 48237. Email journal correspondence, other than manuscript submissions, to ea@jmasm.com.

\section{Notice To Advertisers}

Send requests for advertising information to ea@jmasm.com. 